NUREG/CR-2907

BNL-NUREG-51581

Vol. 10

Radioactive Materials Released from Nuclear Power Plants

Annual Report 1989

Prepared by J. Tichler, K. Norden, J. Congemi

Brookhaven National Laboratory

Prepared for

U.S. Nuclear Regulatory Commission 


\section{AVAILABILITY NOTICE}

Availability of Reference Materials Cited in NRC Publications

Most documents cited in NRC publications will be avallable from one of the following sources:

1. The NRC Public Document Room, 2120 L Street, NW., Lower Level, Washington, DC 20555

2. The Superintendent of Documents, U.S. Government Printing Office, P.O. Box 37082, Washington, DC 20013-7082

3. The National Technical Information Service, Springfield, VA 22161

Although the listing that follows represents the majority of documents cited in NRC publications, It is not intended to be exhaustive.

Referenced documents avallable for inspection and copying for a fee from the NRC Public Document Room Include NRC correspondence and internal NRC memoranda; NRC bulletins, circulars, information notices, Inspection and Investigation notices; licensee event reports; vendor reports and correspondence; Commission papers; and applicant and licensee documents and correspondence.

The following documents in the NUREG series are available for purchase from the GPO Sales Program: formal NRC staff and contractor reports, NRC-sponsored conference proceedings, international agreement reports, grant publications, and NRC booklets and brochures. Also available are regulatory guides, NRC regulations in the Code of Federal Regulations, and Nuclear Regulatory Commission Issuances.

Documents avallable from the National Technical Information Service include NUREG-series reports and technical reports prepared by other Federal agencles and reports prepared by the Atomic Energy Commission, forerunner agency to the Nuclear Regulatory Commission.

Documents available from public and special technical libraries include all open literature items, such as books, Journal articles, and transactions. Federal Register notices, Federal and State legislation, and congressional reports can usually be obtained from these libraries.

Documents such as theses, dissertations, foreign reports and translations, and non-NRC conference proceedings are available for purchase from the organization sponsoring the publication cited.

Single copies of NRC draft reports are available free, to the extent of supply, upon written request to the Office of Administration, Distribution and Mail Services Section, U.S. Nuclear Regulatory Commission, Washington, DC 20555.

Copies of industry codes and standards used in a substantive manner in the NRC regulatory process are maintained at the NRC Library, 7920 Norfolk Avenue, Bethesda, Maryland, for use by the public. Codes and standards are usually copyrighted and may be purchased from the originating organization or, if they are American National Standards, from the American National Standards Institute, 1430 Broadway, New York, NY 10018.

\section{DISCLAIMER NOTICE}

This report was prepared as an account of work sponsored by an agency of the United States Government. Neither the United States Government nor any agency thereof, or any of their employees, makes any warranty, expressed or implied, or assumes any legal liability of responsibility for any third party's use, or the results of such use, of any information, apparatus, product or process disclosed in this report, or represents that its use by such third party would not infringe privately owned rights. 


\section{DISCLAIMER}

This report was prepared as an account of work sponsored by an agency of the United States Government. Neither the United States Government nor any agency Thereof, nor any of their employees, makes any warranty, express or implied, or assumes any legal liability or responsibility for the accuracy, completeness, or usefulness of any information, apparatus, product, or process disclosed, or represents that its use would not infringe privately owned rights. Reference herein to any specific commercial product, process, or service by trade name, trademark, manufacturer, or otherwise does not necessarily constitute or imply its endorsement, recommendation, or favoring by the United States Government or any agency thereof. The views and opinions of authors expressed herein do not necessarily state or reflect those of the United States Government or any agency thereof. 


\section{DISCLAIMER}

Portions of this document may be illegible in electronic image products. Images are produced from the best available original document. 
NUREG/CR--2907-Vol.10

TI93 000761

\section{Radioactive Materials Released from Nuclear Power Plants}

Annual Report 1989

Manuscript Completed: August 1992

Date Published: September 1992

Prepared by

J. Tichler, K. Norden, J. Congem

Brookhaven National Laboratory

Upton, NY 11973

\section{Prepared for}

Office of Information Resources Management

U.S. Nuclear Regulatory Commission

Washington, DC 20555

NRC FIN B2234 
1. "Report on Releases of Radioactivity in Effluents and Solid Wastes from Nuclear Power Plants for 1972," Directorate of Regulatory Operations, August 1973.

2. "Summary of Radioactivity Releases in Effluents from Nuclear Power Plants During 1973," NUREG-75/001, January 1975.

3. "Radioactive Materials Released from Nuclear Power Plants, 1974," NUREG-0077, June 1976.

4. "Radioactive Materials Released from Nuclear Power Plants, 1975," NUREG-0218, March 1977.

5. "Radioactive Materials Released from Nuclear Power Plants, 1976," NUREG-0367, March 1978.

6. "Radioactive Materials Released from Nuclear Power Plants, 1977," NUREG-0521, January 1979.

7. "Radioactive Materials Released from Nuclear Power Plants, 1978," NUREG/CR-1497, BNL-NUREG-51192, March 1981.

8. "Radioactive Materials Released from Nuclear Power Plants, 1979," NUREG/CR-2227, BNL-NUREG-51416, November 1981.

9. "Radioactive Materials Released from Nuclear Power Plants, 1980," NUREG/CR-2907, BNL-NUREG-51581, Vo1. 1, January 1983.

10. "Radioactive Materials Released from Nuclear Power Plants, 1981," NUREG/CR-2907, BNL-NUREG-51581, Vol. 2, June 1984.

11. "Radioactive Materials Released from Nuclear Power Plants, 1982," NUREG/CR-2907, BNL-NUREG-51581, Vol. 3, February 1986.

12. "Radioactive Materials Released from Nuclear Power Plants, 1983," NUREG/CR-2907, BNL-NUREG-51581, Vol. 4, August 1986.

13. "Radioactive Materials Released from Nuclear Power Plants, 1984," NUREG/CR-2907, BNL-NUREG-51581, Vo1. 5, August 1987.

14. "Radioactive Materials Released from Nuclear Power Plants, 1985," NUREG/CR-2907, BNL-NUREG-51581, Vo1. 6, January 1988.

15. "Radioactive Materials Released from Nuclear Power Plants, 1986," NUREG/CR-2907, BNL-NUREG-51581, Vo1. 7, November 1988.

16. "Radioactive Materials Released from Nuclear Power Plants, 1987," NUREG/CR-2907, BNL-NUREG-51581, Vo1. 8, October 1989.

17. "Radioactive Materials Released from Nuclear Power Plants, 1988," NUREG/CR-2907, BNL-NUREG-51581, Vo1. 9, July 1991. 


\section{ABSTRACT}

Releases of radioactive materials in airborne and liquid effluents from commercial light water reactors during 1989 have been compiled and reported. The summary data for the years 1970 through 1988 are included for comparison. Data on solid waste shipments as well as selected operating information have been included. This report supplements earlier annual reports issued by the former Atomic Energy Commission and the Nuclear Regulatory Commission. The 1989 release data are summarized in tabular form. Data covering specific radionuclides are summarized. 



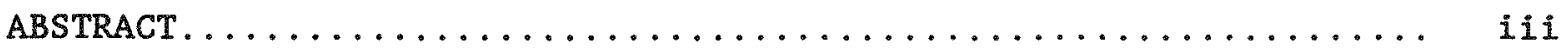

LIST OF TABLES ................................

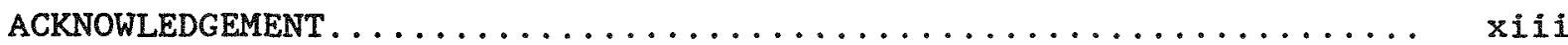

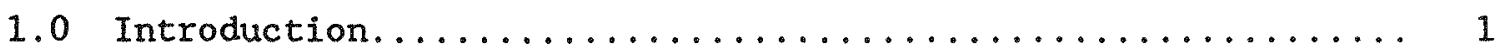

1.1 Purpose.............................. 1

1.2 scope................................ 1

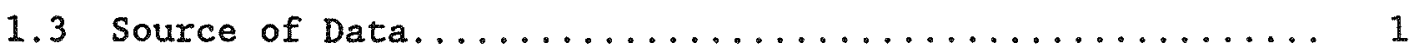

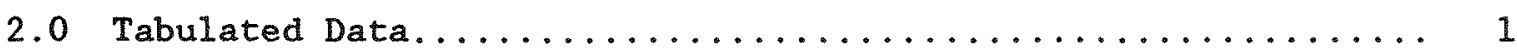

2.1 Airborne and Liquid Effluents................. 1

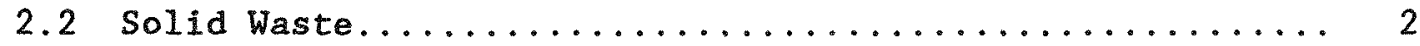

2.3 Energy Generation.......................... 2

2.4 Individual Plant Summaries................. 2

2.5 Numerical Notation......................... 3

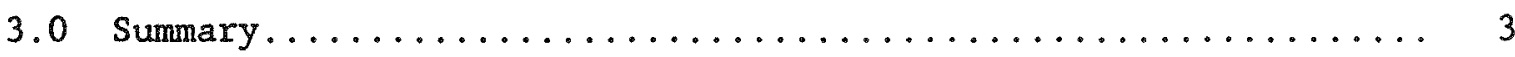

Appendix A - Individual Plant Summaries

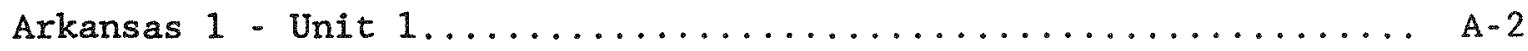

Arkansas $1-$ Unit $2 \ldots \ldots \ldots \ldots \ldots \ldots \ldots \ldots \ldots \ldots \ldots \ldots \ldots$

Beaver Valley $1 \& 2 \ldots \ldots \ldots \ldots \ldots \ldots \ldots \ldots \ldots \ldots \ldots \ldots \ldots \ldots$

Beaver valley $1 \ldots \ldots \ldots \ldots \ldots \ldots \ldots \ldots \ldots \ldots \ldots \ldots \ldots \ldots \ldots \ldots$

Beaver Valley $2 \ldots \ldots \ldots \ldots \ldots \ldots \ldots \ldots \ldots \ldots \ldots \ldots \ldots \ldots \ldots \ldots \ldots$

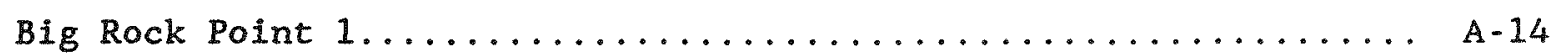

Braidwood $1 \ldots \ldots \ldots \ldots \ldots \ldots \ldots \ldots \ldots \ldots \ldots \ldots \ldots \ldots \ldots \ldots \ldots \ldots \ldots$

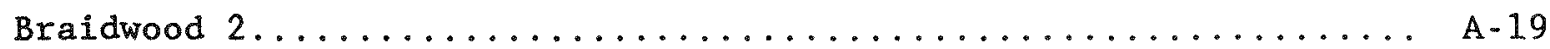


Page

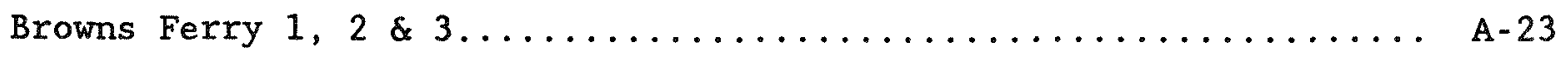

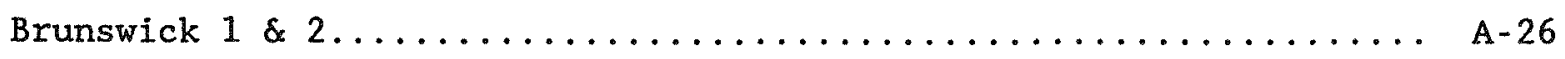

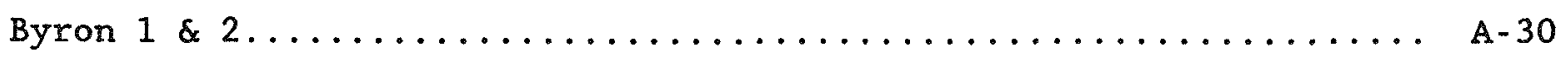

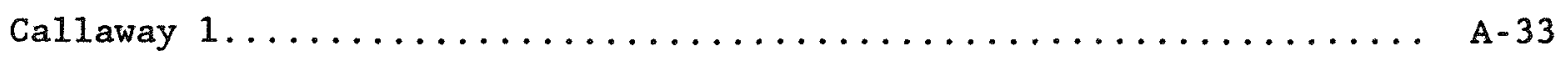

Calvert Cliffs $1 \& 2 \ldots \ldots \ldots \ldots \ldots \ldots \ldots \ldots \ldots \ldots \ldots \ldots \ldots \ldots$

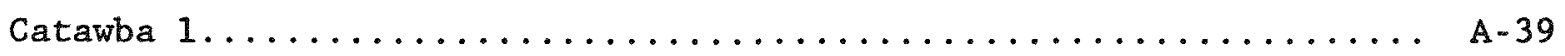

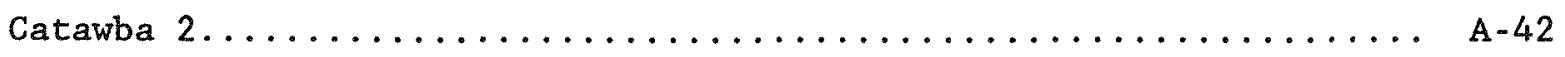

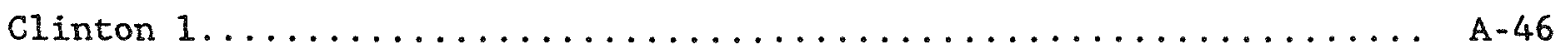

Cook $1 \& 2 \ldots \ldots \ldots \ldots \ldots \ldots \ldots \ldots \ldots \ldots \ldots \ldots \ldots \ldots \ldots \ldots \ldots \ldots \ldots \ldots \ldots$

Cooper.................................

Crystal River $3 \ldots \ldots \ldots \ldots \ldots \ldots \ldots \ldots \ldots \ldots \ldots \ldots \ldots \ldots \ldots \ldots \ldots \ldots$

Davis Besse $1 . \ldots \ldots \ldots \ldots \ldots \ldots \ldots \ldots \ldots \ldots \ldots \ldots \ldots \ldots \ldots \ldots \ldots \ldots \ldots$

Diablo Canyon $1 \& 2 \ldots \ldots \ldots \ldots \ldots \ldots \ldots \ldots \ldots \ldots \ldots \ldots \ldots \ldots \ldots$

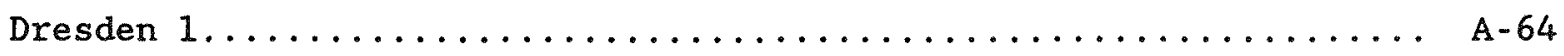

Dresden $2 \& 3 \ldots \ldots \ldots \ldots \ldots \ldots \ldots \ldots \ldots \ldots \ldots \ldots \ldots \ldots \ldots \ldots \ldots \ldots \ldots$

Dresden $1,2 \& 3 \ldots \ldots \ldots \ldots \ldots \ldots \ldots \ldots \ldots \ldots \ldots \ldots \ldots \ldots \ldots \ldots \ldots$

Duane Arnold................................ A-68

Joseph M. Farley $1 \ldots \ldots \ldots \ldots \ldots \ldots \ldots \ldots \ldots \ldots \ldots \ldots \ldots \ldots \ldots \ldots$

Joseph M. Farley $2 \ldots \ldots \ldots \ldots \ldots \ldots \ldots \ldots \ldots \ldots \ldots \ldots \ldots \ldots \ldots$

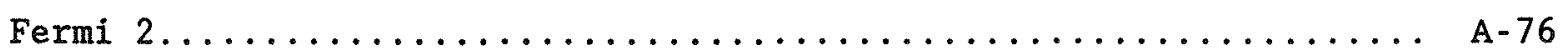

J.A. Fitzpatrick............................ A-79

Fort Calhoun $1 \ldots \ldots \ldots \ldots \ldots \ldots \ldots \ldots \ldots \ldots \ldots \ldots \ldots \ldots \ldots \ldots \ldots$

Fort st. $\operatorname{vrain} . \ldots \ldots \ldots \ldots \ldots \ldots \ldots \ldots \ldots \ldots \ldots \ldots \ldots \ldots \ldots \ldots$

R.E. Ginna.............................. A-86

Grand Gulf $1 \ldots \ldots \ldots \ldots \ldots \ldots \ldots \ldots \ldots \ldots \ldots \ldots \ldots \ldots \ldots \ldots$ 
Haddam Neck.

A-92

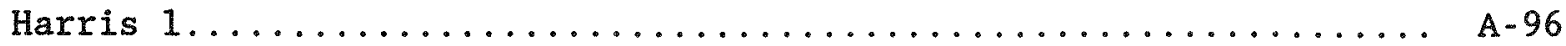

Edwin I. Hatch $1 \& 2 \ldots \ldots \ldots \ldots \ldots \ldots \ldots \ldots \ldots \ldots \ldots \ldots \ldots \ldots \ldots \ldots$

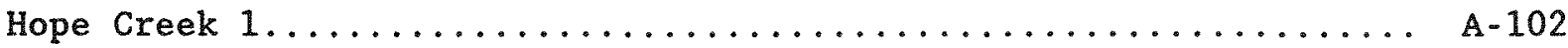

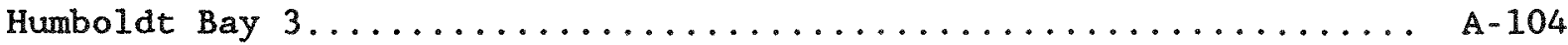

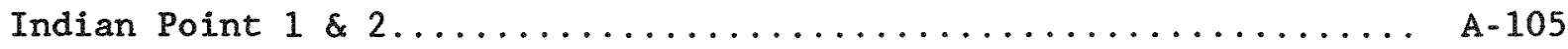

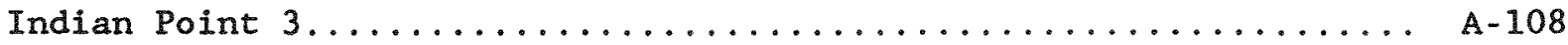

Kewaunee................................. A-111

LaCrosse....................................... A-115

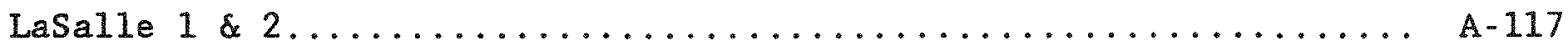

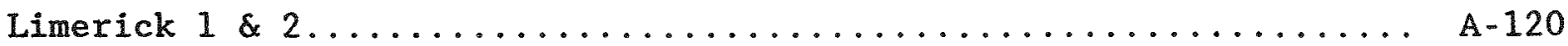

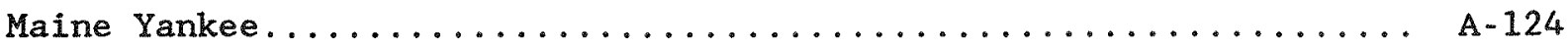

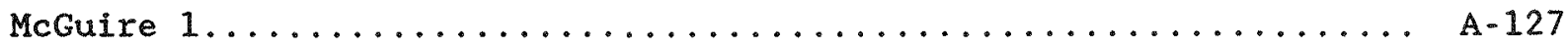

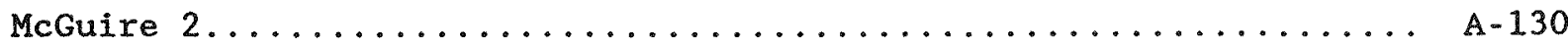

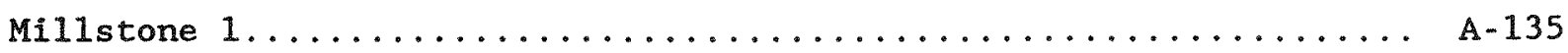

Millstone $2 \ldots \ldots \ldots \ldots \ldots \ldots \ldots \ldots \ldots \ldots \ldots \ldots \ldots \ldots \ldots \ldots \ldots \ldots$

Millstone $3 \ldots \ldots \ldots \ldots \ldots \ldots \ldots \ldots \ldots \ldots \ldots \ldots \ldots \ldots \ldots \ldots$ A. 141

Montice110.................................. A-144

Nine Mile Point $1 \ldots \ldots \ldots \ldots \ldots \ldots \ldots \ldots \ldots \ldots \ldots \ldots \ldots \ldots$ A. 147

Nine Mile Point $2 \ldots \ldots \ldots \ldots \ldots \ldots \ldots \ldots \ldots \ldots \ldots \ldots \ldots$. . . . . . . . . . . .

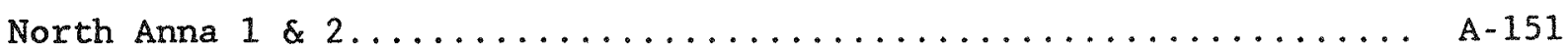

Oconee $1,2 \& 3 \ldots \ldots \ldots \ldots \ldots \ldots \ldots \ldots \ldots \ldots \ldots \ldots \ldots \ldots \ldots \ldots \ldots \ldots \ldots$

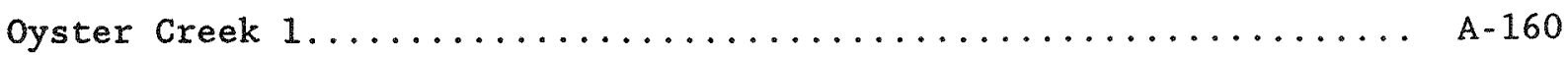

Palisades.................................. A-163

Palo Verde $1 \ldots \ldots \ldots \ldots \ldots \ldots \ldots \ldots \ldots \ldots \ldots \ldots \ldots \ldots \ldots \ldots \ldots \ldots$ 
Palo Verde $2 \ldots \ldots \ldots \ldots \ldots \ldots \ldots \ldots \ldots \ldots \ldots \ldots \ldots \ldots \ldots \ldots$

Palo verde $3 \ldots \ldots \ldots \ldots \ldots \ldots \ldots \ldots \ldots \ldots \ldots \ldots \ldots \ldots \ldots \ldots \ldots \ldots$

Peach Bottom $2 \& 3 \ldots \ldots \ldots \ldots \ldots \ldots \ldots \ldots \ldots \ldots \ldots \ldots \ldots \ldots \ldots$

Perry $1 . \ldots \ldots \ldots \ldots \ldots \ldots \ldots \ldots \ldots \ldots \ldots \ldots \ldots \ldots \ldots \ldots \ldots \ldots \ldots \ldots \ldots$

Pilgrim $1 \ldots \ldots \ldots \ldots \ldots \ldots \ldots \ldots \ldots \ldots \ldots \ldots \ldots \ldots \ldots \ldots \ldots \ldots \ldots \ldots$

Point Beach $1 \& 2 \ldots \ldots \ldots \ldots \ldots \ldots \ldots \ldots \ldots \ldots \ldots \ldots \ldots \ldots \ldots \ldots$

Prairie Island $1 \& 2 \ldots \ldots \ldots \ldots \ldots \ldots \ldots \ldots \ldots \ldots \ldots \ldots \ldots \ldots \ldots$

Quad Cities $1 \& 2 \ldots \ldots \ldots \ldots \ldots \ldots \ldots \ldots \ldots \ldots \ldots \ldots \ldots \ldots$

Rancho Seco $1 \ldots \ldots \ldots \ldots \ldots \ldots \ldots \ldots \ldots \ldots \ldots \ldots \ldots \ldots \ldots \ldots \ldots$

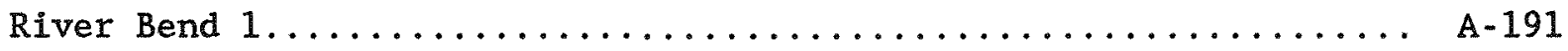

H.B. Robinson $2 \ldots \ldots \ldots \ldots \ldots \ldots \ldots \ldots \ldots \ldots \ldots \ldots \ldots \ldots \ldots$

Salem $1 \ldots \ldots \ldots \ldots \ldots \ldots \ldots \ldots \ldots \ldots \ldots \ldots \ldots \ldots \ldots \ldots \ldots \ldots \ldots$

Salem $2 \ldots \ldots \ldots \ldots \ldots \ldots \ldots \ldots \ldots \ldots \ldots \ldots \ldots \ldots \ldots \ldots \ldots \ldots \ldots$

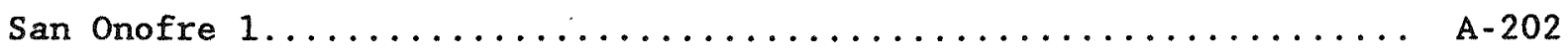

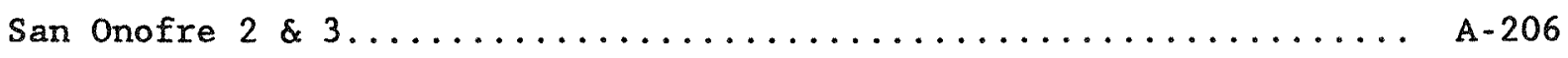

Seabrook $1 . \ldots \ldots \ldots \ldots \ldots \ldots \ldots \ldots \ldots \ldots \ldots \ldots \ldots \ldots \ldots \ldots \ldots$

Sequoyah $1 \& 2 \ldots \ldots \ldots \ldots \ldots \ldots \ldots \ldots \ldots \ldots \ldots \ldots \ldots \ldots \ldots \ldots \ldots \ldots \ldots \ldots$

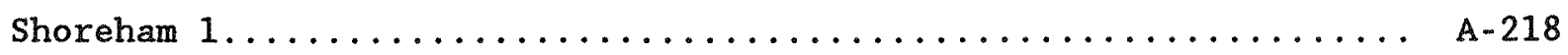

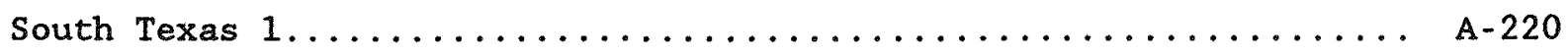

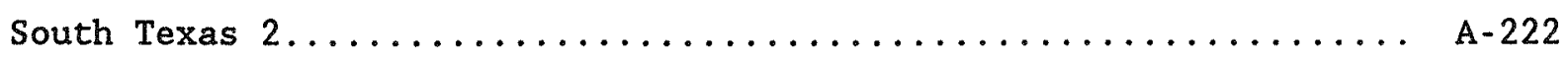

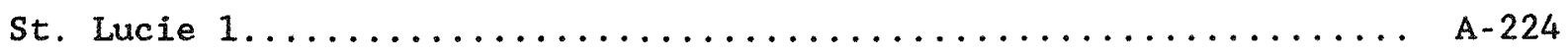

St. Lucie $2 \ldots \ldots \ldots \ldots \ldots \ldots \ldots \ldots \ldots \ldots \ldots \ldots \ldots \ldots \ldots \ldots \ldots \ldots \ldots \ldots$

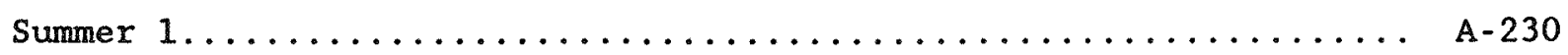

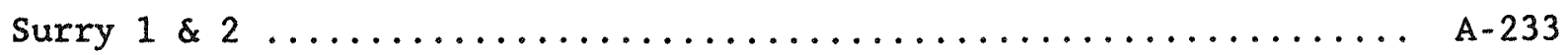

Susquehanna $1 \& 2 \ldots \ldots \ldots \ldots \ldots \ldots \ldots \ldots \ldots \ldots \ldots \ldots \ldots \ldots$ 
Three Mile Island $1 \ldots \ldots \ldots \ldots \ldots \ldots \ldots \ldots \ldots \ldots \ldots \ldots \ldots$ A 239

Three Mile Island $2 \&$ EPICOR II.................... A-24I

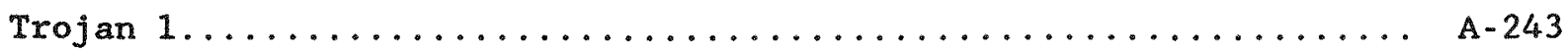

Turkey Point $3 \ldots \ldots \ldots \ldots \ldots \ldots \ldots \ldots \ldots \ldots \ldots \ldots \ldots \ldots$ A. 247

Turkey Point $4 \ldots \ldots \ldots \ldots \ldots \ldots \ldots \ldots \ldots \ldots \ldots \ldots \ldots \ldots \ldots \ldots$

Vermont Yankee $1 \ldots \ldots \ldots \ldots \ldots \ldots \ldots \ldots \ldots \ldots \ldots \ldots \ldots \ldots \ldots$ A. 252

Vogtle $1 \& 2 \ldots \ldots \ldots \ldots \ldots \ldots \ldots \ldots \ldots \ldots \ldots \ldots \ldots \ldots \ldots \ldots \ldots \ldots \ldots \ldots$

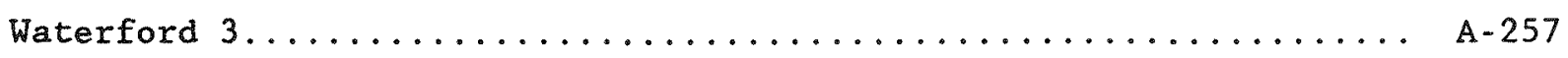

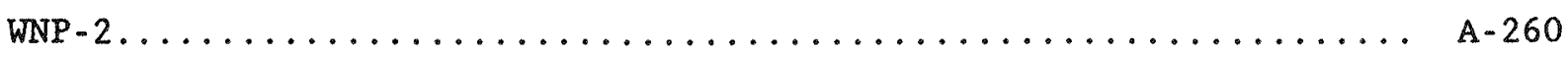

Wolf Creek $1 \ldots \ldots \ldots \ldots \ldots \ldots \ldots \ldots \ldots \ldots \ldots \ldots \ldots \ldots \ldots \ldots$

Yankee Rowe $1 \ldots \ldots \ldots \ldots \ldots \ldots \ldots \ldots \ldots \ldots \ldots \ldots \ldots \ldots \ldots \ldots \ldots$ A. 267

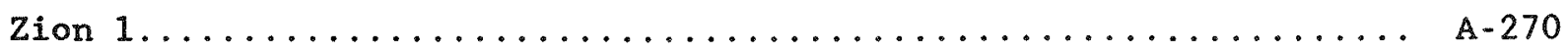

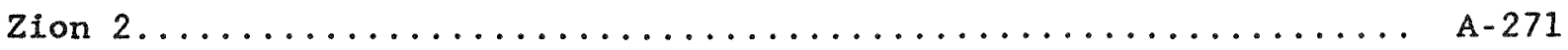

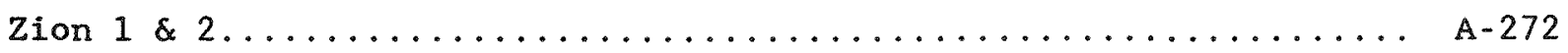



Table 1 Airborne Effluent Comparison by Year, Noble Gases, Boiling Water Reactors...................... 6

TABLE 2 Airborne Effluent Comparison by Year, Noble Gases, Pressurized Water Reactors.....................

TABLE 3 Airborne Effluent Comparison by Year, I-131 and Particulates, Boiling Water Reactors............... 12

TABLE 4 Airborne Effluent Comparison by Year, I-131 and Particulates, Pressurized Water Reactors.............. 14

TABLE 5 Liquid Effluent Comparison by Year, Tritium, Boiling Water Reactors....................... 18

TABLE 6 Liquid Effluent Comparison by Year, Tritium, Pressurized Water Reactors................... 20

TABLE 7 Liquid Effluent Comparison by Year, Mixed Fission and Activation Products, Boiling Water Reactors......... 24

TABLE 8 Liquid Effluent Comparison by Year, Mixed Fission and Activation Products, Pressurized Water Reactors......... 26

TABLE 9 Solid Waste Summary 1989, Boiling Water Reactors....... 30

TABLE 10 Solid Waste Summary 1989, Pressurized Water Reactors..... 31

TABLE 11 Solid Waste Comparison by Year, Boiling Water Reactors... 32

TABLE 12 Solid Waste Comparison by Year, Pressurized Water Reactors.......................... 36

TABLE 13 Net Electrical Energy Generation Comparison by Year, Boiling Water Reactors............... 40

TABLE 14 Net Electrical Energy Generation Comparison by Year, Pressurized Water Reactors.............. 42

TABLE 15 Thermal Energy Generation Comparison by Year, Boiling Water Reactors.................... 46

TABLE 16 Thermal Energy Generation Comparison by Year, Pressurized Water Reactors...................... 49 



\section{ACKNOWLEDGMENT}

Carmen Benkovitz was responsible for the original design of the computer data base in which the effluent data, beginning with the 1978 data, is stored. She was involved in the redesign of the data base when, in 1982, the decision was made to transfer the data base from one computer to another and to change the data base management system being used. 


\subsection{Introduction}

\section{$1.1 \quad$ Purpose}

This report, prepared annually for the staff of the U.S. Nuclear Regulatory Commission, presents measured data on radioactive materials in effluents released from licensed commercial reactor power plants. These data were reported by licensees for plant operations during 1989. This information supplements earlier annual reports issued by the former Atomic Energy Commission and Nuclear Regulatory Commission. ${ }^{1}$

\section{$1.2 \quad$ Scope}

Releases of radioactive materials are governed by $10 \mathrm{CFR}$ Part 20 and 50 and by limits established in the Technical specifications for each facility. The requirement for reporting effluent releases by nuclear power plant operators is described in $10 \mathrm{CFR} 50.36 \mathrm{a}$. Through its office of Nuclear Reactor Regulation, the Nuclear Regulatory Commission maintains a knowledge of radioactive releases from licensed nuclear reactors to ensure that they are within regulatory requirements. This report summarizes data from the licensed nuclear power plants that were declared by the utilities to be in commercial operation as of December 31, 1989. Data are included for several licensed facilities which are permanently or indefinitely shut down (Browns Ferry 1,2,3, Dresden 1, Humboldt Bay, Indian Point 1, LaCrosse and Three Mile Island 2), as well as some plants which were not yet in commercial operation in 1989 (Shoreham and Seabrook).

\subsection{Source of Data}

The information included in this report was obtained from data reported by the 1icensees. Individual licensee reports are available in the NRC Public Document Room, Gelman Building, 2120 L Street, Washington, D.C. 20555 and in local Public Document Rooms located near each licensed facility. Licensee reports varied in the format and extent of information provided.

Data from prior years used in the comparison tables were obtained from the seventeen previous annual summaries.

\section{$2.0 \quad$ Tabulated Data}

\subsection{Airborne and Liquid Effluents}

Tables 1 through 4 list for each reactor, the measured quantities of total noble gases and of I-131 and particulates (with half lives greater than 8 days) released in effluents to the atmosphere during each of the years 1970 through 1989. Tables 5 and 6 list the total measured quantities of tritium released in liquid effluents in each of the years. Tables 7 and 8 list the mixed fission and activation products not including noble gases, tritium and alpha released in liquid effluents in each of the years'.

1 Previous reports in this series are listed on page ii. 


\subsection{Solid Waste}

The total volumes, activity and the number of shipments of solid waste for each plant during 1989 are summarized in Tables 9 and 10 . A comparison for the years 1977 through 1989 is made in Tables 11 and 12 .

\subsection{Energy Generation}

Tables 13 and 14 present a summary of net electrical energy generated by each plant during 1978-1989. Tables 15 and 16 present a summary of the thermal energy generated by each plant during 1989 and previous years from 1972. The reader is cautioned against making simplistic comparisons of radioactive releases with the energy generated because of the many factors which affect the amount of radioactive materials released; factors include the condition of the fuel, primary system integrity, effluent and radioactive waste treatment systems, and the extent to which these systems are used.

\section{$2.4 \quad$ Individual Plant Summaries}

Individual plant summaries are presented in alphabetical order. The summaries include general plant information, power production, effluent and solid waste data, and a summary of specific radionuclides measured in effluents. When the only type of solid waste reported is type "A", this may be because the plant did not break solid waste into different types but reported all types together. The activity released for each nuclide for the year for both airborne and liquid effluents is calculated by summing releases for each quarter. More detailed summaries in the format of Regulatory Guide 1.21 such as were used in the 1978 report $^{2}$ can be made available since all the data for 1978-1989 are stored in a computerized data base.

A wide variation exists in the lists of specific radionuclides reported by utilities (1icensees). Individual licensee Technical Specifications require the measurement and reporting of specific sets of radionuclides and "any others identified." The disparities result becasue of differing analytical methods used by various licensees for their measurements, and their differing operating histories and effluent and emission control methods.

Copies of the summaries included in this report as well as the more detailed summaries maintained in the computer data base were submitted to the licensees for verification before publication. In most cases, the licensees responded either verifying the included data for their plants or providing corrections. Individuals interested in obtaining the more detailed summaries should contact the Office of Information Resources Management of the Nuclear Regulatory Commission.

2"Radioactive Materials Released from Nuclear Power Plants, 1978", NUREG/CR-1497, BNL-NUREG-51192, March, 1981. 


\section{$2.5 \quad$ Notation}

The following notation is used:

$$
\begin{aligned}
& 1.86 \mathrm{E}+06=1.86 \times 10^{6} \\
& 1.86 \mathrm{E}-03=1.86 \times 10^{-3} \\
& N / R=\text { Not Reported } \\
& N / D=\text { Not Detected } \\
& N / A=\text { Not Applicable } \\
& <\text { may actually mean } \leq
\end{aligned}
$$

\subsection{Summary}

Nearly all of the radioactive material reported as being released in effluents are from planned releases. Planned releases result from normal operation or from anticipated operational occurrences. The latter include unplanned releases of radioactive materials from miscellaneous actions such as equipment failure, operator error or procedure error; these releases are not of such consequence as to be considered an accident.

At present, it is difficult to compare effluent releases with those of previous years due to, among other contributors, variability in reporting structure and release requirements. Comparisons with respect to power generation are similarly difficult due to factors which strongly affect the releases such as level of fuel cladding defects, design features of plant radioactive waste treatment systems, operational occurrences and equipment performance.

Though perhaps not identifiable as an important factor at any specific plant from the data in this report, the generic improvement in fuel performance over the last several years has either reduced or has had the potential to reduce the amount of radioactive material released in effluents from most plants. In addition, at Boiling Water Reactors (BWRs), the reduction in the amount of airborne radioactive materials being released at some plants since the early and mid-1970s is due in large part to the installation of augmented offgas (AOG) systems, many of which were required to be installed to meet the provisions of Appendix I to 10CFR Part 50, which was promulgated by the NRC in May 1975. 

TABLES

- 5 - 
Table 1

Airborne Effuents Comparison By Year

Boiling Water Reactors

Fission and Activation Cases (Total Curies)

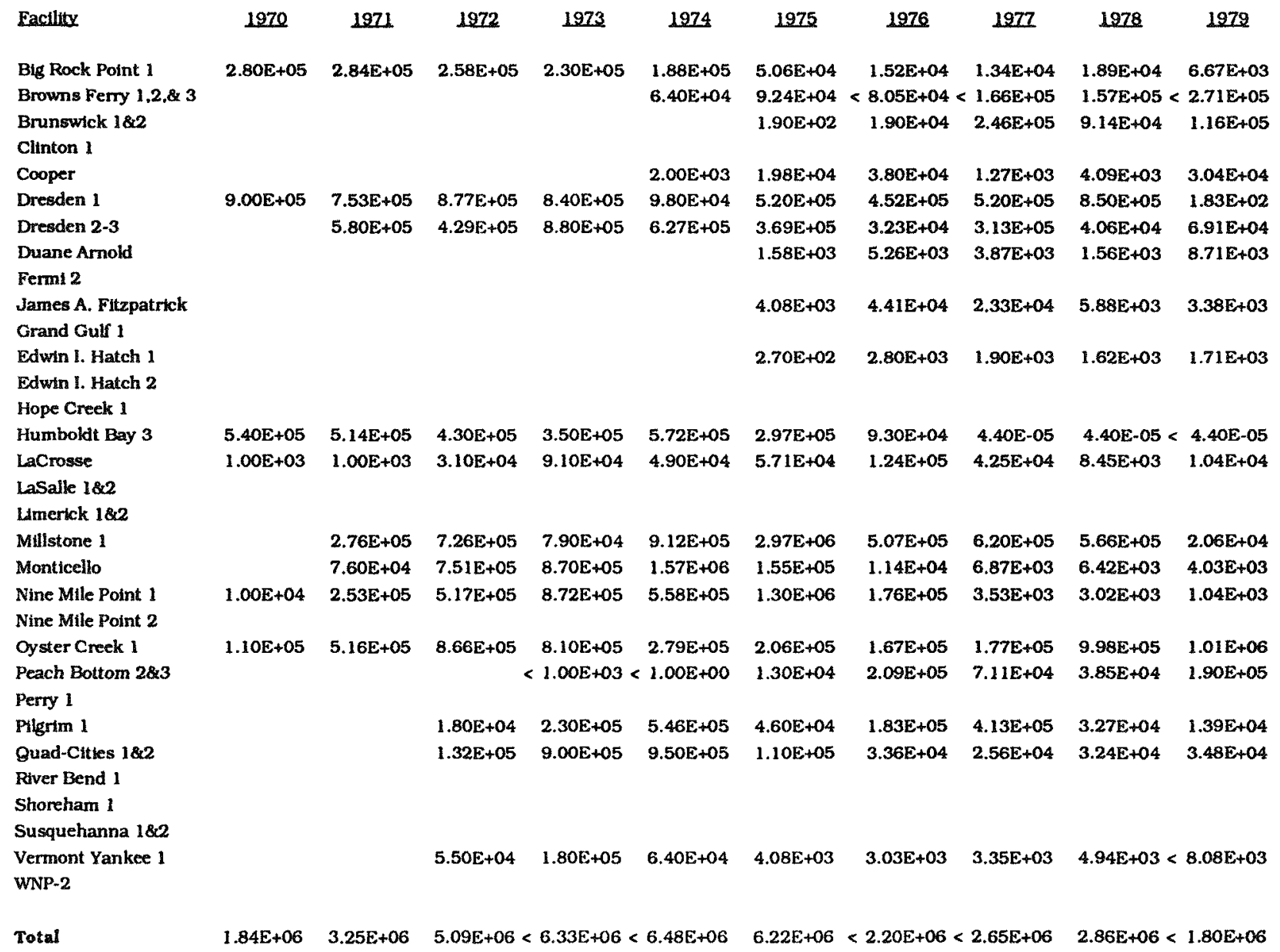

- Fort St. Vrain

$9.30 \mathrm{E}+01$ 
Table 1

Airborne weflueats Comparison By Year

Fismand Activation Gases (Total Curien)

Botling Water Reactors

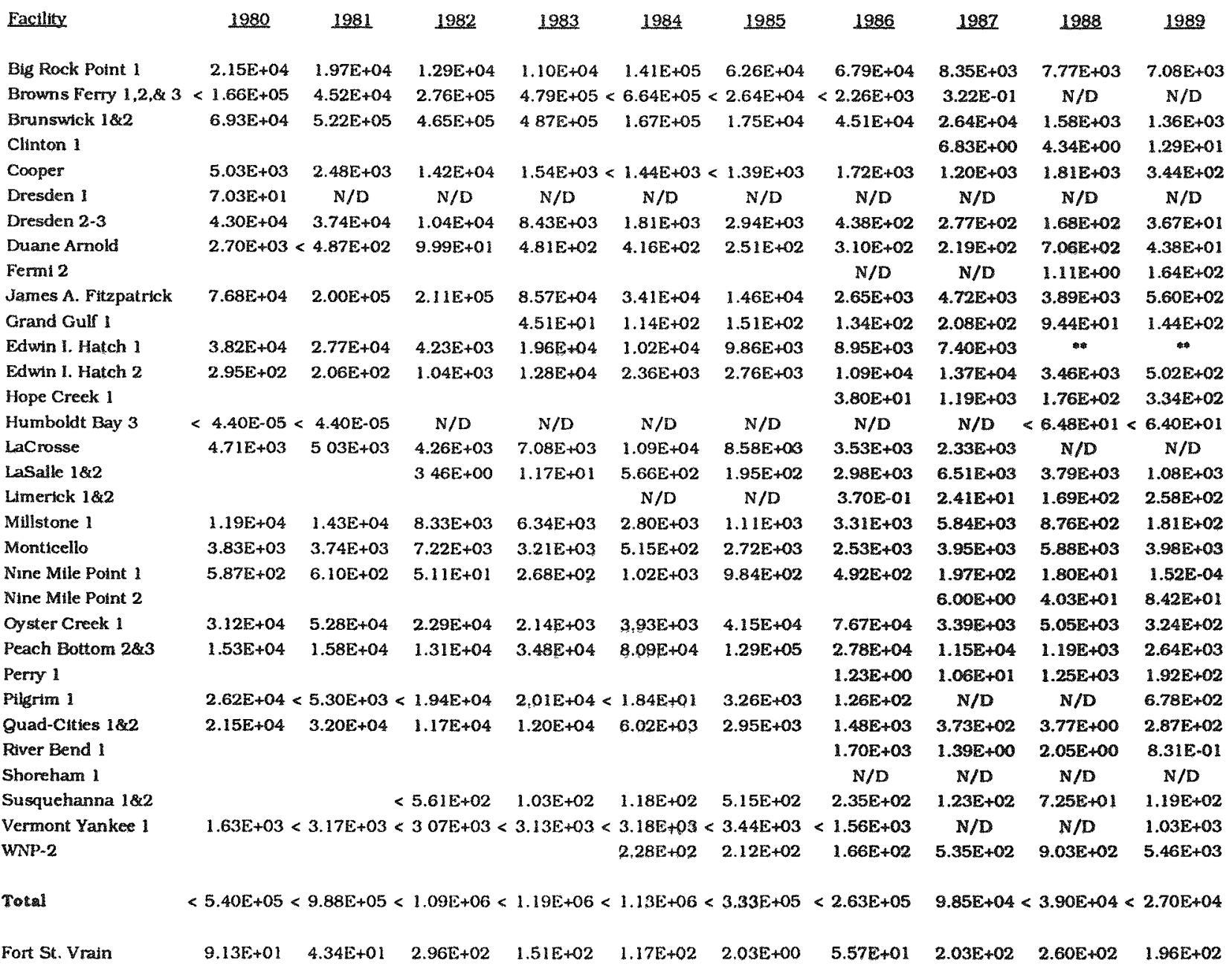

- High temperature gas cookd reactor

- Included with Edwin I. Hatch 2 total

$\mathrm{N} / \mathrm{D}=$ Not Detectable 
Pressurized Water Reactors

Faclitity

Arkansas One 1

Arkansas One 2

Beaver Valley 182

Braidwood 1

Bratowwood 2

Byron 182

Callaway 1

Calvert Cluffs 182

Catawba 1

Catawba 2

Donald C. Cook 182

Crystal River 3

Davis-Besse 1

Dtablo Canyon 182

Joseph M. Fariey 1

Joseph M. Fariey 2

Fort Calhoun 1

R. E. Ginna

Haddam Neck

Harris 1

Indlan Point 1\&2

Indian Point 3

Kewaunee

Maine Yankec

MoGuire 1

MoGuire 2

Millstone 2

Milistone 3

North Anna 182

Oconee $1,2, \& 3$

Palisades

Palo Vende 1

Palo Verde 2

Palo Verde 3

point Beach 182

Pratrie Island $1 \% 2$

Rancho Seco 1

H. B. Robinson 2

Salem 1

Salem 2

San Onofre 1

San Onofre 2-3

Seabrook 1

Sequoyah $1 \& 2$

South Texas 1

South Texas 2

St. Lacie 1

St. Lucie 2

Summer 1

Surry 182

Three Mile Island 1

Three Mile Island 2

TMI 2/Eplcor

- Trojan

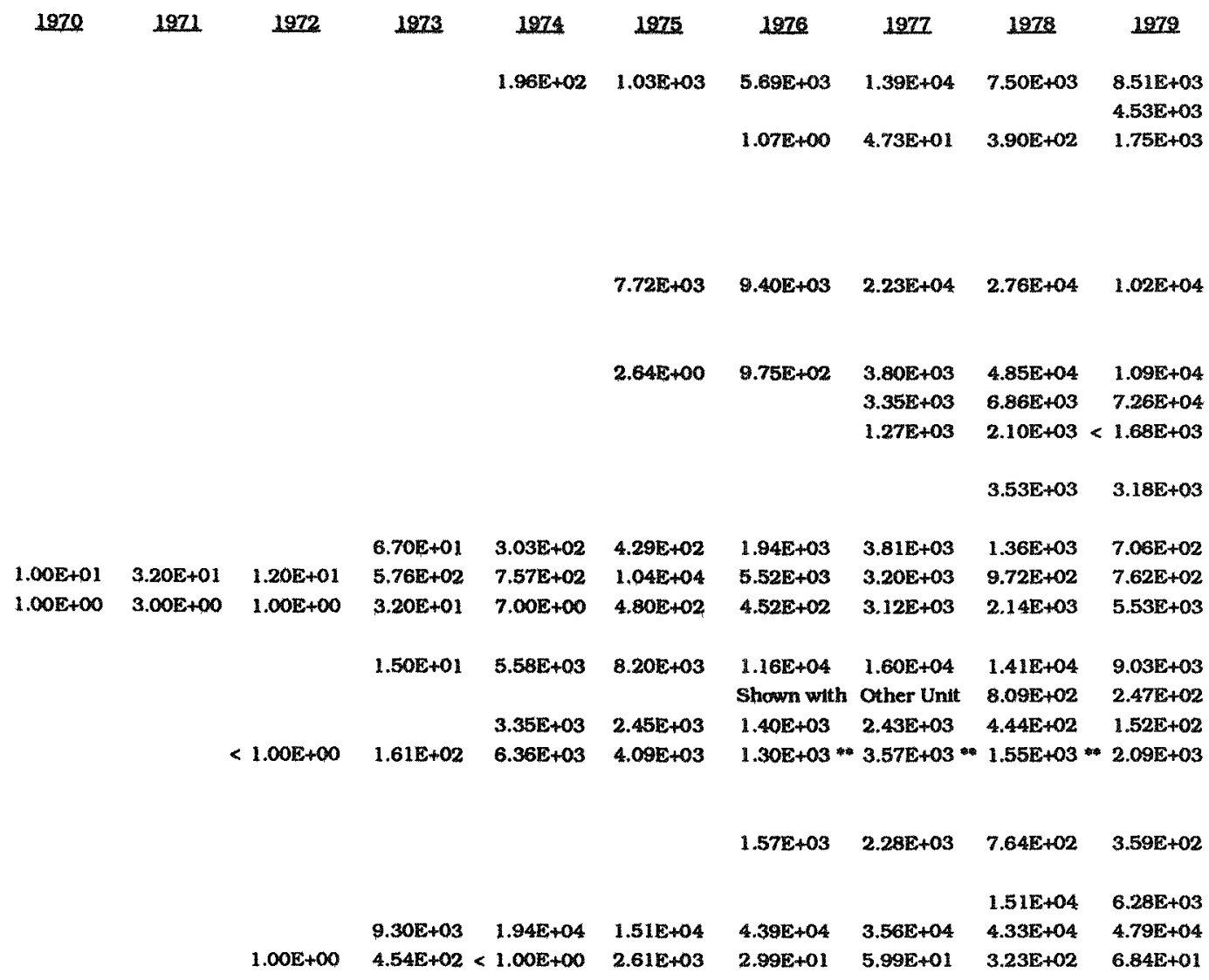

- Changes to the entries for Trajan for 1976 - 1987 represent corrections which were reported and explained in the Trojan July-December 1990 Efluent and Waste Disposal Report.

* Changes to the entries for Maine Yankee for 1977 - 1988 represent corrections which were reported and explained in the Matne Yankee report "Revised Semlannual Efluent Release Report for 770131 - 901231" Docket Date 92/01/08. 
Table 2

Airborne Effients Conazurison Wy Yor

Fismion and Activation Gass (Total Curier)

Pressurized Water Reactors

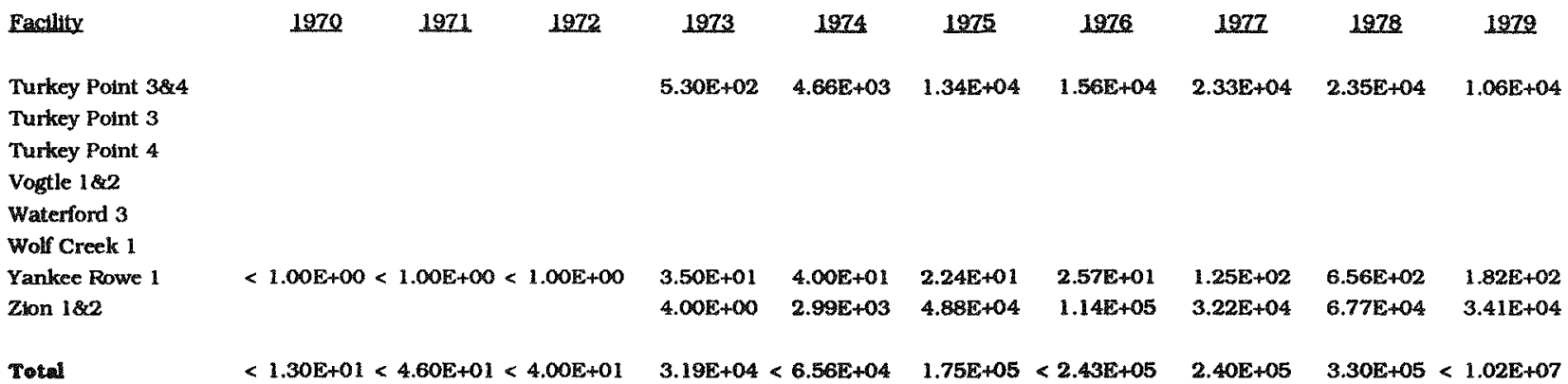


Pressurized Water Reactors

\begin{tabular}{|c|c|c|c|c|c|c|c|c|c|c|}
\hline \multicolumn{11}{|c|}{ Pressurized Water Reactors } \\
\hline Eacluty & 1980 & 1981 & 1982 & 1983 & 1984 & 1985 & 1988 & 1987 & 1988 & 1989 \\
\hline Arkansas One 1 & $3.80 E+04$ & $3.73 E+03$ & $2.10 \mathrm{E}+03$ & $9.83 E+02$ & $2.90 \mathrm{E}+03$ & 8.10E+03 & $1.71 \mathrm{E}+03$ & $3.26 \mathrm{E}+02$ & $1.24 \mathrm{E}+03$ & $2.33 E+03$ \\
\hline Arkansas One 2 & $9.37 \mathrm{E}+03$ & $4.35 \mathrm{E}+03$ & $9.78 \mathrm{E}+03$ & $1.34 \mathrm{E}+03$ & $3.26 \mathrm{E}+03$ & $8.91 E+03$ & $3.46 \mathrm{E}+03$ & $2.06 \mathrm{E}+02$ & $2.16 \mathrm{E}+03$ & $2.76 E+03$ \\
\hline Beaver Valley $1 \& 2$ & $8.64 E+01$ & $8.06 \mathrm{E}+02$ & $1.31 \mathrm{E}+02$ & $1.88 \mathrm{E}+02$ & $1.16 \mathrm{E}+03$ & $3.92 E+01$ & $7.57 \mathrm{E}+01$ & $2.25 E+02$ & $9.41 E+01$ & $1.57 \mathrm{E}+02$ \\
\hline Braidwood 1 & & & & & & & & $2.81 E-01$ & $4.19 \mathrm{E}+01$ & $1.17 \mathrm{E}+03$ \\
\hline Braidwood 2 & & & & & & & & & $3.82 \mathrm{E}+01$ & $5.07 \mathrm{E}+02$ \\
\hline Byron 182 & & & & & & $2.79 E+02$ & $6.36 \mathrm{E}+02$ & $1.30 \mathrm{E}+03$ & $1.78 \mathrm{E}+03$ & $8.16 \mathrm{E}+02$ \\
\hline Callaway 1 & & & & & $2.00 \mathrm{E}+02$ & $1.67 \mathrm{E}+03$ & $5.19 \mathrm{E}+03$ & $2.90 \mathrm{E}+03$ & $6.89 \mathrm{E} 402$ & $7.22 \mathrm{E}+02$ \\
\hline Calvert Chffs 182 & $2.96 \mathrm{E}+03$ & $2.18 \mathrm{E}+03$ & $8.00 \mathrm{E}+03$ & $9.75 E+03$ & $3.83 \mathrm{E}+03$ & $3.98 E+03$ & $7.65 \mathrm{E}+03$ & $4.55 \mathrm{E}+03$ & $5.7 \mathrm{OE}+03$ & $3.28 \mathrm{E}+03$ \\
\hline Catawba 1 & & & & & & $2.77 \mathrm{E}+02$ & $1.36 \mathrm{E}+03$ & $2.41 \mathrm{E}+03$ & $1.56 \mathrm{E}+03$ & $3.15 E+02$ \\
\hline Catawba 2 & & & & & & & $1.36 \mathrm{E}+03$ & $2.41 E+03$ & $1.56 \mathrm{E}+03$ & $3.15 E+02$ \\
\hline Donald C. Cook 182 & $3.76 \mathrm{E}+03$ & $5.42 \mathrm{E}+03$ & $3.88 \mathrm{E}+03$ & $3.28 \mathrm{E}+02$ & $3.50 \mathrm{E}+03$ & $4.94 E+03$ & $3.29 E+02$ & $8.75 E+02$ & $2.58 E+02$ & $1.15 E+02$ \\
\hline Crystal Rơver 3 & $3.65 \mathrm{E}+04$ & $3.96 \mathrm{E}+04$ & $6.85 E+03$ & $3.38 E+03$ & $1.96 \mathrm{E}+03$ & $1.05 E+03$ & $2.76 \mathrm{E}+03$ & $1.105+03$ & $3.41 \mathrm{E}+03$ & $4.54 \mathrm{E}+03$ \\
\hline Davis-Besse 1 & $<3.35 \mathrm{E}+03$ & $1.01 \mathrm{E}+03$ & $5.35 \mathrm{E}+02$ & $9.15 \mathrm{E}+02$ & $5.02 \mathrm{E}+02$ & $1.18 \mathrm{E}+02$ & $5.09 \mathrm{E}-04$ & $3.80 \mathrm{E}+02$ & $1.09 \mathrm{E}+02$ & $3.78 \mathrm{E}+02$ \\
\hline Diablo Canyon 182 & & & & & $5.86 \mathrm{E}-02$ & $5.72 \mathrm{E}+02$ & $2.32 \mathrm{E}+03$ & $7.14 \mathrm{E}+02$ & $3.27 E+02$ & $3.35 \mathrm{E}+02$ \\
\hline Joseph M. Farley 1 & $1.92 \mathrm{E}+04$ & $2.21 \mathrm{E}+02$ & $3.81 E+04$ & $2.20 \mathrm{E}+04$ & $3.73 E+03$ & $1.70 \mathrm{E}+03$ & $1.28 E+03$ & $1.30 E+03$ & $9.60 \mathrm{E}+02$ & $9.92 \mathrm{E}+01$ \\
\hline Joseph M. Farley 2 & & $2.60 \mathrm{E}+00$ & $3.54 \mathrm{E}+03$ & $8.47 \mathrm{E}+02$ & $3.99 \mathrm{E}+03$ & $6.63 \mathrm{E}+02$ & $1.845+03$ & $7.22 \mathrm{E}+02$ & $5.92 \mathrm{E}+02$ & $1.60 \mathrm{E}+02$ \\
\hline Fort Calhoun 1 & $2.97 \mathrm{E}+02$ & $1.22 \mathrm{E}+03$ & $3.46 \mathrm{E}+02$ & $8.79 \mathrm{E}+02$ & $1.52 \mathrm{E}+03$ & $1.48 \mathrm{E}+03$ & $5.68 \mathrm{C}+02$ & $4.23 E+02$ & $7.85 E+02$ & $1.64 \mathrm{E}+02$ \\
\hline R. E. Ginna & $8.61 \mathrm{E}+02$ & $5.46 \mathrm{E}+02$ & $1.95 \mathrm{E}+03$ & $7.12 E+02$ & $2.96 \mathrm{E}+02$ & $4.06 \mathrm{E}+02$ & $2.09 E+02$ & $1.77 \mathrm{E}+02$ & $5.17 E+01$ & $5.11 E+02$ \\
\hline Haddam Neck & $2.68 E+03$ & $1.83 E+03$ & $7.54 \mathrm{E}+02$ & $2.76 E+03$ & $7.52 E+03$ & $2.76 E+03$ & $2.33 E+03$ & $3.58 E+03$ & $2.55 E+03$ & $1.71 E+04$ \\
\hline Harris 1 & & & & & & & & $1.71 \mathrm{E}+03$ & $2.25 \mathrm{E}+03$ & $1.15 E+03$ \\
\hline Indian Point 182 & $9.38 E+03$ & $9.13 \mathrm{E}+03$ & $7.27 E+03$ & $9.58 \mathrm{E}+03$ & $3.78 E+03$ & $1.88 \mathrm{E}+03$ & $2.05 E+03$ & $4.68 \mathrm{E}+03$ & $2.27 \mathrm{E}+02$ & $8.77 \mathrm{E}+01$ \\
\hline Indian Point 3 & $1.11 \mathrm{E}+03$ & $6.57 E+03$ & $2.58 \mathrm{E}+03$ & $5.60 \mathrm{E}+02$ & $1.88 E+03$ & $1.54 \mathrm{E}+03$ & $1.93 E+03$ & $1.82 E+03$ & $3.10 \mathrm{E}+02$ & $3.14 E+02$ \\
\hline Kewaunee & $1.22 \mathrm{E}+02$ & $1.18 \mathrm{E}+02$ & $1.66 \mathrm{E}+02<$ & $2.25 \mathrm{E}+02<$ & $4.04 E+01<$ & $4.97 \mathrm{E}+01$ & $<6.55 \mathrm{E}+01<$ & $3.19 \mathrm{E}+01<$ & $2.91 \mathrm{E}+01$ & $6.52 \mathrm{E}+01$ \\
\hline Maine Yankee & $4.07 \mathrm{E}+03$ & $3.28 \mathrm{E}+02$ & $1.53 \mathrm{E}+03$ * & $5.07 \mathrm{E}+01$ & $1.54 \mathrm{E}+02$ & $4.41 \mathrm{E}+02$ & $1.07 \mathrm{E}+03$ & $8.34 \mathrm{~A}+02$ & $9.19 \mathrm{E}+01$ & $2.02 E+01$ \\
\hline MoGuire I & & $1,58 \mathrm{E}-01$ & $1.65 \mathrm{E}-03$ & $1.60 \mathrm{E}+03$ & $2.28 \mathrm{E}+03$ & $1.93 E+03$ & $1.05 E+03$ & $2.04 \mathrm{E}+03$ & $1.95 \mathrm{E}+03$ & $7.19 E+02$ \\
\hline Mcautre 2 & & & & $1.60 E+03$ & $2.28 E+03$ & $1.93 E+03$ & $1.05 \mathrm{E}+03$ & $2.04 E+03$ & $1.95 E+03$ & $7.19 E+02$ \\
\hline Millstone 2 & $1.33 \mathrm{E}+03$ & $2.24 \mathrm{E}+03$ & $9.09 \mathrm{E}+03$ & $9.06 \mathrm{E}+03$ & $4.19 \mathrm{E}+03$ & $4.00 \mathrm{E}+02$ & $1.02 \mathrm{E}+02$ & $3.97 E+02$ & $6.34 \mathrm{E}+02$ & $2.46 \mathrm{E}+02$ \\
\hline Millstone 3 & & & & & & & $2.39 \mathrm{E}+01$ & $1.05 E+02$ & $8.44 \mathrm{E}+01$ & $2.96 \mathrm{E}+02$ \\
\hline North Anna 182 & $3.50 E+03$ & $5.30 \mathrm{E}+03$ & $4.34 \mathrm{E}+03$ & $2.22 \mathrm{E}+04$ & $1.76 \mathrm{E}+04$ & $8.05 \mathrm{E}+03$ & $5.71 E+03$ & $1.05 \mathrm{E} * 03$ & $4.83 E+02$ & $1.44 \mathrm{E}+03$ \\
\hline Oconee $1,2, \& 3$ & $1.92 E+04$ & $1.63 E+04$ & $2.41 \mathrm{E}+04$ & $2.40 \mathrm{E}+04$ & $2.28 E+04$ & $2.35 E+04$ & $2.43 E+04$ & $1.05 \mathrm{E}+04$ & $2.59 \mathrm{E}+04$ & $8.97 \mathrm{E}+03$ \\
\hline Palisades & $1.40 \mathrm{E}+02$ & $3.00 E+03$ & $7.38 \mathrm{E}+03$ & $3.00 \mathrm{E}+03$ & $2.84 \mathrm{E}+01$ & $3.68 \mathrm{E}+03$ & $1.73 \mathrm{E}+02$ & $1.75 E+03$ & $2.43 \mathrm{E}+03$ & $1.52 \mathrm{E}+02$ \\
\hline Palo Verde 1 & & & & & & $2.53 \mathrm{E}+02$ & $2.67 \mathrm{E}+03$ & $1.27 \mathrm{E}+03$ & $1.84 \mathrm{E}+03$ & $6.41 E+02$ \\
\hline Palo Verde 2 & & & & & & & $1.97 E+03$ & $5.47 E+03$ & $2.97 E+03$ & $4.29 \mathrm{E}+02$ \\
\hline Palo Verde 3 & & & & & & & & 2.52E-02 & $1.36 \mathrm{E}+02$ & $8.34 E+02$ \\
\hline Point Beach $1 \& 2$ & $6.41 \mathrm{E}+02$ & $6.11 \mathrm{E}+02$ & $9.93 E+02$ & $7.68 \mathrm{E}+02$ & $9.30 \mathrm{E}+01$ & $1.16 \mathrm{E}+02$ & $2.78 E+01$ & $4.82 \mathrm{E}+01$ & $8.08 \mathrm{E}+01$ & $1.50 \mathrm{E}+01$ \\
\hline Prairie Island 182 & $2.60 \mathrm{E}+02$ & $4.65 \mathrm{E}+01$ & $5.47 \mathrm{E}+02$ & $2.76 \mathrm{E}+02$ & $7.58 \mathrm{E}+01$ & $4.59 \mathrm{E}+01$ & $3.03 E+01$ & $8.77 \mathrm{E}-01$ & $1.42 \mathrm{E}-01$ & $1.73 E+02$ \\
\hline Rancho Seco 1 & $1.58 \mathrm{E}+03$ & $1.37 \mathrm{E}+03$ & $1.48 \mathrm{E}+03$ & $6.89 \mathrm{E}+02$ & $3.83 E+03$ & $4.67 \mathrm{E}+03$ & $9.30 E+01$ & $2.16 E-02$ & $1.52 \mathrm{E}+03$ & $2.002+03$ \\
\hline H. B. Robinson 2 & $5.82 \mathrm{E}+02$ & $5.13 E+02$ & $1.75 \mathrm{E}+02$ & $2.93 E+02$ & $4.90 \mathrm{E}+01$ & $2.14 \mathrm{E}+03$ & $6.59 \mathrm{E}+02$ & $7.70 \mathrm{E}+02$ & $1.04 E+03$ & $2.79 E+01$ \\
\hline Salem 1 & $7.82 \mathrm{E}+01$ & $1.06 \mathrm{E} * 03$ & $2.34 t+02$ & $1.25 E+02$ & $1.95 \mathrm{E}+02$ & $1.68 \mathrm{E}+03$ & $1.39 E+03$ & $3.64 \mathrm{E}+03$ & $5.29 \mathrm{E}+02$ & $1.39 E+03$ \\
\hline Salem 2 & $7.74 E+\infty 0$ & $6.09 \mathrm{E}+02$ & $1.11 E+03$ & $7.44 E+02$ & $1.81 \mathrm{E}+03$ & $1.15 \mathrm{E}+03$ & $8.56 \mathrm{E}+02$ & $1.06 \mathrm{E}+03$ & $1.18 \mathrm{E}+03$ & $7.30 E+01$ \\
\hline San Onofre 1 & $1.05 E+03$ & $4.17 \mathrm{E}+02$ & $8.61 \mathrm{E}+01$ & $1.06 \mathrm{E}+01$ & $8.62 E+01$ & $3.83 \mathrm{E}+03$ & $4.11 \mathrm{E}+02$ & $9.81 E+02$ & $2.99 E+03$ & $9.05 E+02$ \\
\hline San Onofre 2-3 & & & $6.40 E+\infty$ & $7.43 E+03$ & $4.00 \mathrm{E}+04$ & $2.53 \mathrm{E}+04$ & $8.25 E+03$ & $2.18 \mathrm{E}+04$ & $5.12 E+03$ & $2.46 E+03$ \\
\hline Seabrook 1 & & & & & & & & & & $\mathrm{~N} / \mathrm{D}$ \\
\hline Sequoyah 182 & $3.01 \mathrm{E}+03$ & $9.03 E+03$ & $5.74 \mathrm{E}+03$ & $3.92 E+03$ & $6.68 \mathrm{E}+03$ & $4.57 \mathrm{E}+03$ & $1.21 \mathrm{E}+\infty$ & N/D & $2.25 \mathrm{E}+02$ & $3.85 \mathrm{E}+03$ \\
\hline South Texas 1 & & & & & & & & & $8.64 \mathrm{E}+02$ & $4.45 \mathrm{E}+02$ \\
\hline South Texas 2 & & & & & & & & & & $1.16 E+02$ \\
\hline St. Lucie 1 & $8.97 \mathrm{E}+03$ & $2.30 \mathrm{E}+04$ & $2.33 E+04$ & $2.16 \mathrm{E}+04$ & $3.53 \mathrm{E}+04$ & $5.08 \mathrm{E}+04$ & $3.33 E+04$ & $6.21 E+03$ & $1.42 \mathrm{E}+03$ & $4.53 \mathrm{E}+03$ \\
\hline St. Lucie 2 & & & & $1.25 \mathrm{E}+03$ & $7.68 \mathrm{E}+03$ & $9.55 E+03$ & $9.98 \mathrm{E}+03$ & $8.60 \mathrm{E}+03$ & $9.16 \mathrm{E}+03$ & $2.22 \mathrm{E}+03$ \\
\hline Summer 1 & & & $1.40 \mathrm{E}+02$ & $3.88 \mathrm{E}+02$ & $1.64 \mathrm{E}+01$ & $1.40 \mathrm{E}+02$ & $1.39 \mathrm{E}+01$ & $6.34 \mathrm{E}+02$ & $3.32 \mathrm{E}+02$ & $1.82 E+03$ \\
\hline Surry 182 & $6.17 \mathrm{E}+03$ & $1.41 \mathrm{E}+04$ & $2.11 E+04$ & $5.49 \mathrm{E}+03$ & $6.95 E+03$ & $2.07 \mathrm{E}+03$ & $1.99 \mathrm{E}+03$ & $3.08 \mathrm{E}+02$ & $3.66 \mathrm{E}+02$ & $1.37 \mathrm{E}+02$ \\
\hline Three Mile Island 1 & $4.64 \mathrm{E}-03$ & $5.81 \mathrm{E}-02$ & $7.56 \mathrm{E}-03$ & $2.01 \mathrm{E}+01$ & $3.62 \mathrm{E}-01$ & $1.08 \mathrm{E}+02$ & $3.80 E+03$ & $7.89 \mathrm{E}+02$ & $1.87 \mathrm{E}+03$ & $2.10 \mathrm{E}+03$ \\
\hline Three Mlle Island 2 & $4.72 \mathrm{E}+04$ & $2.88 \mathrm{E}+02$ & $4.89 \mathrm{E}+02$ & $1.73 E+02$ & $2.07 E+02$ & N/D & $2.80 \mathrm{E}-01$ & $\mathrm{~N} / \mathrm{D}$ & $4.40 \mathrm{E}-01$ & $\mathbf{N} / \mathbf{D}$ \\
\hline TMl 2/Eplcor & $2.16 \mathrm{E}+\infty 0$ & $1.84 E+02$ & $4.26 \mathrm{E}+02$ & $3.61 \mathrm{E}+01$ & $3.99 \mathrm{E}+01$ & + & *t & at & + & + \\
\hline Trojan & $4.10 E+02$ & $1.24 \mathrm{E}+03$ & $9.02 \mathrm{E}+02$ & $2.29 \mathrm{E}+02$ & $8.98 \mathrm{E}+02$ & $1.10 \mathrm{E}+03$ & $9.42 \mathrm{E}+02$ & $2.55 \mathrm{E}+02$ & $4.25 E+02$ & $5.94 E+02$ \\
\hline
\end{tabular}

Fiseion and Activation Cwes (Total Curies)

- Changes to the entries for Trojan for 1976-1987 are corrections which were reported and explained in the Trojan July-December 1990 Emuent and Waste Disposal Report.

* Changes to the entries for Maine Yanke for 1977 - 1988 are correctlons which were reported and explained in the Main Yankee report "Revised Semiannual Eflluent and Release Reports for 770131 - $901231^{*}$ Docket Date 92/01/08.

- Included wth Three Mile Island 2 total $\mathrm{N} / \mathrm{D}=$ Not Detectable 
Table 2

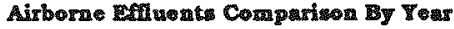

Fission and Activetion Gases (Total Curien)

Pressurized Water Reactors

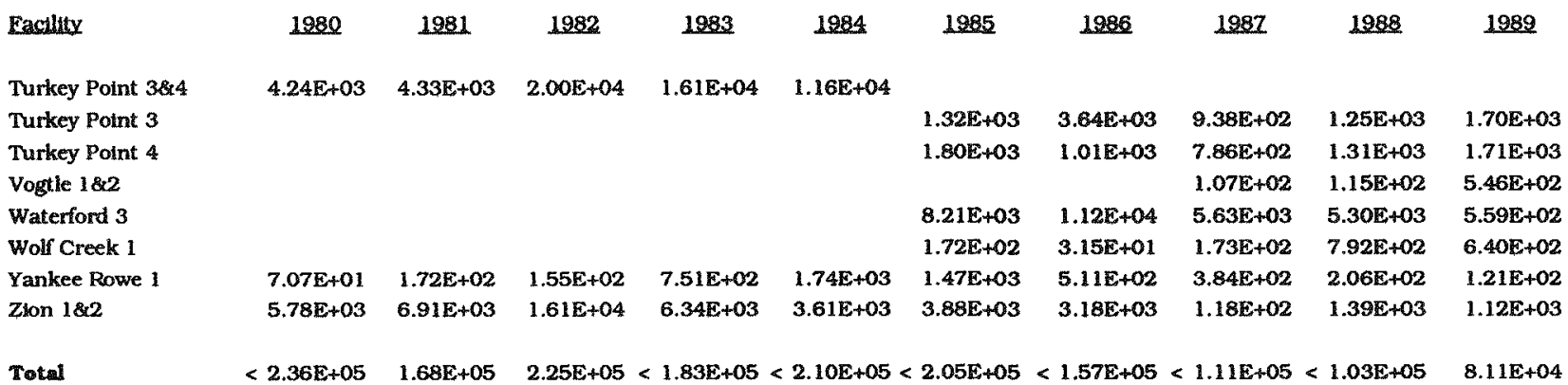


Table 3

Airborne Effluents Comparison By Year

1-131 and Particulatea (Curies)

(Half-Life Equal To or Greater Than 8 Days)

Boiling Water Reactors

\begin{tabular}{|c|c|c|c|c|c|c|c|c|c|c|c|}
\hline Facluty & 1970 & 1971 & 1972 & 1973 & 1974 & 1975 & & 1976 & 1977 & 1978 & 1979 \\
\hline Big Rock Point 1 & $1.30 \mathrm{E}-01$ & $6.10 \mathrm{E}-01$ & $1.50 \mathrm{E}-01$ & $4.60 \mathrm{E}+00$ & $1.60 \mathrm{E}-01$ & $1.20 \mathrm{E}-01$ & & $5.00 \mathrm{E}-02$ & $1.00 \mathrm{E}-02$ & $8.91 \mathrm{E}-03$ & $1.90 \mathrm{E}-03$ \\
\hline Browns Ferry 1,2,\& 3 & & & & & $1.20 \mathrm{E}-01$ & $2.70 \mathrm{E}-01$ & $<$ & $7.00 \mathrm{E}-02$ & $1.04 \mathrm{E}-01$ & $2.27 \mathrm{E}-01$ & $5.03 \mathrm{E}-02$ \\
\hline Brunswick 162 & & & & & $<$ & $1.00 \mathrm{E}-02$ & & $4.60 \mathrm{E}-01$ & $9.32 \mathrm{E}-01$ & $4.07 \mathrm{E}-01$ & $9.52 \mathrm{E}-01$ \\
\hline \multicolumn{12}{|l|}{ Clinton 1} \\
\hline Cooper & & & & & $2.40 \mathrm{E}-01$ & $5.00 \mathrm{E}-02$ & $<$ & $4.00 \mathrm{E}-02<$ & $1.91 \mathrm{E}-02$ & $5.41 \mathrm{E}-03<$ & $1.79 \mathrm{E}-01$ \\
\hline Dresden 1 & $3.30 \mathrm{E}+00$ & $6.70 \mathrm{E}-01$ & $2.75 E+00$ & $4.00 \mathrm{E}-02$ & $6.80 \mathrm{E}-01$ & $9.60 \mathrm{E}-01$ & & $8.40 \mathrm{E}-01$ & $4.93 E+\infty$ & $2.28 \mathrm{E}+00$ & $2.38 \mathrm{E}-02$ \\
\hline Duane Arnold & & & & & & $1.10 \mathrm{E}-03$ & & $8.18 \mathrm{E}-02$ & $2.29 \mathrm{E}-02$ & $3.65 \mathrm{E}-02$ & $3.35 \mathrm{E}-02$ \\
\hline \multicolumn{12}{|l|}{ Fermi 2} \\
\hline James A. Fitzpatrick & & & & & $<$ & $4.00 E-02$ & & $6.80 \mathrm{E}-01$ & $1.73 \mathrm{E}-01$ & $2.79 \mathrm{E}-01$ & $1.42 \mathrm{E}-02$ \\
\hline \multicolumn{12}{|l|}{ Grand Gulf 1} \\
\hline Edwin I. Hatch 1 & & & & & $<$ & $1.00 \mathrm{E}-02$ & $<$ & $1.00 \mathrm{E}-02$ & $5.67 \mathrm{E}-03$ & 4.13E-03 & $2.59 \mathrm{E}-02$ \\
\hline \multicolumn{12}{|l|}{ Edwin 1. Hatch 2} \\
\hline \multicolumn{12}{|l|}{ Hope Creek 1} \\
\hline Lacrosse & $<6.00 \mathrm{E}-02<$ & $1.00 \mathrm{E}-02$ & $7.10 \mathrm{E}-01$ & $2.00 E-01$ & $4.00 \mathrm{E}-02$ & $1.00 \mathrm{E}-01$ & $<$ & $7.06 E-02$ & $1.67 \mathrm{E}-01$ & 2.79E-02 & $2.53 \mathrm{E}-02$ \\
\hline \multicolumn{12}{|l|}{ LaSalle 182} \\
\hline \multicolumn{12}{|l|}{ Limerick 182} \\
\hline Millstone 1 & & $4.00 E+00$ & $1.32 \mathrm{E}+00$ & $2.00 \mathrm{E}-01$ & $3.26 E+00$ & $9.98 \mathrm{E}+00$ & & $2.33 \mathrm{E}+00$ & $4.86 \mathrm{E}+\infty$ & $4.55 \mathrm{E}+00$ & $5.90 \mathrm{E}-01$ \\
\hline Monticello & & $3.60 \mathrm{E}-02$ & $5.78 \mathrm{E}-01$ & $1.20 \mathrm{E}+00$ & $5.70 E+00$ & $3.71 \mathrm{E}+00$ & & $1.71 \mathrm{E}-01$ & $8.51 E-02$ & $5.49 \mathrm{E}-02$ & $3.39 E-02$ \\
\hline Nine Mile Point 1 & $<1.00 \mathrm{E}-02$ & $6.00 \mathrm{E}-02$ & $9.70 E-01$ & $1.98 \mathrm{E}+00$ & $8.90 \mathrm{E}-01$ & $2.78 \mathrm{E}+\infty 0$ & & $2.20 E+00$ & $1.99 \mathrm{E}-01$ & $1.35 \mathrm{E}-01$ & $4.71 E-02$ \\
\hline \multicolumn{12}{|l|}{ Nine Mile Point 2} \\
\hline Oyster Creek 1 & $3.20 \mathrm{E}-01$ & $2.14 E+00$ & $6.48 E+00$ & $7.02 \mathrm{E}+00$ & $3.51 \mathrm{E}+00$ & $5.64 \mathrm{E}+00$ & & $6.39 \mathrm{E}+\infty$ & $9.05 E+00$ & $1.81 E+01$ & $9,32 \mathrm{E}+00$ \\
\hline Peach Bottom $2 \& 3$ & & & $<$ & $1.00 \mathrm{E}-02$ & $1.00 \mathrm{E}-02$ & $4.00 E-02$ & & $9.75 \mathrm{E}-01$ & $2.73 E-01$ & $9.62 \mathrm{E}-02$ & $2.58 \mathrm{E}-01$ \\
\hline \multicolumn{12}{|l|}{ Perry 1} \\
\hline Pilgrtm 1 & & & $3.00 \mathrm{E}-02$ & $4.70 \mathrm{E}-01$ & $1.45 \mathrm{E}+00$ & $2.58 \mathrm{E}+00$ & & $6.74 \mathrm{E}-01$ & $6.90 \mathrm{E}-01$ & $1.81 \mathrm{E}-01$ & $1.45 E-01$ \\
\hline Quad-Cities 182 & & & $7.50 \mathrm{E}-01$ & $5.50 \mathrm{E}+00$ & $8.88 \mathrm{E}+00$ & $1.31 \mathrm{E}+00$ & & $1.33 \mathrm{E}+\infty$ & $1.69 \mathrm{E}+00$ & $2.15 E+00$ & $1.57 \mathrm{E}+00$ \\
\hline \multicolumn{12}{|l|}{ River Bend I } \\
\hline \multicolumn{12}{|l|}{ Shoreham 1} \\
\hline \multicolumn{12}{|l|}{ Susquehanna 182} \\
\hline Total & $<5.77 \mathrm{E}+00<$ & $1.65 \mathrm{E}+01$ & $2.03 \mathrm{E}+01<$ & $2.83 \mathrm{E}+01$ & $3.26 \mathrm{E}+01<$ & $3.30 \mathrm{E}+01$ & & $2.20 \mathrm{E}+01$ & $3.01 \mathrm{E}+01$ & $3.19 E+01<$ & $2.07 \mathrm{E}+01$ \\
\hline
\end{tabular}

- Fort St. Vrain

$6.89 \mathrm{E}-07$ 


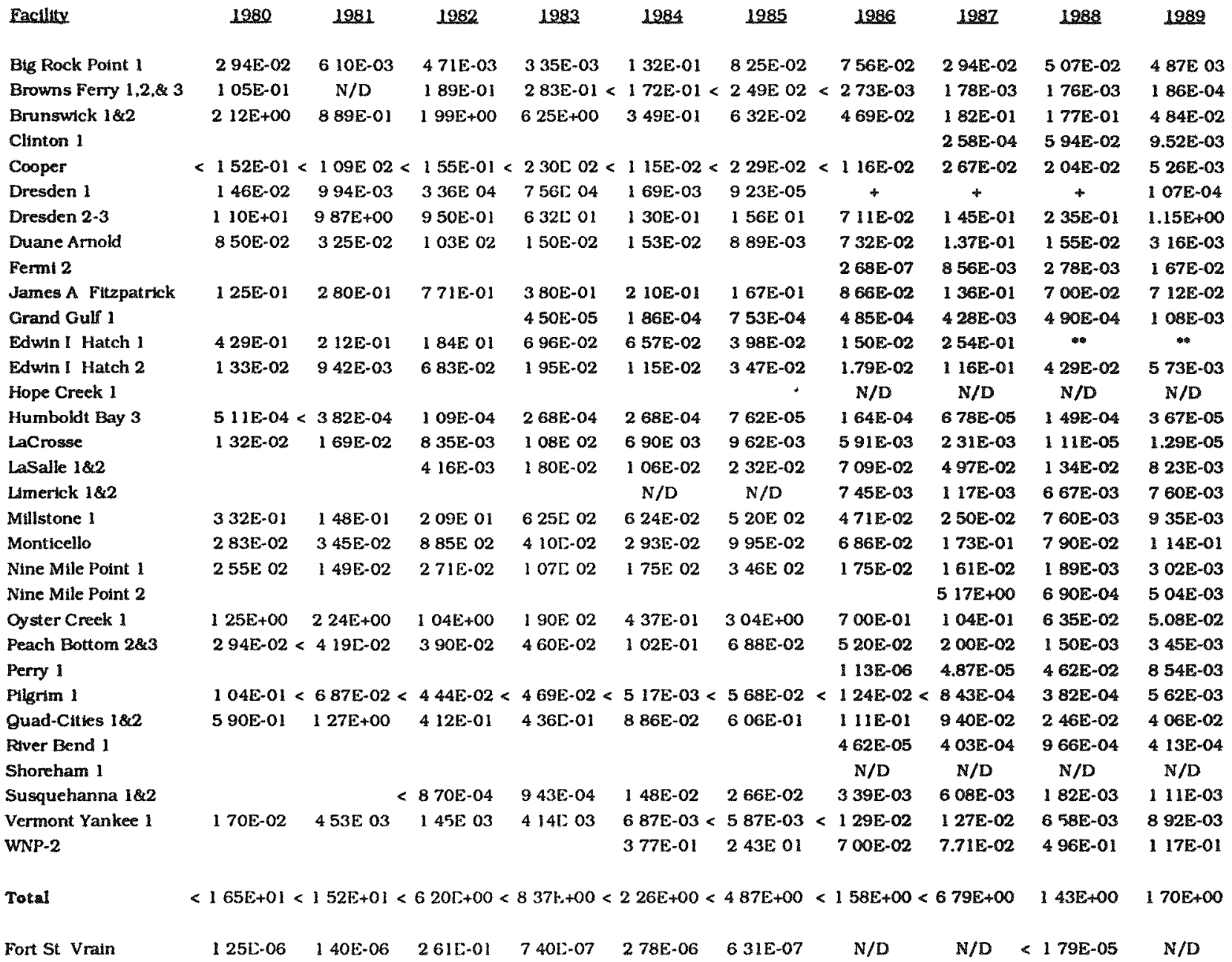


Airborne Ecturent Comperisen Yor

I-131 and Particaliates (Curies)

Pressurized Water Reactors

(Hilf-Life Bqual To ar Oreater Than Dwy)

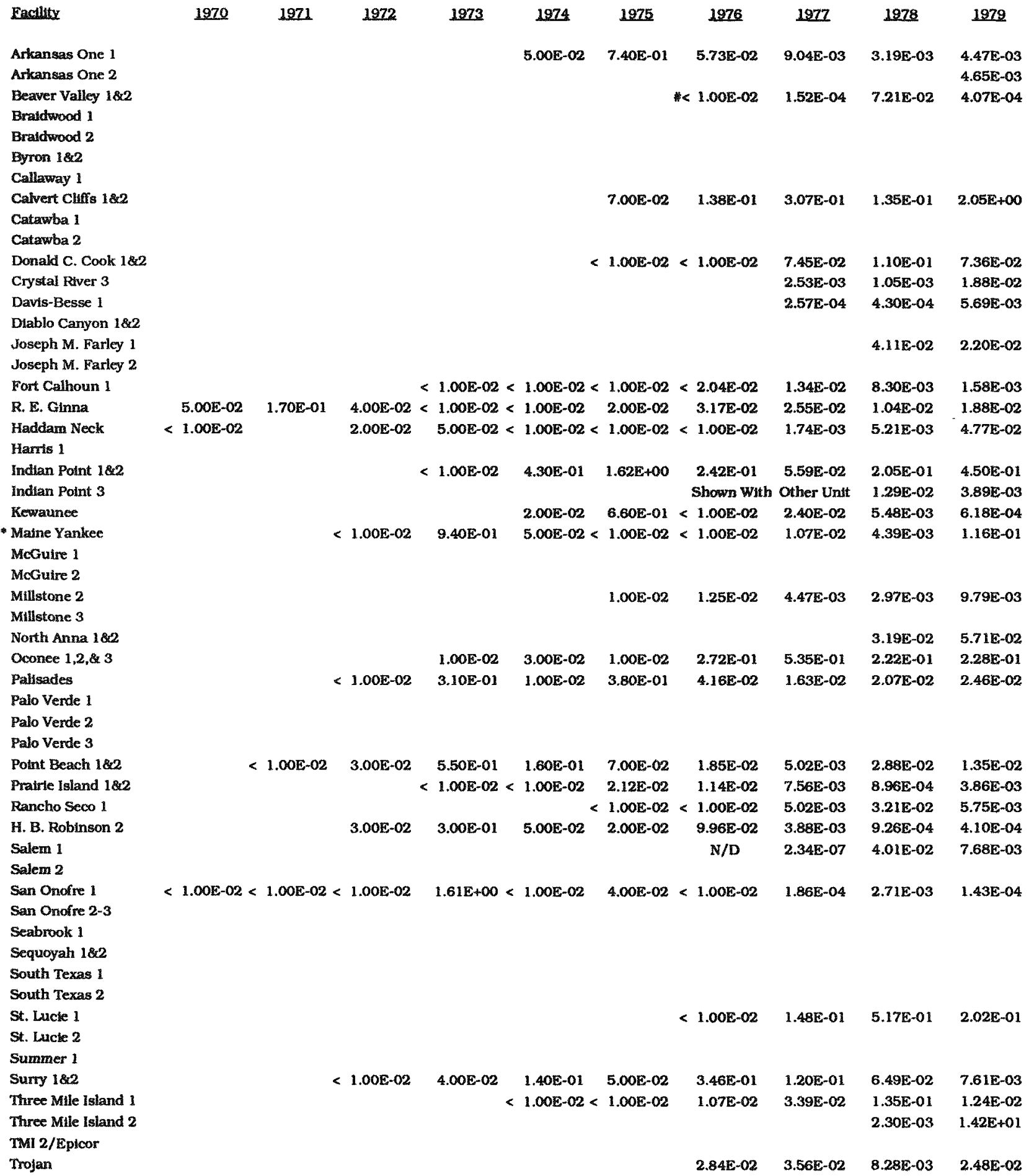

- Changes to the entries for Trojan for 1976-1987 are corrections which were reported and explained in the Trojan July-December 1990 Efluent and Waste Disposal Report.

\# I-131 not included

* Changes to the entries for Malne Yankee for 1977 - 1988 are corrections which were reported and explained in the Maine Yankee report "Revised Semiannual Efluent Release Reports for 770131 - 901231" Docket Date 92/01/08.

$N / D=$ Not Detectable $-14-$ 
Table

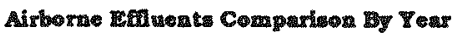

1-131 Ind Particul mte (Curies)

(Half-Life Doqual To or Croter Than Days)

Pressurized Water Reactors

\begin{tabular}{|c|c|c|c|c|c|c|c|c|c|c|}
\hline Facility & 1970 & 1971 & 1972 & 1973 & 1974 & 1975 & 1870 & 1977 & 1978 & 1979 \\
\hline Turkey Point 3\&4 & & & & $6.00 \mathrm{E}-02$ & $3.63 \mathrm{E}+00$ & $4.30 E-01$ & $4.22 \mathrm{E}-01$ & $1.04 E+00$ & $4.59 \mathrm{E}-01$ & $7.91 \mathrm{E}-02$ \\
\hline \multicolumn{11}{|l|}{ Turkey Point 3} \\
\hline \multicolumn{11}{|l|}{ Vogtle $1 \& 2$} \\
\hline \multicolumn{11}{|l|}{ Waterford 3} \\
\hline \multicolumn{11}{|l|}{ Wolf Creek 1} \\
\hline$Z$ Ion 182 & & & & $<1.00 \mathrm{E}-02$ & $1.00 \mathrm{E}-02$ & $1.40 \mathrm{E}-01$ & $9.00 \mathrm{E}-02$ & $5.38 \mathrm{E}-02$ & $8.91 \mathrm{E}-02$ & $6.74 \mathrm{E}-02$ \\
\hline Total & $<8.00 \mathrm{E}-02<$ & $2.00 \mathrm{E}-01<$ & $1.70 \mathrm{E}-01$ & $<4.11 E+\infty 0$ & $5.17 \mathrm{E}^{2}+00$ & $4.35 E+00$ & $<1.936+00$ & $2.53 E+\infty 0$ & $2.27 \mathrm{E}+00$ & $1.78 \mathrm{E}+01$ \\
\hline
\end{tabular}


Airborne Effluent Comparisen By Your

I-131 and Farticulated [Curies)

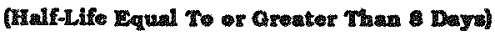

Pressurized Water Reactors

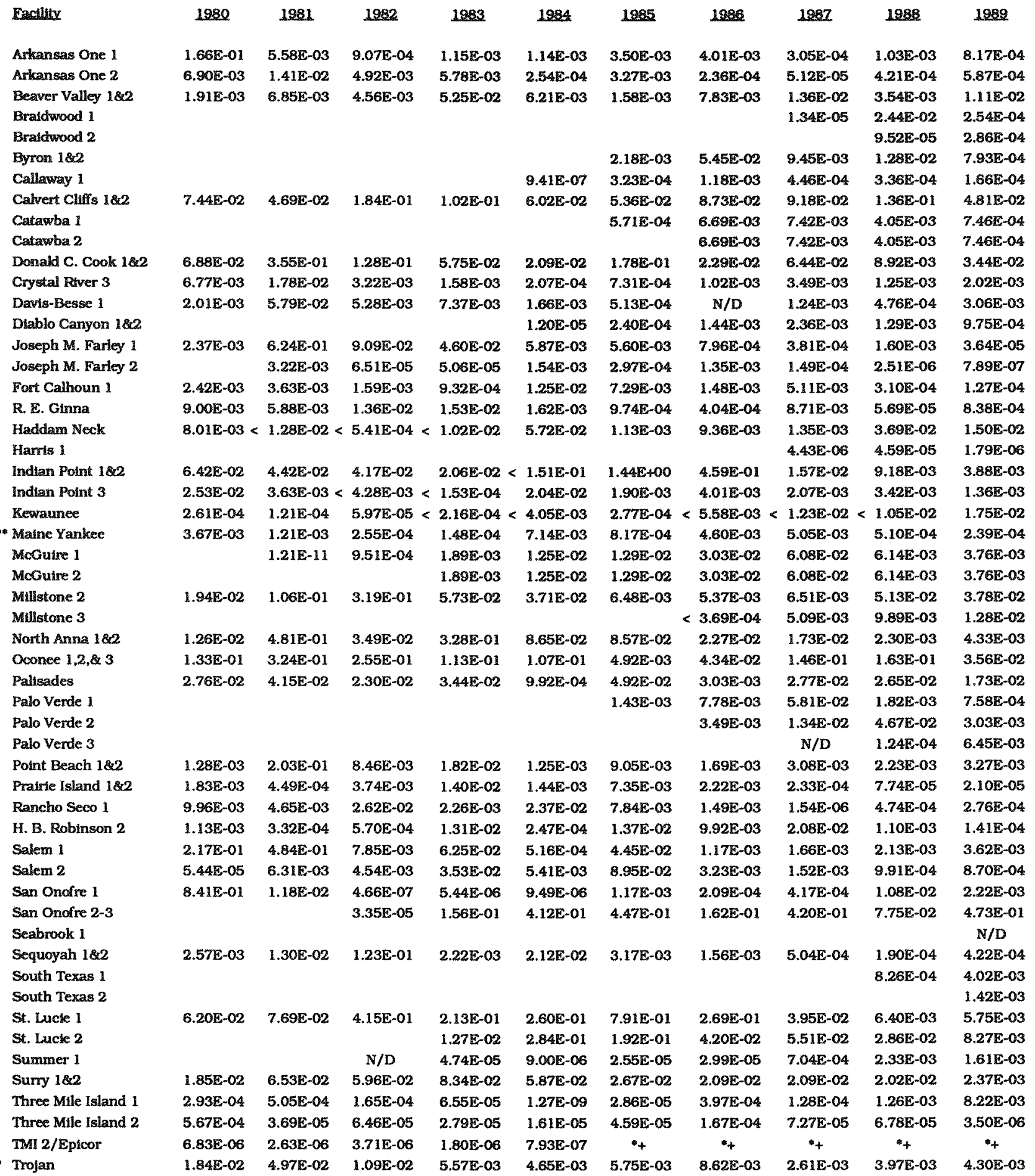

- Changes to the entries for Trojan for 1976-1987 are corrections which were reported and explained in the Trojan July-December 1990 Effluent and Waste Disposal Report.

* Changes to the entries for Maine Yankee for 1977 - 1988 are corrections which are reported and explained in the Maine Yankee report "Revised Semiannual Effluent Release Reports for 770131 - 901231" Docket Date 92/01/08.

* Included with Three Mile Island 2 total $\mathrm{N} / \mathrm{D}=$ Not Detectable 
Table

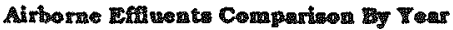

1-131 and Particulates (Curien)

(Half-Life Equal To or Creater Than 8 Day'

Pressurized Water Reactors

\begin{tabular}{|c|c|c|c|c|c|c|c|c|c|c|}
\hline Factlity & 1980 & 1981 & 1982 & 1983 & 1984 & 1985 & 1886 & 1987 & 1988 & 1989 \\
\hline Turkey Point 384 & $7.05 \mathrm{E}-02$ & $2.94 \mathrm{E}-02$ & $2.20 \mathrm{E}-01$ & $1.44 \mathrm{E}-01$ & $2.77 E-02$ & & & & & \\
\hline Turkey Point 3 & & & & & & $7.98 \mathrm{E}-03$ & $1.93 E-02$ & $1.24 \mathrm{E}-02$ & $4.83 E-03$ & $3.10 \mathrm{E}-03$ \\
\hline Vogtle 182 & & & & & & & & $1.99 \mathrm{E}-05$ & $1.75 \mathrm{E}-05$ & $1.25 \mathrm{E}-03$ \\
\hline Waterford 3 & & & & & & $3.48 \mathrm{E}-03$ & $5.30 \mathrm{~L}-03$ & 1.02E-03 & $1.24 \mathrm{E}-03$ & $7.62 \mathrm{E}-04$ \\
\hline Wolf Creek 1 & & & & & & $1.67 \mathrm{E}-06$ & $2.11 \mathrm{E}-04$ & $2.14 E-04$ & $8.36 \mathrm{E}-05$ & $2.31 \mathrm{E}-05$ \\
\hline Zion 182 & $3.00 E-03$ & $1.25 \mathrm{E}-02$ & 8.57E-02 & $2.28 \mathrm{E}-02$ & $4.27 \mathrm{E}-02$ & $2.55 \mathrm{E}-02$ & 4.48E-02 & $4.07 \mathrm{E}-03$ & $1.40 \mathrm{E}-02$ & $2.39 \mathrm{E}-03$ \\
\hline
\end{tabular}


Table 5

Liquid Efluents Comparison By Year

Tritium (Curies)

Boiling Water Reactors

\begin{tabular}{|c|c|c|c|c|c|c|c|c|c|c|}
\hline Eacility & 1970 & 1971 & 1972 & 1923 & 1974 & 1975 & 1976 & 1977 & 1978 & 1979 \\
\hline Big Rock Point 1 & $5.40 E+01$ & $1.03 E+01$ & $1.04 E+01$ & $1.97 \mathrm{E}+01$ & $5.10 \mathrm{E}+00$ & $5.73 E+00$ & $2.41 E+\infty$ & $8.83 E+\infty 0$ & $4.05 \mathrm{E}+00$ & $5.45 \mathrm{E}+00$ \\
\hline Browns Ferry 1.2 .83 & & & & & $2.80 \mathrm{E}+00$ & $1.04 \mathrm{E}+01$ & $<4.02 \mathrm{E}+\infty 0$ & 2. $4 \mathrm{OE}+01$ & $3.08 \mathrm{E}+01$ & $1.32 \mathrm{E}+01$ \\
\hline Brunswick 1\&2 & & & & & & $3.20 \mathrm{E}+\infty 0$ & $5.90 \mathrm{E}+00$ & $8.93 \mathrm{E}+\infty 0$ & $1.41 \mathrm{E}+01$ & $3.09 \mathrm{E}+01$ \\
\hline \multicolumn{11}{|l|}{ Clinton 1} \\
\hline Cooper & & & & & $1.70 E+00$ & $8.25 E+00$ & $8.43 E+00$ & $9.04 \mathrm{E}+\infty$ & $7.51 \mathrm{E}+00$ & $6.63 \mathrm{E}+00$ \\
\hline Dresden 1 & $5.00 \mathrm{E}+\infty 0$ & $8.70 E+00$ & $4.33 \mathrm{E}+01$ & $1.85 \mathrm{E}+01$ & $1.88 \mathrm{E}+01$ & $2.70 \mathrm{E}-01$ & $2.00 \mathrm{E}-02$ & $8.90 \mathrm{E} \cdot 02$ & $1.31 \mathrm{E}+01$ & $1.50 \mathrm{E}+\infty 0$ \\
\hline Duane Arnold & & & & & & $3.30 \mathrm{E}-01$ & $3.40 \mathrm{E}-01$ & $2.13 \mathrm{E}-01$ & $1.19 \mathrm{E}+02$ & $2.90 E-01$ \\
\hline \multicolumn{11}{|l|}{ Ferm 2} \\
\hline Jame A. Futzpatrick & & & & & & $5.03 E+00$ & $4.20 \mathrm{E}+\infty 0$ & $3.35 \mathrm{E}+00$ & $1.90 E+00$ & $1.52 \mathrm{E}+00$ \\
\hline \multicolumn{11}{|l|}{ Grand Gulf I } \\
\hline Edwin I. Hatch 1 & & & & & & $6.12 \mathrm{E}+00$ & $8.98 \mathrm{E}+00$ & $1.20 \mathrm{E}+01$ & $9.00 \mathrm{E}+00$ & $1.23 E+01$ \\
\hline \multicolumn{11}{|l|}{ Edwin I. Hatch 2} \\
\hline \multicolumn{11}{|l|}{ Hope Creek 1} \\
\hline Lacrosse & $2.00 \mathrm{E}+01$ & $9.14 E+01$ & $1.20 \mathrm{E}+02$ & $1.03 E+02$ & $1.15 E+02$ & $1.27 \mathrm{E}+02$ & $4.10 E+01$ & $4.86 \mathrm{E}+01$ & $4.72 \mathrm{E}+01$ & $3.54 E+01$ \\
\hline \multicolumn{11}{|l|}{ LaSalle 182} \\
\hline \multicolumn{11}{|l|}{ Limerick $1 \& 2$} \\
\hline Millstone 1 & & $1.27 \mathrm{E}+01$ & $2.09 \mathrm{E}+01$ & $3.70 E+00$ & $2.41 \mathrm{E}+01$ & $8.03 E+01$ & $2.01 \mathrm{E}+01$ & $4.41 \mathrm{E}+00$ & $3.20 E+00$ & $7.92 E+\infty 0$ \\
\hline Monticello & & $5.92 \mathrm{E}-01<$ & $1.00 \mathrm{E}-01$ & $\mathrm{~N} / \mathrm{D}$ & $\mathrm{N} / \mathrm{D}$ & $\mathrm{N} / \mathrm{D}$ & N/D & $\mathrm{N} / \mathrm{D}$ & $\mathrm{N} / \mathrm{D}$ & N/D \\
\hline Nine Mile Point 1 & $2.00 \mathrm{E}+01$ & $1.24 \mathrm{E}+01$ & $2.78 \mathrm{E}+01$ & $4.65 \mathrm{E}+01$ & $1.87 \mathrm{E}+01$ & $2.81 \mathrm{E}+01$ & $2.46 E+00$ & $2.49 \mathrm{E}+00$ & $\mathrm{~N} / \mathrm{D}$ & $6.78 E+\infty 0$ \\
\hline \multicolumn{11}{|l|}{ Nine Mile Point 2} \\
\hline Oyster Creek 1 & $2.20 \mathrm{E}+01$ & $2.15 \mathrm{E}+01$ & $6.16 \mathrm{E}+01$ & $3.59 \mathrm{E}+01$ & $1.41 \mathrm{E}+01$ & $1.79 \mathrm{E}+01$ & $3.86 \mathrm{E}+01$ & $1.88 \mathrm{E}+01$ & $1.96 \mathrm{E}+01$ & $1.40 E+\infty 0$ \\
\hline Peach Bottom $2 \& 3$ & & & $<$ & $1.00 \mathrm{E}-01$ & $1.00 \mathrm{E}+01$ & $3.08 \mathrm{E}+01$ & $7.37 \mathrm{E}+01$ & $7.09 \mathrm{E}+01$ & $3.24 E+01$ & $4.28 \mathrm{E}+01$ \\
\hline \multicolumn{11}{|l|}{ Perry 1} \\
\hline Pllgrim 1 & & & $4.20 \mathrm{E}+00$ & $4.00 \mathrm{E}-01$ & $1.05 \mathrm{E}+01$ & $1.82 \mathrm{E}+01$ & $4.67 \mathrm{E}+01$ & $3.27 \mathrm{E}+01$ & $2.98 E+00$ & $1.34 \mathrm{E}+01$ \\
\hline Quad-Cities 182 & & & $4.70 E+00$ & $2.45 \mathrm{E}+01$ & $3.40 \mathrm{E}+01$ & $5.37 \mathrm{E}+01$ & $4.98 \mathrm{E}+01$ & $2.64 E+01$ & $1.72 \mathrm{E}+01$ & $1.76 \mathrm{E}+01$ \\
\hline \multicolumn{11}{|l|}{ Rever Bend 1} \\
\hline \multicolumn{11}{|l|}{ Shoreham 1} \\
\hline \multicolumn{11}{|l|}{ Susquehanna $1 \& 2$} \\
\hline Total & $1.59 \mathrm{E}+02$ & $2.04 \mathrm{E}+02<$ & $3.32 \mathrm{E}+02<$ & $3.30 \mathrm{E}+02$ & $3.09 E+02$ & $4.69 \mathrm{E}+02$ & $<3.41 \mathrm{E}+02$ & $2.77 \mathrm{E}+02$ & $3.41 \mathrm{E}+02$ & $2.20 \mathrm{E}+02$ \\
\hline Fort St. Vrain & & & & & & & & & & $1.23 \mathrm{E}+02$ \\
\hline
\end{tabular}


Tritium (Curies)

Bolling Water Reactors

\begin{tabular}{|c|c|c|c|c|c|c|c|c|c|c|}
\hline Pacility & 1980 & 1981 & 1982 & 1983 & 1984 & 1985 & 1986 & 1987 & 1988 & 1989 \\
\hline Big Rock Point 1 & $6.18 \mathrm{E}+00$ & 3.13E+00 & $2.98 \mathrm{E}+00$ & $2.22 \mathrm{E}+01$ & $1.11 E+00$ & $1.27 \mathrm{E}+00$ & $3.51 \mathrm{E}-01$ & $5.85 E-01$ & $3.47 \mathrm{E}-01$ & $6.39 \mathrm{E}-\mathrm{OL}$ \\
\hline Browns Ferny 1,2,83 & $2.18 \mathrm{E}+01$ & $2.42 \mathrm{E}+01$ & $2.398+01$ & $3.20 \mathrm{E}+01$ & $3.18 \mathrm{E}+01$ & $3.31 E+01$ & $7.93 E+\infty$ & $2.03 \mathrm{E}+00$ & $1.46 \mathrm{E}+00$ & $7.01 E-01$ \\
\hline Brunswick 182 & $1.28 \mathrm{E}+01$ & $2.26 \mathrm{E}+01$ & $4.88 \mathrm{E}+01$ & $1.04 \mathrm{E}+02$ & $3.37 \mathrm{E}+01$ & $9.88 \mathrm{E}+00$ & $5.78 \mathrm{E}+\infty 0$ & $1.93 \mathrm{E}+01$ & $3.10 \mathrm{E}+01$ & $1.79 \mathrm{E}+01$ \\
\hline Clinton 1 & & & & & & & & $1.87 E+00$ & $2.90 \mathrm{E}+00$ & $1.49 E+00$ \\
\hline Cooper & $8.77 E+00<$ & $8.37 \mathrm{E}+00<$ & $9.08 E+00<$ & $=7.60 \mathrm{E}+00<$ & $7.20 \mathrm{E}+00<$ & $5.05 E+00$ & $<5.56 E+00$ & $5.02 \mathrm{E}+00$ & 4.17E+00 & $5.45 E+\infty 0$ \\
\hline Dresden 1 & $\mathbb{N} / \mathbb{D}$ & $\mathrm{N} / \mathrm{O}$ & $N / D$ & $\mathrm{~N} / \mathrm{D}$ & $N / D$ & $\mathrm{~N} / \mathrm{D}$ & $\mathrm{N} / \mathrm{D}$ & $\mathrm{N} / \mathrm{D}$ & 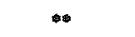 & $\rightarrow$ \\
\hline Duane Arnold & $N / D$ & $\mathrm{~N} / \mathrm{D}$ & $2.25 \mathrm{E}-05$ & $\mathrm{~N} / \mathrm{D}$ & $1.41 \mathrm{E}-06$ & $3.57 E-02$ & $N / D$ & $\mathrm{~N} / \mathrm{D}$ & $\mathrm{N} / \mathrm{D}$ & $\mathrm{N} / \mathrm{D}$ \\
\hline Ferml 2 & & & & & & & $3.00 \mathrm{E}-01$ & $1.05 \mathrm{E}+\infty$ & $9.33 E-01$ & $1.30 \mathrm{E}+00$ \\
\hline James A. Fitzpatrick & $2.81 \mathrm{E}+00$ & $4.11 E+\infty$ & $6.55 \mathrm{E}-01$ & $2.72 E+00$ & $4.77 \mathrm{E}+00$ & $4.20 \mathrm{E}+00$ & $4.99 E+00$ & $2.48 \mathrm{E}+\infty 0$ & $8.87 \mathrm{E}+00$ & $7.32 \mathrm{E}-01$ \\
\hline Grand Gulf 1 & & & & $3.89 \mathrm{E}-03$ & $7.27 \mathrm{E}-01$ & $5.17 \mathrm{E}+00$ & $1.47 \mathrm{E}+01$ & $1.83 E+01$ & $1.34 \mathrm{E}+01$ & $1.32 \mathrm{E}+01$ \\
\hline Edwin I. Hatch 1 & $1.42 \mathrm{E}+01$ & $1.16 \mathrm{E}+01$ & $1.03 E+02$ & $9.47 \mathrm{E}+01$ & $8.02 E+01$ & $3.93 E+01$ & $1.85 E+01$ & $2.01 \mathrm{E}+01$ & + & + \\
\hline Edwin 1. Hatch 2 & $1.07 \mathrm{E}+01$ & $9.28 E+00$ & $3.68 \mathrm{E}+01$ & $3.40 \mathrm{E}+01$ & $2.13 E+01$ & $1.81 E+01$ & $1.01 \mathrm{E}+01$ & $8.10 E+00$ & $4.40 E+01$ & $4.57 \mathrm{E}+01$ \\
\hline Hope Creel 1 & & & & & & & $6.91 E-03$ & $9.53 E+\infty$ & $9.36 \mathrm{E}+00$ & $2.35 \mathrm{E}+01$ \\
\hline osse & $7.20 E+01$ & $7.74 \mathrm{E}+01$ & $5.92 \mathrm{E}+01$ & $1.24 E+02$ & $1.25 E+02$ & $1.28 \mathrm{E}+02$ & $5.75 E+01$ & $4.66 \mathrm{E}+01$ & $4.60 \mathrm{E}+00$ & $2.79 \mathrm{E}+\infty$ \\
\hline Lasalle $1 \& 2$ & & & $9.26 \mathrm{E}-01$ & $4.25 \mathrm{E}+00$ & $1.10 \mathrm{E}+00$ & $3.89 \mathrm{E}-01$ & $1.37 \mathrm{E}-01$ & $1.10 \mathrm{E}+00$ & $1.76 \mathrm{E}+00$ & $1.07 \mathrm{E}+00$ \\
\hline Lmerick 182 & & & & & $\mathrm{~N} / \mathrm{D}$ & $1.15 \mathrm{E}+00$ & $2,06 \mathrm{E}+00$ & $6.02 E+00$ & $\mathrm{~N} / \mathrm{D}$ & $2.70 E+01$ \\
\hline Millstone 1 & $2.73 E+01$ & $2.62 \mathrm{E}+00$ & $6.21 \mathrm{E}+00$ & $8.38 \mathrm{E}+00$ & $8.58 \mathrm{E}+00$ & $1.79 \mathrm{E}+01$ & $5,33 E+\infty$ & $1.78 \mathrm{E}+01$ & $3.78 E+01$ & $4.58 \mathrm{E}+01$ \\
\hline Monticello & $\mathrm{N} / \mathrm{D}$ & $4.17 \mathrm{E}-03$ & $2.70 \mathrm{E}-05$ & $\mathrm{~N} / \mathrm{D}$ & $N / D$ & $N / D$ & $\mathrm{~N} / \mathrm{D}$ & $\mathrm{N} / \mathrm{D}$ & $\mathrm{N} / \mathrm{D}$ & $\mathbb{N} / \mathrm{D}$ \\
\hline Nine Mile Point 1 & $N / D$ & $5.05 E+\infty 0$ & $5.82 E+00$ & $7.89 E+00$ & $\mathrm{~N} / \mathrm{D}$ & $\mathrm{N} / \mathrm{D}$ & $2.19 \mathrm{E}+00$ & $\mathbb{N} / D$ & $N / D$ & $\mathrm{~N} / \mathrm{D}$ \\
\hline Nine Mile Point 2 & & & & & & & & 4.63E-01 & $7.925+00$ & $8.10 \mathrm{E}+00$ \\
\hline Oyster Creek 1 & $1.54 E+02$ & $2.67 E+01$ & $4.95 E+\infty 0$ & $8.76 \mathrm{E}+00$ & $1.03 E+01$ & $\mathbb{N} / \mathbb{D}$ & $1.07 \varepsilon+00$ & $1.96 \mathrm{E}+00$ & $1.62 E+01$ & $3.96 E+\infty 0$ \\
\hline Peach Bottom 283 & $3.73 \mathrm{E}+01$ & $3.68 \mathrm{E}+01$ & $2.37 \mathrm{E}+01$ & $2.02 \mathrm{E}+01$ & $3.58 \mathrm{E}+01$ & $5.04 \mathrm{E}+01$ & $4.46 E+01$ & $4.64 \mathrm{E}+01$ & $9.69 \mathrm{E}+00$ & $2.00 \mathrm{E}+01$ \\
\hline Perry 1 & & & & & & & $2.67 \mathrm{E}-03$ & $3.49 \mathrm{E}+00$ & $7.34 \mathrm{E}+00$ & $6.96 \mathrm{E}+00$ \\
\hline Pilgrim 1 & $4.00 \mathrm{E}+01$ & $3.41 \mathrm{E}+01$ & $5.91 \mathrm{E}+00$ & $1.56 \mathrm{E}+01$ & $1.47 E+01$ & $7.81 \mathrm{E}+00$ & $1.00 \mathrm{E}+01$ & $3.21 E+\infty$ & 5.73E-01 & $2.37 \mathrm{E}+00$ \\
\hline Quad-Cities 182 & $1.03 E+01$ & $1.19 E+01$ & $7.80 \mathrm{E}+00$ & $3.88 E+00$ & $5.42 \mathrm{E}+00$ & $3.41 E+00$ & $6,43 E+\infty 0$ & $6.92 E+00$ & $7.28 \mathrm{E}+00$ & $2.91 E+01$ \\
\hline Fover Bend 1 & & & & & & & $4.56 \mathrm{E}+\infty$ & $6.92 \mathrm{E}+00$ & $9.65 E+00$ & $1.60 \mathrm{E}+01$ \\
\hline Shoreham 1 & & & & & & & $3.80 E-03$ & $6.04 \mathrm{E}-03$ & $\mathrm{~N} / \mathrm{D}$ & $\mathrm{N} / \mathrm{D}$ \\
\hline Susquehanna 182 & & 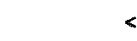 & $8.55 \mathrm{k}-01$ & $8.98 E+00$ & $1.12 \mathrm{E}+01$ & $9.14 \mathrm{E}+00$ & $1.54 E+01$ & $1.87 \mathrm{E}+01$ & $1.45 \mathrm{E}+01$ & $2.74 E+01$ \\
\hline otal & $4.80 E+02<$ & $2.84 E+02<$ & $3.42 E+02<$ & $4.99 E+02<$ & $4.33 E+02<$ & $3.44 \mathrm{E}+02$ & $<2.34 \mathrm{E}+02$ & $2.71 E+02$ & $2.52 E+02$ & $3.21 \mathrm{E}+02$ \\
\hline ort St. Vrain & $2.06 \mathrm{E}+02$ & $2.19 \mathrm{E}+02$ & $2.62 E+02$ & $3.69 E+02$ & $1.24 \mathrm{E}+02$ & $1.53 E+01$ & $1.27 \mathrm{E}+02$ & $5.61 E+01$ & $1.61 E+02$ & $1.12 \mathrm{E}+02$ \\
\hline
\end{tabular}

- High temperature gas cooled reactor

- Included with Dreaden 2-3 total

+ Included whth Edwin I. Hatch 2 tolal

$N / D=$ Not Detectable 


\begin{tabular}{|c|c|c|c|c|c|c|c|c|c|c|}
\hline Facility & 1970 & 1971 & 1972 & 1973 & 1974 & 1975 & 1976 & 1977 & 1978 & 1979 \\
\hline Arkansas One 1 & & & & & $2.56 \mathrm{E}+01$ & $4.60 \mathrm{E}+02$ & $2.12 \mathrm{E}+02$ & $2.45 E+02$ & $2.94 \mathrm{E}+02$ & $1.68 \mathrm{E}+02$ \\
\hline Arkansas One 2 & & & & & & & & & & $5.27 \mathrm{E}+01$ \\
\hline Beaver Valley $1 \& 2$ & & & & & & & $8.60 E+\infty 0$ & $1.08 \mathrm{E}+02$ & $3.49 E+02$ & $9.59 E+01$ \\
\hline \multicolumn{11}{|l|}{ Braldwood 1} \\
\hline \multicolumn{11}{|l|}{ Braldwood 2} \\
\hline \multicolumn{11}{|l|}{ Byron 182} \\
\hline \multicolumn{11}{|l|}{ Callaway 1} \\
\hline Calvert Cliffs 182 & & & & & & $2.63 \mathrm{E}+02$ & $2.74 \mathrm{E}+02$ & $5.75 \mathbb{E}+02$ & $4.56 \mathrm{E}+02$ & $5.14 E+02$ \\
\hline \multicolumn{11}{|l|}{ Catawba 1} \\
\hline \multicolumn{11}{|l|}{ Catawba 2} \\
\hline Donald C. Cook 182 & & & & & & $5.64 E+01$ & $1.92 \mathrm{E}+02$ & $2.86 \mathrm{E}+02$ & $6.24 \mathrm{E}+02$ & $1.22 E+03$ \\
\hline Crystal River 3 & & & & & & & & $1.66 \mathrm{E}+02$ & $1.54 \mathrm{E}+02$ & $1.66 \mathrm{E}+02$ \\
\hline Davis-Besse 1 & & & & & & & & $9.01 \mathrm{E}+00$ & $2.15 \mathrm{E}+02$ & $2.45 \mathbb{E}+02$ \\
\hline \multicolumn{11}{|l|}{ Dlablo Canyon 182} \\
\hline Joseph M. Farley 1 & & & & & & & & & $5.91 E+01$ & $9.40 \mathrm{E}+01$ \\
\hline \multicolumn{11}{|l|}{ Joseph M. Farley 2} \\
\hline Font Calhoun 1 & & & & $1.58 \mathrm{E}+01$ & $1.24 E+02$ & $1.11 E+02$ & $1.22 \mathrm{E}+02$ & $1.57 \mathrm{E}+02$ & $1.50 \mathrm{E}+02$ & $2.58 \mathrm{E}+02$ \\
\hline R. E. Ginna & $1.10 \mathrm{E}+02$ & $1.54 \mathrm{E}+02$ & $1.19 \mathrm{E}+02$ & $2.86 \mathrm{E}+02$ & $1.95 \mathrm{E}+02$ & $2.60 \mathrm{E}+02$ & $2.42 \mathrm{E}+02$ & $1.19 E+02$ & $2.42 E+02$ & $2.40 \mathrm{E}+02$ \\
\hline Haddam Neck & $7.40 \mathrm{E}+03$ & $5.83 E+03$ & $5.89 \mathrm{E}+03$ & $3.90 E+03$ & $2.24 E+03$ & $5.67 \mathrm{E}+03$ & $4.85 \mathrm{E}+03$ & $6.67 \mathrm{E}+03$ & $3.94 E+03$ & $3.55 \mathrm{E}+03$ \\
\hline \multicolumn{11}{|l|}{ Harris 1} \\
\hline Indian Point 1822 & & & & $2.75 \mathrm{E}+01$ & $4.79 E+01$ & $7.94 \mathrm{E}+01$ & $3.32 E+02$ & $3.71 \mathrm{E}+02$ & $5.12 \mathrm{E}+02$ & $3.75 \mathrm{E}+02$ \\
\hline Indtan Point 3 & & & & & & & Shown with & Other Unit & $2.56 \mathrm{E}+02$ & $1.15 E+02$ \\
\hline Kewaunee & & & & & $9.24 \mathrm{E}+01$ & $2.77 \varepsilon+02$ & $1.80 \mathrm{E}+02$ & $2.95 E+02$ & $2.96 \mathrm{E}+02$ & $2.49 \mathrm{E}+02$ \\
\hline Maine Yankee & & & $9.20 \mathrm{E}+00$ & $1.54 \mathrm{E}+02$ & $2.19 \mathrm{E}+02$ & $1.77 \mathrm{E}+02$ & $3.671+02$ & $1.53 E+02$ & $3.15 E+02$ & $2.02 E+02$ \\
\hline \multicolumn{11}{|l|}{ McGuire 1} \\
\hline \multicolumn{11}{|l|}{ Mcoulre 2} \\
\hline Mulstone 2 & & & & & & $7.60 \mathrm{E}+00$ & 2.77E+02 & $2.11 E+02$ & $2.01 E+02$ & $2.54 E+02$ \\
\hline \multicolumn{11}{|l|}{ Mullstone 3} \\
\hline North Anna 182 & & & & & & & & & $2.82 \mathrm{E}+02$ & $3.13 \mathrm{E}+02$ \\
\hline Oconee $1,2, \& 3$ & & & & $7.07 \mathrm{E}+01$ & $3.50 \mathrm{E}+02$ & $3.55 \mathrm{E}+03$ & $2.19 E+03$ & $1.92 \mathrm{E}+03$ & $1.17 E+03$ & $8.94 E+02$ \\
\hline Palisades & & & $2.08 \mathrm{E}+02$ & $1.85 E+02$ & 8. $10 E+\infty 0$ & $4.16 \mathrm{E}+01$ & $9.63 E+\infty 0$ & $5.58 \mathrm{E}+01$ & $1.01 \mathrm{E}+02$ & $1.26 \mathrm{E}+02$ \\
\hline \multicolumn{11}{|l|}{ Palo Verde 1} \\
\hline \multicolumn{11}{|l|}{ Palo Verde 2} \\
\hline \multicolumn{11}{|l|}{ Palo Verde 3} \\
\hline Point Beach $1 \& 2$ & & $2.66 \mathrm{E}+02$ & $5.63 \mathrm{E}+02$ & $5.56 \mathrm{E}+02$ & $8.33 E+02$ & $8.85 E+02$ & $6.94 \mathrm{E}+02$ & $9.99 \mathrm{E}+02$ & $1.29 \mathrm{E}+03$ & $8.92 E+02$ \\
\hline Pratrie Island 182 & & & & $=1.00 \mathrm{E}-01$ & $1.42 \mathrm{E}+02$ & $4.54 \mathrm{E}-01$ & $1.00 \mathrm{E}-01$ & $1.35 E+03$ & $5.51 \mathrm{E}+02$ & $6.25 E+02$ \\
\hline Rancho Seco 1 & & & & & & $1.32 \mathrm{E}+02$ & $\mathrm{~N} / \mathrm{D}$ & $8.55 \mathrm{E}-02$ & N/D & N/D \\
\hline H. B. Robinson 2 & & $1.18 E+02$ & $4.05 E+02$ & $4.32 \mathrm{E}+02$ & $4.49 \mathrm{E}+02$ & $6.24 \mathrm{E}+02$ & $9.80 \mathrm{E}+02$ & $6.85 E+02$ & $4.73 \mathrm{E}+02$ & $4.29 E+02$ \\
\hline Salem 1 & & & & & & & $4.00 \mathrm{E}-02$ & $2.96 \mathrm{E}+02$ & $4.46 \mathrm{E}+02$ & $7.26 \mathrm{E}+02$ \\
\hline \multicolumn{11}{|l|}{ Salem 2} \\
\hline San Onofre 1 & $4.80 \mathrm{E}+03$ & $4.57 \mathrm{E}+03$ & $3.48 \mathrm{E}+03$ & $4.07 \mathrm{E}+03$ & $3.81 E+03$ & $4.00 \mathrm{E}+03$ & $3.39 \mathrm{E}+03$ & $1.79 E+03$ & $2.50 E+03$ & $2.32 \mathrm{E}+03$ \\
\hline San Onolre 2-3 & & & & & & & & & & \\
\hline Seabrook 1 & & & & & & & & & & \\
\hline Sequoyah 182 & & & & & & & & & & \\
\hline South Texas 1 & & & & & & & & & & \\
\hline South Texas 2 & & & & & & & & & & \\
\hline St. Lacie 1 & & & & & & & $1.33 \mathrm{E}+01$ & $2.42 \mathrm{E}+02$ & $1.28 \mathrm{E}+02$ & $1.28 \mathrm{E}+02$ \\
\hline St. Lucle 2 & & & & & & & & & & \\
\hline Summer 1 & & & & & & & & & & \\
\hline Surry 182 & & & $5.00 \mathrm{E}+00$ & $4.88 E+02$ & $2.45 E+02$ & $4.42 \mathrm{E}+02$ & $7.82 \mathrm{E}+02$ & $4.08 \mathrm{E}+02$ & $7.47 \mathbb{E}+02$ & $3.57 \mathrm{E}+02$ \\
\hline Three Mile Island 1 & & & & & $1.30 \mathrm{E}+02$ & $4.63 \mathrm{E}+02$ & $1.89 \mathrm{E}+02$ & $1.92 \mathrm{E}+02$ & $1.55 \mathrm{E}+02$ & $5.59 \mathrm{E}+01$ \\
\hline Three Mile Island 2 & & & & & & & & & $3.83 E+01$ & $7.81 E+01$ \\
\hline TMI 2/Epicor & & & & & & & & & & \\
\hline Trojan & & & & & & & $3.60 \mathrm{E}+01$ & $3.11 \mathrm{E}+02$ & $1.59 \mathrm{E}+02$ & $6.80 E+01$ \\
\hline
\end{tabular}


Tabie 6

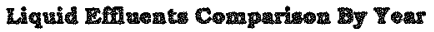

\section{Tritiun (Curios)}

Pressurtzed Water Reactors

Eacility

Turkey Point 384

Turkey Point 3

Turkey Point 4

Vogtle 182

Waterford 3

Wolf Creek 1

Yankee Rowe 1

Zion 1

Zton 2

Total
1970

1971

1972

1973

$3.29 \mathrm{E}+0$
1974

2

$1975 \quad 1976$

$7.97 \mathrm{E}+02 \quad 7.71 \mathrm{E}+02$
1977

$8.24 \mathrm{E}+02$
1978

$1.17 E+03 \quad 9.40 \mathrm{E}+02$

$\begin{array}{rrrrrrrrrr}1.50 \mathrm{E}+03 & 1.68 \mathrm{E}+03 & 8.03 \mathrm{E}+02 & 6.94 \mathrm{E}+02 & 3.14 \mathrm{E}+02 & 2.47 \mathrm{E}+02 & 1.56 \mathrm{E}+02 & 1.39 \mathrm{E}+02 & 1.96 \mathrm{E}+02 & 1.75 \mathrm{E}+02 \\ & & & 1.00 \mathrm{E}-01 & 2.74 \mathrm{E}+02 & 1.03 \mathrm{E}+03 & 7.47 \mathrm{E}+02 & 7.24 \mathrm{E}+02 & 7.25 \mathrm{E}+02 & 6.01 \mathrm{E}+02\end{array}$

$1.38 \mathrm{E}+04 \quad 1.26 \mathrm{E}+04 \quad 1.15 \mathrm{E}+04<1.12 \mathrm{E}+04 \quad 1.01 \mathrm{E}+04 \quad 1.96 \mathrm{E}+04 \quad 1.70 \mathrm{E}+04 \quad 1.94 \mathrm{E}+04 \quad 1.82 \mathrm{E}+04 \quad 1.65 \mathrm{E}+04$ 
Trittung (Curfec)

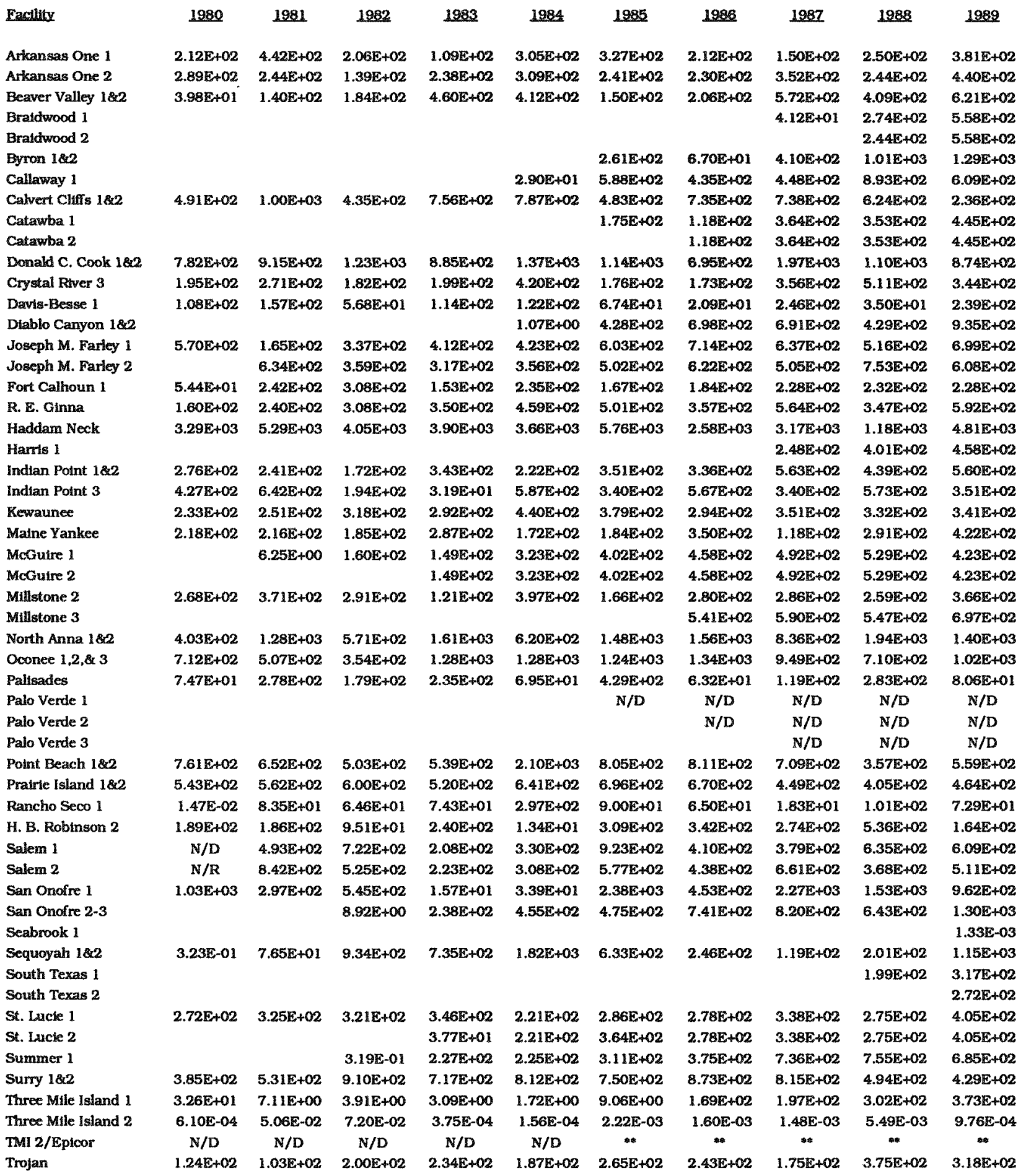

\footnotetext{
* Included with Three Mile Island 2 total 
Talle

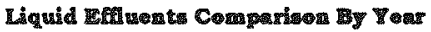

Trited (Curies)

Pressurtzed Water Reactors

\begin{tabular}{|c|c|c|c|c|c|c|c|c|c|c|}
\hline Facility & 1980 & 1981 & 1982 & 1983 & 1984 & $\mathbf{1 9 8 5}$ & 1026 & 1987 & 1988 & 1989 \\
\hline Turkey Point 384 & $7.49 \mathrm{E}+02$ & $1.95 \mathrm{E}+02$ & $6.27 \mathrm{E}+02$ & $7.12 E+02$ & $8.91 \mathrm{E}+02$ & & & & & \\
\hline Turkey Point 3 & & & & & & $4.33 \mathrm{E}+02$ & $3.64 \mathrm{E}+02$ & $2.69 E+02$ & $2.99 \mathrm{E}+02$ & $2.29 \mathrm{E}+02$ \\
\hline Turkey Point 4 & & & & & & $4.33 \mathrm{E}+02$ & $3.648+02$ & $2.69 E+02$ & $2.99 \mathrm{E}+02$ & $2.29 \mathrm{E}+02$ \\
\hline Vogtle $1 \& 2$ & & & & & & & & $3.21 \mathrm{E}+02$ & $3.90 \mathrm{E}+02$ & $9.18 \mathrm{E}+02$ \\
\hline Waterford 3 & & & & & & $2.54 E+01$ & $4.31 \mathrm{E}+02$ & 5.25E+02 & $5.03 E+02$ & $3.58 \mathrm{E}+02$ \\
\hline Wolf Creek 1 & & & & & & $1.83 E+02$ & $3.77 \mathrm{E}+02$ & $3.17 \mathrm{E}+02$ & $4.06 \mathrm{E}+02$ & $5.88 \mathrm{E}+02$ \\
\hline Zion 1 & $7.45 \mathrm{E}+02$ & $6.04 \mathrm{E}+02$ & $6.76 \mathrm{E}+02$ & $1.74 \mathrm{E}+02$ & $1.74 \mathrm{E}+02$ & $1.35 E+02$ & $2.67 \mathrm{E}+02$ & $2.16 \mathrm{E}+02$ & $4.11 E+02$ & $1.81 \mathrm{E}+02$ \\
\hline Zion 2 & & $2.66 E+02$ & $3.77 \mathrm{E}+02$ & $2.56 \mathrm{E}+02$ & $5.112+02$ & $5.21 \mathrm{C}+02$ & $4.46 \mathrm{R}+02$ & $4.40 \mathrm{E}+02$ & $5.581+02$ & $8.66 \mathrm{E}+02$ \\
\hline Toral & $1.37 E+04$ & $1.89 \mathrm{E}+04$ & $1.75 E+04$ & $1.81 E+04$ & $2.27 E+04$ & $2.78 \mathrm{E}+04$ & $2.32 \mathrm{E}+04$ & $2.81 E+04$ & $2.71 E+04$ & $3.40 \mathrm{E}+04$ \\
\hline
\end{tabular}


Table 7

Liquid Efruent Comparizon By Tear

Mixed Fission and Activation Products (Curies)

Boiling Water Reactors

Facility

197

$1972 \quad 1973$

1974

1975

1976

1977

1978

1979

Big Rock Point 1

Brunswick 182

Clinton 1

Cooper

Dresden 1

Dresden 2-3

Duane Arnold

Fermi 2

James A. Futzpatrick

Grand Culf 1

Cdwin I. Hatch 1

Edwin 1. Hatch 2

Hope Creek 1

Humboldt Bay 3

LaCrosse

$4.70 \mathrm{E}+00 \quad 3.50 \mathrm{E}+00 \quad 1.10 \mathrm{E}+00 \quad 2.70 \mathrm{E}+00$

$8.00 \mathrm{E}-01$

2.02E+100

$2.70 \mathrm{E}+00$

$1.89 \mathrm{E}+00$

7.7OE-0

$3.920-01$

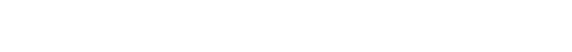

$8.20 \mathrm{E}+00 \quad 6.20 \mathrm{E}+00 \quad 6.80 \mathrm{E}+00 \quad 9.20 \mathrm{E}+00$

$1.40 \mathrm{E}+00 \quad 1.74 \mathrm{E}+00$

$2.30 \mathrm{E}+01 \quad 2.20 \mathrm{E}+01 \quad 2.59 \mathrm{E}+01$

6.906400

$6.90 \mathrm{E}+00 \quad 8.40 \mathrm{E}-01$

$1.19 \mathrm{E}+0$

2.74E-01 9.03E-01

$$
\begin{aligned}
& < \\
& \text { 8.10E-01 } 1.21 \mathrm{E}+00
\end{aligned}
$$$$
<1.00 \mathrm{E}-02<1.00 \mathrm{E}-02
$$$$
6.00 \mathrm{E}-02 \quad 4.00 \mathrm{E}-02 \quad 2.50 \mathrm{E}+01 \quad 4.03 \mathrm{E}-02 \quad 4.82 \mathrm{E}-02
$$

$\begin{array}{llllllllll}2.40 \mathrm{E}+00 & 1.80 \mathrm{E}+00 & 1.40 \mathrm{E}+\infty 0 & 2.40 \mathrm{E}+00 & 4.40 \mathrm{E}+00 & 3.79 \mathrm{E}+00 & 9.90 \mathrm{E}-01 & 9.17 \mathrm{E}-01 & 1.95 \mathrm{E}-01 & 9.55 \mathrm{E}-02\end{array}$ $\begin{array}{llllllllll}6.40 \mathrm{E}+00 & 1.71 \mathrm{E}+01 & 4.85 \mathrm{E}+01 & 3.59 \mathrm{E}+01 & 1.31 \mathrm{E}+01 & 1.42 \mathrm{E}+01 & <5.78 \mathrm{E}+00 & 2.13 \mathrm{E}+01 & 8.86 \mathrm{E}+00 & 1.67 \mathrm{E}+00\end{array}$

LaSalle 182

Limerick $18: 2$

Milstone 1

Monticello

Nine Mile Point 1

Nine Mlle Point 2

Oyster Creek 1

Peach Botton 283

Perry 1

Plgrim 1

Quad-Cities 182

River Bend 1

Shoreham 1

Susquehanna 182

Vermont Yankee 1

WNP-2

Total

$6.82 \mathrm{E}+01<1.16 \mathrm{E}+02<1.80 \mathrm{E}+02<1.77 \mathrm{E}+02 \quad 3.29 \mathrm{E}+02<2.80 \mathrm{E}+02<4.72 \mathrm{E}+01 \quad 6.58 \mathrm{E}+01 \quad 4.10 \mathrm{E}+01<4.49 \mathrm{E}+01$

Fort St. Vrain

$1.89 \mathrm{E}-04$

\footnotetext{
- High temperature gas cooled reactor N/D $=$ Not Detectable
} 
Table 7

Liquid Effuents Coraparimon By Yess

Mixed Fission and Activation Product (Curies)

Bolling Water Reactors

\begin{tabular}{|c|c|c|c|c|c|c|c|c|c|c|}
\hline Eacllity & 1980 & 1981 & 1982 & 1983 & 1984 & 1985 & 1986 & 1987 & 1988 & 1989 \\
\hline Big Rock Point 1 & $7.82 \mathrm{E}-01$ & $3.91 \mathrm{E}-01$ & $2.60 \mathrm{E}-01$ & $7.82 \mathrm{E}-02$ & $1.48 E-01$ & $1.53 \mathrm{E}-01$ & $2.09 \mathrm{E}-02$ & $2.73 \mathrm{E}-01$ & $2.18 \mathrm{E}-01$ & $2.32 E-01$ \\
\hline Browns Ferry $1,2,83$ & $9.38 \mathrm{E}+00$ & $2.24 \mathrm{E} \div 00$ & $5.36 \mathrm{E}+01$ & $1.28 \mathrm{E}+01$ & $6.30 \mathrm{E}+00$ & $1.34 \mathrm{E}+00$ & $5.39 \mathrm{~L}-01$ & $3.25 \mathrm{E}-01$ & $2.42 \mathrm{E}-01$ & $1.71 \mathrm{E}-01$ \\
\hline Brunswick $1 \& 2$ & $1.26 \mathrm{E}+00$ & $2.20 \mathrm{E}+00$ & $2.32 \mathrm{E} 400$ & $1.08 \mathrm{E}+00$ & $5.65 \mathrm{E}-01$ & $1.15 \mathrm{E}-01$ & $1.26 \mathrm{E}-01$ & $7.15 \mathrm{E}-01$ & $8.32 \mathrm{E}-01$ & $1.56 \mathrm{E}+\infty 0$ \\
\hline Clinton 1 & & & & & & & & $1.54 E-02$ & $1.10 \mathrm{E}-01$ & $1.74 \mathrm{E}-02$ \\
\hline Cooper & $<1.10 \mathrm{E}+01$ & $<3.61 E+00<$ & $<5.44 E+00<$ & $1.23 E t 01<$ & $6.30 \mathrm{C}+00<$ & $1.30 \mathrm{E}+01$ & $<7.40 E+00$ & $2.25 \mathrm{E} 400$ & $2.33 E+00$ & $2.19 E+00$ \\
\hline Dresden 1 & $N / D$ & $\mathrm{~N} / \mathrm{D}$ & $\mathrm{N} / \mathrm{D}$ & $N / D$ & $\mathrm{~N} / \mathrm{D}$ & $N / D$ & N/D & $\mathrm{N} / \mathrm{D}$ & + & + \\
\hline Duane Amold & $\mathrm{N} / \mathrm{D}$ & $\mathrm{N} / \mathrm{D}$ & $4.16 \mathrm{E}-06$ & NN/D & $1.90 \mathrm{E}-09$ & $8.24 E-04$ & $\mathrm{~N} / \mathrm{D}$ & $\mathrm{N} / \mathrm{D}$ & $\mathrm{N} / \mathrm{D}$ & $\mathbb{N} / \mathbf{D}$ \\
\hline Ferm1 2 & & & & & & & $3.67 \mathrm{E}-03$ & $2.10 \mathrm{E}-02$ & $7.41 E-02$ & $1.68 \mathrm{E}-01$ \\
\hline James A. Fitzpatrick & $1.51 \mathrm{E}+\infty$ & $2.51 E+\infty 0$ & $6.50 \mathrm{E}-01$ & $7.71 \mathrm{E}-01$ & $9.79 \mathrm{E} \cdot 02$ & $1.80 E-01$ & $1.92 \mathrm{E}-02$ & $7.84 E-02$ & $4.86 \mathrm{E}-02$ & $5.46 \mathrm{E}-02$ \\
\hline Grand Gulf 1 & & & & $4.42 \mathrm{E}-03$ & $3.16 E .02$ & $2.13 \mathrm{E}-01$ & $3.01 \mathrm{E}-01$ & $3.64 E-01$ & $3.96 \mathrm{E}-01$ & $3.20 \mathrm{E}-01$ \\
\hline Edwin 1. Hatch 1 & $6.83 \mathrm{E}-02$ & $3.73 \mathrm{E}-01$ & $7.00 \mathrm{E}-01$ & $9.09 \mathrm{E}-01$ & $1.05 E+00$ & $4.80 \mathrm{E}-01$ & $4.88 \mathrm{E}-01$ & $6.85 E^{3}-01$ & 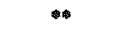 & ( \\
\hline Edwin 1. Hatch 2 & $4.57 \mathrm{E}-02$ & $1.63 \mathrm{E}-01$ & $1.83 \mathrm{E}_{-01}$ & $3.29 \mathrm{E}-01$ & $2.67 \mathrm{E}-01$ & 2.63E-01 & $3.02 \mathrm{C}-01$ & 1.30E-01 & $9.83 \mathrm{E}-0$. & $2.486-01$ \\
\hline Hope Creek 1 & & & & & & & $7.56 \mathrm{E}-01$ & $1.62 E+\infty 0$ & $7.24 E-01$ & $1.05 \mathrm{E}+00$ \\
\hline LaCrosse & $2.13 E+\infty$ & $2.26 \mathrm{E}-01$ & $5.83 E+\infty$ & $3.75 E+00$ & $3.26 \mathrm{E}+00$ & $1.83 E+\infty 0$ & $5.00 E+00$ & $1.16 E+\infty$ & $4.47 \mathrm{E}-01$ & $1.69 \mathrm{E}-01$ \\
\hline Lasalle 182 & & & $9.82 \mathrm{E}-01$ & $8.60 \mathrm{E}+00$ & $8.48 \mathrm{E}-02$ & $3.84 E+00$ & $1.78 \mathrm{E}-02$ & $8.898-01$ & $1.10 \mathrm{E}+01$ & $4.01 E+01$ \\
\hline Limerick 182 & & & & & $6.45 \mathrm{E}-04$ & $2.18 E-02$ & $5.74 \mathrm{E}-03$ & $7.45 E-02$ & $\mathrm{~N} / \mathrm{D}$ & $1.12 E-01$ \\
\hline Millstone 1 & $7.24 \mathrm{E}-01$ & $3.94 \mathrm{E}-01$ & $1.15 E+\infty$ & $8.08 \mathrm{E}-01$ & $3.78 E-02$ & $4.66 E-01$ & $7.73 \mathrm{E}-01$ & $1.14 \mathrm{E}+00$ & $1.08 \mathrm{E}+00$ & $9.065-01$ \\
\hline Monticello & $N / D$ & $3.11 \mathrm{E}-06$ & $5.80 \mathrm{E}_{-07}$ & N/D & $\mathrm{N} / \mathrm{D}$ & N/D & $N / D$ & $N / D$ & $N / D$ & $\mathrm{~N} / \mathrm{D}$ \\
\hline Nine Mile Point 1 & $N / D$ & $5.35 E+\infty$ & $2.51 \mathrm{E}-03$ & $1.11 \mathrm{E}-02$ & $\mathbb{N} / \mathrm{D}$ & $\mathrm{N} / \mathrm{D}$ & $<6.70 \mathrm{E}-04$ & $\mathrm{~N} / \mathrm{D}$ & $\mathrm{N} / \mathrm{O}$ & $\mathrm{N} / \mathrm{D}$ \\
\hline Nine Mlle Point 2 & & & & & & & & $1.30 \mathrm{E}+\infty$ & $3.08 \mathrm{E}+00$ & $2.20 \mathrm{E}-01$ \\
\hline Oyster Creek 1 & $5.06 \mathrm{E}-01$ & $2.48 \mathrm{E}-01$ & $8.10 \mathrm{E}-02$ & $3.63 \mathrm{E}-03$ & $6.84 E-03$ & $N / D$ & $\mathrm{~N} / \mathrm{D}$ & $6.63 \mathrm{E}-03$ & $2.68 \mathrm{E}-02$ & $5.01 \mathbb{E}-02$ \\
\hline Peach Bottom $2 \& 3$ & $1.90 \mathrm{E}+00$ & $1.97 E+\infty$ & $9.33 E+\infty$ & $2.24 \mathrm{E}+00$ & $6.15 E+00$ & $2.16 \mathrm{E}+\infty$ & $4.59 \mathrm{E}-01$ & $3.31 \mathrm{C}-01$ & $2.02 \mathrm{E}-01$ & 1.13E-01 \\
\hline Perry 1 & & & & & & & $3.67 \mathrm{E}-03$ & $1.47 E-02$ & $2.50 \mathrm{E}-01$ & $1.16 E+\infty$ \\
\hline Pilgrim 1 & $2.73 E+00$ & $1.94 \mathrm{E}+00$ & $8.72 \mathrm{E}-01$ & $9.35 \mathrm{E}-01$ & $4.75 \mathrm{E}+00$ & $1.06 \mathrm{E}+00$ & $<2.11 \mathrm{E} 01<$ & $1.47 \mathrm{E}+00$ & $3.56 \mathrm{E}-02$ & $2.498-02$ \\
\hline Quad-Cities 182 & $1.31 \mathrm{E}+01$ & $3.27 \mathrm{E}+00$ & $4.03 \mathrm{E}-01$ & $1.37 \mathrm{E}-01$ & $7.23 E_{-}-02$ & $1.46 \mathrm{E}+00$ & $2.36 \mathrm{E}-01$ & $7.10 \mathrm{E}-02$ & $5.60 \mathrm{E}-02$ & $4.84 \mathrm{E}-01$ \\
\hline River Bend 1 & & & & & & & $1.06 \mathrm{E}-01$ & $7.96 \mathrm{E}-02$ & $5.58 \mathrm{E}-01$ & $1.11 \mathrm{E}+\infty$ \\
\hline Shoreham 1 & & & & & & & $7.17 \mathrm{E}-03$ & $3.41 \mathrm{E}-03$ & $1.98 \mathrm{E} \cdot 05$ & $1.78 \mathrm{E}-05$ \\
\hline Susquehanna 182 & & $<$ & $=1.99 \mathrm{E}-01$ & $2.49 E+00$ & $1.45 \mathrm{E}-01$ & $6.35 \mathrm{E}-01$ & $7.92 \mathrm{E}-01$ & $3.12 \mathrm{E}-01$ & $9.48 \mathrm{E}-02$ & $1.02 \mathrm{E}-01$ \\
\hline Total & $<4.60 \mathrm{E}+01$ & $<2.51 \mathrm{E}+01<$ & $=8.24 \mathrm{E}+01<$ & $4.74 E+01$ & $2.96 \mathrm{E}+01<$ & $2.94 \mathrm{E}+01$ & $<1.79 \mathrm{E}+01<$ & $1.37 \mathrm{E}+01$ & $2.29 E+01$ & $1.16 \mathrm{E}+01$ \\
\hline Fort St. Vr & $6.37 \mathrm{E}-05$ & $3.64 \mathrm{E}-04$ & 4.34E-04 & $1.73 E-02$ & $1.27 \mathrm{E}-03$ & $1.84 \mathrm{E}-03$ & $2.30 \mathrm{E}-05$ & $1.18 \mathrm{E}-06$ & $1.69 \mathrm{E}-04$ & $1.22 \mathrm{E}-05$ \\
\hline
\end{tabular}

- High temperature gas cooled reactor

- Included with Edwin 1. Hatch 2 total

+ Included with Dresden 2-3 total

$\mathrm{N} / \mathrm{D}=\mathrm{Not}$ Detectable 
Tasle

Hequid Efivente Comparisom Toar

Pressurized Water Reactors

Mixed Fismion and Actration Froducts (Curlea)

\begin{tabular}{|c|c|c|c|c|c|c|c|c|c|c|}
\hline Eactity & 1970 & 1971 & 1972 & 1973 & 1974 & 1975 & 1979 & 1977 & 1978 & 1979 \\
\hline Arkansas One 1 & & & & & $6.50 \mathrm{E}+\infty 0$ & $3.11 \mathrm{E}+00$ & $1.31 \mathrm{E}+01$ & $4.50 \mathrm{E}+00$ & $6.05 E+00$ & $3.09 \mathrm{E}+00$ \\
\hline Arkansas One 2 & & & & & & & & & & $1.30 E+00$ \\
\hline Beaver Valley $1 \& 2$ & & & & & & & $1.70 \mathrm{E}-01$ & $6.52 \mathrm{E}-01$ & $2.63 \mathrm{E}-01$ & $1.21 \mathrm{E}-01$ \\
\hline \multicolumn{11}{|l|}{ Braldwood 1} \\
\hline \multicolumn{11}{|l|}{ Bratowood 2} \\
\hline \multicolumn{11}{|l|}{ Byron 182} \\
\hline \multicolumn{11}{|l|}{ Callaway 1} \\
\hline Calvert Cliffs 182 & & & & & & $1.44 \mathrm{E}+00$ & $1.18 \mathrm{E}+\infty$ & $3.48 E+00$ & $6.13 E+\infty$ & $7.80 \mathrm{E}+\infty 0$ \\
\hline \multicolumn{11}{|l|}{ Catawba I } \\
\hline \multicolumn{11}{|l|}{ Catawba 2} \\
\hline Donald C. Cook 182 & & & & & & $2.60 \mathrm{E}-01$ & $1.87 \mathrm{E}+\infty$ & $1.52 E+00$ & $1.48 E+\infty 0$ & $2.58 \mathrm{E}+00$ \\
\hline Crystal Plver 3 & & & & & & & & $1.54 \mathrm{E}-02$ & $2.96 \mathrm{E}-02$ & $4.16 \mathrm{E}-01$ \\
\hline Davls-Besse 1 & & & & & & & & $2.60 E-02$ & $9.01 \mathrm{E}-02$ & $4.28 \mathrm{E}-02$ \\
\hline \multicolumn{11}{|l|}{ Dtablo Canyon 182} \\
\hline Joseph M. Farley 1 & & & & & & & & & $1.03 \mathrm{E}-01$ & $5.86 \mathrm{E}-02$ \\
\hline \multicolumn{11}{|l|}{ Joseph M. Farley 2} \\
\hline Fort Calhoun 1 & & & & $<1.00 \mathrm{E}-01$ & $2.30 \mathrm{E}+00$ & $3.60 \mathrm{E}-01$ & $5.50 \mathrm{E}-01$ & $3.63 \mathrm{E}-01$ & $5.95 \mathrm{E}-01$ & $2.45 \mathrm{E}-01$ \\
\hline R. G. Ginna & $1.00 \mathrm{E}+01$ & $9.00 \mathrm{E}-01$ & $3.00 \mathrm{E}-01$ & $1.00 \mathrm{E} 01$ & $1.00 \mathrm{E}-01$ & $4.20 \mathrm{E}-01$ & $6.90 \mathrm{E}-01$ & $6.47 \mathrm{E}-02$ & $6.07 E-02$ & 8.63E-02 \\
\hline Haddam Neck & $6.70 \mathrm{E}+00$ & $5.90 \mathrm{E}+00$ & $4.80 \mathrm{E}+00$ & $3.00 E+00$ & $2.20 \mathrm{E}+\infty 0$ & $1.20 \mathrm{E}+00$ & $1.30 \mathrm{E}-01$ & $1.71 \mathrm{E}+00$ & $9.50 \mathrm{E}-01$ & $8.67 \mathrm{E}-01$ \\
\hline \multicolumn{11}{|l|}{ Harris 1} \\
\hline Indian Point 182 & & & & $2.20 \mathrm{E}+00$ & $4.20 \mathrm{E}+00$ & $4.93 \mathrm{E}+00$ & $<4.98 \mathrm{E}+00$ & $3.02 \mathrm{C}+00$ & $1.99 \mathrm{E}+00$ & $1.94 \mathrm{E}+\infty$ \\
\hline Indian Point 3 & & & & & & & Shown With & Other Unit & $1.03 E+00$ & $4.02 \mathrm{E}-01$ \\
\hline Kewaunce & & & & & $4,00 \mathrm{E}-01$ & $7.20 \mathrm{E}-01$ & $2.83 \mathrm{E}+00$ & $1.26 \mathrm{E}+\infty$ & $6.99 \mathrm{E}-01$ & $8.94 \mathrm{E}-01$ \\
\hline Matne Yankee & & & $<1.00 \mathrm{E}-01$ & $<1.00 \mathrm{E}-01$ & $4.00 \mathrm{~F}+00$ & $3.21 \mathrm{E}+00$ & $<2.84 \mathrm{E}+\infty$ & $4.42 E+01$ & $1.04 \mathrm{E}-01$ & $4.63 \mathrm{E}-01$ \\
\hline \multicolumn{11}{|l|}{ McGulre 1} \\
\hline \multicolumn{11}{|l|}{ MoGuire 2} \\
\hline Mallatone 2 & & & & & & $2.00 E-02$ & $2.60 \mathrm{E}-01$ & $1.56 \mathrm{E}+00$ & $2.79 E+00$ & $4.87 E+00$ \\
\hline \multicolumn{11}{|l|}{ Millstone 3} \\
\hline North Anna 182 & & & & & & & & & $2.68 \mathrm{E}-01$ & $5.89 \mathrm{E}-01$ \\
\hline Oconee $1,2,8,3$ & & & & $2.80 \mathrm{E}+00$ & $1.90 \mathrm{E}+00$ & $5.05 \mathrm{E}+00$ & $7.93 \mathrm{E}+00$ & $3.62 \mathrm{E}+01$ & $6.51 \mathrm{E}+00$ & $9.24 \mathrm{E}-01$ \\
\hline Paltsades & & & $6.80 \mathrm{E}+00$ & $2.78 \mathrm{E}+01$ & $5.90 \mathrm{E}+00$ & $3.45 E+00$ & $4.40 \mathrm{E}-01$ & $9.29 \mathrm{E}-02$ & $9.65 \mathrm{E}-02$ & $1.28 \mathrm{E}-01$ \\
\hline \multicolumn{11}{|l|}{ Palo Verde 3} \\
\hline Point Beach 182 & & $1.00 E-01$ & $1.50 \mathrm{E}+00$ & $8.00 E-01$ & $2.00 \mathrm{E}-01$ & $2.34 E+00$ & $3.24 \mathrm{E}+\infty$ & $1.50 \mathrm{E}+00$ & $6.86 \mathrm{E}-01$ & $7.25 \mathrm{E}-01$ \\
\hline Prairie Island 182 & & & & $<1.00 \mathrm{E}-01<$ & $1.00 \mathrm{E}-01$ & $4.50 \mathrm{E}-01$ & $1.00 \mathrm{E}-01$ & $1.33 E-02$ & $4.94 E-03$ & $9.00 \mathrm{E}-03$ \\
\hline Rancho Seco 1 & & & & & $<$ & $1.00 \mathrm{E}-02$ & $\mathrm{~N} / \mathrm{D}$ & $\mathrm{N} / \mathrm{D}$ & $\mathrm{N} / \mathrm{D}$ & $\mathrm{N} / \mathrm{D}$ \\
\hline H. B. Robinson 2 & & $7.00 \mathrm{E}-01$ & $8.00 \mathrm{E}-01$ & $6.00 \mathrm{E}-01$ & $2.50 \mathrm{E}+00$ & $4.50 \mathrm{E}-01$ & $3.80 \mathrm{E}-01$ & $3.29 \mathrm{E}-01$ & $1.78 \mathrm{E}-01$ & $2.99 E-01$ \\
\hline Salem 1 & & & & & & & $<1.00 \mathrm{E}-02$ & $2.88 \mathrm{E}+00$ & $4.02 \mathrm{E}+00$ & $3.98 \mathrm{E}+00$ \\
\hline \multicolumn{11}{|l|}{ Salem 2} \\
\hline San Onofre 1 & $7.60 \mathrm{E}+00$ & $1.50 \mathrm{E}+00$ & $3.03 \mathrm{E}+01$ & $1.60 \mathrm{E}+01$ & $5.00 \mathrm{E}+00$ & $1.22 \mathrm{E}+00$ & $7.43 E+00$ & $9.84 \mathrm{E}+00$ & $1.18 \mathrm{E}+01$ & $1.10 \mathrm{E}+01$ \\
\hline San Onofre 2-3 & & & & & & & & & & \\
\hline Seabrook 1 & & & & & & & & & & \\
\hline Sequoyah 182 & & & & & & & & & & \\
\hline South Texas 1 & & & & & & & & & & \\
\hline South Texas 2 & & & & & & & & & & \\
\hline St. Lucie 1 & & & & & & & $8.00 E-02$ & $5.80 \mathrm{E}+00$ & $2.80 \mathrm{E}+00$ & $2.67 \mathrm{E}+\infty 0$ \\
\hline St. Lucle 2 & & & & & & & & & & \\
\hline Summer 1 & & & & & & & & & & \\
\hline Surry 182 & & & $2.00 \mathrm{E}-01$ & $1.00 \mathrm{E}-01$ & $3.80 \mathrm{E}+\infty$ & $9.27 \mathrm{E}+00$ & $3.37 \mathrm{E}+01$ & $6.55 \mathrm{E}+01$ & $2.41 E+\infty$ & $2.53 \mathrm{E}+\infty$ \\
\hline Three Mile Island 1 & & & & & $1.30 \mathrm{E}+00$ & $7.00 \mathrm{E}-02$ & $1.00 \mathrm{E}-01$ & $1.94 \mathrm{E}-01$ & $6.14 E-01$ & $4.91 \mathrm{E}-01$ \\
\hline Three Mile Island 2 & & & & & & & & & $3.92 \mathrm{E}-01$ & $3.31 \mathrm{E}-01$ \\
\hline TMI 2/Epicor & & & & & & & & & & \\
\hline Trojan & & & & & & & $2.77 \mathrm{E}+\infty$ & $4.19 \mathrm{E}+00$ & $7.07 \mathrm{E}-01$ & $5.55 \mathrm{E}-01$ \\
\hline
\end{tabular}


Tละ

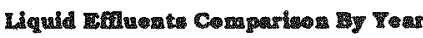

Mixed Fimaion and Actvetion Prod wet (Curien)

Pressurked Water Reactors

\begin{tabular}{|c|c|c|c|c|c|c|c|c|c|c|}
\hline Cacllity & 1970 & 1971 & 1972 & 1973 & 1974 & 1975 & 1976 & 1977 & 1978 & 1979 \\
\hline Turkey Point 384 & & & & $<1.00 \mathrm{E}-01$ & $1.60 \mathrm{E}+00$ & $3.07 \mathrm{E}+00$ & $<8.65 \mathrm{E}+00$ & $8.90 \mathrm{E}+00$ & $3.32 \mathrm{E}+00$ & $4.10 \mathrm{E}-01$ \\
\hline \multicolumn{11}{|l|}{ Turkey Point 3} \\
\hline \multicolumn{11}{|l|}{ Turkey Point 4} \\
\hline Yankee Rowe 1 & $<1.00 \mathrm{E}-01<$ & $1.00 \mathrm{E}-01<$ & $1.00 \mathrm{E}-01$ & $<1.00 \mathrm{E}-01$ & $1.00 \mathrm{E}-01$ & 2.00E-02 & $<1.00 \mathrm{~g}-02$ & $1.80 \mathrm{E}-02$ & $8.14 E-02$ & $1.17 \mathrm{E}-02$ \\
\hline Zton 1 & & & & $<1.00 \mathrm{E}-01$ & $<1.00 \mathrm{E}-01<$ & $1.00 \mathbb{E}-02$ & $1.60 \mathrm{E}-01$ & $9.50 \mathrm{E}-01$ & $9.51 \mathrm{E}-01$ & $7.00 \mathrm{E}-01$ \\
\hline \multicolumn{11}{|l|}{ Zlon 2} \\
\hline Tot 1 & $<2.44 E+01<$ & $9.20 \mathrm{E}+00<$ & $4.49 E+01$ & $<5.40 \mathrm{E}+01$ & $<.22 E+01<$ & $4.11 \mathrm{E}+01$ & $<9.36 \mathrm{E}+01$ & $1.55 \mathrm{E}+02$ & $5.72 \mathrm{E}+01$ & $5.05 E+01$ \\
\hline
\end{tabular}




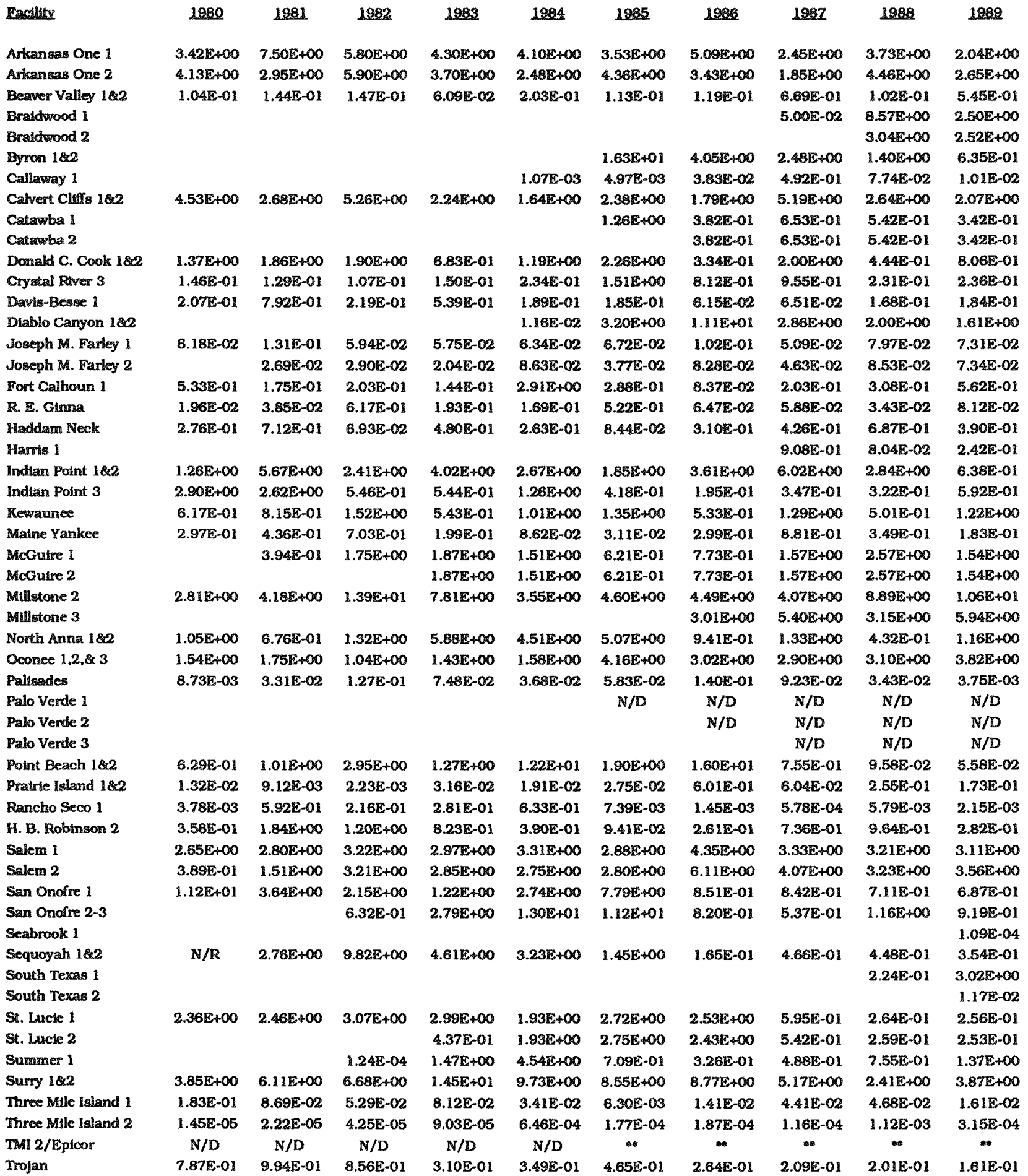

\footnotetext{
* Included wth Three Mile Island 2 total N/D $=$ Not Detectable $N / R=$ Not Reported
} 
Table

Liquid Eftrents Comparimon

Mixed Fismion and Actiwation Product (Curiow)

Pressurized Water Reactors

\begin{tabular}{|c|c|c|c|c|c|c|c|c|c|c|}
\hline Facility & 1980 & 1981. & 1982 & 1983 & 1984 & 1985 & 1986 & 1987 & 1988 & 1989 \\
\hline Turkey Point 384 & 6.78E-01 & $3.03 \mathrm{E}-01$ & $1.68 \mathrm{E}+\infty 0$ & $1.13 E+\infty 0$ & $2.27 \mathrm{E}-01$ & & & & & \\
\hline Turkey Point 3 & & & & & & $4.48 \mathrm{E}-01$ & $2.53 \mathrm{E}-01$ & $3.74 \mathrm{E}-01$ & $3.27 \mathrm{E}-01$ & $1.58 \mathrm{E}-01$ \\
\hline Turkey Point 4 & & & & & & $4.48 \mathrm{E}-01$ & $2.53 \mathrm{E}-01$ & $3.74 \mathrm{E}-01$ & $3.26 \mathrm{E}-01$ & $1.58 \mathrm{E}-01$ \\
\hline Vogtle 182 & & & & & & & & $5.77 \mathrm{E}-01$ & $1.66 \mathrm{E}+\infty 0$ & 4.03E-01 \\
\hline Waterford 3 & & & & & & $2.88 \mathrm{E}-01$ & $4.02 \mathrm{E}+00$ & $1.28 \mathrm{E}+00$ & $1.41 \mathrm{E}+00$ & $1.28 E+\infty$ \\
\hline Wolf Creek 1 & & & & & & $6.35 \mathrm{E}-01$ & $2.26 \mathrm{E}+00$ & $2.90 \mathrm{E}-01$ & $3.79 E-01$ & $7.23 \mathrm{E}-01$ \\
\hline Yankee Rowe 1 & $1.75 E-02$ & $1.43 \mathrm{E}-02$ & 9.53E-03 & $1.30 \mathrm{E}-02$ & $3.06 \mathrm{E}-02$ & $1.69 \mathrm{E}-02$ & $1.36 \mathrm{E}-02$ & $1.56 \mathrm{E}-02$ & $7.10 \mathrm{e}-02$ & $4.88 \mathrm{E}-03$ \\
\hline Zion 1 & $4.74 \mathrm{E}-01$ & $1.61 \mathrm{E}+00$ & $7.22 \mathrm{E}-01$ & $1.50 \mathrm{E}+00$ & $6.82 \mathrm{E}+00$ & $3.24 \mathrm{E}-01$ & 5.57E-01 & $7.53 \mathrm{E}-01$ & $1.61 E+00$ & $9.07 \mathrm{E}-01$ \\
\hline Zion 2 & & $1.05 \mathrm{E}+\infty$ & $1.65 E+\infty$ & $1.15 E+\infty$ & $7.06 \mathrm{E}+00$ & $2.05 \mathrm{E}+00$ & $1.04 E+00$ & $8.20 \mathrm{E}-01$ & $1.97 \mathbb{E}+00$ & $2.57 \mathrm{E}+00$ \\
\hline Cotal & $4.89 \mathrm{E}+01$ & $6.05 E+01$ & $8.17 E+01$ & $7.72 \mathrm{E}+01$ & $1.02 \mathrm{E}+02$ & $1.02 \mathrm{E}+02$ & $9.79 \mathrm{E}+01$ & $6.99 \mathrm{E}+01$ & $7.60 \mathrm{E}+01$ & $6.95 \mathrm{E}+01$ \\
\hline
\end{tabular}


Table 0

Dolid W Wumary 190

Bolling Water Reactors

Volume Activity No. of

Eactlity

Cubic.Meters

(Cundes) Shinments

Big Rock Potnt 1

Browns Ferry 1,2,83

Brunswick 182

$7.35 \mathrm{E}+01$

$3.71 E+02 \quad 12$

$5.84 \mathrm{E}+02$

$2.95 \mathrm{E}+02$

$6.06 \mathrm{E}+03$

$3.99 \mathrm{E}+02$

$1.89 \mathrm{E}+03$

$3.06 \mathrm{E}+02$

Cooper

$2.92 \mathrm{E}+02$

$2.24 \mathrm{E}+03$

$2.54 E+03$

$1.69 \mathrm{E}+04$

Duane Arnold

Fermi 2

$1.46 \mathrm{E}+02$

$3.66 \mathrm{E}+02$

$2.50 \mathrm{E}+02$

$2.72 \mathrm{E}+02$

8.53E+02

$1.67 \mathrm{E}+02$

$0.00 \mathrm{E}+00$

$6.7 \mathrm{AE}+00$

$8.80 \mathrm{E}+02$

$5.76 \mathrm{E}+02$

$4.28 \mathrm{E}+02$

$2.48 \mathrm{E}+02$

$2.37 \mathrm{E}+02$

$4.22 \mathrm{E}+02$

4.20E+02

$7.01 \mathrm{E} \nrightarrow 02$

$0.39 E+04$

$2.06 \mathrm{E}+02$

$1.91 \mathrm{E}+03$

$5.2715+02$

$0.00 E+00$

$3.21 \mathrm{E}+01$

$4.36 \mathrm{E}+03$

$3.40 \mathrm{OE}+04$

$1.992+04$

$5.97 \mathrm{E}+04$

$2.48 \mathrm{E}+02$

$4.895+02$

2.33E+05

$1.73 E+03$

$9.18 \mathrm{E}+02$

$9.68 \mathrm{E}+02$

$2.02 \mathrm{E}+02$

$9.79 \mathrm{E}+02$

$5.16 \mathrm{E}+02$

$2.76 \mathrm{c}+02$

$1.33 E+05$

$8.41 \mathrm{E}+02$

$1.79 \mathrm{E}+01$

$3.51 \mathrm{E}-02$

$1.34 E+03$

$4.28 \mathrm{E}+02$

$4.84 \mathrm{E}+\mathrm{CO}$

$2.15 E+00$

1. $10 \mathrm{E}+03$

45

91

48

31

112

17

39

39

45

109

28

0

5

136

183

52

23

176

33

71

238

39

42

93

67

1

45

1

WNP-2

$3.64 \mathrm{E}+02$

$6.17 \mathrm{E}+05$

1857

- Fort St. Vrain

$1.39 E+04$

$1.08 E+03$

- High temperature gas cooled reactor 


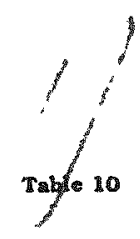

Solid Waste Suxurary 1989

Pressunzed Water Reactors

$\begin{array}{ccc}\text { Volume } & \text { Activity } & \text { No. O } \\ \text { Eacility } & \text { (CubicMeters) } & \text { (Cuniesl }\end{array}$

\begin{tabular}{|c|c|c|c|}
\hline Arkansas One 182 & $2.22 \mathrm{E}+02$ & $2.96 \mathrm{E}+02$ & 32 \\
\hline Beaver Valley 182 & $1.96 E+03$ & $1.35 \mathrm{E}+03$ & 43 \\
\hline Braidwood 182 & $3.10 \mathrm{E}+02$ & $3.89 \mathrm{E}+02$ & 17 \\
\hline Byrom $1 \& 2$ & $3.65 \mathrm{E}+02$ & $1.28 E+03$ & 52 \\
\hline Callaway 1 & $2.09 E+02$ & $6.00 \mathrm{E}+02$ & 22 \\
\hline Calvert Clifs 182 & $2.07 E+02$ & $4.14 \mathrm{E}+02$ & 10 \\
\hline Catawba 182 & $2.16 \mathrm{E}+02$ & $3.17 \mathrm{E}+02$ & 16 \\
\hline Donald C. Cook 182 & $3.88 \mathrm{E}+02$ & $1.17 \mathrm{E}+03$ & 130 \\
\hline Crystal Rover 3 & $3.47 E+02$ & $2.40 E+03$ & 24 \\
\hline Davis-Besse 1 & $1.18 \mathrm{E}+02$ & $2.08 \mathrm{E}+02$ & $\mathbf{8}$ \\
\hline Diablo Canyon $1 \& 2$ & $1.87 E+02$ & $4.29 \mathrm{E}+02$ & 45 \\
\hline Joseph M. Farley 182 & $4.85 \mathrm{E}+02$ & $4.00 \mathrm{E}+02$ & 30 \\
\hline Fort Calhoun 1 & $1.75 \mathrm{E}+\mathrm{O}_{2}$ & $8.76 E+00$ & 10 \\
\hline R.E.Ginna & $2.33 E+02$ & $7.99 E+01$ & 24 \\
\hline Haddam Neck & $1.53 E+02$ & $6.55 E+02$ & 24 \\
\hline Harris I & $1.60 E+02$ & $2.54 E+01$ & 43 \\
\hline Indian Point 182 & $4.78 \mathrm{E}+02$ & 3.60E +02 & 41 \\
\hline Indian Point 3 & $5.77 \mathrm{E}+02$ & $3.50 \mathrm{E}+02$ & 27 \\
\hline Kewaunee & $7.00 E+01$ & $7.74 E+02$ & 6 \\
\hline Maine Yankee & $1.95 E+02$ & $2.36 \mathrm{E}+02$ & 71 \\
\hline McGuire 1822 & $4.36 \mathrm{E}+02$ & $6.32 E+02$ & 16 \\
\hline Millstone 2 & $2.47 E+02$ & $5.55 \mathrm{E}+02$ & 18 \\
\hline Mullstone 3 & $1.47 \mathrm{E}+02$ & $7.37 \mathrm{E}+02$ & 12 \\
\hline North Anna $1 \& 2$ & $6.77 E+02$ & $1.72 \mathrm{E}+03$ & 44 \\
\hline Oconee $1,2.83$ & $4.25 \mathrm{E}+02$ & $1.46 \mathrm{E}+03$ & 80 \\
\hline Palisades & $2.19 \mathrm{E}+02$ & $8.32 \mathrm{E}+03$ & 28 \\
\hline Palo Verde $1,2,83$ & $8.74 E+02$ & $6.74 E+02$ & 66 \\
\hline Point Beach $1 \& 2$ & $1.06 \mathrm{E}+02$ & $2.54 \mathrm{E}+02$ & 27 \\
\hline Pratrie Island 182 & $1.25 \mathrm{E}+02$ & $1.03 E+02$ & 19 \\
\hline Rancho Seco 1 & $2.44 E+02$ & $3.27 \mathrm{E}+02$ & 22 \\
\hline H.B.Robinson 2 & $9.69 \mathrm{E}+01$ & $1.86 E+02$ & 47 \\
\hline Salem 182 & $1.22 \mathrm{E}+02$ & $5.65 \mathrm{E}+04$ & 43 \\
\hline San Onofre & $0.00 \mathrm{E}+00$ & $0.00 \mathrm{E}+00$ & 61 \\
\hline San Onofre 1 & $1.19 E+02$ & $1.72 E \div 03$ & 0 \\
\hline San Onofre 2-3 & $3.28 \mathrm{E}+02$ & $2.72 E+0.3$ & 0 \\
\hline Seabrook I & $0.00 E+\infty$ & $0.00 \mathrm{E}+00$ & 0 \\
\hline Sequoyah 182 & $4.65 \mathrm{E}+02$ & $2.64 \mathrm{E}+03$ & 91 \\
\hline South Texas 182 & $5.03 E+01$ & $7.24 \mathrm{E}+00$ & 9 \\
\hline St. l.ucie 182 & $3.18 E+02$ & $1.69 E+02$ & 72 \\
\hline Summer 1 & $1.40 E_{0}+02$ & $3.76 \mathrm{E}+02$ & 60 \\
\hline Surry 182 & $5.38 E+02$ & $1.31 \mathrm{E}+03$ & 34 \\
\hline Three Mile Island I & $3.32 E+02$ & $5.05 E+01$ & 24 \\
\hline Three Mile Island 2 & $1.18 E+03$ & $1.39 E+04$ & 54 \\
\hline TMI 2/Epicor & 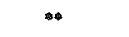 & p & $\Rightarrow$ \\
\hline Trojan & $2.59 \mathrm{E} \uparrow 02$ & $4.47 \mathrm{E}+02$ & 18 \\
\hline Turkey Point $3 \& 4$ & $3.46 E+02$ & $2.26 \mathrm{E} 400$ & 35 \\
\hline Vogtie 182 & $1,00 \mathrm{E}+02$ & $1.51 E+01$ & 13 \\
\hline Wateriond 3 & $7.61 \mathrm{E}+02$ & $4.07 E+02$ & 19 \\
\hline Wolf Creek 1 & $1.51 \mathrm{E}+02$ & $1.26 \mathrm{E}+03$ & 11 \\
\hline Yanke Rowe 1 & $2.98 \mathrm{E}+02$ & $1.78 E+01$ & 19 \\
\hline Zion 182 & $2.14 \mathrm{E}+02$ & $3.58 E+03$ & 35 \\
\hline Total & $1.64 \mathrm{E}+04$ & $1.12 \mathrm{E}+05$ & 1652 \\
\hline
\end{tabular}

- Represents solid waste shipped by plant but not broken down into untts $1,2,8$ i 3

* Included with Three Mile island 2 totals 
Table 11

Dolid Waste Comparimen By Tear

Bolling Watcr Reactors

Eacility

13tg Rock Point I

Browns Ferry $1,2,83$

Brrunswick 182

Clinton 1

Cooper

Dresden 1,2,\& 3

Duane Amold

Fermi 2

James A. Ftzpatrick

Grand Gulf 1

Edwin 1. Hatch 1

Edwin 1. Hatch 2

Hope Creek 1

Humboldt Bay 3

bacrosse

Lasalle 182

Monticello

Nine Mile Point 1

Nine Mue Point 2

Oyster Creek 1

Peach Bottom 283

Perry 1

Pilgrim 1

Quad-Cities $1 \& 2$

River Bend I

Shorcham 1

Susquehanna 182

Vermont Yankee

WNP-2

Tot

- Fort St. Vrain
Limerick 18.2

Millstone 1

Volume (Cublc Meters) - Activity (Curies) 1978

1979 1980

\begin{tabular}{|c|c|c|c|c|c|c|c|}
\hline $7.22 E+01$ & $9.68 \mathrm{E}+02$ & $3.10 \mathrm{E}+01$ & $2.56 \mathrm{E}+01$ & $8.99 E+01$ & $2.77 \mathrm{E}+02$ & $4.20 \mathrm{E}+01$ & $3.09 \mathrm{E}+01$ \\
\hline $1.82 E+03$ & $1.102+04$ & $2.90 E+03$ & $1.33 \mathrm{E}+03$ & $2.29 E+03$ & $4.17 \mathrm{E}+03$ & $2.49 E+03$ & $6.46 \mathrm{E}+03$ \\
\hline $2.47 E+03$ & $3.24 E+03$ & $2.02 E+03$ & $2.14 E+03$ & $3.09 \mathrm{E}+03$ & $4.29 \mathrm{E}+03$ & $6.73 E+03$ & $7.55 \mathrm{E}+03$ \\
\hline $2.83 E+02$ & $2.85 \mathrm{E}+02$ & $3.29 \mathrm{E}+02$ & $3.84 \mathrm{E}+02$ & $5.65 E+02$ & $9.69 \mathrm{E}+01$ & $4.35 E+02$ & $7.05 \mathrm{E}+02$ \\
\hline $2.25 E+03$ & $1.13 \mathrm{E}+04$ & $1.77 \mathrm{E}+03$ & $1.88 \mathrm{E}+03$ & $1.04 E+03$ & $8.45 E+02$ & $1.16 \mathrm{E}+03$ & $4.46 \mathrm{E}+03$ \\
\hline $5.45 E+02$ & $4.98 \mathrm{E}+02$ & $1.10 \mathrm{E}+03$ & $1.86 \mathrm{E}+03$ & $7.99 E+02$ & $8.01 E+02$ & $7.35 \mathrm{E}+02$ & $7.00 \mathrm{E}+02$ \\
\hline $1.23 E+03$ & $6.17 \mathrm{E}+03$ & $8.70 \mathrm{E}+02$ & $3.19 \mathrm{E}+02$ & $8.04 E+02$ & $1.06 \mathrm{E}+03$ & $7.50 \mathrm{E}+02$ & $8.86 \mathrm{E}+02$ \\
\hline \multirow[t]{2}{*}{$5.39 \mathrm{E}+02$} & $3.81 E+02$ & $7.50 \mathrm{E}+02$ & $1.09 \mathrm{E}+04$ & $9.78 E+02$ & $2.70 \mathrm{E}+02$ & $4.64 E+02$ & $9.62 \mathrm{E}+02$ \\
\hline & & & & & & $2.59 \mathrm{E}+02$ & 8.27E+01 \\
\hline $3.77 E+02$ & $2.00 E+01$ & $1.78 E+02$ & $7.91 \mathrm{E}-01$ & $9,06 E+01$ & $3.35 E+03$ & $8.20 \mathrm{E}+01$ & $6.95 \mathrm{E}+01$ \\
\hline $4.65 \mathrm{E}+00$ & $5.88 E+02$ & $3.80 \mathrm{E} \div 01$ & $6.18 E+01$ & $5.09 E+00$ & $1.25 E+02$ & $4.32 E+01$ & $2.02 \mathrm{E}+01$ \\
\hline $1.77 \mathrm{E}+03$ & $3.03 E+03$ & $2.00 E+03$ & $8.15 E+04$ & $2.11 E+03$ & $1.16 \mathrm{E}+03$ & $2.30 E+03$ & $2.36 \mathrm{E}+03$ \\
\hline $5.73 E+02$ & $2.91 \mathrm{E}+04$ & $4.99 \mathrm{E}+02$ & $6.35 E+04$ & $4.74 \mathrm{E}+02$ & $1.31 \mathrm{E}+04$ & $7.42 \mathrm{E}+02$ & $7.57 \mathrm{E}+02$ \\
\hline $6.65 \mathrm{E}+02$ & $2.51 \mathrm{E}+04$ & $3.85 \mathrm{E}+02$ & $2.24 \mathrm{E}+04$ & $4.97 E+02$ & $1.52 \mathrm{E}+03$ & $8.14 E+02$ & $2.32 E+04$ \\
\hline $1.74 E+03$ & $2.73 E+02$ & $1.54 \mathrm{E}+03$ & $1.15 E+03$ & 1.13E+03 & $1.34 \mathrm{E}+03$ & $2.03 E+03$ & $1.32 \mathrm{E}+03$ \\
\hline $2.52 \mathrm{E}+03$ & $1.82 \mathrm{E}+03$ & $1.96 E \downarrow 03$ & $4.97 \mathrm{E}+03$ & $2.40 \mathrm{E} 403$ & $8.03 \mathrm{E}+03$ & $2.64 E+03$ & $6.6918+03$ \\
\hline $5.84 E+02$ & $5.70 \mathrm{E}+03$ & $1.97 E+03$ & $4.92 \mathrm{E}+04$ & $3.03 E+03$ & $2.22 \mathrm{E}+04$ & $2.94 E+03$ & $1.60 \mathrm{E}+03$ \\
\hline $1.20 \mathrm{E}+03$ & $7.53 \mathrm{E}+03$ & $1.34 \mathrm{E}+03$ & $3.27 \mathrm{E}+03$ & $7.82 E+02$ & $4.26 \mathrm{E}+03$ & $1.67 \mathrm{E}+03$ & $4.07 E+03$ \\
\hline $1.08 E+02$ & $1.76 \mathrm{E}+02$ & $3.99 \mathrm{E}+02$ & $5.39 E+04$ & $2.71 E+02$ & $9.99 E+02$ & $4.84 E+02$ & $9.20 \mathrm{E}+02$ \\
\hline \multirow[t]{2}{*}{$1.88 E+04$} & $1.07 E+05$ & $2.01 E+04$ & $2.99 \mathrm{E}+05$ & $2.04 E+04$ & $6.79 \mathrm{E}+04$ & $2.68 \mathrm{E}+04$ & $6.28 \mathrm{E}+04$ \\
\hline & & & & $0.00 E+O O$ & $0.00 \mathrm{E}+00$ & $0.00 E+\infty 0$ & $0.00 E+00$ \\
\hline
\end{tabular}

- High temperature gas cooled reactor 
Table 11

Solid Waste Comparison By Year

Hoiling Water Reactors

Volume (Cubie Meters) - Activity (Curtes)

\begin{tabular}{|c|c|c|c|c|c|c|c|c|}
\hline Eacilitx & \multicolumn{2}{|c|}{1981} & \multicolumn{2}{|c|}{1982} & \multicolumn{2}{|c|}{1983} & \multicolumn{2}{|c|}{1984} \\
\hline Big Rock Point 1 & $1.44 \mathrm{E}+02$ & $3.17 \mathrm{E}+02$ & $1.09 \mathrm{E}+02$ & $4.33 \mathrm{E}+00$ & $1.01 \mathrm{E}+02$ & $2.74 E+02$ & $3.67 E+01$ & $2.13 \mathrm{E}+00$ \\
\hline Browns Ferty $1,2,83$ & $2.23 \mathrm{E}+0.3$ & $4.78 \mathrm{E} \uparrow 03$ & $5.91 \mathrm{E}+03$ & $5.51 E+03$ & $3.72 \mathrm{E}+03$ & $6.90 \mathrm{E} \rightarrow 03$ & $1.92 \mathrm{E}+03$ & $2.15 E+03$ \\
\hline Brunswick 182 & $4.30 \mathrm{E}+03$ & $7.47 \mathrm{E}+03$ & $3.53 E+03$ & $5.50 E+03$ & $3.51 \mathrm{E}+03$ & $8.36 \mathrm{E}+03$ & $1.37 \mathrm{E}+03$ & $3.45 \mathrm{E}+03$ \\
\hline \multicolumn{9}{|l|}{ Clinton 1} \\
\hline Cooper & $4.99 E+02$ & $4.43 \mathrm{E}+02$ & $4.45 \mathrm{E}+02$ & $4.27 \mathrm{E}+02$ & $5.03 E+02$ & $8.53 E+02$ & $4.37 E+02$ & $4.915+02$ \\
\hline Dresden $1,2,8,3$ & $1.14 \mathrm{E}+03$ & $4.596+03$ & $8.99 E+02$ & $1.66 \mathrm{E}+05+$ & $1.42 \mathrm{E}+03$ & $2.91 E+03$ & $1.26 \mathrm{E}+03$ & $4.37 \mathrm{E}+03$ \\
\hline Duane Amold & $6.97 \mathrm{E}+02$ & $1.07 E_{+}+03$ & $4.57 \mathrm{E}+02$ & $1.27 \mathrm{E}+03$ & $6.81 \mathrm{E}+02$ & $1.44 \mathrm{E}+03$ & $2.686+02$ & $9.13 E+02$ \\
\hline \multicolumn{9}{|l|}{ Ferm1 2} \\
\hline James A. Fizpatrick & $8.61 E+02$ & $1.63 \mathrm{E}+03$ & $1.64 \mathrm{E} * 03$ & $7.89 \mathrm{E}+02$ & $7.11 E+02$ & $7.03 E+02$ & $4.31 \mathrm{E}+02$ & $1.26 \mathrm{E}+03$ \\
\hline Grand Gulf 1 & & & & & $3.12 E+02$ & $7.21 \mathrm{E}+00$ & $4.312+02$ & $9.09 \mathrm{E}+00$ \\
\hline Edwin I. Hatch 1 & $1.29 E+03$ & $4.46 \mathrm{E}+03$ & $9.13 \mathrm{E}+02$ & $3.10 \mathrm{E}+03$ & $1.87 \mathrm{E}+03$ & $2.27 E+03$ & $2.50 \mathrm{C}+03$ & $2.58 E+03$ \\
\hline Edwin I. Hatch 2 & $1.40 E+03$ & $3.05 \mathrm{E}+02$ & $7.79 \mathrm{E}+02$ & $9.40 \mathrm{E}+02$ & * & 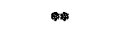 & 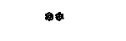 & 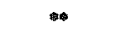 \\
\hline \multicolumn{9}{|l|}{ Hope Creek 1} \\
\hline Humboldt Bay 3 & $8.43 E+01$ & $5.46 \mathrm{E}-01$ & $7.71 \mathrm{E}+01$ & $1.34 \mathrm{E}+00$ & $2.78 \mathrm{E}+01$ & $1.75 E+04$ & $6.56 \mathrm{E}+01$ & $7.29 \mathrm{E}+00$ \\
\hline LaCrosse & $4.82 \mathrm{E}+00$ & $6.11 E+01$ & $3.53 E+01$ & $5.26 \mathrm{E}+01$ & $1.20 E+01$ & $1.88 \mathrm{E}+02$ & $4.22 \mathrm{E}+01$ & $1.93 E+02$ \\
\hline LaSalle 182 & & & $0.00 \mathrm{E}+00$ & $0.00 E+00$ & $6.83 E+02$ & $3.01 \mathrm{E}+01$ & $8.40 E+02$ & $1.80 \mathrm{E}+02$ \\
\hline Limerick $1 \& 2$ & & & & & & & $0.00 \mathrm{E}+00$ & $0.00 E+00$ \\
\hline Millstone 1 & $1.96 \mathrm{E}+03$ & $1.82 E+03$ & $9.77 \mathrm{E}+02$ & $1.08 \mathrm{E}+03$ & $6.93 E+02$ & $6.81 E+02$ & $9.40 \mathrm{E}+\mathrm{O} 2$ & $1.97 \mathrm{E}+03$ \\
\hline Monticello & $5.54 \mathrm{E}+02$ & $4.42 \mathrm{E}+02$ & $7.50 E+02$ & $3.89 E+03$ & $3.57 \mathrm{E}+02$ & $4.43 E+04$ & $1.24 \mathrm{E}+03$ & $5.73 E+02$ \\
\hline Nine Mile Point 1 & $5.31 E+02$ & $1.72 \mathrm{E}+03$ & $5.76 E+02$ & $7.07 \mathrm{E}+03$ & $7.21 \mathrm{E} 402$ & $5.42 E+04$ & $6.29 E+02$ & $1.34 \mathrm{E}+04$ \\
\hline \multicolumn{9}{|l|}{ Nine Mule Point 2} \\
\hline Oyster Creek 1 & $1.78 \mathrm{E}+03$ & $4.21 E+02$ & $9.96 E+02$ & $4.67 \mathrm{E}+03$ & $1.00 E+03$ & $5.61 E+02$ & $1.39 \mathrm{E}+03$ & $4.39 E+04$ \\
\hline Peach Bottom 283 & $2.34 E+03$ & $5.33 E_{403}$ & $3.23 E+03$ & $4.51 \mathrm{E}+03$ & $2.68 \mathrm{E}+03$ & $2.248+04$ & $2.26 \mathrm{E}+03$ & $9.22 \mathrm{E}+04$ \\
\hline \multicolumn{9}{|l|}{ Perry 1} \\
\hline Pilgrim 1 & $1.06 E+03$ & $9.38 \mathrm{E}+02$ & $2.28 \mathrm{E}+03$ & $9.59 E+02$ & $6.65 \mathrm{E}+02$ & $1.48 E+03$ & $3.12 \mathrm{E}+03$ & $1.54 \mathrm{E} * 03$ \\
\hline Quad-Cfties 182 & $1.72 E+03$ & $5.16 \mathrm{E}+03$ & $.46 \mathrm{E}+03$ & $3.98 E+03$ & $1.58 \mathrm{E} \downarrow 03$ & $5.85 E+03$ & $1.35 \mathrm{E}+03$ & $4.06 E+04$ \\
\hline \multicolumn{9}{|l|}{ River Bend 1} \\
\hline \multicolumn{9}{|l|}{ Shoreharn 1} \\
\hline Susquehanna $1 \& 2$ & & & $4.51 \mathrm{E}+01$ & $6.52 \mathrm{E}-02$ & $1.26 \mathrm{E}+03$ & $2.84 \mathrm{E}+02$ & $1.30 E+03$ & $9.27 \mathrm{E}+02$ \\
\hline Vermont Yankee 1 & $4.39 E+02$ & $1.11 \mathrm{E}+03$ & $4.51 \mathrm{E}+02$ & $2.09 E+02$ & $4.15 \mathrm{E}+02$ & $5.75 \mathrm{E}+04$ & $3.48 E+02$ & $2.85 \mathrm{E}+02$ \\
\hline WNPי-2 & & & & & & & $3.87 \mathrm{E}+02$ & $3.58 \mathrm{E}+01$ \\
\hline Total & $2.30 E+04$ & $4.2 \mathrm{IE}+04$ & $2.56 E+04$ & $2.10 \mathrm{E}+05$ & $2.29 \mathrm{E}+04$ & $2.29 \mathrm{E}+05$ & $2.26 \mathrm{E}+04$ & $2.11 E+05$ \\
\hline Fort St. Vrain & $0.00 E+00$ & $0.00 \mathrm{E}+00$ & $0.00 E+00$ & $0.00 E+00$ & $2.39 E+01$ & $1.84 E+01$ & $0.00 E+00$ & $0.00 \mathrm{E}+00$ \\
\hline
\end{tabular}

- High tempcrature gas cooled reactor

+ Includes 12 shipments of poison curtains (irradiated components) to Barnwell, SC

- Included with Edwin I. Hatch 1 totals 
Tabie 11

Solid W aste Comparimon By Year

Boiling Water Reactors

Volume (Cubic Meters) - Activity (Curies)

\begin{tabular}{|c|c|c|c|c|c|c|c|c|}
\hline Facility & & & & 86 & & 1987 & & 1988 \\
\hline Bigs Rock Point 1 & $5.22 \mathrm{E}+01$ & $1.14 \mathrm{E}+02$ & $9.46 E+\infty$ & $2.52 E+02$ & $7.40 \mathrm{E}+01$ & $2.30 \mathrm{E}+03$ & $4.44 \mathrm{E}+01$ & $7.28 \mathrm{E}+02$ \\
\hline Browns Ferty $1,2,83$ & $2.30 E+03$ & $3.08 \mathrm{E}+03$ & $1.36 \mathrm{E}+03$ & $1.49 E+03$ & $1.32 \mathrm{E}+03$ & $6.44 E+02$ & $7.81 E+02$ & $5.72 \mathrm{E}+02$ \\
\hline Brunswick 182 & $1.32 \mathrm{E}+03$ & $2.50 \mathrm{E}+03$ & $9.35 \mathrm{E}+02$ & $9.83 E+03$ & $8.43 E+02$ & $4.482+04$ & $6.89 \mathrm{E}+02$ & $3.07 \mathrm{E}+03$ \\
\hline Clinton 1 & & & & & $5.10 \mathrm{E}+01$ & $1.41 \mathrm{E}-01$ & $2.87 \mathrm{E}+02$ & $6.14 \mathrm{E}+01$ \\
\hline Cooper & $6.35 \mathrm{E}+02$ & $2.98 E+04$ & $4.49 E+02$ & $5.83 E+02$ & $3.41 E+02$ & $3.60 \mathrm{E}+02$ & $3.09 \mathrm{E}+02$ & $1.47 \mathrm{E}+02$ \\
\hline Dresden 1.2 .83 & $1.05 \mathrm{E}+04$ & $6.63 E+04$ & $2.14 E+03$ & $3.74 E+04$ & $1.80 \mathrm{E}+03$ & $8.26 \mathrm{E}+02$ & $2.35 E+03$ & $1.54 \mathrm{E}+03$ \\
\hline Duane Amold & $7.93 E+02$ & $5.24 E+02$ & $2.17 E+02$ & $2.15 E+04$ & $4.94 E+02$ & $2.62 \mathrm{E}+02$ & $2.12 E+02$ & $4.06 \mathrm{E}+02$ \\
\hline Fermit 2 & & & $1.48 E+02$ & $2.12 \mathrm{E} 401$ & $2.36 \mathrm{E}+02$ & $9.37 E+01$ & $2.38 E+02$ & $2.81 E+02$ \\
\hline James A. Fitzpatrick & $7.77 \mathrm{E}+02$ & $7.79 \mathrm{E}+02$ & $4.62 \mathrm{E}+02$ & $7.21 E+02$ & $5.13 E+02$ & $6.44 E+02$ & $3.81 E+02$ & $1.32 E+04$ \\
\hline Grand Gulf 1 & $6.02 \mathrm{E}+02$ & $2.60 \mathrm{E}+02$ & $4.39 E+02$ & $1.36 \mathrm{E}+03$ & $3.92 E+02$ & $1.65 \mathrm{E}+03$ & $4.99 E+02$ & $7.15 E+02$ \\
\hline Edwin 1. Hatch 1 & $2.04 \mathrm{E}+03$ & $3.83 E+04$ & $1.36 E+03$ & $8.82 E+02$ & $7.78 E+02$ & $1.82 E+03$ & $8.36 E+02$ & $2.02 E+03$ \\
\hline Edwin I. Hatch 2 & 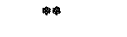 & * & $*$ & $\$$ & $*$ & 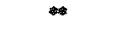 & 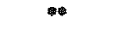 & - \\
\hline Hope Creek 1 & & & $8.45 E+01$ & $5.14 E+00$ & $4.21 E+02$ & $3.63 E+02$ & $2.91 E+02$ & $3.23 \mathrm{E}+03$ \\
\hline Humboldt Bay 3 & $8.31 \mathrm{E}+02$ & $2.60 \mathrm{E}+02$ & $5.99 \mathrm{E}+02$ & $3.50 \mathrm{E}+02$ & $0.00 E+\infty 0$ & $0.00 E+00$ & $3.99 E+01$ & $9.91 \mathrm{E}-02$ \\
\hline LaCrosse & $6.30 \mathrm{E}+01$ & $2.35 E+02$ & $4.81 E+00$ & $7.78 \mathrm{E}+01$ & $2.93 £+01$ & $2.86 \mathrm{E}-03$ & $6.52 \mathrm{E}+\infty 0$ & $7.03 E+01$ \\
\hline LaSalle 182 & $1.21 \mathrm{E}+03$ & $4.87 E+02$ & $8.02 E+02$ & $1.20 E+03$ & $7.66 E \div 02$ & $2.30 \mathrm{E}+03$ & $9.25 E+02$ & $3.38 \mathrm{E}+03$ \\
\hline Limerick $1 \& 2$ & $3.06 E+02$ & $2.06 \mathrm{E}+01$ & $5.76 \mathrm{E}+02$ & $7.53 E+02$ & $3.81 E+02$ & $2.15 E+03$ & $8.95 \mathrm{E}+02$ & $9.70 \mathrm{E}+02$ \\
\hline Millstone I & 1.17E+03 & $9.36 \mathrm{E}+04$ & $7.00 E+02$ & $7.85 \mathrm{E}+02$ & $6.66 \mathrm{E}+02$ & $5.05 E+02$ & $2.79 \mathrm{E}+02$ & $9.54 \mathrm{E}+04$ \\
\hline Monticello & $5.44 E+02$ & $4.87 \mathrm{E}+0.3$ & $2.94 E+02$ & $2.81 E+04$ & $2.19 E+02$ & $5.66 \mathrm{E}+02$ & $1.48 \mathrm{E}+02$ & $2.93 E+02$ \\
\hline Nine Mile Point 1 & $5.75 E+02$ & $6.80 E+03$ & $1.08 \mathrm{E}+03$ & $7.27 E+02$ & $5.07 \mathrm{E}+02$ & $2.30 \mathrm{E}+02$ & $2.72 \mathrm{E}+02$ & $3.07 E+02$ \\
\hline Nine Mile Point 2 & & & & & $9.89 \mathrm{E}+01$ & $1.14 \mathrm{E}+01$ & $3.78 \mathrm{E}+02$ & $3.88 \mathrm{E}+02$ \\
\hline Oyster Creek 1 & $4.62 E+02$ & $6.30 \mathrm{E}+02$ & $5.92 \mathrm{E}+02$ & $7.96 E+02$ & $2.36 \mathrm{E}+02$ & $3.48 \mathrm{E}+04$ & $1.81 E+02$ & $6.29 \mathrm{E}+03$ \\
\hline Peach Boltom $2 \& 3$ & $2.33 E+03$ & $1.21 \mathrm{E}_{4} 05$ & $1.49 E+03$ & $1.88 \mathrm{E}+04$ & $1.58 \mathrm{E}+03$ & $3.89 \mathrm{E} 403$ & $8.38 E+02$ & $1.19 E+03$ \\
\hline Perry 1 & & & $0.00 \mathrm{E}+00$ & $0.00 E+00$ & $4.89 \mathrm{E}+02$ & $4.52 \mathrm{E}+01$ & $4.95 E+02$ & $5.59 \mathrm{E}+02$ \\
\hline Plgrim 1 & $1.41 E+03$ & $7.48 E+04$ & $6.01 \mathrm{E}+02$ & $4.38 E+02$ & $5.27 E+02$ & $3.15 E+02$ & $2.72 E+02$ & $2.19 E+02$ \\
\hline Quad-Cules 182 & $1.33 E+03$ & $5.53 \mathrm{E}+04$ & $1.32 \mathrm{E}+03$ & $2.14 \mathrm{E}+03$ & $9.19 \mathrm{E}+02$ & $2.90 \mathrm{E}+04$ & $9.25 \mathrm{E}+02$ & $6.26 \mathrm{E}+02$ \\
\hline Rtver Bend 1 & & & $4.63 E+02$ & $7.9918+01$ & $4.07 \mathrm{E}+02$ & $3.45 \mathrm{E}+02$ & $3.06 E+02$ & $3.50 \mathrm{E}+02$ \\
\hline Shoreham 1 & & & $4.47 E+01$ & $1.47 \mathrm{E}-01$ & $6.26 E+01$ & $9.57 \mathrm{E}-02$ & $6.26 \mathrm{E}+01$ & $1.86 \mathrm{E}-02$ \\
\hline Susquehanna 182 & $1.13 E+03$ & $2.07 E+03$ & $8.68 \mathrm{E}+02$ & $2.53 E+03$ & $7.18 \mathrm{E}+02$ & $2.11 E+03$ & $1.33 E+03$ & $2.65 E+03$ \\
\hline Vermont Yankee 1 & $5.43 E+02$ & $1.73 \mathrm{E}+04$ & $3.10 \mathrm{E}+02$ & $3.33 E \div 02$ & $2.23 E+02$ & $1.19 E+04$ & $1.73 E+02$ & $4.25 \mathrm{E}+02$ \\
\hline WNP-2 & $4.02 E+02$ & $2.96 \mathrm{E}+02$ & $3.02 E+02$ & $5.07 E+02$ & $3.75 E+02$ & $1.09 \mathrm{E}+03$ & $4.70 E+02$ & $1.01 E+03$ \\
\hline Total & $3.13 E+04$ & $5.19 E+05$ & $1.77 \mathrm{E}+04$ & $1.32 \mathrm{E}+05$ & $1.55 E+04$ & $1.43 E+05$ & $1.49 \mathrm{E}+04$ & $1.40 E+05$ \\
\hline Fort St. Vrain & $1.10 E+02$ & $4.19 \mathrm{E}+02$ & $0.00 E+00$ & $0.00 \mathrm{E}+00$ & $3.02 E+01$ & $1.03 E+02$ & $7.00 \mathrm{E}+00$ & $3.10 \mathrm{E}-01$ \\
\hline
\end{tabular}

- Iligh temperature gas cooled reactor

* Included wath Eawin I. Hatch 1 totals 
Tabie 11

Solid Waste Comaparison By Year

Boiling Water Reactors

Volume (Cubic Meters) - Activity (Curies)

Eacility

1989

\begin{tabular}{|c|c|c|}
\hline Big Rock Point 1 & $7.35 \mathrm{E}+01$ & $3.71 E+02$ \\
\hline 13rowns Ferty $1,2,83$ & $5.84 E+02$ & $2.95 E+02$ \\
\hline Brunswick 1\&2 & $6.20 \mathrm{E}+02$ & $6.06 E+03$ \\
\hline Clinton 1 & $3.99 \mathrm{E}+02$ & $1.89 \mathrm{E}+03$ \\
\hline Cooper & $2.92 E+02$ & $3.06 E+02$ \\
\hline Dresden 1.2 .83 & $2.24 \mathrm{E}+03$ & $2.54 \mathrm{E}+03$ \\
\hline Duane Arnold & $1.46 \mathrm{E}+02$ & $1.69 \mathrm{E} * 04$ \\
\hline Fermi 2 & $3.66 \mathrm{E} * 02$ & $7.01 E+02$ \\
\hline James A. Fitzpatrick & $2.50 E+02$ & $9.39 \mathrm{E}+04$ \\
\hline Grand Gulf I & $2.72 \mathrm{E}+02$ & $2.06 \mathrm{E}+02$ \\
\hline Edwin 1. Hatch I & $8.53 E+02$ & $1.91 \mathrm{E}+03$ \\
\hline Edwin 1. Hatch 2 & $m$ & $*$ \\
\hline Hope Creek I & $1.67 \mathrm{E}+02$ & $5.27 \mathrm{E} \star 02$ \\
\hline Humboldt Bay 3 & $0.00 \mathrm{E}+00$ & $0.00 \mathrm{E}+00$ \\
\hline Lacrosse & $6.74 E+00$ & $3.21 E+01$ \\
\hline LaSalle $1 \& 2$ & $8.80 E+02$ & $4.36 \mathrm{E}+03$ \\
\hline Limerick 182 & $5.76 \mathrm{E}+02$ & $3.40 E+04$ \\
\hline Millstone 1 & $4.28 \mathrm{E}+02$ & $1.99 \mathrm{E}+04$ \\
\hline Monticello & $2.48 \mathrm{E}+02$ & $5.97 \mathrm{E}+04$ \\
\hline Nine Mile Point & $2.37 \mathrm{E}+02$ & $2.48 E+02$ \\
\hline Nine Mile Point 2 & $4.22 \mathrm{E}+02$ & $4.89 E+02$ \\
\hline Oyster Creek 1 & $4.20 \mathrm{E}+02$ & $2.33 E+05$ \\
\hline Peach Bottom 283 & $8.92 \mathrm{E}+02$ & $1.73 E+03$ \\
\hline Perry 1 & $9.68 \mathrm{E}+02$ & $9.18 \mathrm{E}+02$ \\
\hline Pilgrim 1 & $2.02 \mathrm{E}+02$ & $2.76 \mathrm{E}+02$ \\
\hline Quad-Citles 182 & $9.79 \mathrm{E}+02$ & $1.33 E+05$ \\
\hline River Bend 1 & $5.16 E+02$ & $8.41 E+02$ \\
\hline Shoreham 1 & $1.79 \mathrm{E}+01$ & $3.51 \mathrm{E}-02$ \\
\hline Susquehanna 182 & $4.28 E+02$ & $1.34 E+03$ \\
\hline Vermont Yankee 1 & $4.84 E+00$ & $2.15 \mathrm{E}+00$ \\
\hline WNP-2 & $3.64 \mathrm{E}+02$ & $1.10 \mathrm{E}+03$ \\
\hline Total & $1.39 E+04$ & $6.17 \mathrm{E}+05$ \\
\hline Fort St. Vrain & $4.57 \mathrm{E}+00$ & $1.08 \mathrm{E}+03$ \\
\hline
\end{tabular}

- High temperature gas cooled reactor

* Included with Edwin I. Hatch 1 totals 
Table 12

Solid Wast Compmrison By Year

Volume (Cubic Meters) - Activity (Curies)

\begin{tabular}{|c|c|c|c|c|c|c|c|c|}
\hline \multirow{2}{*}{$\begin{array}{l}\text { Eacility } \\
\text { Arkansas One } 1822\end{array}$} & \multicolumn{2}{|c|}{1977} & \multicolumn{2}{|c|}{1978} & \multicolumn{2}{|r|}{1979} & \multicolumn{2}{|r|}{1980} \\
\hline & $3.17 E+02$ & $1.26 \mathrm{E}+02$ & N/R & N/R & $\mathbf{N} / \mathbf{R}$ & $N / R$ & $\mathbf{N} / \mathbf{R}$ & $\mathbf{N} / \mathbf{R}$ \\
\hline Beaver Vallcy $1 \& 2$ & $2.67 \mathrm{E}+02$ & $8.18 E+\infty 0$ & $4.39 E+02$ & $2.25 E+02$ & $2.441+02$ & $2.95 E+02$ & $2.84 E+02$ & $5.34 E+02$ \\
\hline \multicolumn{9}{|l|}{ Braidwood 182} \\
\hline \multicolumn{9}{|l|}{ Byron 182} \\
\hline \multicolumn{9}{|l|}{ Callaway 1} \\
\hline Calvert Cltfs $1 \& 2$ & $3.09 \mathrm{E} \div 02$ & $9.83 E+02$ & $6.03 E+02$ & $1.12 E+03$ & $4.32 \mathrm{E}+02$ & $9.71 \mathrm{E}+02$ & $2.51 E+02$ & $1.48 \mathrm{E}+04$ \\
\hline \multicolumn{9}{|l|}{ Catawba 182} \\
\hline Donald C. Cook $1 \& 2$ & $6.84 E+02$ & $8.28 \mathrm{E}+01$ & $1.28 \mathrm{E}+03$ & $2.25 \varepsilon+02$ & $1.09 E+03$ & $3.37 E+02$ & $2.10 \mathbb{E}+03$ & $1.04 E+03$ \\
\hline Crystal River 3 & $4.48 E+02$ & $3.48 \mathrm{E}+00$ & $6.87 \mathrm{E}+02$ & $2.72 E+04$ & $1.24 \mathrm{E}+03$ & $1.20 \mathrm{E}+03$ & $9.27 E+02$ & $2.05 \mathrm{E}+03$ \\
\hline Davis-Besse 1 & $0.00 \mathrm{E}+00$ & $0.00 \mathrm{E}+00$ & $3.40 \mathrm{E}+02$ & $3.30 \mathrm{E}+00$ & $2.60 \mathrm{E}+02$ & $2.86 E+00$ & $3.30 \mathrm{E}+02$ & $3.00 E+01$ \\
\hline \multicolumn{9}{|l|}{ Drablo Canyon $1 \& 2$} \\
\hline Joseph M Farley $1 \& 2$ & & & $2.69 E+02$ & $5.72 \mathrm{E}+00$ & $1.11 E+03$ & $2.32 \mathrm{E}+02$ & $4.41 \mathrm{E}+02$ & $2.26 E+02$ \\
\hline Fant Calhoun 1 & $5.97 \mathrm{E}+02$ & $6.46 \mathrm{E}+02$ & $5.84 E+02$ & $1.06 \mathrm{E}+02$ & $2.44 E+02$ & $2.99 \mathrm{E}+01$ & $4.06 \mathrm{E}+02$ & $1.32 E+03$ \\
\hline R. E. Ginna & $3.49 E+02$ & $6.90 \mathrm{E}+02$ & $5.96 \mathrm{E}+01$ & $6.27 E+02$ & $3.08 E+02$ & $1.53 E+02$ & $4.00 \mathrm{E}+02$ & $4.60 \mathrm{E}, 402$ \\
\hline Haddam Neck & $1.68 \mathrm{E}+03$ & $8.41 E+02$ & $2.29 \mathrm{E}+02$ & $1.44 E+02$ & $1.29 \mathrm{E}+03$ & $3.05 E+02$ & $1.26 \mathrm{E}+03$ & $4.89 \mathrm{E}+02$ \\
\hline \multicolumn{9}{|l|}{ Harris 1} \\
\hline Indlan Point $18: 2$ & $1.06 \mathrm{E}+03$ & $1.45 \mathrm{E}+03$ & $8.43 E+03$ & $2.37 \mathrm{E}+03$ & $1.17 E+03$ & $2.16 E+03$ & $1.03 E+03$ & $3.32 E+02$ \\
\hline Indtan Point 3 & \multicolumn{2}{|c|}{ Shown With Oher Unit } & $5.94 \mathrm{E}+02$ & $6.49 \mathrm{E}+01$ & $2.25 E+02$ & $1.63 E+02$ & $3.47 \mathrm{E}+02$ & $2.02 \mathrm{E}+02$ \\
\hline Kewaunee & $3.37 \mathrm{E}+01$ & $3.66 \mathrm{E}+02$ & $7.98 \mathrm{E}+01$ & $1.50 E+03$ & $1.7 \mathrm{OE}+02$ & $3.54 E+02$ & $1.03 E+02$ & $1.37 \mathrm{E}+03$ \\
\hline Maine Yankee & $1.84 E+02$ & $1.53 E+04$ & $5.81 E+02$ & 4.142 .03 & $3.63 \mathrm{E}+02$ & $2.77 \mathrm{E}+03$ & $4.57 E+02$ & $4.79 \mathrm{E}+03$ \\
\hline \multicolumn{9}{|l|}{ McGuire 182} \\
\hline Mullstone 2 & $9.35 E+01$ & $5.80 \mathrm{E}+01$ & $1.55 E+02$ & $1.70 \mathrm{E}+01$ & $2.46 \mathrm{E}+02$ & $1.78 \mathrm{E}+03$ & $7.51 \mathrm{E}+00$ & $2.28 \mathrm{E}+02$ \\
\hline \multicolumn{9}{|l|}{ Millistone 3} \\
\hline North Anna $1 \& 2$ & & & $2.14 \mathrm{E}+01$ & $3.59 E+00$ & $2.95 \mathrm{E}+02$ & $5.89 E+01$ & $2.64 \mathrm{E}+02$ & $1.54 E+02$ \\
\hline Oconee $1,2,83$ & $1.07 \mathrm{E}+03$ & $7.37 \mathrm{E}+03$ & $1.58 \mathrm{E}+03$ & $5.93 \mathrm{E}+03$ & $1.63 E+03$ & $2.59 E+03$ & $1.32 \mathrm{E}+03$ & $2.91 \mathrm{E}+03$ \\
\hline Palisades & $4.43 E+02$ & $8.71 E+01$ & $7.17 E+02$ & $3.40 E+03$ & $6.84 \mathrm{E}+02$ & $3.92 \mathrm{E}+02$ & $7.31 \mathrm{E}+02$ & $1.18 \mathrm{E}+02$ \\
\hline \multicolumn{9}{|l|}{ Palo Verde $1,2,83$} \\
\hline Point Beach 182 & $6.84 \mathrm{E}+03$ & $5.68 E+02$ & $1.61 \mathrm{E}+02$ & $1.51 \mathrm{E}+03$ & $2.69 E+02$ & $1.22 \mathrm{E}+03$ & $4.49 \mathrm{E}+02$ & $9.35 E+02$ \\
\hline Prairte Island 182 & $6.43 E+02$ & $2.46 \mathrm{E}+02$ & $1.95 E+02$ & $1.53 E+02$ & $1.99 \mathrm{E}+01$ & $8.83 E+01$ & $5.25 \mathrm{E}+02$ & $1.98 E+02$ \\
\hline Rancho Seco I & $5.06 \mathrm{E}+01$ & $1.21 \mathrm{E}+03$ & $1.29 \mathrm{E}+02$ & $1.27 \mathrm{E}+03$ & $1.01 \mathrm{E}+02$ & $4.03 E+00$ & $4.60 \mathrm{E}+02$ & $1.12 \mathrm{E}+02$ \\
\hline H. B. Robinson 2 & $2.59 \mathrm{E}+02$ & $1.24 \mathrm{E}+03$ & $8.22 E+02$ & $2.40 E+02$ & $8.34 E+02$ & $8.72 E+01$ & $3.99 \mathrm{E}+03$ & $3.08 E+02$ \\
\hline Sallem 182 & $4.25 \mathrm{E}+02$ & $2.20 \mathrm{E}+00$ & $2.27 E+02$ & $1.94 \mathrm{E}+02$ & $6.86 \mathrm{E}+02$ & $1.28 \mathrm{E} \uparrow 02$ & $1.01 E+03$ & $4.59 \mathrm{E}+02$ \\
\hline \multicolumn{9}{|l|}{ San Onofre } \\
\hline San Onofre 1 & $3.68 \mathrm{E}+02$ & $6.02 E+01$ & $1.31 \mathrm{E}+02$ & $7.17 E+00$ & $8.35 \mathrm{E}+01$ & $9.24 E+01$ & $7.12 \mathrm{E}+02$ & $4.35 E+02$ \\
\hline \multicolumn{9}{|l|}{ San Onolre 2-3 } \\
\hline \multicolumn{9}{|l|}{ Scabrook 1} \\
\hline Sequoyah 182 & & & & & & & $N / R$ & $N / R$ \\
\hline South Texas 182 & & & & & & & & \\
\hline St. Lucie 182 & $3.85 E \div 02$ & $3.27 E+0.3$ & $3.58 \mathrm{E}+02$ & $1.26 E+04$ & $3.08 E+02$ & $1.79 E+02$ & $3.12 \mathrm{E}+02$ & $7.46 \mathrm{E}+02$ \\
\hline Summer 1 & & & & & & & & \\
\hline Surry 182 & $7.93 E+02$ & $6.10 E+02$ & $6.03 E+02$ & $5.66 E+02$ & $2.74 E+03$ & $3.45 \mathrm{E}+02$ & $2.01 \mathrm{E}+03$ & $7.06 \mathrm{E}+02$ \\
\hline Threc Mile Island I & $2.18 E+02$ & $4.73 E+01$ & $3.89 \mathrm{E}+02$ & $2.34 \mathrm{E}+02$ & $7.51 \mathrm{E}+02$ & $3.12 \mathrm{E}+01$ & $4.62 \mathrm{E}+02$ & $2.30 E+02$ \\
\hline Three Mike Island 2 & & & Shown With & Other Unit & Shown With & Other Unit & $7.67 \mathrm{E}+02$ & $1.26 \mathrm{E}+02$ \\
\hline TMI 2/Epicor & & & & & & & $0.00 E+00$ & $0.00 E+\infty 0$ \\
\hline Trojan & $1.01 E+02$ & $8.31 E+01$ & $2.26 \mathrm{E}+02$ & $4.48 E+02$ & $6.37 E+02$ & $3.30 \mathrm{E}+02$ & $5.14 \mathrm{E}+02$ & $4.59 \mathrm{E}+01$ \\
\hline Turkey Point 3\&4 & $1.07 \mathrm{E}+03$ & $4.26 \mathrm{E}+02$ & $1.75 E+03$ & $1.72 E+03$ & $9.20 \mathrm{E}+02$ & $2.48 E+02$ & $7.24 \mathrm{E}+02$ & $1.61 \mathrm{E}+02$ \\
\hline Vogtle 182 & & & & & & & & \\
\hline Waterford 3 & & & & & & & & \\
\hline Wolf Creek 1 & & & & & & & & \\
\hline Yankee Rowe 1 & $2.81 E+02$ & $3.54 E+00$ & $2.60 \mathrm{E}+02$ & $9.75 \mathrm{E}+00$ & $2.36 \mathrm{E}+02$ & $1.63 \mathrm{E}+02$ & $2.07 \mathrm{E}+02$ & $9.57 \mathrm{E}+01$ \\
\hline Zion 182 & $1.97 \mathrm{E}+03$ & $2.25 \mathrm{E}+02$ & $1.63 \mathrm{E} \div 03$ & $1.86 \mathrm{E}+03$ & $5.97 E+02$ & $2.69 \varepsilon+03$ & $1.64 \mathrm{E}+03$ & $2.55 \mathrm{E}+03$ \\
\hline Total & $2.09 E+04$ & $3.60 \mathrm{E}+04$ & $2.35 E+04$ & $6.79 E+04$ & $1.92 \mathrm{E}+04$ & $1.94 E+04$ & $2.44 E+04$ & $3.82 E+04$ \\
\hline
\end{tabular}

- Represents sold waste shipped by plant but not broken down into units $1,2, \& 3$

$N / R=$ Not Reported 
Table 12

Solıd Waste Coraporison By Year

Prossurved Waler keactors

Volumic (Cubic Meters) Activity (Curies)

\begin{tabular}{|c|c|c|c|c|c|c|c|c|}
\hline Eacility & \multicolumn{2}{|c|}{1981} & \multicolumn{2}{|c|}{1982} & \multicolumn{2}{|r|}{1983} & \multicolumn{2}{|r|}{1984} \\
\hline Arkansas One 182 & $N / R$ & $N / R$ & $N / R$ & $\mathbb{N} / \mathbf{R}$ & $706 E+02$ & $209 \mathbb{E}+03$ & $810 E+02$ & $146 E+03$ \\
\hline Beaver Volley $1 \& 2$ & $213 E+02$ & $930 E+01$ & $294 E+02$ & $383 E^{*}+02$ & $219 \mathrm{E}+02$ & $475 \mathrm{E}+02$ & $156 E+02$ & $711 \mathrm{E}+02$ \\
\hline \multicolumn{9}{|l|}{ Braidwood 182} \\
\hline \multicolumn{9}{|l|}{ Byron 182} \\
\hline Callaway 1 & & & & & & & $000 E+00$ & $0002+00$ \\
\hline Calvert cliffs $1 \& 2$ & $500 E+02$ & $986 E+01$ & $157 E+02$ & $916 \mathrm{E}_{6}+02$ & $506 E+02$ & $107 \mathrm{E}+02$ & $528 E+02$ & $3776+04$ \\
\hline \multicolumn{9}{|l|}{ Catawba 182} \\
\hline Donald C Cook $1 \& 2$ & $963 E+02$ & $143 E+03$ & $714 \mathrm{E}+02$ & $845 E+02$ & $668 \mathrm{E}+02$ & $201 E+03$ & $94 E+02$ & $669 \mathrm{E}+02$ \\
\hline Crystal River 3 & $127 E+03$ & $138 E+03$ & $662 \mathrm{E}+02$ & $628 E^{\circ}+02$ & $540 \mathrm{OE}+02$ & $155 \mathrm{E}+03$ & $411 E+02$ & $115 \mathrm{E}+03$ \\
\hline Davis Besse 1 & $325 E+02$ & $395 E+01$ & $000 \mathrm{E}+00$ & 0 OOE +00 & $113 E+02$ & $637 \mathrm{E}+02$ & $151 \mathrm{E}+02$ & $473 \mathrm{E}+02$ \\
\hline Diablo Canyon $1 \& 2$ & & & & & & & $0.00 E+\infty 0$ & $000 E+00$ \\
\hline Josx.ph M Fariky I\&2 & $564 \mathrm{E}+02$ & $720 \mathrm{E}+02$ & $346 \mathrm{E}+02$ & $103 E+02$ & $441 \mathrm{E}+02$ & $105 \mathrm{E}+03$ & $562 \mathrm{E}+02$ & $298 E+02$ \\
\hline Fort Calhoun 1 & $253 E+02$ & $101 E+02$ & $342 E+02$ & $354 \mathrm{E}+01$ & $465 \mathrm{E}+02$ & $700 E+02$ & $393 E+02$ & $717 \mathrm{E}+01$ \\
\hline R E Ginna & $376 \mathrm{E}+02$ & $635 \mathrm{E}+02$ & $489 \mathrm{C}+02$ & $202 E+02$ & $336 \mathrm{E}+02$ & $521 \mathrm{c}+02$ & $252 \mathrm{E}+02$ & $323 \mathrm{E}+02$ \\
\hline lladdam Seck & $438 E+02$ & $661 \mathrm{E}+02$ & $312 \mathrm{E}+02$ & $257 E+02$ & $652 \mathrm{E}+02$ & I $52 \mathrm{E}+03$ & $428 E+02$ & $375 \mathrm{E}+02$ \\
\hline \multicolumn{9}{|l|}{ Harris 1} \\
\hline Induan Point $1 \& 2$ & $158 \mathrm{E}+03$ & $171 E_{403}$ & $117 \mathrm{E}+03$ & $646 E+03$ & $129 \mathrm{E}+03$ & $212 E+03$ & $981 \mathrm{E}+02$ & $203 E+03$ \\
\hline Indlan Point 3 & $317 E_{0}+02$ & $640 \mathrm{E}+01$ & $379 E+02$ & $614 E+01$ & $316 \mathrm{E}+02$ & $732 \mathrm{E}+02$ & $153 \mathrm{E}+02$ & $412 E+02$ \\
\hline Kewaunee & $738 \mathrm{E}+01$ & $198 E+02$ & $673 E+01$ & $2748+02$ & $552 E+01$ & $685 E+02$ & $632 E+01$ & $160 \mathrm{E}+03$ \\
\hline Maine Yankee & $414 E+02$ & $167 \varepsilon+03$ & $220 E+02$ & $309 E+01$ & $337 \mathrm{E}+02$ & $103 \mathrm{E}+02$ & $349 E+02$ & $359 \mathrm{E}+02$ \\
\hline MLGuire $1 \& 2$ & $198 E+01$ & $131 \mathrm{EO} 01$ & $991 E+01$ & $643 E+00$ & $244 E+02$ & $282 \mathrm{E}+01$ & $414 E+02$ & $1898+03$ \\
\hline Millstone 2 & $163 E+01$ & $321 \mathrm{E}+02$ & $685 E+\infty$ & $484 E+02$ & $448 \mathrm{E}+01$ & $258 \mathrm{E}+02$ & 6 OSE +01 & $110 E+05$ \\
\hline \multicolumn{9}{|l|}{ Millstone 3} \\
\hline North Anrial 182 & $302 \mathrm{E}+02$ & $262 \mathrm{E}+03$ & $421 E+02$ & $305 \mathrm{E}+02$ & $539 E+02$ & $187 E \nrightarrow 03$ & $900 \mathrm{E}+02$ & $953 E+02$ \\
\hline Oconee $1,2 \& 3$ & $248 E+03$ & $112 E+04$ & $306 E+03$ & $109 E+04$ & $116 E+03$ & $284 E+03$ & $936 E+02$ & $617 \mathrm{E}+03$ \\
\hline Palisades & $854 E+02$ & $157 \mathrm{E}+04$ & $708 \mathrm{E}+02$ & $798 \mathrm{E}+01$ & $575 E+02$ & $256 E+04$ & $448 E+02$ & $258 E+02$ \\
\hline \multicolumn{9}{|l|}{ Palo Verde 1,283} \\
\hline Point Beach $1 \& 2$ & $177 E+02$ & $487 \mathrm{E}+02$ & $252 \mathrm{E}+02$ & $946 E+02$ & $711 \mathrm{E}+02$ & $112 \mathrm{E}+03$ & $712 \mathrm{E}+02$ & $164 \mathrm{E}+03$ \\
\hline Pratrie Island $1 \& 2$ & $297 \mathrm{E}+02$ & $564 E+01$ & $991 \mathrm{E}+01$ & $364 \mathrm{C}+02$ & $239 \mathrm{E}+02$ & $192 \mathrm{E}+02$ & $419 E+01$ & $119 \mathrm{E}+01$ \\
\hline Rancho Seco 1 & $231 E+02$ & $144 E+02$ & $240 E+02$ & $466 \mathrm{E}+02$ & $272 E+02$ & $225 E+02$ & $425 \mathrm{E}+02$ & $460 E+01$ \\
\hline II B Robinson 2 & $902 \mathrm{E}+02$ & $188 \mathrm{~L}+01$ & $138 \mathrm{~L}+03$ & $638 \mathrm{E}+01$ & $109 E+03$ & $462 \mathrm{E}+01$ & $305 E+03$ & $195 E+02$ \\
\hline Salem 182 & $936 E+02$ & $114 \mathrm{E}+03$ & $191 E+03$ & $319 \mathrm{E} \div 02$ & $207 E+03$ & $299 E+02$ & $152 E+03$ & $623 \mathrm{E}+02$ \\
\hline \multicolumn{9}{|l|}{ San Onofre } \\
\hline San Onofre 1 & $162 E+03$ & $126 \mathrm{E}+03$ & $927 \mathrm{E}+02$ & $752 E_{4}+01$ & $333 E+02$ & $227 E+02$ & $291 E+02$ & $154 E+01$ \\
\hline San Onofre 23 & & & $000 E+00$ & 0 OOE +00 & $189 \mathrm{E}+02$ & $798 \mathrm{C}+\infty 0$ & $2022+02$ & $549 \mathrm{C}+02$ \\
\hline \multicolumn{9}{|l|}{ Scabrook 1} \\
\hline Sequoyah 182 & $161 \mathrm{E}+02$ & $292 E+01$ & $358 E+02$ & $228 E+02$ & $693 \mathrm{E}+02$ & $230 E+03$ & $967 \mathrm{E}+02$ & $243 E+03$ \\
\hline \multicolumn{9}{|l|}{ South 7 exas 182} \\
\hline St Lucle 182 & $250 E+02$ & $296 E+02$ & $307 E+02$ & $795 \mathrm{E}+02$ & $620 \mathrm{E}+02$ & $939 \mathrm{E}+04$ & $122 E+03$ & $636 \mathrm{E}+04$ \\
\hline Summer 1 & & & $000 E+00$ & 0 OOE +00 & $925 E+01$ & $137 E+01$ & $480 E+02$ & $155 E+02$ \\
\hline Surry $1 \& 2$ & $280 E+03$ & $136 E+03$ & $217 E+03$ & $989 E+02$ & $308 E+03$ & $356 \mathrm{E}+03$ & $945 E+02$ & $116 \mathrm{E}+03$ \\
\hline Thre Mik Island I & $798 \mathrm{C}+02$ & $234 C+02$ & $532 E+02$ & $891 \mathrm{E}+00$ & $605 \mathrm{E}+02$ & $684 E+02$ & $434 E+502$ & $418 E+02$ \\
\hline Three Mile Island 2 & $274 E+02$ & $5\|1 E+0\|$ & $180 E+02$ & $122 E+01$ & $316 \mathrm{E}+02$ & $517 E+05$ & $2566+02$ & $989 E+03$ \\
\hline TMI 2/ Spkor & $151 \mathrm{E}+02$ & $350 E+02$ & $000 \mathrm{C}+00$ & 0 OOL +00 & $223 E+02$ & $462 E+04$ & $453 E+00$ & $235 E-01$ \\
\hline Trujan & $375 E+02$ & $104 \mathrm{E}+03$ & $217 E+01$ & $287 E+02$ & $228 E+02$ & $167 E+03$ & $230 E+02$ & $585 E+01$ \\
\hline ] urkcy Point 384 & $125 \mathrm{E}+03$ & $117 \mathrm{E}+02$ & $101 E+03$ & $113 E+03$ & $121 \mathrm{E}+03$ & $926 \mathrm{E}+02$ & $850 \mathrm{E}+02$ & $191 \mathrm{E}+03$ \\
\hline \multicolumn{9}{|l|}{ Vogtle 182} \\
\hline \multicolumn{9}{|l|}{ Watcrford 3} \\
\hline \multicolumn{9}{|l|}{ Wolf Cresk 1} \\
\hline Yankec Rowe 1 & $308 E \nless 02$ & $679 E+01$ & $209 E+02$ & $281 E+01$ & $158 E+02$ & $512 E+00$ & $200 E+02$ & $163 \mathrm{E}+02$ \\
\hline Zion 182 & $153 E+03$ & $344 \mathrm{E}+03$ & $882 E+02$ & $217 E+03$ & $921 E+02$ & $297 \mathrm{E}+03$ & $643 \mathrm{E}+02$ & $262 \mathrm{E}+03$ \\
\hline Total & $230 E+04$ & $487 \mathrm{E}+04$ & $199 E+04$ & $299 E+04$ & $223 E+04$ & $716 \mathrm{E}+05$ & $210 \bar{E}+04$ & $252 \mathrm{E}+05$ \\
\hline
\end{tabular}

- Represents solid waste shipped by plant but not broken down fnio units 12 \& 3

$\mathrm{N} / \mathrm{R}=$ Not Reported 
Tabie 12

Solid Wate Comparion By Yor

Pressurized Water Reactors

Volume (Cuble Meters) - Activity (Curies)

\begin{tabular}{|c|c|c|c|c|c|c|c|c|}
\hline Eacillity & \multicolumn{2}{|c|}{1985} & \multicolumn{2}{|c|}{1986} & \multicolumn{2}{|c|}{1987} & \multicolumn{2}{|r|}{1988} \\
\hline Arkansas One 182 & $6.88 E+02$ & $1.75 E+03$ & $1.21 E+02$ & $2.18 E+02$ & $5.23 E+02$ & $1.63 \mathrm{E}+03$ & $1.97 \mathrm{E}+02$ & $8.22 \mathrm{E}+02$ \\
\hline Beaver Valley 182 & $1.56 \mathrm{E}+02$ & $9.71 \mathrm{E}+01$ & $9.49 \mathrm{E}+01$ & $4.45 E+02$ & $7.65 \mathrm{E}+01$ & $3.22 \mathrm{E}+01$ & $2.33 E+02$ & $4.29 E+02$ \\
\hline Braldwood 182 & & & & & $0.00 \mathrm{E}+00$ & $0.005+00$ & $8.32 \mathrm{E}+01$ & $3.51 E+00$ \\
\hline Byron 182 & $1.78 \mathrm{E}+02$ & $1.39 \mathrm{E}+01$ & $3.18 \mathrm{E}+02$ & $9.90 \mathrm{E}+01$ & $3.06 \mathrm{E} \uparrow 02$ & $8.65 \mathrm{E}+02$ & $3.09 E+02$ & $5.095+02$ \\
\hline Callaway 1 & $1.39 \mathrm{E}+02$ & $6.29 \mathrm{E}+00$ & $1.68 \mathrm{E}+02$ & $1.91 \mathrm{E}+01$ & $1.98 \mathrm{E}+02$ & $3.13 \mathrm{E}+02$ & $9.58 \mathrm{E}+01$ & $9.46 \mathrm{E}+02$ \\
\hline Calvert Clifs 182 & $3.89 \mathrm{E}+02$ & $1.51 \mathrm{E}+0 \mathrm{~s}$ & $2.12 \mathrm{E}+\mathrm{O} 2$ & $4.51 \mathrm{E}+02$ & $2.44 \mathrm{E}+02$ & $6.4 \mathrm{in}+02$ & $5.25 \mathrm{E}+01$ & $1.12 \mathrm{E}+03$ \\
\hline Catawba 182 & $3.48 E+01$ & $6.90 \mathrm{E}-02$ & $1.93 \mathrm{E}+02$ & $1.33 \mathrm{E}+01$ & $2.73 E+02$ & $2.79 E+02$ & $2.56 \mathrm{E}+02$ & $7.05 E+02$ \\
\hline Donald C. Cook $1 \& 2$ & $8.28 E+02$ & $2.00 \mathrm{E} \leftrightarrow 03$ & $5.28 \mathrm{E}+02$ & $1.59 \mathrm{E}+03$ & $4.63 E+02$ & $2.30 \mathrm{E}+03$ & $2.46 \varepsilon+02$ & $5.58 \mathrm{E}+02$ \\
\hline Crystal River 3 & $4.98 \mathrm{E}+02$ & $4.60 E+03$ & $3.64 E+02$ & $1.35 \approx+03$ & $2.90 \mathrm{E}+02$ & $6.75 E+02$ & $2.26 \mathrm{E}+02$ & $1.07 \mathrm{E}+03$ \\
\hline Davis-Besse 1 & $1.97 \mathrm{E} 402$ & $9.58 \mathrm{e}+01$ & $1.40 \mathrm{E}+02$ & $2.19 \mathrm{E}+00$ & $8.48 \mathrm{E}+01$ & $3.20 \mathrm{E}+00$ & $1.72 \mathrm{E}+02$ & $1.76 \mathrm{E}+02$ \\
\hline Dlablo Canyon $1 \& 2$ & $3.11 \mathrm{E}+01$ & $4.40 \mathrm{E}+01$ & $9.06 \mathrm{E}+01$ & $6.97 \mathrm{E} 400$ & $1.65 E+02$ & $1.21 \mathrm{C}+02$ & 2. $10 E+02$ & $3.92 \mathrm{E}+02$ \\
\hline Joseph M. Farky $1 \& 2$ & $4.95 E+02$ & $8.20 E+02$ & $2.45 E+02$ & $1.80 \mathrm{E}+03$ & $5.32 E+02$ & $3.34 E+02$ & $4.96 \mathrm{E}+02$ & $1.47 E+03$ \\
\hline Fort Calhoun 1 & $3.43 \mathrm{E}+02$ & $2.24 \mathrm{E}+02$ & $1.16 \mathrm{E}+02$ & $2.82 E+01$ & $1.26 E+02$ & $5.47 \mathrm{E}+02$ & $4.87 \mathrm{E}+01$ & $1.75 \mathrm{E}+01$ \\
\hline R. E. Ginna & $2.23 E+02$ & $1.19 \mathrm{E}+02$ & $1.12 \mathrm{E}+02$ & $1.39 \mathrm{E}+02$ & $1.65 E+02$ & $2.16 \mathrm{~B}+02$ & $1.87 \mathrm{E}+02$ & $3.80 \mathrm{E}+02$ \\
\hline Haddam Neck & $1.73 E+02$ & $5.33 E+01$ & $4.14 E+02$ & $5.86 E+02$ & $3.20 \mathrm{E}+02$ & $5.45 E+02$ & $1.31 \mathrm{E}+02$ & $3.37 \mathrm{E}+02$ \\
\hline Harris 1 & & & & & $1.05 \mathrm{E}+02$ & $2.61 E+00$ & $1.50 E+02$ & $1.05 E+01$ \\
\hline Indtan Point 182 & $6.89 E+02$ & $5.75 \mathrm{E}+02$ & $5.30 E+02$ & $2.52 \mathrm{E}+02$ & $2.30 \mathrm{E}+02$ & $8.34 \mathrm{E}+02$ & $2.41 E+02$ & $4.67 E+02$ \\
\hline Indian Point 3 & $2.39 \mathrm{E}+02$ & $5.49 E+02$ & $8.29 \mathrm{E}+01$ & $2.58 \mathrm{E}+01$ & $3.17 \mathrm{E}+02$ & $3.33 E+02$ & $1.82 E+02$ & $3.57 \mathrm{E}+02$ \\
\hline Kewaunee & $7.77 \mathrm{E}+01$ & $9.56 \mathrm{E}+02$ & $5.31 \mathrm{E}+01$ & $1.33 \mathrm{E}+02$ & $8.25 \mathrm{E}+01$ & $4.58 E+02$ & $7.49 \mathrm{E}+01$ & $4.83 \mathrm{E}+02$ \\
\hline Matne Yankee & $3.59 E+02$ & $1.11 E+02$ & $1.96 E+02$ & $1.64 \mathrm{E}+02$ & $7.50 E+01$ & $1.90 \mathrm{E}+02$ & $1.37 \mathrm{E}+02$ & $4.36 \mathrm{E}+02$ \\
\hline McGutre 182 & $6.60 \mathrm{E}+02$ & $1.97 \mathrm{E}+02$ & $7.83 E+02$ & $6.73 E+02$ & $6.92 \mathrm{E}+02$ & $3.23 E+02$ & $5.16 E+02$ & $6.49 E+02$ \\
\hline Millst one 2 & $2.87 \mathrm{E}+01$ & $6.16 \mathrm{E}+03$ & $8.85 \mathrm{E}+01$ & $6.17 \mathrm{E}+03$ & $1.38 \mathrm{E}+02$ & $2.13 E+04$ & $1.59 \mathrm{E}+02$ & $5.06 \mathrm{E}+01$ \\
\hline Millstone 3 & & & $5.47 \mathrm{E}+00$ & $5.17 \mathrm{E}-01$ & $9.95 \mathrm{E}+01$ & $5.91 E+01$ & $1.63 E+02$ & $5.34 \mathrm{E}+02$ \\
\hline North Anna 182 & $6.50 \mathrm{E}+02$ & $2.90 \mathrm{E}+02$ & $5.30 E+02$ & $7.97 E+02$ & $4.89 E+02$ & $1.64 \mathrm{E}+03$ & $2.95 \mathrm{E}+02$ & $7.71 E+02$ \\
\hline Oconee $1,2,83$ & $4.33 E+02$ & $1.41 \mathrm{E} 403$ & $7.60 \mathrm{E}+02$ & $8.51 E+02$ & $8.53 \mathrm{E}+02$ & 2.01E+03 & $7.23 E+02$ & $1.42 E+04$ \\
\hline Pallsades & $4.76 E+02$ & $1.83 \mathrm{E}+02$ & $2.39 \mathrm{E}+02$ & $2.65 E+02$ & $2.23 E+02$ & $1.06 \mathrm{E}+02$ & $1.87 \mathrm{E}+02$ & $1.38 E+03$ \\
\hline Palo Verde $1,2,83$ & $8.42 \mathrm{E}+01$ & $6.80 \mathrm{E}+01$ & $1.16 \mathrm{E}+02$ & $4.22 E+01$ & $4.63 \mathrm{E}+02$ & $9.76 E+02$ & $7.78 \mathrm{E}+02$ & $7.785+02$ \\
\hline Point Beach 182 & $2.81 E+02$ & $1.25 \mathrm{E}+03$ & $1.08 E+02$ & $1.35 \mathrm{E}+03$ & $1.55 E+02$ & $1.43 E+03$ & $1.95 E+02$ & $6.61 E+02$ \\
\hline Prairie Island 182 & $1.73 \mathrm{E}+02$ & $4.02 \mathrm{E}+02$ & $1.28 \mathrm{E}+02$ & $1.55 E+02$ & $1.42 \mathrm{E}+02$ & $4.30 \mathrm{c}+02$ & $5.68 \mathrm{E}+01$ & $1.38 E+02$ \\
\hline Rancho Seco 1 & $9.76 \mathbb{E}+02$ & $1.57 \mathrm{E}+03$ & $1.56 \mathrm{E}+02$ & $1.00 \mathrm{E}+03$ & $1.59 \mathrm{E}+02$ & $3.51 E+00$ & $3.46 \mathrm{E}+02$ & $6.25 \mathrm{E}+01$ \\
\hline II. B. Robinson 2 & $6.42 \mathrm{E}+02$ & $3.35 E+03$ & $4.53 E+02$ & $1.58 E+02$ & $1.01 \mathrm{E}+02$ & $2.59 E+02$ & $8.42 \mathrm{E}+01$ & $3.76 E+02$ \\
\hline Salem $1 \& 2$ & $4.55 E+02$ & $2.02 E+03$ & $4.71 E+02$ & $4.53 E+02$ & $3.29 E+02$ & $9.69 \mathrm{E}+\mathrm{O} 2$ & $3.59 \mathrm{E}+02$ & $3.80 \mathrm{E}+02$ \\
\hline San Onofre & $1.56 \mathrm{E}+01$ & $8.96 \mathrm{E}-01$ & $2.33 E+\infty 0$ & $4.65 E-01$ & $4.04 E+00$ & $4.32 E-01$ & $4.24 \mathrm{E}-01$ & $1.63 \mathrm{E}-01$ \\
\hline San Onofre 1 & $1.80 \mathrm{E}+02$ & $6.04 \mathrm{E}+00$ & $2.51 \mathrm{E}+02$ & $3.82 E+02$ & $3.69 \mathrm{E}+01$ & $4.98 \mathrm{E}+01$ & $3.08 \mathrm{E}+01$ & $4.06 \mathrm{E}+00$ \\
\hline San Onoíre $2-3$ & $5.45 E+02$ & $1.72 \mathrm{E}+03$ & $2.94 E+02$ & $1.93 E+02$ & $2.45 \mathrm{E}+02$ & $2.71 E+02$ & $2.60 \mathrm{E}+02$ & $2.55 E+03$ \\
\hline \multicolumn{9}{|l|}{ Seabrook 1} \\
\hline Sequoyah $1 \& 2$ & $7.52 E+02$ & $2.45 \mathrm{E}+03$ & $4.27 E+02$ & $1.33 E+04$ & $4.12 \mathrm{E}+02$ & $9.78 \mathrm{E}+02$ & $6.07 \mathrm{E}+02$ & $2.92 \mathrm{E}+02$ \\
\hline South Texas 182 & & & & & & & $0.00 E+00$ & $0.00 E+00$ \\
\hline St. Lucie 182 & $5.45 \mathrm{E}+02$ & $1.59 E+03$ & $4.60 \mathrm{E}+02$ & $2.13 E+03$ & $3.53 E+02$ & $1.05 E+03$ & $4.67 \mathrm{E}+02$ & $1.29 \mathrm{E}+04$ \\
\hline Summer 1 & $4.46 \mathrm{E}+02$ & $1.30 \mathrm{E}+02$ & $1.12 \mathrm{E}+02$ & $1.50 E+01$ & $3.80 \mathrm{E}+02$ & $6.76 E+02$ & $1.61 E+02$ & $1.22 E+02$ \\
\hline Surry 182 & $2.02 \mathrm{E}+03$ & $1.21 \mathrm{E}+03$ & $6.39 \mathrm{E}+02$ & $1.16 \mathrm{E}+03$ & $5.15 E+02$ & $2.94 E+04$ & $7.30 \mathrm{E}+02$ & $1.94 E+02$ \\
\hline Three Mile Island I & $4.69 E+02$ & $1.94 \mathrm{E}+01$ & $2.13 \mathrm{E}+02$ & $7.70 \mathrm{~F}+00$ & $2.49 E+02$ & $2.38 E+02$ & $2.37 \mathrm{E}+02$ & $6.92 \mathrm{E}+02$ \\
\hline Three Mile Island 2 & $4.83 E+02$ & $6.35 E+03$ & $3.29 \mathrm{E}+02$ & $5.81 \mathrm{~F}+01$ & $6.59 E+02$ & $3.18 E+02$ & $9.17 \mathrm{E}+02$ & $7.18 \mathrm{E}+03$ \\
\hline TMI 2/Epicor & $*$ & 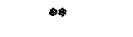 & 4* & * & 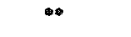 & - & * & 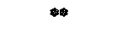 \\
\hline Trojan & $3.09 E+02$ & $3.52 \mathrm{E}+03$ & $2.49 E+02$ & $6.25 E+02$ & $3.31 \mathrm{E}+02$ & $4.09 \mathrm{E}+02$ & $2.48 E+02$ & $4.33 E+02$ \\
\hline Turkcy Point $3 \& 4$ & $6.08 \mathrm{E}+02$ & $1.50 \mathrm{E}+03$ & $3.23 F+02$ & $8.87 \mathrm{E}+01$ & $3.07 E+02$ & $9.32 \mathrm{E}+02$ & $1.07 \mathrm{E}+02$ & $7.05 E+02$ \\
\hline Vogtle $1 \& 2$ & & & & & $0.00 \mathrm{E}+00$ & $0.00 E+00$ & $4.72 \mathrm{E}+01$ & $2.55 \mathrm{E}+01$ \\
\hline Waterford 3 & $2.82 \mathrm{E}+02$ & $3.39 \mathrm{E}+01$ & $1.74 \mathrm{E}+02$ & $3.75 E+01$ & $3.83 E+02$ & $1.78 \mathrm{E}+02$ & $2.81 \mathrm{E}+02$ & $4.43 E+03$ \\
\hline Wolf Creek 1 & $0.00 E+00$ & $0.00 E+00$ & $1.73 E+02$ & $1.48 E+02$ & $1.28 \mathrm{E}+02$ & $1.15 \mathrm{E}+01$ & $1.24 \mathrm{E}+02$ & $1.27 \mathrm{E}+03$ \\
\hline Yanke Rowe I & $2.00 \mathrm{E}+02$ & $2.68 \mathrm{E}+02$ & $1.12 E+02$ & $5.21 E+00$ & $1.86 \mathrm{E}+02$ & $8.83 E+00$ & $1.22 \mathrm{E}+02$ & $8.85 E+03$ \\
\hline Zion 182 & $6.73 E+02$ & $2.69 E+03$ & $3.31 E+02$ & $6.74 \mathrm{E}+02$ & $4.11 \mathrm{E}+02$ & $7.13 E+02$ & $3.59 \mathrm{E}+02$ & $2.50 E+03$ \\
\hline Total & $1.81 \mathrm{E}+04$ & $6.55 E+04$ & $1.19 \mathrm{E}+04$ & $3.81 E+04$ & $1.30 E+04$ & $7.51 \mathrm{E}+04$ & $1.23 E+04$ & $7.28 \mathrm{E}+04$ \\
\hline
\end{tabular}

Represents solid waste shipped by plant but not broken down into untts $1,2,23$

- Included wth Three Mile island 2 totals 
Table 12

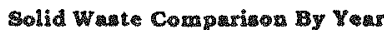

Pressuriked Water Reactors

Volume (Cuble Meters) - Activity (Curtes)

Eacility 1989

\begin{tabular}{|c|c|c|}
\hline Arkansas One 182 & $222 \mathrm{E}+02$ & $296 E+02$ \\
\hline Beaver Valley $18: 2$ & $196 E^{2}+03$ & $135 E+03$ \\
\hline Braidwood $18: 2$ & $310 E+02$ & $389 E+02$ \\
\hline Byron 182 & $365 \mathrm{E}+02$ & $123 E+03$ \\
\hline Callaway 1 & $209 E+02$ & $600 \mathrm{E}+02$ \\
\hline Calvert ChIs $1 \& 2$ & $207 E+02$ & $4148+02$ \\
\hline Calawba $1 \& 2$ & $216 \mathrm{E}+02$ & $317 E+02$ \\
\hline Donald C Cook 182 & $388 \mathrm{E}+02$ & $117 \mathrm{E}+03$ \\
\hline Crystal River 3 & $347 E+02$ & $240 \mathrm{E}+03$ \\
\hline Davis-Besse 1 & $118 \mathrm{E}+02$ & $208 \mathrm{E}+02$ \\
\hline Diablo Canyon 182 & $1876+02$ & $429 E+02$ \\
\hline Joseph M I arky $1 \& 2$ & $485 E+02$ & $400 E+02$ \\
\hline Fort Calhoun 1 & $175 E+02$ & $876 \mathrm{E}+00$ \\
\hline R E Ginna & $233 E+02$ & $799 \mathrm{E}+01$ \\
\hline Haddarn Neck & $153 \mathrm{E}+02$ & $655 \mathrm{E}+02$ \\
\hline Harns 1 & $160 \mathrm{E}+02$ & $254 E+01$ \\
\hline Indan Point 182 & $478 \mathrm{E}+02$ & $360 \mathrm{E}+02$ \\
\hline Indian Point 3 & $577 E+02$ & $350 E+02$ \\
\hline Kewaunee & $700 E+01$ & $774 \mathrm{E}+02$ \\
\hline Maine Yankee & $195 \mathrm{E}+02$ & $236 E+02$ \\
\hline McGuire 182 & $436 \mathrm{E}+02$ & $6326+02$ \\
\hline Millstone 2 & $247 E+02$ & $555 \mathrm{E}+02$ \\
\hline Millstone 3 & $147 \mathrm{E}+02$ & $7375+02$ \\
\hline North Anna $1 \& 2$ & $677 E+02$ & $172 \mathrm{E}+03$ \\
\hline Oconce $1,2, \& 3$ & $425 E+02$ & $146 E_{403}$ \\
\hline Pahsades & $219 E+02$ & $832 \mathrm{E}+03$ \\
\hline Palo Verde $1,2.83$ & $874 E+02$ & $674 \mathrm{E}+02$ \\
\hline Point Beach 182 & $106 \mathrm{E}+02$ & $254 \mathrm{E}+02$ \\
\hline Praine Iskand 182 & $125 \mathrm{E}+02$ & $103 E+02$ \\
\hline Raneho Seco 1 & $244 E+02$ & $327 \mathrm{E}+02$ \\
\hline II B Robinson 2 & $969 E+01$ & $186 \mathrm{E}+02$ \\
\hline Salem 182 & $122 \%+02$ & $565 \mathrm{E}+04$ \\
\hline San Onofre & 0 OOE+ +00 & $000 \mathrm{E}+00$ \\
\hline San Onofrc 1 & $119 \mathrm{E}+02$ & $172 \mathrm{E}+03$ \\
\hline San Onofre 23 & $328 \mathrm{E}+02$ & $272 \mathrm{E}+03$ \\
\hline Seabrook 1 & 0 OOE +00 & $000 E+\infty$ \\
\hline Sequoyah $18: 2$ & $465 E_{0}+02$ & $264 E+03$ \\
\hline South Texas 182 & $503 E+01$ & $724 \mathrm{E}+00$ \\
\hline St Lucie 1 \&2 & $318 E+02$ & $169 \mathrm{E}+02$ \\
\hline Summer 1 & $140 E^{2}+02$ & $376 \mathbb{E}+02$ \\
\hline Surry 182 & $538 \mathrm{E}+02$ & $131 \mathrm{C}+03$ \\
\hline Three Mile Island 1 & $332 \mathrm{E}+02$ & $505 E+01$ \\
\hline Three Mile Island 2 & $118 E+03$ & $139 \mathrm{E}+04$ \\
\hline TMi 2/lipkor & \% & . \\
\hline Trojan & $259 \mathfrak{E}+02$ & $447 \mathrm{E}+02$ \\
\hline Turkey Point 3\&4 & $3466+02$ & $226 \mathrm{E}+\infty$ \\
\hline Vogtle $1 \& 2$ & $100 \mathrm{E}+02$ & $151 \mathrm{E}+01$ \\
\hline Waterford 3 & $761 E+02$ & $407 \mathrm{E}+02$ \\
\hline Woif Creek 1 & $151 E+02$ & $126 \mathrm{E}+03$ \\
\hline Yankee Rowe 1 & $298 E+02$ & $1786+01$ \\
\hline Zion 182 & $214 \mathrm{E}+02$ & $358 E+03$ \\
\hline Total & $164 \mathrm{E}+04$ & $112 \mathrm{E}+05$ \\
\hline
\end{tabular}

Represents solid waste shipped by plant but not broken down into units $1,2,8$, 3

* Included with Three Mile Island 2 totals 


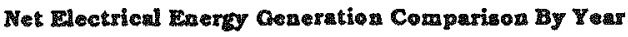

\begin{tabular}{|c|c|c|c|c|c|c|c|c|}
\hline Solling Water Re & \multicolumn{7}{|c|}{ Megawt Hour: } & \multirow[b]{2}{*}{1983} \\
\hline Faclity & $\begin{array}{c}\text { Initial } \\
\text { coticality }\end{array}$ & $\begin{array}{c}\text { Commerclal } \\
\text { Operation }\end{array}$ & 1978 & 1979 & 1980 & 1981 & 1982. & \\
\hline Big Rock Point I & $09 / 27 / 62$ & $03 / 29 / 63$ & $4.011 \mathrm{E}+05$ & $1.14 E+05$ & $4.05 \mathrm{E}+05$ & $4.70 \mathrm{E}+05$ & $3.60 \mathrm{E}+05$ & $3.49 \mathrm{E}+05$ \\
\hline Browns Ferry 1 & $08 / 17 / 73$ & $08 / 01 / 74$ & $1.69 E+07$ & $2.04 \mathrm{E}+07$ & $6.06 \mathrm{E}+06$ & $4.41 E+06$ & $7.88 E+06$ & $2.18 E+06$ \\
\hline Browns Ferry 2 & $07 / 20 / 74$ & $03 / 01 / 75$ & & & $5.62 \mathrm{E}+06$ & $7.47 \mathrm{E}+06$ & $4.45 \mathrm{E}+06$ & 6.395406 \\
\hline Browns Ferry 3 & $08 / 08 / 76$ & $03 / 01 / 77$ & & & $6.94 \mathrm{E}+06$ & $6.26 E+06$ & $4.89 E+06$ & $5.396+06$ \\
\hline Brunswick 1 & $10 / 08 / 76$ & $03 / 18 / 77$ & $9.91 E+06$ & $6.82 E+06$ & $3.94 E+06$ & $2.56 E+06$ & $2.92 E+06$ & $1.395+06$ \\
\hline Brunswtek 2 & $03 / 20 / 75$ & $11 / 03 / 75$ & & & $1.86 \mathrm{E}+05$ & $3.28 E+06$ & $1.91 \mathrm{E}+06$ & $3.94 E+\infty$ \\
\hline Clinton 1 & $02 / 27 / 87$ & $11 / 24 / 87$ & & & & & & \\
\hline Cooper & $02 / 21 / 74$ & $07 / 01 / 74$ & $4.892+06$ & $4.99 \mathrm{E}+06$ & $3.79 E+06$ & 3.85E+06 & $5.28 E+06$ & $3.34 \mathrm{E}+06$ \\
\hline Dresden 1 & $10 / 15 / 59$ & $07 / 04 / 60$ & $7.595+05$ & $0.00 E+00$ & $0.00 E+00$ & $0.00 E+00$ & $0.001+00$ & $0.00 \mathrm{E}+00$ \\
\hline Dresden 2 & $01 / 07 / 70$ & $06 / 09 / 70$ & 9.53E $\$ 06$ & $8.42 E+06$ & $4.58 E+06$ & $3.41 E+06$ & $5.12 E+06$ & $3.40 \mathrm{E}+06$ \\
\hline Dresden 3 & $01 / 31 / 71$ & $11 / 16 / 71$ & & & $4.33 E+06$ & $5.188+06$ & $3.89 \mathrm{E}+06$ & $4.15 E+06$ \\
\hline Duane Arnold & $03 / 23 / 74$ & $02 / 01 / 75$ & $1.23 E^{2}+06$ & $2.90 E+06$ & $2.80 \mathrm{E}+06$ & $2.22 E+06$ & $2.28 \mathrm{E} 406$ & $2.325+06$ \\
\hline Fermi 2 & $06 / 21 / 85$ & $01 / 23 / 66$ & & & & & & \\
\hline James A. Fitzpatrick & $11 / 17 / 74$ & $07 / 28 / 75$ & $4.201+06$ & $2.96 \mathrm{E}+06$ & $4.33 \mathrm{E}+06$ & $4.78 \mathrm{E}+06$ & $4.96 E+06$ & $4.63 E+06$ \\
\hline Grand Gulf 1 & $08 / 18 / 82$ & $07 / 01 / 85$ & & & & & & $0.00 E+00$ \\
\hline Edwin I. Hatch 1 & $09 / 12 / 74$ & $12 / 31 / 75$ & $4.77 E+06$ & $5.10 E+06$ & $4.79 E+06$ & $2.76 E+06$ & $2.88 \mathrm{E}+06$ & $3.96 \mathrm{E}+06$ \\
\hline Edwin 1. Hatch 2 & $07 / 04 / 78$ & $09 / 05 / 79$ & & & $3.64 E+06$ & $4.48 E+06$ & $3.73 E+06$ & $3.81 \mathrm{E}+06$ \\
\hline Hope Creek I & $06 / 28 / 86$ & $12 / 20 / 86$ & & & & f & & \\
\hline llumboldt Bay 3 & $02 / 16 / 63$ & $08 / / 63$ & $0.00 E+00$ & $0.00 \mathrm{E}+00$ & $0.00 \mathrm{E}+00$ & $0.00 \mathrm{E}+00$ & $0.00 E+\infty$ & $0.00 E+\infty$ \\
\hline LaCrosse & $07 / 11 / 67$ & $11 / 01 / 69$ & $1.74 E+05$ & $2.01 \mathrm{E}+05$ & $2.15 E+06$ & $2.41 E+05$ & $1.38 E+05$ & $2.01 \mathrm{E}+05$ \\
\hline LaSalle 1 & $06 / 21 / 82$ & $01 / 01 / 84$ & & & & & $4.61 E+05$ & $1.64 E+06$ \\
\hline Lasalle 2 & $03 / 10 / 84$ & $10 / 19 / 84$ & & & & & & \\
\hline Limertck I & $12 / 22 / 84$ & $02 / 01 / 86$ & & & & & & \\
\hline Limerick 2 & $08 / 12 / 89$ & $01 / 08 / 90$ & & & & & & \\
\hline Milstone 1 & $10 / 26 / 70$ & $03 / 01 / 71$ & $4.65 E+06$ & $4.225+06$ & $3.40 E+06$ & $2.52 E+06$ & $4.08 E+06$ & $5.35 \mathrm{E}+06$ \\
\hline Monticelio & $12 / 10 / 70$ & $06 / 30 / 71$ & $3.868+06$ & $4.40 E+06$ & $3.45 E+06$ & $3.26 \mathrm{E}+06$ & $2.421++06$ & $4.15 E+06$ \\
\hline Nine Mile Point 1 & $09 / 05 / 69$ & $12 / 01 / 69$ & $4.47 E+06$ & $3.00 E+06$ & $4.54 E+06$ & $3.27 \mathrm{E}+06$ & $1.13 E+06$ & $2.80 E 406$ \\
\hline Nine Mile Point 2 & $05 / 23 / 87$ & $04 / 05 / 88$ & & & & & & \\
\hline Dyster Creck I & $05 / 03 / 89$ & $12 / 01 / 69$ & $3.65 \mathrm{E}+06$ & $4.56 \mathrm{E}+06$ & $1.96 E+06$ & $2.63 E+06$ & $2.01 E+06$ & $2.05 \mathrm{E}+05$ \\
\hline Peach Bottom 2 & $09 / 16 / 73$ & $07 / 05 / 74$ & $1.38 \mathrm{E}+07$ & $1.47 E+07$ & $4.34 E+06$ & $6.63 E+06$ & $4.79 E+06$ & $4.45 E+06$ \\
\hline Peach Bottom 3 & $08 / 07 / 74$ & $12 / 23 / 74$ & & & $7.23 E+06$ & $3.13 \mathrm{E}+06$ & $8.53 E+06$ & $2.42 \mathrm{E}+06$ \\
\hline Perry 1 & $06 / 06 / 86$ & $11 / 18 / 87$ & & & & & & \\
\hline PAlgrim I & $06 / 16 / 72$ & $12 / 01 / 72$ & $4.38 E+06$ & $4.84 \mathrm{E}+06$ & $3.04 E+06$ & $3.44 E+06$ & $3.29 \mathrm{E}+06$ & $4.71 \mathrm{E}+06$ \\
\hline Quad-Citles 1 & $10 / 18 / 71$ & $02 / 18 / 73$ & $9.15 \mathrm{E}+06$ & $8.76 E+06$ & $3.44 \mathrm{E}+06$ & $5.73 E+06$ & $3.24 \mathrm{E}+06$ & $5.78 E+06$ \\
\hline Quad-Cities 2 & $04 / 26 / 72$ & $03 / 10 / 73$ & & & $3.61 \mathrm{E}+06$ & $3.77 \mathrm{E}+06$ & $5.06 \mathrm{E}+06$ & $3.15 E+06$ \\
\hline Rtwer Bend 1 & $10 / 31 / 85$ & $06 / 16 / 86$ & & & & & & \\
\hline Shoreham 1 & $02 / 15 / 85$ & & & & & & & \\
\hline Susquchanma 1 & $09 / 10 / 82$ & $06 / 08 / 83$ & & & & & $3.21 \mathrm{E}+05$ & $3.54 E+06$ \\
\hline Susquehanna 2 & $05 / 08 / 84$ & $02 / 12 / 85$ & & & & & & \\
\hline Vermont Yankee I & $03 / 24 / 72$ & $11 / 30 / 72$ & $3.24 \mathrm{E}+06$ & $3.45 \mathrm{E}+06$ & $2.98 E+06$ & $3.57 \mathrm{E}+06$ & $4.17 \mathrm{~B}+06$ & $2.87 \mathrm{E}+06$ \\
\hline WNP-2 & $01 / 19 / 84$ & $12 / 13 / 84$ & & & & & & \\
\hline Total & & & $1.00 E+08$ & $9.98 \mathrm{E}+07$ & $9.16 E+07$ & $8.93 E+07$ & $9.02 \mathrm{E}+07$ & $8.65 E+07$ \\
\hline 1. Vrain 1 & $01 / 31 / 74$ & $07 / 01 / 79$ & $6.09 \mathrm{E}+05$ & $1.24 \mathrm{E}+05$ & $6.76 \mathrm{E}+05$ & $7.55 E+05$ & $5.69 E+05$ & $7.48 E+05$ \\
\hline
\end{tabular}

- High temperature gas cooled reactor 


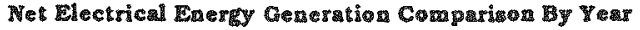

Hegarat Hours

Botling Watcr Reactors

Iniltal Commercial

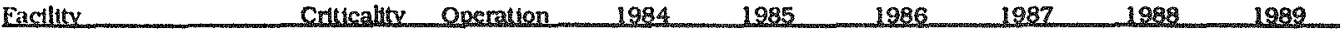

Big Rock Point 1

Browns ferry 1

Browns Ferty 2

Browns Ferry 3

Brunswick 1

Brunsuick 2

Clinton 1

Cooper

Dresden 1

Dresden 2

Dresden 3

Duane Arnold

Fermi 2

James A. Fitzpatrick

Grand Guif I

Edwn ?. llatch 1

Edwin I. Hatch 2

Hope Cresk 1

Humboldt Bay 3

LaCrosse

Lasalle

Lasalle 2

Limerick 1

Lmerick 2

Millstone 1

Monticello

Nine Mile Point 1

Nine Mile Point 2

Oyster Creek 1

Peach Bottorn 2

Peach Bottom 3

perty 1

Pilgrim 1

Quad-Cities 1

guad-Cities 2

River Bend I

Shoreham 1

Susquehanna 1

Susquehanna 2

Vermont Yankee 1

WNP-2

Total

- Fort St. Vrain 1
$09 / 27 / 62 \quad 03 / 29 / 63 \quad 4.18 E+05 \quad 3.62 E+05 \quad 5.06 E+05 \quad 3.75 E+05 \quad 3.84 E+05 \quad 4.17 E+05$

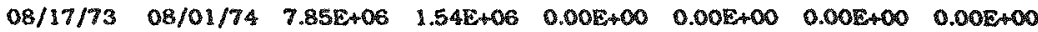
$\begin{array}{llllllll}07 / 20 / 74 & 03 / 01 / 75 & 4.04 \mathrm{E}+06 & 0.00 \mathrm{E}+00 & 0.00 \mathrm{E}+00 & 0.00 \mathrm{E}+00 & 0.00 \mathrm{E}+\infty & 0.00 \mathrm{E}+00\end{array}$

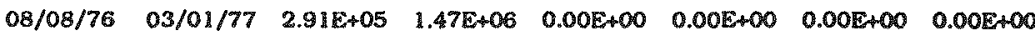

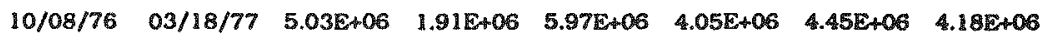
$\begin{array}{llllllll}03 / 20 / 75 & 11 / 03 / 75 & 1.39 E+06 & 5.02 E+06 & 2.91 E+06 & 5.69 E+06 & 3.92 E+06 & 4.19 E+06\end{array}$ $02 / 27 / 87 \quad 11 / 24 / 87 \quad 6.84 \mathrm{E}+05 \quad 5.86 \mathrm{E}+06 \quad 2.86 \mathrm{E} 406$

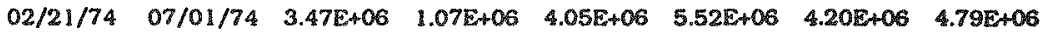
$\begin{array}{llllll}10 / 15 / 59 & 07 / 04 / 60 & 4.46 \mathrm{E}+06 & 0.00 \mathrm{E}+00 & 0.00 \mathrm{E}+00 & 0.00 \mathrm{E}+00\end{array}$

$01 / 07 / 70 \quad 06 / 09 / 70 \quad 2.11 \mathrm{E}+06 \quad 3.09 \mathrm{E}+06 \quad 4.65 \mathrm{E}+06 \quad 3.34 \mathrm{E}+06 \quad 4.32 \mathrm{E}+06 \quad 4.75 \mathrm{E}+06$

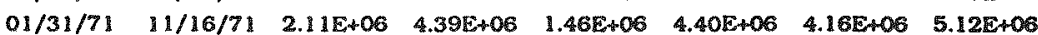

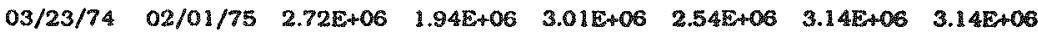
$06 / 21 / 85 \quad 01 / 23 / 86 \quad 0.00 \mathrm{E}+00 \quad 1.39 \mathrm{E}+06 \quad 4.06 \mathrm{E}+06 \quad 5.22 \mathrm{E}+06$ $11 / 17 / 74 \quad 07 / 28 / 75 \quad 4.90 E+06 \quad 4.17 E+06 \quad 6.02 E+06 \quad 4.20 E+06 \quad 4.36 E+06 \quad 6.16 E+06$

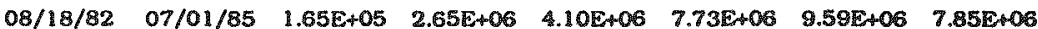
$09 / 12 / 74 \quad 12 / 31 / 75 \quad 3.60 \mathrm{E}+06 \quad 4.76 \mathrm{E}+06 \quad 3.65 \mathrm{E}+06 \quad 5.08 \mathrm{E}+06 \quad 4.11 \mathrm{E}+06 \quad 6.48 \mathrm{E}+06$ $\begin{array}{llllllll}07 / 04 / 78 & 09 / 05 / 79 & 1.88 \mathrm{E}+06 & 5.38 \mathrm{E}+06 & 3.62 \mathrm{E}+06 & 5.76 \mathrm{E}+06 & 4.25 \mathrm{E}+06 & 4.14 \mathrm{E}+06\end{array}$ $\begin{array}{lllllll}06 / 28 / 86 & 12 / 20 / 86 & 1.03 \mathrm{E}+06 & 7.28 \mathrm{E}+06 & 6.99 \mathrm{E}+06 & 6.61 \mathrm{E}+06\end{array}$ $02 / 16 / 63 \quad 08 / \quad / 63 \quad 0.00 \mathrm{E}+00 \quad 0.00 \mathrm{E}+00 \quad 0.00 \mathrm{E}+00 \quad 0.00 \mathrm{E}+\infty$

$07 / 11 / 67 \quad 11 / 01 / 69 \quad 3.19 E+05 \quad 3.23 E+05 \quad 1.57 E+05$

$06 / 21 / 82 \quad 01 / 01 / 84 \quad 5.21 E+06 \quad 4.81 \mathrm{E}+06 \quad 2.02 \mathrm{E}+06 \quad 4.08 \mathrm{E}+06 \quad 5.44 \mathrm{E}+06 \quad 6.16 \mathrm{E}+06$ $\begin{array}{llllllll}03 / 10 / 84 & 10 / 19 / 84 & 1.39 E+06 & 3.43 E+06 & 5.72 E+06 & 4.54 E+06 & 5.66 E+06 & 6.50 E+06\end{array}$

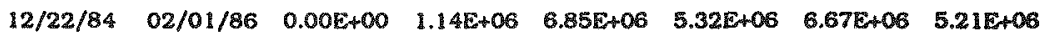
$08 / 12 / 89 \quad 01 / 08 / 90$ $1.06 \mathrm{E}+06$

$10 / 26 / 70 \quad 03 / 01 / 71 \quad 4.32 \mathrm{E}+06 \quad 4.59 \mathrm{E}+06 \quad 5.25 \mathrm{E}+06 \quad 4.38 \mathrm{E}+06 \quad 5.54 \mathrm{E}+06 \quad 4.64 \mathrm{E}+06$

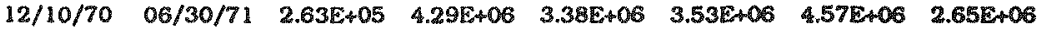
$09 / 05 / 69 \quad 12 / 01 / 69 \quad 3.64 \mathrm{E}+06 \quad 4.93 \mathrm{E}+06 \quad 3.15 \mathrm{E}+06 \quad 4.62 \mathrm{E}+06 \quad 0.00 \mathrm{E}+00 \quad 0.00 \mathrm{E}+00$ $\begin{array}{llllll}05 / 23 / 87 & 04 / 05 / 88 & 2.61 E+05 & 2.51 E+06 & 4.25 E+06\end{array}$ $05 / 03 / 69 \quad 12 / 01 / 69 \quad 2.79 E+05 \quad 3.75 E+06 \quad 1.30 E+06 \quad 3.11 E+06 \quad 3.54 E+06 \quad 2.40 E+06$ $09 / 16 / 73 \quad 07 / 05 / 74 \quad 2.43 \mathrm{E}+06 \quad 2.33 \mathrm{E}+06 \quad 6.90 \mathrm{E}+06 \quad 1.55 \mathrm{E}+06 \quad 0.00 \mathrm{E}+00 \quad 3.86 \mathrm{E}+06$

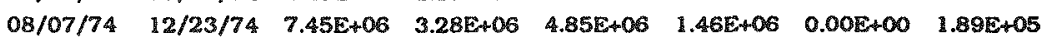

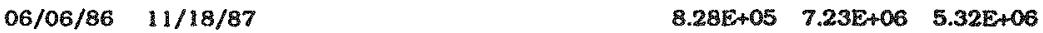

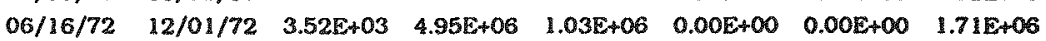
$10 / 18 / 71 \quad 02 / 18 / 73 \quad 3.35 E+06 \quad 6.07 E+06 \quad 4.42 \mathrm{E}+06 \quad 4.46 \mathrm{E}+06 \quad 5.66 \mathrm{E}+06 \quad 4.28 \mathrm{E}+06$ $04 / 26 / 72 \quad 03 / 10 / 73 \quad 4.98 \mathrm{E}+06 \quad 4.56 \mathrm{E}+06 \quad 4.72 \mathrm{E}+06 \quad 4.95 \mathrm{E}+06 \quad 4.18 \mathrm{E}+06 \quad 5.74 \mathrm{E} 406$ $\begin{array}{lllllll}10 / 31 / 85 & 06 / 16 / 86 & 3.00 \mathrm{E}+06 & 4.96 \mathrm{E}+06 & 7.25 \mathrm{E}+06 & 4.79 \mathrm{E}+06\end{array}$ $02 / 15 / 85$

$\begin{array}{llllllll}09 / 10 / 82 & 06 / 08 / 83 & 6.09 E+06 & 5.26 \mathrm{E}+06 & 5.83 \mathrm{E}+06 & 6.13 \mathrm{E}+06 & 8.41 \mathrm{E}+06 & 6.47 \mathrm{E}+06\end{array}$ $\begin{array}{llllllll}05 / 08 / 84 & 02 / 12 / 85 & 9.32 \mathrm{E}+05 & 6.95 \mathrm{E}+06 & 5.45 \mathrm{E}+06 & 8.60 \mathrm{E}+06 & 5.90 \mathrm{E}+06 & 6.77 \mathrm{E}+06\end{array}$ $\begin{array}{llllllll}03 / 24 / 72 & 11 / 30 / 72 & 3.34 \mathrm{E}+06 & 3.00 \mathrm{E}+06 & 2.06 \mathrm{E}+06 & 3.54 \mathrm{E}+06 & 4.11 \mathrm{E}+06 & 3.61 \mathrm{E}+06\end{array}$ $01 / 19 / 84 \quad 12 / 13 / 84 \quad 4.10 \mathrm{E}+05 \quad 5.18 \mathrm{E}+06 \quad 5.18 \mathrm{E}+06 \quad 5.40 \mathrm{E}+06 \quad 6.00 \mathrm{E}+06 \quad 6.12 \mathrm{E}+06$ $8.88 \mathrm{E}+07 \quad 1.07 \mathrm{E}+08 \quad 1.12 \mathrm{E}+08 \quad 1.30 \mathrm{E}+08 \quad 1.46 \mathrm{E}+08 \quad 1.48 \mathrm{E}+08$ $01 / 31 / 74 \quad 07 / 01 / 79 \quad 5.67 \mathrm{E}+04 \quad 0.00 \mathrm{E}+00 \quad 5.20 \mathrm{E}+04 \quad 1.81 \mathrm{E}+05 \quad 6.60 \mathrm{E}+05$

- High temperature gas cooled reactor 
Table 14

Net Electrical Eoergy Generation Comparimon By Year

\begin{tabular}{|c|c|c|c|c|c|c|c|c|}
\hline \multicolumn{9}{|c|}{ Megatmatt Hours } \\
\hline Eacilit & $\begin{array}{c}\text { Initial } \\
\text { Criticality } \\
\end{array}$ & $\begin{array}{l}\text { Commercial } \\
\text { Operation }\end{array}$ & 1978 & 1979 & 1980 & 1981 & 1982 & 1983 \\
\hline Arkansas One 1 & $08 / 06 / 74$ & $12 / 19 / 74$ & $5.25 \mathrm{E}+06$ & $3.32 \mathrm{E}+06$ & $3.78 E+06$ & $4.90 \mathrm{E}+06$ & $3.72 E+06$ & $3.225+06$ \\
\hline Arkansas One 2 & $12 / 05 / 78$ & $03 / 26 / 80$ & $3.98 E+03$ & $8.81 \mathrm{E}+05$ & $3.65 E+06$ & $4.32 \mathrm{E}+06$ & 3.812406 & $4.43 E+06$ \\
\hline Beaver Valley I & $05 / 10 / 76$ & $10 / 01 / 76$ & $2.48 \mathrm{E}+06$ & $1.79 \mathrm{E}+06$ & $3.01 \mathrm{E}+05$ & $4.66 \mathrm{E}+06$ & $2.69 \mathrm{E}+06$ & $4.68 \mathrm{E}+06$ \\
\hline Beaver Valley 2 & $08 / 04 / 87$ & $11 / 17 / 87$ & & & & & & \\
\hline Braddwood 1 & $05 / 29 / 87$ & $07 / 29 / 88$ & & & & & & \\
\hline Braidwood 2 & $03 / 08 / 88$ & $10 / 17 / 88$ & & & & & & \\
\hline Byron 1 & $02 / 02 / 85$ & $09 / 16 / 85$ & & & & & & \\
\hline Byron 2 & $01 / 09 / 87$ & $08 / 21 / 87$ & & & & & & \\
\hline Callaway 1 & $10 / 02 / 84$ & $12 / 19 / 84$ & & & & & & \\
\hline Calvert Clufs 1 & $10 / 07 / 74$ & $05 / 08 / 75$ & $9.91 E+06$ & $9.68 \mathrm{E}+06$ & $4.53 \mathrm{E}+06$ & $6.11 E+06$ & $5.36 \mathrm{E} 406$ & $5.57 \mathrm{E}+06$ \\
\hline Calvert Cliffs 2 & $11 / 30 / 76$ & $04 / 01 / 77$ & & & $6.41 E+06$ & $5.42 \mathrm{E}+06$ & $5.00 E+06$ & $6.11 E+\infty 6$ \\
\hline Catawba I & $01 / 07 / 85$ & $06 / 29 / 85$ & & & & & & \\
\hline Catawba 2 & $05 / 08 / 86$ & $08 / 19 / 86$ & & & & & & \\
\hline Donald C. Cook 1 & $01 / 18 / 75$ & $08 / 27 / 75$ & $1.01 E+07$ & $1.16 \mathrm{E}+07$ & $6.46 \mathrm{E}+06$ & $6.78 \mathrm{E}+06$ & $5.35 E+06$ & $5.29 \mathrm{E} \downarrow 06$ \\
\hline Donald C. Cook 2 & $03 / 10 / 78$ & $07 / 01 / 78$ & & & $6.70 \mathrm{E}+06$ & $6.38 \mathrm{E}+06$ & $7.005+06$ & $7.01 \mathrm{E}+06$ \\
\hline Crystal River 3 & $01 / 14 / 77$ & $03 / 13 / 77$ & $2.59 \mathrm{E}+06$ & $3.76 \mathrm{E}+06$ & $3.35 E+06$ & $4.01 \mathrm{E}+06$ & $4.92 E+06$ & $3.77 \mathrm{E}+06$ \\
\hline Davis-Besse I & $08 / 12 / 77$ & $07 / 31 / 78$ & $2.61 \mathrm{E}+06$ & $3.13 E+06$ & $2.09 \mathrm{E}+06$ & $4.36 \mathrm{E}+06$ & $3.22 \mathrm{E}+06$ & $4.88 \mathrm{E}+06$ \\
\hline Diablo Canyor 1 & $04 / 29 / 84$ & $05 / 07 / 85$ & & & & & & \\
\hline Diablo Canyon 2 & $09 / 19 / 85$ & $03 / 13 / 86$ & & & & & & \\
\hline Joseph M. Faricy I & $08 / 09 / 77$ & $12 / 01 / 77$ & $5.92 \mathrm{E}+06$ & $1.74 E+06$ & $4.60 \mathrm{E}+06$ & $2.62 \mathrm{E}+06$ & $5.22 E+06$ & $5.26 \mathrm{E}+06$ \\
\hline Joseph M. Farley 2 & $05 / 05 / 81$ & $07 / 30 / 81$ & & & & $2.92 E+06$ & $5.30 \mathrm{E}+06$ & $5.98 \mathrm{E}+06$ \\
\hline Fort Calhoun 1 & $08 / 06 / 73$ & $06 / 20 / 74$ & $2.85 E+06$ & $3.67 E+06$ & $2.01 E+06$ & $2.15 \mathrm{E}+06$ & $3.48 E+06$ & $2.75 E+06$ \\
\hline R. E. Girna & $11 / 08 / 69$ & $07 / 01 / 70$ & $3.22 E+06$ & $2.96 E+06$ & $3.09 \mathrm{E}+06$ & $3.32 \mathrm{E}+06$ & $2.41 \mathrm{E}+06$ & $3.04 \mathrm{E}+06$ \\
\hline Haddam Neck & $07 / 24 / 67$ & $01 / 01 / 68$ & $4.71 E+06$ & $4.12 E+06$ & $3.56 E+06$ & $4.06 E+06$ & $4.54 \mathrm{E}+06$ & $3.78 \mathrm{E}+06$ \\
\hline Harris 1 & $01 / 03 / 87$ & $05 / 02 / 87$ & & & & & & \\
\hline Indian Point 1 & $08 / 02 / 62$ & $10 / / 62$ & $4.37 E+06$ & $4.80 \mathrm{E}+06$ & $0.00 \mathrm{E}+00$ & $0.00 \mathrm{E}+00$ & $0.00 E+00$ & $0.00 E+00$ \\
\hline Indian Point 2 & $05 / 22 / 73$ & $08 / 01 / 74$ & & & $4.26 \mathrm{E}+06$ & $3.06 \mathrm{E}+06$ & $4.45 E+06$ & $5.90 \mathrm{E}+08$ \\
\hline Indian Point 3 & $04 / 06 / 76$ & $08 / 30 / 76$ & $5.46 E+06$ & $4.79 \mathrm{E}+06$ & $3.07 \mathrm{E}+06$ & $3.03 E+06$ & $1.44 E+06$ & $6.07 \mathrm{E}+04$ \\
\hline Kewaunee & $03 / 07 / 74$ & $06 / 16 / 74$ & $3.89 \mathrm{E}+06$ & $3.44 \mathrm{E}+06$ & $3.63 E+06$ & $3.77 \mathrm{E}+06$ & $3.82 \mathrm{E}+06$ & $3.71 E+06$ \\
\hline Maine Yankee & $10 / 23 / 72$ & $12 / 28 / 72$ & $5.35 \mathrm{E}+06$ & $4.54 \mathrm{E}+06$ & $4.40 E+06$ & $5.21 \mathrm{E}+06$ & $4.52 E+06$ & $0.00 E+00$ \\
\hline McGutre 1 & $08 / 08 / 81$ & $12 / 01 / 81$ & & & & $1.91 E+04$ & $4.30 E+06$ & $4.63 E+06$ \\
\hline McGuire 2 & $05 / 08 / 83$ & $03 / 01 / 84$ & & & & & & $0.00 \mathrm{E}+\infty$ \\
\hline Millstone 2 & $10 / 17 / 75$ & $12 / 26 / 75$ & $4.50 \mathrm{E}+06$ & $4.36 \mathrm{E}+06$ & $4.88 \mathrm{E}+06$ & $6.09 \mathrm{E}+06$ & $5.01 \mathrm{E}+06$ & $2.45 E+06$ \\
\hline Millstone 3 & $01 / 23 / 86$ & $04 / 23 / 86$ & & & & & & \\
\hline North Anna 1 & $04 / 05 / 78$ & $06 / 06 / 78$ & $3.66 \mathrm{E}+06$ & $4.19 \mathrm{E}+06$ & $5.63 E+06$ & $4.64 \mathrm{E}+06$ & $2.40 \mathrm{E}+06$ & $5.31 E+06$ \\
\hline North Anna 2 & $06 / 12 / 80$ & $12 / 14 / 80$ & & & $3.50 \mathrm{E}+05$ & $5.65 E+06$ & $4.05 E^{\circ}+06$ & $5.80 \mathrm{E}+06$ \\
\hline Oconee 1 & $04 / 19 / 73$ & $07 / 15 / 73$ & $1.59 E+07$ & $1.32 E+07$ & $5.12 E+06$ & $3.00 E+06$ & $5.15 E+06$ & $5.67 \mathrm{E}+06$ \\
\hline Oconee 2 & $11 / 11 / 73$ & $09 / 09 / 74$ & & & $3.88 \mathrm{E}+06$ & $5.19 E+06$ & $3.44 E+06$ & $5.14 E+\infty 6$ \\
\hline Oconee 3 & $09 / 05 / 74$ & $12 / 16 / 74$ & & & $5.22 \mathrm{E}+06$ & $5.64 E+06$ & $2.12 E+06$ & $7.10 E+06$ \\
\hline Palisades & $05 / 24 / 71$ & $12 / 31 / 71$ & $2.62 \mathrm{E}+06$ & $3.43 E+06$ & $2.38 \mathrm{E}+06$ & $3.46 E+06$ & $3.35 E+06$ & $3.77 E+06$ \\
\hline Palo Verde 1 & $05 / 25 / 85$ & $02 / 13 / 86$ & & & & & & \\
\hline Palo Verde 2 & $04 / 18 / 86$ & $09 / 19 / 86$ & & & & & & \\
\hline Palo Verde 3 & $10 / 25 / 87$ & $01 / 08 / 88$ & & & & & & \\
\hline Point Beach 1 & $11 / 02 / 70$ & $12 / 21 / 70$ & $7.65 \mathrm{E}+06$ & $6.77 \mathrm{E}+06$ & $2.48 \mathrm{E}+06$ & $2.61 \mathrm{E}+06$ & $2.70 E+\infty 6$ & $2.38 \mathrm{E}+06$ \\
\hline Point Beach 2 & $05 / 30 / 72$ & $10 / 01 / 71$ & & & $3.59 \mathrm{E}+06$ & $3.72 \mathrm{E}+06$ & $3.61 E+06$ & $3.02 E+06$ \\
\hline Prairie Island 1 & $12 / 01 / 73$ & $12 / 16 / 73$ & $7.73 E+06$ & $7.10 \mathrm{E}+06$ & $3.11 E+06$ & $3.84 E+06$ & $3.92 \mathrm{E}+06$ & $3.89 E+06$ \\
\hline Prairie Island 2 & $12 / 17 / 74$ & $12 / 21 / 74$ & & & $3.47 \mathrm{E} 406$ & $3.09 \mathrm{E}+06$ & $3.86 \mathrm{E}+06$ & $3.725+06$ \\
\hline Rancho Scco 1 & $09 / 16 / 74$ & $04 / 17 / 75$ & $4.992+06$ & $5.71 \mathrm{E}+06$ & $4.42 \mathrm{E}+06$ & $2.63 \mathrm{E}+06$ & $3.37 \mathrm{E}+06$ & $2.85 E+06$ \\
\hline 11. B. Robinson 2 & $09 / 20 / 70$ & $03 / 07 / 71$ & $3.98 \mathrm{E}+06$ & $4.00 \mathrm{E} \rightarrow 06$ & $3.21 \mathrm{E}+06$ & $3.50 \mathrm{E}+06$ & $2.25 \mathrm{E}+06$ & $3.35 \mathrm{E}+06$ \\
\hline Silem 1 & $12 / 11 / 76$ & $06 / 30 / 77$ & $4.53 E+06$ & $2.04 E+06$ & $5.68 \mathrm{E}+06$ & $6.19 E+06$ & $4.09 E+06$ & $5.38 E+06$ \\
\hline Salem 2 & $08 / 08 / 80$ & $10 / 13 / 81$ & & & $0.00 E+\infty 0$ & $1.63 E+06$ & $7.94 E+06$ & $7.44 E+05$ \\
\hline Sun Onofre 1 & $06 / 14 / 67$ & $01 / 01 / 68$ & $2.68 \mathrm{E}+06$ & $3.36 E+06$ & $8.17 E+05$ & $7.798+05$ & $5.105+05$ & $0.00 E+00$ \\
\hline San Onofre 2 & $07 / 26 / 82$ & $08 / 08 / 83$ & & & & & $1.26 \mathrm{E}+05$ & $3.76 E+06$ \\
\hline San Onofre 3 & $08 / 29 / 83$ & $04 / 01 / 84$ & & & & & & $9.97 \mathrm{E}+0.5$ \\
\hline Seabrook 1 & $06 / 13 / 89$ & $08 / 19 / 90$ & & & & & & \\
\hline Sequoyah 1 & $07 / 05 / 80$ & $07 / 01 / 81$ & & & $5.18 \mathrm{E}+05$ & $2.53 \mathrm{E}+06$ & $4.91 \mathrm{E}+06$ & $7.34 E+06$ \\
\hline Sequoyah 2 & $11 / 05 / 81$ & $06 / 01 / 82$ & & & & & & $6.69 \mathrm{E}+06$ \\
\hline South Texas 1 & $03 / 08 / 88$ & $08 / 25 / 88$ & & & & & & \\
\hline South Texas 2 & $03 / 12 / 89$ & $06 / 19 / 89$ & & & & & & \\
\hline
\end{tabular}


Table 14

Net Electrical Eucrey Generation Comparimon By Y

\begin{tabular}{|c|c|c|c|c|c|c|c|c|}
\hline \multicolumn{9}{|c|}{ 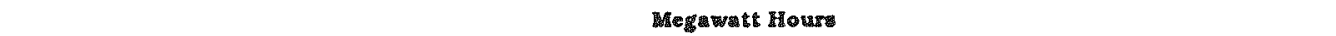 } \\
\hline Eacilitx & $\begin{array}{l}\text { Initial } \\
\text { Cnticality }\end{array}$ & $\begin{array}{c}\text { Commercial } \\
\text { Opemation }\end{array}$ & 1978 & 1979 & 1980 & 1981 & 1982 & 1983 \\
\hline St. Lucie 1 & $04 / 22 / 76$ & $12 / 21 / 76$ & $5.00 \mathrm{E}+06$ & $4.88 \mathrm{E}+06$ & $5.20 E+06$ & $4.95 E+06$ & $6.78 \mathrm{E}+06$ & $1.07 \mathrm{E}+06$ \\
\hline St. Lucte 2 & $06 / 02 / 83$ & $08 / 08 / 83$ & & & & & & $2.40 \mathrm{E}+06$ \\
\hline Surnmer 1 & $10 / 22 / 82$ & $01 / 01 / 84$ & & & & & $1.91 E+05$ & $4.33 E+06$ \\
\hline Surry 1 & $07 / 01 / 72$ & $12 / 22 / 72$ & $1.01 \mathrm{E}+07$ & $2.87 \mathrm{E}+06$ & $2.47 E+06$ & $2.38 \mathrm{E}+06$ & $5.48 \mathrm{E}+06$ & $3.52 \mathrm{EA06}$ \\
\hline Surry 2 & $03 / 07 / 73$ & $05 / 01 / 73$ & & & $2.24 \mathbb{E}+06$ & $5.15 \mathrm{E}+06$ & $5.49 E+06$ & $4.09 E+06$ \\
\hline Three Mile Island 1 & $06 / 05 / 74$ & $09 / 02 / 74$ & $5.67 \mathrm{E}+06$ & $8.48 \mathrm{C}+05$ & $0.00 E+00$ & $0.00 E+00$ & $0.00 \mathrm{E}+00$ & $0.00 \mathrm{E}+00$ \\
\hline Three Mile Island 2 & $03 / 28 / 78$ & $12 / 30 / 78$ & $5.77 \mathrm{E} * 05$ & $N / R$ & $0.00 E+00$ & $0.00 E+00$ & $0.00 \mathrm{E}+00$ & $0.002+00$ \\
\hline Trojan & $12 / 15 / 75$ & $05 / 20 / 76$ & $1.67 \mathrm{E}+06$ & $5.27 E+06$ & $6.07 \mathrm{E}+06$ & $6.42 E+06$ & $4.80 E+06$ & $4.08 E+06$ \\
\hline Turkey Point 3 & $10 / 20 / 72$ & $12 / 14 / 72$ & $8.29 \mathrm{E}+06$ & $6.71 E+06$ & $4.39 E+06$ & $9.125+05$ & $3.77 E+06$ & $4.33 E+06$ \\
\hline Turkey Point 4 & $06 / 11 / 73$ & $09 / 07 / 73$ & & & $3.85 \mathrm{E}+06$ & $4.50 E+06$ & $3.84 \mathrm{E}+06$ & $2.97 \mathrm{E}+06$ \\
\hline Vogtk I & $03 / 09 / 87$ & $05 / 31 / 87$ & & & & & & \\
\hline Vogtle 2 & $03 / 28 / 89$ & $05 / 20 / 89$ & & & & & & \\
\hline Waterford 3 & $03 / 04 / 85$ & $09 / 24 / 85$ & & & & & & \\
\hline Wolf Creek 1 & $05 / 22 / 85$ & $09 / 03 / 85$ & & & & & & \\
\hline Yankee Rowe 1 & $08 / 19 / 60$ & $07 / 01 / 61$ & $1.19 \mathrm{E}+06$ & $1.23 \mathrm{E}+06$ & $2.92 \mathrm{E}+05$ & $8.85 \mathrm{C}^{2}+05$ & $8.82 E_{6}+05$ & $1.34 \mathrm{E}+06$ \\
\hline Zion 1 & $06 / 19 / 73$ & $12 / 31 / 73$ & $1.35 E^{\circ}+07$ & $1.03 E+07$ & $6.51 \mathrm{E}+06$ & $6.19 E+06$ & $4.70 \mathrm{E}+06$ & $4.02 \mathrm{E}+06$ \\
\hline Zion 2 & $12 / 24 / 73$ & $09 / 17 / 74$ & & & $5.28 \mathrm{E}+06$ & $5.26 \mathrm{E}+06$ & $5.16 \mathrm{E}+06$ & $6.18 \mathrm{E}+06$ \\
\hline rotal & & & $1.73 E+08$ & $1.50 \mathrm{E}+08$ & $1.57 E+08$ & $1.78 \mathrm{~b}+08$ & $1.84 \mathrm{E}+08$ & $1.98 \mathrm{E}+08$ \\
\hline
\end{tabular}




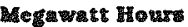

Pressurized Water Reactors

Initial Commercial

Eacility $\quad$\begin{tabular}{cccccccc} 
Critically & Operation & 1984 & 1985 & 1986 & 1987 & 1988 & 1989 \\
\hline
\end{tabular}

Arkansas One 1

Arkansas One 2

Beaver Valley 1

Beaver Valley 2

Braidwood 1

Bratcwood 2

Byron 1

Byron 2

Callaway 1

Calvert Cliffs 1

Calvert Cliffs 2

Catawba 1

Catawba 2

Donald C. Cook 1

Donald C. Cook 2

Crystal River 3

Davis-Besse 1

Drablo Canyon 1

Diablo Canyon 2

Joseph M. Farley I

Joseph M. Fariey 2

Fort Calhoun

R. E. Cinna

Haddam Neck

Harris 1

Indlan Point 1

Indian Point 2

Indian Point 3

Kewaunee

Mane Yankee

McGutre 1

McGuire 2

Millstone 2

Millstone 3

North Anna

North Anna 2

Oconee 1

Oconee 2

Oconee 3

Palisades

Palo Verde 1

Palo Verde 2

Palo Verde 3

Point Beach 1

Point Beach 2

Pratrite Island

Prairte Island 2

Rancho Seco I

I1. B. Robinson 2

Salem 1

Salem 2

San Onofre 1

San Onofre 2

San Onofre 3

Seabrook

Sequoyah 1

Sequoyah 2

South Texas 1

South Texas 2

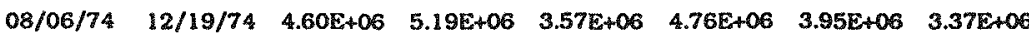

$\begin{array}{lllllllll}12 / 05 / 78 & 03 / 26 / 80 & 6.20 \mathrm{E}+06 & 4.70 \mathrm{E}+06 & 5.31 \mathrm{E}+06 & 6.61 \mathrm{E}+06 & 4.95 \mathrm{E}+06 & 5.47 \mathrm{E}+06\end{array}$

$05 / 10 / 76 \quad 10 / 01 / 76 \quad 4.75 \mathrm{E}+06 \quad 5.90 \mathrm{E}+06 \quad 4.78 \mathrm{E}+06 \quad 5.62 \mathrm{E}+06 \quad 4.98 \mathrm{E}+06 \quad 3.79 \mathrm{E}+06$

08/04/87 $11 / 17 / 87 \quad 7.38 \mathrm{E}+05 \quad 6.48 \mathrm{E}+06 \quad 4.54 \mathrm{E}+06$

$\begin{array}{llllll}05 / 29 / 87 & 07 / 29 / 88 & 1.46 \mathrm{E}+06 & 3.42 \mathrm{E}+06 & 4.63 \mathrm{E}+06\end{array}$

$\begin{array}{lllll}03 / 08 / 88 & 10 / 17 / 88 & 1.35 E+06 & 7.14 E+06\end{array}$

$\begin{array}{lllllll}02 / 02 / 85 & 09 / 16 / 85 & 1.016406 & 7.40 \mathrm{E}+06 & 5.33 \mathrm{E}+06 & 6.29 \mathrm{E}+06 & \mathbf{8 . 9 5 E + 0 6}\end{array}$

$\begin{array}{llllll}01 / 09 / 87 & 08 / 21 / 87 & 1.97 \mathrm{E}+06 & 6.36 \mathrm{E}+06 & 6.06 \mathrm{E}+06\end{array}$

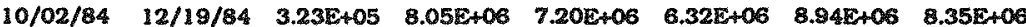

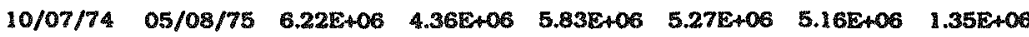

$\begin{array}{llllllll}11 / 30 / 76 & 04 / 01 / 77 & 5.34 E+06 & 5.61 E+06 & 7.01 \mathrm{E}+06 & 4.83 \mathrm{E}+06 & 6.60 \mathrm{E}+06 & 4.53 \mathrm{E}+06\end{array}$

$\begin{array}{llllllll}01 / 07 / 85 & 06 / 29 / 85 & 3.44 E+06 & 5.18 E+06 & 6.38 E+06 & 7.63 E+06 & 7.76 E+06\end{array}$

$\begin{array}{llllll}05 / 08 / 86 & 08 / 19 / 86 & 1.30 \mathrm{E}+06 & 7.17 \mathrm{E}+06 & 6.17 \mathrm{E}+06 & 6.51 \mathrm{E}+06\end{array}$

$01 / 18 / 75 \quad 08 / 27 / 75 \quad 7.75 E+06 \quad 2.12 E+06 \quad 6.65 E+06 \quad 5.03 E+06 \quad 7.47 E+06 \quad 5.43 E+06$

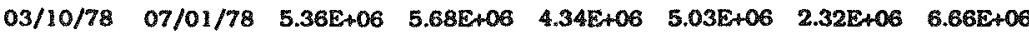

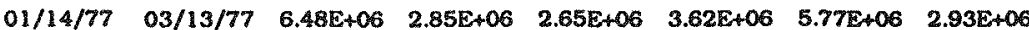

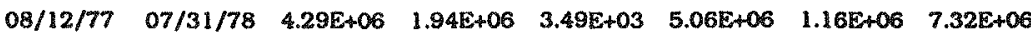

$\begin{array}{llllllll}04 / 29 / 84 & 05 / 07 / 85 & 2.04 \mathrm{E}+05 & 5.23 \mathrm{E}+06 & 5.29 \mathrm{E}+06 & 8.28 \mathrm{E}+06 & 5.26 \mathrm{E}+06 & 7.20 \mathrm{E}+06\end{array}$

$\begin{array}{lllllll}09 / 19 / 85 & 03 / 13 / 86 & 5.41 \mathrm{E}+06 & 6.55 E+06 & 5.72 E+06 & 6.23 \mathrm{E}+06 & 8.62 E+06\end{array}$

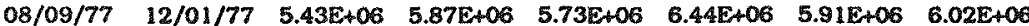

$\begin{array}{lllllllll}05 / 05 / 81 & 07 / 30 / 81 & 6.62 E+06 & 5.47 \mathrm{E}+06 & 5.96 \mathrm{E}+06 & 4.90 \mathrm{E}+06 & 7.17 \mathrm{E}+06 & 5.62 \mathrm{E}+06\end{array}$

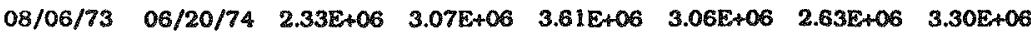

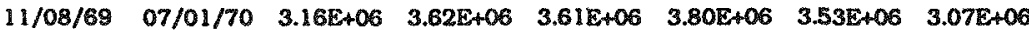

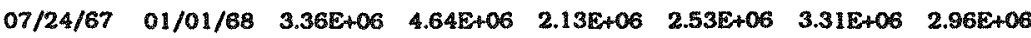

$\begin{array}{llllll}01 / 03 / 87 & 05 / 02 / 87 & 3.38 \mathrm{E}+06 & 5.33 \mathrm{E}+06 & 5.63 \mathrm{E}+06\end{array}$

$08 / 02 / 62 \quad 10 / \quad / 62 \quad 0.00 \mathrm{E}+00 \quad 0.00 \mathrm{E}+00 \quad 0.00 \mathrm{E}+00 \quad 0.00 \mathrm{E}+00$

05/22/73 $08 / 01 / 74 \quad 2.89 E+06 \quad 6.67 \mathrm{E}+06 \quad 3.81 \mathrm{E}+06 \quad 5.15 \mathrm{E}+06 \quad 6.06 \mathrm{E}+06 \quad 4.47 \mathrm{E}+06$

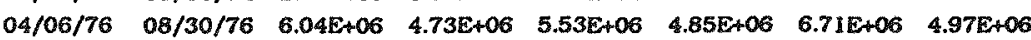

$03 / 07 / 74 \quad 06 / 16 / 74 \quad 3.81 \mathrm{E}+06 \quad 3.70 \mathrm{E}+06 \quad 3.85 \mathrm{E}+06 \quad 4.01 \mathrm{E}+06 \quad 3.91 \mathrm{E}+06 \quad 3.74 \mathrm{E}+06$

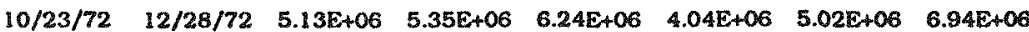

$\begin{array}{lllllllll}08 / 08 / 81 & 12 / 01 / 81 & 6.42 \mathrm{E}+06 & 6.78 \mathrm{E}+06 & 5.16 \mathrm{E}+06 & 7.35 \mathrm{E}+06 & 7.39 \mathrm{E}+06 & 7.80 \mathrm{E}+06\end{array}$

$\begin{array}{lllllllll}05 / 08 / 83 & 03 / 01 / 84 & 6.56 \mathrm{E}+06 & 5.60 \mathrm{E}+06 & 6.21 \mathrm{E}+06 & 7.57 \mathrm{E}+06 & 8.05 \mathrm{E}+06 & 7.41 \mathrm{E}+06\end{array}$

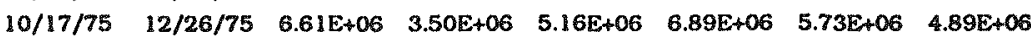

$\begin{array}{lllllll}01 / 23 / 86 & 04 / 23 / 86 & 5.86 \mathrm{E}+06 & 6.74 \mathrm{E}+06 & 7.67 \mathrm{E}+06 & 7.08 \mathrm{E}+06\end{array}$

$\begin{array}{llllllll}04 / 05 / 78 & 06 / 06 / 78 & 3.78 \mathrm{E}+06 & 5.80 \mathrm{E}+06 & 6.31 \mathrm{E}+06 & 3.57 \mathrm{E}+06 & 6.90 \mathrm{E}+06 & 4.30 \mathrm{E}+06\end{array}$

$\begin{array}{lllllllll}06 / 12 / 80 & 12 / 14 / 80 & 4.72 \mathrm{E}+06 & 6.81 \mathrm{E}+06 & 6.02 \mathrm{E}+06 & 5.65 \mathrm{E}+06 & 7.88 \mathrm{E}+06 & 5.90 \mathrm{E}+06\end{array}$

$\begin{array}{lllllllll}04 / 19 / 73 & 07 / 15 / 73 & 6.17 \mathrm{E}+06 & 7.07 \mathrm{E}+06 & 4.78 \mathrm{E}+06 & 5.03 \mathrm{E}+06 & 7.19 \mathrm{E}+06 & 5.94 \mathrm{E}+06\end{array}$

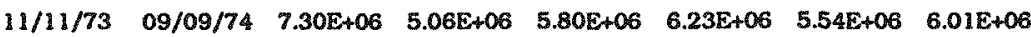

$\begin{array}{llllllll}09 / 05 / 74 & 12 / 16 / 74 & 5.35 \mathrm{E}+06 & 4.86 \mathrm{E}+06 & 6.06 \mathrm{E}+06 & 5.08 \mathrm{E}+06 & 5.97 \mathrm{E}+06 & 6.34 \mathrm{E}+06\end{array}$

$\begin{array}{lllllllll}05 / 24 / 71 & 12 / 31 / 71 & 8.12 \mathrm{E}+05 & 5.30 \mathrm{E}+06 & 8.41 \mathrm{E}+05 & 2.63 \mathrm{E}+06 & 3.44 \mathrm{E}+06 & 3.64 \mathrm{E}+06\end{array}$

$\begin{array}{llllllll}05 / 25 / 85 & 02 / 13 / 86 & 1.13 \mathrm{E}+06 & 5.85 \mathrm{E}+06 & 5.27 \mathrm{E}+06 & 6.67 \mathrm{E}+06 & 1.80 \mathrm{E}+06\end{array}$

$\begin{array}{lllllll}04 / 18 / 86 & 09 / 19 / 86 & 2.65 \mathrm{E}+06 & 8.19 \mathrm{C}+06 & 6.75 \mathrm{E}+06 & 4.70 \mathrm{E}+06\end{array}$

$\begin{array}{llllll}10 / 25 / 87 & 01 / 08 / 88 & 3.20 \mathrm{E}+05 & 3.55 \mathrm{E}+04 & 1.33 \mathrm{E}+06\end{array}$

$\begin{array}{llllllll}11 / 02 / 70 & 12 / 21 / 70 & 3.11 \mathrm{E}+06 & 3.35 \mathrm{E}+06 & 3.77 \mathrm{E}+06 & 3.57 \mathrm{E}+06 & 3.83 \mathrm{E}+06 & 3.61 \mathrm{E}+06\end{array}$

$\begin{array}{lllllllll}05 / 30 / 72 & 10 / 01 / 71 & 3.51 \mathrm{E}+06 & 3.60 \mathrm{E}+06 & 3.42 \mathrm{E}+06 & 3.61 \mathrm{E}+06 & 3.72 \mathrm{E}+06 & 3.49 \mathrm{E}+06\end{array}$

$12 / 01 / 73 \quad 12 / 16 / 73 \quad 4.16 \mathrm{E}+06 \quad 3.68 \mathrm{E}+06 \quad 3.82 \mathrm{E}+06 \quad 3.59 \mathrm{E}+06 \quad 3.82 \mathrm{E}+06 \quad 4.39 \mathrm{E}+06$

$12 / 17 / 74 \quad 12 / 21 / 74 \quad 3.91 \mathrm{E}+06 \quad 3.61 \mathrm{E}+06 \quad 3.86 \mathrm{E}+06 \quad 4.43 \mathrm{E}+06 \quad 3.89 \mathrm{E}+06 \quad 3.89 \mathrm{E}+06$

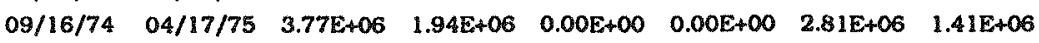

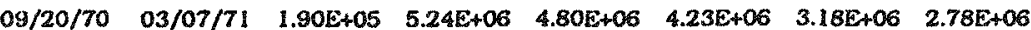

$12 / 11 / 76 \quad 06 / 30 / 77 \quad 2.13 \mathrm{E}+06 \quad 9.01 \mathrm{E}+06 \quad 7.08 \mathrm{E}+06 \quad 6.21 \mathrm{E}+06 \quad 7.41 \mathrm{E}+06 \quad 6.21 \mathrm{E}+06$

$\begin{array}{lllllllll}08 / 08 / 80 & 10 / 13 / 81 & 3.20 \mathrm{E}+06 & 5.02 \mathrm{E}+06 & 5.31 \mathrm{E}+06 & 6.17 \mathrm{E}+06 & 5.97 \mathrm{E}+06 & 7.82 \mathrm{E}+06\end{array}$

$\begin{array}{llllllll}06 / 14 / 67 & 01 / 01 / 68 & 2.62 \mathrm{E}+05 & 2.46 \mathrm{E}+06 & 8.74 \mathrm{E}+05 & 2.71 \mathrm{E}+06 & 1.37 \mathrm{E}+06 & 1.17 \mathrm{E}+06\end{array}$

$07 / 26 / 82 \quad 08 / 08 / 83 \quad 5.27 \mathrm{E}+06 \quad 5.15 \mathrm{E}+06 \quad 6.38 \mathrm{E}+06 \quad 6.23 \mathrm{E}+06 \quad 9.00 \mathrm{E}+06 \quad 5.22 \mathrm{E}+06$

$\begin{array}{llllllll}08 / 29 / 83 & 04 / 01 / 84 & 4.10 E+06 & 3.71 E+06 & 6.76 E+06 & 7.52 E+06 & 6.13 E+06 & 8.84 E+06\end{array}$

$06 / 13 / 89 \quad 08 / 19 / 90$

$\begin{array}{llllllll}07 / 05 / 80 & 07 / 01 / 81 & 6.10 E+06 & 4.06 E+06 & 0.00 E+00 & 0.00 E+00 & 6.71 E+04 & 9.55 E+06\end{array}$

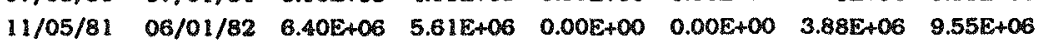

$\begin{array}{lll}03 / 08 / 88 & 08 / 25 / 88 & 2.79 E+06 \quad 6.28 E+06\end{array}$

$\begin{array}{lll}03 / 12 / 89 & 06 / 19 / 89 & 3.02 \mathrm{E}+\infty\end{array}$ 
Net Electrical Eacry Generation Comparison By $\mathrm{Y}$ enr

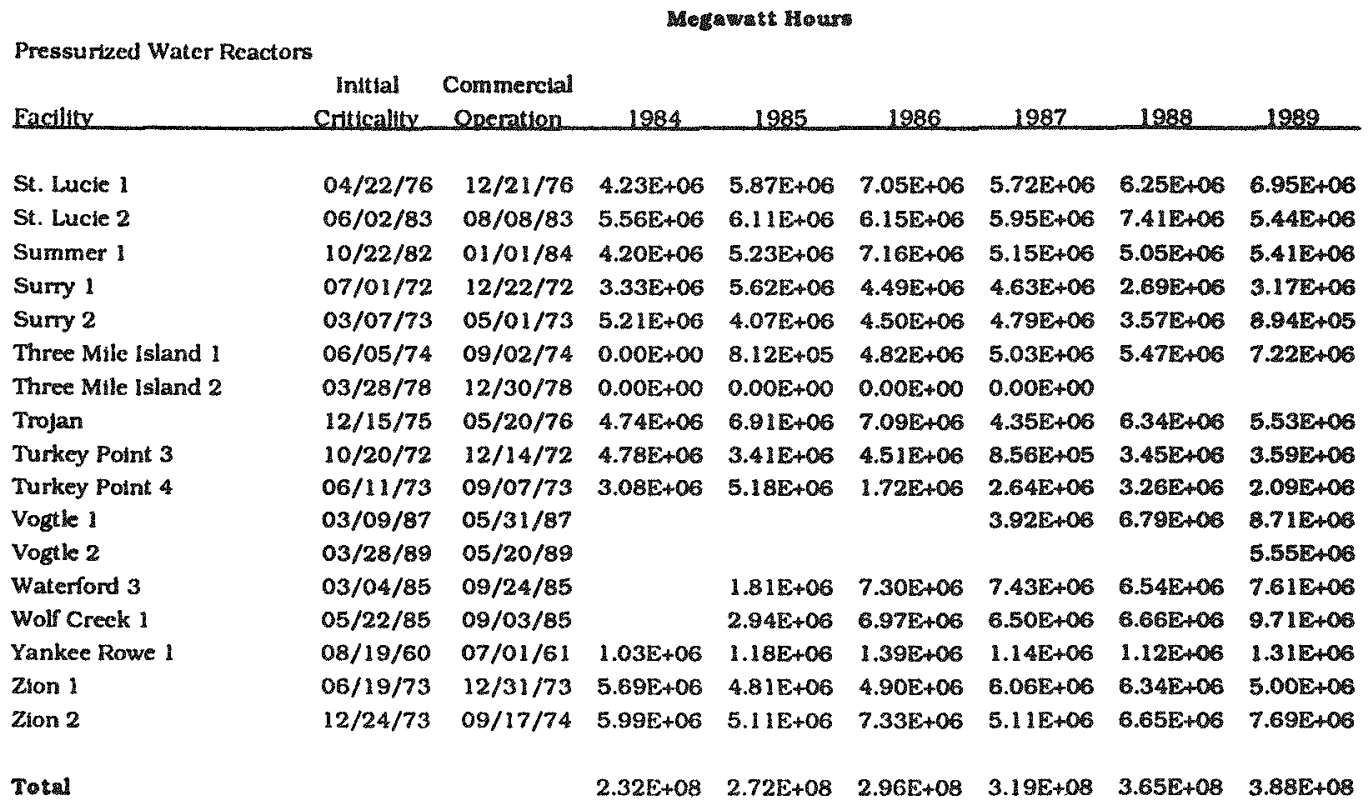


Thermal 2uergy Generation Comparian By Yenr

\begin{tabular}{|c|c|c|c|c|c|c|c|c|}
\hline Bolling Water Reactors & \multicolumn{7}{|c|}{ Hegratt Houx } & \multirow[b]{2}{*}{1977} \\
\hline Faclity & $\begin{array}{c}\text { Initial } \\
\text { Criticality }\end{array}$ & $\begin{array}{l}\text { Commerctal } \\
\text { operation. }\end{array}$ & 1972 & 1973 & 1974 & 1975 & 1976 & \\
\hline Big Rock Point I & $09 / 27 / 62$ & $03 / 29 / 63$ & $1.20 E+06$ & $1.41 \mathrm{E}+06$ & $1.13 E+06$ & $9.80 \mathrm{E}+05$ & $8.30 E+05$ & 1.232106 \\
\hline Browns Ferry 1 & $08 / 17 / 73$ & $08 / 01 / 74$ & & $1.37 \mathrm{E}+06$ & $1.64 E+07$ & $8.75 E+06$ & $1.34 E+07$ & $5.37 \mathrm{E}+07$ \\
\hline Browns Ferry 2 & $07 / 20 / 74$ & $03 / 01 / 75$ & & & & & & \\
\hline Browns Ferry 3 & $08 / 08 / 76$ & $03 / 01 / 77$ & & & & & & \\
\hline Brunswick 1 & $10 / 08 / 76$ & $03 / 18 / 77$ & & & & $4.72 E+06$ & $7.81 \mathrm{E}+06$ & $1.59 \mathrm{E}+07$ \\
\hline Brunswick 2 & $03 / 20 / 75$ & $11 / 03 / 75$ & & & & & & \\
\hline Clinton 1 & $02 / 27 / 87$ & $11 / 24 / 87$ & & & & & & \\
\hline Cooper & $02 / 21 / 74$ & $07 / 01 / 74$ & & & $6.908+06$ & $1.24 \mathrm{ENO}$ & $1.19 E+07$ & $1.45 E+07$ \\
\hline Dresden 1 & $10 / 15 / 59$ & $07 / 04 / 60$ & $3.76 E+06$ & $2.43 E+06$ & $1.36 \mathrm{E}+06$ & $2.56 \mathrm{E}+06$ & $3.42 E+06$ & $2.21 \pm+06$ \\
\hline Dresden 2 & $01 / 07 / 70$ & $06 / 09 / 70$ & $2.52 E+07$ & $2.83 E+07$ & $2.18 \mathrm{E}+07$ & $1.708+07$ & $2.74 E+07$ & $2.89 E+07$ \\
\hline Dresden 3 & $01 / 31 / 71$ & $11 / 16 / 71$ & & & & & & \\
\hline Duane Amold & $03 / 23 / 74$ & $02 / 01 / 75$ & & & & $7.42 E+06$ & $8.02 E+06$ & $9.32 \mathrm{E}-06$ \\
\hline Fermi 2 & $06 / 21 / 85$ & $01 / 23 / 86$ & & & & & & \\
\hline James A. Fitzpatrick & $11 / 17 / 74$ & $07 / 28 / 75$ & & & & $6.81 \mathrm{E}+06$ & $1.26 \mathrm{E}+07$ & 1.18E 407 \\
\hline Grand Gulf I & $08 / 18 / 82$ & $07 / 01 / 85$ & & & & & & \\
\hline Edwh I. Hatch 1 & $09 / 12 / 74$ & $12 / 31 / 75$ & & & & $9.75 \mathrm{E}+06$ & $1.38 \mathrm{E}+07$ & $1.228+07$ \\
\hline Edwin 1. Hatch 2 & $07 / 04 / 78$ & $09 / 05 / 79$ & & & & & & \\
\hline Hope Creek 1 & $06 / 28 / 86$ & $12 / 20 / 86$ & & & & s & & \\
\hline Humboldt Bay 3 & $02 / 16 / 63$ & $08 / / 63$ & $1.25 \mathrm{E}+06$ & $1.47 \mathrm{E}+06$ & $1.27 \mathrm{E}+06$ & $1.32 E+06$ & $6.80 E+05$ & $0.00 \mathrm{E}+00$ \\
\hline LaCrosse & $07 / 11 / 67$ & $11 / 01 / 69$ & $8.20 \mathrm{E}+05$ & $6.90 E+05$ & $1.08 \mathrm{E}+06$ & $9.20 \mathrm{E}+05$ & $6.10 \mathrm{E}+05$ & $3.43 E+05$ \\
\hline LaSalle 1 & $06 / 21 / 82$ & $01 / 01 / 84$ & & & & & & \\
\hline LaSalle 2 & $03 / 10 / 84$ & $10 / 19 / 84$ & & & & & & \\
\hline Limerick 1 & $12 / 22 / 84$ & $02 / 01 / 86$ & & & & & & \\
\hline Lmerick 2 & $08 / 12 / 89$ & $01 / 08 / 90$ & & & & & & \\
\hline Millstone 1 & $10 / 26 / 70$ & $03 / 01 / 71$ & $9.69 \mathrm{E}+06$ & $5.96 \mathrm{E}+06$ & $1.12 \mathrm{E}+07$ & $1.21 \mathrm{E}+07$ & $1.16 E+07$ & $1.48 \mathrm{E}+07$ \\
\hline Mont tcello & $12 / 10 / 70$ & $06 / 30 / 71$ & $1.10 \mathrm{E}+07$ & $9.90 E+06$ & $8.28 \mathrm{E}+06$ & $8.88 \mathrm{E}+06$ & $1.23 E+07$ & $1.106+07$ \\
\hline Nine Mile Point 1 & $09 / 05 / 69$ & $12 / 01 / 69$ & $1.00 \mathrm{E}+07$ & $1.105+07$ & $1.05 \mathrm{E}+07$ & $9.68 \mathrm{E}+06$ & $1.31 \mathrm{E}+07$ & $9.15 E+06$ \\
\hline Nine Mile Point 2 & $05 / 23 / 87$ & $04 / 05 / 88$ & & & & & & \\
\hline Oyster Creek 1 & $05 / 03 / 69$ & $12 / 01 / 69$ & $1.30 \mathrm{E}+07$ & $1.09 \mathrm{E}+07$ & $1.13 \mathrm{E}+07$ & $9.81 \mathrm{E}+06$ & $1.18 \mathrm{E}+07$ & $9.82 \mathrm{E}+06$ \\
\hline Peach Botlom 2 & $09 / 16 / 73$ & $07 / 05 / 74$ & & & $1.23 \mathrm{E}+07$ & $3.34 \mathrm{E}+07$ & $3.72 \mathrm{E}+07$ & $2.86 \mathrm{E}+07$ \\
\hline Peach Bottom 3 & $08 / 07 / 74$ & $12 / 23 / 74$ & & & & & & \\
\hline Perry 1 & $06 / 06 / 86$ & $11 / 18 / 87$ & & & & & & \\
\hline Pilgrim 1 & $06 / 16 / 72$ & $12 / 01 / 72$ & $2.65 \mathrm{E}+06$ & $1.25 \mathrm{E}+07$ & $6.00 E+06$ & $8.10 \mathrm{E}+06$ & $7.60 E+06$ & $8.26 \mathrm{E}+06$ \\
\hline Quad-Cities 1 & $10 / 18 / 71$ & $02 / 18 / 73$ & $1.25 \mathrm{E}+07$ & $3.17 E+07$ & $2.61 E+07$ & $2.31 E+07$ & $2.59 \mathrm{E}+07$ & $2.68 \mathrm{E}+07$ \\
\hline guad-Cittes 2 & $04 / 26 / 72$ & $03 / 10 / 73$ & & & & & & \\
\hline River Bend 1 & $10 / 31 / 85$ & $06 / 16 / 86$ & & & & & & \\
\hline Shoreham 1 & $02 / 15 / 85$ & $\cdot$ & & & & & & \\
\hline Susquehanna 1 & $09 / 10 / 82$ & $06 / 08 / 83$ & & & & & & \\
\hline Susquehanma 2 & $05 / 08 / 84$ & $02 / 12 / 85$ & & & & & & \\
\hline Vermont Yankee I & $03 / 24 / 72$ & $11 / 30 / 72$ & $1.48 \mathrm{E}+06$ & $6.08 E+06$ & $8.20 E+06$ & $1.13 E+07$ & $1.02 \mathrm{E}+07$ & $1.11 \mathrm{E}+07$ \\
\hline WNP-2 & $01 / 19 / 84$ & $12 / 13 / 84$ & & & & & & \\
\hline Total & & & $9.26 \mathrm{E}+07$ & $1.24 \mathrm{E}+08$ & $1.44 \mathrm{E}+08$ & $1.89 \mathrm{E}+08$ & $2.30 \mathrm{E}+08$ & $2.70 E+08$ \\
\hline Fort St. Vrain 1 & $01 / 31 / 74$ & 779 & & & & & & \\
\hline
\end{tabular}

\footnotetext{
- High temperature gas cooled reactor
} 
Inttal Commerclal

\begin{tabular}{|c|c|c|c|c|c|c|c|c|}
\hline Eacilly & Criticalite & Operation & 1978 & 1979 & 1980 & 1981 & 1982 & 1983 \\
\hline Big Rock Point 1 & $09 / 27 / 62$ & $03 / 29 / 63$ & $137 \mathrm{E}+06$ & $396 \mathrm{E}+0 \mathrm{~S}$ & $140 E+06$ & $163 \mathrm{E}+06$ & $120 E+06$ & $114 E+06$ \\
\hline Browns Ferry 1 & $08 / 17 / 73$ & $08 / 01 / 74$ & $532 E+07$ & $631 \mathrm{E}+07$ & $192 \mathrm{E}+07$ & $136 \mathrm{E}+07$ & $249 \mathrm{E}+07$ & $678 \mathrm{E}+06$ \\
\hline Browns Ferry 2 & $07 / 20 / 74$ & $03 / 01 / 75$ & & & $1745+07$ & $231 E+07$ & $138 E+07$ & $197 \mathrm{E}+07$ \\
\hline Browns Ferty 3 & $08 / 08 / 76$ & $03 / 01 / 77$ & & & $213 E+07$ & $195 E+07$ & $155 E+07$ & $170 \mathrm{E}+07$ \\
\hline Brunswick & $10 / 08 / 76$ & $03 / 18 / 77$ & $307 \mathrm{E}+07$ & $212 E+07$ & $123 E+07$ & $829 E+06$ & $948 \mathrm{E}+06$ & $453 E+06$ \\
\hline Brunswick 2 & $03 / 20 / 75$ & $11 / 03 / 75$ & & & $538 E+06$ & $104 \mathrm{E}+07$ & $630 E^{3}+06$ & $123 E+07$ \\
\hline Clinton 1 & $02 / 27 / 87$ & $11 / 24 / 87$ & & & & & & \\
\hline Cooper & $02 / 21 / 74$ & $07 / 01 / 74$ & I $54 \mathrm{E}+07$ & $158 E+07$ & $136 \mathrm{C}+07$ & $139 E+07$ & $164 E A 07$ & $105 E \div 07$ \\
\hline Dresden 1 & $10 / 15 / 59$ & $07 / 04 / 60$ & $273 \Gamma+06$ & 0 OOE +00 & $000 E+00$ & 0 OOE +00 & $000 E+\infty$ & 0 OOE+00 \\
\hline Dresden 2 & $01 / 07 / 70$ & $06 / 09 / 70$ & $313 E+07$ & $277 \mathrm{E}+07$ & $157 \mathrm{E}+07$ & $113 \mathrm{E}+07$ & $169 \mathrm{E}+07$ & $113 \mathrm{E}+07$ \\
\hline Dresden 3 & $01 / 31 / 71$ & $11 / 16 / 71$ & & & $142 E+07$ & $1715+07$ & $127 E+07$ & $137 \mathrm{E}+07$ \\
\hline Duane Arnold & $03 / 23 / 74$ & $02 / 01 / 75$ & $396 \mathrm{E}+06$ & $907 \mathrm{E}+06$ & $887 \mathrm{E}+06$ & $705 \mathrm{E}+06$ & $732 E+06$ & $738 \mathrm{E}+06$ \\
\hline Fermi 2 & $06 / 21 / 85$ & $01 / 23 / 86$ & & & & & & \\
\hline James A Fitzpatrick & $11 / 17 / 74$ & $07 / 28 / 75$ & $130 \mathrm{E}+07$ & $897 \mathrm{E}+06$ & $130 \mathrm{E}+07$ & $142 E+07$ & $151 E+07$ & $1425+07$ \\
\hline Grand Gull 1 & $08 / 18 / 82$ & $07 / 01 / 85$ & & & & & & 0 OOE+DO \\
\hline Edwin I Hatch I & $09 / 12 / 74$ & $12 / 31 / 75$ & $337 E+07$ & $162 E_{6} 407$ & $154 \mathrm{E}+07$ & $8975+06$ & $942 E+06$ & $129 \mathrm{E}+07$ \\
\hline Edwin 1 Hatch 2 & $07 / 04 / 78$ & $09 / 05 / 79$ & & & $116 E+07$ & $147 \mathrm{E} \div 07$ & $118 \mathrm{E}+07$ & $119 \mathrm{E}+07$ \\
\hline Hope Creek 1 & $06 / 28 / 86$ & $12 / 20 / 86$ & & & & $d$ & & \\
\hline Humboldt Bay 3 & $02 / 16 / 63$ & $08 / 163$ & $000 E+00$ & $000 E+00$ & 0 OOE +00 & $000 \mathrm{E}+00$ & 0 O0E +00 & $000 E+00$ \\
\hline LaCrosse & $07 / 11 / 67$ & $11 / 01 / 69$ & $654 E+05$ & $748 E+05$ & $800 \mathrm{C}+05$ & $911 E+05$ & $527 E+05$ & $760 \mathrm{E}+05$ \\
\hline LaSalle 1 & $06 / 21 / 82$ & $01 / 01 / 84$ & & & & & $2095+06$ & $586 E+06$ \\
\hline Lasalle 2 & $03 / 10 / 84$ & $10 / 19 / 84$ & & & & & & \\
\hline Limerick 1 & $12 / 22 / 84$ & $02 / 01 / 86$ & & & & & & \\
\hline Limerick 2 & $08 / 12 / 89$ & $01 / 08 / 90$ & & & & & & \\
\hline Millstone ! & $10 / 26 / 70$ & $03 / 01 / 71$ & $143 E+07$ & $130 \mathrm{E}+07$ & $104 E+07$ & $860 \mathrm{E}+06$ & $136 \mathbb{E}+07$ & $164 E+07$ \\
\hline Monticello & $12 / 10 / 70$ & $06 / 30 / 71$ & $118 E+07$ & $135 E+07$ & $107 E+07$ & $101 \mathrm{E}+07$ & $768 \mathrm{E}+06$ & $130 \mathrm{E}+07$ \\
\hline Nine Mile Point & $09 / 05 / 69$ & $12 / 01 / 69$ & 1.395007 & $967 E+06$ & $141 \mathrm{E}+07$ & $1015+07$ & $342 E+06$ & 872506 \\
\hline Nine Mile Point 2 & $05 / 23 / 87$ & $04 / 05 / 88$ & & & & & & \\
\hline Oyster Creck I & $05 / 03 / 69$ & $12 / 01 / 69$ & $110 E 407$ & $138 \mathrm{E}+07$ & $627 \mathrm{E}+06$ & $844 E+06$ & $679 E+06$ & $923 \mathrm{E}+05$ \\
\hline Peach Bottom 2 & $09 / 16 / 73$ & $07 / 05 / 74$ & $439 E+07$ & $458 E+07$ & $137 E+07$ & $208 \mathrm{E}+07$ & $1532+07$ & $1406+07$ \\
\hline Peach Bottom 3 & $08 / 07 / 74$ & $12 / 23 / 74$ & & & $226 \mathrm{E}+07$ & 9856406 & $265 E+07$ & $782 E+06$ \\
\hline Perty 1 & $06 / 06 / 86$ & $11 / 18 / 87$ & & & & & & \\
\hline Pilgrim 1 & $06 / 16 / 72$ & $12 / 01 / 72$ & $133 E+07$ & $147 E+07$ & $920 \mathrm{E}+06$ & $105 E+07$ & $990 \varepsilon+06$ & $1425+07$ \\
\hline guad Cities 1 & $10 / 18 / 71$ & $02 / 18 / 73$ & $314 E+07$ & $300 \mathrm{E} \uparrow 07$ & $117 \mathrm{E}+07$ & $1886+07$ & $112 \mathrm{E}+07$ & $1896+07$ \\
\hline Quad Cities 2 & $04 / 26 / 72$ & $03 / 10 / 73$ & & & $122 \mathrm{E}+07$ & $127 \vec{E}+07$ & $167 \mathrm{E}+07$ & $108 \mathrm{E}+07$ \\
\hline Rwer Bend 1 & $10 / 31 / 85$ & $06 / 16 / 86$ & & & & & & \\
\hline Shoreham I & $02 / 15 / 85$ & & & & & & & \\
\hline Susquehanna 1 & $09 / 10 / 82$ & $06 / 08 / 83$ & & & & & $116 \mathrm{E}+06$ & $112 \mathrm{E} * 07$ \\
\hline Susquehanna 2 & $05 / 08 / 84$ & $02 / 12 / 85$ & & & & & & \\
\hline Vermont Yankee 1 & $03 / 24 / 72$ & $11 / 30 / 72$ & $100 E+07$ & $108 E+07$ & $938 \mathrm{E}+06$ & 1. $13 E+07$ & $131 \mathrm{E}+07$ & $912 \mathrm{E}+06$ \\
\hline WNP 2 & $01 / 19 / 84$ & $12 / 13 / 84$ & & & & & & \\
\hline Total & & & $336 E+08$ & $314 E+08$ & $290 E+08$ & $286 E+08$ & $289 E+08$ & $275 E+08$ \\
\hline Fort St Vrain 1 & $01 / 31 / 74$ & $07 / 01 / 79$ & $204 \mathrm{~F}+06$ & $4786+05$ & $223 E+06$ & $223 \mathrm{E}+06$ & $186 E+06$ & $258 \mathrm{E} \uparrow 06$ \\
\hline
\end{tabular}

- Hgh temperature gas cooled reactor 


\begin{tabular}{|c|c|c|c|c|c|c|c|c|}
\hline 3oil & \multicolumn{7}{|c|}{ Megamate Hours } & \multirow[b]{2}{*}{1989} \\
\hline Facility & $\begin{array}{c}\text { Inttal } \\
\text { Criticality }\end{array}$ & $\begin{array}{c}\text { Commerclal } \\
\text { Oneration }\end{array}$ & 1984 & 1985 & 1986 & 1987 & 1988 & \\
\hline Btg Rock Point 1 & $09 / 27 / 62$ & $03 / 29 / 63$ & $1.37 \mathrm{E}+06$ & $1.198+06$ & $1.66 \mathrm{E}+06$ & $1.23 E+06$ & $1.26 \mathrm{E}+06$ & $1.14 \mathrm{E}+07$ \\
\hline Browns Ferry 1 & $08 / 17 / 73$ & $08 / 01 / 74$ & $2.45 \mathrm{E}+07$ & $4.95 \mathrm{E}+06$ & $0.00 \mathrm{E}+00$ & $0.00 E+00$ & $0.00 E+00$ & $0.00 E+00$ \\
\hline Browns Ferry 2 & $07 / 20 / 74$ & $03 / 01 / 75$ & $1.31 \mathrm{E}+07$ & $0.00 E+\infty$ & $0.00 \mathrm{E}+00$ & $0.00 E+00$ & $0.002+00$ & $0.00 E+00$ \\
\hline Browns Ferry 3 & $08 / 08 / 76$ & $03 / 01 / 77$ & $9.11 E+05$ & $4.65 \mathrm{E}+06$ & $0.00 \mathrm{E}+00$ & $0.001+00$ & $0.00 E+00$ & $0.00 \mathrm{E}+00$ \\
\hline Brunswick 1 & $10 / 08 / 76$ & $03 / 18 / 77$ & $1.56 \mathrm{E}+07$ & $6.06 E+06$ & $1.89 E+07$ & $1.29 E+07$ & $1.43 \mathrm{E}+07$ & $1.32 E * 07$ \\
\hline Brunswick 2 & $03 / 20 / 75$ & $11 / 03 / 75$ & $4.53 \mathrm{E}+06$ & $1.57 \mathrm{E}+07$ & $9.43 E+06$ & $1.83 \mathrm{E}+07$ & $1.24 \mathrm{E}+07$ & $1.34 \mathrm{E}+07$ \\
\hline Chinton 1 & $02 / 27 / 87$ & $11 / 24 / 87$ & & & & $2.15 E+06$ & $1.86 \mathrm{E}+07$ & $9.26 \mathrm{E}+06$ \\
\hline Cooper & $02 / 21 / 74$ & $07 / 01 / 74$ & $1.09 E+07$ & $3.42 F+06$ & $1.26 \mathrm{E}+07$ & $1.72 \mathrm{E}+07$ & $1.31 E+07$ & $1.50 E+07$ \\
\hline Dresden 1 & $10 / 15 / 59$ & $07 / 04 / 60$ & $1.46 \mathrm{E}+07$ & $0.00 E+00$ & $0.00 E+00$ & $0.00 \mathrm{E}+\infty 0$ & & \\
\hline Dresden 2 & $01 / 07 / 70$ & $06 / 09 / 70$ & $7.10 E+06$ & $1.03 E+07$ & $1.52 \mathrm{E}+07$ & $1.11 E+07$ & $1.45 \mathrm{E}+07$ & $1.56 \mathrm{E}+07$ \\
\hline Dresden 3 & $01 / 31 / 71$ & $11 / 16 / 71$ & $7.10 E+06$ & $1.47 \mathrm{E}+07$ & $5.04 E+06$ & $1.46 \mathrm{E}+07$ & $1.36 \mathrm{E} 407$ & $1.67 \mathrm{E}+07$ \\
\hline Duane Arnold & $03 / 23 / 74$ & $02 / 01 / 75$ & $8.71 \mathrm{E}+06$ & $6.15 E+06$ & $9.48 \mathrm{E}+06$ & $7.968+06$ & $9.97 \mathrm{E}+06$ & $1.00 \mathrm{D}+07$ \\
\hline Fermi 2 & $06 / 21 / 85$ & $01 / 23 / 86$ & & & $2.23 E+05$ & $5.99 E+06$ & $1.30 \mathrm{E}+07$ & $1.63 \mathrm{E}+07$ \\
\hline James A. Fitzpatrick & $11 / 17 / 74$ & $07 / 28 / 75$ & $1.52 \mathrm{E}+07$ & $1.28 E+07$ & $1.84 \mathrm{E}+07$ & $1.31 \mathrm{E}+07$ & $1.34 \mathrm{E}+07$ & $1.88 \mathrm{E}+07$ \\
\hline Grand Gull 1 & $08 / 18 / 82$ & $07 / 01 / 85$ & $8.80 \mathrm{E}+05$ & $9.80 E+06$ & $1.47 \mathrm{E}+07$ & $2.55 \mathrm{E}+07$ & $3.05 \mathrm{E}+07$ & $2.51 E+07$ \\
\hline Edwin I. Hatch I & $09 / 12 / 74$ & $12 / 31 / 75$ & $1.202+07$ & $1.53 E+07$ & $1.18 E+07$ & $1.65 \mathrm{E}+07$ & $1.35 E+07$ & $2.09 \mathrm{E}+07$ \\
\hline Edwin 1. Hatch 2 & $07 / 04 / 78$ & $09 / 05 / 79$ & $5.99 \mathrm{E}+06$ & $1.70 E+07$ & $1.19 E+07$ & $1.83 E+07$ & $1.36 E+07$ & $1.35 E+07$ \\
\hline Hope Creek 1 & $06 / 28 / 86$ & $12 / 20 / 86$ & & & $3.62 \mathrm{E}+06$ & $2.29 \mathrm{E}+07$ & $2.22 \mathrm{E}+07$ & $2.10 E+07$ \\
\hline Humboldt Bay 3 & $02 / 16 / 63$ & $08 / 163$ & $0.00 \mathrm{E}+00$ & $0.00 \mathrm{E}+\infty 0$ & $0.00 E+00$ & $0.00 \mathrm{E}+00$ & & \\
\hline LaCrosse & $07 / 11 / 67$ & $11 / 01 / 69$ & $1.07 \mathrm{E}+06$ & $1.11 E+06$ & $5.58 E+05$ & & & \\
\hline Lasalle 1 & $06 / 21 / 82$ & $01 / 01 / 84$ & $2.30 \mathrm{E}+07$ & $1.54 E+07$ & $6.54 \mathrm{E}+06$ & $1.31 \mathrm{E}+07$ & $1.69 \mathrm{E}+07$ & $1.91 E+07$ \\
\hline Lasalle 2 & $03 / 10 / 84$ & $10 / 19 / 84$ & $4.51 \mathrm{E}+06$ & $1.10 E+07$ & 1.80E+07 & $1.43 E+07$ & $1.81 \mathrm{E}+07$ & $2.04 E+07$ \\
\hline Ltmerick 1 & $12 / 22 / 84$ & $02 / 01 / 86$ & $0.00 E+00$ & $4.42 E+06$ & $2.16 \mathrm{E}+07$ & $1.73 \mathrm{E}+07$ & $2.19 \mathrm{E}+07$ & $1.71 \mathrm{EA07}$ \\
\hline Limerick 2 & $08 / 12 / 89$ & $01 / 08 / 90$ & & & & & & $3.54 E+06$ \\
\hline Millstone 1 & $10 / 26 / 70$ & $03 / 01 / 71$ & $1.34 E+07$ & $1.42 E+07$ & $1.61 \mathrm{E}+07$ & $1.34 E+07$ & $1.70 E+07$ & $1.42 E+07$ \\
\hline Monticello & $12 / 10 / 70$ & $06 / 30 / 71$ & $8.98 \mathrm{E}+05$ & $1.31 E+07$ & $1.04 E+07$ & $1.10 \mathrm{E}+07$ & $1.43 E+07$ & $8.48 E+06$ \\
\hline Nine Mile Point 1 & $09 / 05 / 69$ & $12 / 01 / 69$ & $1.12 E+07$ & $1.52 \mathrm{E}+07$ & $9.76 \mathrm{E}+06$ & $1.42 \mathrm{E}+07$ & $0.00 E+\infty 0$ & $0.00 E+00$ \\
\hline Nine Mile Point 2 & $05 / 23 / 87$ & $04 / 05 / 88$ & & & & $1.53 E+06$ & $8.32 \mathrm{E}+06$ & $1.41 E+07$ \\
\hline Oyster Creek I & $05 / 03 / 69$ & $12 / 01 / 69$ & $1.04 E+06$ & $1.16 E+07$ & $4.12 E+06$ & $9.69 \mathrm{E}+06$ & $1.09 \mathrm{E}+07$ & $7.75 \mathrm{E}+06$ \\
\hline Peach Bottom 2 & $09 / 16 / 73$ & $07 / 05 / 74$ & $7.87 \mathrm{E}+06$ & 7.85E+06 & $2.16 \mathrm{E}+07$ & $4.98 E+06$ & $0.00 E+00$ & 1.25EA07 \\
\hline Peach Bottom 3 & $08 / 07 / 74$ & $12 / 23 / 74$ & $2.32 E+07$ & $1.08 E+07$ & $1.55 E+07$ & $4.76 \mathrm{E}+06$ & $0.00 E+00$ & $8.84 E+05$ \\
\hline Perry 1 & $06 / 06 / 86$ & $11 / 18 / 87$ & & & & $2.56 \mathrm{E}+06$ & $2.23 E+07$ & $1.62 \mathrm{E}+07$ \\
\hline Pligrtm 1 & $06 / 16 / 72$ & $12 / 01 / 72$ & $4.99 \mathrm{E}+05$ & $1.50 E+07$ & $3.09 \mathrm{E}+06$ & $0.00 E+00$ & $0.00 \mathrm{E}+\infty$ & $5.48 E+06$ \\
\hline Quad-Citles I & $10 / 18 / 71$ & $02 / 18 / 73$ & $1.06 \mathrm{E}+07$ & $1.92 \mathrm{E}+07$ & $1.41 \mathrm{E} \leftarrow 07$ & $1.42 \mathrm{E}+07$ & $1.83 E+07$ & $1.40 E+07$ \\
\hline Quad-Cities 2 & $04 / 26 / 72$ & $03 / 10 / 73$ & $1.615+07$ & $1.46 \mathrm{E}+07$ & $1.52 \mathrm{E}+07$ & $1.60 \mathrm{E}+07$ & $1.36 E+07$ & $1.85 \mathrm{E}+07$ \\
\hline Rwer Bend I & $10 / 31 / 85$ & $06 / 16 / 86$ & & & $9.85 E+06$ & $1.55 E+07$ & $2.25 E+07$ & $1.53 E+07$ \\
\hline Shorehara 1 & $02 / 15 / 85$ & & & & & & & \\
\hline Susquehanna 1 & $09 / 10 / 82$ & $06 / 08 / 83$ & $1.94 \mathrm{E}+07$ & $1.70 E+07$ & $1.87 \mathrm{E}+07$ & $1.98 \mathrm{E}+07$ & $2.65 E+07$ & $2.05 E+07$ \\
\hline Susquehanna 2 & $05 / 08 / 84$ & $02 / 12 / 85$ & $3.236+06$ & $2.20 E+07$ & $1.74 E+07$ & $2.72 E+07$ & $1.87 E+07$ & $2.14 E+07$ \\
\hline Vermont Yankee 1 & $03 / 24 / 72$ & $11 / 30 / 72$ & $1.04 \mathrm{E}+07$ & $9.55 E+06$ & $6.57 \mathrm{E}+06$ & $1.11 \mathrm{E}+07$ & $1.30 \mathrm{E}+07$ & $1.13 E+07$ \\
\hline WNP-2 & $01 / 19 / 84$ & $12 / 13 / 84$ & $1.21 E+06$ & $1.64 \mathrm{E}+07$ & $1.61 E+07$ & $1.67 E+07$ & $1.87 \mathrm{E}+07$ & $1.92 \mathrm{E}+07$ \\
\hline Total & & & $2.90 \mathrm{E}+08$ & $3.40 E+08$ & $3.58 E+08$ & $4.15 E+08$ & $4.65 E+08$ & $4.80 E+08$ \\
\hline Fort St. Vrain I & $01 / 31 / 74$ & $07 / 01 / 79$ & $3.40 E+05$ & $3.34 \mathrm{E}+0.4$ & $3.70 \mathrm{E}+05$ & $6.68 \mathrm{E}+05$ & $1.95 E+06$ & \\
\hline
\end{tabular}

\footnotetext{
- High ternperature gas cookd reactor
} 
Table 16

Thermal Earry Generation Comparison By Year

\begin{tabular}{|c|c|c|c|c|c|c|c|c|}
\hline \multicolumn{9}{|c|}{ Att Hours } \\
\hline Factlity & $\begin{array}{c}\text { Intial } \\
\text { Criticalty }\end{array}$ & $\begin{array}{c}\text { Commercial } \\
\text { Operatton }\end{array}$ & 1972 & 1973 & 1974 & 1925 & 1976 & 1977 \\
\hline Arkansas One 1 & $08 / 06 / 74$ & $12 / 19 / 74$ & & & $1.99 \mathrm{E}+06$ & $1.54 E+07$ & $1.21 E+07$ & $1.64 \mathrm{E}+07$ \\
\hline Arkansas One 2 & $12 / 05 / 78$ & $03 / 26 / 80$ & & & & & & \\
\hline Beaver Valley 1 & $05 / 10 / 76$ & $10 / 01 / 76$ & & & & & $1.97 \mathrm{E}+06$ & $1.01 \mathrm{E}+07$ \\
\hline Beaver Valley 2 & $08 / 04 / 87$ & $11 / 17 / 87$ & & & & & & \\
\hline Bratdwood 1 & $05 / 29 / 87$ & $07 / 29 / 88$ & & & & & & \\
\hline Braddwood 2 & $03 / 08 / 88$ & $10 / 17 / 88$ & & & & & & \\
\hline Byron 1 & $02 / 02 / 85$ & $09 / 16 / 85$ & & & & & & \\
\hline Byron 2 & $01 / 09 / 87$ & $08 / 21 / 87$ & & & & & & \\
\hline Callaway 1 & $10 / 02 / 84$ & $12 / 19 / 84$ & & & & & & \\
\hline Calvert Cliffs I & $10 / 07 / 74$ & $05 / 08 / 75$ & & & & $1.40 E+07$ & $1.98 \mathrm{E}+07$ & $2.97 \mathrm{E}+07$ \\
\hline Calvert Ciffs 2 & $11 / 30 / 76$ & $04 / 01 / 77$ & & & & & & \\
\hline Catawba 1 & $01 / 07 / 85$ & $06 / 29 / 85$ & & & & 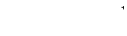 & & \\
\hline Catawba 2 & $05 / 08 / 86$ & $08 / 19 / 86$ & & & & & & \\
\hline Domald C. Cook 1 & $01 / 18 / 75$ & $08 / 27 / 75$ & & & & $1.46 \mathrm{E}+07$ & $2.15 \mathrm{E}+07$ & $1.55 \mathrm{E}+07$ \\
\hline Donald C. Cook 2 & $03 / 10 / 78$ & $07 / 01 / 78$ & & & & & & \\
\hline Crystal Ruver 3 & $01 / 14 / 77$ & $03 / 13 / 77$ & & & & & & $1.26 \mathrm{E}+07$ \\
\hline Davis-Besse 1 & $08 / 12 / 77$ & $07 / 31 / 78$ & & & & & & $1.66 \mathrm{E}+06$ \\
\hline Diablo Canyon I & $04 / 29 / 84$ & $05 / 07 / 85$ & & & & & & \\
\hline Dublo Canyon 2 & $08 / 19 / 85$ & $03 / 13 / 86$ & & & & & & \\
\hline Joseph M. Farley 1 & $08 / 09 / 77$ & $12 / 01 / 77$ & & & & & & \\
\hline Joseph M. Farley 2 & $05 / 05 / 81$ & $07 / 30 / 81$ & & & & & & \\
\hline Fort Calhoun 1 & $08 / 06 / 73$ & $06 / 20 / 74$ & & $2.03 E+06$ & $7.58 E+06$ & $6.71 \mathrm{E}+06$ & $7.15 E+06$ & $9.40 \mathrm{E}+06$ \\
\hline R. E. Gmna & $11 / 08 / 69$ & $07 / 01 / 70$ & $7.71 E+06$ & $1.08 \mathrm{E}+07$ & $6.71 \mathrm{E}+06$ & $9.71 \mathrm{E}+06$ & $6.98 \mathrm{E} \times 06$ & $1.11 \mathrm{E}+07$ \\
\hline Haddam Neck & $07 / 24 / 67$ & $01 / 01 / 68$ & $1.38 E+07$ & $7.73 \mathrm{E}+06$ & $1.42 E+07$ & $1.34 \mathrm{E}+07$ & $1.30 \mathrm{E}+07$ & $1.31 \mathrm{E} * 07$ \\
\hline Harris 1 & $01 / 03 / 87$ & $05 / 02 / 87$ & & & & & & \\
\hline Indian Point 1 & $08 / 02 / 62$ & $10 / / 62$ & & $1.47 E+06$ & $1.15 E+07$ & $1.64 \mathrm{E}+07$ & $7.60 E+06$ & $3.50 \mathrm{E}+07$ \\
\hline Indian Point 2 & $05 / 22 / 73$ & $08 / 01 / 74$ & & & & & & \\
\hline Indian Point 3 & $04 / 06 / 76$ & $08 / 30 / 76$ & & & & & & \\
\hline Kewaunee & $03 / 07 / 74$ & $06 / 16 / 74$ & & & $5.03 \mathrm{E}+06$ & $1.08 E+07$ & $1.08 E+07$ & $1.1112+07$ \\
\hline Maine Yankee & $10 / 23 / 72$ & $12 / 28 / 72$ & $1.44 \mathrm{E}+06$ & $1.08 E+07$ & $1.14 E+07$ & $1.47 \mathrm{E}+07$ & $1.94 \mathrm{E}+07$ & $1.65 \mathrm{E}+07$ \\
\hline Mcculre 1 & $08 / 08 / 81$ & $12 / 01 / 81$ & & & & & & \\
\hline McGuire 2 & $05 / 08 / 83$ & $03 / 01 / 84$ & & & & & & \\
\hline Millstone 2 & $10 / 17 / 75$ & $12 / 26 / 75$ & & & & $6.40 E+0.5$ & $1.52 E+07$ & $1.42 E+07$ \\
\hline Millstone 3 & $01 / 23 / 86$ & $04 / 23 / 86$ & & & & & & \\
\hline North Anna 1 & $04 / 05 / 78$ & $06 / 06 / 78$ & & & & & & \\
\hline North Anna 2 & $06 / 12 / 80$ & $12 / 14 / 80$ & & & & & & \\
\hline Oconee 1 & $04 / 19 / 73$ & $07 / 15 / 73$ & & $6.62 \mathrm{E}+06$ & $1.70 E+07$ & $4.68 \mathrm{E}+07$ & $3.97 \mathrm{E}+07$ & $4.00 \mathrm{E}+07$ \\
\hline Oconee 2 & $11 / 11 / 73$ & $09 / 09 / 74$ & & & & & & \\
\hline Dconee 3 & $09 / 05 / 74$ & $12 / 16 / 74$ & & & & & & \\
\hline Palisades & $05 / 24 / 71$ & $12 / 31 / 71$ & $5.91 \mathrm{E}+06$ & $7.80 E+06$ & $4.00 \mathrm{E}+05$ & $8.91 \mathrm{E}+06$ & $9.66 \mathrm{E} 406$ & $1.73 \mathrm{E}+07$ \\
\hline Palo Verde I & $05 / 25 / 85$ & $02 / 13 / 86$ & & & & & & \\
\hline Palo Verde 2 & $04 / 18 / 86$ & $09 / 19 / 86$ & & & & & & \\
\hline Palo Verde 3 & $10 / 25 / 87$ & $01 / 08 / 88$ & & & & & & \\
\hline Point Beach 1 & $11 / 02 / 70$ & $12 / 21 / 70$ & $9.96 E^{\circ}+06$ & $1.84 E+07$ & $2.045+07$ & $2.09 E+07$ & $2.18 E+07$ & $2.23 E+07$ \\
\hline Point Beach 2 & $05 / 30 / 72$ & $10 / 01 / 71$ & & & & & & \\
\hline Praírie lsland 1 & $12 / 01 / 73$ & $12 / 16 / 73$ & & $1.28 E+05$ & $5.26 \mathrm{E}+06$ & $2.25 \mathrm{E}+07$ & $2.06 \mathrm{E}+07$ & $2.46 E+07$ \\
\hline Praurle 1sland 2 & $12 / 17 / 74$ & $12 / 21 / 74$ & & & & & & \\
\hline Rancho Seco 1 & $09 / 16 / 74$ & $04 / 17 / 75$ & & & & $4.11 E+06$ & $6.91 E+06$ & $1.81 \mathrm{E}+07$ \\
\hline H. B. Robinson 2 & $09 / 20 / 70$ & $03 / 07 / 71$ & $1.55 \mathrm{E} \rightarrow 07$ & $1.25 \mathrm{E}+07$ & $1.56 \mathrm{E}+07$ & $1.36 \mathrm{E}+07$ & $1.59 \mathrm{E}+07$ & $1.43 E+07$ \\
\hline Salern 1 & $12 / 11 / 76$ & $06 / 30 / 77$ & & & & & $5.00 E+04$ & $6.708+06$ \\
\hline Salem 2 & $08 / 08 / 80$ & $10 / 13 / 81$ & & & & & & \\
\hline San Onorre 1 & $06 / 14 / 67$ & $01 / 01 / 68$ & $8.53 E+06$ & $7.09 \mathrm{E}+06$ & $9.73 E+06$ & $1.00 \mathrm{E}+07$ & $7.75 \mathrm{E}+06$ & $7.29 \mathrm{E}+06$ \\
\hline San Onofre 2 & $07 / 26 / 82$ & $08 / 08 / 83$ & & & & & & \\
\hline San Onofre 3 & $08 / 29 / 83$ & $04 / 01 / 84$ & & & & & & \\
\hline Seabrook 1 & $06 / 13 / 89$ & $08 / 19 / 90$ & & & & & & \\
\hline Sequoyah 1 & $07 / 05 / 80$ & $07 / 01 / 81$ & & & & & & \\
\hline Sequoyah 2 & $11 / 05 / 81$ & $06 / 01 / 82$ & & & & & & \\
\hline South Texas 1 & $03 / 08 / 88$ & $08 / 25 / 88$ & & & & & & \\
\hline South Texas 2 & $03 / 12 / 89$ & $06 / 19 / 89$ & & & & & & \\
\hline
\end{tabular}


rable 16

Thermal Energy Generation Comparison By Year

Megawatt Hours

Pressurized Water Reactors

Initial Commercial

Facility Coticalitx Operation

$1972-1973$

\begin{tabular}{l}
$1974 \quad 1975 \quad 1976 \quad 1977$ \\
\hline
\end{tabular}

St. Lucie 1

$04 / 22 / 76 \quad 12 / 21 / 76$

$06 / 02 / 83 \quad 08 / 08 / 83$

$10 / 22 / 82 \quad 01 / 01 / 84$

$\begin{array}{llllllll}07 / 01 / 72 & 12 / 22 / 72 & 1.29 \mathrm{E}+06 & 2.26 \mathrm{E}+07 & 1.92 \mathrm{E}+07 & 2.90 \mathrm{E}+07 & 2.51 \mathrm{E}+07 & 3.10 \mathrm{E}+07\end{array}$

Surty 1

$03 / 07 / 73 \quad 05 / 01 / 73$

$06 / 05 / 74 \quad 09 / 02 / 74$

$03 / 28 / 78 \quad 12 / 30 / 78$

$12 / 15 / 75 \quad 05 / 20 / 76$

$\begin{array}{llllllll}10 / 20 / 72 & 12 / 14 / 72 & 2.90 \mathrm{E}+05 & 1.53 \mathrm{E}+07 & 2.55 \mathrm{E}+07 & 2.78 \mathrm{E}+07 & 2.68 \mathrm{E}+07 & 2.70 \mathrm{E}+07\end{array}$

$06 / 11 / 73 \quad 09 / 07 / 73$

$03 / 09 / 87 \quad 05 / 31 / 87$

$03 / 28 / 89 \quad 05 / 20 / 89$

$03 / 04 / 85 \quad 09 / 24 / 85$

$05 / 22 / 85 \quad 09 / 03 / 85$

$08 / 19 / 60 \quad 07 / 01 / 61 \quad 2.40 \mathrm{E}+06 \quad 3.57 \mathrm{E}+06 \quad 3.07 \mathrm{E}+06 \quad 4.02 \mathrm{E}+06 \quad 4.25 \mathrm{E}+06 \quad 3.52 \mathrm{E}+06$

$06 / 19 / 73 \quad 12 / 31 / 73$

$12 / 24 / 73 \quad 09 / 17 / 74$

$\begin{array}{lllll}2.73 \mathrm{E}+06 & 1.69 \mathrm{E}+07 & 3.28 \mathrm{E}+07 & 3.11 \mathrm{E}+07 & 3.66 \mathrm{E}+07\end{array}$

Total

$6.68 \mathrm{E}+07 \quad 1.30 \mathrm{E}+08 \quad 1.98 \mathrm{E}+08 \quad 3.54 \mathrm{E}+08 \quad 3.67 \mathrm{E}+08 \quad 5.01 \mathrm{E}+08$ 


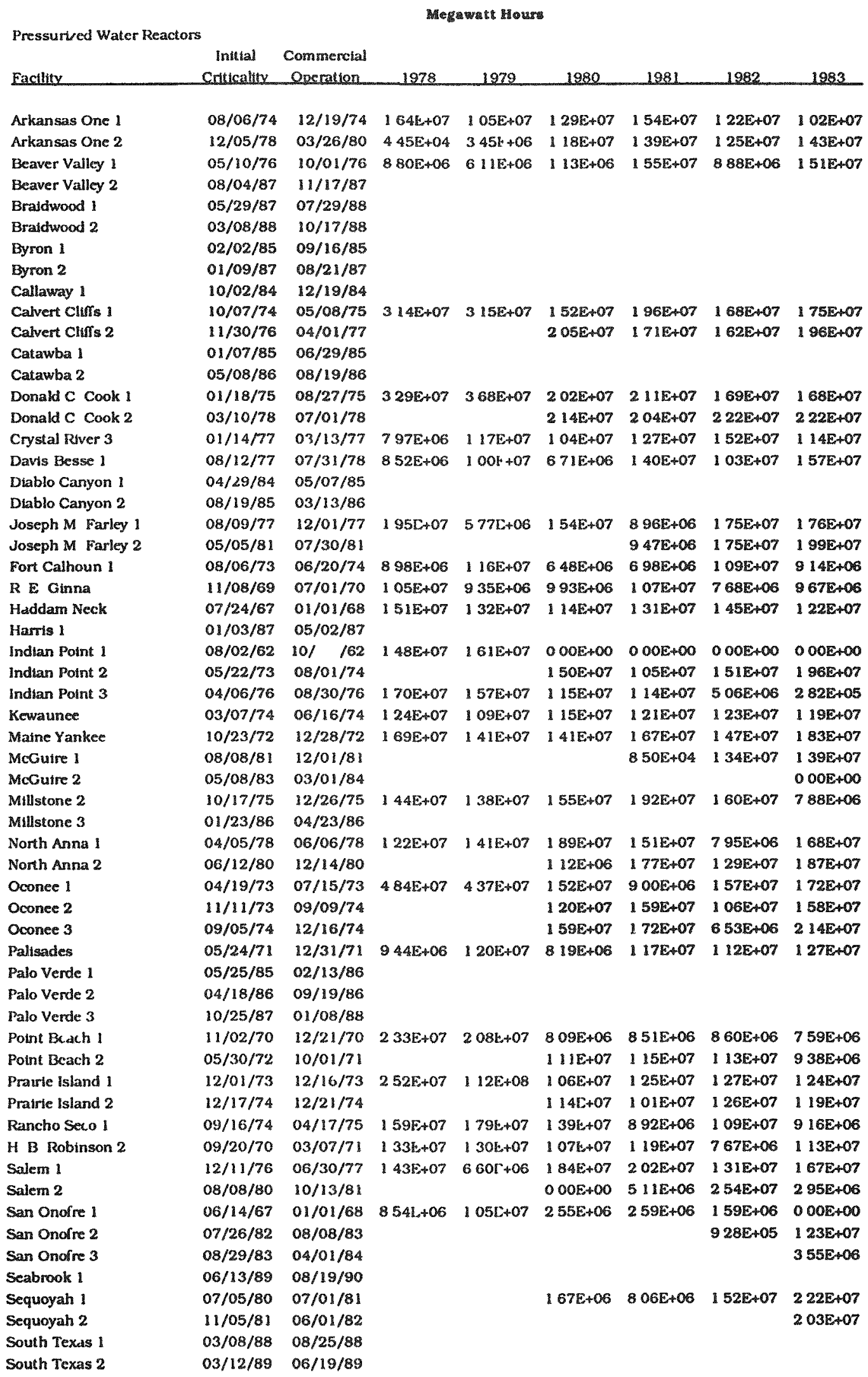


Table 16

Thermal Eneryy Qeneration Comparimon By Year

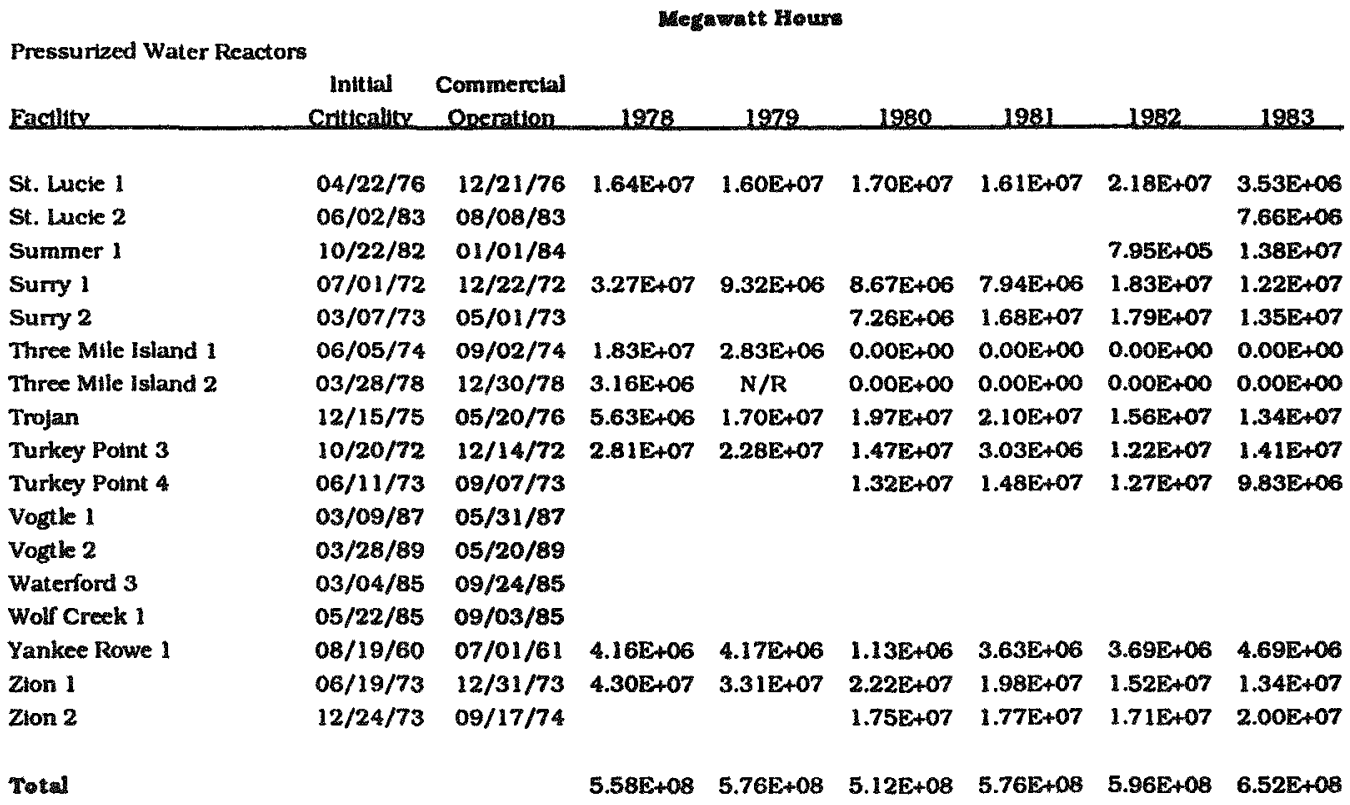

N/R $=$ Not Reported 


\begin{tabular}{|c|c|c|c|c|c|c|c|c|}
\hline \\
\hline Facilit & $\begin{array}{c}\text { Intulal } \\
\text { Critciculto }\end{array}$ & $\begin{array}{l}\text { Commerchal } \\
\text { Opertion }\end{array}$ & 1984 & 1985 & 1986 & 1987 & 1988 & 1989 \\
\hline Arkansas One 1 & $08 / 06 / 74$ & $12 / 19 / 74$ & $1.44 \mathrm{E}+07$ & $1.6218+07$ & $1.1118+07$ & $1.5115+07$ & $24 \mathrm{E}+07$ & $1.072+07$ \\
\hline Arkansas One 2 & $12 / 05 / 78$ & $03 / 26 / 80$ & $1.95 \mathrm{E}+07$ & $1.502+07$ & $1.68 E+07$ & $2.106+07$ & $1.58 \mathrm{E}+07$ & $1.768+07$ \\
\hline Beaver Valley 1 & $05 / 10 / 76$ & $10 / 01 / 76$ & $1.58 E+07$ & $1.96 \mathrm{E}+07$ & $1.555+07$ & $1.84 \mathrm{E}+07$ & $1.645+07$ & $1.278+07$ \\
\hline Beaver Valley 2 & $08 / 04 / 87$ & $1 / 1 / 7 / 87$ & & & & 2.396406 & 2.138+07 & $1.518 * 07$ \\
\hline Braidwood 1 & $05 / 29 / 87$ & $07 / 29 / 88$ & & & & $5.02 E+06$ & $1.0426+07$ & $1.442+07$ \\
\hline Braldwood 2 & $03 / 08 / 88$ & $10 / 17 / 88$ & & & & & 4.081406 & $2.18 \mathrm{C}+07$ \\
\hline Byron 1 & $02 / 02 / 85$ & $09 / 16 / 85$ & & $3.34 \mathrm{E}+06$ & $2.325+07$ & $1.7112+07$ & $1.998+07$ & $2.77 \mathrm{e}+07$ \\
\hline Byron 2 & $01 / 09 / 87$ & $08 / 21 / 87$ & & & & 6.476406 & 2.048507 & 1.871007 \\
\hline Callaway 1 & $10 / 02 / 84$ & $12 / 19 / 84$ & $1.00 \mathrm{E}+06$ & $2.498+07$ & $2.265+07$ & $1.98 \mathrm{E}+07$ & $2.76 E+07$ & $3.15 E+07$ \\
\hline Calvert Cliffs 1 & $10 / 07 / 74$ & $05 / 08 / 75$ & $1.96 \mathrm{E}+07$ & $1.35 \mathrm{E}+07$ & $1.82+07$ & $1.65 \mathrm{E}+07$ & $1.62 \mathrm{E}+07$ & 4.235406 \\
\hline Calvert Chifs 2 & $11 / 30 / 76$ & $04 / 01 / 77$ & 1.695407 & $1.78 E+07$ & $2.18 \mathrm{C}+07$ & $1.50 \mathrm{E}+07$ & 2.07507 & $1.495+06$ \\
\hline Catawba 1 & $01 / 07 / 85$ & $06 / 29 / 85$ & & $1.07 \mathrm{E}+07$ & $1.59 \mathrm{E}+07$ & $1.95 \mathrm{E}+07$ & $2.292+07$ & $2.36 \mathrm{E}+07$ \\
\hline Catawba 2 & $05 / 08 / 86$ & $08 / 19 / 86$ & & & $6.048+06$ & $2.17 \mathrm{C}+07$ & $1.88 \mathrm{E}+07$ & 1.9510407 \\
\hline Donald C. Cook I & $01 / 18 / 75$ & $08 / 27 / 75$ & $2.41 E+07$ & $6.83 \mathrm{EAO6}$ & $2.162+07$ & $1.64 \mathrm{E}_{5}+07$ & 2.42507 & $1.74 E+07$ \\
\hline Donald C. Cook 2 & $03 / 10 / 78$ & $07 / 01 / 78$ & $1.70 \mathrm{E}+07$ & $1.82 \mathrm{E} \cdot 07$ & 1. 448407 & $1.65 \mathrm{E}+07$ & $7.4112+06$ & $2.13 \mathrm{E}+07$ \\
\hline Crystal River 3 & $01 / 14 / 77$ & $03 / 13 / 77$ & $1.97 \mathrm{E}+07$ & $1.17 \mathrm{E}+07$ & $8.146+06$ & $1.11 \mathrm{EnO}$ & $1.78 \mathrm{E}+07$ & $9.148+06$ \\
\hline Davls-Besse 1 & $08 / 12 / 77$ & $07 / 31 / 78$ & $1.39 \mathrm{E}+07$ & $6.315+06$ & $1.29 \mathrm{E} 405$ & $1.65 \mathrm{E}+07$ & $3.912+06$ & $2.358+07$ \\
\hline Diablo Canyon 1 & $04 / 29 / 84$ & $05 / 07 / 85$ & $9.48 \mathrm{E}+05$ & $1.66 \mathrm{E} 407$ & $1.70 \mathrm{E}+07$ & $2.545+07$ & $1.66 \mathrm{C}+07$ & $2.25 E+07$ \\
\hline Dhablo Canyon 2 & $08 / 19 / 85$ & $03 / 13 / 86$ & & $2.12 \mathrm{E}+06$ & $2.11 \mathrm{E}+07$ & $1.82 \mathrm{E}+07$ & $1.98 \mathrm{E}+07$ & $2.685+07$ \\
\hline Joseph M. Farley 1 & $08 / 09 / 77$ & $12 / 01 / 77$ & 1.781607 & $1.90 \mathrm{E}+07$ & $1.85 E 407$ & $2.11 \mathrm{C}+07$ & $1.928+07$ & $1.962 \times 07$ \\
\hline Joseph M. Farley 2 & $05 / 05 / 81$ & $07 / 30 / 81$ & $2.158+07$ & $1.74 \mathrm{E} 607$ & $1.91 \mathrm{E}+07$ & $1.60 \mathrm{E}+07$ & $2.296+07$ & $1.81 \mathrm{E}+07$ \\
\hline Fort Calhoun 1 & $08 / 06 / 73$ & $06 / 20 / 74$ & $7.43 \mathrm{E}+06$ & $9.56 \mathrm{E}+06$ & $1.13 \mathrm{E}^{2}+07$ & $9.486+\infty 6$ & $8.345+06$ & $1.072+07$ \\
\hline R. E. Ginna & $11 / 08 / 69$ & $07 / 01 / 70$ & $1.00 \mathrm{E}+07$ & $1.14 E+07$ & $1.13 E+07$ & 1.192407 & $1.10 \mathrm{~B}+07$ & $9.65 \mathrm{E}+06$ \\
\hline Haddam Neck & $07 / 24 / 67$ & $01 / 01 / 68$ & $1.08 \mathrm{E}+07$ & $1.495+07$ & $7.26 E+06$ & $8.39 \mathrm{e}+06$ & $1.06 \mathrm{E}+07$ & $9.478+06$ \\
\hline Marris 1 & $01 / 03 / 87$ & $05 / 02 / 87$ & & & & $1.12 \mathrm{E}+07$ & 1.7115407 & $1.83 E+07$ \\
\hline Indian Point 1 & $08 / 02 / 62$ & $10 / / 62$ & $0.00 \mathrm{E}+00$ & $0.00 \mathrm{E}+00$ & $0.00 E+00$ & $0.00 \mathrm{E}+00$ & & \\
\hline Indian Point 2 & $05 / 22 / 73$ & $08 / 01 / 74$ & $1.17 \mathrm{E}+07$ & $2.21 \mathrm{E}+07$ & $1.29 \mathrm{E}+07$ & 1.718407 & $1.95 \mathrm{E}+07$ & $1.455+07$ \\
\hline Indian Point 3 & $04 / 06 / 76$ & $08 / 30 / 76$ & $1.935+07$ & $1.51 E+07$ & $1.77 \mathrm{Etor}$ & 1.565407 & 2.14607 & 1.555407 \\
\hline Kewaunce & $03 / 07 / 74$ & $06 / 16 / 74$ & $1.23 \mathrm{E} 407$ & $1.16 \mathrm{E}+0 \%$ & $1.21 \mathrm{E}+407$ & $1.285+07$ & $1.225+07$ & $1.18 \mathrm{E}+07$ \\
\hline Madne Yankee & $10 / 23 / 72$ & $12 / 28 / 72$ & $1.62 \mathrm{E}+07$ & $1.67 \mathrm{E}^{2}+07$ & $1.835 \mathrm{EO}$ & $1.318+07$ & $1.67 \mathrm{E}+07$ & $2.112+07$ \\
\hline McGuire 1 & $08 / 08 / 81$ & $12 / 01 / 81$ & $1.94 E+07$ & $2.06 \mathrm{E}+07$ & $1.56 \mathrm{E}^{2}+07$ & 2.211507 & $2.26 \mathrm{E}+07$ & $2.38 \mathrm{E}+07$ \\
\hline McCulre 2 & $05 / 08 / 83$ & $03 / 01 / 84$ & $1.945+07$ & $1.686+07$ & $1.96 \mathrm{E}+0 \%$ & $2.25 E+07$ & $2.41 \mathrm{E} * 07$ & $2.195+07$ \\
\hline Millstone 2 & $10 / 17 / 75$ & $12 / 26 / 75$ & $2.14 \mathrm{E}^{\mathrm{E}}+07$ & 1.122407 & $1.66 \mathrm{~F}+07$ & $2.18 \mathrm{FOH}$ & $1.81 \mathrm{E}+07$ & $1.55 \mathrm{E} 407$ \\
\hline Milistone 3 & $01 / 23 / 86$ & $04 / 23 / 86$ & & & 2.17E.+07 & $2.05 \mathrm{E}+07$ & $2.33 E 4.07$ & $2.17 \mathrm{E}+07$ \\
\hline North Anna 1 & $04 / 05 / 78$ & $06 / 06 / 78$ & $1.18 E+07$ & $1.81 \mathrm{E}+07$ & $1.98 \mathrm{E}+07$ & $1.14 \mathrm{E}+07$ & $2.20 \mathrm{E} 407$ & $1.37 E+07$ \\
\hline North Anna 2 & $06 / 12 / 80$ & $12 / 14 / 80$ & $1.51 \mathrm{E}+07$ & $2.166+07$ & $1.92 \mathrm{E}+07$ & $1.80 \mathrm{E} * 07$ & $2.50 \mathrm{E}+07$ & $1.88 \mathrm{E}+07$ \\
\hline Oconee 1 & $04 / 19 / 73$ & $07 / 15 / 73$ & $1.86 E+07$ & $2.146+07$ & $1.46 \mathrm{E} \div 07$ & $1.566+07$ & $2.21 \mathrm{E}+07$ & $1.82 \mathrm{E}-07$ \\
\hline Oconee 2 & $11 / 11 / 73$ & $09 / 09 / 74$ & $2.236+07$ & $8.56 \mathrm{E}+07$ & $1.798+07$ & $1.93 \mathrm{E}+07$ & $1.72 \mathrm{E}+07$ & $1.84 E+07$ \\
\hline Oconee 3 & $09 / 05 / 74$ & $12 / 16 / 74$ & $1.63 \mathrm{E}+07$ & $1.49 \mathrm{E}+07$ & $1.88 \mathrm{E}+07$ & $1.556+07$ & $1.80 \mathrm{E}+07$ & $1.94 E+07$ \\
\hline Palisades & $05 / 24 / 7$ & $12 / 31 / 71$ & $2.72 \mathrm{E}+06$ & $1.75 \mathbb{E}^{2}+07$ & $2.76 \mathrm{E}+406$ & $8.83 E+06$ & $1.14 \mathrm{E}+07$ & $1.21 \mathrm{E}+07$ \\
\hline Palo Verde 1 & $05 / 25 / 85$ & $02 / 13 / 86$ & & $4.39 \varepsilon+06$ & $1.82 \mathrm{E}+07$ & $1.61 \mathrm{E}+07$ & $2.03 E+07$ & $5.57 \mathrm{E}+06$ \\
\hline Palo Verde 2 & $04 / 18 / 86$ & $09 / 19 / 86$ & & & $7.02 E+06$ & 2. $49 \mathrm{EE}+07$ & $2.07 E+07$ & $1.47 \mathrm{E}+07$ \\
\hline Palo Verde 3 & $10 / 25 / 87$ & $01 / 08 / 88$ & & & & $1.24 \mathrm{E}+06$ & $3.03 \mathrm{E}+07$ & $4.10 E+06$ \\
\hline Point Beach 1 & $11 / 02 / 70$ & $12 / 21 / 70$ & $9.4118+06$ & $1.020+07$ & $1.15 E+07$ & $1.09 \mathrm{E}+07$ & $1.172+07$ & $1.11 E+07$ \\
\hline Point Beach 2 & $05 / 30 / 72$ & $10 / 01 / 71$ & $1.09 E+07$ & 1.11E+07 & $1.06 \mathrm{E}+07$ & $1.11 \mathrm{E}+07$ & $1.14 E+07$ & $1.05 \mathrm{E}+07$ \\
\hline Pratrie Island I & $12 / 01 / 73$ & $12 / 16 / 73$ & $1.33 E+07$ & $1.18 \mathrm{~g}^{\circ} \mathrm{O}$ & $1.22 \mathrm{E}+07$ & $1.14 E+07$ & $1.23 \mathrm{E}+07$ & 1. $40 \mathrm{ESO} 07$ \\
\hline Prairie Island 2 & $12 / 17 / 74$ & $12 / 21 / 74$ & $248+07$ & $1.15 \mathrm{E}+07$ & $1.23 \mathrm{E}+07$ & $1.41 E+07$ & $1.26 \mathrm{E}+17$ & $1.255+07$ \\
\hline Rancho Seco 1 & $09 / 15 / 74$ & $04 / 17 / 75$ & $1.216+07$ & $6.266+06$ & $0.00 \mathrm{R}+00$ & $0.00 \mathrm{E}+00$ & $9.47 E+06$ & $4.62 E+06$ \\
\hline H. B. Robinson 2 & $09 / 20 / 70$ & $03 / 07 / 71$ & $7.84 \mathrm{E}+05$ & 1.668407 & 1.522507 & $1.358+07$ & $1.06 E+07$ & $8.86 \mathrm{E}+06$ \\
\hline Salem 1 & $12 / 11 / 76$ & $06 / 30 / 77$ & $6.95 \mathrm{E} \star 06$ & $2.77 \mathrm{E}+07$ & $2.24 E+07$ & $1.96 \mathrm{EA07}$ & $2.32 E+07$ & $1.97 \mathrm{E}+07$ \\
\hline Salcm 2 & $08 / 08 / 80$ & $10 / 13 / 81$ & $1.032+07$ & $1.60 \mathrm{~B}+07$ & $1.74 \mathrm{E}+07$ & $1.966+07$ & 1.905007 & $2.44 E \rightarrow 07$ \\
\hline San Ondre 1 & $06 / 14 / 67$ & $01 / 01 / 68$ & $9.248+05$ & $8.12 \mathrm{E}+06$ & $3.00 E+06$ & $8.825+06$ & $4.528+06$ & $3.928+00$ \\
\hline $\operatorname{San}$ Onofre 2 & $07 / 26 / 82$ & $08 / 08 / 83$ & 1.666007 & $1.65 \mathrm{E}+07$ & $2.01 E+07$ & $1.93 E+07$ & $2.758+07$ & $1.638+07$ \\
\hline San Ondre 3 & $08 / 29 / 83$ & $04 / 01 / 84$ & 1.296407 & $1.21 \mathrm{E}+07$ & 2.138 .07 & $2.32 \mathrm{E}+07$ & $1.89 \mathrm{E}+07$ & 2.735407 \\
\hline Seabrook 1 & $06 / 13 / 89$ & $08 / 19 / 90$ & & & & & & \\
\hline Sequoyah I & $07 / 05 / 80$ & $07 / 01 / 81$ & $1.92 E+07$ & $1.246+07$ & $0.00 \mathrm{E}+00$ & $0.00 E+00$ & $5.16 \mathrm{E}+05$ & $2.88 \mathrm{E}+07$ \\
\hline Sequoyah 2 & $11 / 05 / 81$ & $06 / 01 / 82$ & $1.96 \mathrm{C}+07$ & $1.71 \mathrm{E}+07$ & $0.00 \mathrm{E}+00$ & $0.00 E+00$ & $1.26 \mathrm{E}+07$ & $2.88 \mathrm{C}+07$ \\
\hline South Texas 1 & $03 / 08 / 88$ & $08 / 25 / 88$ & & & & & $8.815+06$ & 1.9960707 \\
\hline South Texas I & $03 / 12 / 89$ & $06 / 19 / 89$ & & & & & & $9.52 \mathrm{C}+06$ \\
\hline
\end{tabular}


Table 16

Thermal Eaeray Ceneration Comparieon By Year

Megawatt Hours

Pressurized Water Reactors

Initial Commercial

\begin{tabular}{|c|c|c|c|c|c|c|c|c|}
\hline Eacllity & Crticalty & Operation & 1984 & 1985 & 1986 & 1987 & 1988 & 1982 \\
\hline St. Lucie 1 & $04 / 22 / 76$ & $12 / 21 / 76$ & $1.35 \mathrm{E}+07$ & $1.88 \mathrm{E}+07$ & $2.23 E+07$ & $1.81 E+07$ & $1.97 \mathrm{E}+07$ & $2.19 E+07$ \\
\hline St. Lucie 2 & $06 / 02 / 83$ & $08 / 08 / 83$ & $1.77 E+07$ & $1.93 \mathrm{E}+07$ & $1.9418+07$ & $1.90 E+07$ & $2.35 E+07$ & $1.73 \mathrm{E}+07$ \\
\hline Summer 1 & $10 / 22 / 82$ & $01 / 01 / 84$ & $1.33 \mathrm{E}+07$ & $1.65 \mathrm{E}+07$ & $2.26 \mathrm{E}+07$ & $1.64 \mathrm{E}+07$ & $1.61 \mathrm{E}+07$ & $1.76 \mathrm{E}+07$ \\
\hline Surry 1 & $07 / 01 / 72$ & $12 / 22 / 72$ & $1.11 \mathrm{E}+07$ & $1.79 \mathrm{E}+07$ & $1.42 \mathrm{E}+07$ & $1.47 E+07$ & $8.45 \mathrm{E}+06$ & $9.95 E+06$ \\
\hline Surry 2 & $03 / 07 / 73$ & $05 / 01 / 73$ & $1.73 E+07$ & $1.33 E+07$ & $1.45 E+07$ & $1.54 \mathrm{E}+07$ & $1.16 \mathrm{E}+07$ & $2.87 E+06$ \\
\hline Three Mile Island I & $06 / 05 / 74$ & $09 / 02 / 74$ & $0.00 E+00$ & $2.82 \mathrm{E} \uparrow 06$ & $1.56 \mathrm{E}+07$ & $1.56 \mathrm{E}+07$ & $1.69 \mathrm{E}+07$ & $2.22 \times+07$ \\
\hline Three Mile Island 2 & $03 / 28 / 78$ & $12 / 30 / 78$ & $0.00 E+\infty 0$ & $0.00 \mathrm{E}+\infty 0$ & $0.00 E+\infty 0$ & $0.00 \mathrm{E}+00$ & & \\
\hline Trojan & $12 / 15 / 75$ & $05 / 20 / 76$ & $1.54 \mathrm{E}+07$ & $2.25 E+07$ & $2.29 \mathrm{E}+07$ & $1.39 \mathrm{E}+07$ & $1.98 E+07$ & $1.73 E+07$ \\
\hline Turkey Point 3 & $10 / 20 / 72$ & $12 / 14 / 72$ & $1.56 \mathrm{E}+07$ & $1.11 E+07$ & $1.46 \mathrm{E}+07$ & $2.96 \mathrm{E}+06$ & $1.14 \mathrm{E}+07$ & $1.18 E+07$ \\
\hline Turkey Point 4 & $06 / 11 / 73$ & $09 / 07 / 73$ & $1.04 E+07$ & $1.69 \mathrm{E}+07$ & $5.73 E \wedge 06$ & $8.69 \mathrm{E}+06$ & $1.06 \mathrm{E}+07$ & $7.140+06$ \\
\hline Vogtle 1 & $03 / 09 / 87$ & $05 / 31 / 87$ & & & & $1.27 E+07$ & $2.18 \mathrm{E}+07$ & $2.76 E+07$ \\
\hline Vogtle 2 & $03 / 28 / 89$ & $05 / 20 / 89$ & & & & & & $1.722+07$ \\
\hline Wateriond 3 & $03 / 04 / 85$ & $09 / 24 / 85$ & & $5.64 \mathrm{E}+06$ & $2.27 \mathrm{E}+07$ & $2.31 E+07$ & $2.06 \mathrm{E}+07$ & $2.37 E+07$ \\
\hline Wolf Creek 1 & $05 / 22 / 85$ & $09 / 03 / 85$ & & $8.87 E+06$ & $2.09 E+07$ & $1.97 \mathrm{E}+07$ & $2.01 \mathrm{E}+07$ & $2.88 E+07$ \\
\hline Yankee Rowe 1 & $08 / 19 / 60$ & $07 / 01 / 61$ & $3.61 E+06$ & $4.19 \mathrm{E}+06$ & $4.90 E^{2}+06$ & $4.03 E+06$ & $4.03 E+06$ & $4.66 \mathrm{E}+06$ \\
\hline Zion 1 & $06 / 19 / 73$ & $12 / 31 / 73$ & $1.83 E+07$ & $1.85 \mathrm{E}+07$ & $1.58 \mathrm{E}+07$ & $1.88 \mathrm{E}+07$ & $1.97 \mathrm{C}+07$ & $1.56 E+07$ \\
\hline Zion 2 & $12 / 24 / 73$ & $09 / 17 / 74$ & $1.92 E+07$ & $2.16 \mathrm{E}+07$ & $2.30 \mathrm{E}+07$ & $1.61 \mathrm{E}+07$ & $2.08 E+07$ & $2.40 \mathrm{E}+07$ \\
\hline rotal & & & $7.37 \mathrm{E}+08$ & $8.58 \mathrm{E}+08$ & $9.38 \mathrm{E}+08$ & $1.01 E+09$ & $1.18 \mathrm{E}+09$ & $1.21 E+09$ \\
\hline
\end{tabular}


APPENDIX A

A- 1 
EFFLUENT AND WASTE DISPOSAL ANNUAL REPORT FOR YEAR 1989 AIRBORNE AND LIQUID EFFLUENTS

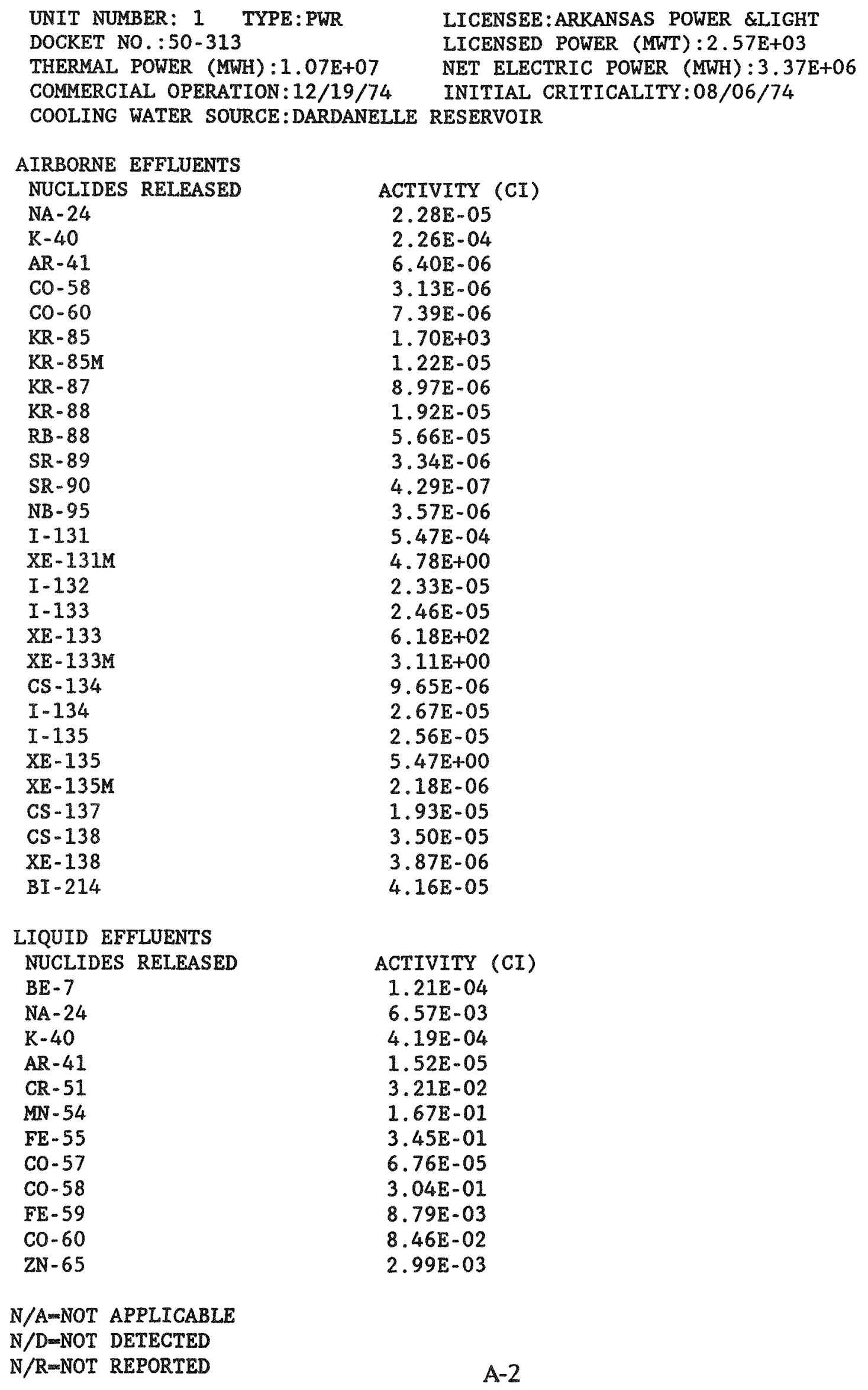


EFFLUENT AND WASTE DISPOSAL ANNUAL REPORT FOR YEAR 1989 AIRBORNE AND LIQUID EFFLUENTS

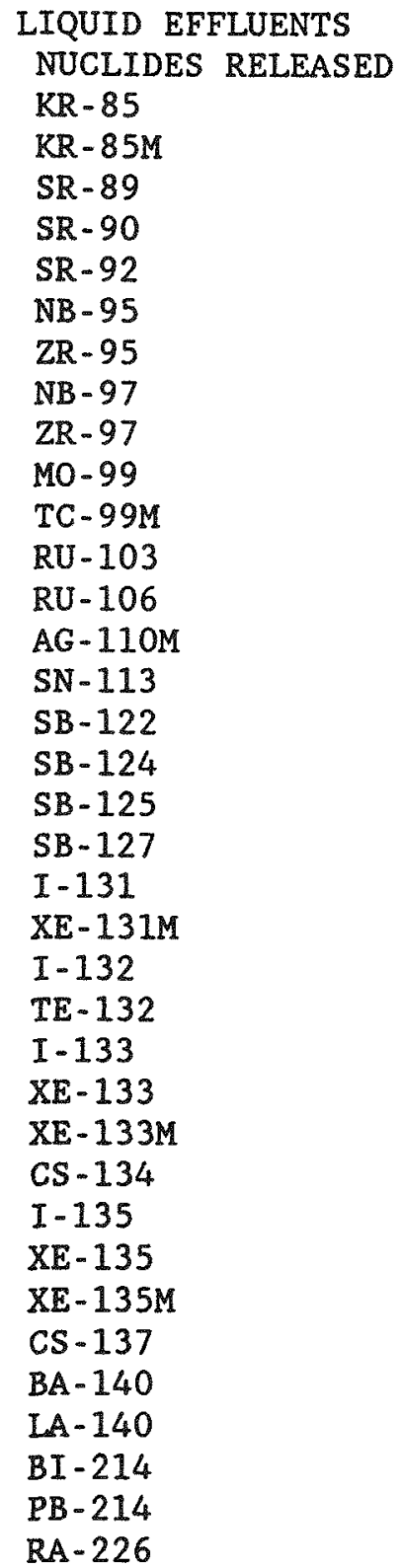

$$
\begin{aligned}
& \text { ACTIVITY (CI) } \\
& \text { 1.53E-01 } \\
& \text { 1. } 37 \mathrm{E}-04 \\
& \text { 2.81E-03 } \\
& \text { 8.24E-04 } \\
& \text { 3. } 54 \mathrm{E}-03 \\
& \text { 8.15E-03 } \\
& \text { 8.93E-03 } \\
& \text { 2. 36E-04 } \\
& 7.05 E-04 \\
& 4.01 E-04 \\
& 1.00 E-03 \\
& \text { 4. } 29 \mathrm{E}-03 \\
& 4.18 \mathrm{E}-04 \\
& 2.43 E-01 \\
& \text { 9.34E-04 } \\
& 3.84 E-03 \\
& \text { 2. } 20 \mathrm{E}-02 \\
& 2.41 \mathrm{E}-01 \\
& \text { 2. 30E-04 } \\
& 2.07 E-01 \\
& \text { 3. } 76 \mathrm{E}-01 \\
& \text { 7. } 94 \mathrm{E}-04 \\
& 7.05 E-04 \\
& \text { 7. } 01 \mathrm{E}-05 \\
& \text { 2. } 27 \mathrm{E}+01 \\
& \text { 1. } 32 \mathrm{E}-01 \\
& \text { 8. 29E-02 } \\
& 1.08 \mathrm{E}-05 \\
& \text { 3. } 55 \mathrm{E}-03 \\
& \text { 1. } 11 \mathrm{E}-03 \\
& \text { 2. } 21 E-01 \\
& \text { 1. } 28 \mathrm{E}-04 \\
& \text { 3. } 49 \mathrm{E}-02 \\
& 4.05 E-04 \\
& 5.79 E-08 \\
& 2.03 E-08
\end{aligned}
$$

4

TOTAL AIRBORNE TRITIUM RELEASE $1.62 E+01$

TOTAL LIQUID TRITIUM RELEASE $3.81 \mathrm{E}+02$

VOLUME OF LIQUID WASTE RELEASED (PRIOR TO DILUTION) IITERS $1.13 E+07$ VOLUME OF DILUTION WATER USED DURING PERIOD 
EFFLUENT AND WASTE DISPOSAL ANNUAL REPORT FOR YEAR 1989 AIRBORNE AND LIQUID EFFLUENTS

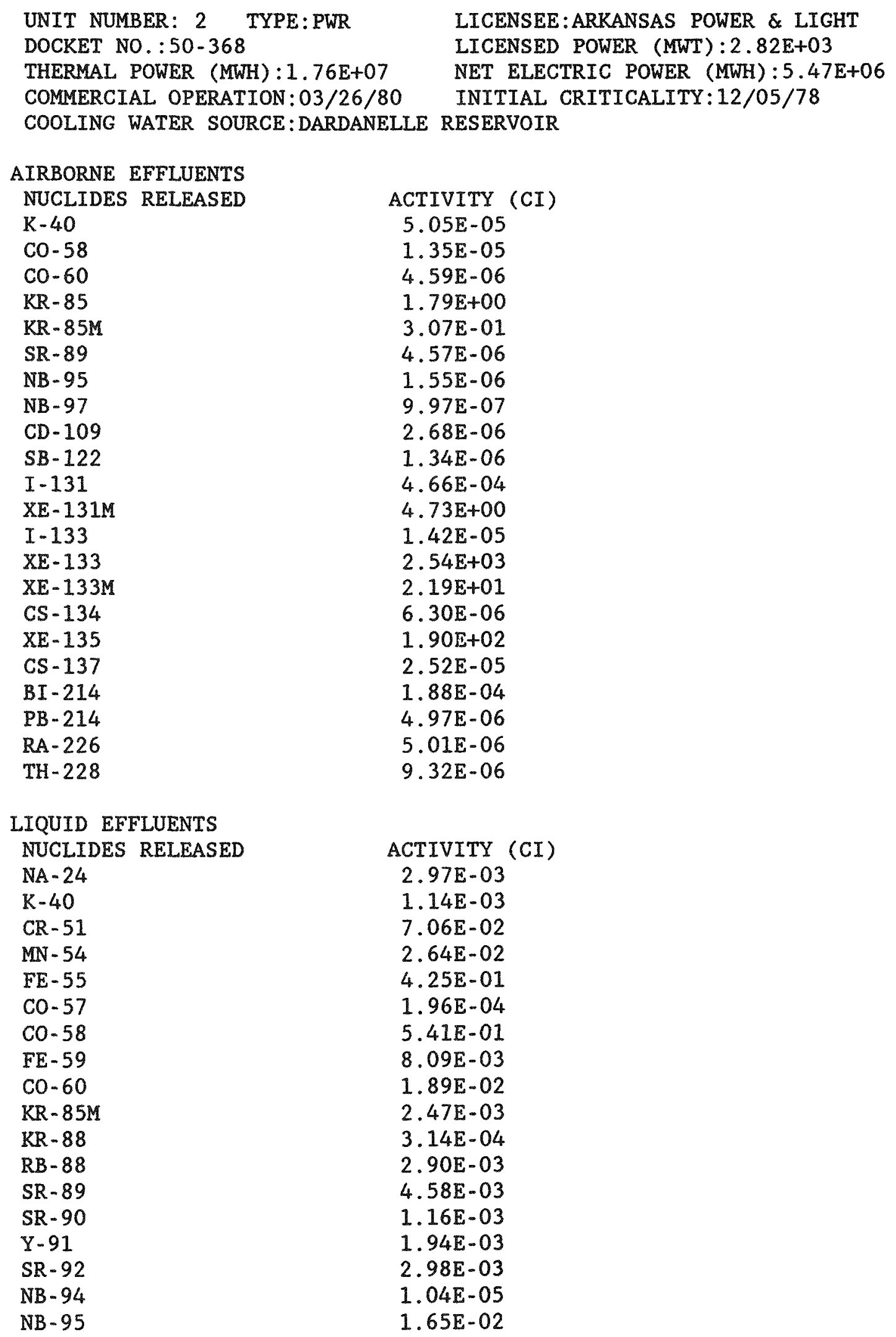

N/A=NOT APPLICABLE 
EFFLUENT AND WASTE DISPOSAL ANNUAL REPORT FOR YEAR 1989 AIRBORNE AND LIQUID EFFLUENTS

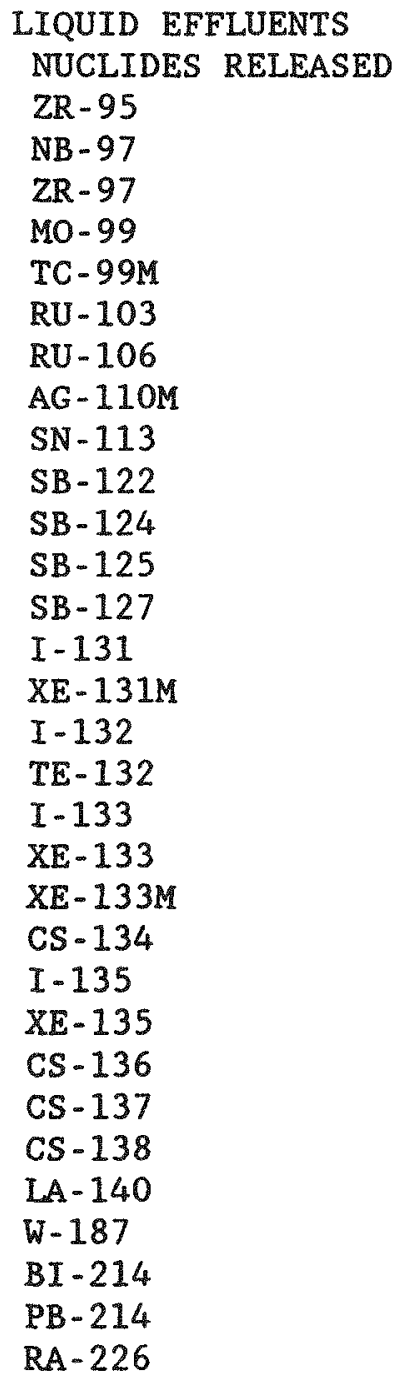

ACTIVITY (CI)

1.71E-02

1. $40 \mathrm{E}-04$

$2.47 E-04$

$2.48 \mathrm{E}-02$

$2.50 \mathrm{E}-02$

1. $39 E-03$

$1.17 \mathrm{E}-03$

2. $28 \mathrm{E}-01$

4. $12 \mathrm{E}-03$

2. $29 \mathrm{E}-02$

1. $90 \mathrm{E}-01$

$5.03 E-01$

2.71E-03

3. $01 \mathrm{E}-01$

3. $01 E-01$

$2.48 \mathrm{E}-03$

$5.76 E-03$

2. $81 \mathrm{E}-03$

2. $22 E+01$

$1.75 E-01$

4. $48 \mathrm{E}-02$

$1.34 \mathrm{E}-04$

$6.66 \mathrm{E}-02$

$1.83 \mathrm{E}-04$

1. $36 \mathrm{E}-01$

$2.82 E-03$

$5.32 \mathrm{E}-03$

3. $83 \mathrm{E}-04$

4. $30 \mathrm{E}-03$

$1.60 \mathrm{E}-04$

$4.85 E-05$

TOTAL AIRBORNE TRITIUM RELEASE $1.25 E+01$

TOTAL LIQUID TRITIUM RELEASE $4.40 \mathrm{E}+02$

VOLUME OF LIQUID WASTE RELEASED (PRIOR TO DILUTION) LITERS 2.26E+06 VOLUME OF DILUTION WATER USED DURING PERIOD 
EFFLUENT AND WASTE DISPOSAL ANNUAL REPORT FOR YEAR 1989 SOLID EFFLUENTS

SOLID WASTE DISPOSITION NUMBER OF SHIPMENTS

$$
\begin{array}{r}
2 \\
1 \\
12 \\
16 \\
1
\end{array}
$$

$$
\begin{aligned}
& \text { MODE OF TRANSPORTATION } \\
& \text { CASK SHIPMENT } \\
& \text { UNSHIELDED VAN/TRUCK } \\
& \text { UNSHIELDED VAN/TRUCK } \\
& \text { UNSHIELDED VAN/TRUCK } \\
& \text { UNSHIELDED VAN/TRUCK }
\end{aligned}
$$

ESTIMATE OF MAJOR NUCLIDE COMPOSITION (BY TYPE OF WASTE)

\section{DESTINATION BARNWELL SC BEATTY NV OAK RIDGE TN PITTSBURGH PA WAMPUM PA}

JAN-JUNE JULY-DEC

A

$$
\begin{aligned}
& \text { AG-110M } \\
& \text { CO-58 } \\
& \text { CO-60 } \\
& \text { CS-134 } \\
& \text { CS-137 } \\
& \text { FE-55 } \\
& M N-54 \\
& \text { NI }-63 \\
& \text { SB-125 } \\
& \text { TE-125M } \\
& \text { AG-110M } \\
& \text { CO-58 } \\
& \text { CO-60 } \\
& \text { CR-51 } \\
& \text { CS-134 } \\
& \text { CS }-137 \\
& \text { FE }-55 \\
& M N-54 \\
& \text { NB-95 } \\
& \text { NI }-63 \\
& \text { ZR- } 95
\end{aligned}
$$$$
\text { B }
$$

\section{TYPE OF WASTE}
A. SPENT RESINS, FILTER SLUDGES, EVAPORATOR BOTTOMS, ETC.
B. DRY COMPRESSIBLE WASTE, CONTAMINATED EQUIPMENT, ETC.
C. IRRADIATED COMPONENTS, CONTROL RODS, ETC.
D. OTHER

$\begin{array}{lc}\text { UNIT } & \text { YEAR TOTAL } \\ \text { M3 } & 1.83 \mathrm{E}+01 \\ \text { CI } & 2.83 \mathrm{E}+02 \\ \text { M3 } & 2.03 \mathrm{E}+02 \\ \text { CI } & 1.26 \mathrm{E}+01 \\ \text { M3 } & 0.00 \mathrm{E}+00 \\ \text { CI } & 0.00 \mathrm{E}+00 \\ \text { M3 } & 0.00 \mathrm{E}+00 \\ \text { CI } & 0.00 \mathrm{E}+00\end{array}$


EFFLUENT AND WASTE DISPOSAL ANNUAL REPORT FOR YEAR 1989 AIRBORNE AND LIQUID EFFLUENTS
UNIT NUMBER: 1 TYPE:PWR
DOCKET NO. : $50-334$
THERMAL POWER (MWH) : $1.27 \mathrm{E}+07$
COMMERCIAL OPERATION : $10 / 01 / 76$
COOLING WATER SOURCE:OHIO RIVER
UNIT NUMBER: 2 TYPE:PWR
DOCKET NO. : 50-412
THERMAL POWER (MWH) : $1.51 \mathrm{E}+07$
COMMERCIAL OPERATION: $11 / 17 / 87$
COOLING WATER SOURCE:OHIO RIVER
AIRBORNE EFFLUENTS
NUCLIDES RELEASED
AR -41
CO -58
ACTIVITY (CI)
Co- 60
$6.12 \mathrm{E}-01$
$1.19 \mathrm{E}-05$
$\mathrm{KR}-85$
$7.59 \mathrm{E}-06$
$\mathrm{KR}-85 \mathrm{M}$
7. $31 \mathrm{E}-01$
I- 131
$1.61 \mathrm{E}-02$
$\mathrm{XE}-131 \mathrm{M}$
$2.23 E-05$
I -133
$\mathrm{XE}-133$
$X E-133 M$
1. $56 \mathrm{E}-01$
3. $13 E-06$
$\mathrm{XE}-135$
1. $21 \mathrm{E}+01$
CS -137
$6.11 \mathrm{E}-02$
CE-144
$5.52 \mathrm{E}-01$
3. $60 \mathrm{E}-04$
1. $18 \mathrm{E}-07$

\section{LIQUID EFFLUENTS}
NUCLIDES RELEASED
ACTIVITY (CI)
NA- 24
CR -51
1.65E-05
MN -54
4. $95 \mathrm{E}-02$
FE -55
$6.33 \mathrm{E}-03$
CO- 57
2.14E-01
CO- 58
$7.43 \mathrm{E}-04$
FE -59
1. $48 \mathrm{E}-01$
CO -60
$5.24 E-03$
NB -95
$6.87 \mathrm{E}-02$
ZR-NB -95
1. $83 \mathrm{E}-03$
NB -97
1. $91 \mathrm{E}-03$
MO- 99
2.14E-03
TC-99M
3.17E-06
RU-103
3. $10 \mathrm{E}-06$
AG-110M
4. $91 \mathrm{E}-04$
SB - 124
$4.81 \mathrm{E}-03$
SB-125
$7.82 \mathrm{E}-03$
I -131
I -133
$\mathrm{XE}-133$
3. $31 \mathrm{E}-02$
1. $91 \mathrm{E}-04$
$1.54 \mathrm{E}-05$
CS -134
3. $74 \mathrm{E}-03$
$\mathrm{XE}-135$
3. $21 \mathrm{E}-05$
5. $36 \mathrm{E}-04$

LICENSEE: DUQUESNE LIGHT

LICENSED POWER (MWT) $: 2.65 \mathrm{E}+03$

NET ELECTRIC POWER (MWH) : $3.79 \mathrm{E}+06$

INITIAL CRITICALITY:05/10/76

LICENSEE: DUQUESNE LIGHT

LICENSED POWER (MWT): $2.65 E+03$

NET ELECTRIC POWER (MWH):4.54E+06

INITIAL CRITICALITY:08/04/87

N/A-NOT APPLICABLE

N/D-NOT DETECTED

$\mathrm{N} / \mathrm{R}=\mathrm{NOT}$ REPORTED 
EFFLUENT AND WASTE DISPOSAL ANNUAL REPORT FOR YEAR 1989 AIRBORNE AND LIQUID EFFLUENTS

LIQUID EFFLUENTS

NUCLIDES RELEASED

CS -137

BA-LA- 140
ACTIVITY (CI)

8. 31E-04

4. $29 \mathrm{E}-05$

TOTAL AIRBORNE TRITIUM RELEASE

$8.05 \mathrm{E}+01$

TOTAL LIQUID TRITIUM RELEASE

$6.21 E+02$

VOLUME OF LIQUID WASTE RELEASED (PRIOR TO DILUTION)

VOLUME OF DILUTION WATER USED DURING PERIOD

LITERS

$9.31 E+06$

LITERS $7.03 \mathrm{E}+09$ 
EFFLUENT AND WASTE DISPOSAL ANNUAL REPORT FOR YEAR 1989 SOLID EFFLUENTS

SOLID WASTE DISPOSITION

$\begin{array}{cl}\text { NUMBER OF SHIPMENTS } & \text { MODE OF TRANSPORTATION } \\ 9 & \text { TRUCK } \\ 34 & \text { TRUCK }\end{array}$

DESTINATION

BARNWELL SC

OAK RIDGE TN

ESTIMATE OF MAJOR NUCLIDE COMPOSITION (BY TYPE OF WASTE)

JAN-JUNE JULY-DEC

A

AG-110M

$A M-241$

BA -140

C -14

CE-141

CE $-144 / P R-144$

CL- 36

CM-242

CM- $243 / 244$

CO- 57

CO- 58

CO -60

CR -51

CS -134

CS -137

FE -55

FE- 59

H-3

I -129

I- 131

$\mathrm{MN}-54$

NB -95

NI -59

NI -63

P-32

PU-238

PU $-239 / 240$

PU- 241

SB-124

SB- 125

SR- 89

SR -90

TC- 99

$\mathrm{ZN}-65$

ZR-95

B

$A G-110 M$

$1.81 \mathrm{E}-03 \quad 1.04 \mathrm{E}-06$

1.67E-06 1.43E-05

8.47E-06 1.59E-04

2.84E-02 3.00E-02

7. $26 \mathrm{E}-05$

$2.87 \mathrm{E}-03$

$0.00 \mathrm{E}+00$

1.03E-05 1.52E-04

4.21E-06 3.61E-05

1.38E-01 2.11E-01

$7.46 \mathrm{E}+01 \quad 5.57 \mathrm{E}+01$

$6.57 \mathrm{E}+00 \quad 1.46 \mathrm{E}+01$

3.56E-05 3.54E-01

1.79E-02 1.33E-02

3. 30E-02 5.60E-02

1. $33 \mathrm{E}+01 \quad 1.53 \mathrm{E}+01$

1.27E-05 9.66E-02

5.33E-01 3.35E-01

4.62E-05 1.16E-05

5.82E-04 8.09E-03

$1.54 \mathrm{E}-01 \quad 9.56 \mathrm{E}+00$

$2.12 E-04 \quad 1.51 E-03$

7.21E-04 2.19E-02

$3.17 E+00 \quad 2.57 E+00$

$1.50 \mathrm{E}+00 \quad 1.19 \mathrm{E}-02$

7.47E-06 5.08E-05

1.28E-05 2.39E-05

3. $57 \mathrm{E}-03 \quad 3.49 \mathrm{E}-03$

$6.19 \mathrm{E}-03 \quad 3.55 \mathrm{E}-01$

$1.45 \mathrm{E}-04 \quad 7.13 \mathrm{E}-02$

3. $32 E-05 \quad 7.43 E-02$

3.12E-05 1.01E-05

3. $59 \mathrm{E}-01$

1.75E-04 1.49E-03

2.91E-01 
INSTALLATION : BEAVER VALLEY

EFFLUENT AND WASTE DISPOSAL ANNUAL REPORT FOR YEAR 1989 SOLID EFFLUENTS

ESTIMATE OF MAJOR NUCLIDE COMPOSITION (BY TYPE OF WASTE)

\section{$B$}

AM-241

$B A-133$

$\mathrm{BA}-140$

$\mathrm{BE}-7$

C -14

CD-109

CE -139

CE- 141

CE-144/PR - 144

CL- 36

CM- 242

CM $-243 / 244$

$\mathrm{CO}-57$

CO- 58

CO -60

CR -51

CS -134

CS -137

FE- 55

FE- 59

$\mathrm{H}-3$

HG -203

I -129

$I-131$

MN -54

NB-95

NI -59

NI -63

P - 32

PB -210

PR -147

PU- 238

PU-239/240

PU -241

RU -103

SB- 124

SB- 125

SN -113

SR -89

SR -90

TC- 99

TH -230

$\mathrm{Y}-88$
JAN-JUNE JULY-DEC

9.35E-03 1.21E-03

5. $31 \mathrm{E}-02 \quad 7.87 \mathrm{E}-03$

$3.55 E+00$

4.25E-01 5.09E-01

3. $88 \mathrm{E}-06$

$2.25 E-09$

1. $98 \mathrm{E}-02$

5.65E-04 3.92E-02

$7.20 \mathrm{E}-07$

$9.94 \mathrm{E}-04 \quad 2.73 \mathrm{E}-03$

8.03E-03 1.99E-03

4.65E-04 1.48E-02

$2.63 \mathrm{E}+01 \quad 1.43 \mathrm{E}+01$

$1.05 \mathrm{E}+01 \quad 1.34 \mathrm{E}+01$

4. $90 E+00 \quad 2.69 E+00$

$9.55 \mathrm{E}-01 \quad 9.70 \mathrm{E}-01$

$1.74 \mathrm{E}+00 \quad 2.22 \mathrm{E}+00$

$3.47 \mathrm{E}+01 \quad 2.36 \mathrm{E}+01$

$6.25 E-01 \quad 8.83 E-02$

$5.83 E-01 \quad 2.15 E+00$

$4.97 \mathrm{E}-13$

7.70E-03 1.52E-02

9. 50E-02

$1.79 \mathrm{E}+00$

$5.43 E-01$

$4.91 E+00$

$8.16 \mathrm{E}-01$

$0.00 \mathrm{E}+00$

4. $96 \mathrm{E}-01$

2. $50 \mathrm{E}+00$

3. $53 \mathrm{E}+01$

$3.09 E+00$

1. $30 \mathrm{E}+00$

$1.04 \mathrm{E}-04$

$7.04 E-09$

1. $30 \mathrm{E}-02 \quad 3.06 \mathrm{E}-03$

$6.93 E-03 \quad 1.62 E-03$

4.27E-01 6.83E-02

1. $27 \mathrm{E}-12$

1. $69 \mathrm{E}-02$

$6.95 \mathrm{E}-01 \quad 8.86 \mathrm{E}-01$

1. $55 \mathrm{E}-09$

2.52E-02 1.70E-02

3.52E-03 2.79E-02

9.51E-03 1.10E-02

7. 20E-08

1. $39 \mathrm{E}-09$ 
EFFLUENT AND WASTE DISPOSAL ANNUAL REPORT FOR YEAR 1989 SOLID EFFLUENTS

ESTIMATE OF MAJOR NUCLIDE COMPOSITION (BY TYPE OF WASTE)

B

$$
\text { ZR-95 }
$$

\section{TYPE OF WASTE}

A. SPENT RESINS, FILTER SLUDGES, EVAPORATOR BOTTOMS , ETC.

B. DRY COMPRESSIBLE WASTE, CONTAMINATED EQUIPMENT, ETC.

C. IRRADIATED COMPONENTS, CONTROL RODS, ETC.

D. OTHER

*VOLUME PRIOR TO REDUCTION (2.29E+2 M3 AFTER REDUCTION)
JAN-JUNE JULY-DEC
8
$1.78 \mathrm{E}+00$
$3.12 \mathrm{E}-01$

UNIT YEAR TOTAL

M3 $4.30 E+01$

CI $\quad 1.32 \mathrm{E}+03$

M3 $1.92 \mathrm{E}+03 *$

CI $\quad 3.04 \mathrm{E}+01$

M3 $0.00 \mathrm{E}+00$

CI $\quad 0.00 \mathrm{E}+00$

M3

CI 


\section{EFFLUENT AND WASTE DISPOSAL ANNUAL REPORT FOR YEAR 1989 AIRBORNE AND LIQUID EFFLUENTS}

\begin{tabular}{|c|c|c|}
\hline UNIT NUMBER: & & LICENSEI \\
\hline DOCKET NO. : 50-334 & & LICENSED \\
\hline THERMAL POWER (MWL & & NET ELECI \\
\hline COMMERCIAL OPERATI & & INITIAL \\
\hline COOLING WATER SOUR & IVER & \\
\hline AIRBORNE EFFLUENTS & & \\
\hline NUCLIDES RELEASED & $\mathrm{ACT}$ & $\operatorname{VITY}(\mathrm{CI})$ \\
\hline $\mathrm{CO}-58$ & & $3 E-04$ \\
\hline Co- 60 & & $7 E-03$ \\
\hline KR -85 & & $3 E+00$ \\
\hline $\mathrm{KR}-85 \mathrm{M}$ & & $4 E-03$ \\
\hline MO-99 & & $8 E-07$ \\
\hline TC-99M & & $1 E-07$ \\
\hline I- 131 & & $3 E-04$ \\
\hline$X E-131 M$ & & $8 E+00$ \\
\hline$I-133$ & & $5 E-05$ \\
\hline$X E-133$ & & $6 E+02$ \\
\hline $\mathrm{XE}-133 \mathrm{M}$ & & $5 E-02$ \\
\hline CS -134 & & $4 E-05$ \\
\hline$X E-135$ & & $O E+00$ \\
\hline$X E-135 M$ & & $7 E-04$ \\
\hline CS -137 & & $7 E-05$ \\
\hline
\end{tabular}


EFFLUENT AND WASTE DISPOSAL ANNUAL REPORT FOR YEAR 1989 AIRBORNE AND LIQUID EFFLUENTS

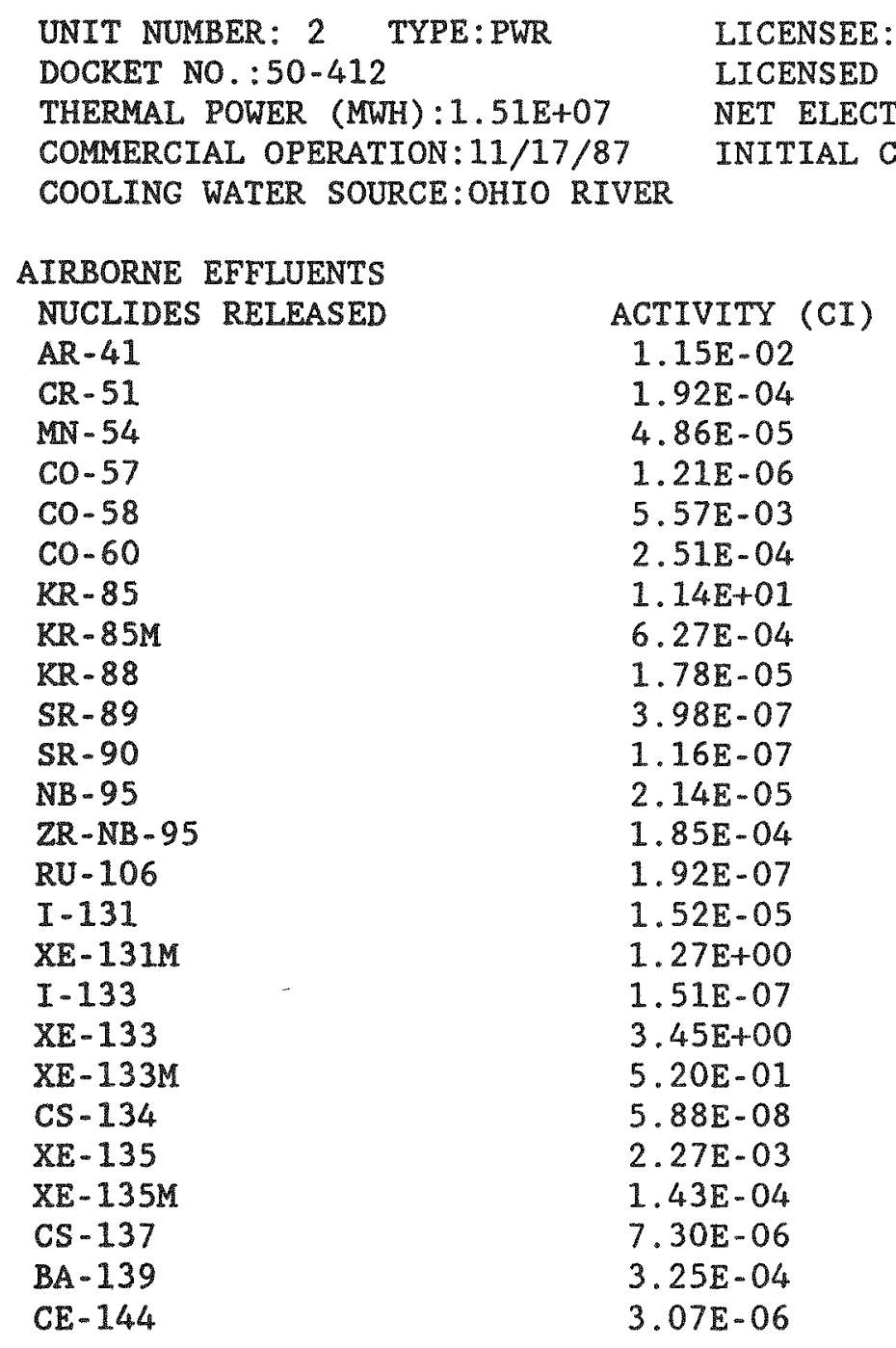


EFFLUENT AND WASTE DISPOSAL ANNUAL REPORT FOR YEAR 1989 AIRBORNE AND LIQUID EFFLUENTS

\begin{tabular}{|c|c|}
\hline \multicolumn{2}{|c|}{ UNIT NUMBER: 1 TYPE:BWR } \\
\hline \multirow{2}{*}{\multicolumn{2}{|c|}{$\begin{array}{l}\text { LICENSED POWER (MWT) }: 2.40 \mathrm{E}+02 \\
\text { NET ELECTRIC POWER (MWH) }: 4.17 \mathrm{E}+05\end{array}$}} \\
\hline & NET ELECTRIC POWER (MWH) :4.17E+05 \\
\hline COMMERCIAL OPERATI & 63 INITIAL CRITICALITY:09/27/62 \\
\hline \multicolumn{2}{|c|}{ COOLING WATER SOURCE: LAKE MICHIGAN } \\
\hline \multicolumn{2}{|l|}{ AIRBORNE EFFLUENTS } \\
\hline NUCLIDES RELEASED & ACTIVITY (CI) \\
\hline$N-13$ & $1.60 \mathrm{E}+03$ \\
\hline NA -24 & $9.47 E-04$ \\
\hline$C R-51$ & $6.77 \mathrm{E}-04$ \\
\hline MN -54 & $1.73 \mathrm{E}-04$ \\
\hline Co-58 & $3.63 E-06$ \\
\hline FE -59 & $1.68 \mathrm{E}-05$ \\
\hline $\mathrm{CO}-60$ & $2.48 \mathrm{E}-04$ \\
\hline $\mathrm{ZN}-65$ & $3.27 E-05$ \\
\hline BR -82 & $1.14 \mathrm{E}-03$ \\
\hline KR-85M & $9.91 E+01$ \\
\hline $\mathrm{KR}-87$ & $4.91 \mathrm{E}+02$ \\
\hline KR -88 & $3.12 E+02$ \\
\hline SR -89 & $1.28 \mathrm{E}-04$ \\
\hline SR-90 & $5.67 \mathrm{E}-06$ \\
\hline SR-91 & $1.60 \mathrm{E}-03$ \\
\hline NB-95 & $2.51 E-07$ \\
\hline MO-99 & $7.17 E-05$ \\
\hline AG-110M & $4.17 \mathrm{E}-05$ \\
\hline $\mathrm{I}-131$ & $2.60 \mathrm{E}-03$ \\
\hline$I-133$ & $1.88 \mathrm{E}-02$ \\
\hline$X E-133$ & $4.05 E+01$ \\
\hline CS -134 & $8.85 E-05$ \\
\hline$I-135$ & $5.47 E-03$ \\
\hline$X E-135$ & $4.65 E+02$ \\
\hline $\mathrm{XE}-135 \mathrm{M}$ & $7.91 \mathrm{E}+02$ \\
\hline CS -137 & $3.68 \mathrm{E}-04$ \\
\hline$X E-138$ & $3.28 E+03$ \\
\hline$B A-140$ & $4.52 E-04$ \\
\hline LA -140 & $1.29 \mathrm{E}-03$ \\
\hline UNIDENTIFIED & $3.47 E-05$ \\
\hline LIQUID EFFLUENTS & \\
\hline NUCLIDES RELEASED & ACTIVITY (CI) \\
\hline CR -51 & $3.67 \mathrm{E}-03$ \\
\hline MN - 54 & $5.77 \mathrm{E}-02$ \\
\hline $\mathrm{CO}-58$ & $1.53 E-03$ \\
\hline FE -59 & $1.09 \mathrm{E}-02$ \\
\hline $\mathrm{Co}-60$ & $5.97 E-02$ \\
\hline $\mathrm{ZN}-65$ & $2.88 \mathrm{E}-03$ \\
\hline SR-89 & $2.88 \mathrm{E}-04$ \\
\hline SR -90 & $3.64 \mathrm{E}-04$ \\
\hline SR -92 & $1.25 \mathrm{E}-05$ \\
\hline MO-99 & $6.85 E-05$ \\
\hline
\end{tabular}


EFFLUENT AND WASTE DISPOSAL ANNUAL REPORT FOR YEAR 1989 AIRBORNE AND LIQUID EFFLUENTS

\section{LIQUID EFFLUENTS}

NUCLIDES RELEASED

AG-110M

SN -113

$\mathrm{SB}-124$

CS -134

CS -137

UNIDENTIFIED

$$
\begin{aligned}
& \text { ACTIVITY (CI) } \\
& 9.81 \mathrm{E}-04 \\
& 1.18 \mathrm{E}-04 \\
& 2.12 \mathrm{E}-04 \\
& 3.14 \mathrm{E}-03 \\
& 2.45 \mathrm{E}-02 \\
& 6.59 \mathrm{E}-02
\end{aligned}
$$

TOTAL AIRBORNE TRITIUM RELEASE 5.21E+00

TOTAL LIQUID TRITIUM RELEASE $6.39 \mathrm{E}-01$

VOLUME OF LIQUID WASTE RELEASED (PRIOR TO DILUTION) LITERS $8.44 E+05$

VOLUME OF DILUTION WATER USED DURING PERIOD 
EFFLUENT AND WASTE DISPOSAL ANNUAL REPORT FOR YEAR 1989 SOLID EFFLUENTS

SOLID WASTE DISPOSITION

NUMBER OF SHIPMENTS MODE OF TRANSPORTATION 12

$N / R$

TYPE OF WASTE

A. SPENT RESINS, FILTER SLUDGES, EVAPORATOR BOTTOMS, ETC.

B. DRY COMPRESSIBLE WASTE, CONTAMINATED EQUIPMENT, ETC.

C. IRRADIATED COMPONENTS, CONTROL RODS, ETC.

D. OTHER
DESTINATION

BARNWELL SC

UNIT YEAR TOTAL

M3 $2.83 \mathrm{E}+01$

CI $\quad 2.69 \mathrm{E}+02$

M3 $3.74 E+01$

CI $\quad 2.58 \mathrm{E}+00$

M3 $0.00 \mathrm{E}+00$

CI $\quad 0.00 E+00$

M3 $\quad 7.84 \mathrm{E}+00$

CI $\quad 9.99 \mathrm{E}+01$ 
EFFLUENT AND WASTE DISPOSAL ANNUAL REPORT FOR YEAR 1989 AIRBORNE AND LIQUID EFFLUENTS

LIQUID EFFLUENTS
NUCLIDES RELEASED
SB -124
SB -125
$I-131$
$I-132$
$I-133$
$X E-133$
$X E-133 M$
$C S-134$
$I-134$
$X E-135$
$C S-136$
$C S-137$
$C S-138$
$B A / L A-140$
$C E-144$
ACTIVITY (CI)
$1.76 \mathrm{E}-02$
$1.12 \mathrm{E}-02$
9.14E-03
$3.00 \mathrm{E}-02$
3. $48 \mathrm{E}-05$
$2.21 E-01$
$4.86 \mathrm{E}-04$
$6.19 \mathrm{E}-02$
$2.72 \mathrm{E}-02$
$4.29 E-03$
$4.73 E-05$
$7.68 \mathrm{E}-02$
$1.29 \mathrm{E}-04$
$1.31 \mathrm{E}-03$
$3.44 E-04$

TOTAL AIRBORNE TRITIUM RELEASE $8.72 \mathrm{E}+00$

TOTAL LIQUID TRITIUM RELEASE $5.58 \mathrm{E}+02$

$\begin{array}{llll}\text { VOLUME OF LIQUID WASTE RELEASED (PRIOR TO DILUTION) } & \text { LITERS } & 6.00 E+07 \\ \text { VOLUME OF DIIUTION WATER USED DURING PERIOD } & \text { LITERS } & 2.68 E+10\end{array}$ 
EFFLUENT AND WASTE DISPOSAL ANNUAL REPORT FOR YEAR 1989 AIRBORNE AND LIQUID EFFLUENTS

\begin{tabular}{|c|c|}
\hline \multicolumn{2}{|r|}{ LICENSEE: COMMONWEALTH EDISON } \\
\hline \multicolumn{2}{|r|}{ LICENSED POWER (MWT) $: 3.41 E+03$} \\
\hline \multicolumn{2}{|r|}{ NET ELECTRIC POWER (MWH) :7.14E+06 } \\
\hline \multicolumn{2}{|c|}{ COMMERCIAL OPERATION: $10 / 17 / 88$ INITIAL CRITICALITY:03/08/88 } \\
\hline \multicolumn{2}{|c|}{ COOLING WATER SOURCE: KANKAKEE RIVER } \\
\hline \multicolumn{2}{|l|}{ AIRBORNE EFFLUENTS } \\
\hline NUCLIDES RELEASED & ACTIVITY (CI) \\
\hline$A R-41$ & $5.67 \mathrm{E}-01$ \\
\hline $\mathrm{MN}-54$ & $1.60 \mathrm{E}-06$ \\
\hline $\mathrm{CO}-58$ & $5.07 E-05$ \\
\hline $\mathrm{CO}-60$ & $1.42 E-05$ \\
\hline $\mathrm{KR}-85$ & $1.48 \mathrm{E}-01$ \\
\hline $\mathrm{KR}-85 \mathrm{M}$ & $1.71 \mathrm{E}-01$ \\
\hline $\mathrm{KR}-87$ & $2.17 \mathrm{E}-04$ \\
\hline $\mathrm{KR}-88$ & $5.40 \mathrm{E}-04$ \\
\hline $\mathrm{RU}-103$ & $3.71 E-08$ \\
\hline$I-131$ & $2.19 E-04$ \\
\hline$X E-131 M$ & $1.06 \mathrm{E}+00$ \\
\hline$I-133$ & $7.46 \mathrm{E}-05$ \\
\hline $\mathrm{XE}-133$ & $4.52 E+02$ \\
\hline $\mathrm{XE}-133 \mathrm{M}$ & $3.75 E-01$ \\
\hline$I-134$ & $2.81 E-06$ \\
\hline$I-135$ & $2.74 E-03$ \\
\hline$X E-135$ & $5.33 E+01$ \\
\hline CS -137 & $9.14 E-07$ \\
\hline $\mathrm{BA} / \mathrm{LA}-140$ & $1.97 E-07$ \\
\hline \multicolumn{2}{|l|}{ LIQUID EFFLUENTS } \\
\hline NUCLIDES RELEASED & ACTIVITY (CI) \\
\hline$B E-7$ & $1.75 \mathrm{E}-05$ \\
\hline NA -24 & $1.13 E-03$ \\
\hline$S C-46$ & $2.41 E-05$ \\
\hline CR -51 & $6.90 E-02$ \\
\hline$M N-54$ & $5.43 E-02$ \\
\hline Co-57 & $4.49 E-03$ \\
\hline $\mathrm{Co}-58$ & $1.85 E+00$ \\
\hline FE -59 & $3.52 \mathrm{E}-02$ \\
\hline $\mathrm{CO}-60$ & $1.61 E-01$ \\
\hline$N I-65$ & $2.51 E-03$ \\
\hline $\mathrm{ZN}-65$ & $2.52 E-03$ \\
\hline AS -76 & $1.36 \mathbb{E}-05$ \\
\hline $\mathrm{BR}-82$ & $1.39 \mathrm{E}-02$ \\
\hline $\mathrm{KR}-88$ & $8.93 E-03$ \\
\hline SR-91 & $4.59 E-06$ \\
\hline SR-92 & $5.75 \mathrm{E}-06$ \\
\hline NB- 95 & $2.54 E-02$ \\
\hline $2 R-95$ & $1.31 \mathrm{E}-02$ \\
\hline $2 R-97$ & $1.96 \mathrm{E}-02$ \\
\hline MO-99 & $5.10 E-04$ \\
\hline RU-103 & $1.05 E-04$ \\
\hline
\end{tabular}


EFFLUENT AND WASTE DISPOSAL ANNUAL REPORT FOR YEAR 1989 AIRBORNE AND LIQUID EFFLUENTS

LIQUID EFFLUENTS

NUCLIDES RELEASED

RU -105

AG-110M

SN -113

$\mathrm{SB}-122$

$\mathrm{SB}-124$

$\mathrm{SB}-125$

I - 131

I -132

$I-133$

$\mathrm{XE}-133$

$\mathrm{XE}-133 \mathrm{M}$

CS -134

I -134

XE-135

CS -136

CS -137

CS -138

BA/LA- 140

CE-144

\section{ACTIVITY (CI)}

1.39E-05

$1.97 \mathrm{E}-03$

$2.22 \mathrm{E}-03$

4.08E-06

1. $76 \mathrm{E}-02$

3. $81 \mathrm{E}-02$

$9.14 \mathrm{E}-03$

3. $00 \mathrm{E}-02$

3. $48 \mathrm{E}-05$

$2.21 E-01$

$4.86 E-04$

$6.19 \mathrm{E}-02$

2. $72 \mathrm{E}-02$

$4.29 \mathrm{E}-03$

4.73E-05

$7.68 \mathrm{E}-02$

1. $29 \mathrm{E}-04$

1. $31 \mathrm{E}-03$

3. $44 \mathrm{E}-04$

TOTAL AIRBORNE TRITIUM RELEASE 3.90E +00

TOTAL LIQUID TRITIUM RELEASE $5.58 E+02$

VOLUME OF LIQUID WASTE RELEASED (PRIOR TO DILUTION) IITERS $6.00 E+07$

VOLUME OF DILUTION WATER USED DURING PERIOD

LITERS $\quad 2.68 \mathrm{E}+10$ 
SOLID WASTE DISPOSITION NUMBER OF SHIPMENTS 3

4

MODE OF TRANSPORTATION EXCLUSIVE USE VEHICLE EXCLUSIVE USE VEHICLE EXCLUSIVE USE VEHICLE

ESTIMATE OF MAJOR NUCLIDE COMPOSITION (BY TYPE OF WASTE)
DESTINATION

CHEM-NUCLEAR BARNWELL SC SEG OAK RIDGE TN US ECOLOGY RICHLAND WA
A
$\mathrm{BE}-7$
$\mathrm{CO}-57$
Co- 58
CO- 60
CR -51
CS -134
CS -137
FE -55
$\mathrm{FE}-59$
$\mathrm{H}-3$
$\mathrm{MN}-54$
NB -95
NI -63
SB- 125
$\mathrm{SN}-113$
ZE -59
$\mathrm{ZN}-65$
ZR-95
B
C- 14
CO- 57
$\mathrm{CO}-58$
CO- 60
CR -51
CS -134
CS -137
FE -55
FE - 59
MN -54
NB-95
NI -63
PU -238
PU- 239
PU -241
SR -90

$\begin{array}{lll}8 & 7.63 \mathrm{E}+00 & 2.20 \mathrm{E}-01 \\ 8 & & 2.60 \mathrm{E}-01 \\ 8 & 3.43 \mathrm{E}+01 & 5.24 \mathrm{E}+01 \\ 8 & 3.17 \mathrm{E}+00 & 1.60 \mathrm{E}+01 \\ 8 & 2.27 \mathrm{E}+00 & \\ 8 & & 4.30 \mathrm{E}-01 \\ 8 & & 1.14 \mathrm{E}+00 \\ 8 & 3.26 \mathrm{E}+01 & 9.92 \mathrm{E}+00 \\ 8 & 1.76 \mathrm{E}+00 & \\ 8 & 1.22 \mathrm{E}+00 & \\ 8 & 4.08 \mathrm{E}+00 & 9.36 \mathrm{E}+00 \\ 8 & 1.11 \mathrm{E}+00 & 6.50 \mathrm{E}-01 \\ 8 & 1.62 \mathrm{E}+00 & 8.14 \mathrm{E}+00 \\ 8 & & 5.50 \mathrm{E}-01 \\ 8 & 7.97 \mathrm{E}+00 & 9.00 \mathrm{E}-02 \\ 8 & & 1.60 \mathrm{E}-01 \\ 8 & & 2.15 \mathrm{E}-01 \\ 8 & & 3.20 \mathrm{E}-01 \\ 8 & & \\ 8 & 1.37 \mathrm{E}+00 & 1.90 \mathrm{E}-02 \\ 8 & & 8.80 \mathrm{E}-02 \\ 8 & 4.51 \mathrm{E}+01 & 3.95 \mathrm{E}+01 \\ 8 & 2.00 \mathrm{E}+01 & 4.13 \mathrm{E}+00 \\ 8 & 1.20 \mathrm{E}-01 & \\ 8 & & 2.50 \mathrm{E}-01 \\ 8 & & 4.93 \mathrm{E}-01 \\ 8 & & 3.42 \mathrm{E}+01 \\ 8 & 9.35 \mathrm{E}+00 & 7.55 \mathrm{E}-01 \\ 8 & 1.74 \mathrm{E}+00 & 1.52 \mathrm{E}+01 \\ 8 & 1.54 \mathrm{E}+01 & 9.62 \mathrm{E}-01 \\ 8 & 1.36 \mathrm{E}+00 & 4.26 \mathrm{E}+00 \\ 8 & 2.55 \mathrm{E}+00 & 4.51 \mathrm{E}-03 \\ 8 & & 5.38 \mathrm{E}-03 \\ 8 & & 1.59 \mathrm{E}-01 \\ 8 & & 1.30 \mathrm{E}-02\end{array}$


EFFLUENT AND WASTE DISPOSAL ANNUAL REPORT FOR YEAR 1989 SOLID EFFLUENTS

TYPE OF WASTE

A. SPENT RESINS, FILTER SLUDGES, EVAPORATOR BOTTOMS, ETC.

B. DRY COMPRESSIBLE WASTE, CONTAMINATED EQUIPMENT, ETC.

C. IRRADIATED COMPONENTS, CONTROL RODS, ETC.

D. OTHER
UNIT YEAR TOTAL

M3 6.31E+01*

CI $3.85 \mathrm{E}+02 *$

M3 $2.47 \mathrm{E}+02$

CI $\quad 4.57 E+00$

M3 $0.00 \mathrm{E}+00$

CI $\quad 0.00 E+00$

M3

CI

*TYPE A. WASTE INCLUDES SMOKE DETECTORS (1.38E-01 M3, 7.58E-05 CI.) 
EFFLUENT AND WASTE DISPOSAL ANNUAL REPORT FOR YEAR 1989 AIRBORNE AND LIQUID EFFLUENTS

\begin{tabular}{|c|c|}
\hline UNIT NUMBER: 1 & LICENSEE:TENNESSEE VALLEY AUTHORITY \\
\hline DOCKET NO.: $50-259$ & LICENSED POWER (MWT) $: 3.29 E+03$ \\
\hline THERMAL POWER (MWH) :0.00E+00 & NET ELECTRIC POWER (MWH):0.00E+00 \\
\hline COMMERCIAL OPERATION: $08 / 01 / 74$ & INITIAL CRITICALITY:08/17/73 \\
\hline COOLING WATER SOURCE:TENNESSEE & IVER \\
\hline UNIT NUMBER: 2 TYPE:BWR & LICENSEE:TENNESSEE VALLEY AUTHORITY \\
\hline DOCKET NO.: $50-260$ & LICENSED POWER (MWT) $: 3.29 E+03$ \\
\hline THERMAL POWER (MWH) :0.00E +00 & NET ELECTRIC POWER (MWH) :0.00E+00 \\
\hline COMMERCIAL OPERATION:03/01/75 & INITIAL CRITICALITY:07/20/74 \\
\hline COOLING WATER SOURCE:TENNESSEE & IVER \\
\hline UNIT NUMBER: 3 TYPE:BWR & LICENSEE:TENNESSEE VALLEY AUTHORITY \\
\hline DOCKET NO. : 50-296 & LICENSED POWER (MWT) $: 3.29 \mathrm{E}+03$ \\
\hline THERMAL POWER (MWH) :0.00E+00 & NET ELECTRIC POWER (MWH):0.00E+00 \\
\hline COMMERCIAL OPERATION:03/01/77 & INITIAI CRITICALITY:08/08/76 \\
\hline COOLING WATER SOURCE:TENNESSEE & IVER \\
\hline AIRBORNE EFFLUENTS & \\
\hline NUCLIDES RELEASED & $\operatorname{VITY}(C I)$ \\
\hline$C 0-60$ & $4 E-04$ \\
\hline $\operatorname{CS}-137$ & $3 E-05$ \\
\hline LIQUID EFFLUENTS & \\
\hline NUCLIDES RELEASED & $\operatorname{VITY}(\mathrm{CI})$ \\
\hline $\mathrm{MN}-54$ & $2 E-05$ \\
\hline $\mathrm{CO}-57$ & $0 \mathrm{E}-06$ \\
\hline$c 0-60$ & $1 E-02$ \\
\hline $2 N-65$ & $9 E-03$ \\
\hline$S B-125$ & $1 E-04$ \\
\hline $\operatorname{CS}-134$ & $5 E-02$ \\
\hline CS-137 & $3 E-01$ \\
\hline$C E-144$ & $7 E-05$ \\
\hline
\end{tabular}

TOTAL AIRBORNE TRITIUM RELEASE 2.00E-01

TOTAL LIQUID TRITIUM RELEASE 7.01E-01

VOLUME OF LIQUID WASTE RELEASED (PRIOR TO DILUTION) LITERS 4.40E+07

VOLUME OF DILUTION WATER USED DURING PERIOD $\quad$ LITERS $1.64 E+11$ 
EFFLUENT AND WASTE DISPOSAL ANNUAL REPORT FOR YEAR 1989 SOLID EFFLUENTS

SOLID WASTE DISPOSITION NUMBER OF SHIPMENTS 43

MODE OF TRANSPORTATION

DESTINATION SOLE USE TRUCK

SOLE USE TRUCK

BARNWELL SC

RICHLAND WA

IRRADIATED FUEL SHIPMENTS (DISPOSITION)

NUMBER OF SHIPMENTS MODE OF TRANSPORTATION 0

N/A

DESTINATION

N/A

JAN-JUNE JULY-DEC

A

Co -60

CS -134

CS -137

FE -55

$\mathrm{MN}-54$

OTHERS

ZN -65

MAJOR NUCLIDE COMPOSITION (BY TYPE OF WASTE)

B

AG-110M

CO- 60

CR -51

CS -134

CS -137

FE -55

FE -59

MN -54

NB- 95

OTHERS

$\mathrm{ZN}-65$

D

AG-110M

CO -60

CR -51

CS -134

CS -137

FE -55

FE -59

MN -54

NB -95

OTHERS

ZN -65

$\begin{array}{lll}8 & 4.53 E+01 & 3.51 E+01 \\ 8 & 4.00 E+00 & 6.77 E+00 \\ 8 & 1.77 E+01 & 2.10 E+01 \\ 8 & 2.77 E+01 & 3.19 E+01 \\ 8 & 4.10 E-02 & 1.70 E-01 \\ 8 & 2.80 E+00 & 2.88 E+00 \\ 8 & 2.30 E+00 & 2.18 E+00 \\ & & \\ 8 & 1.30 E+00 & 8.65 E-01 \\ 8 & 1.78 E+01 & 2.81 E+01 \\ 8 & 1.15 E+01 & 7.79 E+00 \\ 8 & 6.80 E+00 & 5.05 E+00 \\ 8 & 6.80 E+00 & 6.72 E+00 \\ 8 & 2.45 E+01 & 3.15 E+01 \\ 8 & 1.30 E+00 & 8.24 E-01 \\ 8 & 4.20 E+00 & 2.93 E+00 \\ 8 & 2.20 E+00 & 1.42 E+00 \\ 8 & 5.20 E+00 & 1.58 E+00 \\ 8 & 1.84 E+01 & 1.33 E+01 \\ & & \\ 8 & 1.33 E+00 & 1.33 E+00 \\ 8 & 1.88 E+01 & 1.88 E+01 \\ 8 & 1.21 E+01 & 1.21 E+01 \\ 8 & 7.20 E+00 & 7.20 E+00 \\ 8 & 7.20 E+00 & 7.20 E+00 \\ 8 & 2.57 E+01 & 2.57 E+01 \\ 8 & 1.34 E+00 & 1.34 E+00 \\ 8 & 4.43 E+00 & 4.43 E+00 \\ 8 & 2.31 E+00 & 2.31 E+00 \\ 8 & 2.21 E-01 & 2.21 E-01 \\ 8 & 1.94 E+01 & 1.94 E+01\end{array}$


EFFLUENT AND WASTE DISPOSAL ANNUAL REPORT FOR YEAR 1989 SOLID EFFLUENTS

TYPE OF WASTE

A. SPENT RESINS, FILTER SLUDGES, EVAPORATOR BOTTOMS, ETC.

B. DRY COMPRESSIBLE WASTE, CONTAMINATED EQUIPMENT, ETC.

C. IRRADIATED COMPONENTS, CONTROL RODS, ETC.

D. OTHER ABSORBED OIL MATERIAL
UNIT YEAR TOTAL

M3 $1.82 \mathrm{E}+02$

CI $\quad 2.61 \mathrm{E}+02$

M3 $3.39 E+02$

CI $\quad 3.04 E+01$

M3 $0.00 E+00$

CI $\quad 0.00 E+00$

M3 6.30E+01

CI $3.15 \mathrm{E}+00$ 
EFFLUENT AND WASTE DISPOSAL ANNUAL REPORT FOR YEAR 1989 AIRBORNE AND LIQUID EFFLUENTS
UNIT NUMBER: 1 TYPE:BWR
LICENSEE: CAROLINA POWER \&LIGHT
DOCKET NO. : $50-325$
LICENSED POWER (MWT) $: 2.44 \mathrm{E}+03$
THERMAL POWER (MWH) : $1.32 \mathrm{E}+07$
COMMERCIAL OPERATION:03/18/77
COOLING WATER SOURCE: CAPE FEAR RIVER
NET ELECTRIC POWER (MWH):4.18E+06
INITIAL CRITICALITY: 10/08/76
UNIT NUMBER: 2 TYPE:BWR
DOCKET NO. : 50-324
LICENSEE: CAROLINA POWER \&LIGHT
THERMAL POWER (MWH) : $1.34 E+07$
COMMERCIAL OPERATION:11/03/75
COOLING WATER SOURCE:CAPE FEAR RIVER
LICENSED POWER (MWT) $: 2.44 E+03$
NET ELECTRIC POWER (MWH) :4.19E+06
INITIAL CRITICALITY:03/20/75
AIRBORNE EFFLUENTS
NUCLIDES RELEASED
AR -41
CR -51
MN -54
$\mathrm{CO}-57$
$\mathrm{CO}-58$
FE -59
CO- 60
ACTIVITY (CI)
$\mathrm{KR}-85 \mathrm{M}$
$5.18 \mathrm{E}+00$
2. $44 E-02$
2. 11E-03
2.99E-06
$5.89 E-04$
9. $64 \mathrm{E}-05$
KR -87
3. $46 \mathrm{E}-03$
$6.78 \mathrm{E}+01$
KR -88
$7.66 \mathrm{E}+00$
SR -89
5. $56 \mathrm{E}+01$
SR -90
9. $64 E-04$
NB -95
RU-103
I-131
I -132
I- 133
$\mathrm{XE}-133$
CS -134
$7.00 \mathrm{E}-06$
I- 134
I-135
$\mathrm{XE}-135$
$8.45 \mathrm{E}-06$
$6.27 \mathrm{E}-06$
1. 51E-02
4. 29E-02
$7.78 \mathrm{E}-02$
$3.44 E+02$
4. $23 E-05$
$3.92 E-03$
$8.70 \mathrm{E}-02$
$\mathrm{XE}-135 \mathrm{M}$
2. $60 \mathrm{E}+02$
CS -137
1. $10 \mathrm{E}+02$
$\mathrm{XE}-137$
1. $36 \mathrm{E}-04$
$\mathrm{XE}-138$
3. $79 \mathrm{E}+02$
1. $29 E+02$
BA -140
1. $43 E-03$
LA -140
$1.02 \mathrm{E}-03$
CE- 141
1. $67 \mathrm{E}-06$
CE -144
$2.16 \mathrm{E}-05$
HF- 181
1. $10 \mathrm{E}-05$
LIQUID EFFLUENTS
NUCLIDES RELEASED
ACTIVITY (CI)
NA -24
1. $17 \mathrm{E}-02$
AR -41
4. $21 \mathrm{E}-05$
CR -51
$8.82 E-01$
MN -54
$1.40 \mathrm{E}-01$ 
EFFLUENT AND WASTE DISPOSAL ANNUAL REPORT FOR YEAR 1989 AIRBORNE AND LIQUID EFFLUENTS

\section{LIQUID EFFLUENTS}

NUCLIDES RELEASED

FE -55

MN -56

$\mathrm{CO}-58$

$\mathrm{FE}-59$

CO- 60

$\mathrm{ZN}-65$

AS -76

KR $-85 \mathrm{M}$

SR -89

SR -90

$\mathrm{Y}-91$

Y-91M

SR-92

$\mathrm{Y}-92$

NB-95

NB -97

MO-99

TC - $99 \mathrm{M}$

TC -104

RU-105

AG-110M

TE-129M

I -131

I -132

TE-132

I -133

XEE -133

$\mathrm{XE}-133 \mathrm{M}$

CS -134

I -134

I -135

$\mathrm{XE}-135$

$\mathrm{XE}-135 \mathrm{M}$

CS -136

CS -137

CS -138

LA -140

CE -141

LA- 142

CE -144

$\mathrm{HF}-181$

W- 187

NP -239

\section{ACTIVITY (CI)}

7. 22E-03

$1.17 \mathrm{E}-03$

4.14E-02

3.07E-03

2. $28 \mathrm{E}-01$

4. $87 \mathrm{E}-04$

1. $95 E-03$

8. $46 \mathrm{E}-06$

$9.97 \mathrm{E}-04$

3.12E- 04

$9.61 \mathrm{E}-04$

$7.32 E-04$

$9.79 E-05$

1. $00 \mathrm{E}-02$

4. 16E-05

8.63E-04

1. $06 \mathrm{E}-04$

4. $94 E-02$

1. $67 \mathrm{E}-04$

$6.89 E-04$

1. 13E-04

$6.56 \mathrm{E}-05$

$7.89 \mathrm{E}-03$

$7.86 \mathrm{E}-05$

1. $88 \mathrm{E}-04$

$5.15 \mathrm{E}-03$

4. 28E-02

1. $73 \mathrm{E}-04$

$7.98 \mathrm{E}-02$

4. 04E-05

$1.62 \mathrm{E}-03$

$2.02 E-01$

$1.70 \mathrm{E}-03$

$8.05 E-04$

$7.68 \mathrm{E}-02$

4. 50E-05

$4.35 E-04$

8.47E-06

1. $24 E-04$

2. $39 E-04$

1. $85 \mathrm{E}-05$

2. 18E-03

1. $55 \mathrm{E}-03$ 
EFFLUENT AND WASTE DISPOSAL ANNUAL REPORT FOR YEAR 1989 AIRBORNE AND LIQUID EFFLUENTS

TOTAL AIRBORNE TRITIUM RELEASE 9.17E+00

TOTAL LIQUID TRITIUM RELEASE 1.79E+01

VOLUME OF LIQUID WASTE RELEASED (PRIOR TO DILUTION)

LITERS

$3.75 E+07$

VOLUME OF DILUTION WATER USED DURING PERIOD

LITERS

$1.12 \mathrm{E}+11$ 
INSTALLATION : BRUNSWICK

EFFLUENT AND WASTE DISPOSAL ANNUAL REPORT FOR YEAR 1989 SOLID EFFLUENTS

SOLID WASTE DISPOSITION NUMBER OF SHIPMENTS 71 13 2
MODE OF TRANSPORTATION SOLE USE SOLE USE SOLE USE
DESTINATION CNSI/BARNWELL SC SEG/OAK RIDGE TN US ECOLOGY/HANFORD WA

DESTINATION CP\&L/SHNPP (NON - BURIAL)

ESTIMATE OF MAJOR NUCLIDE COMPOSITION (BY TYPE OF WASTE)

\section{MODE OF TRANSPORTATION} SOLE USE/RAIL

A

$\mathrm{CO}-58$

CO- 60

CR -51

CS -134

CS -137

FE -55

$\mathrm{MN}-54$

B

NI -63

CO -60

CS -134

CS -137

FE -55

MN -54

NI -63

$\mathrm{C}$

CO -60

FE- 55

NI -63

$\mathrm{SB}-125$

D

CO -60

CS -137

FE -55

$\mathrm{H}-3$

TYPE OF WASTE

A. SPENT RESINS, FILTER SLUDGES, EVAPORATOR BOTTOMS, ETC.

B. DRY COMPRESSIBLE WASTE, CONTAMINATED EQUIPMENT, ETC.

C. IRRADIATED COMPONENTS, CONTROL RODS, ETC.

D. OTHER

OIL

JAN-JUNE JULY - DEC

$\begin{array}{lll}8 & 1.80 \mathrm{E}+00 & 1.77 \mathrm{E}+00 \\ 8 & 1.94 \mathrm{E}+01 & 1.54 \mathrm{E}+01 \\ 8 & 1.30 \mathrm{E}+01 & 1.09 \mathrm{E}+01 \\ 8 & 2.83 \mathrm{E}+00 & 2.64 \mathrm{E}+00 \\ 8 & 2.97 \mathrm{E}+00 & 2.65 \mathrm{E}+00 \\ 8 & 4.85 \mathrm{E}+01 & 5.83 \mathrm{E}+01 \\ 8 & 9.43 \mathrm{E}+00 & 7.39 \mathrm{E}+00 \\ 8 & 1.87 \mathrm{E}+00 & 1.02 \mathrm{E}+00 \\ & 1.94 \mathrm{E}+01 & 1.09 \mathrm{E}+01 \\ 8 & 1.05 \mathrm{E}+00 & \\ 8 & 1.27 \mathrm{E}+00 & 1.28 \mathrm{E}+00 \\ 8 & 1.93 \mathrm{E}+01 & 8.43 \mathrm{E}+01 \\ 8 & 6.991 \\ 8 & 6.55 \mathrm{E}+00 & 2.37 \mathrm{E}+00 \\ 8 & 9.70 \mathrm{E}-01 & \\ & & \\ 8 & 3.14 \mathrm{E}+01 & 2.99 \mathrm{E}+01 \\ 8 & 1.35 \mathrm{E}+01 & 1.43 \mathrm{E}+01 \\ 8 & 1.32 \mathrm{E}+00 & 1.34 \mathrm{E}+00 \\ 8 & 5.37 \mathrm{E}+01 & 5.43 \mathrm{E}+01 \\ 8 & & 3.03 \mathrm{E}+01 \\ 8 & & 1.87 \mathrm{E}+00 \\ 8 & & 6.56 \mathrm{E}+01 \\ 8 & & 9.40 \mathrm{E}-01\end{array}$

UNIT YEAR TOTAL

M3 2.20E+02

CI $\quad 3.57 \mathrm{E}+03$

M3 $3.60 \mathrm{E}+02 *$

CI $\quad 1.82 \mathrm{E}+01$

M3 $1.76 \mathrm{E}+01$

CI $2.47 \mathrm{E}+03$

M3 $2.17 \mathrm{E}+01$

CI $\quad 6.47 \mathrm{E}-03$

*VOLUMES REPORTED WERE AFTER COMPACTION 
EFFLUENT AND WASTE DISPOSAL ANNUAL REPORT FOR YEAR 1989 AIRBORNE AND LIQUID EFFLUENTS

\author{
UNIT NUMBER: 1 TYPE:PWR \\ DOCKET NO.: $50-454$ \\ THERMAL POWER (MWH) :2.77E+07 \\ COMMERCIAL OPERATION:09/16/85 \\ COOLING WATER SOURCE:ROCK RIVER \\ UNIT NUMBER: 2 TYPE:PWR \\ DOCKET NO. : $50-455$ \\ THERMAL POWER (MWH) $: 1.87 \mathrm{E}+07$ \\ COMMERCIAL OPERATION:08/21/87
}

COOLING WATER SOURCE:ROCK RIVER
LICENSEE: COMMONWEALTH EDISON CO. IICENSED POWER (MWT) $: 3.41 \mathrm{E}+03$ NET ELECTRIC POWER (MWH) $: 8.95 E+06$ INITIAL CRITICALITY:02/02/85

LICENSEE: COMMONWEALTH EDISON CO. LICENSED POWER (MWT) $: 3.41 E+03$ NET ELECTRIC POWER (MWH) $: 6.06 \mathrm{E}+06$ INITIAL CRITICALITY:01/09/87
AIRBORNE EFFLUENTS
NUCLIDES RELEASED
AR -41
CO- 60
$\mathrm{KR}-85$
$\mathrm{KR}-85 \mathrm{M}$
$\mathrm{KR}-87$
$\mathrm{KR}-88$
$I-131$
$\mathrm{XE}-131 \mathrm{M}$
ACTIVITY (CI)
$I-132$
$\mathrm{I}-133$
$\mathrm{XE}-133$
$X E-133 M$
$3.59 \mathrm{E}-01$
$3.40 \mathrm{E}-05$
1. $33 \mathrm{E}+01$
2. $51 \mathrm{E}-01$
1. $14 \mathrm{E}-03$
$4.18 \mathrm{E}-01$
$7.59 \mathrm{E}-04$
$7.26 \mathrm{E}+00$
1. $10 \mathrm{E}-04$
$1.44 \mathrm{E}-03$
$7.84 \mathrm{E}+02$
$5.08 \mathrm{E}+00$
I-135
$8.08 \mathrm{E}-04$
$\mathrm{XE}-135$
$5.97 E+00$
LIQUID EFFLUENTS
NUCLIDES RELEASED
ACTIVITY (CI)
AR -41
CR -51
$2.61 \mathrm{E}-05$
5. $79 \mathrm{E}-02$
MN -54
2. $61 \mathrm{E}-02$
$\mathrm{FE}-55$
$\mathrm{CO}-57$
$\mathrm{CO}-58$
FE- 59
Co- 60
$\mathrm{ZN}-65$
$\mathrm{BR}-82$
NB -95
ZR-95
MO-99
MO- $99 / \mathrm{TC}-99 \mathrm{M}$
$2.47 \mathrm{E}-06$
4. $78 \mathrm{E}-04$
$2.41 E-01$
$2.41 \mathrm{E}-02$
1.69E-01
$1.62 \mathrm{E}-03$
1. $61 \mathrm{E}-04$
$2.70 \mathrm{E}-02$
$1.45 \mathrm{E}-02$
$8.08 \mathrm{E}-05$
$\mathrm{RU}-103$
$A G-110 M$
$1.69 E-04$
$3.93 \mathrm{E}-05$
$1.08 \mathrm{E}-03$
IN-113M
$4.76 E-05$
$\mathrm{SN}-113$
SB-124
$1.07 E-03$
$8.56 E-04$
SB-125
1.23E-02
$\mathrm{I}-131$
$2.58 \mathrm{E}-03$

$N / A=N O T$ APPLICABLE

N/D-NOT DETECTED

$\mathrm{N} / \mathrm{R}=\mathrm{NOT}$ REPORTED 
EFFLUENT AND WASTE DISPOSAL ANNUAL REPORT FOR YEAR 1989 AIRBORNE AND LIQUID EFFLUENTS

LIQUID EFFLUENTS
NUCLIDES RELEASED
XE-131M
I-132
BA-133
$I-133$
XE-133
XE $-133 \mathrm{M}$
CS -134
XE -135
CS -137
BA $/ L A-140$
CE-144
HF-181

ACTIVITY (CI)
$6.19 \mathrm{E}-03$
$2.71 \mathrm{E}-03$
$4.01 \mathrm{E}-05$
$5.03 \mathrm{E}-05$
$1.36 \mathrm{E}+00$
$5.26 \mathrm{E}-03$
$2.13 \mathrm{E}-02$
$7.24 \mathrm{E}-04$
$2.83 \mathrm{E}-02$
$1.25 \mathrm{E}-03$
$1.29 \mathrm{E}-03$
$2.62 \mathrm{E}-04$

6.19E-03

2.71E-03

4.01E-05

$5.03 E-05$

$1.36 \mathrm{E}+00$

$5.26 \mathrm{E}-03$

$2.13 \mathrm{E}-02$

$7.24 \mathrm{E}-04$

$.83 \mathrm{E}-02$

$1.29 E-03$

$2.62 E-04$

TOTAL AIRBORNE TRITIUM RELEASE 1.84E+02

TOTAL LIQUID TRITIUM RELEASE 1.29E+03

VOLUME OF LIQUID WASTE RELEASED (PRIOR TO DILUTION) LITERS $3.17 E+07$ VOLUME OF DILUTION WATER USED DURING PERIOD 
SOLID WASTE DISPOSITION NUMBER OF SHIPMENTS 37

3

12

ESTIMATE OF MAJOR NUCLIDE COMPOSITION (BY TYPE OF WASTE)

A

AM -241

C. 14

$\mathrm{CM}-244$

$\mathrm{CO}-58$

CO -60

CS -134

CS -137

FE -55

$\mathrm{H}-3$

I- 129

$\mathrm{MN}-54$

NI -63

PU -238

PU-239

PU- 241

SR -90

TC- 99

B

C -14

CO -58

CO- 60

CS -134

CS -137

FE -55

I -129

MN -54

NI -63

$\mathrm{SR}-90$

TC-99
MODE OF TRANSPORTATION

EXCLUSIVE USE

EXCLUSIVE USE

EXCLUSIVE USE
DESTINATION
BARNWELL SC
RICHLAND WA
VENDOR FOR COMPACTION

JAN-JUNE JULY-DEC

$\begin{array}{lll}8 & 3.70 \mathrm{E}-02 & 5.00 \mathrm{E}-03 \\ 8 & 5.10 \mathrm{E}-01 & 5.80 \mathrm{E}-01 \\ 8 & 3.70 \mathrm{E}-02 & 1.30 \mathrm{E}-02 \\ 8 & 3.30 \mathrm{E}+01 & 4.87 \mathrm{E}+01 \\ 8 & 6.60 \mathrm{E}+00 & 9.91 \mathrm{E}+00 \\ 8 & 1.71 \mathrm{E}+01 & 9.67 \mathrm{E}+00 \\ 8 & 2.30 \mathrm{E}+01 & 9.51 \mathrm{E}+00 \\ 8 & 7.40 \mathrm{E}+00 & 1.14 \mathrm{E}+01 \\ 8 & 8.33 \mathrm{E}+00 & 1.10 \mathrm{E}-01 \\ 8 & 9.20 \mathrm{E}-02 & 4.00 \mathrm{E}-03 \\ 8 & 2.61 \mathrm{E}+00 & 8.20 \mathrm{E}+00 \\ 8 & 1.72 \mathrm{E}-01 \\ 8 & 1.27 \mathrm{E}+00 & 1.00 \mathrm{E}-03 \\ 8 & 9.00 \mathrm{E}-03 & 2.00 \mathrm{E} \\ 8 & 9.00 \mathrm{E}-03 & 4.00 \mathrm{E}-03 \\ 8 & 4.00 \mathrm{E}-04 & 1.00 \mathrm{E}-03 \\ 8 & 2.00 \mathrm{E}-03 & 4.00 \mathrm{E}-03 \\ 8 & 5.00 \mathrm{E}-02 & 1.74 \mathrm{E}+00 \\ 8 & & \\ 8 & 7.70 \mathrm{E}+00 & 4.30 \mathrm{E}+00 \\ 8 & 1.20 \mathrm{E}+01 & 1.33 \mathrm{E}+01 \\ 8 & & 1.55 \mathrm{E}+01 \\ 8 & & 1.30 \mathrm{E}+00 \\ 8 & 3.30 \mathrm{E}+00 & 1.40 \mathrm{E}+00 \\ 8 & 5.80 \mathrm{E}+00 & 6.06 \mathrm{E}+01 \\ 8 & 4.66 \mathrm{E}+01 & 1.00 \mathrm{E}-04 \\ 8 & & 1.50 \mathrm{E}+00 \\ 8 & 4.00 \mathrm{E}+00 & 2.06 \mathrm{E}+00 \\ 8 & 8.40 \mathrm{E}+00 & 3.80 \mathrm{E}-02 \\ 8 & & 5.00 \mathrm{E}-03 \\ 8 & & \end{array}$

TYPE OF WASTE

A. SPENT RESINS, FILTER SLUDGES, EVAPORATOR BOTTOMS, ETC.

B. DRY COMPRESSIBLE WASTE, CONTAMINATED EQUIPMENT, ETC.

C. IRRADIATED COMPONENTS, CONTROL RODS, ETC.

D. OTHER

VAN

$\begin{array}{lc}\text { UNIT } & \text { YEAR TOTAL } \\ \text { M3 } & 1.64 \mathrm{E}+02 \\ \text { CI } & 1.27 \mathrm{E}+03 \\ \text { M3 } & 1.83 \mathrm{E}+02 * \\ \text { CI } & 1.13 \mathrm{E}+01 \\ \text { M3 } & \\ \text { CI } & \\ \text { M3 } & 1.78 \mathrm{E}+01 \\ \text { CI } & 8.60 \mathrm{E}-01\end{array}$

$N / A=N O T$ APPLICABLE

$N / D=N O T$ DETECTED

N/R-NOT REPORTED 
EFFLUENT AND WASTE DISPOSAL ANNUAL REPORT FOR YEAR 1989 AIRBORNE AND LIQUID EFFLUENTS

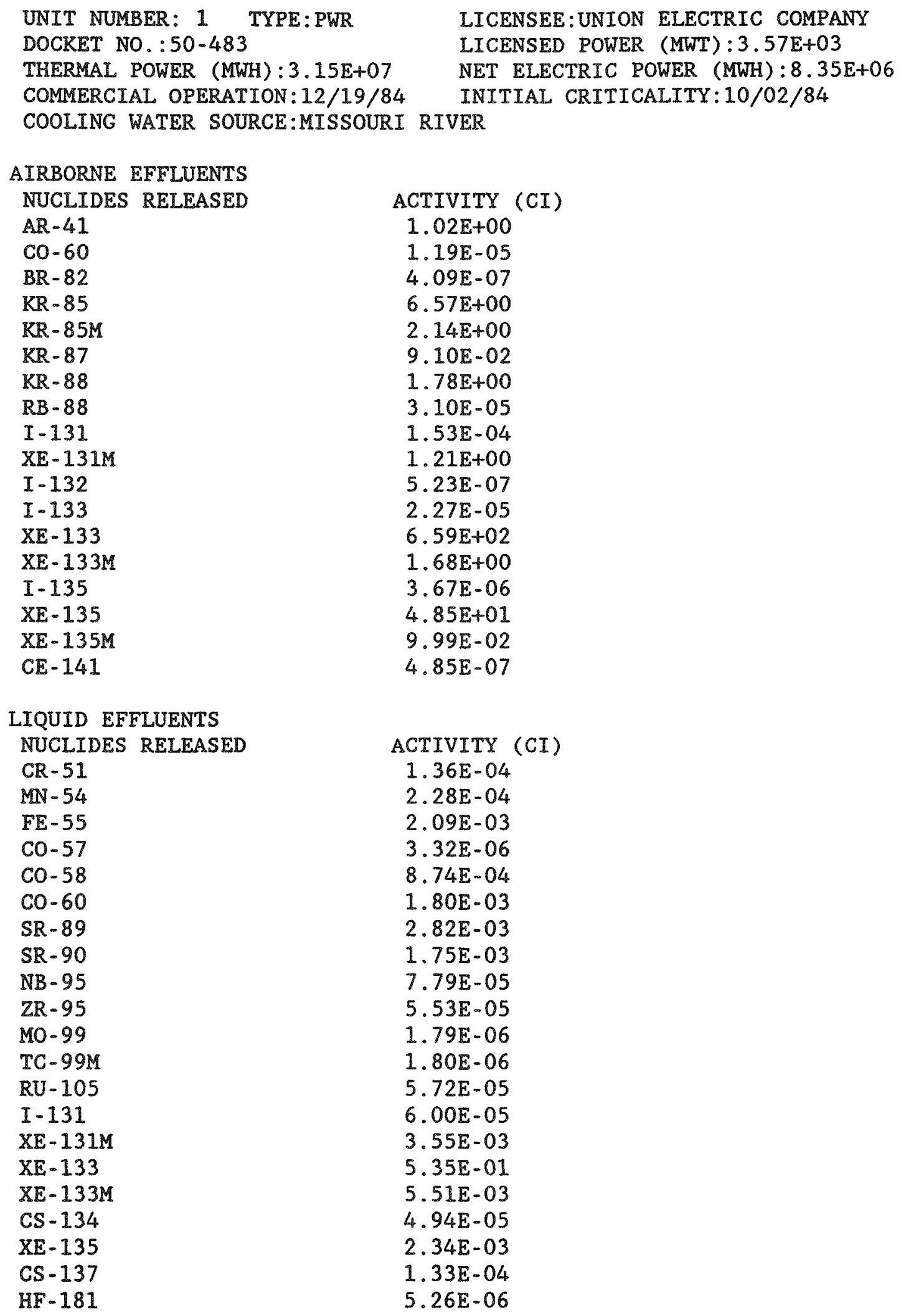


EFFLUENT AND WASTE DISPOSAL ANNUAL REPORT FOR YEAR 1989 AIRBORNE AND LIQUID EFFLUENTS

TOTAL AIRBORNE TRITIUM RELEASE $3.89 \mathrm{E}+01$

TOTAL LIQUID TRITIUM RELEASE

$6.10 \mathrm{E}+02$

VOLUME OF LIQUID WASTE RELEASED (PRIOR TO DILUTION) LITERS

$8.69 \mathrm{E}+07$

VOLUME OF DILUTION WATER USED DURING PERIOD

LITERS

$3.04 \mathrm{E}+09$ 
EFFLUENT AND WASTE DISPOSAL ANNUAL REPORT FOR YEAR 1989 SOLID EFFLUENTS

SOLID WASTE DISPOSITION

NUMBER OF SHIPMENTS MODE OF TRANSPORTATION

$\begin{aligned} 5 & \text { CASK } \\ 1 & \text { TRUCK } \\ 4 & \text { TRUCK } \\ 2 & \text { TRUCK } \\ 10 & \text { TRUCK }\end{aligned}$

IRRADIATED FUEL SHIPMENTS (DISPOSITION)

NUMBER OF SHIPMENTS MODE OF TRANSPORTATION 0 N/A

ESTIMATE OF MAJOR NUCLIDE COMPOSITION (BY TYPE OF WASTE)

A $\mathrm{CO}-58$

$\mathrm{CO}-60$

CS -134

CS -137

FE -55

$\mathrm{H}-3$

MN -54

NB-95

NI -63

B

C -14

CO- 58

CO- 60

CR -51

FE -55

MN -54

$\mathrm{NB}-95$

NI -63

ZR-95
TYPE OF WASTE

A. SPENT RESINS, FILTER SLUDGES, EVAPORATOR BOTTOMS, ETC.

B. DRY COMPRESSIBLE WASTE, CONTAMINATED EQUIPMENT, ETC.

C. IRRADIATED COMPONENTS, CONTROL RODS, ETC.

D. OTHER

\author{
DESTINATION \\ BARNWELL SC \\ BARNWELL SC \\ OAK RIDGE TN (QUADREX) \\ OAK RIDGE TN (SEG) \\ RICHLAND WA
}

\section{DESTINATION}

N/A

JAN-JUNE JULY-DEC

$\begin{array}{lll}8 & & 5.30 \mathrm{E}+00 \\ 8 & 2.69 \mathrm{E}+01 & 2.77 \mathrm{E}+01 \\ 8 & 8.70 \mathrm{E}+00 & 1.80 \mathrm{E}+00 \\ 8 & 2.05 \mathrm{E}+01 & 4.00 \mathrm{E}+00 \\ 8 & 2.63 \mathrm{E}+01 & 4.06 \mathrm{E}+01 \\ 8 & & 1.90 \mathrm{E}+00 \\ 8 & 3.23 \mathrm{E}+00 & 1.30 \mathrm{E}+00 \\ 8 & & 1.00 \mathrm{E}+00 \\ 8 & & 1.44 \mathrm{E}+01 \\ 8 & 1.30 \mathrm{E}+01 & \\ & & 2.60 \mathrm{E}+00 \\ 8 & & 1.93 \mathrm{E}+01 \\ 8 & 2.72 \mathrm{E}+01 & 1.43 \mathrm{E}+01 \\ 8 & 1.20 \mathrm{E}+01 & 1.50 \mathrm{E}+00 \\ 8 & 2.90 \mathrm{E}+00 & 5.02 \mathrm{E}+01 \\ 8 & 4.55 \mathrm{E}+01 & 5.70 \mathrm{E}+00 \\ 8 & 3.60 \mathrm{E}+00 & 3.00 \\ 8 & 3.20 \mathrm{E}+00 & 1.80 \mathrm{E}+00 \\ 8 & 3.20 \mathrm{E}+00 & 4.70 \mathrm{E}+00 \\ 8 & 2.30 \mathrm{E}+00 & 1.50 \mathrm{E}+00\end{array}$

UNIT YEAR TOTAL
M3
$1.06 \mathrm{E}+02$
CI $\quad 5.94 E+02$
M3 $1.03 \mathrm{E}+02 *$
CI $\quad 6.40 \mathrm{E}+00$
M3
CI
M3
CI

*VOLUME LISTED IS AFTER REDUCTION. 
EFFLUENT AND WASTE DISPOSAL ANNUAL REPORT FOR YEAR 1989 AIRBORNE AND LIQUID EFFLUENTS

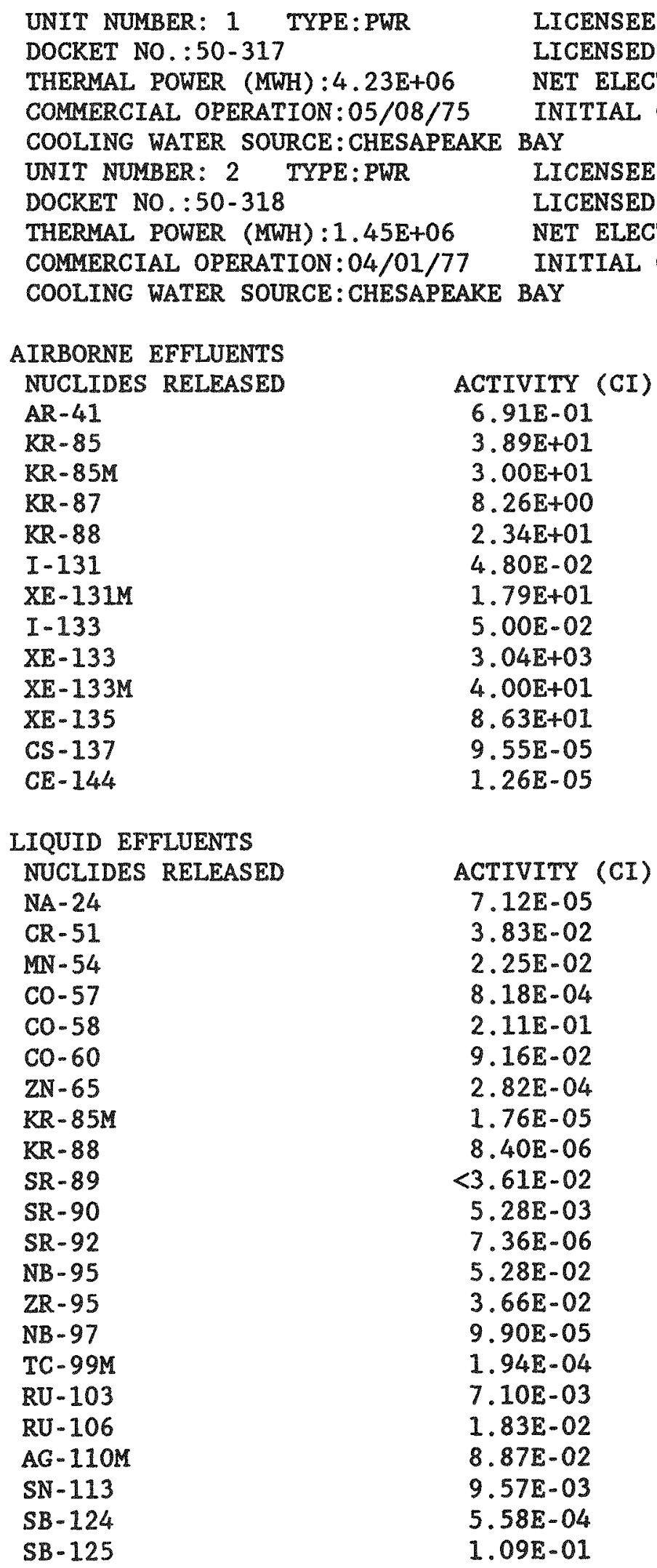

N/A-NOT APPLICABLE $N / D=N O T$ DETECTED 
EFFLUENT AND WASTE DISPOSAL ANNUAL REPORT FOR YEAR 1989 AIRBORNE AND LIQUID EFFLUENTS

LIQUID EFFLUENTS
NUCLIDES RELEASED
I-131
XE-131M
I-133
XE-133
XE $-133 \mathrm{M}$
CS -134
$X E-135$
CS -136
CS -137
CE -139
BA-140
LA -140
CE -141
CE -144

LIQUID EFFLUENTS

NUCLIDES RELEASED

$I-131$

$\mathrm{XE}-131 \mathrm{M}$

I -133

$\mathrm{XE}-133$

$\mathrm{XE}-133 \mathrm{M}$

CS -134

CE-144
ACTIVITY (CI)
$2.92 \mathrm{E}-02$
9.92E-05
2.01E-03
2. $94 \mathrm{E}-01$
$2.00 \mathrm{E}-04$
$3.19 \mathrm{E}-01$
$6.24 \mathrm{E}-04$
$7.92 \mathrm{E}-05$
$9.49 \mathrm{E}-01$
$2.46 \mathrm{E}-04$
4. $47 \mathrm{E}-06$
$1.18 \mathrm{E}-03$
$8.41 E-04$
4. $02 E-02$

$\begin{array}{ll}\text { TOTAL AIRBORNE TRITIUM RELEASE } & 2.04 E+01 \\ \text { TOTAL LIQUID TRITIUM RELEASE } & 2.36 \mathrm{E}+02\end{array}$

VOLUME OF LIQUID WASTE RELEASED (PRIOR TO DILUTION) IITERS $1.47 \mathrm{E}+07$

VOLUME OF DILUTION WATER USED DURING PERIOD

LITERS $1.86 \mathrm{E}+12$ 
EFFLUENT AND WASTE DISPOSAL ANNUAL REPORT FOR YEAR 1989 SOLID EFFLUENTS

SOLID WASTE DISPOSITION NUMBER OF SHIPMENTS 4
6

ESTIMATE OF MAJOR NUCLIDE COMPOSITION (BY TYPE OF WASTE)

A

$\mathrm{CO}-58$

$\mathrm{CO}-60$

CS -134

CS -137

FE- 55

$\mathrm{MN}-54$

NI -63

SB- 125

B

$\mathrm{CO}-58$

Co- 60

CS -134

CS -137

FE- 55

NI -63
MODE OF TRANSPORTATION

MOTOR SURFACE TRANSIT

MOTOR SURFACE TRANSIT
DESTINATION

BARNWELL SC

SEG OAK RIDGE TN

JAN-JUNE JULY-DEC

$\begin{array}{lll}8 & 9.90 \mathrm{E}+00 & 1.24 \mathrm{E}+01 \\ 8 & 2.70 \mathrm{E}+00 & 2.50 \mathrm{E}+00 \\ 8 & 1.86 \mathrm{E}+01 & 1.95 \mathrm{E}+01 \\ 8 & 5.90 \mathrm{E}+01 & 5.48 \mathrm{E}+01 \\ 8 & 2.60 \mathrm{E}+00 & 2.50 \mathrm{E}+00 \\ 8 & 1.50 \mathrm{E}+00 & \\ 8 & 3.50 \mathrm{E}+00 & 3.70 \mathrm{E}+00 \\ 8 & & 2.40 \mathrm{E}+00 \\ & & \\ 8 & 3.20 \mathrm{E}+00 & 3.20 \mathrm{E}+00 \\ 8 & 1.40 \mathrm{E}+01 & 1.40 \mathrm{E}+01 \\ 8 & 8.30 \mathrm{E}+00 & 8.30 \mathrm{E}+00 \\ 8 & 2.10 \mathrm{E}+01 & 2.10 \mathrm{E}+01 \\ 8 & 4.10 \mathrm{E}+01 & 4.10 \mathrm{E}+01 \\ 8 & 8.90 \mathrm{E}+00 & 8.90 \mathrm{E}+00\end{array}$

UNIT YEAR TOTAL

M3 1.23E+01

CI $\quad 3.39 \mathrm{E}+01$

M3 1.91E+02 *

CI 1.12E+01

M3

CI

M3 $3.60 \mathrm{E}+00$

CI $\quad 3.69 \mathrm{E}+02$

$*$ COMPACTED VOLUME 
EFFLUENT AND WASTE DISPOSAL ANNUAL REPORT FOR YEAR 1989 AIRBORNE AND LIQUID EFFLUENTS

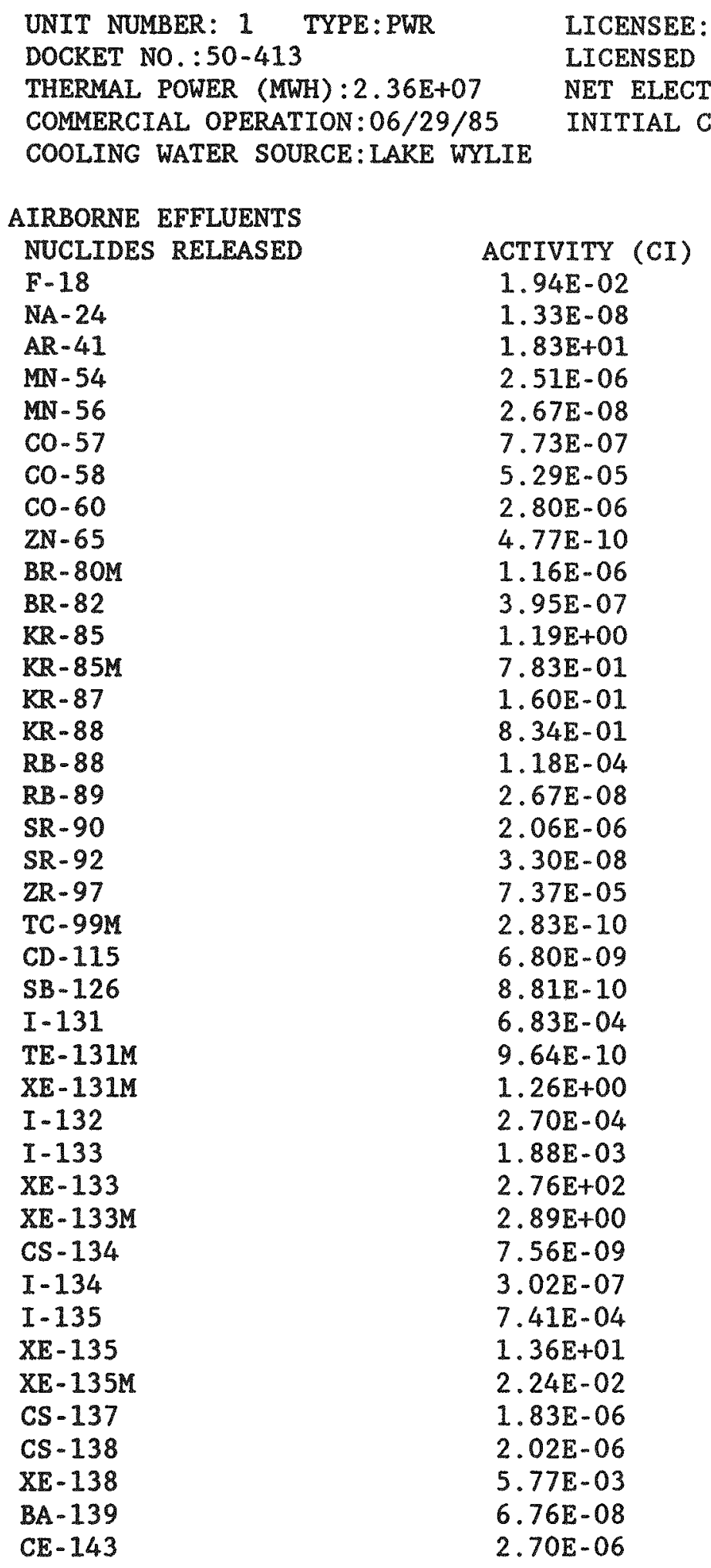


EFFLUENT AND WASTE DISPOSAL ANNUAL REPORT FOR YEAR 1989 AIRBORNE AND LIQUID EFFLUENTS

\begin{tabular}{|c|c|}
\hline $\begin{array}{l}\text { LIQUID EFFLUENTS } \\
\text { NUCLIDES RELEASED }\end{array}$ & ACTTVTTY (CT) \\
\hline $\mathrm{BE}-7$ & $3.02 \mathrm{E}-04$ \\
\hline$F-18$ & $1.82 E-03$ \\
\hline$N A-24$ & $1.63 E-04$ \\
\hline$A R-41$ & $2.02 E-06$ \\
\hline CR -51 & $1.93 E-02$ \\
\hline $\mathrm{MN}-54$ & $7.22 E-03$ \\
\hline$F E-55$ & $1.07 \mathrm{E}-01$ \\
\hline $\mathrm{CO}-57$ & $8.23 E-05$ \\
\hline $\mathrm{CO}-58$ & $7.46 E-02$ \\
\hline$F E-59$ & $2.57 E-03$ \\
\hline $\mathrm{CO}-60$ & $2.31 E-02$ \\
\hline $\mathrm{ZN}-65$ & $1.41 E-05$ \\
\hline$K R-85$ & $4.68 \mathrm{E}-04$ \\
\hline $\mathrm{KR}-87$ & $1.19 E-06$ \\
\hline $\mathrm{KR}-88$ & $4.00 E-06$ \\
\hline $\mathrm{RB}-88$ & $2.03 E-04$ \\
\hline$S R-89$ & $2.27 E-05$ \\
\hline SR-91 & $8.30 E-06$ \\
\hline SR-92 & $4.63 E-05$ \\
\hline$Y-92$ & $1.89 \mathrm{E}-05$ \\
\hline NB-95 & $2.27 E-03$ \\
\hline $2 R-95$ & $1.25 \mathrm{E}-03$ \\
\hline NB- 97 & $4.04 E-04$ \\
\hline ZR-97 & $9.01 E-05$ \\
\hline MO- 99 & $1.52 \mathrm{E}-05$ \\
\hline TC-99M & $4.72 E-04$ \\
\hline RU-103 & $1.21 \mathrm{E}-04$ \\
\hline$A G-110 M$ & $8.85 E-05$ \\
\hline $\mathrm{SN}-113$ & $1.45 E-04$ \\
\hline$S B-122$ & $2.02 \mathrm{E}-04$ \\
\hline$S B-124$ & $6.30 E-03$ \\
\hline$S B-125$ & $3.10 E-02$ \\
\hline$S B-126$ & $4.62 E-06$ \\
\hline$I-131$ & $3.36 E-03$ \\
\hline$I-132$ & $2.66 \mathrm{E}-06$ \\
\hline$I-133$ & $9.47 \mathrm{E}-04$ \\
\hline $\mathrm{XE}-133$ & $7.44 \mathrm{E}-02$ \\
\hline$X E-133 M$ & $5.27 E-04$ \\
\hline CS -134 & $2.37 E-03$ \\
\hline$I-135$ & $1.03 E-04$ \\
\hline $\mathrm{XE}-135$ & $1.33 \mathrm{E}-02$ \\
\hline$X E-135 M$ & $2.44 \mathrm{E}-06$ \\
\hline CS - 136 & $8.93 E-06$ \\
\hline CS -137 & $4.25 \mathrm{E}-03$ \\
\hline CS -138 & $3.44 E-02$ \\
\hline $\mathrm{BA}-140$ & $9.46 \mathrm{E}-05$ \\
\hline$L A-140$ & $1.41 E-03$ \\
\hline$C E-143$ & $2.52 E-06$ \\
\hline
\end{tabular}


EFFLUENT AND WASTE DISPOSAL ANNUAL REPORT FOR YEAR 1989 AIRBORNE AND LIQUID EFFLUENTS

\author{
LIQUID EFFLUENTS \\ NUCLIDES RELEASED \\ W- 187 \\ TL-208 \\ BI -214 \\ $\mathrm{PB}-214$ \\ $A C-228$ \\ TH -228
}
ACTIVITY (CI)
1.37E-05
3. $30 \mathrm{E}-05$
$2.72 \mathrm{E}-04$
$2.29 \mathrm{E}-04$
$5.72 \mathrm{E}-06$
$1.58 \mathrm{E}-02$

TOTAL AIRBORNE TRITIUM RELEASE 2.38E+01

TOTAL LIQUID TRITIUM RELEASE $4.45 \mathrm{E}+02$

VOLUME OF LIQUID WASTE RELEASED (PRIOR TO DILUTION) LITERS $1.22 E+08$ VOLUME OF DILUTION WATER USED DURING PERIOD LITERS $1.05 \mathrm{E}+11$ 
EFFLUENT AND WASTE DISPOSAL ANNUAL REPORT FOR YEAR 1989 AIRBORNE AND LIQUID EFFLUENTS

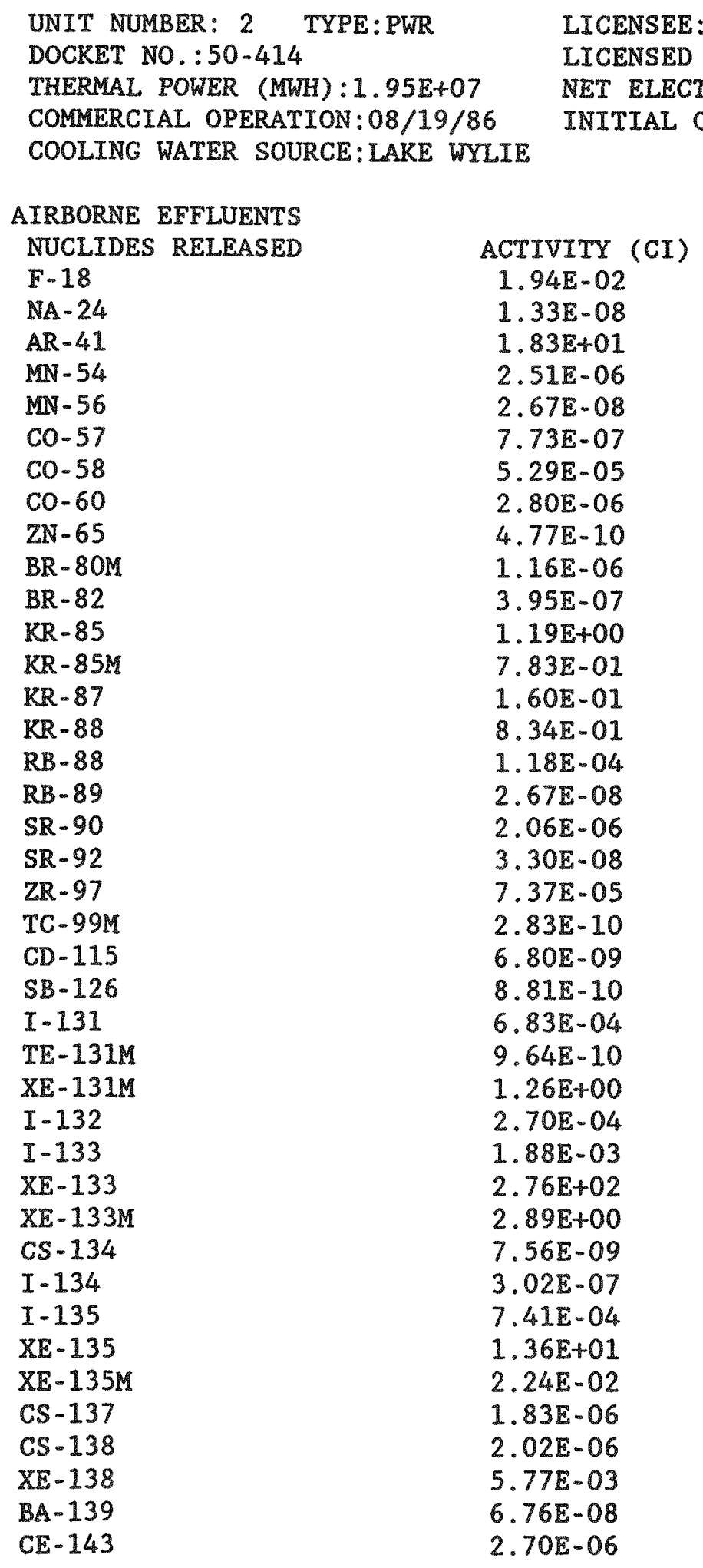


EFFLUENT AND WASTE DISPOSAL ANNUAL REPORT FOR YEAR 1989 AIRBORNE AND LIQUID EFFLUENTS

\section{LIQUID EFFLUENTS}

NUCLIDES RELEASED

$\mathrm{BE}-7$

F- 18

NA -24

AR -41

$\mathrm{CR}-51$

$\mathrm{MN}-54$

FE -55

CO- 57

CO- 58

FE- 59

CO-60

ZN-65

KR -85

KR -87

KR -88

RB -88

SR -89

SR -91

SR -92

$\mathrm{Y}-92$

NB-95

ZR-95

NB -97

ZR-97

MO- 99

TC-99M

RU - 103

AG-110M

SN-113

SB-122

SB-124

SB- 125

SB- 126

I- 131

I -132

I -133

$\mathrm{XE}-133$

$\mathrm{XE}-133 \mathrm{M}$

CS -134

I -135

XE-135

$X E-135 M$

CS -136

CS -137

CS -138

BA- 140

LA- 140

CE-143

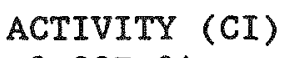

1. $82 \mathrm{E}-03$

1. $63 \mathrm{E}-04$

$2.02 E-06$

$1.93 \mathrm{E}-02$

7. $22 \mathrm{E}-03$

$1.07 \mathrm{E}-01$

8. 23E-05

$7.46 \mathrm{E}-02$

$2.57 E-03$

2. $31 E-02$

$1.41 \mathrm{E}-05$

4. $68 \mathrm{E}-04$

1. $19 \mathrm{E}-06$

4. $00 \mathrm{E}-06$

$2.03 E-04$

$2.27 E-05$

8. 30E-06

$4.63 \mathrm{E}-05$

$1.89 \mathrm{E}-05$

$2.27 \mathrm{E}-03$

1. $25 \mathrm{E}-03$

4.04E- 04

$9.01 \mathrm{E}-05$

$1.52 \mathrm{E}-05$

4.72E-04

1. $21 \mathrm{E}-04$

$8.85 \mathrm{E}-05$

$1.45 \mathrm{E}-04$

$2.02 \mathrm{E}-04$

$6.30 \mathrm{E}-03$

3. $10 \mathrm{E}-02$

4. $62 \mathrm{E}-06$

3. $36 \mathrm{E}-03$

$2.66 E-06$

$9.47 \mathrm{E}-04$

$7.44 \mathrm{E}-02$

$5.27 \mathrm{E}-04$

$2.37 E-03$

1. $03 \mathrm{E}-04$

1. $33 \mathrm{E}-02$

$2.44 \mathrm{E}-06$

8. $93 \mathrm{E}-06$

$4.25 \mathrm{E}-03$

$3.44 \mathrm{E}-02$

$9.46 \mathrm{E}-05$

$1.41 E-03$

$2.52 \mathrm{E}-06$ 
EFFLUENT AND WASTE DISPOSAL ANNUAL REPORT FOR YEAR 1989 AIRBORNE AND LIQUID EFFLUENTS

\author{
LIQUID EFFLUENTS \\ NUCLIDES RELEASED \\ W-187 \\ TL-208 \\ BI -214 \\ PB- 214 \\ AC -228 \\ TH -228
}
ACTIVITY (CI)
1.37E-05
3. $30 \mathrm{E}-05$
2. $72 \mathrm{E}-04$
$2.29 \mathrm{E}-04$
$5.72 E-06$
$1.58 \mathrm{E}-02$

TOTAL AIRBORNE TRITIUM RELEASE TOTAL LIQUID TRITIUM RELEASE

\section{$2.38 \mathrm{E}+01$}

$4.45 E+02$

$\begin{array}{llll}\text { VOLUME OF LIQUID WASTE RELEASED (PRIOR TO DILUTION) } & \text { LITERS } & 1.22 E+08 \\ \text { VOLUME OF DILUTION WATER USED DURING PERIOD } & \text { LITERS } & 1.05 E+11\end{array}$ 
SOLID WASTE DISPOSITION

NUMBER OF SHIPMENTS MODE OF TRANSPORTATION

$+16$

$\mathrm{N} / \mathrm{R}$

+DOES NOT INCLUDE BROKERED TOTALS

ESTIMATE OF MAJOR NUCLIDE COMPOSITION (BY TYPE OF WASTE)

A

$$
\begin{aligned}
& \text { C- } 14 \\
& \mathrm{CO}-57 \\
& \mathrm{CO}-58 \\
& \text { CO- } 60 \\
& \text { CS }-134 \\
& \text { CS }-137 \\
& \text { FE- } 55 \\
& \text { I-131 } \\
& \text { LA- } 140 \\
& \mathrm{MN}-54 \\
& \text { NI }-63 \\
& \text { SB- } 122 \\
& X E-133
\end{aligned}
$$

B

D

TYPE OF WASTE

A. SPENT RESINS, FILTER SLUDGES, EVAPORATOR BOTTOMS, ETC.

B. DRY COMPRESSIBLE WASTE, CONTAMINATED EQUIPMENT, ETC.

C. IRRADIATED COMPONENTS, CONTROL RODS, ETC.

D. OTHER

DEWATERED MECH. FLTRS .
DESTINATION

$\mathrm{N} / \mathrm{R}$
JAN-JUNE JULY-DEC

8

\%

8

8

8

8

8

8

8

8

8

8

$6.90 E+00$

$1.67 \mathrm{E}+01$

$7.20 \mathrm{E}+00$

1. $52 \mathrm{E}+01$

2. $20 \mathrm{E}+01$

(20.01

$1.99 E+01$

1. $13 \mathrm{E}+01$

$2.16 \mathrm{E}-04$

2. $00 \mathrm{E}+00$

3. $83 E+01$

$1.09 \mathrm{E}+01$

$6.30 E+00$

1. $21 \mathrm{E}+01$

1. $44 E+01$

$1.00 E-02$

$1.96 \mathrm{E}-04$

$7.40 \mathrm{E}+00$

$7.10 E+00$

$1.96 \mathrm{E}-04$

$6.67 E-04$

$\begin{array}{lll}7.95 E+00 & 7.61 E+00 \\ & 2.41 E+01 & 2.44 E+01 \\ & 1.20 E+00 & 1.00 E+00 \\ & 5.55 E-01 & 5.57 E+01 \\ 8 & 7.85 E+00 & 7.87 E+00 \\ 8 & 2.50 E+00 & 2.49 E+00\end{array}$

$\begin{array}{lll}8 & 1.31 E+01 & 1.31 E+01 \\ 8 & 2.93 E+01 & 2.93 E+01 \\ 8 & 3.92 E+01 & 3.92 E+01 \\ 8 & 1.28 E+01 & 1.28 E+01 \\ 8 & 4.30 E+00 & 4.30 E+00\end{array}$

UNIT YEAR TOTAL

M3 4.03E+01

CI $\quad 1.11 E+02$

M3 $1.53 \mathrm{E}+02 *$

CI $\quad 1.27 \mathrm{E}+01$

M3 $0.00 \mathrm{E}+00$

CI $\quad 0.00 E+00$

M3 2.27E+01

CI $1.93 \mathrm{E}+02$

*TOTAL VOLUME INCLUDES COMPACTED AND NON-COMPACTED VOLUMES 
EFFLUENT AND WASTE DISPOSAL ANNUAL REPORT FOR YEAR 1989 AIRBORNE AND LIQUID EFFLUENTS

\begin{tabular}{|c|c|c|}
\hline \multicolumn{2}{|c|}{ UNIT NUMBER: 1 TYPE:BWR } & LICENSEE:ILLINOIS POWER \\
\hline \multicolumn{2}{|c|}{ DOCKET NO. : $50-461$} & LICENSED POWER (MWT):2.89E+03 \\
\hline \multirow{2}{*}{\multicolumn{2}{|c|}{$\begin{array}{l}\text { THERMAL POWER (MWH) : } 9.26 E+06 \\
\text { COMMERCIAL OPERATION:11/24/87 }\end{array}$}} & NET ELECTRIC POWER (MWH) $: 2.86 E+06$ \\
\hline & & INITIAL CRITICALITY:02/27/87 \\
\hline \multicolumn{3}{|c|}{ COOLING WATER SOURCE:SALT CREEK } \\
\hline \multicolumn{3}{|l|}{ AIRBORNE EFFLUENTS } \\
\hline NUCLIDES RELEASED & \multicolumn{2}{|c|}{ ACTIVITY (CI) } \\
\hline$C R-51$ & \multicolumn{2}{|c|}{$8.15 E-03$} \\
\hline $\mathrm{MN}-54$ & \multicolumn{2}{|c|}{$3.28 \mathrm{E}-04$} \\
\hline $\mathrm{CO}-58$ & \multicolumn{2}{|c|}{$3.05 E-04$} \\
\hline$C 0-60$ & \multicolumn{2}{|c|}{$4.84 E-04$} \\
\hline $\mathrm{KR}-87$ & \multicolumn{2}{|c|}{$7.64 \mathrm{E}+00$} \\
\hline SR-89 & \multicolumn{2}{|c|}{$8.19 E-05$} \\
\hline$A G-110 M$ & \multicolumn{2}{|c|}{$5.16 E-06$} \\
\hline$I=131$ & \multicolumn{2}{|c|}{$1.67 \mathrm{E}-04$} \\
\hline I-133 & \multicolumn{2}{|c|}{$1.54 \mathrm{E}-04$} \\
\hline$X E-135$ & \multicolumn{2}{|c|}{$5.31 E+00$} \\
\hline \multicolumn{3}{|l|}{ LIQUID EFFLUENTS } \\
\hline NUCLIDES RELEASED & \multicolumn{2}{|c|}{ ACTIVITY (CI) } \\
\hline $\mathrm{CR}-51$ & \multicolumn{2}{|c|}{$2.60 \mathrm{E}-03$} \\
\hline $\mathrm{MN}-54$ & \multicolumn{2}{|c|}{$2.75 \mathrm{E}-03$} \\
\hline$F E-55$ & \multicolumn{2}{|c|}{$1.89 E-03$} \\
\hline $\mathrm{CO}-58$ & \multicolumn{2}{|c|}{$2.18 \mathrm{E}-03$} \\
\hline $\mathrm{CO}-60$ & \multicolumn{2}{|c|}{$7.91 E-03$} \\
\hline$A G-110 M$ & \multicolumn{2}{|c|}{$2.38 \mathrm{E}-05$} \\
\hline
\end{tabular}

TOTAL AIRBORNE TRITIUM RELEASE TOTAL LIQUID TRITIUM RELEASE
$8.69 E-01$

$1.49 \mathrm{E}+00$
VOLUME OF LIQUTD WASTE RELEASED (PRIOR TO DILUTION)

VOLUME OF DILUTION WATER USED DURING PERIOD
LITERS $3.12 E+06$

LITERS $\quad 2.90 \mathrm{E}+08$ 
EFFLUENT AND WASTE DISPOSAL ANNUAL REPORT FOR YEAR 1989 SOLID EFFLUENTS

SOLID WASTE DISPOSITION NUMBER OF SHIPMENTS 6 42 TRUCK TRUCK

IRRADIATED FUEL SHIPMENTS (DISPOSITION) NUMBER OF SHIPMENTS MODE OF TRANSPORTATION 0 N/A

ESTIMATE OF MAJOR NUCLIDE COMPOSITION (BY TYPE OF WASTE)

A

CO- 60

$\mathrm{CR}-51$

FE -55

FE -59

MN -54

OTHER

$\mathrm{B}$

$A G-110 M$

$\mathrm{CO}-58$

CO -60

CR -51

FE -55

MN -54

OTHER

TYPE OF WASTE

A. SPENT RESINS, FILTER SLUDGES, EVAPORATOR BOTTOMS, ETC.

B. DRY COMPRESSIBLE WASTE, CONTAMINATED EQUIPMENT, ETC.

C. IRRADIATED COMPONENTS, CONTROL RODS, ETC.

D. OTHER
DESTINATION
BARNWELL SC
RICHLAND WA

DESTINATION

$\mathrm{N} / \mathrm{A}$

JAN-JUNE JULY-DEC

$\begin{array}{lll}8 & 1.36 \mathrm{E}+01 & 9.58 \mathrm{E}+00 \\ 8 & & 2.81 \mathrm{E}+01 \\ 8 & 7.08 \mathrm{E}+01 & 5.35 \mathrm{E}+01 \\ 8 & 1.35 \mathrm{E}+00 & \\ 8 & 1.13 \mathrm{E}+01 & 6.39 \mathrm{E}+00 \\ 8 & 5.82 \mathrm{E}-01 & 3.60 \mathrm{E}-01\end{array}$

$1.25 \mathrm{E}+01$

$2.29 E+00 \quad 3.17 E+00$

8. $23 E+00 \quad 1.46 E+01$

$2.41 E+00 \quad 2.11 E+00$

$7.22 E+01 \quad 4.95 E+01$

$1.21 E+01 \quad 1.52 E+01$

$2.85 E+00$

UNIT YEAR TOTAL

M3 $2.90 \mathrm{E}+02$

CI $\quad 1.89 \mathrm{E}+03$

M3 $\quad 1.09 \mathrm{E}+02$ *

CI $2.20 \mathrm{E}+00$

M3 $0.00 \mathrm{E}+00$

CI $\quad 0.00 \mathrm{E}+00$

M3

CI

*VOLUME AFTER REDUCTION (5.66E+02 M3 PRIOR TO REDUCTION) 
INSTALLATION:DONALD C. COOK LOCATION:11 MI SSW ST. JOSEPH, MI

EFFLUENT AND WASTE DISPOSAL ANNUAL REPORT FOR YEAR 1989 AIRBORNE AND LIQUID EFFLUENTS

LIQUID EFFLUENTS
NUCLIDES RELEASED
SB-122
SB-124
SB-125
$I-131$
$X E-131 M$
$I-133$
$X E-133$
$X E-133 M$
$C S-134$
$X E-135$
$C S-136$
$C S-137$

LIQUID EFFLUENTS

NUCLIDES RELEASED

SB- 122

$S B-124$

$\mathrm{SB}-125$

$I-131$

$\mathrm{XE}-131 \mathrm{M}$

$X E^{-133}$

$\mathrm{XE}-133 \mathrm{M}$

CS -134

CS -136

CS -137
ACTIVITY (CI)
1.84E-03
$5.08 \mathrm{E}-02$
$1.78 \mathrm{E}-02$
7. $76 E-03$
$8.35 \mathrm{E}-04$
$5.31 \mathrm{E}-04$
7. $63 \mathrm{E}-02$
$5.62 \mathrm{E}-04$
$1.95 E-02$
$2.89 E-04$
$3.08 \mathrm{E}-03$
3. $35 E-02$

TOTAL AIRBORNE TRITIUM RELEASE $1.76 \mathrm{E}+01$

TOTAL LIQUID TRITIUM RELEASE
$8.74 \mathrm{E}+02$

VOLUME OF LIQUID WASTE RELEASED (PRIOR TO DILUTION) LITERS 5.16E+08

VOLUME OF DILUTION WATER USED DURING PERIOD

LITERS $\quad 3.02 \mathrm{E}+12$ 
INSTALLATION: DONALD C . COOK

EFFLUENT AND WASTE DISPOSAL ANNUAL REPORT FOR YEAR 1989

SOLID EFFLUENTS

SOLID WASTE DISPOSITION

$\begin{array}{cl}\text { NUMBER OF SHIPMENTS } & \text { MODE OF TRANSPORTATION } \\ 127 & \text { TRUCK } \\ 3 & \text { TRUCK }\end{array}$

ESTIMATE OF MAJOR NUCLIDE COMPOSITION (BY TYPE OF WASTE)

\section{DESTINATION \\ BARNWELL SC \\ RICHLAND WA}

JAN-JUNE JULY-DEC

A

$$
\begin{aligned}
& C O-58 \\
& C O-60 \\
& C S-134 \\
& C S-137 \\
& F E-55 \\
& M N-54 \\
& N I-63 \\
& S B-125
\end{aligned}
$$

B

C. -14

$\mathrm{CO}-58$

Co- 60

CS -134

CS -137

FE -55

H -3

NI -63
8

1. $10 \mathrm{E}+01$

$2.00 \mathrm{E}+01$

2.00E+01

$3.00 E+01$

$3.00 \mathrm{E}+00$

1. $30 \mathrm{E}+01$

3. $00 \mathrm{E}+00$

4.00E+00

$2.80 \mathrm{E}+01$

1. $70 \mathrm{E}+01$

4. $90 \mathrm{E}+01$

$2.00 E+00$
3. $30 \mathrm{E}+01$

1. $40 \mathrm{E}+01$

$9.00 \mathrm{E}+00$

1. $70 \mathrm{E}+01$

1. $10 \mathrm{E}+01$

$8.00 \mathrm{E}+00$

$8.00 \mathrm{E}+00$

$5.00 E+00$

$2.00 \mathrm{E}+00$

1. $60 \mathrm{E}+01$

9. $00 \mathrm{E}+00$

2. $50 \mathrm{E}+01$

$2.40 E+01$

$1.90 \mathrm{E}+01$

TYPE OF WASTE

A. SPENT RESINS, FILTER SLUDGES, EVAPORATOR

UNIT YEAR TOTAL BOTTOMS, ETC.

B. DRY COMPRESSIBLE WASTE, CONTAMINATED EQUIPMENT, ETC.

C. IRRADIATED COMPONENTS, CONTROL RODS, ETC.

M3 4.17E+01

CI $\quad 1.16 \mathrm{E}+03$

M3 $3.46 \mathrm{E}+02$

CI $\quad 1.57 \mathrm{E}+01$

M3

CI

M3

CI 
EFFLUENT AND WASTE DISPOSAL ANNUAL REPORT FOR YEAR 1989 AIRBORNE AND LIQUID EFFLUENTS
UNIT NUMBER: 1 TYPE: BWR
LICENSEE: NEBRASKA PUBLIC POWER DISTRICT
DOCKET NO. : 50-298
LICENSED POWER (MWT) :2.38E+03
THERMAL POWER (MWH) $: 1.50 \mathrm{E}+07$
COMMERCIAL OPERATION:07/01/74
COOLING WATER SOURCE:MISSOURI RIVER
NET ELECTRIC POWER (MWH) $: 4.79 \mathrm{E}+06$
INITIAL CRITICALITY:02/21/74
AIRBORNE EFFLUENTS
NUCLIDES RELEASED
$\mathrm{KR}-83 \mathrm{M}$
ACTIVITY (CI)
$\mathrm{KR}-85$
$2.66 \mathrm{E}+00$
$\mathrm{KR}-85 \mathrm{M}$
1. $50 \mathrm{E}+01$
$\mathrm{KR}-87$
5. $12 \mathrm{E}+00$
$\mathrm{KR}-88$
$1.64 \mathrm{E}+01$
$\mathrm{RB}-88$
$1.68 \mathrm{E}+01$
KR -89
4. 16E-03
RB -89
$7.49 \mathrm{E}+01$
SR-91
$1.36 E-03$
$\mathrm{Y}-91 \mathrm{M}$
1. $18 \mathrm{E}-04$
AG-110M
$7.66 \mathrm{E}-05$
$\mathrm{I}-131$
1. $29 \mathrm{E}-05$
I -132
5. $23 \mathrm{E}-03$
TE- 132
2. 16E-05
I -133
$8.99 \mathrm{E}-05$
$\mathrm{XE}-133$
1.07E-04
$\mathrm{XE}-133 \mathrm{M}$
2. $13 E+01$
I -135
2. 25E-01
$\mathrm{XE}-135$
2. $94 \mathrm{E}-04$
$\mathrm{XE}-135 \mathrm{M}$
2. $33 E+01$
$\mathrm{XE}-137$
$8.16 \mathrm{E}+00$
CS -138
$8.95 E+01$
$\mathrm{XE}-138$
1. $56 \mathrm{E}-02$
$B A-140$
7. $10 \mathrm{E}+01$
$1.98 \mathrm{E}-05$
LIQUID EFFLUENTS
NUCLIDES RELEASED
NA -24
CR -51
MN -54
FE -55
CO- 58
FE- 59
CO- 60
SR -89
SR -90
TC-99M
ACTIVITY (CI)
AG-110M
$3.55 \mathrm{E}-04$
$2.83 \mathrm{E}-02$
$2.21 \mathrm{E}-01$
$3.22 \mathrm{E}-01$
$4.85 \mathrm{E}-02$
$5.34 E-04$
$1.02 \mathrm{E}+00$
$5.22 E-02$
$4.28 \mathrm{E}-03$
$7.55 E-05$
$S B-125$
3. $32 \mathrm{E}-02$
I -131
$3.75 \mathrm{E}-04$
I -133
$1.48 \mathrm{E}-02$
$6.26 \mathrm{E}-04$
CS -134
1. $81 \mathrm{E}-01$
CS -136
8. $34 \mathrm{E}-04$ 


\section{EFFLUENT AND WASTE DISPOSAL ANNUAL REPORT FOR YEAR 1989} AIRBORNE AND LIQUID EFFLUENTS

LIQUID EFFLUENTS

NUCLIDES RELEASED

CS -137

BA -140

LA -140

\author{
ACTIVITY (CI) \\ 2. $62 \mathrm{E}-01$ \\ $2.36 \mathrm{E}-03$ \\ $1.10 \mathrm{E}-03$
}

TOTAL AIRBORNE TRITIUM RELEASE $0.00 E+00$

TOTAL LIQUID TRITIUM RELEASE $5.45 E+00$

VOLUME OF LIQUID WASTE RELEASED (PRIOR TO DILUTION) LITERS $8.84 E+06$

VOLUME OF DILUTION WATER USED DURING PERIOD

LITERS $\quad 8.16 \mathrm{E}+11$ 
EFFLUENT AND WASTE DISPOSAL ANNUAL REPORT FOR YEAR 1989 SOLID EFFLUENTS

SOLID WASTE DISPOSITION NUMBER OF SHIPMENTS 2
28 MODE OF TRANSPORTATION EXCLUSIVE USE VEHICLE EXCLUSIVE USE VEHICLE

IRRADIATED FUEL SHIPMENTS (DISPOSITION) NUMBER OF SHIPMENTS MODE OF TRANSPORTATION 1 RAIL

ESTIMATE OF MAJOR NUCLIDE COMPOSITION (BY TYPE OF WASTE)

A

$A G-110 M$

BA-LA- 140

C- 14

CO- 58

CO- 60

CR -51

CS -134

CS -137

CU- 242

FE- 55

FE- 59

H -3

I- 131

MN -54

NI - 59

NI -63

PU-241

SR -90

TC -99

B

AM- 241

CO- 60

CS -134

CS -137

CU- 242

FE- 55

$\mathrm{MN}-54$

NI -63

PU- 241

SB- 125

SR -90

\author{
DESTINATION \\ BEATTY NV \\ RICHLAND WA
}

DESTINATION

MORRIS IL

JAN-JUNE JULY-DEC

$\begin{array}{lll}8 & 5.22 \mathrm{E}-01 & 9.99 \mathrm{E}-01 \\ 8 & 2.86 \mathrm{E}-02 & 1.97 \mathrm{E}-02 \\ 8 & 5.75 \mathrm{E}-01 & 2.83 \mathrm{E}-01 \\ 8 & 4.04 \mathrm{E}+00 & 1.27 \mathrm{E}+00 \\ 8 & 2.87 \mathrm{E}+01 & 4.22 \mathrm{E}+01 \\ 8 & 8.41 \mathrm{E}-01 & 5.40 \mathrm{E}-02 \\ 8 & 3.27 \mathrm{E}+00 & 6.24 \mathrm{E}+00 \\ 8 & 4.13 \mathrm{E}+00 & 1.12 \mathrm{E}+01 \\ 8 & 1.10 \mathrm{E}-04 & 7.27 \mathrm{E}-05 \\ 8 & 4.48 \mathrm{E}+01 & 2.63 \mathrm{E}+01 \\ 8 & 5.69 \mathrm{E}-02 & \\ 8 & 4.57 \mathrm{E}-02 & 1.76 \mathrm{E}-02 \\ 8 & 2.52 \mathrm{E}-01 & 5.82 \mathrm{E}-02 \\ 8 & 1.17 \mathrm{E}+01 & 1.06 \mathrm{E}+01 \\ 8 & & 1.80 \mathrm{E}-03 \\ 8 & 9.92 \mathrm{E}-01 & 8.26 \mathrm{E}-01 \\ 8 & 7.53 \mathrm{E}-03 & 5.21 \mathrm{E}-03 \\ 8 & 8.26 \mathrm{E}-03 & 1.31 \mathrm{E}-02 \\ 8 & 1.24 \mathrm{E}-03 & 8.12 \mathrm{E}-04 \\ & & \\ 8 & 3.14 \mathrm{E}-02 & \\ 8 & 5.69 \mathrm{E}+01 & 5.69 \mathrm{E}+01 \\ 8 & 4.16 \mathrm{E}-01 & 4.16 \mathrm{E}-01 \\ 8 & 1.64 \mathrm{E}+00 & 1.65 \mathrm{E}+00 \\ 8 & 1.50 \mathrm{E}-05 & 1.50 \mathrm{E}-05 \\ 8 & 3.87 \mathrm{E}+01 & 3.88 \mathrm{E}+01 \\ 8 & 6.82 \mathrm{E}-01 & 6.84 \mathrm{E}-01 \\ 8 & 6.00 \mathrm{E}-01 & 6.02 \mathrm{E}-01 \\ 8 & 7.73 \mathrm{E}-03 & 7.75 \mathrm{E}-03 \\ 8 & 9.45 \mathrm{E}-01 & 9.50 \mathrm{E}-01 \\ 8 & 5.76 \mathrm{E}-04 & 5.78 \mathrm{E}-04\end{array}$


INSTALLATION : COOPER

EFFLUENT AND WASTE DISPOSAL ANNUAL REPORT FOR YEAR 1989 SOLID EFFLUENTS

\section{TYPE OF WASTE}
A. SPENT RESINS, FILTER SLUDGES, EVAPORATOR BOTTOMS, ETC.
B. DRY COMPRESSIBLE WASTE, CONTAMINATED EQUIPMENT, ETC.
C. IRRADIATED COMPONENTS, CONTROL RODS, ETC.
D. OTHER

$\begin{array}{lc}\text { UNIT } & \text { YEAR TOTAL } \\ \text { M3 } & 1.24 E+02 \\ \text { CI } & 3.06 E+02 \\ \text { M3 } & 1.68 E+02 \\ \text { CI } & 4.09 E-01 \\ \text { M3 } & \\ \text { CI } & \\ \text { M3 } & \\ \text { CI } & \end{array}$


EFFLUENT AND WASTE DISPOSAL ANNUAL REPORT FOR YEAR 1989 AIRBORNE AND LIQUID EFFLUENTS

\begin{tabular}{|c|c|}
\hline \multicolumn{2}{|l|}{ UNIT NUMBER: 3 TYPE:PWR } \\
\hline \multicolumn{2}{|r|}{ LICENSED POWER (MWT):2.54E+03 } \\
\hline \multicolumn{2}{|r|}{ NET ELECTRIC POWER (MWH) :2.93E+06 } \\
\hline \multirow{2}{*}{\multicolumn{2}{|c|}{ COMMERCIAL OPERATION:03/13/77 INITIAL CRITICALITY:01/14/77 }} \\
\hline & \\
\hline \multicolumn{2}{|l|}{ AIRBORNE EFFLUENTS } \\
\hline NUCLIDES RELEASED & VITY (CI) \\
\hline $\mathrm{KR}-85$ & $5 E+01$ \\
\hline $\mathrm{KR}-85 \mathrm{M}$ & $8 E+01$ \\
\hline $\mathrm{KR}-87$ & $9 E+00$ \\
\hline $\mathrm{KR}-88$ & $1 E+00$ \\
\hline SR -89 & $2 E-06$ \\
\hline SR-90 & $1 E-07$ \\
\hline$I-131$ & $2 E-03$ \\
\hline $\mathrm{XE}-131 \mathrm{M}$ & $7 E+01$ \\
\hline$I-133$ & $4 E-04$ \\
\hline $\mathrm{XE}-133$ & $1 E+03$ \\
\hline $\mathrm{XE}-133 \mathrm{M}$ & $.0 E+01$ \\
\hline $\operatorname{CS}-134$ & $8 E-09$ \\
\hline $\mathrm{XE}-135$ & $5 E+02$ \\
\hline $\mathrm{XE}-135 \mathrm{M}$ & $3 E-01$ \\
\hline CS -137 & $1 E-09$ \\
\hline$C E-143$ & $7 E-07$ \\
\hline \multicolumn{2}{|l|}{ LIQUID EFFLUENTS } \\
\hline NUCLIDES RELEASED & VITY (CI) \\
\hline NA -24 & $8 \mathrm{E}-06$ \\
\hline$C R-51$ & $\mathrm{OE}-02$ \\
\hline$M N-54$ & $6 E-03$ \\
\hline$F E-55$ & $7 E-02$ \\
\hline Co- 58 & $9 E-02$ \\
\hline FE -59 & $7 E-04$ \\
\hline Co- 60 & OE -02 \\
\hline $\mathrm{KR}-85$ & $6 E-02$ \\
\hline $\mathrm{KR}-85 \mathrm{M}$ & $8 E-03$ \\
\hline $\mathrm{KR}-88$ & $4 E-04$ \\
\hline$R B-88$ & $4 E-03$ \\
\hline$S R-89$ & $2 E-04$ \\
\hline SR-90 & $8 E-04$ \\
\hline SR -92 & $6 E-03$ \\
\hline$Y-92$ & OE-04 \\
\hline NB -95 & $7 E-03$ \\
\hline ZR-95 & $7 E-04$ \\
\hline ZR-97 & $8 E-04$ \\
\hline MO-99 & $4 E-03$ \\
\hline TC.99M & $9 E-03$ \\
\hline TE-99M & $8 E-03$ \\
\hline RU-103 & $4 E-04$ \\
\hline $\mathrm{RU}-106$ & $5 E-04$ \\
\hline$A G-110 M$ & $8 E-03$ \\
\hline
\end{tabular}

N/A $=$ NOT APPLICABLE N/D-NOT DETECTED $\mathrm{N} / \mathrm{R}-\mathrm{NOT}$ REPORTED 
EFFLUENT AND WASTE DISPOSAL ANNUAL REPORT FOR YEAR 1989

AIRBORNE AND LIQUID EFFLUENTS

LIQUID EFFLUENTS
NUCLIDES RELEASED
I-131
XE $-131 M$
$T E-132$
$I-133$
XE -133
$X E-133 M$
CS -134
XE -135
CS -137
BA -139
BA-140
LA -140

ACTIVITY (CI)

7.40E-03

$3.20 E-01$

$1.02 E=04$

$1.21 \mathrm{E}-03$

$4.21 E+01$

$4.09 E-01$

$4.86 E-03$

$2.07 E-01$

$1.09 E-02$

$3.17 \mathrm{E}-04$

$5.27 \mathrm{E}-05$

$7.15 E-03$

TOTAL AIRBORNE TRITIUM RELEASE $3.43 E+01$

TOTAL LIQUID TRITIUM RELEASE $3.44 \mathrm{E}+02$

VOLUME OF LIQUID WASTE RELEASED (PRIOR TO DILUTION) LITERS 3.03E+07 VOLUME OF DILUTION WATER USED DURING PERIOD

LITERS $7.18 \mathrm{E}+10$ 
INSTALLATION : CRYSTAL RIVER

EFFLUENT AND WASTE DISPOSAL ANNUAL REPORT FOR YEAR 1989

SOLID EFFLUENTS

SOLID WASTE DISPOSITION NUMBER OF SHIPMENTS 17

7
MODE OF TRANSPORTATION TRUCK EXCLUSIVE USE TRUCK EXCLUSIVE USE

IRRADIATED FUEL SHIPMENTS (DISPOSITION)

NUMBER OF SHIPMENTS MODE OF TRANSPORTATION

0

N/A

ESTIMATE OF MAJOR NUCLIDE COMPOSITION (BY TYPE OF WASTE)

A

$\mathrm{CO}-58$

$\mathrm{CO}-60$

CS -134

CS -137

FE -55

$\mathrm{MN}-54$

NI -63

B

C -14

$\mathrm{CO}-58$

$\mathrm{CO}-60$

CR -51

CS -137

FE -55

NI -63

DESTINATION

CHEM NUCLEAR BARNWELL SC SEG OAK RIDGE TN

DESTINATION

N/A

JAN-JUNE JULY-DEC

$\begin{array}{lll}7 & 7.20 \mathrm{E}+00 & 3.17 \mathrm{E}+00 \\ & 7.23 \mathrm{E}+01 & 1.26 \mathrm{E}+01 \\ & 3.60 \mathrm{E}+00 & 1.80 \mathrm{E}+01 \\ 8 & 7.60 \mathrm{E}+00 & 3.97 \mathrm{E}+01 \\ & & 1.24 \mathrm{E}+01 \\ 8 & 5.70 \mathrm{E}+00 & \\ & & 1.00 \mathrm{E}+01 \\ 8 & & 2.30 \mathrm{E}+00 \\ 8 & 1.51 \mathrm{E}+01 & \\ 8 & 2.14 \mathrm{E}+01 & 1.42 \mathrm{E}+01 \\ 8 & 4.67 \mathrm{E}+00 & \\ 8 & 4.35 \mathrm{E}+01 & 2.77 \mathrm{E}+01 \\ 8 & 1.21 \mathrm{E}+01 & 7.26 \mathrm{E}+01\end{array}$

UNIT YEAR TOTAL

M3 $6.65 \mathrm{E}+01$

CI $2.40 \mathrm{E}+03$

M3 2.81E+02

CI $\quad 4.94 \mathrm{E}+00$

M3

$\mathrm{CI}$

$\mathrm{M} 3$

CI 
EFFLUENT AND WASTE DISPOSAL ANNUAL REPORT FOR YEAR 1989 AIRBORNE AND LIQUID EFFLUENTS

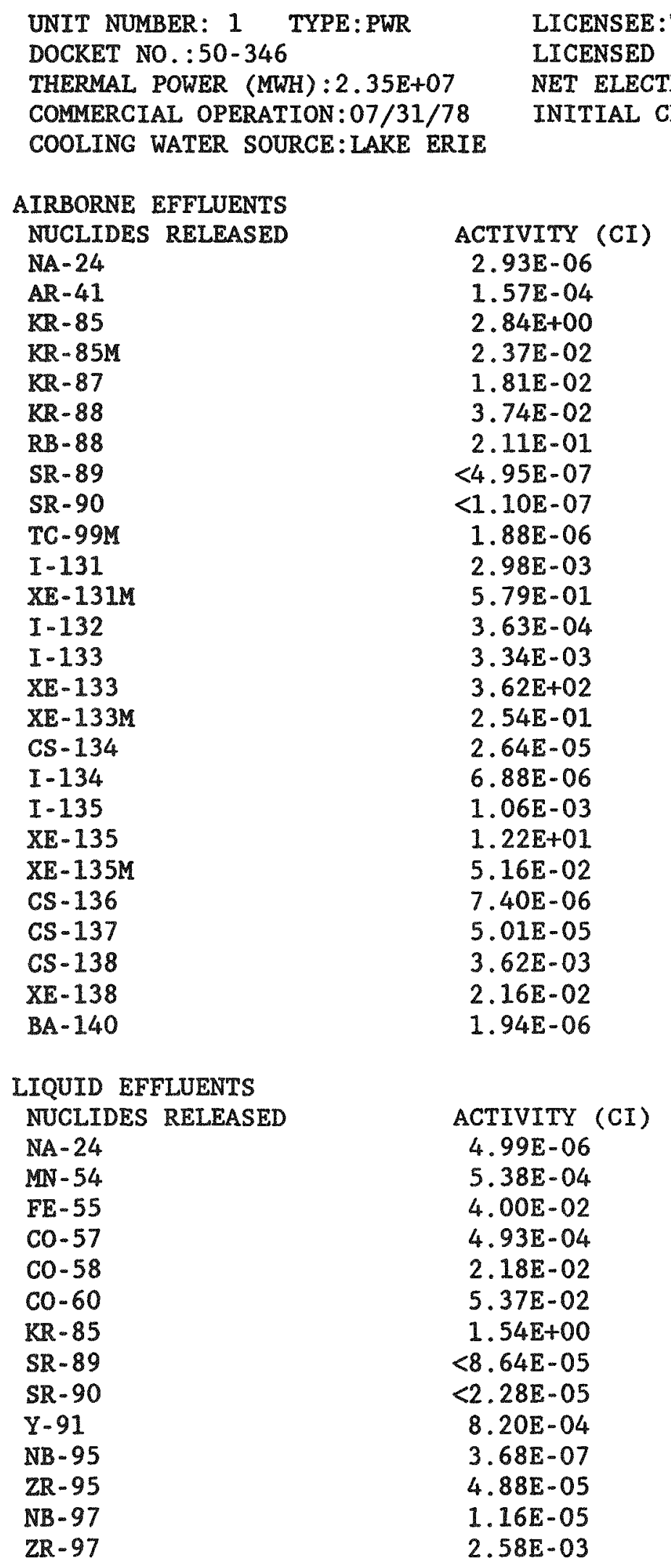

N/A $=$ NOT APPLICABLE

N/D $=$ NOT DETECTED

N/R $=$ NOT REPORTED 
EFFLUENT AND WASTE DISPOSAL ANNUAL REPORT FOR YEAR 1989 AIRBORNE AND LIQUID EFFLUENTS

\author{
LIQUID EFFLUENTS \\ NUCLIDES RELEASED \\ MO-99 \\ TC-99M \\ RU -106 \\ AG-110M \\ $\mathrm{SN}-113$ \\ $\mathrm{SB}-125$ \\ $I-131$ \\ $\mathrm{XE}-131 \mathrm{M}$ \\ BA- -133 \\ I -133 \\ $\mathrm{XE}-133$ \\ $\mathrm{XE}-133 \mathrm{M}$ \\ CS -134 \\ I- 135 \\ $\mathrm{XE}-135$ \\ CS -136 \\ CS -137 \\ LA- 140 \\ CE- 144 \\ W-187
}

ACTIVITY (CI)
$4.41 E-04$
$1.42 E-03$
$2.35 E-05$
$5.10 E-02$
$1.47 E-04$
$3.45 E-03$
$2.81 E-03$
$8.46 E-03$
$2.00 E-06$
$4.24 E-04$
$8.67 E-02$
$4.85 E-03$
$7.96 E-04$
$9.83 E-06$
$2.34 E-03$
$2.46 E-06$
$2.55 E-03$
$1.93 E-06$
$5.77 E-04$
$6.98 E-06$

4.41E-04

1. $42 \mathrm{E}-03$

2. $35 \mathrm{E}=05$

$5.10 \mathrm{E}-02$

$1.47 \mathrm{E}-04$

$3.45 \mathrm{E}-03$

2.81E-03

$.46 \mathrm{E}-03$

4. 24E-04

8.67E-02

$4.85 E-03$

$7.96 \mathrm{E}-04$

$9.83 E-06$

$2.34 \mathrm{E}-03$

$2.46 \mathrm{E}-06$

$2.55 \mathrm{E}-03$

1. $93 \mathrm{E}-06$

$6.98 \mathrm{E}-06$

TOTAL AIRBORNE TRITIUM RELEASE 1.89E+01

TOTAL LIQUID TRITIUM RELEASE $2.39 \mathrm{E}+02$

VOLUME OF LIQUID WASTE RELEASED (PRIOR TO DILUTION) IITERS 4.99E+06

VOLUME OF DILUTION WATER USED DURING PERIOD

LITERS $\quad 1.24 \mathrm{E}+09$ 
INSTALLATION: DAVIS - BESSE

EFFLUENT AND WASTE DISPOSAL ANNUAL REPORT FOR YEAR 1989 SOLID EFFLUENTS

SOLID WASTE DISPOSITION NUMBER OF SHIPMENTS 8 MODE OF TRANSPORTATION TRUCK

ESTIMATE OF MAJOR NUCLIDE COMPOSITION (BY TYPE OF WASTE)

A CS -137

$\mathrm{FE}-55$

B

FE- 55

D

FE- 55
DESTINATION

BARNWELL SC

JAN-JUNE JULY-DEC

\& $\quad 5.06 \mathrm{E}+01$

$3.45 E+01$

8 $5.03 \mathrm{E}+01$

8

$5.02 E+01$

8

$5.92 E+01$

UNIT YEAR TOTAL

M3 6.27E+01

CI $1.83 \mathrm{E}+02$

M3 5.21E+01

CI $1.72 \mathrm{E}+00$

M3

CI

M3

CI $2.31 \mathrm{E}+01$ 
EFFLUENT AND WASTE DISPOSAL ANNUAL REPORT FOR YEAR 1989 AIRBORNE AND LIQUID EFFLUENTS

UNIT NUMBER: 1 TYPE: PWR

DOCKET NO. : 50-275

THERMAL POWER (MWH) :2.25E +07

COMMERCIAL OPERATION:05/07/85

COOLING WATER SOURCE:PACIFIC OCEAN

UNIT NUMBER: 2 TYPE:PWR

DOCKET NO.: 50-323

THERMAL POWER (MWH) :2.69E+07

COMMERCIAL OPERATION:03/13/86

COOLING WATER SOURCE:PACIFIC OCEAN
LICENSEE:PACIFIC GAS \& ELECTRIC CO LICENSED POWER (MWT) : $3.34 E+03$ NET ELECTRIC POWER (MWH) :7.20E+06 INITIAL CRITICALITY:04/29/84

LICENSEE:PACIFIC GAS \& ELECTRIC CO LICENSED POWER (MWT) :3.41E+03 NET ELECTRIC POWER (MWH):8.62E+06 INITIAL CRITICALITY:08/19/85

\section{AIRBORNE EFFLUENTS}

NUCLIDES RELEASED

AR -41

CO- 57

$\mathrm{CO}-58$

$\mathrm{CO}-60$

$\mathrm{KR}-85$

$\mathrm{KR}-85 \mathrm{M}$

$\mathrm{KR}-87$

$\mathrm{KR}-88$

$\mathrm{I}-131$

$\mathrm{XE}-131 \mathrm{M}$

$I-133$

$\mathrm{XE}-133$

$\mathrm{XE}-133 \mathrm{M}$

$X E-135$

$\mathrm{XE}-135 \mathrm{M}$

CS -137

$\mathrm{XE}-138$

\section{LIQUID EFFLUENTS}

NUCLIDES RELEASED

BE -7

NA -24

CR -51

$\mathrm{MN}-54$

FE -55

$\mathrm{MN}-56$

$\mathrm{CO}-57$

$\mathrm{CO}-58$

FE -59

CO- 60

$\mathrm{BR}-82$

$\mathrm{KR}-85$

SR -89

SR -91

SR-92

ZR -95

MO-99

RU-103

\author{
ACTIVITY (CI) \\ $1.94 \mathrm{E}+00$ \\ 1. $17 \mathrm{E}-06$ \\ 2. 61E-05 \\ $2.89 E-05$ \\ 3. $44 \mathrm{E}+01$ \\ 1. $30 \mathrm{E}-01$ \\ 4. $70 \mathrm{E}-02$ \\ 9.76E-02 \\ $9.15 \mathrm{E}-04$ \\ 1. $27 E+00$ \\ 4. $58 \mathrm{E}-04$ \\ $2.77 E+02$ \\ $7.45 \mathrm{E}-01$ \\ $1.91 \mathrm{E}+01$ \\ 3. 19E-02 \\ $3.93 \mathrm{E}-06$ \\ 7. $32 \mathrm{E}-02$
}

ACTIVITY (CI)

$1.21 \mathrm{E}-03$

$1.79 \mathrm{E}-04$

7. $21 \mathrm{E}-02$

$1.97 \mathrm{E}-02$

2. 61E-01

9.30E-09

$1.87 \mathrm{E}-03$

$5.43 \mathrm{E}-01$

$9.22 \mathrm{E}-03$

2. 39E-01

$1.44 E-04$

$2.04 E-03$

$3.15 \mathrm{E}-04$

$1.25 \mathrm{E}-05$

$5.83 \mathrm{E}-06$

$1.67 \mathrm{E}-02$

$7.35 \mathrm{E}-04$

1.23E-04

N/A =NOT APPLICABLE

$N / D$-NOT DETECTED

N/R=NOT REPORTED 
EFFLUENT AND WASTE DISPOSAL ANNUAL REPORT FOR YEAR 1989 AIRBORNE AND LIQUID EFFLUENTS

LIQUID EFFLUENTS

NUCLIDES RELEASED

AG-110M

SN -113

SN $-117 \mathrm{M}$

$S B-122$

$S B-124$

$\mathrm{SB}-125$

$\mathrm{TE}-129 \mathrm{M}$

I -131

I -132

TE -132

I -133

$\mathrm{XE}-133$

$\mathrm{XE}-133 \mathrm{M}$

CS -134

I -134

I -135

$\mathrm{XE}-135$

CS -136

CS -137

LA - 140

$C E-141$

CE-144

W-187

\section{ACTIVITY (CI)}

3. $15 \mathrm{E}-03$

1. $28 \mathrm{E}-03$

$2.70 \mathrm{E}-05$

1. $56 \mathrm{E}-04$

1. $28 \mathrm{E}-02$

1. $23 \mathrm{E}-01$

2. $96 \mathrm{E}-03$

8. $33 E-02$

$4.26 \mathrm{E}-06$

9.13E-0.5

4. $81 \mathrm{E}-03$

$1.49 \mathrm{E}-01$

$4.25 E-04$

$9.51 \mathrm{E}-02$

$5.70 \mathrm{E}-09$

$2.79 \mathrm{E}-04$

1. $55 \mathrm{E}-03$

4.97E-04

$1.19 \mathrm{E}-01$

$5.70 \mathrm{E}-04$

1. $80 \mathrm{E}-05$

$7.21 E-04$

$2.89 \mathrm{E}-06$

TOTAL AIRBORNE TRITIUM RELEASE 3.82E+01

TOTAL LIQUID TRITIUM RELEASE $9.35 \mathrm{E}+02$

VOLUME OF LIQUID WASTE RELEASED (PRIOR TO DILUTION) LITERS $4.48 E+08$

VOLUME OF DILUTION WATER USED DURING PERIOD

LITERS $\quad 6.87 \mathrm{E}+11$ 
INSTALLATION : DIABLO CANYON

EFFLUENT AND WASTE DISPOSAL ANNUAL REPORT FOR YEAR 1989

SOLID EFFLUENTS

SOLID WASTE DISPOSITION

NUMBER OF SHIPMENTS

35

10

OF TRANSPORTATION

TRUCK

TRUCK

IRRADIATED FUEL SHIPMENTS (DISPOSITION)

NUMBER OF SHIPMENTS MODE OF TRANSPORTATION

N/A

ESTIMATE OF MAJOR NUCLIDE COMPOSITION (BY TYPE OF WASTE)

A

CO- 58

CO- 60

CR-51

CS -134

CS -137

FE -55

B

NI -63

CO- 58

CO- 60

FE- 55

H -3

NI -63
DESTINATION

BARNWELL SC

RICHLAND WA
DESTINATION
N/A
TYPE OF WASTE
A. SPENT RESINS, FILTER SLUDGES, EVAPORATOR BOTTOMS, ETC.
B. DRY COMPRESSIBLE WASTE, CONTAMINATED EQUIPMENT, ETC.
C. IRRADIATED COMPONENTS, CONTROL RODS, ETC.
D. OTHER

JAN-JUNE JULY-DEC

$\begin{array}{lll}8 & & 7.47 \mathrm{E}+00 \\ 8 & 3.30 \mathrm{E}+01 & 8.11 \mathrm{E}+00 \\ 3.39 \mathrm{E}+00 \\ 8 & 1.60 \mathrm{E}+01 & 2.26 \mathrm{E}+00 \\ 8 & 1.78 \mathrm{E}+01 & 3.70 \mathrm{E}+00 \\ 8 & & 6.55 \mathrm{E}+01 \\ 8 & 1.77 \mathrm{E}+01 & 3.94 \mathrm{E}+00 \\ & & \\ 8 & & 4.54 \mathrm{E}+00 \\ 8 & 1.50 \mathrm{E}+01 & 7.89 \mathrm{E}+00 \\ 8 & 8.30 \mathrm{E}+01 & 7.82 \mathrm{E}+01 \\ 8 & & 1.20 \mathrm{E}+00 \\ 8 & & 4.63 \mathrm{E}+0 \mathrm{C}\end{array}$

UNIT YEAR TOTAL

M3 $3.19 \mathrm{E}+01$

CI $4.20 \mathrm{E}+02$

M3 $1.55 \mathrm{E}+02$

CI $\quad 8.83 E+00$

M3

CI

M3

CI 
EFFLUENT AND WASTE DISPOSAL ANNUAL REPORT FOR YEAR 1989 AIRBORNE AND LIQUID EFFLUENTS
UNIT NUMBER: 1 TYPE:BWR
DOCKET NO.: $50-10$
THERMAL POWER (MWH):
COMMERCIAL OPERATION:07/04/60
COOLING WATER SOURCE:KANKAKEE RIVER

$\begin{array}{lc}\text { AIRBORNE EFFLUENTS } & \\ \text { NUCLIDES RELEASED } & \text { ACTIVITY (CI) } \\ \text { MN }-54 & 1.26 \mathrm{E}-05 \\ \text { FE }-55 & 5.08 \mathrm{E}-06 \\ \text { CO-60 } & 2.90 \mathrm{E}-05 \\ \text { SR }-89 & 1.76 \mathrm{E}-05 \\ \text { SR }-90 & 8.75 \mathrm{E}-08 \\ \text { CS }-137 & 4.26 \mathrm{E}-05\end{array}$

LICENSEE : COMMON WEALTH EDISON

LICENSED POWER (MWT) : $7.00 E+02$

NET ELECTRIC POWER (MWH): 
EFFLUENT AND WASTE DISPOSAL ANNUAL REPORT FOR YEAR 1989 AIRBORNE AND LIQUID EFFLUENTS

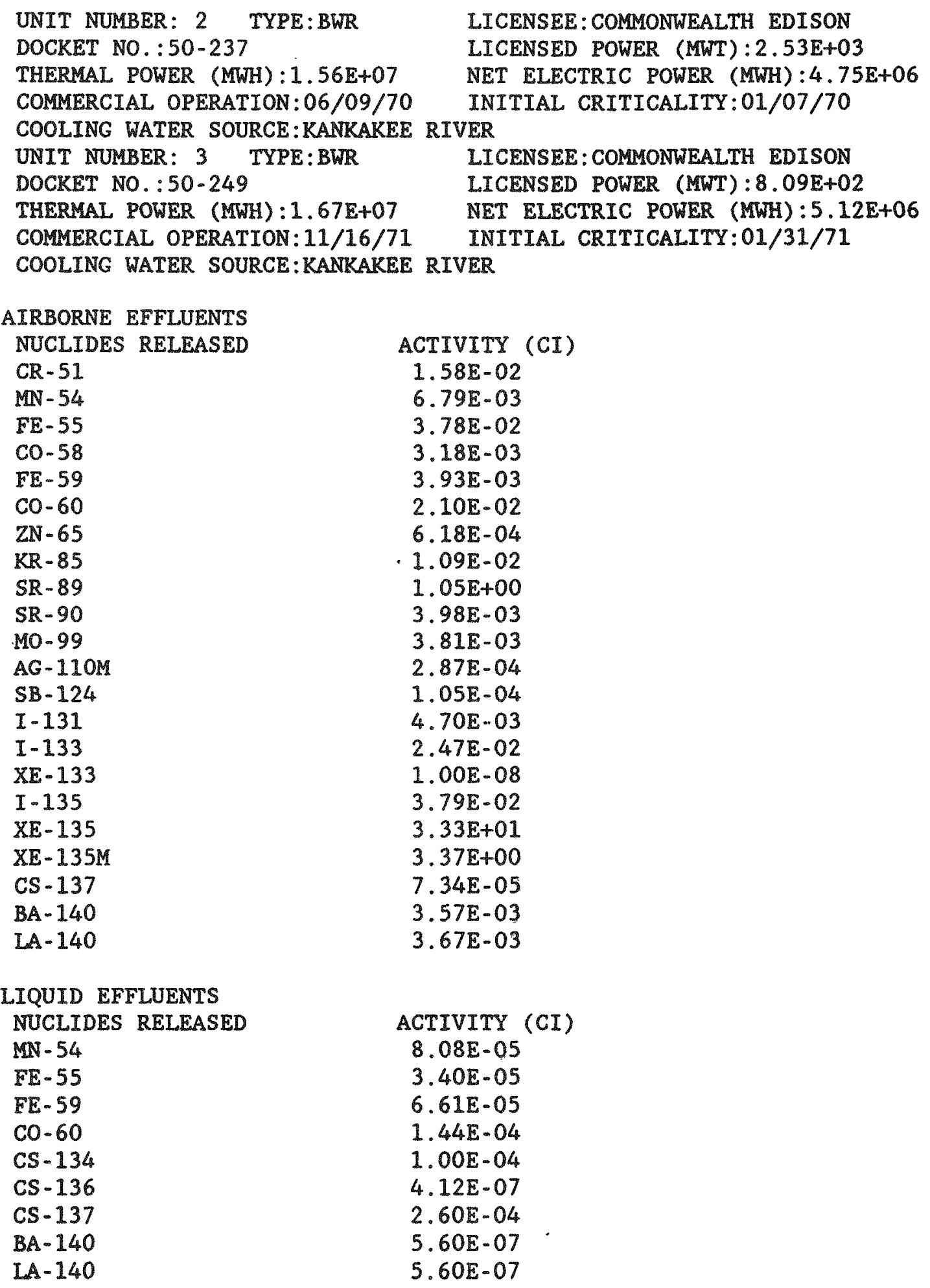


EFFLUENT AND WASTE DISPOSAL ANNUAL REPORT FOR YEAR 1989 AIRBORNE AND LIQUID EFFLUENTS

\begin{tabular}{|c|c|}
\hline UNTT NIIMBFR - & \\
\hline DOCKET NO.: $50-10$ & LICENSED POWER (MWT) $: 7.00 E+02$ \\
\hline THERMAL POWER (MWH): & NET ELECTRIC POWER (MWH): \\
\hline COMMERCIAL OPERATION:07/04/60 & INITIAL CRITICALITY:10/15/59 \\
\hline COOLING WATER SOURCE:KANKAKEE & VER \\
\hline UNIT NUMBER: 2 TYPE:BWR & LICENSEE : COMMONWEALTH EDISON \\
\hline DOCKET NO.: $50-237$ & LICENSED POWER (MWT): $2.53 E+03$ \\
\hline THERMAL POWER (MWH) :1.56E+07 & NET ELECTRIC POWER (MWH) :4.75E+06 \\
\hline COMMERCIAL OPERATION:06/09/70 & INITIAL CRITICALITY:01/07/70 \\
\hline COOLING WATER SOURCE:KANKAKEE & VER \\
\hline UNIT NUMBER: 3 TYPE:BWR & LICENSEE : COMMONWEALTH EDISON \\
\hline DOCKET NO.: $50-249$ & LICENSED POWER (MWT):8.09E+02 \\
\hline THERMAL POWER (MWH) :1.67E+07 & NET ELECTRIC POWER (MWH) :5.12E+06 \\
\hline COMMERCIAL OPERATION: $11 / 16 / 71$ & INITIAL CRITICALITY:01/31/71 \\
\hline COOLING WATER SOURCE:KANKAKEE & VER \\
\hline LIQUID EFFLUENTS & \\
\hline NUCLIDES RELEASED & $\operatorname{VITY}(C I)$ \\
\hline$C R-51$ & $2 E-03$ \\
\hline$M N-54$ & $6 \mathrm{E}-01$ \\
\hline$F E-55$ & $6 \mathrm{E}-02$ \\
\hline $\mathrm{Co}-58$ & $3 E-03$ \\
\hline$F E-59$ & $3 E-03$ \\
\hline $\mathrm{co}-60$ & $1 E-01$ \\
\hline CU-64 & $5 E-06$ \\
\hline $\mathrm{ZN}-65$ & OE-O5 \\
\hline AS - 76 & $1 E-05$ \\
\hline$K R-88$ & $4 E-05$ \\
\hline$R B-88$ & $7 E-04$ \\
\hline $\mathrm{SR}-90$ & $5 E-04$ \\
\hline NB-95 & $1 E-05$ \\
\hline TC-99M & $7 E-06$ \\
\hline$S B-124$ & $3 E-02$ \\
\hline$X E-133$ & $0 E-04$ \\
\hline $\operatorname{CS}-134$ & $1 E-05$ \\
\hline$I-135$ & OE -05 \\
\hline$X E-135$ & $1 E-04$ \\
\hline $\mathrm{CS}-137$ & $2 E-02$ \\
\hline $\operatorname{CS}-138$ & $2 E-05$ \\
\hline$L A-140$ & $2 E-06$ \\
\hline $\mathrm{MF}-181$ & $7 \mathrm{E}-05$ \\
\hline
\end{tabular}

TOTAL AIRBORNE TRITIUM RELEASE TOTAL LIQUID TRITIUM RELEASE
$1.46 \mathrm{E}+01$

$1.83 \mathrm{E}+01$
VOLUME OF LIQUID WASTE RELEASED (PRIOR TO DILUTION) VOLUME OF DILUTION WATER USED DURING PERIOD
LITERS $2.60 \mathrm{E}+07$

LITERS $9.53 \mathrm{E}+10$

N/A=NOT APPLICABLE 
INSTALLATION:DRESDEN $1,2 \& 3$

EFFLUENT AND WASTE DISPOSAL ANNUAL REPORT FOR YEAR 1989

SOLID EFFLUENTS

SOLID WASTE DISPOSITION NUMBER OF SHIPMENTS 66

10

12

13

11
MODE OF TRANSPORTATION

MOTOR FREIGHT(EXCL. USE)

MOTOR FREIGHT (EXCL. USE)

MOTOR FREIGHT (EXCL. USE)

MOTOR FREIGHT(EXCL. USE)

MOTOR FREIGHT(EXCL. USE)

ESTIMATE OF MAJOR NUCLIDE COMPOSITION (BY TYPE OF WASTE)

A

Co -60

CS -137

FE -55

MN -54

NI -63

OTHER

B

Co- 60

CS -137

FE- 55

MN- 54

OTHER

C

Co- 60

CS -137

FE -55

MN -54

TYPE OF WASTE

A. SPENT RESINS, FILTER SLUDGES, EVAPORATOR BOTTOMS, ETC.

B. DRY COMPRESSIBLE WASTE, CONTAMINATED EQUIPMENT, ETC.

C. IRRADIATED COMPONENTS, CONTROL RODS, ETC.

D. OTHER

DESTINATION

BARNWELL SC

CNSI, CHANNON IL

RICHLAND WA

SEG, OAK RIDGE TN

WEST. DDR, MADISON PA

JAN-JUNE JULY-DEC

$\begin{array}{lll} & 6.72 \mathrm{E}+01 & 5.41 \mathrm{E}+01 \\ & & 1.09 \mathrm{E}+01 \\ & 1.89 \mathrm{E}+01 & 2.12 \mathrm{E}+01 \\ & 1.21 \mathrm{E}+01 & 5.64 \mathrm{E}+00 \\ 8 & 8.05 \mathrm{E}-01 & 6.46 \mathrm{E}+00 \\ & 9.95 \mathrm{E}-01 & \\ & 2.66 \mathrm{E}+01 & 2.53 \mathrm{E}+01 \\ 8 & 9.81 \mathrm{E}-01 & 1.18 \mathrm{E}+01 \\ 8 & 6.31 \mathrm{E}+01 & 5.99 \mathrm{E}+01 \\ 8 & 7.99 \mathrm{E}+00 & 1.14 \mathrm{E}+00 \\ & 1.33 \mathrm{E}+00 & \\ & & \\ 8 & & 2.02 \mathrm{E}+01 \\ 8 & & 6.38 \mathrm{E}+00 \\ 8 & & 1.72 \mathrm{E}+00\end{array}$

UNIT YEAR TOTAL

M3 $3.64 \mathrm{E}+02$

CI $2.50 \mathrm{E}+03$

M3 $1.86 \mathrm{E}+03$

CI $\quad 3.38 \mathrm{E}+01$

M3 $1.42 \mathrm{E}+01$

CI $3.97 \mathrm{E}+00$

M3

CI 
EFFLUENT AND WASTE DISPOSAL ANNUAL REPORT FOR YEAR 1989 AIRBORNE AND LIQUID EFFLUENTS
UNIT NUMBER: 1 TYPE:BWR
LICENSEE: IOWA ELECTRIC LIGHT \& POWER
DOCKET NO.: 50-331 LICENSED POWER (MWT) $: 1.66 \mathrm{E}+03$
THERMAL POWER (MWH) : $1.00 E+07$
COMMERCIAL OPERATION:02/01/75
NET ELECTRIC POWER (MWH) :3.14E+06
COOLING WATER SOURCE:CEDAR RAPIDS RIVER
AIRBORNE EFFLUENTS
NUCLIDES RELEASED
ACTIVITY (CI)
$\mathrm{N}-13$
CR -51
$2.17 \mathrm{E}+00$
$\mathrm{MN}-54$
1. $37 \mathrm{E}-03$
CO- 58
$1.46 \mathrm{E}-04$
Co- 60
$2.20 \mathrm{E}-04$
KR -85
SR -89
1. $16 \mathrm{E}-03$
SR -90
2. 24E-03
I- 131
$2.68 \mathrm{E}-05$
I- 132
$1.27 \mathrm{E}-06$
$1.85 \mathrm{E}-04$
I -133
3. $30 \mathrm{E}-06$
$\mathrm{XE}-133$
$2.69 \mathrm{E}-04$
CS -134
1. $10 \mathrm{E}+00$
I -134
1. 58E-05
I-135
3. 10E-05
$\mathrm{XE}-135$
$6.55 E-05$
$\mathrm{XE}-135 \mathrm{M}$
1. $85 \mathrm{E}+01$
CS-137
2. $20 E+01$
BA-LA - 140
3. $41 E-05$
CE- 141
$4.20 \mathrm{E}-07$
$2.80 \mathrm{E}-07$

TOTAL AIRBORNE TRITIUM RELEASE 1.66E+01

TOTAL LIQUID TRITIUM RELEASE

$0.00 E+00$

VOLUME OF LIQUID WASTE RELEASED (PRIOR TO DILUTION)

VOLUME OF DILUTION WATER USED DURING PERIOD

LITERS N/A

LITERS N/A 
EFFLUENT AND WASTE DISPOSAL ANNUAL REPORT FOR YEAR 1989 SOLID EFFLUENTS

SOLID WASTE DISPOSITION

$\begin{array}{cl}\text { NUMBER OF SHIPMENTS } & \text { MODE OF TRANSPORTATION } \\ 12 & \text { TRUCK(EXCL. USE) } \\ 3 & \text { TRUCK (EXCL. USE) } \\ 2 & \text { TRUCK (EXCL. USE) }\end{array}$

DESTINATION

BARNWELL SC

OAK RIDGE TN

RICHLAND WA

ESTIMATE OF MAIOR NUCLIDE COMPOSITION (BY TYPE OF WASTE)

JAN-JUNE JULY-DEC

A

AG-110M, I-134, NB-94, RH-105, SB-125, SR-90, ZN-65

C. 14

CM- 242

$\mathrm{CO}-58$

CO- 60

$\mathrm{CR}-51$

CS -134

CS -137

$\mathrm{FE}-55$

$\mathrm{H}-3$

$\mathrm{MN}-54$

NI -63

PU -241

SR-90, FE- 59, CO-57, ZN-65, RH- 105, NI-63, SR-89

TRU

B

C -14

CE-144, CR-51, CO-58, CS-137, CS-134, FE- 59, NI - 59,

$\mathrm{SR}-90, \mathrm{ZN}-65$

CM- 242

$\mathrm{CO}-58$

CO- 60

$\mathrm{CR}-51$

$\mathrm{CS}-137, \mathrm{CS}-134, \mathrm{zN}-65$

FE -55

FE- 59

I -129

MN -54

NI -63

PU. 241

TC- 99

TRU

홍

1.47E-02

8.21E-01

$6.35 E-05$

8.93E-02

3. $61 \mathrm{E}+00$

$2.53 E+00$

$2.48 \mathrm{E}+01$

$4.08 \mathrm{E}+01$

$2.89 E+00$

1. $22 \mathrm{E}+00$

1.11E+01

$2.89 E+00$

$9.69 E+00$

4. $18 \mathrm{E}+00$

$3.55 \mathrm{E}+01$

$3.99 E+01$

$6.80 \mathrm{E}-03$

2. 70E-02

1. $10 \mathrm{E}+01$

$5.55 \mathrm{E}+00$

7. $18 \mathrm{E}-03$

1. $99 E+00$

1. $36 \mathrm{E}+00$

$6.69 E-06$

$3.40 \mathrm{E}-04$

$5.86 E-04$

8

$7.89 E-01$

3. 77E-01

4. 89E-03

1. $36 E-04$

1. $71 E+00$

$3.06 E+01$

$2.30 E+01$

$2.16 E+01$

$1.19 \mathrm{E}+00$

$3.11 E+01$

9.41E-01

$7.07 E+01$

$9.38 \mathrm{E}+00$

6. $60 \mathrm{E}-06$

$2.97 E+00$

3. $88 \mathrm{E}+00$

4.89E-01

1. $33 E+00$

$4.892-01$

2.11E-03

$9.57 E-03$

1. $84 \mathrm{E}-05$

C

C. 14

CM- 242

CO- 60

$1.27 \mathrm{E}-03$

8.67E-08

$4.28 \mathrm{E}+01$ 
INSTALLATION : DUANE ARNOLD

EFFLUENT AND WASTE DISPOSAL ANNUAL REPORT FOR YEAR 1989 SOLID EFFLUENTS

ESTIMATE OF MAJOR NUCLIDE COMPOSITION (BY TYPE OF WASTE)

JAN-JUNE JULY-DEC

$\mathrm{C}$

$\mathrm{CS}-137, \mathrm{NB}-94, \mathrm{NI}-59, \mathrm{SR}-90$

$\mathrm{FE}-55$

$\mathrm{H}-3$

I-129

MN -54

NI -63

PU -24.1

TC-99

TRU

D

CE-144, CS $-134, C S-137, C R-51,2 N-65$

CM- 242

$\mathrm{CO}-58$

CO- 60

CR- 51

$\mathrm{CS}-137, \mathrm{CS}-134, \mathrm{ZN}-65$

$\mathrm{FE}-55$

FE -59

MN -54

NI -63

PU -241

TRU

TYPE OF WASTE

A. SPENT RESINS, FILTER SLUDGES, EVAPORATOR BOTTOMS, ETC.

B. DRY COMPRESSIBLE WASTE, CONTAMINATED EQUIPMENT, ETC.

C. IRRADIATED COMPONENTS, CONTROL RODS, ETC.

D. OTHER

WET TRASH

\begin{tabular}{|c|c|c|}
\hline & & $5.34 E-02$ \\
\hline 8 & & $4.87 E+01$ \\
\hline & & $1.73 E-02$ \\
\hline & & $4.60 E-1$ \\
\hline 8 & & $4.84 E+0$ \\
\hline & & $3.59 E+0$ \\
\hline 8 & & $6.98 \mathrm{E}-0$ \\
\hline 8 & & $5.11 E-0$ \\
\hline 8 & & $8.86 E-0$ \\
\hline 8 & & $8.00 E-0$ \\
\hline 8 & $4.90 E-03$ & $3.77 \mathrm{E}-0$ \\
\hline 응 & $1.71 E+00$ & $1.59 \mathrm{E}+0$ \\
\hline 8 & $3.06 \mathrm{E}+01$ & $1.76 \mathrm{E}+0$ \\
\hline z & $2.17 E+01$ & \\
\hline क & $1.20 \mathrm{E}+00$ & \\
\hline 8 & $3.10 \mathrm{E}+01$ & $5.94 E+0$ \\
\hline$\frac{8}{8}$ & $9.40 \mathrm{E}-01$ & $1.94 E+0$ \\
\hline 8 & $9.40 \mathrm{E}+00$ & $5.14 \mathrm{E}+0$ \\
\hline 8 & $2.98 \mathrm{E}+00$ & $1.35 \mathrm{E}+0$ \\
\hline 8 & $4.90 \mathrm{E}-01$ & $3.51 E-0$ \\
\hline & $9.56 \mathrm{E}-03$ & $1.07 E-0$ \\
\hline
\end{tabular}

UNIT YEAR TOTAL

M3 $5.63 \mathrm{E}+01$

CI $\quad 9.78 \mathrm{E}+02$

M3 $7.87 \mathrm{E}+01$

CI $4.91 \mathrm{E}+01$

M3 $\quad 1.63 E+00$

CI $\quad 1.59 \mathrm{E}+04$

M3 $9.73 \mathrm{E}+00$

CI $\quad 1.17 \mathrm{E}+00$ 
EFFLUENT AND WASTE DISPOSAL ANNUAL REPORT FOR YEAR 1989 AIRBORNE AND LIQUID EFFLUENTS

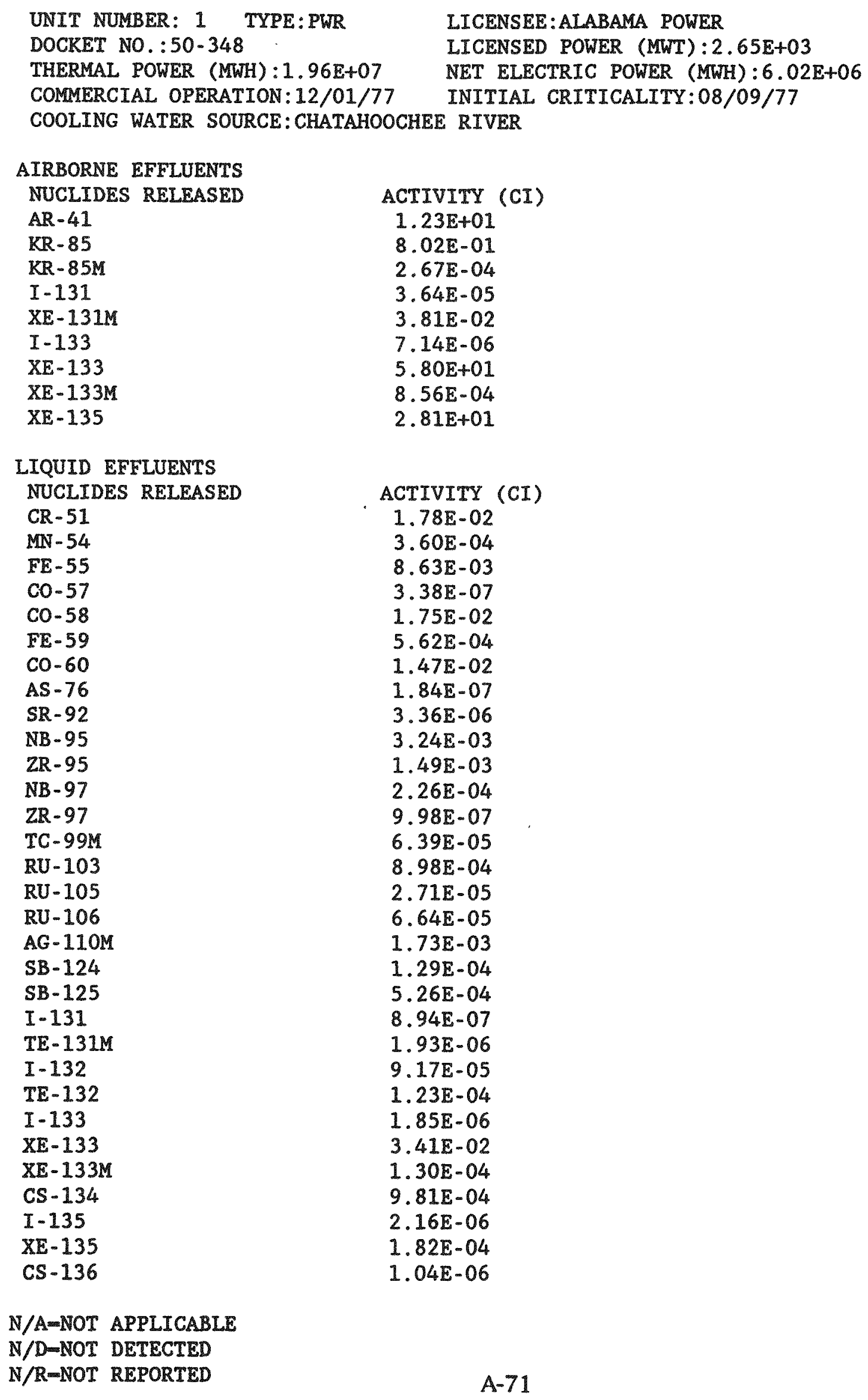


EFFLUENT AND WASTE DISPOSAL ANNUAL REPORT FOR YEAR 1989 AIRBORNE AND LIQUID EFFLUENTS

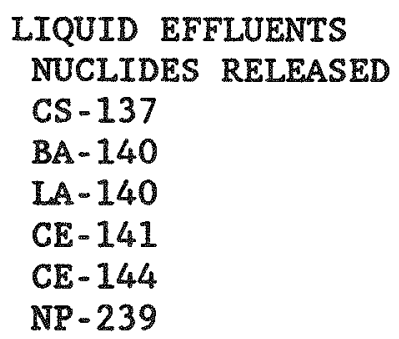

TOTAL AIRBORNE TRITIUM RELEASE $1.15 E+02$

TOTAL LIQUID TRITIUM RELEASE $6.99 \mathrm{E}+02$

$$
\begin{aligned}
& \text { ACTIVITY (CI) } \\
& 3.95 E-03 \\
& 1.01 E-06 \\
& 6.34 E-06 \\
& 4.85 E-06 \\
& 7.82 E-06 \\
& 2.16 E-05
\end{aligned}
$$
$\begin{array}{lllll}\text { VOLUME OF LIQUID WASTE RELEASED (PRIOR TO DILUTION) } & \text { LITERS } & 3.84 E+08 \\ \text { VOLUME OF DILUTION WATER USED DURING PERIOD } & \text { LITERS } & 5.78 E+10\end{array}$ 
EFFLUENT AND WASTE DISPOSAL ANNUAL REPORT FOR YEAR 1989 AIRBORNE AND LIQUID EFFLUENTS

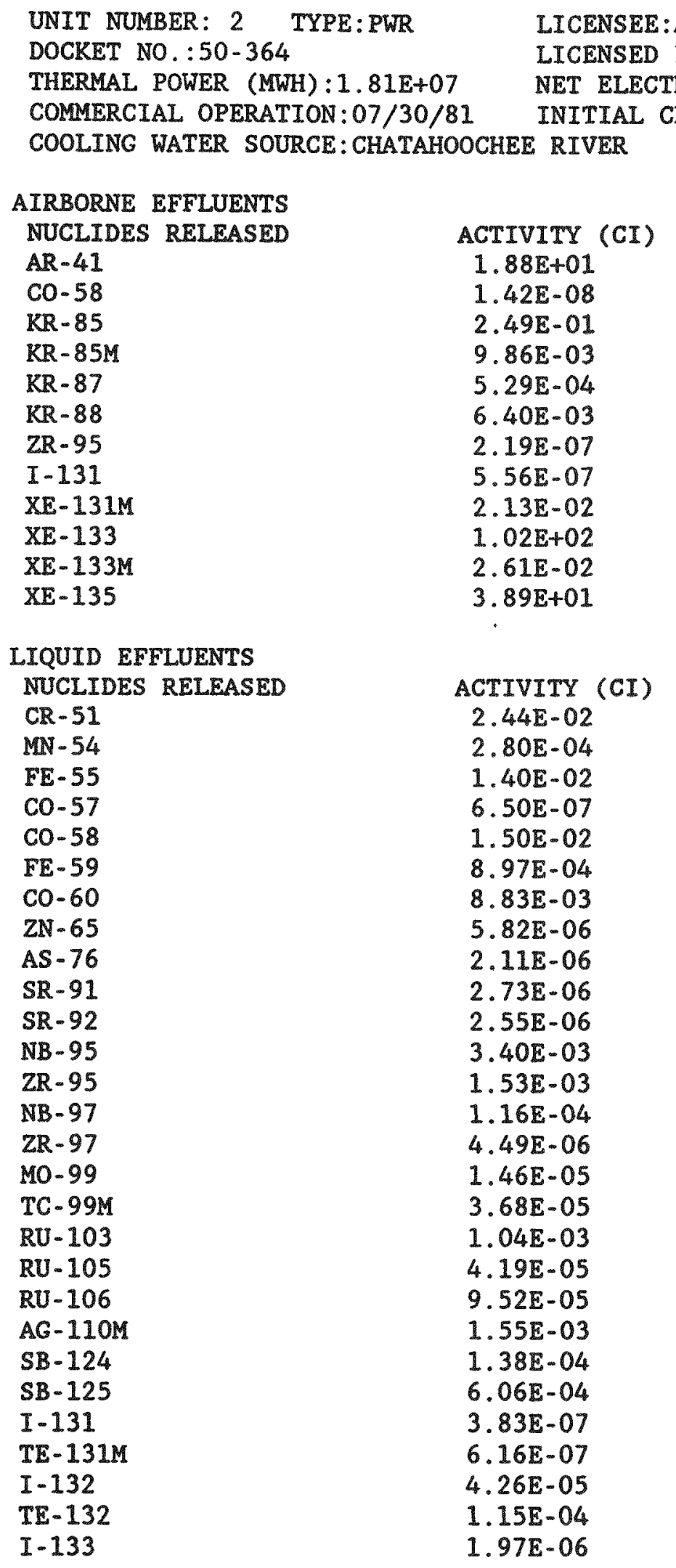


EFFLUENT AND WASTE DISPOSAL ANNUAL REPORT FOR YEAR 1989 AIRBORNE AND LIQUID EFFLUENTS

LIQUID EFFLUENTS
NUCLIDES RELEASED
XE-133
XE $-133 \mathrm{M}$
$\mathrm{CS}-134$
$\mathrm{I}-135$
$\mathrm{XE}-135$
$\mathrm{CS}-136$
$\mathrm{CS}-137$
$\mathrm{LA}-140$
$\mathrm{CE}-141$
$\mathrm{~W}-187$
NP -239

LIQUID EFFLUENTS

$\mathrm{XE}-133$

$\mathrm{XE}-133 \mathrm{M}$

CS -134

I- 135

$\mathrm{XE}-135$

CS -136

CS -137

LA -140

W-187

NP -239
ACTIVITY (CI)
$2.64 \mathrm{E}-02$
$7.57 \mathrm{E}-05$
3. $60 \mathrm{E}-04$
1. $90 \mathrm{E}-06$
$1.73 E-04$
$9.77 E-07$
8.18E- 04
$2.80 \mathrm{E}-06$
4. 20E-05
1.63E-06
$3.76 E-05$

TOTAL AIRBORNE TRITIUM RELEASE $9.33 E+01$

TOTAL LIQUID TRITIUM RELEASE $6.08 \mathrm{E}+02$

VOLUME OF LIQUID WASTE RELEASED (PRIOR TO DILUTION) LITERS 4.13E+08

VOLUME OF DILUTION WATER USED DURING PERIOD

LITERS $\quad 5.97 \mathrm{E}+10$ 
INSTALLATION: JOSEPH M. FARLEY

EFFLUENT AND WASTE DISPOSAL ANNUAL REPORT FOR YEAR 1989 SOLID EFFLUENTS

SOLID WASTE DISPOSITION NUMBER OF SHIPMENTS 10

MODE OF TRANSPORTATION CHEM-NUC TRANSPORT

19 HITTMAN TRANSPORT HITTMAN TRANSPORT

ESTIMATE OF MAJOR NUCLIDE COMPOSITION (BY TYPE OF WASTE)

DESTINATION BARNWELL SC BARNWELL SC RICHLAND WA

JAN-JUNE JULY-DEC

A

C- 14

$\mathrm{CO}-58$

CO-60

CR- 51

CS -134

CS -137

FE- 55

H- 3

MN -54

NB -95

NI -63

SB-124

B

C. 14

$\mathrm{CE}-144$

CO- 58

CO- 60

CR- 51

CS -137

FE -55

MN -54

NB- 95

NI -63

PU-241

ZR-95

$\begin{array}{lll} & & 1.67 \mathrm{E}+01 \\ & 1.15 \mathrm{E}+00 & 1.42 \mathrm{E}+01 \\ & 5.09 \mathrm{E}+01 & 4.98 \mathrm{E}+00 \\ & 2.65 \mathrm{E}+00 & 1.78 \mathrm{E}+00 \\ & 4.96 \mathrm{E}+00 & 3.08 \mathrm{E}+00 \\ & 4.83 \mathrm{E}+00 & 6.95 \mathrm{E}+00 \\ & & 2.00 \mathrm{E}+01 \\ & & 1.76 \mathrm{E}+01 \\ 8 & 3.04 \mathrm{E}+01 & 1.03 \mathrm{E}+01 \\ & 1.86 \mathrm{E}+00 & \\ & 1.18 \mathrm{E}+00 & 1.71 \mathrm{E}+00 \\ 8 & 2.77 \mathrm{E}+00 & \\ 8 & 1.45 \mathrm{E}+01 & 8.31 \mathrm{E}+00 \\ & 1.95 \mathrm{E}+01 & 2.89 \mathrm{E}+01 \\ 8 & 5.22 \mathrm{E}+00 & \\ 8 & 3.12 \mathrm{E}+01 & 4.79 \mathrm{E}+00 \\ 8 & 1.98 \mathrm{E}+00 & \\ 8 & 7.14 \mathrm{E}+00 & 1.01 \mathrm{E}+00 \\ 8 & 4.66 \mathrm{E}+00 & 6.45 \mathrm{E}+00 \\ 8 & 3.19 \mathrm{E}+00 & \\ 8 \mathrm{E}+00 & \end{array}$

UNIT YEAR TOTAL

M3 $1.34 \mathrm{E}+02$

CI $3.88 \mathrm{E}+02$

M3 $3.51 E+02$

CI $1.23 \mathrm{E}+01$

M3

CI

M3

CI 


\section{EFFLUENT AND WASTE DISPOSAL ANNUAL REPORT FOR YEAR 1989} AIRBORNE AND LIQUID EFFLUENTS

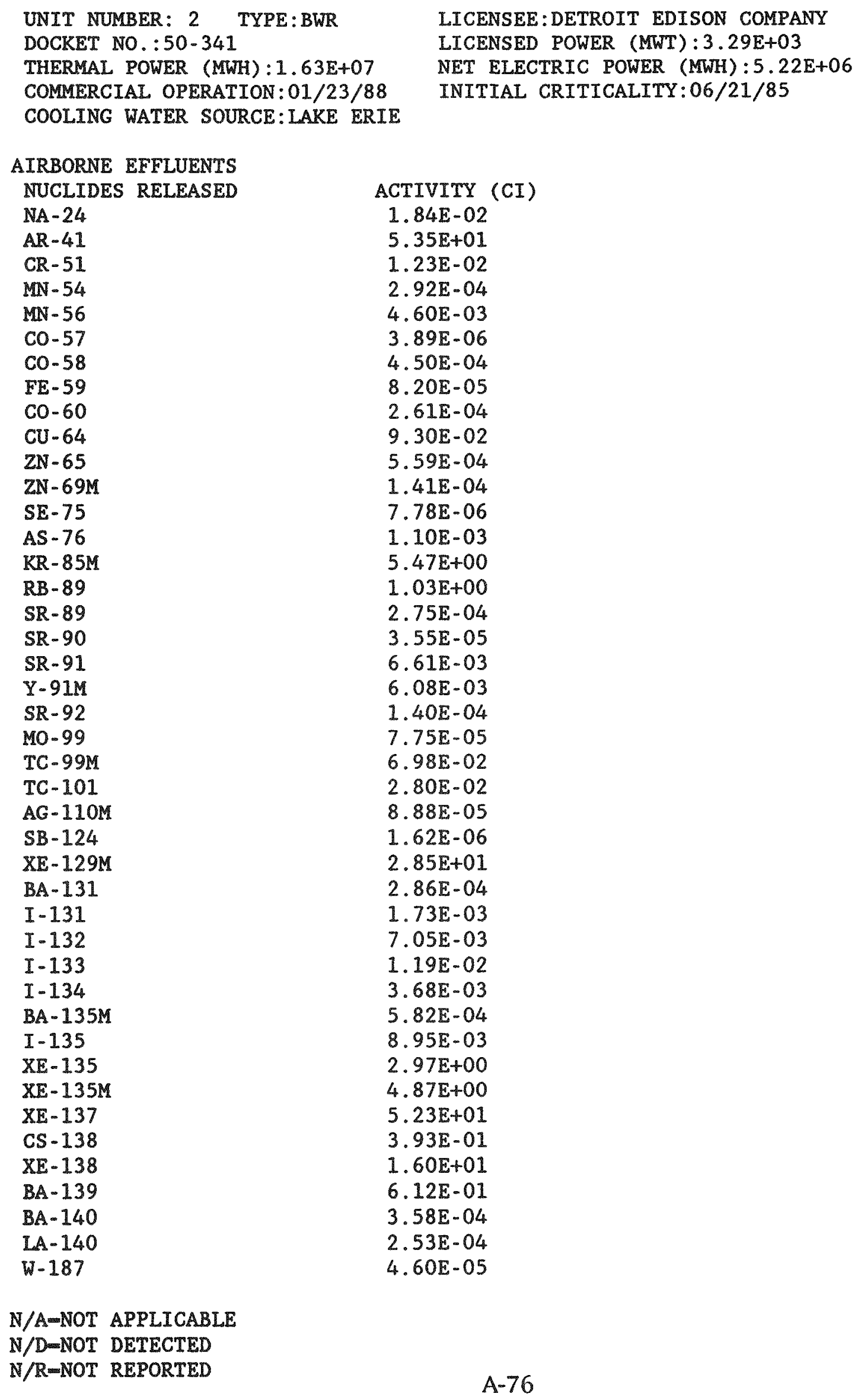


EFFLUENT AND WASTE DISPOSAL ANNUAL REPORT FOR YEAR 1989 AIRBORNE AND LIQUID EFFLUENTS

$\begin{array}{lc}\text { AIRBORNE EFFLUENTS } & \\ \text { NUCLIDES RELEASED } & \text { ACTIVITY (CI) } \\ \text { RE-188 } & 8.00 \mathrm{E}-04 \\ & \\ \text { LIQUID EFFLUENTS } & \\ \text { NUCLIDES RELEASED } & \text { ACTIVITY (CI) } \\ \text { NA-24 } & 1.09 E-02 \\ \text { CR-51 } & 6.31 E-02 \\ \text { MN-54 } & 2.31 E-02 \\ \text { FE-55 } & 3.64 E-03 \\ \text { CO-57 } & 4.17 E-06 \\ \text { CO-58 } & 1.59 E-02 \\ \text { FE-59 } & 3.88 E-03 \\ \text { CO-60 } & 1.75 E-02 \\ \text { ZN-65 } & 1.80 E-02 \\ \text { AS-76 } & 5.63 E-04 \\ \text { SR-89 } & 1.99 E-04 \\ \text { SR-90 } & 1.01 E-05 \\ \text { MO-99 } & 1.28 E-03 \\ \text { TC-99M } & 4.20 E-03 \\ \text { AG-110M } & 5.98 E-04 \\ \text { SB-124 } & 1.31 E-04 \\ \text { SB-125 } & 6.45 E-05 \\ \text { BA-131 } & 3.92 E-03 \\ \text { I-131 } & 6.18 E-05 \\ \text { BA-133 } & 3.32 E-05 \\ \text { I-133 } & 3.02 E-04 \\ \text { XE-133 } & 5.90 E-05 \\ \text { BA-135M } & 4.55 E-05 \\ \text { XE-135 } & 5.64 E-05 \\ \text { W-187 } & 3.42 E-05 \\ \text { RE-188 } & 3.92 E-04 \\ & \end{array}$

TOTAL AIRBORNE TRITIUM RELEASE $0.00 E+00$

TOTAL LIQUID TRITIUM RELEASE $1.30 \mathrm{E}+00$

VOLUME OF LIQUID WASTE RELEASED (PRIOR TO DILUTION) LITERS $2.61 E+06$

$\begin{array}{lll}\text { VOLUME OF DILUTION WATER USED DURING PERIOD } & \text { LITERS } & 3.71 E+10\end{array}$ 
EFFLUENT AND WASTE DISPOSAL ANNUAL REPORT FOR YEAR 1989 SOLID EFFLUENTS

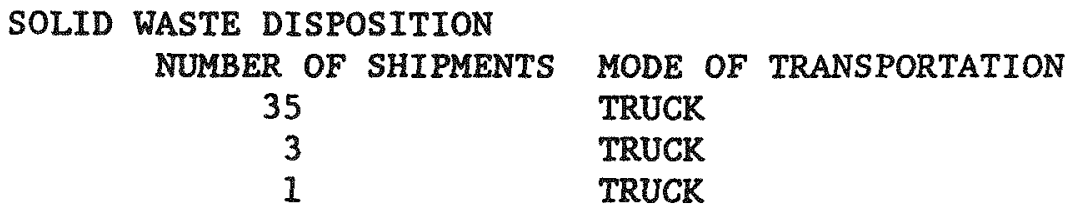

ESTIMATE OF MAJOR NUCLIDE COMPOSITION (BY TYPE OF WASTE)

A
AG-110M
$\mathrm{BA}-131$
C - 14
$\mathrm{CE}-144$
CO- 58
CO -60
CR -51
FE -55
$F E-59$
H- 3
I -129
MN- 54
NB. 95
NI -63
SN-113
TC-99
ZN- 65
ZR-95
C- 14
Co- 58
CO- 60
CR- 51
CS -137
FE-55
FE- 59
MN- 54
NI -63
ZN-65

B

TYPE OF WASTE
A. SPENT RESINS, FILTER SLUDGES, EVAPORATOR BOTTOMS, ETC.
B. DRY COMPRESSIBLE WASTE, CONTAMINATED EQUIPMENT, ETC.
C. IRRADIATED COMPONENTS, CONTROL RODS, ETC.
D. OTHER

\begin{abstract}
DESTINATION
BARNWELL SC

CHANNAHON IL

OAK RIDGE TN
\end{abstract}

JAN-JUNE JULY-DEC

$\begin{array}{rrr}8 & 6.00 \mathrm{E}-01 & 1.20 \mathrm{E}+00 \\ 8 & <1.00 \mathrm{E}-01 & \\ 8 & 3.00 \mathrm{E}-01 & <1.00 \mathrm{E}-01 \\ 8 & & <1.00 \mathrm{E}-01 \\ 8 & 8.30 \mathrm{E}+00 & 5.30 \mathrm{E}+00 \\ 8 & 7.30 \mathrm{E}+00 & 3.90 \mathrm{E}+00 \\ 8 & 3.62 \mathrm{E}+01 & 6.37 \mathrm{E}+01 \\ 8 & 2.73 \mathrm{E}+01 & 1.16 \mathrm{E}+01 \\ 8 & 5.00 \mathrm{E}-01 & <1.00 \mathrm{E}-01 \\ 8 & <1.00 \mathrm{E}-01 & <1.00 \mathrm{E}-01 \\ 8 & <1.00 \mathrm{E}-01 & <1.00 \mathrm{E}-01 \\ 8 & 9.40 \mathrm{E}+00 & 2.90 \mathrm{E}+00 \\ 8 & 4.00 \mathrm{E}-01 & <1.00 \mathrm{E}-01 \\ 8 & 2.00 \mathrm{E}-01 & \\ 8 & & 3.00 \mathrm{E}-01 \\ 8 & <1.00 \mathrm{E}-01 & <1.00 \mathrm{E}-01 \\ 8 & 9.50 \mathrm{E}+00 & 1.10 \mathrm{E}+01 \\ 8 & 2.00 \mathrm{E}-01 & <1.00 \mathrm{E}-01 \\ & & \\ 8 & 1.16 \mathrm{E}+01 & 3.10 \mathrm{E}+00 \\ 8 & 6.60 \mathrm{E}+00 & 2.60 \mathrm{E}+00 \\ 8 & 1.02 \mathrm{E}+01 & 1.24 \mathrm{E}+01 \\ 8 & 8.70 \mathrm{E}+00 & 3.10 \mathrm{E}+00 \\ 8 & <1.00 \mathrm{E}-01 & \\ 8 & 3.88 \mathrm{E}+01 & 6.65 \mathrm{E}+01 \\ 8 & <1.00 \mathrm{E}-01 & 1.60 \mathrm{E}+00 \\ 8 & 1.46 \mathrm{E}+01 & 9.00 \mathrm{E}+00 \\ 8 & 3.00 \mathrm{E}+00 & 8.00 \mathrm{E}-01 \\ 8 & 6.40 \mathrm{E}+00 & 1.00 \mathrm{E}+00\end{array}$

UNIT YEAR TOTAL

M3 2.83E+02

CI 7.01E+02

M3 8.29E+01*

CI 4.71E-01

M3

CI

M3

*VOLUME PRIOR TO REDUCTION

N/A-NOT APPLICABLE

N/D-NOT DETECTED

$N / R=N O T$ REPORTED 
EFFLUENT AND WASTE DISPOSAL ANNUAL REPORT FOR YEAR 1989

AIRBORNE AND LIQUID EFFLUENTS

TOTAL AIRBORNE TRITIUM RELEASE $1.26 \mathrm{E}+01$

TOTAL LIQUID TRITIUM RELEASE 7.32E-01

VOLUME OF LIQUID WASTE RELEASED (PRIOR TO DILUTION)

VOLUME OF DILUTION WATER USED DURING PERIOD
LITERS

LITERS
$3.40 E+05$

$7.43 E+11$ 
INSTALLATION:JAMES A. FITZPATRICK

EFFLUENT AND WASTE DISPOSAL ANNUAL REPORT FOR YEAR 1989 SOLID EFFLUENTS

SOLID WASTE DISPOSITION NUMBER OF SHIPMENTS 33

MODE OF TRANSPORTATION TRUCK

6

TRUCK

ESTIMATE OF MAJOR NUCLIDE COMPOSITION (BY TYPE OF WASTE)

DESTINATION
BARNWELL SC
RICHLAND WA

JAN - JUNE

JULY-DEC

A

C - 14

CO- 58

CO- 60

CR -51

CS -134

CS -137

FE -55

MN -54

NI -63

ZN-65

B

$\mathrm{CO}-58$

CO- 60

CR -51

CS -134

CS -137

FE -55

MN -54

NI -63

$\mathrm{ZN}-65$

C

C -14

CO- 60

FE- 55

$\mathrm{H}-3$

MN -54

NI -59

NI -63

TYPE OF WASTE

A. SPENT RESINS, FILTER SLUDGES, EVAPORATOR BOTTOMS, ETC.

B. DRY COMPRESSIBLE WASTE, CONTAMINATED EQUIPMENT, ETC.

C. IRRADIATED COMPONENTS, CONTROL RODS, ETC.

D. OTHER
$1.29 \mathrm{E}-01$

$1.30 \mathrm{E}+00$

$4.86 \mathrm{E}+01$

1. $80 \mathrm{E}+00$

$4.90 \mathrm{E}+00$

$7.80 \mathrm{E}+00$

$2.26 \mathrm{E}+01$

9. $30 \mathrm{E}+00$

$4.25 E-01$

1. $90 \mathrm{E}+00$

$6.67 \mathrm{E}-03$

1.90E-01

1. $77 \mathrm{E}+01$

4. 70E-01

$1.45 \mathrm{E}+00$

2. $60 \mathrm{E}+00$

$9.00 \mathrm{E}+00$

2. $90 \mathrm{E}+00$

1.75E-01

6. $37 E+01$

8 $1.20 \mathrm{E}+00$

$5.14 E+01$

3. $99 \mathrm{E}+01$

$3.00 \mathrm{E}+00$

2. $30 \mathrm{E}+00$

2. $20 E+00$

1. $78 \mathrm{E}+00$

$6.15 \mathrm{E}+00$

4. $75 E+00$

2. $46 \mathrm{E}+01$

1. $92 E+01$

7. $30 \mathrm{E}+00$

5. $70 \mathrm{E}+00$

1. $20 \mathrm{E}+00$

3. $00 \mathrm{E}+00$

2. $57 E+01$

8
8
8
8
8
8

1.00E-02

1.00E-02

3. $55 \mathrm{E}+01$

3. $49 \mathrm{E}+01$

$5.91 \mathrm{E}+01$

$6.00 \mathrm{E}+01$

2. $90 \mathrm{E}-01$

4. $60 \mathrm{E}-01$

1. $24 \mathrm{E}+00$

$1.42 \mathrm{E}+00$

2. $00 \mathrm{E}-02$

2. $00 \mathrm{E}-02$

3. $71 E+00$

3. $14 \mathrm{E}+00$
CI $1.22 \mathrm{E}+01$

M3 $6.50 \mathrm{E}+00$

CI $\quad 9.26 \mathrm{E}+04$

M3

CI 
EFFLUENT AND WASTE DISPOSAL ANNUAL REPORT FOR YEAR 1989 AIRBORNE AND LIQUID EFFLUENTS

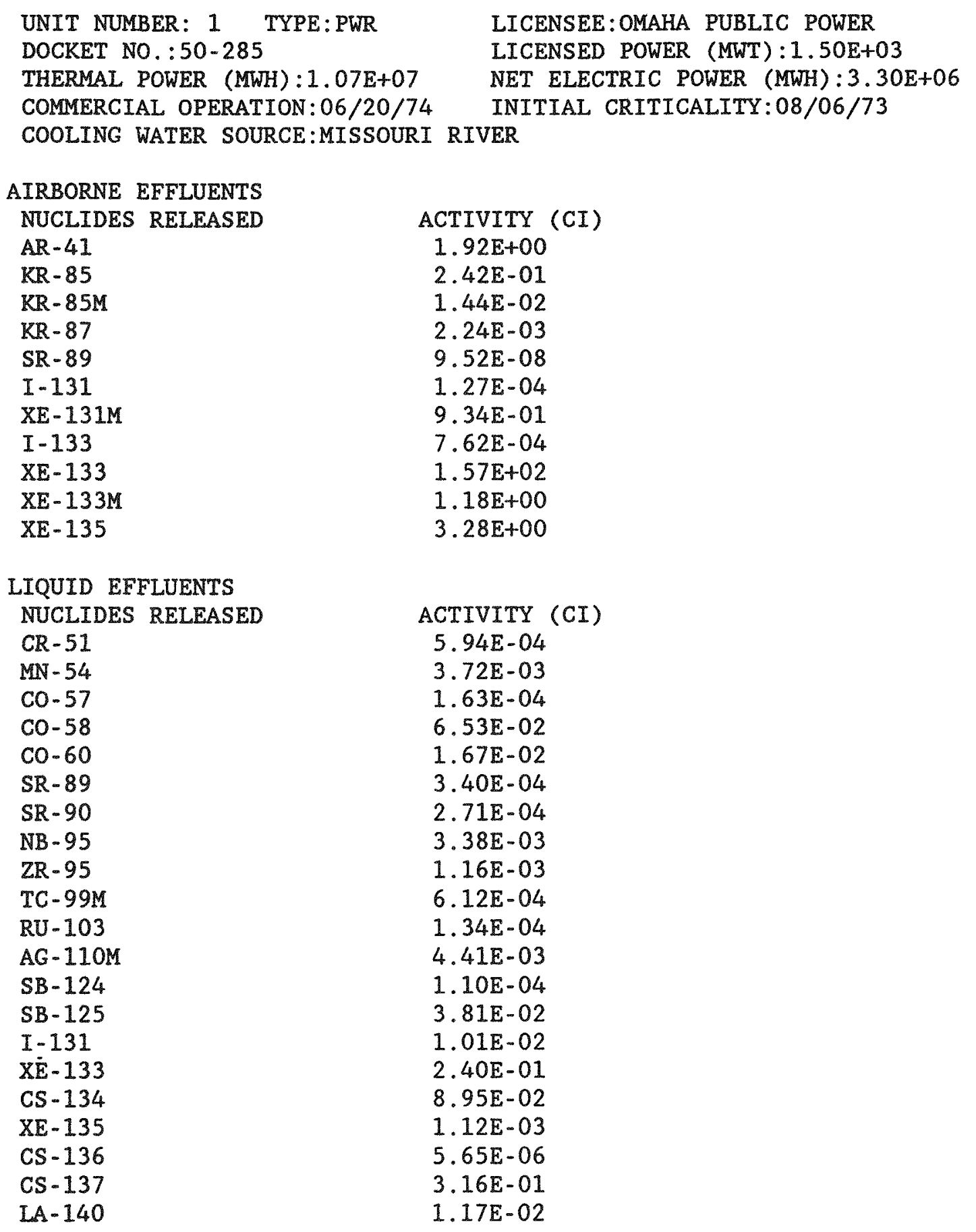

TOTAL AIRBORNE TRITIUM RELEASE 2.25E-01

TOTAL LIQUID TRITIUM RELEASE $2.28 \mathrm{E}+02$

VOLUME OF LIQUID WASTE RELEASED (PRIOR TO DILUTION) LITERS 1.55E+08

VOLUME OF DILUTION WATER USED DURING PERIOD

LITERS $\quad 7.03 E+11$ 
SOLID WASTE DISPOSITION

NUMBER OF SHIPMENTS MODE OF TRANSPORTATION 10

CLOSED SOLE USE VEH.

ESTIMATE OF MAJOR NUCLIDE COMPOSITION (BY TYPE OF WASTE)

B

AG-110M

CE -144

$\mathrm{CO}-58$

$\mathrm{CO}-60$

CS -134

CS -137

$\mathrm{RH}-106$

$\mathrm{RU}-103$

TC- 99

\section{TYPE OF WASTE}

A. SPENT RESINS, FILTER SLUDGES, EVAPORATOR BOTTOMS, ETC.

B. DRY COMPRESSIBLE WASTE, CONTAMINATED EQUIPMENT, ETC.

C. IRRADIATED COMPONENTS, CONTROL RODS, ETC.

D. OTHER

\section{DESTINATION \\ RICHLAND WA}

JAN-JUNE JULY-DEC

$\begin{array}{lll}8 & 2.80 \mathrm{E}+00 & 2.80 \mathrm{E}+00 \\ 8 & 2.10 \mathrm{E}+00 & 2.10 \mathrm{E}+00 \\ 8 & 1.26 \mathrm{E}+01 & 1.26 \mathrm{E}+01 \\ 8 & 1.20 \mathrm{E}+00 & 1.20 \mathrm{E}+00 \\ \& & 6.10 \mathrm{E}+00 & 6.10 \mathrm{E}+00 \\ \& & 6.54 \mathrm{E}+01 & 6.54 \mathrm{E}+01 \\ \& & 1.90 \mathrm{E}+00 & 1.90 \mathrm{E}+00 \\ \& & 2.90 \mathrm{E}+00 & 2.90 \mathrm{E}+00 \\ \& & 5.00 \mathrm{E}+00 & 5.00 \mathrm{E}+00\end{array}$

UNIT YEAR TOTAL

M3 $0.00 \mathrm{E}+00$

CI $\quad 0.00 \mathrm{E}+00$

M3 $1.75 \mathrm{E}+02$

CI $\quad 8.76 \mathrm{E}+00$

M3

CI

M3

CI 


\section{EFFLUENT AND WASTE DISPOSAL ANNUAL REPORT FOR YEAR 1989 AIRBORNE AND LIQUID EFFLUENTS}

$\begin{array}{lc}\text { UNIT NUMBER: } 1 \text { TYPE:HTG } & \text { LICENSEE: } \\ \text { DOCKET NO.: } 50-267 & \text { LICENSED } \\ \text { THERMAL POWER (MWH): } & \text { NET ELECTRI } \\ \text { COMMERCIAL OPERATION :07/01/79 } & \text { INITIAL CRI } \\ \text { COOLING WATER SOURCE: SOUTH } & \text { PLATTE RIVER } \\ & \\ \text { AIRBORNE EFFLUENTS } & \\ \text { NUCLIDES RELEASED } & \text { ACTIVITY (CI) } \\ \text { KR-85 } & 2.68 E+00 \\ \text { KR-85M } & 1.28 E+00 \\ \text { KR-87 } & 1.31 E-01 \\ \text { KR-88 } & 1.20 E+00 \\ \text { XE-131M } & 9.67 E-01 \\ \text { XE-133 } & 1.81 E+02 \\ \text { XE-133M } & 1.72 E+00 \\ \text { XE-135 } & 7.63 E+00 \\ \text { XE-135M } & 5.52 E-03 \\ & \\ \text { LIQUID EFFLUENTS } & \\ \text { NUCLIDES RELEASED } & \\ \text { CO-60 } & \text { ACTIVITY (CI) } \\ \text { CS-137 } & 2.60 E-06 \\ & 9.63 E-06\end{array}$

TOTAL AIRBORNE TRITIUM RELEASE $3.72 \mathrm{E}+00$

TOTAL LIQUID TRITIUM RELEASE $1.12 \mathrm{E}+02$

VOLUME OF LIQUID WASTE RELEASED (PRIOR TO DILUTION) LITERS $6.46 \mathrm{E}+07$

VOLUME OF DILUTION WATER USED DURING PERIOD $\quad$ LITERS $2.19 \mathrm{E}+09$ 
INSTALLATION:FORT ST. VRAIN

EFFLUENT AND WASTE DISPOSAL ANNUAL REPORT FOR YEAR 1989

SOLID EFFLUENTS

SOLID WASTE DISPOSITION

NUMBER OF SHIPMENTS MODE OF TRANSPORTATION

3

HIGHWAY

ESTIMATE OF MAJOR NUCLIDE COMPOSITION (BY TYPE OF WASTE)

B

AG - 110M

CS -134

CS -137

$\mathrm{H}-3$

$M N-54$

C

CO- 60

FE -55

H- 3

MN -54

NI -59

NI -63
DESTINATION

BEATTY NV

JAN-JUNE JULY-DEC $\begin{array}{ll}8 & 1.22 \mathrm{E}+01 \\ 8 & 7.31 \mathrm{E}+00 \\ 8 & 1.34 \mathrm{E}+01 \\ 8 & 5.11 \mathrm{E}+01 \\ 8 & 1.59 \mathrm{E}+01 \\ & \\ 8 & 2.30 \mathrm{E}+01 \\ 8 & 4.07 \mathrm{E}+01 \\ 8 & 8.30 \mathrm{E}+00 \\ 8 & 2.00 \mathrm{E}-01 \\ 8 & 2.00 \mathrm{E}-01 \\ 8 & 2.76 \mathrm{E}+01\end{array}$

UNIT YEAR TOTAL

M3 $0.00 \mathrm{E}+00$

CI $\quad 0.00 \mathrm{E}+00$

M3 $2.86 \mathrm{E}+00$

CI $\quad 1.16 \mathrm{E}-02$

M3 $1.71 \mathrm{E}+00$

CI $1.08 \mathrm{E}+03$

M3

CI

*VOLUME BEFORE COMPACTION 
EFFLUENT AND WASTE DISPOSAL ANNUAL REPORT FOR YEAR 1989 AIRBORNE AND LIQUID EFFLUENTS
UNIT NUMBER: 1 TYPE:PWR
LICENSEE: ROCHESTER GAS\&ELEC
DOCKET NO.: 50-244
LICENSED POWER (MWT) $: 1.52 \mathrm{E}+03$
THERMAL POWER (MWH) $: 9.65 \mathrm{E}+06$
NET ELECTRIC POWER (MWH):3.07E+06
COMMERCIAL OPERATION:07/01/70
INITIAL CRITICALITY: $11 / 08 / 69$
COOLING WATER SOURCE:LAKE ONTARIO
AIRBORNE EFFLUENTS
NUCLIDES RELEASED
AR -41
$\mathrm{CO}-58$
ACTIVITY (CI)
CO -60
$1.47 \mathrm{E}+00$
$2.18 \mathrm{E}-05$
$\mathrm{KR}-85$
$3.99 \mathrm{E}-05$
$\mathrm{KR}-85 \mathrm{M}$
$6.34 \mathrm{E}-01$
KR -87
$6.13 \mathrm{E}-01$
KR -88
I -131
XE-131M
1. $90 \mathrm{E}-01$
1. $88 \mathrm{E}-01$
$4.48 E-04$
I- 133
$4.85 \mathrm{E}+00$
$X E-133$
2. $64 \mathrm{E}-04$
4. $69 E+02$
$\mathrm{XE}-133 \mathrm{M}$
2. $23 \mathrm{E}+00$
$X E-135$
2. $87 \mathrm{E}+01$
$\mathrm{XE}-135 \mathrm{M}$
$1.54 \mathrm{E}+00$
CS -137
$6.87 \mathrm{E}-06$
$\mathrm{XE}-138$
7. $35 \mathrm{E}-01$
UNIDENTIFIED
3. $22 \mathrm{E}-04$

\begin{tabular}{|c|c|}
\hline NUCLIDES RELFASED & ACTTVTTY (CT) \\
\hline$C R-51$ & $7.95 E-06$ \\
\hline MN - 54 & $2.65 \mathrm{E}-04$ \\
\hline$F E-55$ & $3.11 E-04$ \\
\hline CO- 58 & $7.83 E-03$ \\
\hline$C O-60$ & $3.38 \mathrm{E}-03$ \\
\hline $\mathrm{ZN}-65$ & $9.29 \mathrm{E}-09$ \\
\hline SR - 89 & $4.04 E-05$ \\
\hline SR-90 & $9.77 \mathrm{E}-06$ \\
\hline$Z R-N B-95$ & $1.56 \mathrm{E}-04$ \\
\hline MO-99 & $8.16 E-05$ \\
\hline AG-110M & $5.66 \mathrm{E}-04$ \\
\hline$S B-124$ & $1.20 \mathrm{E}-02$ \\
\hline SB-125 & $6.15 E-03$ \\
\hline$I-131$ & $1.21 \mathrm{E}-02$ \\
\hline$I-133$ & $1.37 E-02$ \\
\hline$X E-133$ & $5.34 E-04$ \\
\hline CS -134 & $2.05 E-03$ \\
\hline$I-135$ & $6.24 E-03$ \\
\hline$X E-135$ & $3.03 E-06$ \\
\hline CS -136 & $3.35 E-05$ \\
\hline $\operatorname{CS}-137$ & $1.61 E-02$ \\
\hline$B A-L A-140$ & $3.07 E-05$ \\
\hline$C E-141$ & $1.54 \mathrm{E}-04$ \\
\hline
\end{tabular}

N/A=NOT APPLICABLE

$N / D=N O T$ DETECTED

N/R-NOT REPORTED 
EFFLUENT AND WASTE DISPOSAL ANNUAL REPORT FOR YEAR 1989 AIRBORNE AND LIQUID EFFLUENTS

TOTAL AIRBORNE TRITIUM RELEASE $8.64 \mathrm{E}+01$

TOTAL LIQUID TRITIUM RELEASE $5.92 \mathrm{E}+02$

VOLUME OF LIQUID WASTE RELEASED (PRIOR TO DILUTION) LITERS

1. $19 \mathrm{E}+08$

VOLUME OF DILUTION WATER USED DURING PERIOD

LITERS

$5.59 \mathrm{E}+11$ 
EFFLUENT AND WASTE DISPOSAL ANNUAL REPORT FOR YEAR 1989 SOLID EFFLUENTS

SOLID WASTE DISPOSITION

NUMBER OF SHIPMENTS MODE OF TRANSPORTATION 24

HIGHWAY VEHICLE

ESTIMATE OF MAJOR NUCLIDE COMPOSITION (BY TYPE OF WASTE)

A

$\mathrm{CO}-58$

CO- 60

CS -134

CS -137

FE- 55

$\mathrm{H}-3$

$\mathrm{MN}-54$

NI -63

SB-125

B

C -14

Co- 58

CO -60

CS -137

FE -55

NI -63

SR -90

\section{TYPE OF WASTE}
A. SPENT RESINS, FILTER SLUDGES, EVAPORATOR BOTTOMS, ETC.
B. DRY COMPRESSIBLE WASTE, CONTAMINATED EQUIPMENT, ETC.
C. IRRADIATED COMPONENTS, CONTROL RODS, ETC.
D. OTHER

DESTINATION

BARNWELL SC

$$
\text { JAN-JUNE JULY-DEC }
$$

$\begin{array}{lll}8 & 2.40 \mathrm{E}+00 & 2.40 \mathrm{E}+00 \\ 8 & 3.70 \mathrm{E}+01 & 3.70 \mathrm{E}+01 \\ 8 & 5.90 \mathrm{E}+00 & 5.90 \mathrm{E}+00 \\ 8 & 2.50 \mathrm{E}+01 & 2.50 \mathrm{E}+01 \\ 8 & 1.10 \mathrm{E}+01 & 1.10 \mathrm{E}+01 \\ 8 & 1.60 \mathrm{E}+00 & 1.60 \mathrm{E}+00 \\ 8 & 3.00 \mathrm{E}+00 & 3.00 \mathrm{E}+00 \\ 8 & 1.00 \mathrm{E}+01 & 1.00 \mathrm{E}+01 \\ 8 & 1.30 \mathrm{E}+00 & 1.30 \mathrm{E}+00 \\ & & \\ 8 & 2.00 \mathrm{E}+00 & 2.20 \mathrm{E}+00 \\ 8 & 6.00 \mathrm{E}+00 & 6.00 \mathrm{E}+00 \\ 8 & 1.60 \mathrm{E}+01 & 1.60 \mathrm{E}+01 \\ 8 & 1.00 \mathrm{E}+00 & 1.00 \mathrm{E}-01 \\ 8 & 3.90 \mathrm{E}+01 & 4.00 \mathrm{E}+01 \\ 8 & 3.30 \mathrm{E}+01 & 3.40 \mathrm{E}+01 \\ 8 & 2.00 \mathrm{E}+00 & 2.00 \mathrm{E}-01\end{array}$

UNIT YEAR TOTAL

M3 1.30E+02

CI $\quad 6.88 \mathrm{E}+01$

M3 1.03E+02*

CI $1.11 E+01$

M3

CI

M3

CI

*AFTER VOLUME REDUCTION 


\section{EFFLUENT AND WASTE DISPOSAL ANNUAL REPORT FOR YEAR 1989} AIRBORNE AND LIQUID EFFLUENTS

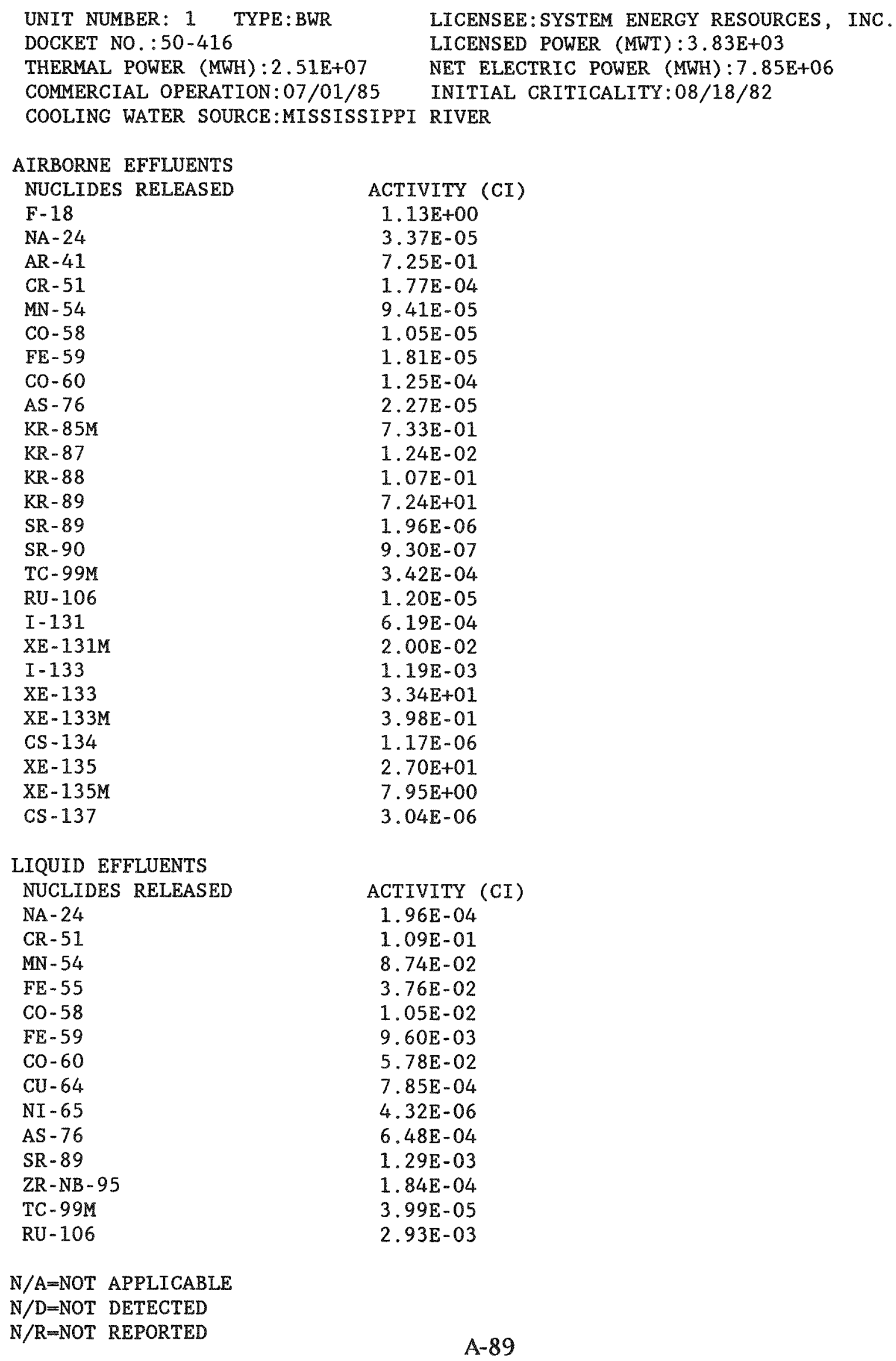

RU -106 
EFFLUENT AND WASTE DISPOSAL ANNUAL REPORT FOR YEAR 1989 AIRBORNE AND LIQUID EFFLUENTS

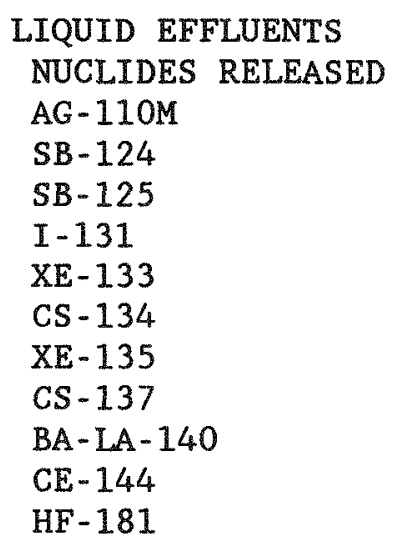
ACTIVITY (CI)
$6.91 \mathrm{E}-04$
7.61E-05
1. $20 \mathrm{E}-05$
7. $58 \mathrm{E}-05$
$2.51 \mathrm{E}-04$
2. $30 \mathrm{E}-04$
$3.90 \mathrm{E}-04$
2. $76 E-04$
3. $30 \mathrm{E}-06$
$1.13 \mathrm{E}-04$
$2.42 \mathrm{E}-05$

TOTAL AIRBORNE TRITIUM RELEASE $3.34 \mathrm{E}+00$

TOTAL LIQUID TRITIUM RELEASE 1.32E+01

VOLUME OF LIQUID WASTE RELEASED (PRIOR TO DILUTION) LITERS $1.13 E+08$

VOLUME OF DILUTION WATER USED DURING PERIOD

LITERS $\quad 4.09 E+09$ 
INSTALLATION: GRAND GULF

EFFLUENT AND WASTE DISPOSAL ANNUAL REPORT FOR YEAR 1989 SOLID EFFLUENTS

SOLID WASTE DISPOSITION NUMBER OF SHIPMENTS 27

1

MODE OF TRANSPORTATION

DESTINATION TRUCK

TRUCK

BARNWELL SC

BEATTY NV

17 TRUCK

SEG, BARNWELL SC

ESTIMATE OF MAJOR NUCLIDE COMPOSITION (BY TYPE OF WASTE)

JAN-JUNE JULY-DEC

A

$\mathrm{CO}-58$

$\mathrm{CO}-60$

$\mathrm{CR}-51$

FE- 55

MN-54

OTHERS

B

$\mathrm{CO}-58$

$\mathrm{CO}-60$

CR-51

FE -55

FE -59

MN -54

OTHERS

$\begin{array}{lll}8 & 2.00 \mathrm{E}+00 & 2.00 \mathrm{E}+00 \\ 8 & 2.60 \mathrm{E}+01 & 3.20 \mathrm{E}+01 \\ 8 & 3.00 \mathrm{E}+00 & \\ 8 & 3.40 \mathrm{E}+01 & 3.60 \mathrm{E}+01 \\ 8 & 2.60 \mathrm{E}+01 & 2.40 \mathrm{E}+01 \\ 8 & 3.00 \mathrm{E}+00 & 6.00 \mathrm{E}+00 \\ & & \\ 8 & 2.00 \mathrm{E}+00 & \\ 8 & 2.10 \mathrm{E}+01 & 2.30 \mathrm{E}+01 \\ 8 & 9.00 \mathrm{E}+00 & 5.00 \mathrm{E}+00 \\ 8 & 2.40 \mathrm{E}+01 & 2.60 \mathrm{E}+01 \\ 8 & 1.00 \mathrm{E}+01 & 9.00 \mathrm{E}+00 \\ 8 & 3.40 \mathrm{E}+01 & 3.60 \mathrm{E}+01 \\ 8 & & 1.00 \mathrm{E}+00\end{array}$

TYPE OF WASTE

A. SPENT RESINS, FILTER SLUDGES, EVAPORATOR BOTTOMS, ETC.

B. DRY COMPRESSIBLE WASTE, CONTAMINATED EQUIPMENT, ETC.

UNIT YEAR TOTAL

M3 $1.63 \mathrm{E}+02$

CI $2.00 \mathrm{E}+02$

M3 1.09E+02*

CI $\quad 5.73 E+00$

C. IRRADIATED COMPONENTS, CONTROL RODS, ETC.

M3

CI

D. OTHER

M3

CI

*ACTUAL COMPACTED BURIAL VOLUME AFTER VOL. REDUCTION BY SEG 
EFFLUENT AND WASTE DISPOSAL ANNUAL REPORT FOR YEAR 1989 AIRBORNE AND LIQUID EFFLUENTS

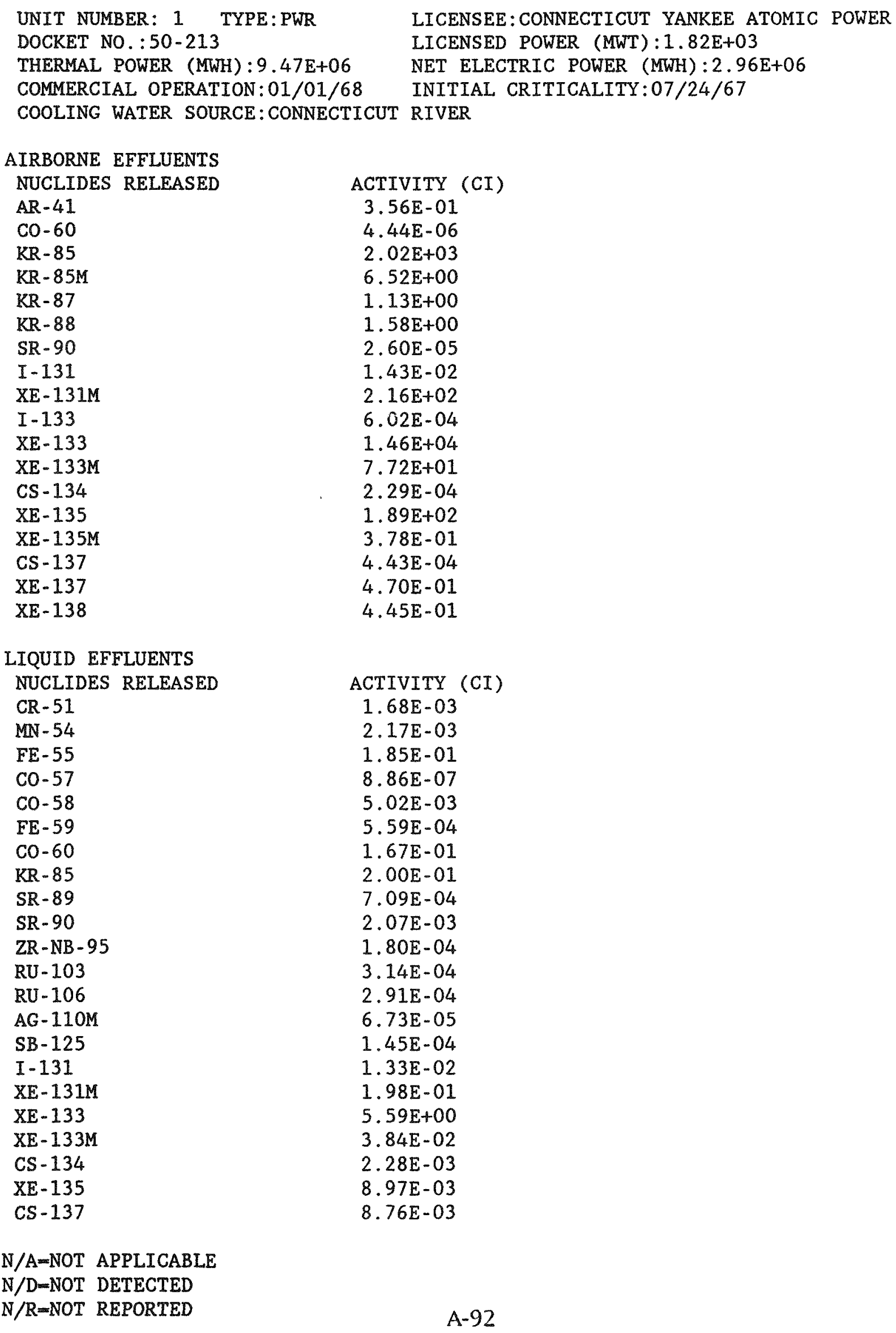


EFFLUENT AND WASTE DISPOSAL ANNUAL REPORT FOR YEAR 1989 AIRBORNE AND LIQUID EFFLUENTS

LIQUID EFFLUENTS NUCLIDES RELEASED

$B A-I A-140$
ACTIVITY (CI)

3. $00 \mathrm{E}-05$
TOTAL AIRBORNE TRITIUM RELEASE $1.37 E+02$

TOTAL LIQUID TRITIUM RELEASE
VOLUME OF LIQUID WASTE RELEASED (PRIOR TO DILUTION) VOLUME OF DILUTION WATER USED DURING PERIOD
LITERS $\quad 1.09 \mathrm{E}+08$

LITERS $3.78 \mathrm{E}+11$ 
EFFLUENT AND WASTE DISPOSAL ANNUAL REPORT FOR YEAR 1989 SOLID EFFLUENTS

SOLID WASTE DISPOSITION NUMBER OF SHIPMENTS 14

MODE OF TRANSPORTATION TRUCK

10 TRUCK

ESTIMATE OF MAJOR NUCLIDE COMPOSITION (BY TYPE OF WASTE)

A

AM-241

C- 14

CE-144

CM- 242

CM-243

CM-244

$\mathrm{CO}-57$

$\mathrm{CO}-58$

CO- 60

CS -134

CS -137

FE -55

$\mathrm{H}-3$

I -129

MN -54

NI -63

PU-238

PU-239

PU-240

PU-241

SR -90

TC-99

$B$

AM-241

$\mathrm{C}-14$

CM- 242

CM- 243

CM-244

CO- 58

CO- 60

CS -134

CS -137

FE- 55

H -3

I -129

NI -63

NP- 237
DESTINATION

BARNWELL SC

RICHLAND WA

JAN-JUNE JULY-DEC

1.29E-02

4. $57 \mathrm{E}-03$

$2.41 E-01$

8.10E-02

1. $00 \mathrm{E}+00$

7. $40 \mathrm{E}-02$

9.75E-05

$6.85 \mathrm{E}-03$

$2.03 E-03$

$6.85 E-03$

$2.03 E-03$

1.00E-01

6.00E-03

3. $60 \mathrm{E}+00$

2. $97 \mathrm{E}+00$

2. $11 \mathrm{E}+01$

$2.07 E+01$

4. $00 \mathrm{E}+00$

1. $89 \mathrm{E}+01$

1. $30 \mathrm{E}+01$

2. $27 \mathrm{E}+01$

4. $74 \mathrm{E}+01$

2. $65 E+01$

$2.00 \mathrm{E}-01$

2. 10E-02

$2.05 E-04$

$6.29 E-05$

1. $50 \mathrm{E}+00$

2. $32 E+00$

7. $22 \mathrm{E}+00$

3. $80 \mathrm{E}+00$

2. 20E-02

3. $29 \mathrm{E}-03$

$3.29 \mathrm{E}-03$

1. 10E-02

$5.00 \mathrm{E}-01$

1. $66 \mathrm{E}-03$

2. $05 \mathrm{E}-03$

$3.00 \mathrm{E}-01$

1. 10E-04

1. $49 \mathrm{E}-01$

$2.93 E-04$

1. $91 \mathrm{E}-02$

$2.47 E-01$

1. $92 \mathrm{E}-02$

1. $65 \mathrm{E}-02$

2. $47 \mathrm{E}-01$

1. $12 \mathrm{E}-02$

1. $65 \mathrm{E}-02$

1. 12E-02

1. $12 \mathrm{E}-02$

2. $98 E+00$

1. $12 \mathrm{E}-02$

1. $03 \mathrm{E}+01$

$2.98 \mathrm{E}+00$

1. $41 \mathrm{E}+00$

$1.03 \mathrm{E}+01$

$7.54 \mathrm{E}+00$

$1.41 \mathrm{E}+00$

$6.18 \mathrm{E}+01$

7. $55 \mathrm{E}+00$

4. $57 \mathrm{E}-01$

$6.18 \mathrm{E}+0 \mathrm{I}$

1. $51 \mathrm{E}-01$

$3.25 \mathrm{E}-01$

$5.87 \mathrm{E}+00$

1. $51 \mathrm{E}-01$

3. $19 \mathrm{E}-03$

$5.87 \mathrm{E}+00$

$3.20 \mathrm{E}-03$ 
EFFLUENT AND WASTE DISPOSAL ANNUAL REPORT FOR YEAR 1989 SOLID EFFLUENTS

ESTIMATE OF MAJOR NUCLIDE COMPOSITION (BY TYPE OF WASTE)

JAN-JUNE JULY-DEC

B

PU -238

PU -239

PU -240

PU -241

SR -90

TC-99

$\begin{array}{lll}8 & 1.88 \mathrm{E}-01 & 1.89 \mathrm{E}-01 \\ 8 & 2.23 \mathrm{E}-02 & 2.24 \mathrm{E}-02 \\ 8 & 2.23 \mathrm{E}-02 & 2.24 \mathrm{E}-02 \\ 8 & 8.85 \mathrm{E}+00 & 8.86 \mathrm{E}+00 \\ 8 & 9.04 \mathrm{E}-02 & 9.06 \mathrm{E}-02 \\ 8 & 2.86 \mathrm{E}-02 & 2.87 \mathrm{E}-02\end{array}$

TYPE OF WASTE

A. SPENT RESINS, FILTER SLUDGES, EVAPORATOR

UNIT YEAR TOTAL BOTTOMS, ETC.

M3 2.73E+01

CI $\quad 6.35 \mathrm{E}+02$

B. DRY COMPRESSIBLE WASTE, CONTAMINATED

M3 $1.26 E+02$

EQUIPMENT, ETC.

CI $\quad 2.01 \mathrm{E}+01$

C. IRRADIATED COMPONENTS, CONTROL

M3

RODS, ETC.

CI

D. OTHER

M3

CI 
EFFLUENT AND WASTE DISPOSAL ANNUAL REPORT FOR YEAR 1989 AIRBORNE AND LIQUID EFFLUENTS

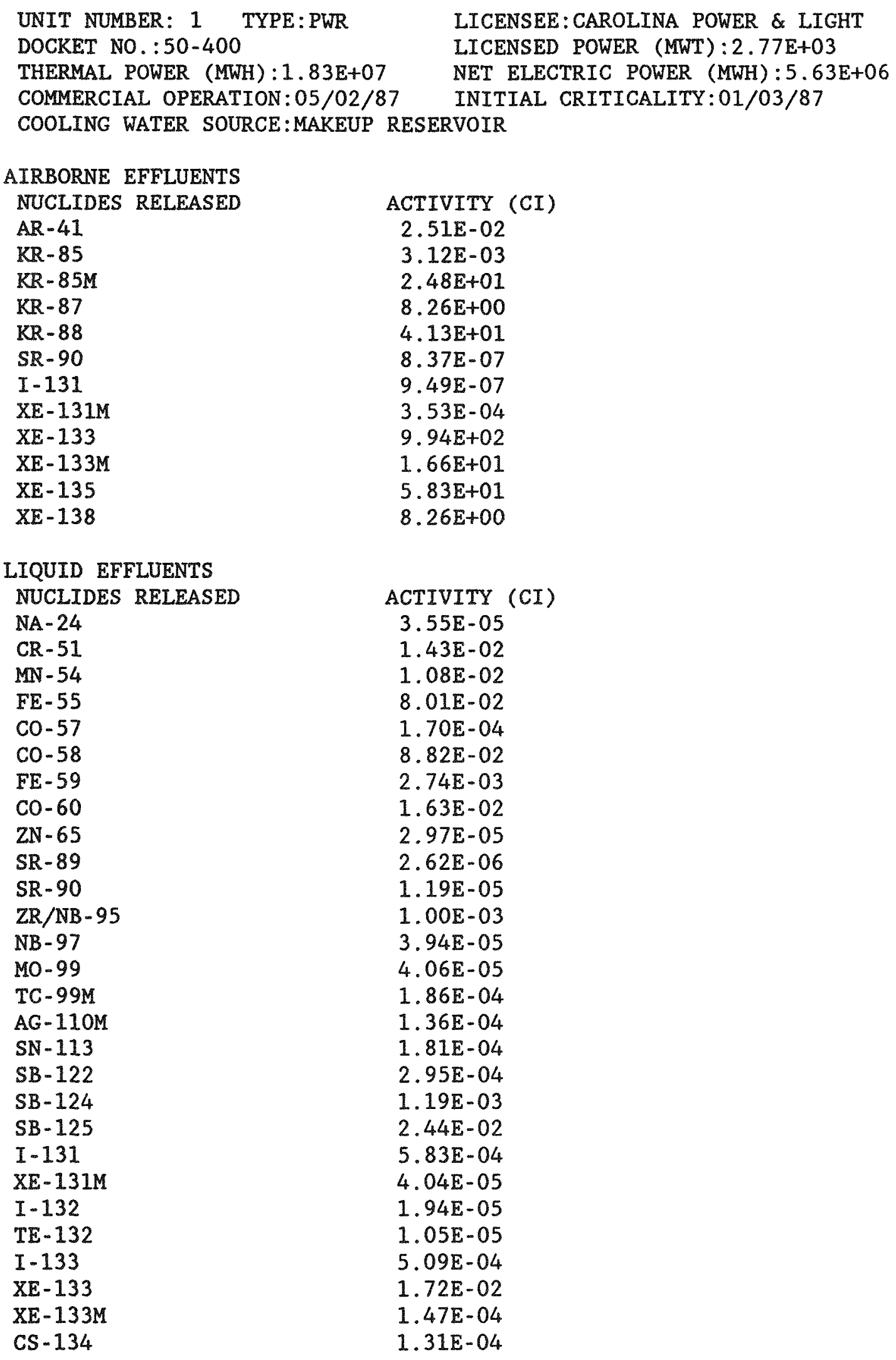

$N / A=N O T$ APPLICABLE $N / D=N O T$ DETECTED N/R-NOT REPORTED 
EFFLUENT AND WASTE DISPOSAL ANNUAL REPORT. FOR YEAR 1989 AIRBORNE AND LIQUID EFFLUENTS

\author{
LIQUID EFFLUENTS \\ NUCLIDES RELEASED \\ $\mathrm{XE}-135$ \\ $\mathrm{CS}-137$ \\ CS -138 \\ CE -141 \\ PR -144 \\ $\mathrm{HF}-181$ \\ W-187
}

ACTIVITY (CI)

$3.02 E-04$

$3.83 \mathrm{E}-04$

$1.47 \mathrm{E}-05$

$7.79 \mathrm{E}-06$

$2.61 E-04$

$2.85 \mathrm{E}-05$

$1.99 \mathrm{E}-05$

TOTAL AIRBORNE TRITIUM RELEASE $0.00 E+00$

TOTAL LIQUID TRITIUM RELEASE $4.58 \mathrm{E}+02$

VOLUME OF LIQUID WASTE RELEASED (PRIOR TO DILUTION-) LITERS $7.28 E+07$

VOLUME OF DILUTION WATER USED DURING PERIOD IITERS $1.43 \mathrm{E}+10$ 
EFFLUENT AND WASTE DISPOSAL ANNUAL REPORT FOR YEAR 1989 SOLID EFFLUENTS

SOLID WASTE DISPOSITION

$\begin{array}{cl}\text { NUMBER OF SHIPMENTS } & \text { MODE OF TRANSPORTATION } \\ 16 & \text { TRUCK } \\ 27 & \text { TRUCK }\end{array}$

ESTIMATE OF MAJOR NUCLIDE COMPOSITION (BY TYPE OF WASTE)

DESTINATION
BARNWELL SC
BARNWELL SC VIA SEG

JAN-JUNE JULY-DEC

A

C -14

Co- 58

CO- 60

FE -55

$\mathrm{H}-3$

$M N-54$

NI -63

SB-125

B

$\mathrm{AM}-241$

C -14

CM-244

$\mathrm{CO}-58$

CO- 60

CR -51

CS -137

FE -55

FE- 59

$\mathrm{H}-3$

MN -54

NB -95

NI -63

ZR -95

\section{TYPE OF WASTE}

A. SPENT RESINS, FILTER SLUDGES, EVAPORATOR BOTTOMS, ETC.

B. DRY COMPRESSIBLE WASTE, CONTAMINATED EQUIPMENT, ETC.

C. IRRADIATED COMPONENTS, CONTROL RODS, ETC.

D. OTHER

$\begin{array}{lll}8 & 2.60 \mathrm{E}+00 & 9.00 \mathrm{E}-02 \\ 8 & 1.59 \mathrm{E}+01 & 2.01 \mathrm{E}+01 \\ 8 & 1.25 \mathrm{E}+01 & 8.66 \mathrm{E}+00 \\ 8 & 3.09 \mathrm{E}+01 & 5.89 \mathrm{E}+01 \\ 8 & 1.36 \mathrm{E}+01 & 3.90 \mathrm{E}+00 \\ 8 & 1.37 \mathrm{E}+01 & 5.29 \mathrm{E}+00 \\ 8 & 1.07 \mathrm{E}+01 & 3.09 \mathrm{E}+00 \\ 8 & 1.00 \mathrm{E}-02 \\ 8 & 1.00 \mathrm{E}-01 & 1 \\ & & \\ 8 & 1.05 \mathrm{E}-03 & 5.87 \mathrm{E}-05 \\ 8 & 5.66 \mathrm{E}-01 & 5.00 \mathrm{E}-02 \\ 8 & 1.53 \mathrm{E}-03 & 8.72 \mathrm{E}-05 \\ 8 & 9.80 \mathrm{E}+00 & 2.15 \mathrm{E}+00 \\ 8 & 1.81 \mathrm{E}+00 & 6.23 \mathrm{E}+00 \\ 8 & 1.10 \mathrm{E}+00 & 1.70 \mathrm{E}-01 \\ 8 & 1.34 \mathrm{E}-03 & 1.00 \mathrm{E}-02 \\ 8 & 7.56 \mathrm{E}+01 & 8.53 \mathrm{E}+01 \\ 8 & 7.41 & 1.00 \mathrm{E}-02 \\ 8 & 2.21 \mathrm{E}-01 & 1.35 \mathrm{E}+00 \\ 8 & 1.48 \mathrm{E}+00 & 1.74 \mathrm{E}+00 \\ 8 & 3.51 \mathrm{E}+00 & 5.50 \mathrm{E}-01 \\ 8 & 7.68 \mathrm{E}-01 & 2.00 \mathrm{E}+00 \\ 8 & 5.77 \mathrm{E}+00 & 4.60 \mathrm{E}-01\end{array}$

UNIT YEAR TOTAL

M3 $\quad 1.03 \mathrm{E}+02$

CI $\quad 1.75 \mathrm{E}+01$

M3 5.78E+01*

CI $\quad 7.89 E+00$

M3

CI

M3

CI 
EFFLUENT AND WASTE DISPOSAL ANNUAL REPORT FOR YEAR 1989 AIRBORNE AND LIQUID EFFLUENTS

\begin{tabular}{|c|c|}
\hline UNIT NUMBER: 1 & LICENSEE:GEORGIA POWER \\
\hline DOCKET NO.: $50-321$ & LICENSED POWER (MWT) $: 2.44 E+03$ \\
\hline THERMAL POWER (MWH) :2.09E+07 & NET ELECTRIC POWER (MWH) :6.48E+06 \\
\hline COMMERCIAL OPERATION : $12 / 31 / 75$ & INITIAL CRITICALITY:09/12/74 \\
\hline COOLING WATER SOURCE:ALTAMAHA & A RIVER \\
\hline UNIT NUMBER: 2 TYPE:BWR & LICENSEE: GEORGIA POWER \\
\hline DOCKET NO.: $50-366$ & LICENSED POWER (MWT) $: 2.44 \mathrm{E}+03$ \\
\hline THERMAL POWER (MWH) $: 1.35 E+07$ & NET ELECTRIC POWER (MWH) :4.14E+06 \\
\hline COMMERCIAL OPERATION:09/05/79 & INITIAL CRITICALITY:07/04/78 \\
\hline COOLING WATER SOURCE:ALTAMAHA & A RIVER \\
\hline AIRBORNE EFFLUENTS & \\
\hline NUCLIDES RELEASED & ACTIVITY (CI) \\
\hline$A R-41$ & $2.99 \mathbb{E}+00$ \\
\hline $\mathrm{CR}-51$ & $4.44 E-04$ \\
\hline$M N-54$ & $5.02 E-06$ \\
\hline $\mathrm{CO}-58$ & $4.50 E-05$ \\
\hline $\mathrm{CO}-60$ & $1.00 E-04$ \\
\hline $\mathrm{ZN}-65$ & $6.49 E-04$ \\
\hline$K R-83 M$ & $9.43 \mathrm{E}-02$ \\
\hline $\mathrm{KR}-85 \mathrm{M}$ & $6.08 E+00$ \\
\hline $\mathrm{KR}-87$ & $7.34 \mathrm{E}+00$ \\
\hline $\mathrm{KR}-88$ & $4.16 E+00$ \\
\hline$S R-89$ & $3.02 E-04$ \\
\hline SR -90 & $1.26 \mathrm{E}-06$ \\
\hline NB-95 & $2.29 E-09$ \\
\hline MO-99 & $2.70 E-05$ \\
\hline$I-131$ & $3.26 \mathrm{E}-03$ \\
\hline $\mathrm{XE}-131 \mathrm{M}$ & $1.26 E-05$ \\
\hline I - 133 & $1.73 E-02$ \\
\hline$X E-133$ & $1.57 \mathrm{E}+02$ \\
\hline$I-135$ & $2.31 E-02$ \\
\hline$X E-135$ & $1.38 E+02$ \\
\hline$X E-135 M$ & $6.22 \mathrm{E}+01$ \\
\hline CS - 137 & $1.68 \mathrm{E}-05$ \\
\hline $\mathrm{XE}-138$ & $1.25 E+02$ \\
\hline$B A-140$ & $8.64 E-04$ \\
\hline LA -140 & $1.54 E-03$ \\
\hline$C E-141$ & $4.04 E-05$ \\
\hline$C E-144$ & $2.18 \mathrm{E}-06$ \\
\hline LIQUID EFFLUENTS & \\
\hline NUCLIDES RELEASED & ACTIVITY (CI) \\
\hline $\mathrm{NA}-24$ & $6.49 E-03$ \\
\hline AR -41 & $6.02 E-06$ \\
\hline $\mathrm{CR}-51$ & $5.37 \mathrm{E}-03$ \\
\hline MN - 54 & $6.92 E-03$ \\
\hline$M N-56$ & $1.76 E-05$ \\
\hline $\mathrm{CO}-58$ & $2.69 E-03$ \\
\hline FE -59 & $1.37 E-04$ \\
\hline$C 0-60$ & $2.30 E-02$ \\
\hline
\end{tabular}

$N / A=N O T$ APPLICABLE 
EFFLUENT AND WASTE DISPOSAL ANNUAL REPORT FOR YEAR 1989 AIRBORNE AND LIQUID EFFLUENTS

\begin{tabular}{|c|c|}
\hline NUCLIDES RELEASED & ACTIVITY (CI) \\
\hline $\mathrm{CU}-64$ & $1.01 E-02$ \\
\hline $\mathrm{ZN}-65$ & $4.20 \mathrm{E}-02$ \\
\hline AS -76 & $3.93 E-04$ \\
\hline $\mathrm{KR}-85$ & $6.01 E-04$ \\
\hline $\mathrm{KR}-85 \mathrm{M}$ & $3.85 E-06$ \\
\hline $\mathrm{KR}-87$ & $4.44 E-06$ \\
\hline $\mathrm{KR}-88$ & $6.66 \mathrm{E}-06$ \\
\hline $\mathrm{RB}-88$ & $2.35 E-05$ \\
\hline SR-89 & $1.68 \mathrm{E}-03$ \\
\hline SR-91 & $1.07 \mathrm{E}-04$ \\
\hline$Y-91 M$ & $1.78 E-03$ \\
\hline SR - 92 & $7.42 E-05$ \\
\hline NB -95 & $2.68 E-05$ \\
\hline NB-97 & $2.80 E-04$ \\
\hline MO-99 & $3.02 E-04$ \\
\hline TC-99M & $1.89 E-03$ \\
\hline $\mathrm{SB}-125$ & $6.11 E-05$ \\
\hline$I-131$ & $5.49 E-03$ \\
\hline$I-132$ & $1.06 \mathrm{E}-04$ \\
\hline$I-133$ & $6.19 E-03$ \\
\hline$X E-133$ & $1.33 \mathrm{E}-02$ \\
\hline $\mathrm{XE}-133 \mathrm{M}$ & $2.15 E-04$ \\
\hline CS -134 & $3.62 E-02$ \\
\hline$I-134$ & $6.89 E-05$ \\
\hline$I-135$ & $9.72 E-04$ \\
\hline$X E-135$ & $4.24 E-02$ \\
\hline$X E-135 M$ & $3.91 E-03$ \\
\hline CS -136 & $7.47 \mathrm{E}-05$ \\
\hline CS -137 & $8.69 E-02$ \\
\hline $\mathrm{BA}-139$ & $2.33 E-05$ \\
\hline$B A-140$ & $3.38 E-05$ \\
\hline$L A=140$ & $1.35 E-04$ \\
\hline$C E-141$ & 3. $32 E-05$ \\
\hline$N P-239$ & $8.82 E-03$ \\
\hline
\end{tabular}

TOTAL AIRBORNE TRITIUM RELEASE $5.41 \mathrm{E}+01$

TOTAL LIQUID TRITIUM RELEASE 4.57E+01

VOLUME OF LIQUID WASTE RELEASED (PRIOR TO DILUTION) LITERS $3.29 E+07$

VOLUME OF DILUTION WATER USED DURING PERIOD $\quad$ LITERS $7.22 \mathrm{E}+09$ 
INSTALLATION:EDWIN I. HATCH $1 \& 2$

EFFLUENT AND WASTE DISPOSAL ANNUAL REPORT FOR YEAR 1989 SOLID EFFLUENTS

SOLID WASTE DISPOSITION NUMBER OF SHIPMENTS 31

33

13

32

MODE OF TRANSPORTATION
CASK
CASK
VAN/BOXES
VAN

VAN

ESTIMATE OF MAJOR NUCLIDE COMPOSITION (BY TYPE OF WASTE)

A Co- 60

CS -137

FE-55

OTHER

$\mathrm{ZN}-65$

B

Co -60

CS -137

FE- 55

OTHER

$\mathrm{ZN}-65$

D

CO -60

CS -137

$\mathrm{FE}-55$

NI -63

OTHER

$\mathrm{ZN}-65$

TYPE OF WASTE

A. SPENT RESINS, FILTER SLUDGES, EVAPORATOR BOTTOMS, ETC.

B, DRY COMPRESSIBLE WASTE, CONTAMINATED EQUIPMENT, ETC.

C. IRRADIATED COMPONENTS, CONTROL RODS, ETC.

D. OTHER CONTROL ROD DRIVE FLTRS*

DESTINATION

BARNWELL \& SEG OAK RIDGE TN BARNWELL SC

BARNWELL SC

BARNWELL \& SEG OAK RIDGE TN

JAN-JUNE JULY-DEC

$\begin{array}{lll}8 & 1.03 \mathrm{E}+01 & 1.34 \mathrm{E}+01 \\ 8 & 3.00 \mathrm{E}+00 & 2.39 \mathrm{E}+00 \\ 8 & 1.41 \mathrm{E}+01 & 1.75 \mathrm{E}+01 \\ 8 & 1.76 \mathrm{E}+01 & 1.22 \mathrm{E}+01 \\ 8 & 5.50 \mathrm{E}+01 & 5.45 \mathrm{E}+01 \\ & & \\ -8 & 3.81 \mathrm{E}+01 & 2.75 \mathrm{E}+01 \\ 8 & 1.04 \mathrm{E}+01 & 8.32 \mathrm{E}+00 \\ 8 & 3.90 \mathrm{E}+00 & 4.42 \mathrm{E}+00 \\ 8 & 1.19 \mathrm{E}+01 & 9.68 \mathrm{E}+00 \\ 8 & 3.57 \mathrm{E}+01 & 5.01 \mathrm{E}+01 \\ & & \\ 8 & & 5.49 \mathrm{E}+01 \\ 8 & & 4.10 \mathrm{E}-02 \\ 8 & & 2.50 \mathrm{E}-02 \\ 8 & & 3.29 \mathrm{E}+00 \\ 8 & & 3.28 \mathrm{E}+01 \\ 8 & & 8.91 \mathrm{E}+00\end{array}$

UNIT YEAR TOTAL

M3 2.01E+02

CI $\quad 1.88 \mathrm{E}+03$

M3 $6.30 \mathrm{E}+02$

CI $\quad 5.52 \mathrm{E}+00$

M3

CI

M3 2.20E+01

CI $1.99 \mathrm{E}+01$

*ALSO, DAW COMPACTED AT SEG (ACTUAL VOL. BURIED) \& SOLIDIFIED OIL 
EFFLUENT AND WASTE DISPOSAL ANNUAL REPORT FOR YEAR 1989 AIRBORNE AND LIQUID EFFLUENTS

UNIT NUMBER: 1 TYPE:BWR

DOCKET NO.: $50-354$

THERMAL POWER (MWH) $: 2.10 \mathrm{E}+07$

COMMERCIAL OPERATION: $12 / 20 / 86$

COOLING WATER SOURCE:DELAWARE RIVER

AIRBORNE EFFLUENTS

NUCLIDES RELEASED

$\mathrm{KR}-83 \mathrm{M}$

$\mathrm{KR}-85 \mathrm{M}$

$\mathrm{KR}-87$

$\mathrm{KR}-88$

$\mathrm{KR}-89$

$\mathrm{XE}-133$

$\mathrm{XE}-135$

$\mathrm{XE}-135 \mathrm{M}$

$X E-137$

$\mathrm{XE}-138$

\section{LIQUID EFFLUENTS}

NUCLIDES RELEASED

NA -24

CR -51

$M N-54$

FE -55

$\mathrm{CO}-58$

FE- 59

CO- 60

$\mathrm{ZN}-65$

AS -76

SR -90

SR-92

NB- 95

NB -97

TC-99M

AG-110M

SB-125

I- 131

XE-133

$X E-135$

CS -137
LICENSEE: PUBLIC SERV ELEC \& GAS CO OF NJ LICENSED POWER (MWT) $: 3.29 \mathrm{E}+03$ NET ELECTRIC POWER (MWH) $: 6.61 \mathrm{E}+06$ INITIAL CRITICALITY:06/28/86
ACTIVITY (CI)
$3.14 \mathrm{E}+00$
$3.14 \mathrm{E}+00$
$1.26 \mathrm{E}+01$
$1.26 \mathrm{E}+01$
$8.48 \mathrm{E}+01$
$2.74 \mathrm{E}+01$
$1.57 \mathrm{E}+01$
$1.79 \mathrm{E}+01$
$9.75 \mathrm{E}+01$
$5.97 \mathrm{E}+01$

ACTIVITY (CI)

9.28E-03

$1.85 \mathrm{E}-01$

$2.02 E-01$

$2.06 \mathrm{E}-01$

$1.69 \mathrm{E}-02$

$5.77 \mathrm{E}-02$

4. $56 \mathrm{E}-02$

$3.22 \mathrm{E}-01$

$2.44 \mathrm{E}-05$

$3.01 E-05$

$3.83 E-04$

4. 33E- 06

$2.14 E-05$

$9.99 \mathrm{E}-05$

$1.13 \mathrm{E}-03$

$5.82 \mathrm{E}-05$

1. $33 \mathrm{E}-05$

$4.58 \mathrm{E}-04$

$2.68 \mathrm{E}-03$

$3.58 \mathrm{E}-05$

TOTAL AIRBORNE TRITIUM RELEASE 2.76E+01

TOTAL LIQUID TRITIUM RELEASE 2.35E+01

VOLUME OF LIQUID WASTE RELEASED (PRIOR TO DILUTION) VOLUME OF DILUTION WATER USED DURING PERIOD
LITERS

LITERS
$1.75 \mathrm{E}+07$

4. $44 E+10$

$N / A=N O T$ APPLICABLE

$N / D=$ NOT DETECTED

N/R $=$ NOT REPORTED 
SOLID WASTE DISPOSITION NUMBER OF SHIPMENTS 27 1 TRUCK

TRUCK

IRRADIATED FUEL SHIPMENTS (DISPOSITION)

NUMBER OF SHIPMENTS MODE OF TRANSPORTATION N/A

ESTIMATE OF MAJOR NUCLIDE COMPOSITION (BY TYPE OF WASTE)

A
C -14
CE -144
CO- 58
CO -60
CR -51
CS -137
FE -55
FE- 59
MN -54
NI -59
NI -63
ZN- 65
C -14
CO- 58
CO- 60
CR -51
FE- 55
FE - 59
MN -54
ZN- 65

B

TYPE OF WASTE
A. SPENT RESINS, FILTER SLUDGES, EVAPORATOR BOTTOMS, ETC.
B. DRY COMPRESSIBLE WASTE, CONTAMINATED EQUIPMENT, ETC.
C. IRRADIATED COMPONENTS, CONTROL RODS, ETC.
D. OTHER

\author{
DESTINATION \\ BARNWELL SC \\ RICHLAND WA
}

\section{DESTINATION \\ N/A}

$$
\text { JAN-JUNE JULY-DEC }
$$

$\begin{array}{lll}8 & & 1.00 \mathrm{E}-01 \\ 8 & 1.20 \mathrm{E}-01 & 5.00 \mathrm{E}-01 \\ 8 & 2.80 \mathrm{E}-01 & \\ 8 & 1.98 \mathrm{E}+00 & 2.90 \mathrm{E}+00 \\ 8 & 3.34 \mathrm{E}+00 & 3.20 \mathrm{E}+00 \\ 8 & & 2.00 \mathrm{E}-01 \\ 8 & & \\ 8 & 8.35 \mathrm{E}+00 & 2.05 \mathrm{E}+01 \\ 8 & 7.40 \mathrm{E}-01 & 3.00 \mathrm{E}-01 \\ 8 & 4.16 \mathrm{E}+00 & 4.30 \mathrm{E}+00 \\ 8 & & 6.00 \mathrm{E}-01 \\ 8 & 1.50 \mathrm{E}-01 & 5.00 \mathrm{E}-01 \\ 8 & 8.09 \mathrm{E}+01 & 6.85 \mathrm{E}+01 \\ & & \\ 8 & 4.00 \mathrm{E}-02 & 2.00 \mathrm{E}-01 \\ 8 & 2.21 \mathrm{E}+00 & 1.40 \mathrm{E}+00 \\ 8 & 8.96 \mathrm{E}+00 & 2.20 \mathrm{E}+00 \\ 8 & 7.15 \mathrm{E}+00 & 1.10 \mathrm{E}+01 \\ 8 & 1.23 \mathrm{E}+01 & 2.73 \mathrm{E}+01 \\ 8 & 2.91 \mathrm{E}+00 & 2.30 \mathrm{E}+00 \\ 8 & 2.90 \mathrm{E}+00 \\ 8 & 8.52 \mathrm{E}+00 & 5.80 \mathrm{E}+00 \\ 8 & 6.07 \mathrm{E}+01 & 4.98 \mathrm{E}+01\end{array}$

*VOLUME AFTER COMPACTION BY SEG 
EFFLUENT AND WASTE DISPOSAL ANNUAL REPORT FOR YEAR 1989 AIRBORNE AND LIQUID EFFLUENTS

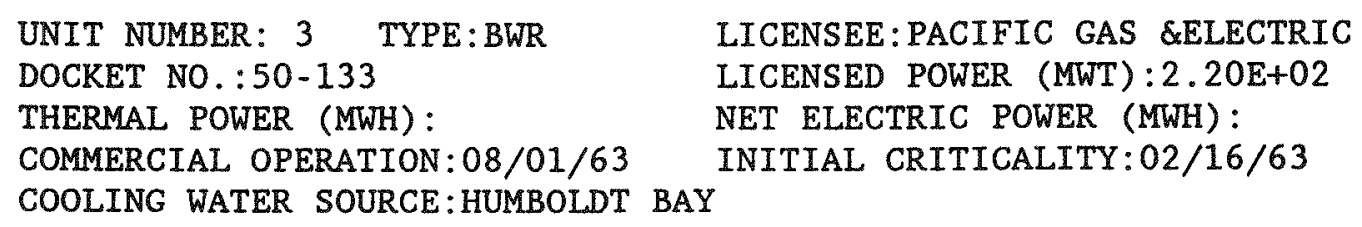

SR -90

$2.38 \mathrm{E}-05$

$<6.40 \mathrm{E}+01$

CS -134

$<4.54 \mathrm{E}-07$

CS -137

$<1.58 \mathrm{E}-05$

UNIDENTIFIED

$1.28 \mathrm{E}-05$

$<1.58 \mathrm{E}-05$

LIQUID EFFLUENTS

NUCLIDES RELEASED

CO- 60

SR -90

CS -134

CS -137

UNIDENTIFIED

ACTIVITY (CI)

$8.79 E-04$

$2.50 \mathrm{E}-04$

$3.42 \mathrm{E}-0.5$

$7.25 \mathrm{E}-03$

$<1.04 \mathrm{E}-05$

TOTAL AIRBORNE TRITIUM RELEASE <3.94E-02

TOTAL LIQUID TRITIUM RELEASE 1.14E-03

VOLUME OF LIQUID WASTE RELEASED (PRIOR TO DILUTION)

VOLUME OF DILUTION WATER USED DURING PERIOD
LITERS

LITERS
$3.71 \mathrm{E}+05$

$6.79 \mathrm{E}+10$ 
EFFLUENT AND WASTE DISPOSAL ANNUAL REPORT FOR YEAR 1989 AIRBORNE AND LIQUID EFFLUENTS

LIQUID EFFLUENTS
NUCLIDES RELEASED
TC-99M
RU-103
RU-106
AG-110M
SB-124
SB-125
$I-131$
$I-132$
TE-132
$I-133$
$X E-133$
$C S-134$
$I-134$
$I-135$
XE-135
$X E-135 M$
$C S-137$
$C S-138$
BA -139
LA -140

ACTIVITY (CI)
$5.21 E-04$
$3.01 E-05$
$4.38 E-03$
$6.32 E-06$
$6.84 E-03$
$2.88 E-02$
$1.14 E-02$
$4.76 E-02$
$3.98 E-05$
$8.37 E-02$
$7.51 E-03$
$7.52 E-05$
$3.68 E-02$
$8.14 E-02$
$7.34 E-03$
$4.18 E-02$
$2.30 E-02$
$1.01 E-02$
$5.01 E-03$
$6.74 E-05$

TOTAL AIRBORNE TRITIUM RELEASE 3.04E-01

TOTAL LIQUID TRITIUM RELEASE 5.60E+02

VOLUME OF LIQUID WASTE RELEASED (PRIOR TO DILUTION) LITERS $1.87 \mathrm{E}+08$ VOLUME OF DILUTION WATER USED DURING PERIOD LITERS $9.04 E+11$ 
INSTALIATION: INDIAN POINT $1-2$

EFFLUENT AND WASTE DISPOSAL ANNUAL REPORT FOR YEAR 1989 SOLID EFFLUENTS

SOLID WASTE DISPOSITION

NUMBER OF SHIPMENTS MODE OF TRANSPORTATION 41

TRUCK

ESTIMATE OF MAJOR NUCLIDE COMPOSITION (BY TYPE OF WASTE)

A

CO- 58

$\mathrm{CO}-60$

CS -134

CS -137

FE -55

$\mathrm{H}-3$

$\mathrm{MN}-54$

NI -63

OTHERS

SB -125

B

C. -14

Co- 58

CO- 60

CS -134

CS -137

FE -55

$\mathrm{H}-3$

I -129

MN -54

NB- 95

NI -63

PU -241

SR -90

ZR -95

\section{TYPE OF WASTE}
A. SPENT RESINS, FILTER SLUDGES, EVAPORATOR BOTTOMS, ETC.
B. DRY COMPRESSIBLE WASTE, CONTAMINATED EQUIPMENT, ETC.
C. IRRADIATED COMPONENTS, CONTROL RODS, ETC.
D. OTHER

DESTINATION
BARNWELL SC

JAN-JUNE JULY-DEC

\begin{tabular}{|c|c|c|}
\hline$\frac{8}{8}$ & $3.24 E+01$ & $2.74 E+01$ \\
\hline 6 & $4.29 E+01$ & $1.70 \mathrm{E}+01$ \\
\hline$\frac{8}{8}$ & $2.30 E+00$ & $9.00 E-01$ \\
\hline 8 & $4.90 E+00$ & $3.16 E+01$ \\
\hline 8 & $6.30 \mathrm{E}+00$ & $4.10 E+00$ \\
\hline 8 & & $1.20 E+00$ \\
\hline 8 & 1. $30 \mathrm{E}+00$ & $2.05 E+00$ \\
\hline 8 & $9.30 \mathrm{E}+00$ & 1. $14 \mathrm{E}+01$ \\
\hline 웅 & $6.00 \mathrm{E}-01$ & $8.50 E-01$ \\
\hline 융 & & $3.50 \mathrm{E}+00$ \\
\hline 8 & $3.00 \mathrm{E}-01$ & $3.00 \mathrm{E}-01$ \\
\hline 8 & $9.40 E+00$ & $9.10 E+00$ \\
\hline 8 & $2.53 E+01$ & $2.49 \mathrm{E}+01$ \\
\hline 8 & $7.90 E+00$ & $7.70 E+00$ \\
\hline 용 & $1.68 \mathrm{E}+01$ & $1.63 E+01$ \\
\hline$\%$ & $7.80 \mathrm{E}+00$ & $7.60 E+00$ \\
\hline 왕 & $1.03 E+01$ & 1. $00 \mathrm{E}+01$ \\
\hline 8 & $1.00 \mathrm{E}-03$ & $1.00 E-03$ \\
\hline 8 & $3.20 \mathrm{E}+00$ & $3.10 E+00$ \\
\hline 8 & $9.00 \mathrm{E}-01$ & $9.00 E-01$ \\
\hline 8 & $1.38 E+01$ & 1. $34 \mathrm{E}+01$ \\
\hline 8 & & $2.50 E+00$ \\
\hline 8 & $2.00 E-01$ & $2.00 E-01$ \\
\hline$\frac{8}{8}$ & $4.10 \mathrm{E}+00$ & $4.00 E+00$ \\
\hline
\end{tabular}

UNIT YEAR TOTAL

M3 $3.13 \mathrm{E}+01$

CI $\quad 3.34 \mathrm{E}+02$

M3 $4.46 \mathrm{E}+02 *$

CI $2.62 \mathrm{E}+01$

M3

CI

M3

CI

*VOLUME AFTER REDUCTION 
EFFLUENT AND WASTE DISPOSAL ANNUAL REPORT FOR YEAR 1989

AIRBORNE AND LIQUID EFFLUENTS

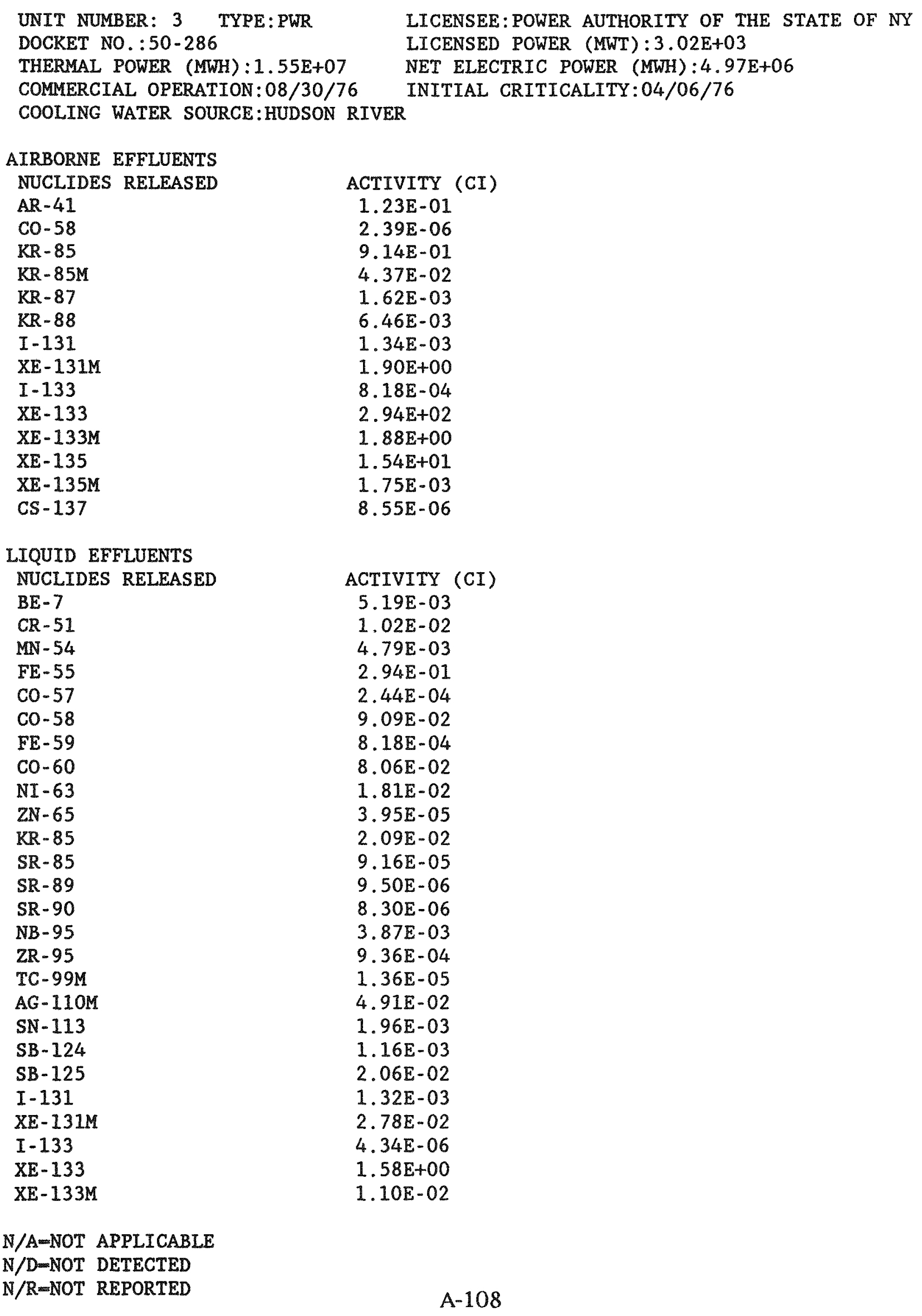


EFFLUENT AND WASTE DISPOSAL ANNUAL REPORT FOR YEAR 1989 AIRBORNE AND LIQUTD EFFLUENTS

LIQUID EFFLUENTS NUCLIDES RELEASED

CS -134

$X E-135$

CS -137

LA -140
ACTIVITY (CI)

$3.29 E-03$

$8.26 E-03$

$3.91 \mathrm{E}-03$

$5.52 E-04$

TOTAL AIRBORNE TRITIUM RELEASE 2.21E+00

TOTAL LIQUID TRITIUM RELEASE $3.51 E+02$

VOLUME OF LIQUID WASTE RELEASED (PRIOR TO DILUTION) LITERS 9.30E+06

VOLUME OF DILUTION WATER USED DURING PERIOD

LITERS $\quad 7.98 \mathrm{E}+11$ 
INSTALLATION: INDIAN POINT 3

EFFLUENT AND WASTE DISPOSAL ANNUAL REPORT FOR YEAR 1989 SOLID EFFLUENTS

SOLID WASTE DISPOSITION NUMBER OF SHIPMENTS

$\begin{array}{rr}2 & \text { TRUCK } \\ 10 & \text { TRUCK } \\ 2 & \text { TRUCK } \\ 13 & \text { TRUCK }\end{array}$

ESTIMATE OF MAJOR NUCLIDE COMPOSITION (BY TYPE OF WASTE)

\author{
DESTINATION \\ ALARON, WAMPUM PA \\ BARNWELL SC \\ QUADREX, OAK RIDGE TN \\ SEG, OAK RIDGE TN
}

$$
\text { JAN-JUNE JULY-DEC }
$$
A
CO- 58
$\mathrm{CO}-60$
$\mathrm{CR}-51$
CS -134
CS -137
FE -55
MN -54
NB- 95
NI -63
ZR-95
B
$\mathrm{CO}-58$
$\mathrm{CO}-60$
CS -137
FE -55
NI -63
D
Co- 58
CO- 60
CS -137
FE -55
NI -63

TYPE OF WASTE

A. SPENT RESINS, FILTER SLUDGES, EVAPORATOR BOTTOMS, ETC.

B. DRY COMPRESSIBLE WASTE, CONTAMINATED EQUIPMENT, ETC.

C. IRRADIATED COMPONENTS, CONTROL RODS, ETC.

D. OTHER DRY COMPRESS. EQUIP/NOL. RED.
$1.40 \mathrm{E}+01$
$3.65 \mathrm{E}+01$
1. $10 \mathrm{E}+01$
1. $51 E+01$
$1.50 \mathrm{E}+00$
$5.30 \mathrm{E}+00$
$2.00 \mathrm{E}+01$
2. $50 \mathrm{E}+00$
1. $80 E+01$
$3.90 \mathrm{E}+00$
$2.60 \mathrm{E}+01$
2. $39 \mathrm{E}+01$
$1.40 \mathrm{E}+00$
$1.80 \mathrm{E}+00$
$5.90 E+00$
1. $40 E+00$
5. $10 \mathrm{E}+00$
1. $20 \mathrm{E}+00$
$5.00 \mathrm{E}+00$
2. $80 \mathrm{E}+01$
2. $80 \mathrm{E}+01$
2. $00 \mathrm{E}+00$
2. $00 \mathrm{E}+00$
$5.90 \mathrm{E}+01$
5. $90 \mathrm{E}+01$
$5.00 \mathrm{E}+00$
5. $00 \mathrm{E}+00$
$5.00 \mathrm{E}+00$
2. $80 \mathrm{E}+01$
2. $80 \mathrm{E}+01$
$2.00 \mathrm{E}+00$
$2.00 \mathrm{E}+00$
$5.90 \mathrm{E}+01$
5. $90 \mathrm{E}+01$
$5.00 \mathrm{E}+00$
$5.00 \mathrm{E}+00$

\% $\quad 5.00 \mathrm{E}+00$

\& $5.00 \mathrm{E}+00$

UNIT YEAR TOTAL
M3 $3.71 \mathrm{E}+01$
CI $3.96 E+01$
M3 1.13E+01
CI $3.06 \mathrm{E}+02$
M3
CI
M3
CI
$5.29 E+02$
4. $70 \mathrm{E}+00$ 
EFFLUENT AND WASTE DISPOSAL ANNUAL REPORT FOR YEAR 1989 AIRBORNE AND LIQUID EFFLUENTS

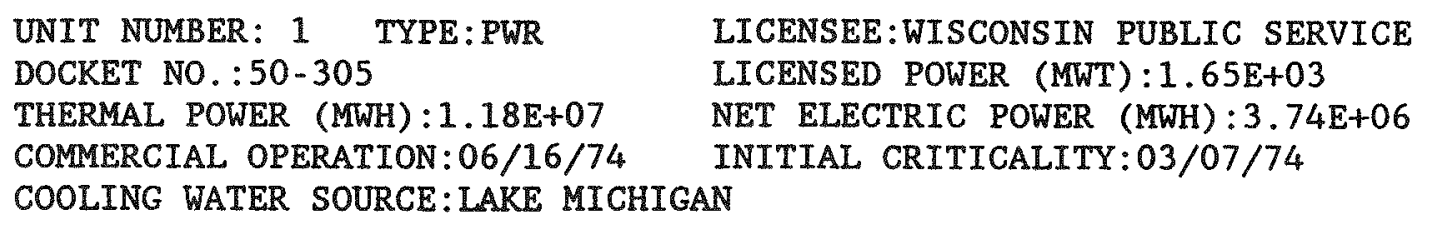

1. $71 \mathrm{E}-04$

$N / A=N O T$ APPLICABLE 
EFFLUENT AND WASTE DISPOSAL ANNUAL REPORT FOR YEAR 1989 AIRBORNE AND LIQUID EFFLUENTS

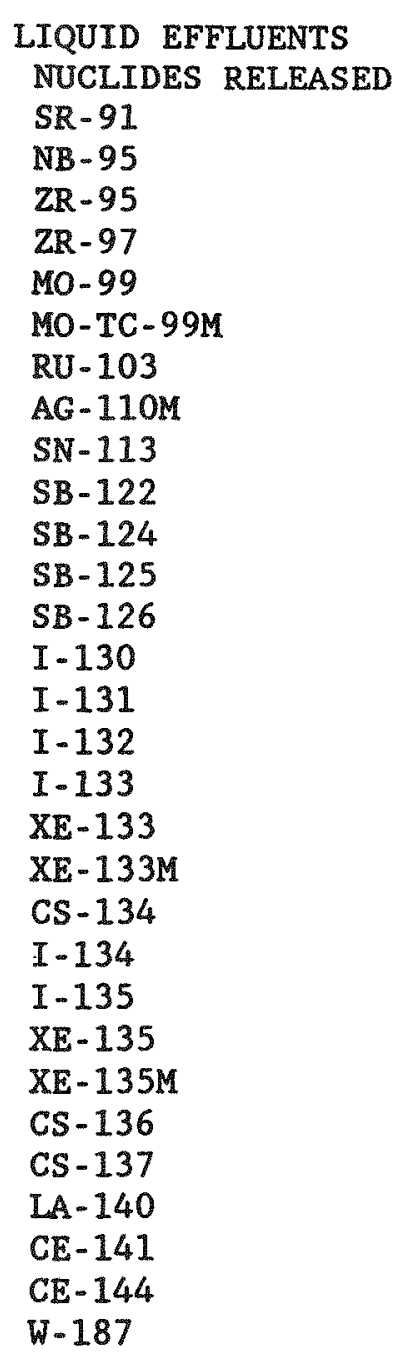

LIQUID EFFLUENTS

SR -91

NB-95

$\mathrm{ZR}-95$

MO-99

MO-TC-99M

$\mathrm{RU}-103$

AG-110M

SN -113

$\mathrm{SB}-122$

$\mathrm{SB}-124$

$S B-125$

I -131

I -132

$I-133$

$\mathrm{XE}-133 \mathrm{M}$

CS -134

$I-135$

$\mathrm{XE}-135$

$\mathrm{XE}-135 \mathrm{M}$

CS -136

CS -137

LA- 140

W-187
ACTIVITY (CI)
$9.26 E-06$
$1.48 \mathrm{E}-02$
$1.03 \mathrm{E}-02$
3. $98 \mathrm{E}-04$
3. $16 \mathrm{E}-07$
$2.98 \mathrm{E}-05$
$6.95 E-06$
6.12E-02
4. $38 \mathrm{E}-03$
1.11E-04
$1.44 \mathrm{E}-02$
$1.08 \mathrm{E}-02$
1. $92 \mathrm{E}-06$
2. $38 \mathrm{E}-04$
$4.87 \mathrm{E}-03$
8. 87E-04
3. 12E- 03
2. $31 \mathrm{E}-02$
3. $37 \mathrm{E}-04$
$8.77 E-03$
$1.69 \mathrm{E}-04$
$1.52 \mathrm{E}-03$
$1.03 \mathrm{E}-03$
1. $22 \mathrm{E}-03$
1. $65 \mathrm{E}-04$
$7.02 E-03$
$2.49 \mathrm{E}-04$
$2.48 \mathrm{E}-05$
1.17E-04
$9.02 E-04$

TOTAL AIRBORNE TRITIUM RELEASE 1.01E+01 TOTAL LIQUID TRITIUM RELEASE $3.41 \mathrm{E}+02$

VOLUME OF LIQUID WASTE RELEASED (PRIOR TO DILUTION) LITERS 4.69E+06 VOLUME OF DILUTION WATER USED DURING PERIOD

LITERS $4.09 \mathrm{E}+10$ 
EFFLUENT AND WASTE DISPOSAL ANNUAL REPORT FOR YEAR 1989 SOLID EFFLUENTS

SOLID WASTE DISPOSITION NUMBER OF SHIPMENTS

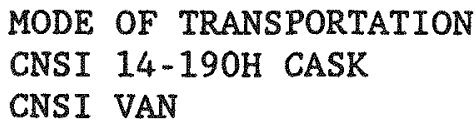
2 CNSI VAN

ESTIMATE OF MAJOR NUCLIDE COMPOSITION (BY TYPE OF WASTE)

A

$$
\begin{aligned}
& \text { AG-110M } \\
& \text { C- } 14 \\
& \text { CM- } 242 \\
& \text { CO-57 } \\
& \text { CO-58 } \\
& \text { CO-60 } \\
& \text { CS }-137 \\
& \text { FE }-55 \\
& \text { MN }-54 \\
& \text { NB- } 94 \\
& \text { NI }-59 \\
& \text { NI }-63 \\
& \text { PU- } 241 \\
& \text { SB-125 } \\
& \text { SR-90 } \\
& \text { TC- } 99 \\
& \text { TRU }
\end{aligned}
$$

B

$A G-110 M$

C -14

CM- 242

$\mathrm{CO}-57$

$\mathrm{CO}-58$

$\mathrm{CO}-60$

CS -134

CS -137

FE -55

$\mathrm{MN}-54$

NB -95

NI -59

NI -63

PU- 241

$\mathrm{SB}-125$

SN -113

SR -90

TRU

ZR-95
DESTINATION

BARNWELL SC

BARNWELL SC

JAN-JUNE JULY-DEC

$$
\begin{array}{ll}
4.05 E-01 & 4.05 E-01 \\
5.01 E-02 & 5.02 E-02 \\
5.11 E-06 & 5.11 E-06 \\
1.58 E-01 & 1.59 E-01 \\
8.80 E-01 & 8.81 E-01 \\
4.19 E+01 & 4.18 E+01 \\
3.93 E-01 & 3.93 E-01 \\
1.42 E+01 & 1.42 E+01 \\
1.05 E+00 & 1.05 E+00 \\
4.14 E-03 & 4.21 E-02 \\
4.09 E-01 & 4.09 E-01 \\
3.55 E+01 & 3.56 E+01 \\
1.34 E-03 & 1.34 E-03 \\
5.06 E+00 & 5.08 E+00 \\
7.72 E-03 & 7.70 E-03 \\
4.73 E-04 & 4.71 E-04 \\
4.33 E-05 & 4.32 E-05
\end{array}
$$
$5.73 E-01$
1. $25 \mathrm{E}-01$
4. $94 E-04$
9.42E-02
1. 54E-03
3. $35 \mathrm{E}+00$
1. $27 \mathrm{E}-01$
1. $76 \mathrm{E}+01$
$1.98 \mathrm{E}+01$
$7.27 \mathrm{E}-02$
$2.83 E+01$
2. $12 \mathrm{E}-01$
2.27E-01
$5.52 \mathrm{E}+01$
$3.97 \mathrm{E}+01$
$1.15 \mathrm{E}+00$
1. $23 E+00$
$1.79 \mathrm{E}+00$
4. 11E-01
2.01E-01
1.75E+01
$9.05 E+00$
$2.44 \mathrm{E}-02$
$7.64 \mathrm{E}-02$
1. $18 \mathrm{E}+00$
3. $83 \mathrm{E}-01$
$2.37 \mathrm{E}-01$
$2.27 \mathrm{E}-01$
1. $16 \mathrm{E}-03$
4. 14E-04
$1.29 \mathrm{E}-03$
8. $62 \mathrm{E}-01$
$1.69 E-01$ 
D

AG-110M

C -14

CM- 242

$\mathrm{CO}-57$

$\mathrm{CO}-58$

$\mathrm{CO}-60$

CR -51

CS -134

CS -137

FE- 55

FE -59

$\mathrm{MN}-54$

NB -94

NB -95

NI -59

NI -63

PU-241

SB- 124

$S B-125$

$\mathrm{SN}-112$

SN -113

SR -90

TRU

ZR-95

\begin{tabular}{|c|c|c|}
\hline 8 & $2.10 E-01$ & \\
\hline ; & $1.39 \mathrm{E}-03$ & \\
\hline 8 & $3.22 E-07$ & $5.99 E-06$ \\
\hline$\frac{8}{8}$ & $6.13 E-05$ & $9.92 \mathrm{E}-02$ \\
\hline 8 & $7.52 \mathrm{E}+01$ & $1.85 E+01$ \\
\hline 8 & 1. $10 \mathrm{E}+00$ & $2.88 E+01$ \\
\hline 8 & $1.52 E+01$ & \\
\hline 8 & & $8.24 E-04$ \\
\hline 8 & $4.73 E-05$ & $3.77 \mathrm{E}-01$ \\
\hline 8 & $4.07 E-01$ & $2.71 E+01$ \\
\hline 8 & $7.10 \mathrm{E}-01$ & \\
\hline 8 & $2.59 E-01$ & $4.91 E-03$ \\
\hline 8 & & $2.30 E-02$ \\
\hline 8 & $3.06 \mathrm{E}+00$ & $4.50 E+00$ \\
\hline 8 & $1.00 \mathrm{E}-02$ & \\
\hline 8 & $9.39 \mathrm{E}-01$ & $1.60 E+01$ \\
\hline 8 & $1.59 E-05$ & $2.96 \mathrm{E}-04$ \\
\hline 8 & $8.70 \mathrm{E}-01$ & \\
\hline 8 & $7.67 \mathrm{E}-04$ & $1.64 E+00$ \\
\hline 8 & $1.54 E-04$ & \\
\hline 8 & & $5.32 \mathrm{E}-01$ \\
\hline 8 & $7.53 E-07$ & \\
\hline 8 & $2.69 E-07$ & $2.20 \mathrm{E}-05$ \\
\hline 8 & $1.93 E+00$ & $2.29 \mathrm{E}+00$ \\
\hline
\end{tabular}

TYPE OF WASTE
A. SPENT RESINS, FILTER SLUDGES, EVAPORATOR BOTTOMS, ETC.
B. DRY COMPRESSIBLE WASTE, CONTAMINATED EQUIPMENT, ETC.
C. IRRADIATED COMPONENTS, CONTROL RODS, ETC.
D. OTHER
NON-COMPR. CONTAM. SCRAP, FILTER

$\begin{array}{lc}\text { UNIT } & \text { YEAR TOTAI } \\ \text { M3 } & 1.04 \mathrm{E}+01 \\ \text { CI } & 6.32 \mathrm{E}+02 \\ \text { M3 } & 4.76 \mathrm{E}+01 \\ \text { CI } & 3.40 \mathrm{E}+00 \\ \text { M3 } & 0.00 \mathrm{E}+00 \\ \text { CI } & 0.00 \mathrm{E}+00 \\ \text { M3 } & 1.20 \mathrm{E}+01 \\ \text { CI } & 1.40 \mathrm{E}+02\end{array}$


EFFLUENT AND WASTE DISPOSAL ANNUAL REPORT FOR YEAR 1989 AIRBORNE AND LIQUID EFFLUENTS

$\begin{array}{lc}\text { UNIT NUMBER: } 1 \text { TYPE:BWR } & \text { LICENSEE: } \\ \text { DOCKET NO.: } 50-409 & \text { LICENSED } \\ \text { THERMAL POWER (MWH) : } & \text { NET ELECTR } \\ \text { COMMERCIAL OPERATION:11/01/69 } & \text { INITIAL CR } \\ \text { COOLING WATER SOURCE:MISSISSIPPI RIVER } \\ \text { AIRBORNE EFFLUENTS } \\ \text { NUCLIDES RELEASED } \\ \text { MN-54 } & \text { ACTIVITY (CI) } \\ \text { CO-60 } & 3.78 E-07 \\ \text { CS-137 } & 7.19 E-06 \\ & 5.38 E-06 \\ \text { LIQUID EFFLUENTS } & \\ \text { NUCLIDES RELEASED } & \\ \text { MN-54 } & \\ \text { FE-55 } & \text { ACTIVITY (CI) } \\ \text { CO-57 } & 6.50 E-03 \\ \text { CO-60 } & 3.76 E-02 \\ \text { ZN-65 } & 1.50 E-05 \\ \text { SR-89 } & 8.74 E-02 \\ \text { SR-90 } & 3.70 E-05 \\ \text { AG-110M } & 1.82 E-04 \\ \text { CS-134 } & 9.63 E-04 \\ \text { CS-137 } & 1.34 E-04 \\ \text { CE-144 } & 3.30 E-04 \\ \text { AM-241 } & 3.53 E-02 \\ & 7.44 E-05 \\ & 3.90 E-05\end{array}$

TOTAL AIRBORNE TRITIUM RELEASE 1.06E+00

TOTAL LIQUID TRITIUM RELEASE $2.79 E+00$

VOLUME OF LIQUID WASTE RELEASED (PRIOR TO DILUTION) LITERS 5.09E+05

VOLUME OF DILUTION WATER USED DURING PERIOD TITERS 7.03E+09 
EFFLUENT AND WASTE DISPOSAL ANNUAL REPORT FOR YEAR 1989 SOLID EFFLUENTS

\section{SOLID WASTE DISPOSITION NUMBER OF SHIPMENTS 4 1 MODE OF TRANSPORTATION SOLE USE SOLE USE}

ESTIMATE OF MAJOR NUCLIDE COMPOSITION (BY TYPE OF WASTE)

A AM-241

C- 14

CM- 242

CM $-243 / 244$

$\mathrm{CO}-60$

CS -137

FE -55

H-3

I- 129

$\mathrm{MN}-54$

NI -59

NI -63

PU -238

PU $-239 / 240$

PU-241

SR -90

TC -99

U- 235

$\mathrm{U}-238$

B

CO- 60

CS -137

FE- 55

MN -54

NI -63

PU- 241

TYPE OF WASTE

A. SPENT RESINS, FILTER SLUDGES, EVAPORATOR BOTTOMS, ETC.

B. DRY COMPRESSIBLE WASTE, CONTAMINATED EQUIPMENT, ETC.

C. IRRADIATED COMPONENTS, CONTROL RODS, ETC.

D. OTHER
DESTINATION

(QUADREX) OAK RIDGE, TN BARNWELL SC

JAN-JUNE JULY-DEC

$3.22 E-03$

$1.31 \mathrm{E}-02$

$1.80 \mathrm{E}-02$

$1.41 E-03$

$5.57 \mathrm{E}+01$

$9.42 \mathrm{E}+00$

$2.52 \mathrm{E}+01$

5. 32E-03

$2.94 E-03$

3. $60 \mathrm{E}+00$

5. $70 \mathrm{E}-02$

$5.01 E+00$

$7.98 \mathrm{E}-03$

4. $48 \mathrm{E}-03$

3. $57 \mathrm{E}-01$

5. $89 \mathrm{E}-01$

$6.11 \mathrm{E}-05$

$2.45 \mathrm{E}-06$

$2.07 \mathrm{E}-06$

4. $38 \mathrm{E}+01$

$2.09 \mathrm{E}+00$

4. $51 \mathrm{E}+01$

$1.83 \mathrm{E}+00$

$6.84 E+00$

$3.60 \mathrm{E}-01$

UNIT YEAR TOTAL

M3 $\quad 4.62 \mathrm{E}+00$

CI $\quad 3.19 \mathrm{E}+01$

M3 $2.12 \mathrm{E}+00 *$

CI $2.23 \mathrm{E}-01$

M3

CI

M3

CI

*VOLUME AFTER REDUCTION (1.70E+1 M3 BEFORE PROCESSING) 
EFFLUENT AND WASTE DISPOSAL ANNUAL REPORT FOR YEAR 1989 AIRBORNE AND LIQUID EFFLUENTS

\begin{abstract}
UNIT NUMBER: 1 TYPE:BWR
DOCKET NO.: $50-373$

THERMAL POWER (MWH) $: 1.91 \mathrm{E}+07$

COMMERCIAL OPERATION:01/01/84

COOLING WATER SOURCE:RESERVOIR UNIT NUMBER: 2 TYPE:BWR

DOCKET NO. : $50-374$

THERMAL POWER (MWH): $2.04 E+07$

COMMERCIAL OPERATION: $10 / 19 / 84$

COOLING WATER SOURCE:RESERVOIR
\end{abstract}

\author{
LICENSEE: COMMONWEALTH EDISON COMPANY \\ LICENSED POWER (MWT) : $3.32 \mathrm{E}+03$ \\ NET ELECTRIC POWER (MWH) :6.16E+06 \\ INITIAL CRITICALITY:06/21/82 \\ LICENSEE: COMMONWEALTH EDISON COMPANY \\ LICENSED POWER (MWT) : $3.32 \mathrm{E}+03$ \\ NET ELECTRIC POWER (MWH) $: 6.50 E+06$ \\ INITIAL CRITICALITY:03/10/84
}

\begin{tabular}{|c|c|}
\hline \multicolumn{2}{|l|}{ AIRBORNE EFFLUENTS } \\
\hline NUCLIDES RELEASED & ACTIVITY (CI) \\
\hline$A R=41$ & $2.35 E-03$ \\
\hline $\mathrm{CR}-51$ & $1.33 E-04$ \\
\hline$M \mathbb{N}-54$ & $2.03 E-04$ \\
\hline $\mathrm{FE}-59$ & $8.98 \mathrm{E}-06$ \\
\hline$C 0-60$ & $2.20 E-03$ \\
\hline $\mathrm{KR}-85 \mathrm{M}$ & $1.52 E+02$ \\
\hline $\mathrm{KR}-87$ & $8.30 E+01$ \\
\hline $\mathrm{KR}-88$ & $3.31 E+02$ \\
\hline$Z R-95$ & $9.01 E-06$ \\
\hline$I-131$ & $5.68 \mathrm{E}-03$ \\
\hline$I-132$ & $6.99 E-03$ \\
\hline$I-133$ & $1.24 E-02$ \\
\hline $\mathrm{XE}-133$ & $4.16 E+02$ \\
\hline$X E-133 M$ & $2.16 \mathrm{E}-06$ \\
\hline$I-134$ & $1.65 E-02$ \\
\hline$I-135$ & $6.29 E-03$ \\
\hline$X E-135$ & $9.39 E+01$ \\
\hline CS -136 & $6.58 \mathrm{E}-06$ \\
\hline
\end{tabular}

LIQUID EFFLUENTS

NUCLIDES RELEASED

NA -24

CR -51

MN- 54

FE -55

CO- 58

FE- 59

CO- 60

ZN-65

SR-89

SR -90

TC-99M

AG-11OM

CS -137
2.35E-03
1. $33 \mathrm{E}-04$
2.03E-04
8. $98 \mathrm{E}-06$
2. 20E-03
1. $52 \mathrm{E}+02$
8. $30 \mathrm{E}+01$
3. $31 E+02$
$9.01 \mathrm{E}-06$
$5.68 \mathrm{E}-03$
$6.99 \mathrm{E}-03$
1. 24E-02
$1.65 \mathrm{E}-02$
6.29E-03
$6.58 \mathrm{E}-06$

ACTIVITY (CI)

4.06E-06

1. 23E-02

4. $47 \mathrm{E}-02$

$2.14 \mathrm{E}-01$

1. $34 \mathrm{E}-03$

1. 11E-03

1. $25 \mathrm{E}-01$

1. $79 \mathrm{E}-03$

$2.29 E-04$

2. $53 \mathrm{E}-05$

1. $10 \mathrm{E}-04$

$6.50 \mathrm{E}-05$

1. $29 \mathrm{E}-04$ 
EFFLUENT AND WASTE DISPOSAL ANNUAL REPORT FOR YEAR 1989 AIRBORNE AND LIQUID EFFLUENTS

TOTAL AIRBORNE TRITIUM RELEASE 5.99E-01

TOTAI LIQUID TRITIUM RELEASE 1.07E+00

VOLUME OF LIQUID WASTE RELEASED (PRIOR TO DILUTION) LITERS $1.51 \mathrm{E}+06$

VOLUME OF DILUTION WATER USED DURING PERIOD

LITERS $1.86 \mathrm{E}+09$ 
EFFLUENT AND WASTE DISPOSAL ANNUAL REPORT FOR YEAR 1989 SOLID EFFLUENTS

SOLID WASTE DISPOSITION

$\begin{array}{cl}\text { NUMBER OF SHIPMENTS } & \text { MODE OF TRANSPORTATION } \\ 16 & \text { HITTMAN } \\ 97 & \text { HITTMAN } \\ 16 & \text { HITTMAN } \\ 7 & \text { HITTMAN }\end{array}$

DESTINATION

BARNWELL SC

BEATTY NV

OAR RIDGE TN

WALTZ MILL PA

ESTIMATE OF MAJOR NUCLIDE COMPOSITION (BY TYPE OF WASTE)

JAN-JUNE JULY-DEC

A

CO- 60

$\mathrm{CR}-51$

FE -55

$\mathrm{MN}-54$

B

CO- 60

$\mathrm{FE}-55$

$M \mathbb{N}-54$

$\begin{array}{lll}8 & 2.49 \mathrm{E}+01 & 2.50 \mathrm{E}+01 \\ 8 & 1.78 \mathrm{E}+00 & 2.00 \mathrm{E}+00 \\ 8 & 6.07 \mathrm{E}+01 & 5.80 \mathrm{E}+01 \\ 8 & 1.27 \mathrm{E}+01 & 1.30 \mathrm{E}+01 \\ & & \\ 8 & 1.00 \mathrm{E}+01 & 1.00 \mathrm{E}+01 \\ 8 & 7.10 \mathrm{E}+01 & 7.10 \mathrm{E}+01 \\ 8 & 6.00 \mathrm{E}+00 & 6.00 \mathrm{E}+00\end{array}$

\section{TYPE OF WASTE}
A. SPENT RESINS, FILTER SLUDGES, EVAPORATOR BOTTOMS, ETC.
B. DRY COMPRESSIBLE WASTE, CONTAMINATED EQUIPMENT, ETC.
C. IRRADIATED COMPONENTS, CONTROL RODS, ETC.
D. OTHER

$\begin{array}{ll}\text { UNIT } & \text { YEAR TOTAL } \\ \text { M3 } & 3.80 \mathrm{E}+02 \\ \text { CI } & 4.32 \mathrm{E}+03 \\ \text { M3 } & 5.00 \mathrm{E}+02 \text { * } \\ \text { CI } & 4.07 \mathrm{E}+01 \\ \text { M3 } & \\ \text { CI } & \\ \text { M3 } & \\ \text { CI } & \end{array}$

*VOLUME PRIOR TO REDUCTION 
EFFLUENT AND WASTE DISPOSAL ANNUAL REPORT FOR YEAR 1989 AIRBORNE AND LIQUID EFFLUENTS

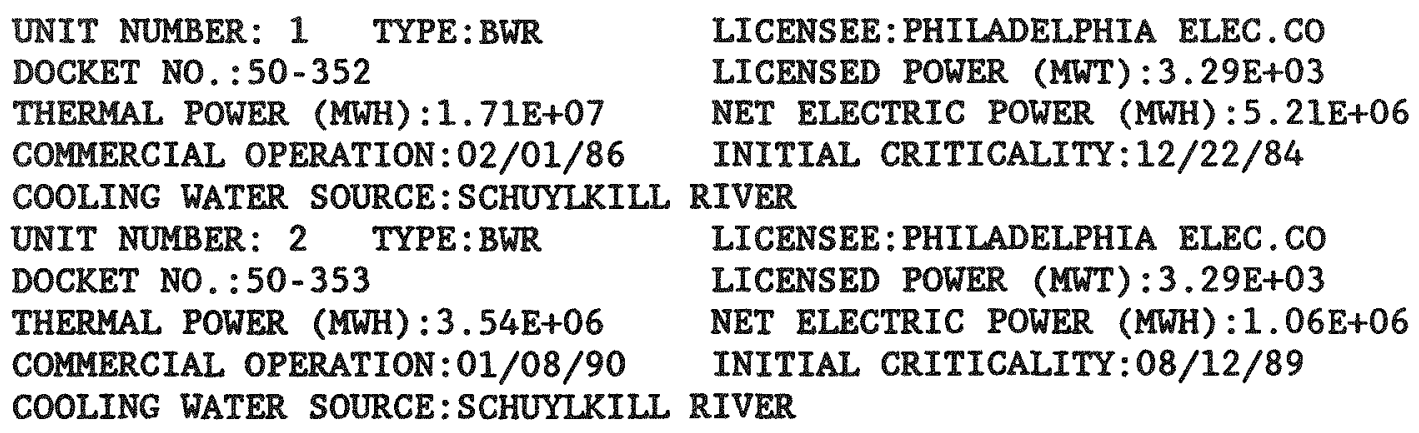

LICENSEE: PHILADELPHIA ELEC.CO LICENSED POWER (MWT) $: 3.29 E+03$ NET ELECTRIC POWER (MWH) :5.21E+06 INITIAL CRITICALITY: $12 / 22 / 84$

LICENSEE : PHILADELPHIA ELEC . CO LICENSED POWER (MWT) : $3.29 E+03$ NET ELECTRIC POWER (MWH):1.06E+06 INITIAL CRITICALITY:08/12/89

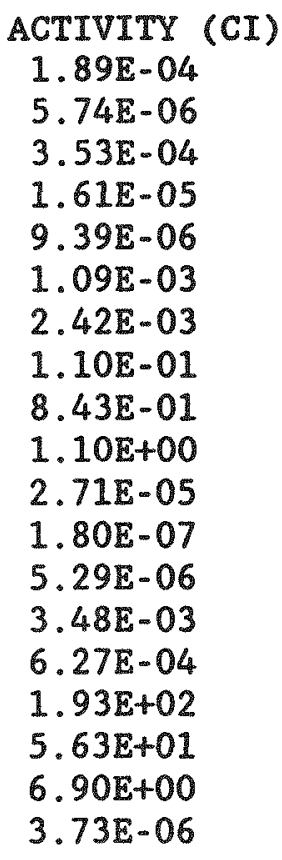


EFFLUENT AND WASTE DISPOSAL ANNUAL REPORT FOR YEAR 1989 AIRBORNE AND LIQUID EFFLUENTS

LIQUID EFFLUENTS
NUCLIDES RELEASED
CS -134
XE -135
CS -137
LA -140
CE -141
CE -144

LIQUID EFFLUENTS

NUCLIDES RELEASED

$\mathrm{CS}-134$

$X E-135$

CS -137

$\mathrm{CE}-141$

$\mathrm{CE}-144$
ACTIVITY (CI)
2.61E-03
$6.97 \mathrm{E}-03$
$3.86 \mathrm{E}-03$
$3.66 \mathrm{E}-05$
$9.20 \mathrm{E}-05$
$1.11 \mathrm{E}-04$

TOTAL AIRBORNE TRITIUM RELEASE 1.79E+01

TOTAL LIQUID TRITIUM RELEASE 2.70E+01

VOLUME OF LIQUID WASTE RELEASED (PRIOR TO DILUTIÓN) LITERS $1.93 \mathrm{E}+07$

VOLUME OF DILUTION WATER USED DURING PERIOD

LITERS $\quad 5.73 \mathrm{E}+08$ 
INSTALLATION : LIMERICK

EFFLUENT AND WASTE DISPOSAL ANNUAL REPORT FOR YEAR 1989 SOLID EFFLUENTS

SOLID WASTE DISPOSITION

NUMBER OF SHIPMENTS MODE OF TRANSPORTATION

71

100

12

TRUCK

TRUCK

TRUCK

ESTIMATE OF MAJOR NUCLIDE COMPOSITION (BY TYPE OF WASTE)

A

$A G-110 M$

$B A-140$

BA-LA- 140

C- 14

CE- 141

CO- 58

CO- 60

CR -51

CS -134

CS -136

CS -137

FE -55

FE -59

H -3

I -131

I -134

$\mathrm{MN}-54$

NB-95

NI -63

P- 32

PU -241

SR -89

SR -90

$\mathrm{ZN}-65$

ZR-95

B

AG-110M

$\mathrm{CO}-57$

Co- 58

CO- 60

CR -51

CS -134

CS -137

FE- 55

$\mathrm{H}-3$

I - 131

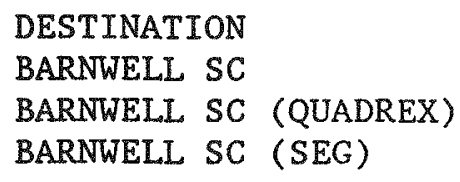

JAN-JUNE JULY-DEC

1. $31 \mathrm{E}-01$

$3.30 E-01$

$3.14 E-01$

7. $62 \mathrm{E}-03$

$4.76 \mathrm{E}+00$

$3.87 \mathrm{E}+00$

$2.40 \mathrm{E}+01$

7. $39 \mathrm{E}+00$

3. $33 \mathrm{E}-01$

$7.33 E+00$

$8.43 E-01$

$3.44 E-02$

$8.08 \mathrm{E}-02$

1. $13 \mathrm{E}+01$

1. $58 \mathrm{E}+00$

$1.01 \mathrm{E}+00$

$1.24 E-01$

$2.95 E-04$

$5.62 E-03$

3. $67 \mathrm{E}+01$

2. $22 \mathrm{E}-01$

8.00E-02

4. $90 \mathrm{E}-01$

4. $23 E+00$

$5.77 \mathrm{E}+00$

$1.07 \mathrm{E}+01$

1. $04 E+01$

$1.08 \mathrm{E}+01$

1. $69 \mathrm{E}+00$

1. 30E-01

$2.67 \mathrm{E}+00$

2. $11 E+00$

5. $70 \mathrm{E}-01$

$2.80 \mathrm{E}-01$

7. $83 E-03$

6.10E-01

$7.12 \mathrm{E}-03$

$4.93 E+01$

3. $00 E-02$

2. $10 \mathrm{E}-02$

3. $60 \mathrm{E}-02$

$5.24 \mathrm{E}+00$

4. $80 \mathrm{E}+00$

$6.08 \mathrm{E}+00$

4. $62 \mathrm{E}+00$

$1.46 \mathrm{E}+01$

$2.87 \mathrm{E}+01$

$1.07 \mathrm{E}+01$

$7.54 \mathrm{E}+00$

1. $11 \mathrm{E}+01$

$7.88 \mathrm{E}+00$

2. $38 \mathrm{E}+00$

$3.13 \mathrm{E}+00$

1. 10E-02

$1.07 \mathrm{E}-01$ 
ESTIMATE OF MAJOR NUCLIDE COMPOSITION (BY TYPE OF WASTE)

JAN-JUNE JULY-DEC

\section{B}

MN -54

NB- 95

NI -63

SR -89

ZN -65

C

Co- 60

FE -55

H- 3

NI -63

UNIDENTIFIED

\section{TYPE OF WASTE}
A. SPENT RESINS, FILTER SLUDGES, EVAPORATOR BOTTOMS, ETC.
B. DRY COMPRESSIBLE WASTE, CONTAMINATED EQUI PMENT, ETC.
C. IRRADIATED COMPONENTS, CONTROL RODS, ETC.
D. OTHER

$\begin{array}{lll}8 & 2.34 \mathrm{E}+00 & 5.05 \mathrm{E}+00 \\ 8 & 1.23 \mathrm{E}+00 & 8.59 \mathrm{E}-01 \\ 8 & & 1.79 \mathrm{E}-01 \\ 8 & 9.43 \mathrm{E}-01 & 6.45 \mathrm{E}-01 \\ 8 & 4.54 \mathrm{E}-01 & 3.64 \mathrm{E}+01 \\ & & \\ 8 & & 2.69 \mathrm{E}+01 \\ 8 & & 7.03 \mathrm{E}+01 \\ 8 & & 1.00 \mathrm{E}-03 \\ 8 & & 1.23 \mathrm{E}+00 \\ 8 & & 1.57 \mathrm{E}+00\end{array}$

$\begin{array}{lc}\text { UNIT } & \text { YEAR TOTAL } \\ \text { M3 } & 4.27 \mathrm{E}+02 \\ \text { CI } & 2.57 \mathrm{E}+03 \\ \text { M3 } & 1.48 \mathrm{E}+02 \\ \text { CI } & 8.44 \mathrm{E}+00 \\ \text { M3 } & 6.37 \mathrm{E}-01 \\ \text { CI } & 3.14 \mathrm{E}+04 \\ \text { M3 } & \\ \text { CI } & \end{array}$

*VOLUME AFTER REDUCTION. PROCESSED BY OFFSITE VENDORS (QUADREX, SEG) 
EFFLUENT AND WASTE DISPOSAL ANNUAL REPORT FOR YEAR 1989 AIRBORNE AND LIQUTD EFFLUENTS
UNIT NUMBER: 1 TYPE:PWR
DOCKET NO. : 50-309
LICENSEE:MAINE YANKEE ATOMIC POWER
THERMAL POWER (MWH) $: 2.11 \mathrm{E}+07$
COMMERCIAL OPERATION: $12 / 28 / 72$
LICENSED POWER (MWT) : $2.70 \mathrm{E}+03$
NET ELECTRIC POWER (MWH):6.94E+06
COOLING WATER SOURCE:BACK RIVER
INITIAL CRITICALITY: $10 / 23 / 72$

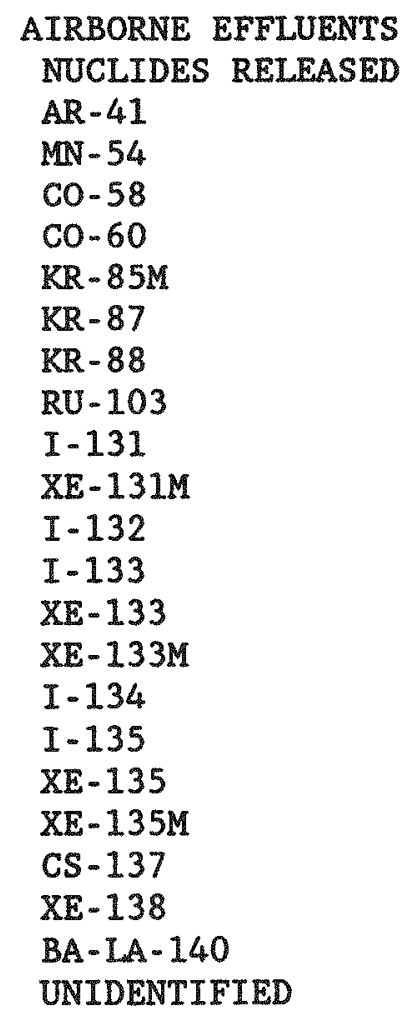

ACTIVITY (CI)

$9.68 \mathrm{E}-02$

$1.75 \mathrm{E}-07$

$1.73 E-05$

$5.69 E-06$

$3.88 \mathrm{E}-02$

$5.64 \mathrm{E}-02$

$2.79 \mathrm{E}-02$

$2.34 \mathrm{E}-05$

1. $89 \mathrm{E}-04$

1. $10 \mathrm{E}-01$

$9.56 \mathrm{E}-05$

$4.70 \mathrm{E}-04$

1. $62 \mathrm{E}+01$

$1.79 \mathrm{E}-01$

$6.28 \mathrm{E}-06$

$1.14 \mathrm{E}-04$

$1.03 E+00$

$1.18 \mathrm{E}-01$

3.71E-06

$5.32 \mathrm{E}-02$

2. $36 \mathrm{E}-10$

2. $26 E+00$

LIQUID EFFLUENTS

NUCLIDES RELEASED

ACTIVITY (CI)

CR- 51

MN -54

4.89E-04

$1.85 \mathrm{E}-03$

FE -55

$2.18 \mathrm{E}-02$

CO- 57

$4.25 E-05$

CO- 58

$2.66 \mathrm{E}-02$

CO- 60

3. $47 E-02$

$\mathrm{ZN}-65$

$\mathrm{KR}-85 \mathrm{M}$

$9.39 \mathrm{E}-08$

KR -87

$2.11 E-06$

KR -88

$1.04 \mathrm{E}-05$

SR-89

$1.66 \mathrm{E}-06$

$7.08 \mathrm{E}-04$

SR-91

Y-91M

ZR-NB-95

$5.13 \mathrm{E}-05$

$5.78 \mathrm{E}-05$

4. 29E-05

MO- 99

TC $-99 \mathrm{M}$

$1.69 \mathrm{E}-04$

RU- 103

$4.15 E-04$

AG-110M

$9.32 \mathrm{E}-07$

$7.69 \mathrm{E}-03$

N/A=NOT APPLICABLE

N/D $=$ NOT DETECTED

$N / R=N O T$ REPORTED 
EFFLUENT AND WASTE DISPOSAL ANNUAL REPORT FOR YEAR 1989 AIRBORNE AND LIQUID EFFLUENTS

$\begin{array}{lr}\text { LIQUID EFFLUENTS } & \\ \text { NUCLIDES RELEASED } & \text { ACTIVITY } \\ \text { SN-113 } & 9.88 \mathrm{E}-05 \\ \text { SB }-124 & 3.44 \mathrm{E}-02 \\ \text { SB-125 } & 2.49 \mathrm{E}-02 \\ \text { I-131 } & 5.00 \mathrm{E}-03 \\ \text { XE-131M } & 6.27 \mathrm{E}-04 \\ \text { TE-132 } & 7.28 \mathrm{E}-07 \\ \text { I-133 } & 1.15 \mathrm{E}-03 \\ \text { TE-133M } & 8.09 \mathrm{E}-06 \\ \text { XE-133 } & 1.89 \mathrm{E}-01 \\ \text { XE-133M } & 2.23 \mathrm{E}-03 \\ \text { CS-134 } & 3.96 \mathrm{E}-03 \\ \text { TE-134 } & 1.74 \mathrm{E}-03 \\ \text { I-135 } & 2.21 \mathrm{E}-05 \\ \text { XE-135 } & 1.69 \mathrm{E}-03 \\ \text { CS-137 } & 1.60 \mathrm{E}-02 \\ \text { BA-139 } & 4.87 \mathrm{E}-05 \\ \text { BA-LA-140 } & 8.26 \mathrm{E}-04 \\ \text { LA-142 } & 1.77 \mathrm{E}-06 \\ \text { CE-144 } & 6.76 \mathrm{E}-06 \\ \text { OTAL AIRBORNE TRITIUM RELEASE } & 1.26 \mathrm{E}+01 \\ \text { OTAL LIQUID TRITIUM RELEASE } & 4.22 \mathrm{E}+02\end{array}$

VOLUME OF LIQUID WASTE RELEASED (PRIOR TO DILUTION) 
EFFLUENT AND WASTE DISPOSAL ANNUAL REPORT FOR YEAR 1989 SOLID EFFLUENTS

\section{SOLID WASTE DISPOSITION NUMBER OF SHIPMENTS 14
$\# \quad 57$ \\ MODE OF TRANSPORTATION \\ TRUCK OVER HIGHWAY \\ TRUCK OVER HIGHWAY}

\#SEG PERFORMS SUPER COMPACTING OF DAW

ESTIMATE OF MAJOR NUCLIDE COMPOSITION (BY TYPE OF WASTE)

A
$B A-137 M$
C. 14
CO- 58
CO- 60
CS -134
CS -137
FE -55
MN -54
NI -63
$S B-125$
$\mathrm{CO}-58$
$\mathrm{CO}-60$
CS -134
CS -137
$\mathrm{FE}-55$
NI -63
SB- 125
ZR-95

B

\section{TYPE OF WASTE}

A. SPENT RESINS, FILTER SLUDGES, EVAPORATOR BOTTOMS, ETC.

B. DRY COMPRESSIBLE WASTE, CONTAMINATED EQUIPMENT, ETC.

C. IRRADIATED COMPONENTS, CONTROL RODS, ETC.

D. OTHER
DESTINATION

BARNWELL SC

SEG, BARNWELL SC
JAN-JUNE JULY-DEC

$\begin{array}{lll}8 & 2.00 \mathrm{E}+00 & \\ 8 & & 1.50 \mathrm{E}+00 \\ 8 & & 3.80 \mathrm{E}+00 \\ 8 & 2.20 \mathrm{E}+01 & 3.31 \mathrm{E}+01 \\ 1.69 \mathrm{E}+01 \\ 8 & & 2.26 \mathrm{E}+01 \\ 8 & 2.00 \mathrm{E}+00 & 1.44 \mathrm{E}+01 \\ \frac{8}{8} & 3.50 \mathrm{E}+01 & 1.20 \mathrm{E}+00 \\ 8 & & \\ 8 & 3.10 \mathrm{E}+01 & 4.40 \mathrm{E}+00 \\ 8 & 1.00 \mathrm{E}+00 & \\ & & \\ 8 & 1.10 \mathrm{E}+01 & 1.10 \mathrm{E}+01 \\ 8 & 2.20 \mathrm{E}+01 & 2.20 \mathrm{E}+01 \\ 8 & 3.00 \mathrm{E}+00 & 3.00 \mathrm{E}+00 \\ 8 & 1.00 \mathrm{E}+01 & 1.00 \mathrm{E}+01 \\ 8 & 2.60 \mathrm{E}+01 & 2.60 \mathrm{E}+01 \\ 8 & 1.40 \mathrm{E}+01 & 1.40 \mathrm{E}+01 \\ 8 & 2.00 \mathrm{E}+00 & 2.00 \mathrm{E}+00 \\ 8 & 5.00 \mathrm{E}+00 & 5.00 \mathrm{E}+00\end{array}$

UNIT YEAR TOTAL

M3 2.33E+01

CI $2.31 \mathrm{E}+02$

M3 1.72E+02*

CI $5.50 E+00$

M3

CI

M3

CI

*VOLUME AFTER COMPACTION 
EFFLUENT AND WASTE DISPOSAL ANNUAL REPORT FOR YEAR 1989 AIRBORNE AND LIQUID EFFLUENTS

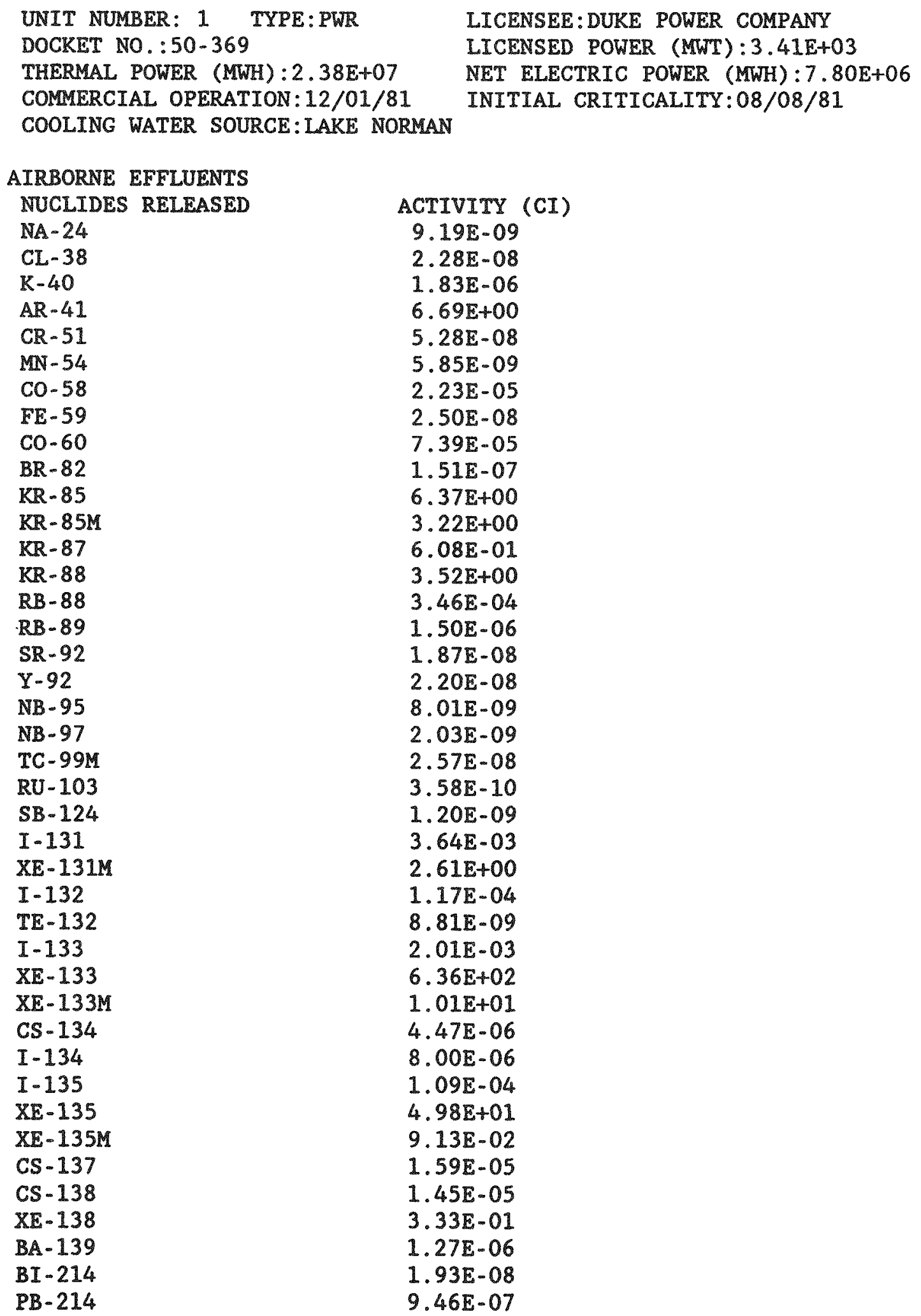


EFFLUENT AND WASTE DISPOSAL ANNUAL REPORT FOR YEAR 1989 AIRBORNE AND LIQUID EFFLUENTS

\begin{tabular}{|c|c|}
\hline \multicolumn{2}{|l|}{ LIQUID EFFLUENTS } \\
\hline NUCLIDES RELEASED & ACTIVITY (CI) \\
\hline$B E-7$ & $4.46 E-05$ \\
\hline$F-18$ & $8.41 E-03$ \\
\hline NA -24 & $7.89 E-04$ \\
\hline $\mathrm{K}-40$ & $1.22 E-03$ \\
\hline$A R-41$ & $2.51 E-06$ \\
\hline CR -51 & $1.19 E-01$ \\
\hline$M N-54$ & $3.80 E-02$ \\
\hline$F E-55$ & $1.87 E-01$ \\
\hline $\mathrm{CO}-57$ & $2.67 \mathrm{E}-03$ \\
\hline Co- 58 & $5.67 E-01$ \\
\hline FE -59 & $7.36 \mathrm{E}-03$ \\
\hline $\mathrm{CO}-60$ & $2.08 \mathrm{E}-01$ \\
\hline NI -65 & $5.50 E-06$ \\
\hline $\mathrm{ZN}-65$ & $6.54 E-04$ \\
\hline$S E-75$ & $2.33 E-05$ \\
\hline$B R-82$ & $1.15 E-04$ \\
\hline $\mathrm{KR}-85$ & $8.93 E-04$ \\
\hline $\mathrm{KR}-85 \mathrm{M}$ & $1.07 \mathrm{E}-04$ \\
\hline $\mathrm{KR}-87$ & $3.45 \mathrm{E}-03$ \\
\hline$K R-88$ & $2.64 E-04$ \\
\hline$R B-88$ & $3.67 \mathrm{E}-03$ \\
\hline $\mathrm{RB}-89$ & $3.66 E-03$ \\
\hline SR- 89 & $6.89 E-05$ \\
\hline SR-91 & $5.79 \mathrm{E}-05$ \\
\hline$Y-91 M$ & $4.55 E-05$ \\
\hline SR-92 & $4.15 E-04$ \\
\hline NB- 95 & $2.24 E-02$ \\
\hline $\mathrm{ZR}-95$ & $1.23 E-02$ \\
\hline NB -97 & $1.16 \mathrm{E}-03$ \\
\hline $\mathrm{ZR}-97$ & $7.00 E-05$ \\
\hline MO-99 & $6.35 E-05$ \\
\hline TC-99M & 1. $30 E-03$ \\
\hline RU-103 & $8.75 E-04$ \\
\hline $\mathrm{RU}-106$ & $2.18 E-03$ \\
\hline$A G-110 M$ & $9.42 \mathrm{E}-03$ \\
\hline $\mathrm{SN}-113$ & $4.84 E-03$ \\
\hline $\mathrm{SB}-122$ & $3.39 E-03$ \\
\hline SB- 124 & $1.75 E-02$ \\
\hline$S B-125$ & $1.49 E-01$ \\
\hline SB-126 & $4.12 E-04$ \\
\hline$I-131$ & $3.41 E-02$ \\
\hline $\mathrm{XE}-131 \mathrm{M}$ & $1.22 \mathrm{E}-02$ \\
\hline$I-132$ & $2.48 \mathrm{E}-03$ \\
\hline$T E-132$ & $3.18 \mathrm{E}-04$ \\
\hline$I-133$ & $2.71 E-02$ \\
\hline$X E-133$ & $2.23 E-01$ \\
\hline$X E-133 M$ & $300 E_{-03}$ \\
\hline CS -134 & $2.94 \mathrm{E}-02$ \\
\hline$\checkmark / A=N O T$ APPLICABLE & \\
\hline N/D-NOT DETECTED & \\
\hline N/R=NOT REPORTED & $A-128$ \\
\hline
\end{tabular}


EFFLUENT AND WASTE DISPOSAL ANNUAL REPORT FOR YEAR 1989 AIRBORNE AND LIQUID EFFLUENTS

LIQUID EFFLUENTS
NUCLIDES RELEASED
I -134
TE -134
I -135
XE -135
XE-135M
CS -136
CS -137
CS -138
BA-139
BA-140
LA-140
CE -141
CE -144
W-187
BI -214
PB- 214
NP -239

LIQUID EFFLUENTS

NUCLIDES RELEASED

I -134

$\mathrm{XE}-135 \mathrm{M}$

CS -136

CS -137

CS -138

BA -139

BA -140

LA -140

$\mathrm{CE}-141$

CE-144

BI 187

PB - 214

NP- 239
ACTIVITY (CI)

$$
\text { 5.06E-04 }
$$

1. $55 E-05$

1. 08E-02

1. $86 \mathrm{E}-02$

1. $53 \mathrm{E}-03$

$6.96 \mathrm{E}-03$

3. $34 \mathrm{E}-02$

1. $94 \mathrm{E}-02$

1. $06 \mathrm{E}-03$

9. $34 \mathrm{E}-05$

1. $33 \mathrm{E}-03$

1. $34 \mathrm{E}-04$

$1.42 \mathrm{E}-03$

1. $50 \mathrm{E}-04$

3. 71E-05

2. $75 \mathrm{E}-04$

$1.78 \mathrm{E}-04$

TOTAL AIRBORNE TRITIUM RELEASE 2.66E+01

TOTAL LIQUID TRITIUM RELEASE $4.23 E+02$ $\begin{array}{llll}\text { VOLUME OF LIQUID WASTE RELEASED (PRIOR TO DILUTION) } & \text { LITERS } & 2.75 E+07 * \\ \text { VOLUME OF DILUTION WATER USED DURING PERIOD } & \text { LITERS } & 3.45 E+12 *\end{array}$

*THESE VOLUMES REPRESENT THE TOTAL DILUTION FLOW FOR BOTH MCGUIRE $1 \& 2$ SINCE THERE IS ONE PRIMARY RELEASE POINT FOR THE SITE. 
EFFLUENT AND WASTE DISPOSAL ANNUAL REPORT FOR YEAR 1989 AIRBORNE AND LIQUID EFFLUENTS

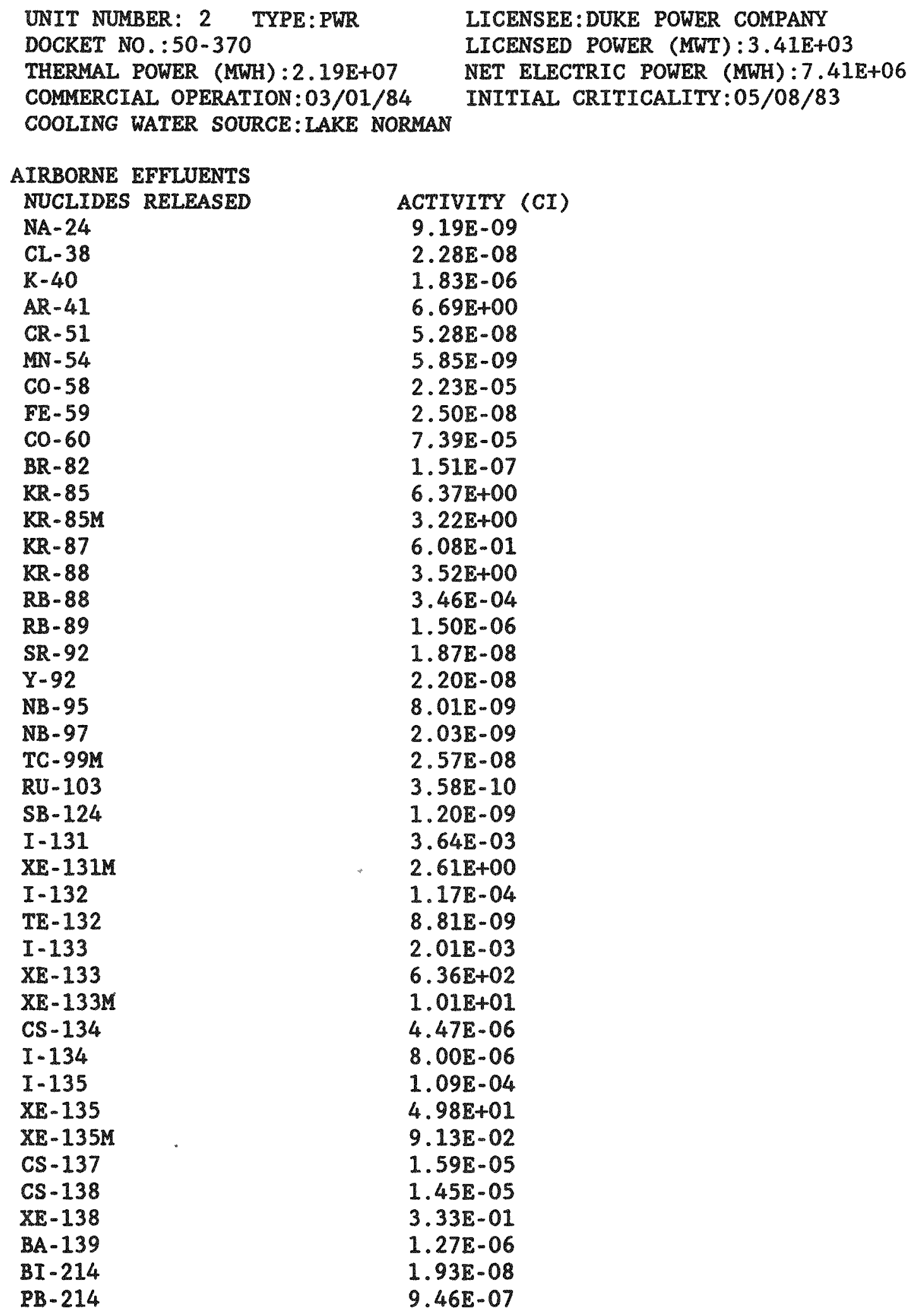


EFFLUENT AND WASTE DISPOSAL ANNUAL REPORT FOR YEAR 1989 AIRBORNE AND LIQUID EFFLUENTS

\begin{tabular}{|c|c|}
\hline NUCLIDES RELEASED & ACTIVITY (CI) \\
\hline $\mathrm{BE}-7$ & $4.46 \mathrm{E}-05$ \\
\hline$F-18$ & $8.41 E-03$ \\
\hline $\mathrm{NA}-24$ & $7.89 \mathrm{E}-04$ \\
\hline$K-40$ & $1.22 E=03$ \\
\hline AR -41 & $2.51 E-06$ \\
\hline CR -51 & $1.19 \mathrm{E}-01$ \\
\hline $\mathbb{M N}-54$ & $3.80 \mathrm{E}-02$ \\
\hline FE -55 & $1.87 \mathrm{E}-01$ \\
\hline $\mathrm{CO}-57$ & $2.67 E-03$ \\
\hline $\mathrm{CO}-58$ & $5.67 E-01$ \\
\hline$F E-59$ & $7.36 E-03$ \\
\hline $\mathrm{Co}-60$ & $2.08 \mathrm{E}-01$ \\
\hline NI -65 & $5.50 \Sigma-06$ \\
\hline $\mathrm{ZN}-65$ & $6.54 E-04$ \\
\hline SE-75 & $2.33 E-05$ \\
\hline$B R-82$ & $1.15 E-04$ \\
\hline $\mathrm{KR}-85$ & $8.93 E-04$ \\
\hline$K R-85 M$ & $1.07 E-04$ \\
\hline KR -87 & $3.45 E-03$ \\
\hline $\mathrm{KR}-88$ & $2.64 E-04$ \\
\hline $\mathrm{RB}-88$ & $3.67 E-03$ \\
\hline RB -89 & $3.66 \mathrm{E}-03$ \\
\hline$S R-89$ & $6.89 E-05$ \\
\hline SR-91 & $5.79 E-05$ \\
\hline$Y-91 M$ & $4.55 \mathrm{E}-0.5$ \\
\hline SR -92 & $4.15 E-04$ \\
\hline $\mathrm{NB}-95$ & $2.24 E-02$ \\
\hline$Z R-95$ & $1.23 E-02$ \\
\hline$N B-97$ & $1.16 \mathrm{E}-03$ \\
\hline ZR-97 & $7.00 \mathrm{E}-05$ \\
\hline MO-99 & $6.35 E-05$ \\
\hline TC-99M & $1.30 E-03$ \\
\hline $\mathrm{RU}-103$ & $8.75 E-04$ \\
\hline RU-106 & $2.18 \mathrm{E}-03$ \\
\hline$A G-110 M$ & $9.42 E-03$ \\
\hline SN-113 & $4.84 E-03$ \\
\hline$S B-122$ & $3.39 E-03$ \\
\hline$S B-124$ & $1.75 \mathrm{E}-02$ \\
\hline$S B-125$ & $1.49 E-01$ \\
\hline$S B-126$ & $4.12 E-04$ \\
\hline$I-131$ & $3.41 E-02$ \\
\hline$X E-131 M$ & $1.22 E-02$ \\
\hline$I-132$ & $2.48 \mathrm{E}-03$ \\
\hline$T E-132$ & $3.18 E-04$ \\
\hline$I=133$ & $2.71 E-02$ \\
\hline $\mathrm{XE}-133$ & $2.23 E-01$ \\
\hline$X E-133 M$ & $3.00 E-03$ \\
\hline $\operatorname{CS}-134$ & $2.94 E-02$ \\
\hline
\end{tabular}


EFFLUENT AND WASTE DISPOSAL ANNUAL REPORT FOR YEAR 1989 AIRBORNE AND LIQUID EFFLUENTS

LIQUID EFFLUENTS
NUCLIDES RELEASED
I-134
TE -134
I-135
XE-135
XE $-135 \mathrm{M}$
CS -136
CS -137
CS -138
BA -139
BA-140
LA-140
CE -141
CE -144
W-187
BI -214
PB -214
NP -239
ACTIVITY (CI)
$5.06 \mathrm{E}-04$
$1.55 E-05$
$1.08 \mathrm{E}-02$
$1.86 \mathrm{E}-02$
$1.53 \mathrm{E}-03$
$6.96 \mathrm{E}-03$
3. $34 \mathrm{E}-02$
$1.94 \mathrm{E}-02$
1.06E-03
9.34E-05
1. $33 \mathrm{E}-03$
1. $34 E-04$
$1.42 \mathrm{E}-03$
$1.50 \mathrm{E}-04$
3. 71E-05
$2.75 E-04$
$1.78 \mathrm{E}-04$

$\begin{array}{ll}\text { TOTAL AIRBORNE TRITIUM RELEASE } & 2.66 \mathrm{E}+01 \\ \text { TOTAL LIOUID TRITIUM RELEASE } & 4.23 \mathrm{E}+02\end{array}$

VOLUME OF LIQUID WASTE RELEASED (PRIOR TO DILUTION) LITERS $2.75 E+07$ * VOLUME OF DILUTION WATER USED DURING PERIOD

*THESE VOLUMES REPRESENT THE TOTAL DILUTION FLOW FOR BOTH MCGUIRE $1 \& 2$ SINCE THERE IS ONE PRIMARY RELEASE POINT FOR THE SITE. 
EFFLUENT AND WASTE DISPOSAL. ANNUAL REPORT FOR YEAR 1989 SOLID EFFLUENTS

SOLID WASTE DISPOSITION NUMBER OF SHIPMENTS MODE OF TRANSPORTATION \# $16 \quad \mathrm{~N} / \mathrm{R}$

\#DOES NOT INCLUDE BROKERED TOTALS

ESTIMATE OF MAJOR NUCLIDE COMPOSITION (BY TYPE OF WASTE)

A

C -14

$\mathrm{CE}-144$

CO- 57

$\mathrm{CO} .58$

$\mathrm{CO}-60$

CR- 51

CS -134

CS-137

FE -55

FE -59

I- 131

$\mathrm{MN}-54$

NB- 95

NI -63

PU -241

SB-122

$S B-125$

$S N-113$

SR -90

$\mathrm{ZN}-65$

ZR-95

B

C -14

Co- 58

$\mathrm{CO}-60$

CR -51

$F E-55$

$\mathrm{H}-3$

MN -54

NB- 95

NI -63

ZR-95

D

C -14

CE- 144

CO- 58

CO- 60

CS -137
JAN-JUNE JULY-DEC

\begin{tabular}{|c|c|c|}
\hline 8 & $5.40 E+00$ & $1.20 \mathrm{E}-01$ \\
\hline 8 & & $2.00 \mathrm{E}-02$ \\
\hline 8 & & $2.00 E-01$ \\
\hline 8 & 2. $30 E+00$ & $2.19 E+01$ \\
\hline 8 & 1. $30 E+00$ & $2.81 E+01$ \\
\hline 8 & & $1.40 E-01$ \\
\hline 8 & $3.34 E+01$ & $8.30 E+00$ \\
\hline 8 & $4.74 E+01$ & $1.46 \mathrm{E}+01$ \\
\hline 8 & & 8. $36 E+00$ \\
\hline 8 & & $2.00 E-02$ \\
\hline$\frac{8}{8}$ & $3.50 E+00$ & $6.19 E-05$ \\
\hline 8 & $1.00 E+00$ & $4.60 E+00$ \\
\hline 8 & $1.10 E+00$ & $9.00 \mathrm{E}-02$ \\
\hline 8 & & 1. $25 E+01$ \\
\hline 8 & & $8.94 E-03$ \\
\hline 8 & & $2.00 E-02$ \\
\hline 8 & $1.10 \mathrm{E}+00$ & $8.40 E-01$ \\
\hline 8 & & $8.00 \mathrm{E}-02$ \\
\hline 8 & $2.60 \mathrm{E}+00$ & $5.00 \mathrm{E}-02$ \\
\hline 8 & & $2.00 \mathrm{E}-02$ \\
\hline 용 & $8.00 \mathrm{E}-01$ & $5.00 E-02$ \\
\hline 8 & $1.90 \mathrm{E}-01$ & $1.90 \mathrm{E}-01$ \\
\hline 8 & $4.63 E+01$ & $4.63 E+01$ \\
\hline 8 & $1.33 E+01$ & 1. $33 E+01$ \\
\hline 8 & $2.18 E+01$ & $2.18 \mathrm{E}+01$ \\
\hline 8 & $9.69 E+00$ & $9.69 \mathrm{E}+00$ \\
\hline 8 & $1.92 E+00$ & $1.92 E+00$ \\
\hline 8 & $1.89 E+00$ & $1.89 E+00$ \\
\hline 8 & $2.26 E+00$ & 2. $26 E+00$ \\
\hline 8 & $1.39 \mathrm{E}+00$ & $1.39 E+00$ \\
\hline 8 & 1. $18 E+00$ & $1.18 \mathrm{E}+00$ \\
\hline 8 & $2.00 E-01$ & $2.00 \mathrm{E}-01$ \\
\hline 8 & $1.50 \mathrm{E}-01$ & $1.50 E-01$ \\
\hline 8 & $7.93 E+00$ & $7.93 E+00$ \\
\hline 2 & $3.49 \mathrm{E}+01$ & $3.49 E+01$ \\
\hline 8 & $4.60 E-01^{\prime}$ & $4.60 E-01$ \\
\hline
\end{tabular}

DESTINATION

$N / R$ 
EFFLUENT AND WASTE DISPOSAL ANNUAL REPORT FOR YEAR 1989 SOLID EFFLUENTS

ESTIMATE OF MAJOR NUCLIDE COMPOSITION (BY TYPE OF WASTE)

JAN-JUNE JULY-DEC

D
$\mathrm{FE}-55$
$\mathrm{MN}-54$
NB -95
NI -63
PU -241
TRU

$\begin{array}{lll}8 & 4.68 \mathrm{E}+01 & 4.68 \mathrm{E}+01 \\ 8 & 2.55 \mathrm{E}+00 & 2.55 \mathrm{E}+00 \\ 8 & 1.80 \mathrm{E}+00 & 1.80 \mathrm{E}+00 \\ 8 & 5.10 \mathrm{E}+00 & 5.10 \mathrm{E}+00 \\ 8 & 8.00 \mathrm{E}-02 & 8.00 \mathrm{E}-02 \\ 8 & 2.00 \mathrm{E}-02 & 2.00 \mathrm{E}-02\end{array}$

TYPE OF WASTE

A. SPENT RESINS, FILTER SLUDGES, EVAPORATOR

UNIT YEAR TOTAL BOTTOMS, ETC.

M3 $1.66 E+02$

CI $\quad 5.88 \mathrm{E}+02$

B. DRY COMPRESSIBLE WASTE, CONTAMINATED

M3

$2.63 \mathrm{E}+02 *$

EQUIPMENT, ETC.

CI $\quad 3.20 \mathrm{E}+01$

C. IRRADIATED COMPONENTS, CONTROL

M3

RODS, ETC.

CI

D. OTHER

M3 $6.44 \mathrm{E}+00$

DEWATERED MECH. FILTERS

CI $\quad 1.20 \mathrm{E}+01$

*VOLUME CONTAINS COMPACTED AND NON-COMPACTED WASTE 
EFFLUENT AND WASTE DISPOSAL ANNUAL REPORT FOR YEAR 1989 AIRBORNE AND LIQUID EFFLUENTS
UNIT NUMBER: 1 TYPE:BWR
LICENSEE: NORTHEAST NUCLEAR ENERGY
DOCKET NO. : 50-245
THERMAL POWER (MWH) : $1.42 \mathrm{E}+07$
COMMERCIAL OPERATION:03/01/71
LICENSED POWER (MWT) : $2.01 E+03$
NET ELECTRIC POWER (MWH):4.64E+06
COOLING WATER SOURCE:LONG ISLAND SOUND
AIRBORNE EFFLUENTS
NUCLIDES RELEASED
$A R-41$
CR -51
MN -54
Co- 58
$\mathrm{CO}-60$
$\mathrm{ZN}-65$
$\mathrm{KR}-85$
$\mathrm{KR}-85 \mathrm{M}$
$\mathrm{KR}-87$
$\mathrm{KR}-88$
SR -89
SR -90
$I-131$
$I-133$
$\mathrm{XE}-133$
$X E-133 M$
$\mathrm{XE}-135$
$\mathrm{XE}-135 \mathrm{M}$
ACTIVITY (CI)
$1.65 \mathrm{E}+00$
1. $33 E-03$
1. $37 \mathrm{E}-04$
1. $31 \mathrm{E}-04$
8. $30 \mathrm{E}-04$
3. $27 \mathrm{E}-03$
$2.47 \mathrm{E}+00$
$1.09 E+01$
1. $65 \mathrm{E}-01$
7. 20E+00
$4.65 \mathrm{E}-04$
9. $22 \mathrm{E}-06$
$2.34 \mathrm{E}-03$
$9.76 \mathrm{E}-03$
$8.97 \mathrm{E}+01$
6. 21E-01
2. $53 E+01$
9. $60 \mathrm{E}+00$
CS -137
$\mathrm{XE}-138$
2.71E-04
3. $35 E+01$
BA -140
5. $72 \mathrm{E}-04$

\section{LIQUID EFFLUENTS}
NUCLIDES RELEASED
NA -24
$\mathrm{CR}-51$
$\mathrm{MN}-54$
FE -55
CO- 58
FE- 59
Co- 60
$\mathrm{ZN}-65$
$\mathrm{ZN}-69 \mathrm{M}$
SR-89
SR -90
NB -97
TC-99M
AG-110M
I-131
I -132
I- 133
XE -133
ACTIVITY (CI)
3. $94 E-06$
7. $34 \mathrm{E}-03$
$6.23 E-02$
$1.43 \mathrm{E}-01$
1. $05 \mathrm{E}-02$
1.41E-02
2. 81E-01
$1.69 \mathrm{E}-01$
5.80E-05
$6.84 \mathrm{E}-04$
$4.36 \mathrm{E}-03$
8.16E-05
1. $52 \mathrm{E}-03$
8.74E-04
$4.88 \mathrm{E}-04$
4. $72 E-06$
1. 11E-03
1. $71 \mathrm{E}-03$
CS -134
$2.32 E-03$

N/A-NOT APPLICABLE 
EFFLUENT AND WASTE DISPOSAL ANNUAL REPORT FOR YEAR 1989 AIRBORNE AND LIQUID EFFLUENTS

\author{
LIQUID EFFLUENTS \\ NUCLIDES RELEASED \\ I-135 \\ $\mathrm{XE}-135$ \\ $\mathrm{XE}-135 \mathrm{M}$ \\ CS -137 \\ CS -138 \\ $\mathrm{BA}-139$ \\ NP -239
}
ACTIVITY (CI)
3. $00 \mathrm{E}-04$
$2.41 E-03$
9.68E-05
$2.06 \mathrm{E}-01$
$4.54 \mathrm{E}-05$
$2.21 \mathrm{E}-0.5$
$5.32 \mathrm{E}-04$

TOTAL AIRBORNE TRITIUM RELEASE $1.15 E+02$

TOTAL LIQUID TRITIUM RELEASE 4.58E+01

VOLUME OF LIQUID WASTE RELEASED (PRIOR TO DILUTION) LITERS $1.74 \mathrm{E}+07$

VOLUME OF DILUTION WATER USED DURING PERIOD

LITERS $\quad 7.40 \mathrm{E}+11$ 
INSTALLATION:MILLSTONE 1

EFFLUENT AND WASTE DISPOSAL ANNUAL REPORT FOR YEAR 1989

SOLID EFFLUENTS

SOLID WASTE DISPOSITION

$$
\text { NUMBER OF SHIPMENTS MODE OF TRANSPORTATION }
$$
41

1

9

\# 1
TRUCK (SOLE USE)

TRUCK (SOLE USE)

TRUCK (SOLE USE)

TRUCK (SOLE USE)
DESTINATION

CHEM-NUC, BARNWELL SC QUADREX, OAK RIDGE TN SEG, OAK RIDGE TN US ECOLOGY, RICHLAND WA

\#NO UNIT ASSIGNMENT, CHARGED TO STATION

TYPE OF WASTE

A. SPENT RESINS, FILTER SLUDGES, EVAPORATOR

UNIT YEAR TOTAL BOTTOMS, ETC.

M3 $\quad 1.66 E+02$

CI $\quad 1.77 \mathrm{E}+03$

B. DRY COMPRESSIBLE WASTE, CONTAMINATED

M3 2.58E+02 *

EQUIPMENT, ETC.

CI $\quad 2.02 E+01$

C. IRRADIATED COMPONENTS, CONTROL

M3 4.40E+00 RODS , ETC.

CI $\quad 1.81 \mathrm{E}+04$

D. OTHER

M3

CI

*TOTAL VOLUME SENT TO BURIAL 
EFFLUENT AND WASTE DISPOSAL ANNUAL REPORT FOR YEAR 1989 AIRBORNE AND LIQUID EFFLUENTS

\begin{tabular}{|c|c|}
\hline \multicolumn{2}{|c|}{ UNIT NUMBER: 2 TYPE:PWR LICENSEE:NORTHEAST NUCLEAR ENERGY } \\
\hline \multicolumn{2}{|c|}{ DOCKET NO.:50-336 LICENSED POWER (MWT) $: 2.70 \mathrm{E}+03$} \\
\hline \multicolumn{2}{|r|}{ NET ELECTRIC POWER (MWH) $: 4.89 E+06$} \\
\hline \multicolumn{2}{|c|}{ COMMERCIAL OPERATION: $12 / 26 / 75$ INITIAL CRITICALITY:10/17/75 } \\
\hline COOLING WATER SOURCE:LONG & ISLAND SOUND \\
\hline \multicolumn{2}{|l|}{ AIRBORNE EFFLUENTS } \\
\hline NUCLIDES RELEASED & ACTIVITY (CI) \\
\hline$A R-41$ & $2.05 \mathrm{E}-05$ \\
\hline Co- 58 & $3.10 \mathrm{E}-07$ \\
\hline $\mathrm{CO}-60$ & $3.60 E-06$ \\
\hline $\mathrm{KR}-85$ & $4.74 E+00$ \\
\hline $\mathrm{KR}-85 \mathrm{M}$ & $6.26 E-03$ \\
\hline $\mathrm{RB}-88$ & $7.87 \mathrm{E}-04$ \\
\hline$S B-125$ & $1.80 \mathrm{E}-07$ \\
\hline I- 131 & $3.78 \mathrm{E}-02$ \\
\hline$X E-131 M$ & $5.34 \mathrm{E}-01$ \\
\hline I-133 & $8.41 E-03$ \\
\hline$X E-133$ & $2.24 \mathrm{E}+02$ \\
\hline $\mathrm{XE}-133 \mathrm{M}$ & $2.04 E-01$ \\
\hline CS -134 & $2.24 E-06$ \\
\hline $\mathrm{XE}-135$ & $1.64 E+01$ \\
\hline $\mathrm{XE}-135 \mathrm{M}$ & $2.71 \mathrm{E}-01$ \\
\hline $\operatorname{cs}-137$ & $6.65 E-06$ \\
\hline \multicolumn{2}{|l|}{ LIQUID EFFLUENTS } \\
\hline NUCLIDES RELEASED & ACTIVITY (CI) \\
\hline NA - 24 & $3.08 E-03$ \\
\hline $\mathrm{CR}-51$ & $2.24 E-01$ \\
\hline $\mathrm{MN}-54$ & $4.92 E-02$ \\
\hline FE -55 & $2.30 \mathrm{E}-01$ \\
\hline $\mathrm{CO}-57$ & 1. $33 E-02$ \\
\hline $\mathrm{CO}-58$ & $6.95 E+00$ \\
\hline$F E-59$ & $7.29 E-03$ \\
\hline Co- 60 & $1.26 \mathrm{E}+00$ \\
\hline $\mathrm{KR}-85$ & $3.65 E-01$ \\
\hline$S R-89$ & $1.40 E-03$ \\
\hline$S R-90$ & $1.59 E-04$ \\
\hline SR -92 & $4.15 \mathrm{E}-02$ \\
\hline NB-95 & $1.13 E-02$ \\
\hline $2 R-95$ & $2.52 E-02$ \\
\hline NB-97 & $1.85 E-01$ \\
\hline MO-99 & $5.87 E-03$ \\
\hline TC-99M & $5.31 E-03$ \\
\hline$R U-103$ & $3.68 E-03$ \\
\hline $\mathrm{RU}-106$ & $1.89 E-03$ \\
\hline$A G-110 M$ & $1.28 E-01$ \\
\hline $\mathrm{SB}-124$ & $1.24 \mathrm{E}-01$ \\
\hline $\mathrm{SB}-125$ & $7.45 E-02$ \\
\hline $\mathrm{I}-131$ & $1.05 E-01$ \\
\hline $\mathrm{XE}-131 \mathrm{M}$ & $5.27 E-02$ \\
\hline \multicolumn{2}{|l|}{ N/Am=NOT APPLICABLE } \\
\hline \multicolumn{2}{|l|}{ N/D=NOT DETECTED } \\
\hline$N / R=N O T$ REPORTED & A-138 \\
\hline
\end{tabular}


EFFLUENT AND WASTE DISPOSAL ANNUAL REPORT FOR YEAR 1989 AIRBORNE AND LIQUID EFFLUENTS

LIQUID EFFLUENTS
NUCLIDES RELEASED
$I-132$
$I-133$
XE -133
$X E-133 M$
$C S-134$
$I-135$
$X E-135$
$C S-136$
$C S-137$
LA -140
LA -141
$C E-144$
PR -144

LIQUID EFFLUENTS NUCLIDES RELEASED

I -132

I -133

$\mathrm{XE}-133$

$\mathrm{XE}-133 \mathrm{M}$

CS -134

PR -144
ACTIVITY (CI)
$2.12 \mathrm{E}-03$
$1.40 \mathrm{E}-02$
$1.42 \mathrm{E}+00$
$4.72 \mathrm{E}-03$
4. $05 \mathrm{E}-01$
$1.57 \mathrm{E}-03$
$2.09 \mathrm{E}-02$
1. $28 \mathrm{E}-02$
$6.90 E-01$
$1.96 \mathrm{E}-03$
$3.54 \mathrm{E}-02$
$9.61 E-04$
$9.45 \mathrm{E}-03$

TOTAL AIRBORNE TRITIUM RELEASE 6.55E+01

TOTAL LIQUID TRITIUM RELEASE $3.66 \mathrm{E}+02$

VOLUME OF LIQUID WASTE RELEASED (PRIOR TO DILUTION) LITERS $1.37 E+08$ VOLUME OF DILUTION WATER USED DURING PERIOD 
INSTALLATION:MILLSTONE 2

EFFLUENT AND WASTE DISPOSAL ANNUAL REPORT FOR YEAR 1989 SOLID EFFLUENTS

SOLID WASTE DISPOSITION NUMBER OF SHIPMENTS 12

MODE OF TRANSPORTATION TRUCK (SOLE USE) TRUCK (SOLE USE) 3
DESTINATION

CHEM - NUC, BARNWELL SC QUADREX, OAK RIDGE TN SEG, OAK RIDGE TN
TXPE OF WASTE

A. SPENT RESINS, FILTER SLUDGES, EVAPORATOR BOTTOMS, ETC.

B. DRY COMPRESSIBLE WASTE, CONTAMINATED EQUIPMENT, ETC.

C. IRRADIATED COMPONENTS, CONTROL RODS, ETC.

D. OTHER
UNIT YEAR TOTAL

M3 $1.68 \mathrm{E}+01$ *

CI $\quad 5.51 E+02$

M3 2.30E $+02 *$

CI $\quad 4.23 \mathrm{E}+00$

M3

$\mathrm{CI}$

M3

CI

*TOTAL VOLUME SENT TO BURIAL 
EFFLUENT AND WASTE DISPOSAL ANNUAL REPORT FOR YEAR 1989 AIRBORNE AND LIQUID EFFLUENTS

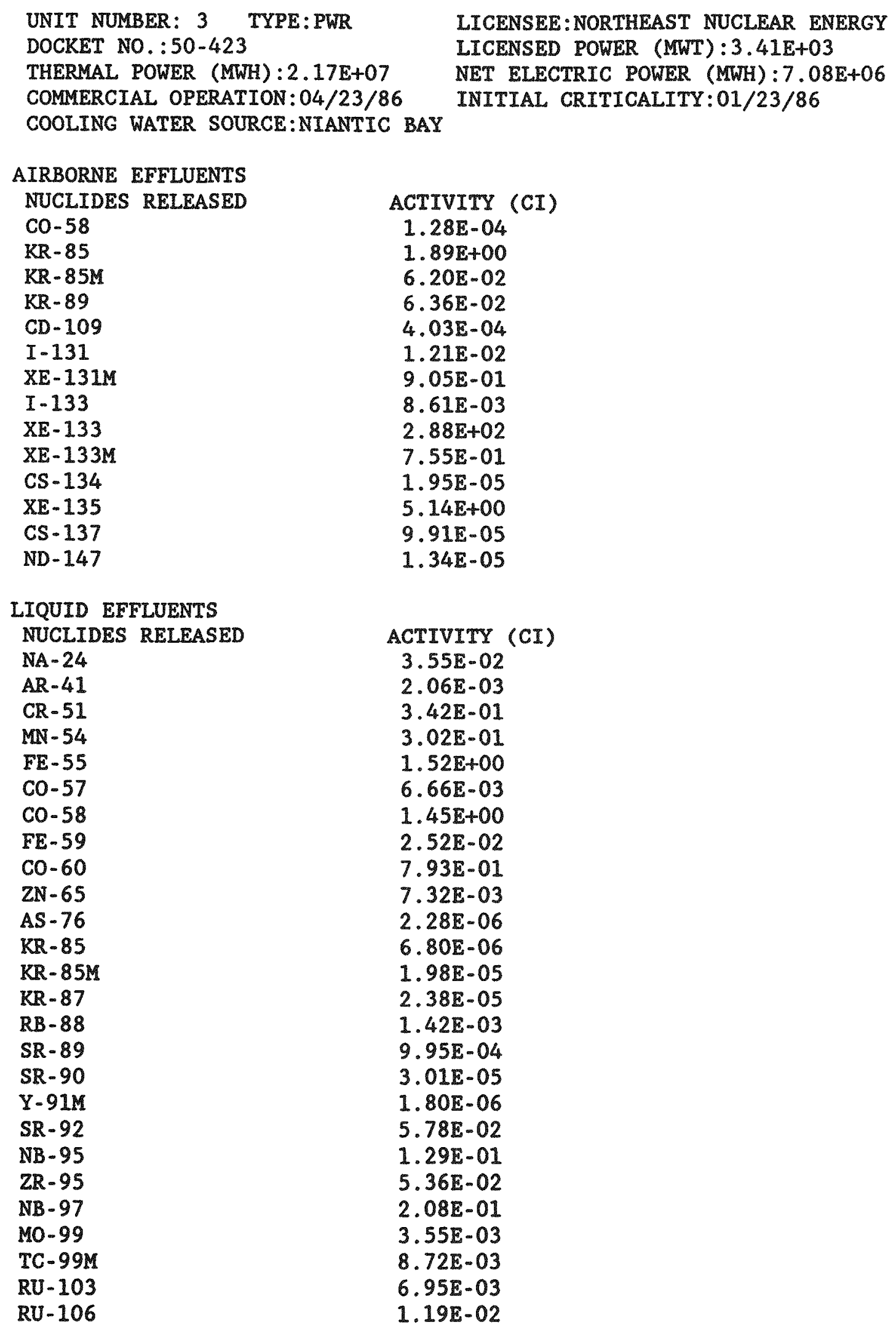

N/A-NOT APPLICABLE

N/D-NOT DETECTED

$N / R=N O T$ REPORTED 
EFFLUENT AND WASTE DISPOSAL ANNUAL REPORT FOR YEAR 1989 AIRBORNE AND LIQUID EFFLUENTS

\begin{tabular}{|c|c|}
\hline \multicolumn{2}{|l|}{ LIQUID EFFLUENTS } \\
\hline NUCLIDES RELEASED & ACTIVITY (CI) \\
\hline$A G-110 M$ & $1.15 \mathrm{E}-01$ \\
\hline$S B-124$ & $6.37 E-03$ \\
\hline$S B-125$ & $6.10 \mathrm{E}-02$ \\
\hline$I-131$ & $1.95 E-01$ \\
\hline$X E-131 M$ & $7.36 E-03$ \\
\hline $1-132$ & $2.41 E-05$ \\
\hline$I-133$ & $1.16 \mathrm{E}-02$ \\
\hline$X E-133$ & $1.38 \mathrm{E}-01$ \\
\hline$X E-133 M$ & $2.50 E-03$ \\
\hline CS -134 & $1.87 \mathrm{E}-01$ \\
\hline $\operatorname{cs}-135$ & $1.12 E-03$ \\
\hline$I-135$ & $2.13 E-03$ \\
\hline$X E-135$ & $3.25 E-02$ \\
\hline$X E-135 M$ & $1.67 \mathrm{E}-03$ \\
\hline$C S-136$ & $8.34 E-03$ \\
\hline CS -137 & $3.28 E-01$ \\
\hline$L A=141$ & $1.13 E-03$ \\
\hline$C E-144$ & $4.23 E-02$ \\
\hline $\mathrm{HF}-181$ & $8.69 E-03$ \\
\hline$W-187$ & $4.87 E-04$ \\
\hline
\end{tabular}

TOTAL AIRBORNE TRITIUM RELEASE 1.84E+01

TOTAL LIQUID TRITIUM RELEASE $6.97 E+02$

VOLUME OF LIQUID WASTE RELEASED (PRIOR TO DILUTION) LITERS $1.86 E+07$ 
INSTALLATION:MILLSTONE 3

EFFLUENT AND WASTE DISPOSAL ANNUAL REPORT FOR YEAR 1989 SOLID EFFLUENTS

SOLID WASTE DISPOSITION

NUMBER OF SHIPMENTS MODE OF TRANSPORTATION

DESTINATION

12

TRUCK (SOLE USE)

CHEM-NUC, BARNWELL SC

TYPE OF WASTE

A. SPENT RESINS, FILTER SLUDGES, EVAPORATOR

UNIT YEAR TOTAL BOTTOMS, ETC.

M3 3.02E+01*

CI $\quad 7.34 \mathrm{E}+02$

B. DRY COMPRESSIBLE WASTE, CONTAMINATED

EQUI PMENT, ETC.

M3 $1.17 \mathrm{E}+02$ *

CI $\quad 3.45 \mathrm{E}+00$

C. IRRADIATED COMPONENTS, CONTROL

M3

RODS, ETC.

D. OTHER

$\mathrm{CI}$

M3

CI

*TOTAL VOLUME SENT TO BURIAL 
EFFLUENT AND WASTE DISPOSAL ANNUAL REPORT FOR YEAR 1989 AIRBORNE AND LIQUID EFFLUENTS
UNIT NUMBER: 1 TYPE: BWR
LICENSEE: NORTHERN STATES POWER
DOCKET NO.: 50-263
LICENSED POWER (MWT) : $1.67 E+03$
THERMAL POWER (MWH) $: 8.48 \mathrm{E}+06$
NET ELECTRIC POWER (MWH) :2.65E+06
COMMERCIAL OPERATION:06/30/71
COOLING WATER SOURCE:MISSISSIPPI RIVER

\begin{tabular}{|c|c|}
\hline \multicolumn{2}{|l|}{ AIRBORNE EFFLUENTS } \\
\hline NUCLIDES RELEASED & ACTIVITY (CI) \\
\hline CR -51 & $1.32 E-03$ \\
\hline$M N-54$ & $1.56 \mathrm{E}-04$ \\
\hline CO- 58 & $1.59 E-04$ \\
\hline FE -59 & $1.87 \mathrm{E}-06$ \\
\hline $\mathrm{CO}-60$ & $2.43 E-03$ \\
\hline$Z N-65$ & $6.66 E-03$ \\
\hline $\mathrm{KR}-85$ & $1.60 \mathrm{E}+02$ \\
\hline $\mathrm{KR}-85 \mathrm{M}$ & $7.73 E+00$ \\
\hline $\mathrm{KR}-87$ & $4.03 E+01$ \\
\hline $\mathrm{KR}-88$ & $2.53 \mathrm{E}+01$ \\
\hline $\mathrm{KR}-89$ & $6.09 E+02$ \\
\hline SR-89 & $2.09 E-03$ \\
\hline $\mathrm{KR}-90$ & $2.07 E+01$ \\
\hline SR-90 & $1.93 E-05$ \\
\hline$R U-103$ & $2.61 E-05$ \\
\hline$I-131$ & $9.09 E-02$ \\
\hline $\mathrm{XE}-131 \mathrm{M}$ & $5.02 E+00$ \\
\hline$I-133$ & $4.40 E-01$ \\
\hline$X E-133$ & $1.10 E+03$ \\
\hline $\mathrm{XE}-133 \mathrm{M}$ & $8.70 \mathrm{E}+00$ \\
\hline $\mathrm{CS}-134$ & $8.58 E-06$ \\
\hline$I-135$ & $5.76 \mathrm{E}-01$ \\
\hline $\mathrm{XE}-135$ & $9.71 E+01$ \\
\hline $\mathrm{XE}-135 \mathrm{M}$ & $1.32 E+02$ \\
\hline CS -137 & $2.17 E-04$ \\
\hline$X E-137$ & $9.71 E+02$ \\
\hline$X E-138$ & $7.44 E+02$ \\
\hline$X E-139$ & $6.14 E+01$ \\
\hline $\mathrm{BA}-140$ & $9.95 E-03$ \\
\hline $\mathrm{CE}-141$ & $9.28 E-05$ \\
\hline$C E-144$ & $7.35 E-07$ \\
\hline
\end{tabular}

TOTAL AIRBORNE TRITIUM RELEASE 9.23E+01

TOTAL LIQUID TRITIUM RELEASE $0.00 E+00$

$\begin{array}{llll}\text { VOLUME OF LIQUID WASTE RELEASED (PRIOR TO DILUTION) } & \text { LITERS } & 0.00 E+00 \\ \text { VOLUME OF DILUTION WATER USED DURING PERIOD } & \text { LITERS } & 0.00 E+00\end{array}$ VOLUME OF DILUTION WATER USED DURING PERIOD 
SOLID WASTE DISPOSITION NUMBER OF SHIPMENTS

$\begin{aligned} 3 & \text { RAILWAY } \\ 11 & \text { TRUCK } \\ 9 & \text { TRUCK }\end{aligned}$

ESTIMATE OF MAJOR NUCLIDE COMPOSITION (BY TYPE OF WASTE)

A

BA -140

CE-141

$\mathrm{CO}-58$

$\mathrm{CO}-60$

$\mathrm{CR}-51$

CS -134

CS -137

FE- 55

FE- 59

I -131

LA - 140

MN -54

RU -103

SR -89

SR -90

$\mathrm{ZN}-65$

ZR-95

B

$\mathrm{BA}-140$

CO -58

$\mathrm{CO}-60$

$\mathrm{CR}-51$

CS -134

CS -137

FE -55

$\mathrm{FE}-59$

I -131

LA -140

$\mathrm{MN}-54$

SR -89

$\mathrm{SR}-90$

$\mathrm{ZN}-65$

C

CO -60

$\mathrm{CR}-51$

FE -55

\section{MODE OF TRANSPORTATION \\ TRUCK \\ TRUCK

MODE OF TRANSPORTATION
RAILWAY
TRUCK
TRUCK

\section{DESTINATION}

CHEM-NUC, BARNWELL SC

CHEM-NUC, BARNWELL SC

US ECOLOGY, RICHLAND WA

$$
\text { JAN-JUNE JULY-DEC }
$$

\begin{tabular}{|c|c|c|}
\hline 8 & $1.93 E+00$ & $8.87 \mathrm{E}-02$ \\
\hline 8 & $3.29 E-02$ & $1.34 \mathrm{E}-01$ \\
\hline 8 & & $1.14 \mathrm{E}+00$ \\
\hline 8 & $2.70 E+01$ & $5.54 E+01$ \\
\hline 8 & $9.75 E-01$ & \\
\hline 8 & $2.57 E+00$ & $9.12 \mathrm{E}-01$ \\
\hline 8 & $1.89 E+01$ & $3.67 E+00$ \\
\hline 8 & $2.94 E+01$ & $2.55 \mathrm{E}+01$ \\
\hline 8 & $5.55 E+00$ & $2.91 E-01$ \\
\hline 8 & $4.49 E+00$ & $8.35 \mathrm{E}-02$ \\
\hline 8 & $7.01 E-01$ & \\
\hline 8 & $1.48 \mathrm{E}+00$ & $5.14 E+00$ \\
\hline 8 & & 1. $23 \mathrm{E}-01$ \\
\hline 8 & $1.81 E-01$ & $4.03 E-01$ \\
\hline 8 & & $1.67 \mathrm{E}-01$ \\
\hline 8 & $3.24 E+00$ & $5.75 E+00$ \\
\hline 8 & & $2.45 \mathrm{E}-02$ \\
\hline 8 & & $4.44 E-01$ \\
\hline 8 & & $5.68 \mathrm{E}-01$ \\
\hline 8 & $2.93 E+01$ & $3.19 \mathrm{E}+01$ \\
\hline 8 & $1.77 \mathrm{E}+00$ & $2.56 \mathrm{E}+00$ \\
\hline 8 & $2.52 E+00$ & $1.44 \mathrm{E}+00$ \\
\hline 8 & $9.50 \mathrm{E}+00$ & $4.75 E+00$ \\
\hline 8 & $3.81 E+01$ & $3.07 \mathrm{E}+01$ \\
\hline 8 & & 7.70E-01 \\
\hline 8 & $1.85 E+00$ & \\
\hline 8 & $1.11 E+00$ & $4.38 E-01$ \\
\hline 8 & $2.83 \mathrm{E}+00$ & 1. $12 \mathrm{E}+01$ \\
\hline 8 & & $1.03 E+00$ \\
\hline 8 & & $3.49 \mathrm{E}-01$ \\
\hline 8 & $3.04 E+00$ & $3.15 \mathrm{E}+00$ \\
\hline 8 & $4.43 E+01$ & \\
\hline 8 & $7.24 \mathrm{E}-01$ & \\
\hline 8 & $4.83 E+01$ & \\
\hline
\end{tabular}


EFFLUENT AND WASTE DISPOSAL ANNUAL REPORT FOR YEAR 1989 SOLID EFFLUENTS

ESTIMATE OF MAJOR NUCLIDE COMPOSITION (BY TYPE OF WASTE)

C $\mathrm{MN}-54$

TYPE OF WASTE
A. SPENT RESINS, FILTER SLUDGES, EVAPORATOR BOTTOMS, ETC.
B. DRY COMPRESSIBLE WASTE, CONTAMINATED EQUIPMENT, ETC.
C. IRRADIATED COMPONENTS, CONTROL RODS, ETC.
D. OTHER

JAN-JUNE JULY-DEC

\% $\quad 3.15 \mathrm{E}+00$

UNIT YEAR TOTAL

M3 $\quad 6.07 \mathrm{E}+01$

CI $\quad 4.55 \mathrm{E}+02$

M3 $1.85 \mathrm{E}+02$ *

CI $\quad 6.85 \mathrm{E}+00$

M3 $2.23 \mathrm{E}+00$

CI $\quad 5.92 E+04$

M3

CI

*VOLUME AFTER COMPACTION 
EFFLUENT AND WASTE DISPOSAL ANNUAL REPORT FOR YEAR 1989 AIRBORNE AND LIQUID EFFLUENTS
UNIT NUMBER: 1 TYPE:BWR
DOCKET NO. : 50-220
THERMAL POWER (MWH) :0.00E +00
COMMERCIAL OPERATION: $12 / 01 / 69$
COOLING WATER SOURCE:LAKE ONTARIO

\author{
AIRBORNE EFFLUENTS \\ NUCLIDES RELEASED \\ $\mathrm{MN}-54$ \\ FE -55 \\ $\mathrm{CO}-60$ \\ $\mathrm{KR}-85$ \\ SR -89 \\ SR -90 \\ $\mathrm{XE}-133$ \\ CS -137
}
ACTIVITY (CI)
$7.92 \mathrm{E}-06$
$2.87 \mathrm{E}-04$
$1.83 \mathrm{E}-03$
$1.31 \mathrm{E}-04$
$2.62 \mathrm{E}-05$
$7.08 \mathrm{E}-06$
$2.11 \mathrm{E}-05$
$8.64 E-04$

LICENSEE: NIAGARA MOHAWK POWER

LICENSED POWER (MWT) :1.85E+03

NET ELECTRIC POWER (MWH):0.00E +00

INITIAL CRITICALITY:09/05/69

TOTAL AIRBORNE TRITIUM RELEASE $7.76 E+00$

TOTAL LIQUID TRITIUM RELEASE $0.00 E+00$

VOLUME OF LIQUID WASTE RELEASED (PRIOR TO DILUTION)

VOLUME OF DILUTION WATER USED DURING PERIOD
LITERS $\quad 0.00 E+00$

LITERS $1.84 E+11$ 
INSTALLATION:NINE MILE POINT 1

EFFLUENT AND WASTE DISPOSAL ANNUAL REPORT FOR YEAR 1989 SOLID EFFLUENTS

SOLID WASTE DISPOSITION

$\begin{array}{cll}\text { NUMBER OF SHIPMENTS } & \text { MODE OF TRANSPORTATION } \\ * \quad 151 & \text { N/R } \\ 24 & \text { TRUCK } \\ 1 & \text { TRUCK }\end{array}$

*WASTE BURIED AFTER VOL. RED. BY SEG, ALARON \& QUADREX

ESTIMATE OF MAJOR NUCLIDE COMPOSITION (BY TYPE OF WASTE)

A

$$
\begin{aligned}
& \text { C-14 } \\
& \text { CO-60 } \\
& \text { CS }-134 \\
& \text { CS-137 } \\
& \text { FE-55 } \\
& \text { MN-54 } \\
& \text { NI }-63 \\
& \text { OTHER } \\
& \text { SR-90 } \\
& \text { AG-110M } \\
& \text { CO-60 } \\
& \text { CS-134 } \\
& \text { CS-137 } \\
& \text { H-3 } \\
& \text { MN-54 } \\
& \text { NI }-63 \\
& \text { OTHER } \\
& \text { CO-60 } \\
& \text { CS }-137 \\
& \text { FE-55 } \\
& \text { H-3 } \\
& \text { OTHER } \\
& \text { PU- } 241
\end{aligned}
$$

B

D

$\begin{array}{lll}8 & 2.45 \mathrm{E}+00 & \\ 8 & 6.92 \mathrm{E}+01 & 3.72 \mathrm{E}+01 \\ 8 & & 3.37 \mathrm{E}-01 \\ 8 & 1.91 \mathrm{E}+01 & 2.99 \mathrm{E}+01 \\ 8 & 3.84 \mathrm{E}+00 & 2.83 \mathrm{E}+01 \\ 8 & 2.60 \mathrm{E}+00 & 1.03 \mathrm{E}+00 \\ 8 & 1.78 \mathrm{E}+00 & 1.99 \mathrm{E}+00 \\ 8 & 1.03 \mathrm{E}+00 & 5.40 \mathrm{E}-01 \\ 8 & & 7.36 \mathrm{E}-01 \\ & & \\ 8 & 2.72 \mathrm{E}+00 & \\ 8 & 5.06 \mathrm{E}+01 & 3.30 \mathrm{E}+01 \\ 8 & 5.80 \mathrm{E}-02 & \\ 8 & 3.30 \mathrm{E}+01 & 5.89 \mathrm{E}+01 \\ 8 & 3.74 \mathrm{E}-01 & 5.28 \mathrm{E}+00 \\ 8 & 2.58 \mathrm{E}+00 & 1.07 \mathrm{E}+00 \\ 8 & 7.75 \mathrm{E}+00 & 8.40 \mathrm{E}-01 \\ 8 & 2.93 \mathrm{E}+00 & 9.10 \mathrm{E}-01 \\ & & \\ 8 & 5.97 \mathrm{E}+00 & \\ 8 & 1.22 \mathrm{E}+01 & \\ 8 & 4.70 \mathrm{E}+00 & \\ 8 & 7.32 \mathrm{E}+01 & \\ 8 & 1.20 \mathrm{E}+00 & \\ 8 & 2.73 \mathrm{E}+00 & \end{array}$

TYPE OF WASTE**
A. SPENT RESINS, FILTER SLUDGES, EVAPORATOR BOTTOMS, ETC.
B. DRY COMPRESSIBLE WASTE, CONTAMINATED EQUIPMENT, ETC.
C. IRRADIATED COMPONENTS, CONTROL RODS, ETC.
D. OTHER
OIL

$\begin{array}{lc}\text { UNIT } & \text { YEAR TOTAL } \\ \text { M3 } & 9.76 \mathrm{E}+01 \\ \text { CI } & 2.46 \mathrm{E}+02 \\ \text { M3 } & 1.34 \mathrm{E}+02 \\ \text { CI } & 2.22 \mathrm{E}+00 \\ \text { M3 } & 0.00 \mathrm{E}+00 \\ \text { CI } & 0.00 \mathrm{E}+00 \\ \text { M3 } & 5.10 \mathrm{E}+00 \\ \mathrm{CI} & 1.25 \mathrm{E}-03\end{array}$

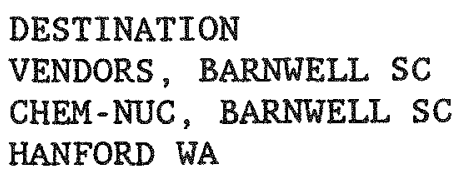

$$
\text { JAN-JUNE JULY-DEC }
$$

$$
\begin{array}{ll}
2.45 \mathrm{E}+00 & \\
6.92 \mathrm{E}+01 & 3.72 \mathrm{E}+01 \\
& 3.37 \mathrm{E}-01 \\
1.91 \mathrm{E}+01 & 2.99 \mathrm{E}+01 \\
3.84 \mathrm{E}+00 & 2.83 \mathrm{E}+01 \\
2.60 \mathrm{E}+00 & 1.03 \mathrm{E}+00 \\
1.78 \mathrm{E}+00 & 1.99 \mathrm{E}+00 \\
1.03 \mathrm{E}+00 & 5.40 \mathrm{E}-01 \\
& 7.36 \mathrm{E}-01
\end{array}
$$

$5.80 \mathrm{E}-02$

3. $30 \mathrm{E}+01$

$5.89 E+01$

1.07

$8.40 E-01$

$9.10 \mathrm{E}-01$

**SOLID WASTE TOTALS DO NOT INCLUDE VENDOR WASTE. 
EFFLUENT AND WASTE DISPOSAL ANNUAL REPORT FOR YEAR 1989 AIRBORNE AND LIQUID EFFLUENTS

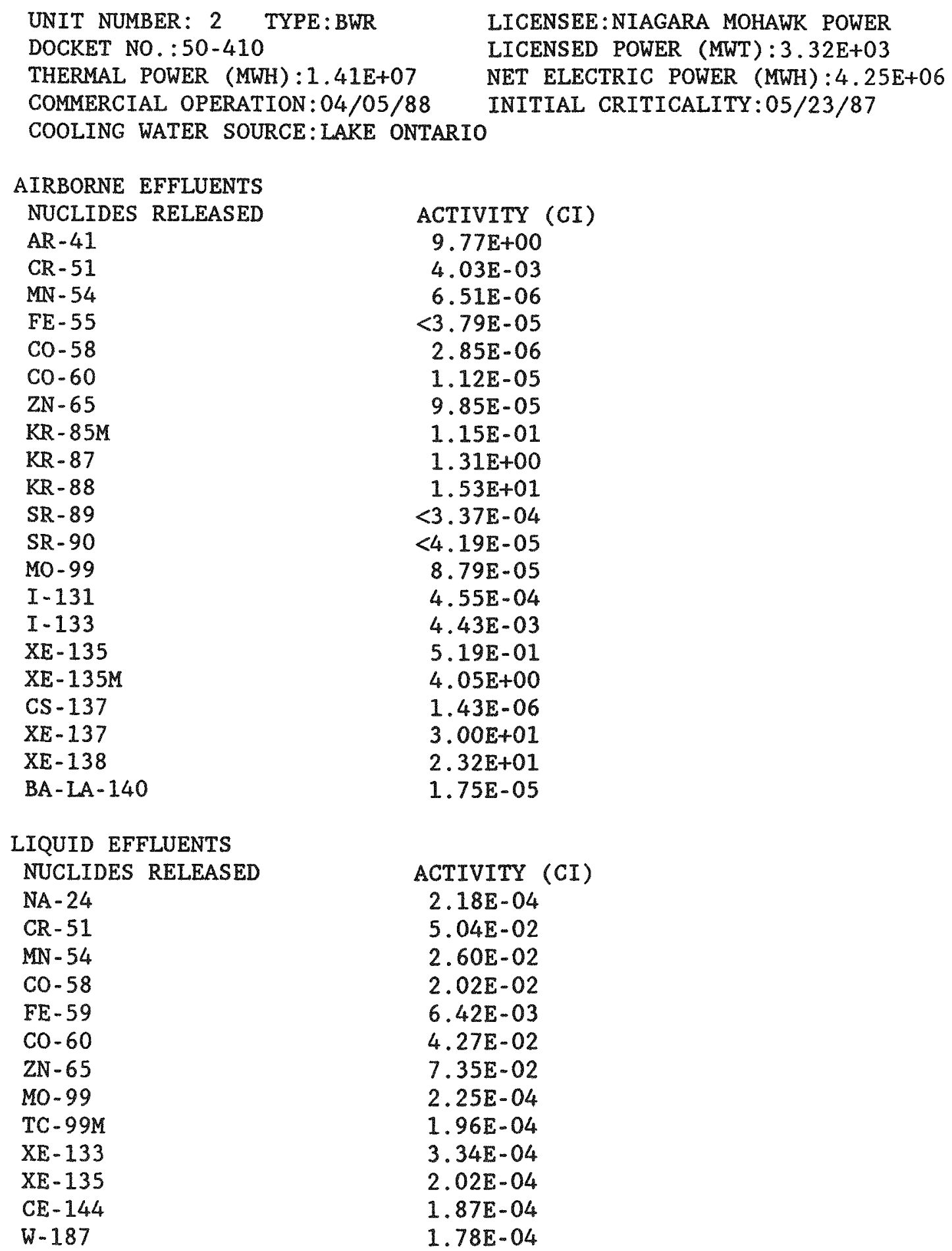

TOTAL AIRBORNE TRITIUM RELEASE 1.00E+01

TOTAL LIQUID TRITIUM RELEASE $8.10 \mathrm{E}+00$

VOLUME OF LIQUTD WASTE RELEASED (PRIOR TO DILUTION) LITERS 1.31E+07

VOLUME OF DILUTION WATER USED DURING PERIOD $\quad$ LITERS $5.62 \mathrm{E}+10$ 
INSTALIATION:NINE MILE POINT 2

EFFLUENT AND WASTE DISPOSAL ANNUAL REPORT FOR YEAR 1989 SOLID EFFLUENTS

SOLID WASTE DISPOSITION NUMBER OF SHIPMENTS 31

MODE OF TRANSPORTATION TRUCK

2 TRUCK

DESTINATION
BARNWELL SC
OAK RIDGE TN

ESTIMATE OF MAJOR NUCLIDE COMPOSITION (BY TYPE OF WASTE) JAN-JUNE JULY-DEC

A Co- 58 CO- 60

$\mathrm{CR}-51$

FE- 55

MN -54

OTHERS

$\mathrm{ZN}-65$

B

CO- 58

CO-60

CR -51

FE -55

MN - 54

OTHERS

ZN -65

D

$\mathrm{CO}-58$

CO- 60

$\mathrm{FE}-55$

MN - 54

OTHERS

$\mathrm{ZN}-65$

$\begin{array}{lll}8 & 6.50 \mathrm{E}+00 & 2.26 \mathrm{E}+00 \\ 8 & 1.66 \mathrm{E}+01 & 4.83 \mathrm{E}+00 \\ 8 & 3.40 \mathrm{E}+00 & 7.14 \mathrm{E}+01 \\ 8 & 2.77 \mathrm{E}+01 & 5.29 \mathrm{E}+00 \\ 8 & 1.12 \mathrm{E}+01 & 2.44 \mathrm{E}+00 \\ 8 & 2.80 \mathrm{E}+00 & 8.80 \mathrm{E}-01 \\ 8 & 3.18 \mathrm{E}+01 & 1.29 \mathrm{E}+01 \\ & & \\ 8 & & 2.30 \mathrm{E}+00 \\ 8 & & 1.89 \mathrm{E}+01 \\ 8 & & 3.46 \mathrm{E}+01 \\ 8 & & 9.87 \mathrm{E}+00 \\ 8 & & 1.31 \mathrm{E}+01 \\ 8 & & 1.63 \mathrm{E}+00 \\ 8 & & 1.96 \mathrm{E}+01\end{array}$

$\begin{array}{rr}8 & 1.33 \mathrm{E}+01 \\ 8 & 3.08 \mathrm{E}+01 \\ 8 & 2.42 \mathrm{E}+00 \\ 8 & 2.91 \mathrm{E}+01 \\ 8 & <1.00 \mathrm{E}+00 \\ 8 & 2.37 \mathrm{E}+01\end{array}$

TYPE OF WASTE

A. SPENT RESINS, FILTER SLUDGES, EVAPORATOR

UNIT YEAR TOTAL BOTTOMS, ETC.

M3 $2.46 \mathrm{E}+02$

CI $\quad 4.77 \mathrm{E}+02$

M3 $3.06 \mathrm{E}+01$

CI $\quad 1.23 \mathrm{E}-01$

EQUIPMENT, ETC.

M3 $0.00 \mathrm{E}+00$

CI $\quad 0.00 \mathrm{E}+00$

RODS, ETC.

M3 $1.45 E+02 *$

D. OTHER

CI $1.13 \mathrm{E}+01$

(NON-COMPACTED TRASH SENT TO PROCESSOR)

*VOLUME BEFORE REDUCTION (8.19E-01 M3 AFTER REDUCTION AT OAK RIDGE) 
EFFLUENT AND WASTE DISPOSAL ANNUAL REPORT FOR YEAR 1989 AIRBORNE AND LIQUID EFFLUENTS

\begin{tabular}{|c|c|c|}
\hline UNIT NUMBER: 1 T & & LICENSEI \\
\hline DOCKET NO. : $50-338$ & & LICENSED \\
\hline THERMAL POWER (MWH & & NET ELEC? \\
\hline COMMERCIAI OPERATI & & INITIAL \\
\hline COOLING WATER SOUR & NNA & \\
\hline UNIT NUMBER: 2 & & LICENSEE \\
\hline DOCKET NO.: $50-339$ & & LICENSED \\
\hline THERMAL POWER (MWH & & NET ELEC? \\
\hline COMMERCIAL OPERATI & & INITIAL \\
\hline COOLING WATER SOUR & NNA & \\
\hline AIRBORNE EFFLUENTS & & \\
\hline NUCLIDES RELEASED & $A C I$ & VITY (CI) \\
\hline $\mathrm{NA}-24$ & & $6 E-08$ \\
\hline$A R-41$ & & $6 \mathrm{E}+00$ \\
\hline$M N-54$ & & $7 E-09$ \\
\hline$F E-55$ & & $4 E-05$ \\
\hline$C 0-58$ & & $1 E-05$ \\
\hline $\mathrm{CO}-60$ & & $1 E-04$ \\
\hline$K R-83 M$ & & $9 \mathrm{E}+00$ \\
\hline $\mathrm{KR}-85$ & & $6 \mathrm{E}+02$ \\
\hline $\mathrm{KR}-85 \mathrm{M}$ & & $1 E+00$ \\
\hline$S R-85$ & & $6 E-06$ \\
\hline $\mathrm{KR}-87$ & & $1 E-01$ \\
\hline $\mathrm{KR}-88$ & & $5 E+00$ \\
\hline$R B-88$ & & $4 E-05$ \\
\hline NB- 95 & & $7 E-08$ \\
\hline RH-106 & & $9 \mathrm{E}-08$ \\
\hline RU-106 & & $5 E-07$ \\
\hline$A G-110 M$ & & $3 E-08$ \\
\hline$S B-122$ & & $0 \mathrm{E}-06$ \\
\hline$S B-125$ & & $O E-06$ \\
\hline$I-131$ & & $6 E-03$ \\
\hline$X E-131 M$ & & $\mathrm{OE}+01$ \\
\hline$I-132$ & & $7 E-08$ \\
\hline$I-133$ & & $3 E-03$ \\
\hline$X E-133$ & & $6 \mathrm{E}+03$ \\
\hline$X E-133 M$ & & $5 E+00$ \\
\hline CS -134 & & $2 E-05$ \\
\hline$I-134$ & & $5 E-08$ \\
\hline$I-135$ & & $8 E-08$ \\
\hline$X E-135$ & & $6 \mathrm{E}+01$ \\
\hline$X E-135 M$ & & $2 E-01$ \\
\hline CS -137 & & $7 E-04$ \\
\hline $\operatorname{CS}-138$ & & $7 E-08$ \\
\hline$X E-138$ & & $6 E-02$ \\
\hline $\mathrm{CE}-143$ & & $8 E-04$ \\
\hline
\end{tabular}


EFFLUENT AND WASTE DISPOSAL ANNUAL REPORT FOR YEAR 1989 AIRBORNE AND LIQUID EFFLUENTS

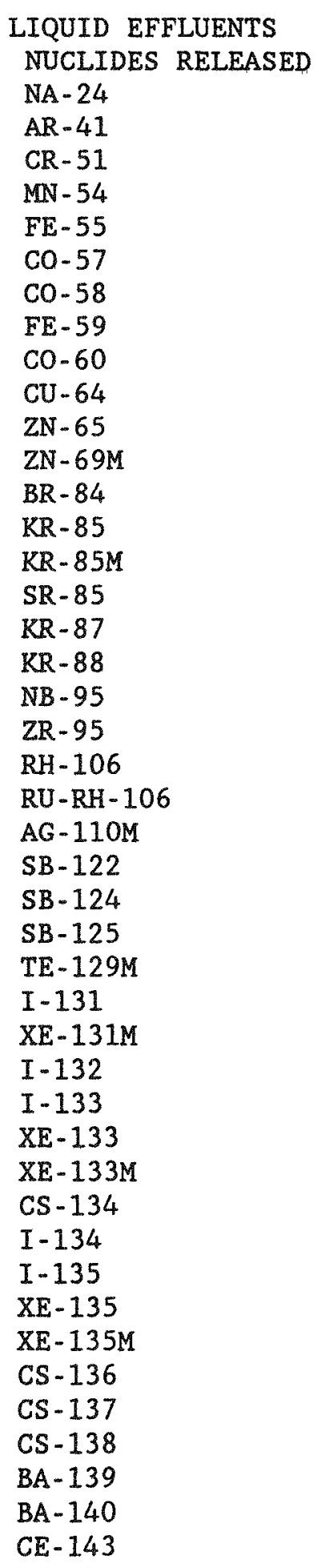

\section{LIQUID EFFLUENTS}

NA -24

$\mathrm{AR}-41$

$\mathrm{MN}-54$

FE -55

$\mathrm{CO}-57$

$\mathrm{CO}-58$

FE -59

CO- 60

CU. -64

$B R-84$

$\mathrm{KR}-85$

SR -85

NB -95

ZR-95

RH -106

RU - RH - 106

AG-110M

$S B-122$

SB -124

SB- 125

TE-129M

$\mathrm{I}-132$

$I-133$

$\mathrm{XE}-133$

$\mathrm{XE}-133 \mathrm{M}$

CS -134

I -134

I -135

$X E-135$

$\mathrm{XE}-135 \mathrm{M}$

CS -138

$B A-140$

CE -143
$9.72 \mathrm{E}-03$
3. $92 \mathrm{E}-03$
1. $00 \mathrm{E}-04$
1. $83 \mathrm{E}-04$
3. 05E-01
9.99E-04
2. $13 \mathrm{E}-01$
7.01E-04
4. $27 \mathrm{E}-06$
1. $46 \mathrm{E}-05$
$9.29 \mathrm{E}-05$
3. $49 \mathrm{E}-02$
$8.55 E-04$
1. $51 \mathrm{E}-04$
$1.06 \mathrm{E}-04$
1. $68 \mathrm{E}-03$
7. $56 \mathrm{E}-03$
1.71E-05
$3.65 E-04$
7. $25 \mathrm{E}-03$
$1.09 \mathrm{E}-01$
5. 12E-03
$4.52 \mathrm{E}-03$
$3.07 \mathrm{E}-01$
2. $60 \mathrm{E}-04$
1. $74 \mathrm{E}-02$
1. $59 \mathrm{E}-02$
$2.06 \mathrm{E}-04$
3. $61 \mathrm{E}-03$
$5.51 \mathrm{E}+00$
4. $34 \mathrm{E}-02$
$6.15 \mathrm{E}-02$
7. $88 \mathrm{E}-08$
$8.52 \mathrm{E}-04$
$6.31 E-02$
$5.09 \mathrm{E}-03$
7. $66 \mathrm{E}-04$
$1.04 \mathrm{E}-01$
4.10E-05
1.77E-04
2. $42 \mathrm{E}-04$
1. $09 \mathrm{E}-04$ 
EFFLUENT AND WASTE DISPOSAL ANNUAL REPORT FOR YEAR 1989

AIRBORNE AND LIQUID EFFLUENTS

TOTAL AIRBORNE TRITIUM RELEASE $1.22 E+02$

TOTAL LIQUID TRITIUM RELEASE 1.40E+03

VOLUME OF LIQUID WASTE RELEASED (PRIOR TO DILUTION)

VOLUME OF DILUTION WATER USED DURING PERIOD
LITERS

LITERS
$2.74 E+08$

$2.43 E+12$ 
EFFLUENT AND WASTE DISPOSAL ANNUAL REPORT FOR YEAR 1989 SOLID EFFLUENTS

SOLID WASTE DISPOSITION NUMBER OF SHIPMENTS 26

5

13

6
5
3

MODE OF TRANSPORTATION TRUCK TRUCK TRUCK

ESTIMATE OF MAJOR NUCLIDE COMPOSITION (BY TYPE OF WASTE)

A

$$
\begin{aligned}
& \text { CO-60 } \\
& \text { CS }-134 \\
& \text { CS }-137 \\
& \text { FE }-55 \\
& \text { MN }-54 \\
& \text { NI }-63 \\
& \text { B } \\
& \text { CO- } 58 \\
& \text { CO-60 } \\
& \text { CR }-51 \\
& \text { CS }-137 \\
& \text { FE }-55 \\
& \text { MN }-54 \\
& \text { NB-95 } \\
& \text { NI }-63 \\
& \text { PU }-241 \\
& \text { RU }-103 \\
& \text { ZR }-95 \\
& D \\
& \text { CE }-144 \\
& \text { CO }-60 \\
& \text { CS }-137
\end{aligned}
$$$$
\text { D }
$$

TYPE OF WASTE
A. SPENT RESINS, FILTER SLUDGES, EVAPORATOR BOTTOMS, ETC.
B. DRY COMPRESSIBLE WASTE, CONTAMINATED EQUIPMENT, ETC.
C. IRRADIATED COMPONENTS, CONTROL RODS, ETC.
D. OTHER
CONTAMINATED OIL

\author{
DESTINATION \\ BARNWELL SC \\ QUADREX, OAK RIDGE TN \\ SEG, OAK RIDGE TN
}

$$
\text { JAN - JUNE JULY - DEC }
$$

$\begin{array}{lll}8 & 4.68 \mathrm{E}+01 & 4.93 \mathrm{E}+01 \\ 8 & 1.55 \mathrm{E}+00 & 1.30 \mathrm{E}+00 \\ 8 & 1.02 \mathrm{E}+01 & 2.62 \mathrm{E}+00 \\ 8 & 1.32 \mathrm{E}+01 & 1.76 \mathrm{E}+01 \\ 8 & 1.07 \mathrm{E}+00 & 1.10 \mathrm{E}+00 \\ 8 & 2.57 \mathrm{E}+01 & 2.65 \mathrm{E}+01 \\ & & \\ 8 & 3.50 \mathrm{E}+00 & 3.52 \mathrm{E}+00 \\ \frac{8}{8} & 2.55 \mathrm{E}+01 & 2.76 \mathrm{E}+01 \\ 8 & 2.77 \mathrm{E}+01 & 2.83 \mathrm{E}+01 \\ 8 & & 1.17 \mathrm{E}+00 \\ 8 & 2.68 \mathrm{E}+01 & 2.52 \mathrm{E}+01 \\ 8 & 1.23 \mathrm{E}+00 & 1.28 \mathrm{E}+00 \\ 8 & 3.41 \mathrm{E}+00 & 2.19 \mathrm{E}+00 \\ 8 & 4.16 \mathrm{E}+00 & 3.57 \mathrm{E}+00 \\ 8 & 1.00 \mathrm{E}+00 & 1.19 \mathrm{E}+00 \\ 8 & 3.55 \mathrm{E}+00 & 3.85 \mathrm{E}+00 \\ 8 & 1.15 \mathrm{E}+00 & \\ 8 & & \\ 8 & & 6.48 \mathrm{E}+01 \\ 8 & & 1.81 \mathrm{E}+01 \\ \frac{8}{8} & & 1.71 \mathrm{E}+01\end{array}$

*NOT REPRESENTATIVE OF ACTUAL VOL. BURIED (VOL. BURIED $57.1 \mathrm{M} 3$ )

\#NOT REPRESENTATIVE OF ACTUAL VOL. BURIED (VOL. BURIED 342.1 M3)

+SHIPPED TO WASTE PROCESSOR FOR INCINERATION (VOL. BURIED O. M3)

$N / A=N O T$ APPLICABLE

$N / D=$ NOT DETECTED

N/R=NOT REPORTED 
EFFLUENT AND WASTE DISPOSAL ANNUAL REPORT FOR YEAR 1989 AIRBORNE AND LIQUID EFFLUENTS

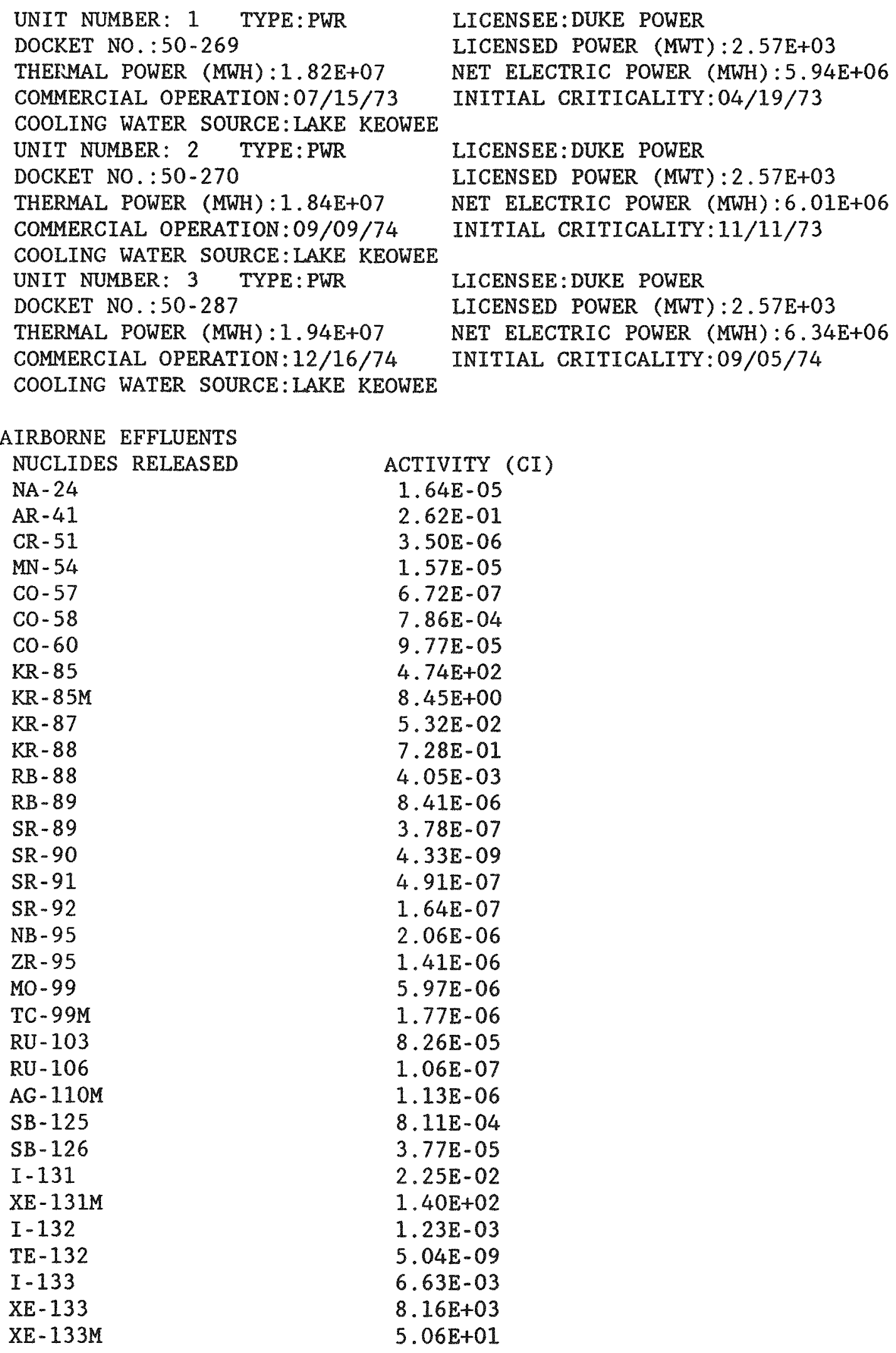


EFFLUENT AND WASTE DISPOSAL ANNUAL REPORT FOR YEAR 1989 AIRBORNE AND LIQUID EFFLUENTS

\author{
AIRBORNE EFFLUENTS \\ NUCLIDES RELEASED \\ CS -134 \\ $\mathrm{I}-134$ \\ $\mathrm{I}-135$ \\ $\mathrm{XE}-135$ \\ $\mathrm{XE}-135 \mathrm{M}$ \\ CS -136 \\ CS -137 \\ CS -138 \\ BA -140 \\ CE -141 \\ CE -143
}

\section{LIQUID EFFLUENTS}

NUCLIDES RELEASED

NA -24

AR -41

CR -51

$\mathrm{MN}-54$

FE -55

$\mathrm{CO}-57$

CO- 58

FE- 59

$\mathrm{CO}-60$

$\mathrm{ZN}-65$

SR -89

SR -90

SR -92

NB-95

ZR -95

NB -97

MO-99

TC-99M

RU -103

RU-106

AG-110M

SN-113

SB- 122

SB-124

SB- 125

SB-126

I -131

$\mathrm{XE}-131 \mathrm{M}$

I -132

TE-132

I -133

$\mathrm{XE}-133$

$\mathrm{XE}-133 \mathrm{M}$

CS -134

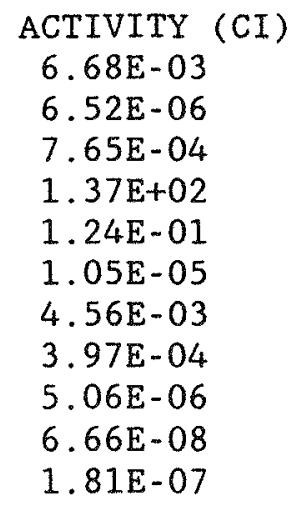

3. $40 \mathrm{E}-02$ 
EFFLUENT AND WASTE DISPOSAL ANNUAL REPORT FOR YEAR 1989 AIRBORNE AND LIQUID EFFLUENTS

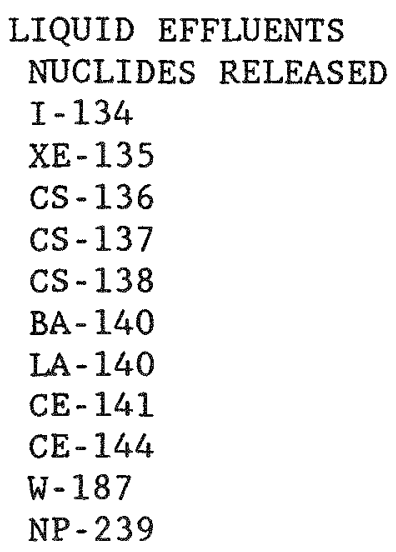

ACTIVITY (CI)
$1.02 \mathrm{E}-04$
$2.11 \mathrm{E}-02$
$1.71 \mathrm{E}-03$
$4.76 \mathrm{E}-02$
$8.57 \mathrm{E}-04$
$6.58 \mathrm{E}-03$
$8.08 \mathrm{E}-02$
$2.86 \mathrm{E}-03$
$3.35 \mathrm{E}-02$
$1.65 \mathrm{E}-04$
$2.66 \mathrm{E}-03$

TOTAL AIRBORNE TRITIUM RELEASE $1.18 \mathrm{E}+02$

TOTAL LIQUID TRITIUM RELEASE $1.02 E+03$

VOLUME OF LIQUID WASTE RELEASED (PRIOR TO DILUTION) LITERS 3.00E+09 VOLUME OF DILUTION WATER USED DURING PERIOD LITERS $6.64 \mathrm{E}+11$ 
INSTALLATION : OCONEE

EFFLUENT AND WASTE DISPOSAL ANNUAL REPORT FOR YEAR 1989 SOLID EFFLUENTS

SOLID WASTE DISPOSITION NUMBER OF SHIPMENTS

MODE OF TRANSPORTATION

$N / R$

\# $\quad 41$

$N / R$

$\mathrm{N} / \mathrm{R}$
DESTINATION

ALARON-BARNWELL SC

$\mathrm{N} / \mathrm{R}$

SEG-BARNWELL SC

\#DOES NOT INCLUDED BROKERED TOTALS

ESTIMATE OF MAJOR NUCLIDE COMPOSITION (BY TYPE OF WASTE)

JAN - JUNE

JULY-DEC

A

AG-110M

C- 14

Co- 58

CO -60

CR -51

CS -134

CS -137

FE -55

H -3

I-131

MN -54

NB- 95

NI -63

$S B-125$

SR -90

XE-131M

$\mathrm{XE}-133$

ZR-95

B

C -14

Co- 58

$\mathrm{CO}-60$

CR- 51

CS -134

CS -137

FE- 55

H -3

NI -63

PU-241

SB-125

SR -90

C

TC- 99

AG- $108 \mathrm{M}$

AG -110

AG - 110M

$\begin{array}{lll}8 & 3.77 \mathrm{E}+00 & 2.66 \mathrm{E}-01 \\ 8 & 7.96 \mathrm{E}-01 & 1.21 \mathrm{E}-01 \\ 8 & 1.62 \mathrm{E}+01 & 9.52 \mathrm{E}+00 \\ 8 & 2.20 \mathrm{E}+01 & 1.81 \mathrm{E}+00 \\ 8 & 7.52 \mathrm{E}-04 & \\ 8 & 2.90 \mathrm{E}+00 & 3.03 \mathrm{E}+01 \\ 8 & 8.63 \mathrm{E}+00 & 4.97 \mathrm{E}+01 \\ 8 & 2.20 \mathrm{E}+01 & 1.91 \mathrm{E}+00 \\ 8 & & 2.10 \mathrm{E}+00 \\ 8 & 3.35 \mathrm{E}-01 & 1.37 \mathrm{E}-01 \\ 8 & 4.67 \mathrm{E}+00 & 7.57 \mathrm{E}-01 \\ 8 & 1.50 \mathrm{E}-03 & \\ 8 & 1.77 \mathrm{E}+01 & 2.57 \mathrm{E}+00 \\ 8 & 8.93 \mathrm{E}-01 & \\ 8 & & 3.80 \mathrm{E}-01 \\ 8 & 7.52 \mathrm{E}-04 & \\ 8 & 7.52 \mathrm{E}-03 & \\ 8 & 7.52 \mathrm{E}-04 & \\ & & \\ 8 & 2.30 \mathrm{E}+00 & 2.30 \mathrm{E}+00 \\ 8 & 2.15 \mathrm{E}+01 & 2.13 \mathrm{E}+01 \\ 8 & 4.00 \mathrm{E}+00 & 4.00 \mathrm{E}+00 \\ 8 & 2.39 \mathrm{E}+00 & 2.31 \mathrm{E}+00 \\ 8 & 2.36 \mathrm{E}+01 & 2.36 \mathrm{E}+01 \\ 8 & 3.36 \mathrm{E}+01 & 3.37 \mathrm{E}+01 \\ 8 & 7.20 \mathrm{E}+00 & 7.20 \mathrm{E}+00 \\ 8 & 7.34 \mathrm{E}-01 & 6.00 \mathrm{E}-01 \\ 8 & 2.80 \mathrm{E}+00 & 2.80 \mathrm{E}+00 \\ 8 & 1.00 \mathrm{E}-01 & 1.00 \mathrm{E}-01 \\ 8 & 1.20 \mathrm{E}+00 & 1.20 \mathrm{E}+00 \\ 8 & 4.00 \mathrm{E}-01 & 4.00 \mathrm{E}-01 \\ 8 & 3.00 \mathrm{E}-01 & 3.00 \mathrm{E}-01 \\ 8 & & \\ 8 & & 3.00 \mathrm{E}-01 \\ 8 & & 8.95 \mathrm{E}+01\end{array}$


EFFLUENT AND WASTE DISPOSAL ANNUAL REPORT FOR YEAR 1989 SOLID EFFLUENTS

ESTIMATE OF MAJOR NUCLIDE COMPOSITION (BY TYPE OF WASTE)

JAN-JUNE JULY-DEC

C

$\mathrm{CO}-58$

$\mathrm{CO}-60$

$\mathrm{CR}-51$

FE -55

MN -54

NI -63

TA -182

D

AG-110M

C -14

CE -144

CO- 58

CO- 60

CS -134

CS -137

FE -55

$\mathrm{H}-3$

I- 131

MN- 54

NI -63

PU- 241

SB- 125

SR -90

TC -99

TYPE OF WASTE

A. SPENT RESINS, FILTER SLUDGES, EVAPORATOR BOTTOMS, ETC.

B. DRY COMPRESSIBLE WASTE, CONTAMINATED

EQUIPMENT, ETC.

8

8

8

8

8

8

8

$\%$

8

8

8

8

8

웅

8

8

8

8

8

8

8

8

8
$3.00 E+00$

8. $70 \mathrm{E}-01$

3. $40 \mathrm{E}-01$

1. $66 \mathrm{E}+00$

2. $30 \mathrm{E}-01$

5. $40 \mathrm{E}-01$

2. $32 \mathrm{E}+00$

3. $80 E+00$

8. $00 \mathrm{E}-01$

2. $35 E+01$

4. $00 \mathrm{E}-01$

1. $62 \mathrm{E}+01$

2. $22 \mathrm{E}+01$

$2.90 \mathrm{E}+00$

$8.40 \mathrm{E}+00$

2. $21 E+01$

2.00E-01

4. $70 \mathrm{E}+00$

$1.78 \mathrm{E}+01$

$9.00 \mathrm{E}-01$

$5.99 \mathrm{E}-01$

2. $38 \mathrm{E}+00$

$3.83 \mathrm{E}+01$

$3.02 E-01$

3. $10 \mathrm{E}+00$

1.71E-01

1. $50 \mathrm{E}+00$

$2.85 E+01$

$5.00 E-01$

4. $01 \mathrm{E}-01$

2.00E-01

C. IRRADIATED COMPONENTS, CONTROL RODS, ETC.

UNIT YEAR TOTAL

M3 $1.49 \mathrm{E}+02$

CI $\quad 1.30 \mathrm{E}+03$

M3 $2.47 \mathrm{E}+02$ *

CI $\quad 6.58 \mathrm{E}+01$

M3 $1.08 \mathrm{E}+00$

CI $2.80 \mathrm{E}+00$

D. OTHER

M3

2.71E+01

DEWATERED MECHANICAL FILTERS

CI

$9.26 \mathrm{E}+01$

*VOLUME CONTAINS COMPACTED AND NON-COMPACTED WASTE 
EFFLUENT AND WASTE DISPOSAL ANNUAL REPORT FOR YEAR 1989 AIRBORNE AND LIQUID EFFLUENTS

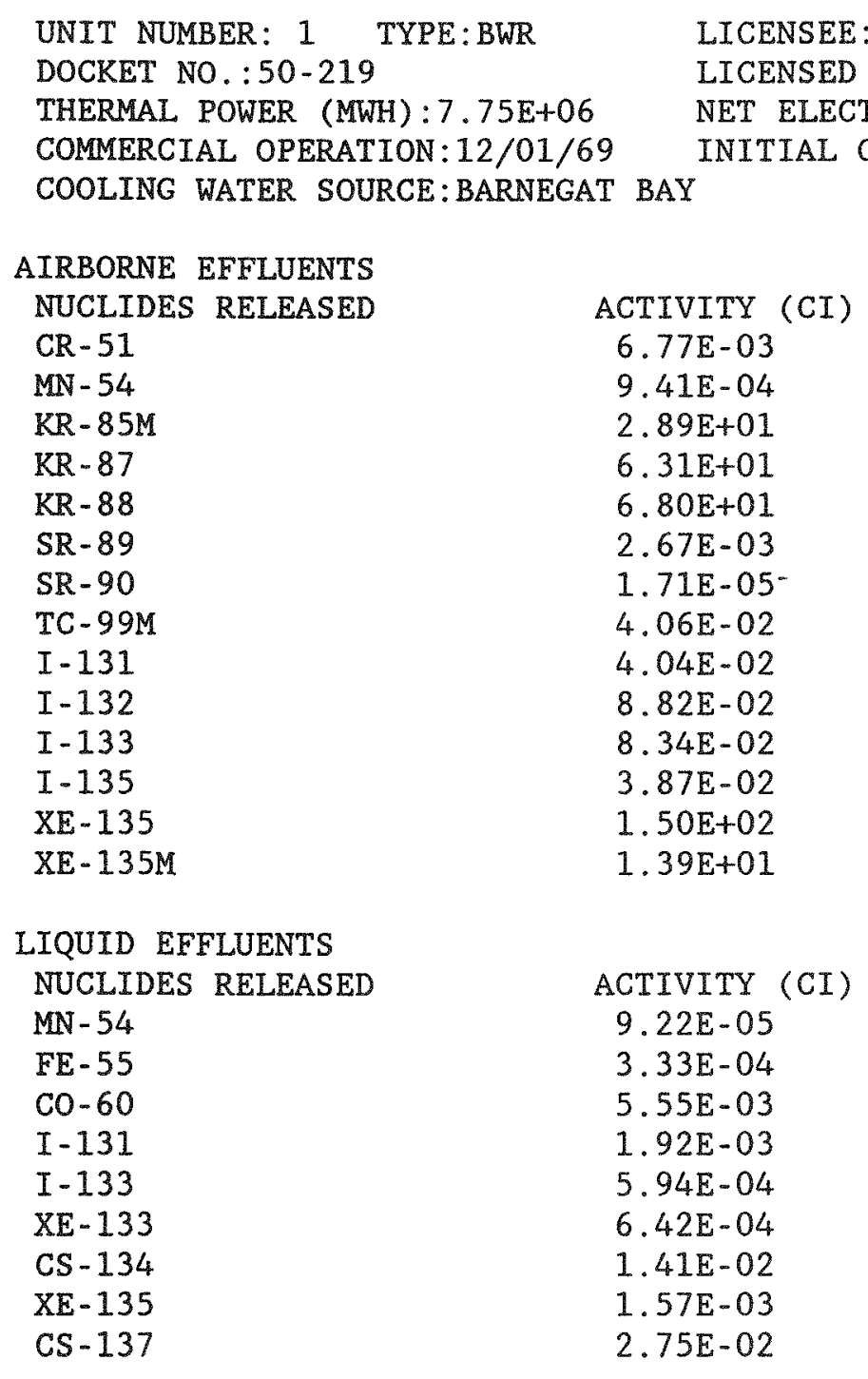

TOTAL AIRBORNE TRITIUM RELEASE $1.03 \mathrm{E}+01$

TOTAL LIQUID TRITIUM RELEASE $3.96 E+00$

VOLUME OF LIQUID WASTE RELEASED (PRIOR TO DILUTION) LITERS 2.98E+06

VOLUME OF DILUTION WATER USED DURING PERIOD

LITERS $\quad 2.19 \mathrm{E}+10$ 
INSTALLATION:OYSTER CREEK

EFFLUENT AND WASTE DISPOSAL ANNUAL REPORT FOR YEAR 1989 SOLID EFFLUENTS

SOLID WASTE DISPOSITION NUMBER OF SHIPMENTS 54

MODE OF TRANSPORTATION TRUCK

17

TRUCK

ESTIMATE OF MAJOR NUCLIDE COMPOSITION (BY TYPE OF WASTE)

DESTINATION

BARNWELL SC

SEG, OAK RIDGE TN

A

C -14

CM- 242

CO -60

CR -51

CS -134

CS -137

FE- 55

H-3

$\mathrm{MN}-54$

NI -63

PU -241

B

SR -90

C - 14

CE-141

CE -144

CO- 58

CO- 60

CR -51

CS -134

CS -137

FE- 55

FE -59

H- 3

LA -140

$\mathrm{MN}-54$

NI -63

PU -241

SR -89

SR -90

$\mathrm{ZN}-65$

$\mathrm{C}$

C. 14

CO- 60

CS -137

FE -55

H -3

$$
\text { JAN-JUNE JULY-DEC }
$$

\begin{tabular}{|c|c|c|}
\hline 8 & $4.63 E-01$ & $1.50 \mathrm{E}-02$ \\
\hline$\frac{8}{6}$ & $3.00 E-03$ & $1.00 \mathrm{E}-03$ \\
\hline$\frac{8}{8}$ & $3.04 E+01$ & $2.52 \mathrm{E}+0$ \\
\hline 8 & $1.08 E+00$ & \\
\hline 8 & 1. $20 \mathrm{E}+01$ & $7.89 E+0$ \\
\hline 8 & $2.78 E+01$ & $2.54 E+C$ \\
\hline 8 & $2.30 E+01$ & $3.66 E+$ \\
\hline 8 & $1.03 \mathrm{E}-01$ & $2.20 E-$ \\
\hline 8 & $4.59 E+00$ & $4.45 E+0$ \\
\hline 8 & $2.87 E-01$ & $2.77 E-C$ \\
\hline 8 & $9.60 \mathrm{E}-02$ & $7.00 \mathrm{E}-0$ \\
\hline 8 & $1.29 E-01$ & $5.70 E-0$ \\
\hline 8 & $2.40 E-03$ & $1.22 E-C$ \\
\hline 8 & $2.00 E-02$ & $1.05 \mathrm{E}-0$ \\
\hline 8 & $2.00 E-02$ & $1.05 E-0$ \\
\hline 8 & $3.88 \mathrm{E}-01$ & $3.15 E-C$ \\
\hline 8 & $2.98 E+01$ & $2.75 E+0$ \\
\hline 8 & $1.08 \mathrm{E}+00$ & $1.08 \mathrm{E}+0$ \\
\hline 8 & $4.30 E+00$ & $4.31 E+0$ \\
\hline 8 & $1.37 E+01$ & $1.17 \mathrm{E}+0$ \\
\hline 8 & $4.60 E+01$ & $5.10 E+0$ \\
\hline 8 & $6.10 \mathrm{E}-02$ & $6.30 \mathrm{E}-02$ \\
\hline$\frac{8}{8}$ & $4.08 \mathrm{E}-03$ & $4.20 E-03$ \\
\hline 8 & $2.49 E-01$ & $2.10 \mathrm{E}-0$ \\
\hline 8 & $3.80 E+00$ & $3.37 E+0$ \\
\hline 8 & 1. $33 \mathrm{E}-01$ & $1.05 E-0$ \\
\hline 8 & $7.10 E-02$ & $4.20 E-0$ \\
\hline 8 & $1.94 \mathrm{E}-01$ & $3.20 E-0$ \\
\hline 8 & $4.10 E-02$ & $8.90 E-0$ \\
\hline 8 & $7.10 \mathrm{E}-02$ & $6.30 E-0$ \\
\hline$\frac{8}{8}$ & & $8.00 \mathrm{E}-03$ \\
\hline 8 & & $3.45 E+0$ \\
\hline 8 & & $9.00 \mathrm{E}-03$ \\
\hline 8 & & $5.61 E+01$ \\
\hline \% & & $6.95 E-0$ \\
\hline
\end{tabular}


EFFLUENT AND WASTE DISPOSAL ANNUAL REPORT FOR YEAR 1989 SOLID EFFLUENTS

ESTIMATE OF MAJOR NUCLIDE COMPOSITION (BY TYPE OF WASTE)

C

$\mathrm{MN}-54$

NI -59

NI -63

D

CO -60

CS -134

CS -137

FE -55

$\mathrm{H}-3$

MN -54

NI -63

PU-241
JAN-JUNE JULY-DEC

4. $23 \mathrm{E}+00$

$3.10 \mathrm{E}-02$

$4.45 \mathrm{E}+00$

$3.03 E+01$

$9.68 \mathrm{E}+00$

$3.65 \mathrm{E}+01$

1. $77 \mathrm{E}+01$

2. 20E-02

$5.05 \mathrm{E}+00$

$6.88 \mathrm{E}-01$

1. $80 \mathrm{E}-02$

\section{TYPE OF WASTE}

A. SPENT RESINS, FILTER SLUDGES, EVAPORATOR BOTTOMS, ETC.

B, DRY COMPRESSIBLE WASTE, CONTAMINATED EQUIPMENT, ETC.

C. IRRADIATED COMPONENTS, CONTROL RODS, ETC.

D. OTHER

OTHER WASTE

UNIT YEAR TOTAL

M3 $1.56 \mathrm{E}+02$

CI $\quad 1.18 \mathrm{E}+03$

M3 2.15E+02*

CI $1.71 \mathrm{E}+01$

M3 4.40E+01

CI $\quad 2.32 \mathrm{E}+05$

M3 $5.10 \mathrm{E}+00$

CI $\quad 2.42 \mathrm{E}+01$

*VOLUME AFTER COMPACTION INCLUDES 199.6 M3 PROCESSED BY SEG 
EFFLUENT AND WASTE DISPOSAL ANNUAL REPORT FOR YEAR 1989 AIRBORNE AND LIQUID EFFLUENTS

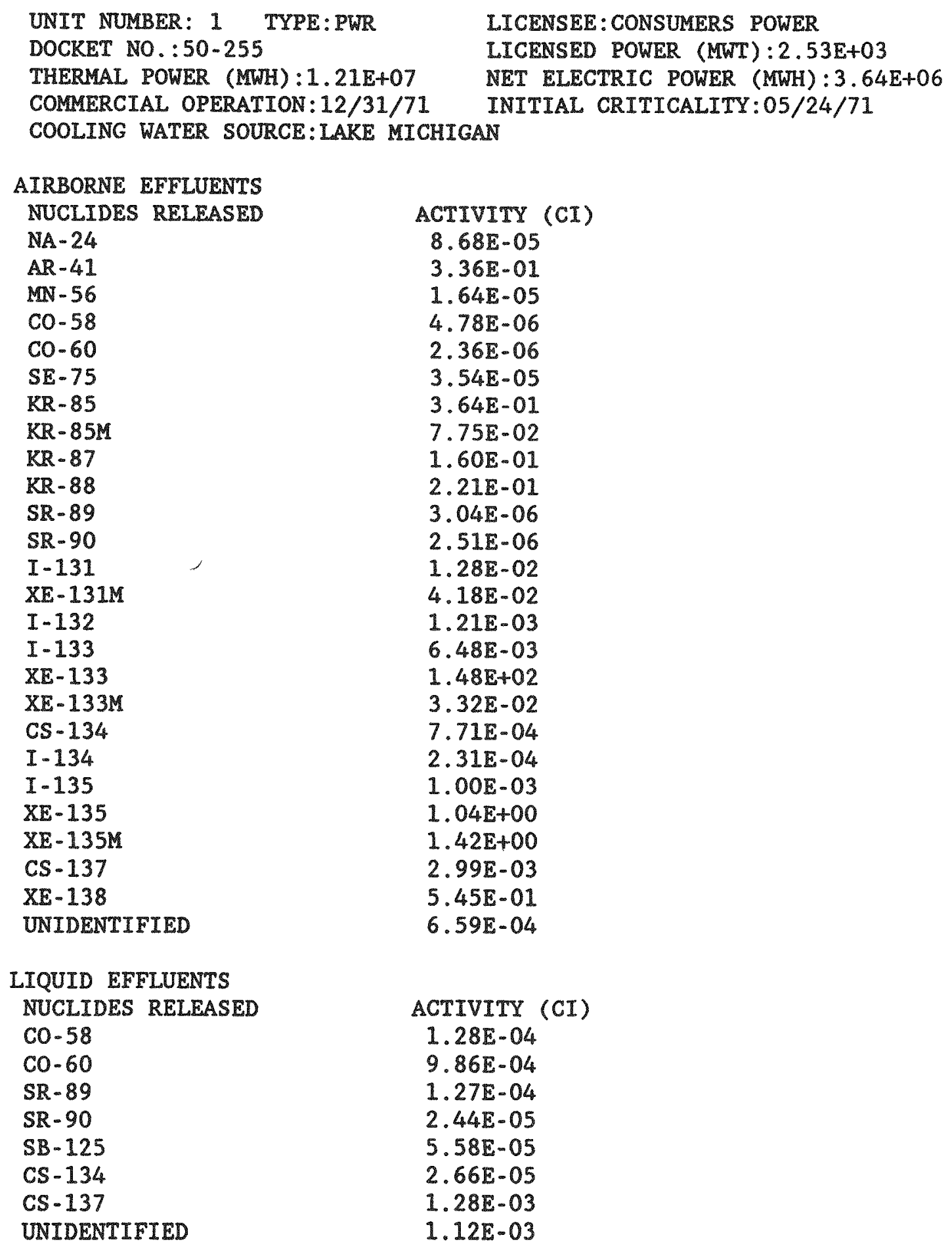

TOTAL AIRBORNE TRITIUM RELEASE 1.12E+01

TOTAL LIQUID TRITIUM RELEASE $8.06 E+01$

VOLUME OF LIQUID WASTE RELEASED (PRIOR TO DILUTION) LITERS 2.81E+06 VOLUME OF DILUTION WATER USED DURING PERIOD 
EFFLUENT AND WASTE DISPOSAL ANNUAL REPORT FOR YEAR 1989 SOLID EFFLUENTS

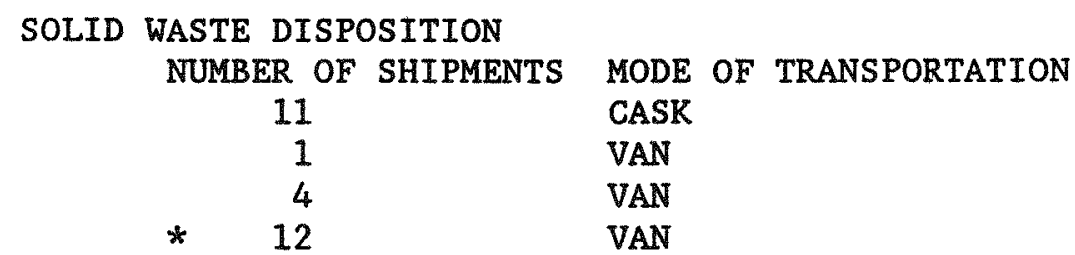

TYPE OF WASTE
A. SPENT RESINS, FILTER SLUDGES, EVAPORATOR BOTTOMS, ETC.
B. DRY COMPRESSIBLE WASTE, CONTAMINATED EQUIPMENT, ETC.
C. IRRADIATED COMPONENTS, CONTROL RODS, ETC.
D. OTHER

\begin{tabular}{|c|c|}
\hline \multicolumn{2}{|c|}{$\begin{array}{l}\text { BARNWELL SC } \\
\text { BEATTY NV } \\
\text { RICHLAND WA }\end{array}$} \\
\hline UNIT & YEAR TOTAL \\
\hline M3 & $4.80 E+01$ \\
\hline CI & $4.19 \mathrm{E}+03$ \\
\hline M3 & $1.70 \mathrm{E}+02$ \\
\hline CI & $1.11 \mathrm{E}+01$ \\
\hline M3 & $4.13 E-01$ \\
\hline CI & $4.11 E+03$ \\
\hline \multicolumn{2}{|l|}{ M3 } \\
\hline \multicolumn{2}{|l|}{$C I$} \\
\hline
\end{tabular}

DESTINATION

BARNWELL SC

BEATTY NV

RICHLAND WA

SEG, BARNWELL SC

*SENT TO SEG FOR VOL. REDUCTION 
EFFLUENT AND WASTE DISPOSAL ANNUAL REPORT FOR YEAR 1989 AIRBORNE AND LIQUID EFFLUENTS

\begin{tabular}{|c|c|}
\hline \multicolumn{2}{|l|}{$\begin{array}{l}\text { UNIT NUMBER: } 1 \text { TYPE:PWR } \\
\text { DOCKET NO }: 50-528\end{array}$} \\
\hline \multicolumn{2}{|l|}{ DOCKET NO.:50-528 } \\
\hline \multicolumn{2}{|r|}{ NET ELECTRIC POWER (MWH): $1.80 \mathrm{E}+06$} \\
\hline \multirow{2}{*}{\multicolumn{2}{|c|}{$\begin{array}{l}\text { COMMERCIAL OPERATION:01/28/86 INITIAL CRITICALITY:05/25/85 } \\
\text { COOLING WATER SOURCE: SEWAGE TREATMENT }\end{array}$}} \\
\hline & \\
\hline \multicolumn{2}{|l|}{ AIRBORNE EFFLUENTS } \\
\hline NUCLIDES RELEASED & VITY (CI) \\
\hline AR -41 & 9 E-01 \\
\hline $\mathrm{MN}-54$ & $1 E-06$ \\
\hline$C 0-57$ & OE -07 \\
\hline Co- 58 & $3 E-05$ \\
\hline$C 0-60$ & $6 \mathrm{E}-05$ \\
\hline$B R-82$ & $1 E-06$ \\
\hline $\mathrm{KR}-85$ & $5 E+01$ \\
\hline $\mathrm{KR}-85 \mathrm{M}$ & $3 E+00$ \\
\hline $\mathrm{KR}-87$ & $7 E+00$ \\
\hline $\mathrm{KR}-88$ & $6 \mathrm{E}+01$ \\
\hline $\mathrm{RB}-88$ & $1 E-03$ \\
\hline$N B-95$ & $9 E-06$ \\
\hline $\mathrm{RU}-103$ & $7 E-05$ \\
\hline$S B-124$ & $3 E-05$ \\
\hline$I-131$ & $5 E-04$ \\
\hline$X E-131 M$ & $1 E+00$ \\
\hline$I-132$ & $9 \mathrm{E}-06$ \\
\hline$I-133$ & OE -04 \\
\hline$X E-133$ & $2 \mathrm{E}+02$ \\
\hline$X E-133 M$ & $5 E-01$ \\
\hline $\operatorname{CS}-134$ & $1 E-08$ \\
\hline$I-134$ & $9 E-07$ \\
\hline$I-135$ & $6 \mathrm{E}-05$ \\
\hline$X E-135$ & $5 E+01$ \\
\hline CS -137 & $3 E-06$ \\
\hline CS -138 & $5 E-0.5$ \\
\hline
\end{tabular}

TOTAL AIRBORNE TRITIUM RELEASE $1.56 \mathrm{E}+02$

TOTAL LIQUID TRITIUM RELEASE $0.00 \mathrm{E}+00$

VOLUME OF LIQUID WASTE RELEASED (PRIOR TO DILUTION) LITERS $0.00 E+00$ VOLUME OF DILUTION WATER USED DURING PERIOD 
EFFLUENT AND WASTE DISPOSAL ANNUAL REPORT FOR YEAR 1989 AIRBORNE AND LIQUID EFFLUENTS

\begin{tabular}{|c|c|}
\hline UNIT NUMBER: & LICENSEE \\
\hline DOCKET NO.: $50-529$ & LICENSED \\
\hline THERMAL POWER (MWH) $: 1.47 \mathrm{E}+07$ & NET ELECT \\
\hline COMMERCIAL OPERATION:09/19/86 & INITIAL $\mathrm{C}$ \\
\hline COOLING WATER SOURCE:SEWAGE I & TREATMENT \\
\hline AIRBORNE EFFLUENTS & \\
\hline NUCLIDES RELEASED & ACTIVITY (CI) \\
\hline AR-41 & $1.77 \mathrm{E}-01$ \\
\hline $\mathrm{CO}-58$ & $3.67 E-05$ \\
\hline$C 0-60$ & $4.04 E-06$ \\
\hline $\mathrm{BR}-82$ & $4.55 E-05$ \\
\hline $\mathrm{KR}-85$ & $3.97 E+00$ \\
\hline $\mathrm{KR}-85 \mathrm{M}$ & $1.74 \mathrm{E}+00$ \\
\hline KR-87 & $7.17 \mathrm{E}-01$ \\
\hline $\mathrm{KR}-88$ & $2.03 E+00$ \\
\hline $\mathrm{RB}-88$ & $1.52 E-04$ \\
\hline SR-90 & $1.34 E-07$ \\
\hline$S B-124$ & $2.57 \mathrm{E}-05$ \\
\hline I - 131 & $2.97 E-03$ \\
\hline $\mathrm{XE}-131 \mathrm{M}$ & $8.14 E-01$ \\
\hline I-133 & $5.43 E-05$ \\
\hline$X E-133$ & $3.84 \mathrm{E}+02$ \\
\hline $\mathrm{XE}-133 \mathrm{M}$ & $3.06 E+00$ \\
\hline$X E-135$ & $3.12 E+01$ \\
\hline$X E-135 M$ & $6.14 E-01$ \\
\hline CS -138 & $1.48 E-06$ \\
\hline $\mathrm{CE}=141$ & $5.08 \mathrm{E}-07$ \\
\hline
\end{tabular}

TOTAL AIRBORNE TRITIUM RELEASE $3.59 \mathrm{E}+02$

TOTAL LIQUID TRITIUM RELEASE $0.00 E+00$

VOLUME OF LIQUTD WASTE RELEASED (PRIOR TO DILUTION) LITERS $0.00 E+00$

VOLUME OF DILUTION WATER USED DURING PERIOD LITERS $0.00 E+00$ 
EFFLUENT AND WASTE DISPOSAL ANNUAL REPORT FOR YEAR 1989 AIRBORNE AND LIQUID EFFLUENTS
UNIT NUMBER: 3 TYPE:PWR
LICENSEE:ARIZONA PUBLIC SERVICE CO. DOCKET NO. : $50-530$
LICENSED POWER (MWT) : $3.80 \mathrm{E}+03$
THERMAL POWER (MWH) :4.10E+06
NET ELECTRIC POWER (MWH) $: 1.33 \mathrm{E}+06$
COMMERCIAL OPERATION:01/08/88
INITIAL CRITICALITY: $10 / 25 / 87$
COOLING WATER SOURCE:SEWAGE TREATMENT
AIRBORNE EFFLUENTS
NUCLIDES RELEASED
AR-41
MN -54
ACTIVITY (CI)
$\mathrm{Co}-58$
$6.90 \mathrm{E}-01$
$\mathrm{CO}-60$
$1.13 \mathrm{E}-06$
$\mathrm{BR}-82$
$6.70 \mathrm{E}-05$
KR -85
$1.42 E-05$
$1.22 E-04$
$\mathrm{KR}-85 \mathrm{M}$
1. $76 \mathrm{E}+02$
$\mathrm{KR}-87$
1. $43 \mathrm{E}-01$
$\mathrm{KR}-88$
$5.03 E-02$
$\mathrm{RB}-88$
1.79E-01
1. $82 \mathrm{E}-02$
SR -89
$1.04 E-07$
SR -90
$3.48 \mathrm{E}-09$
SB -122
$8.73 E-06$
SB-124
3. $60 \mathrm{E}-04$
I-131
$6.00 E-03$
$\mathrm{XE}-131 \mathrm{M}$
$3.94 \mathrm{E}+00$
$I-132$
$4.12 \mathrm{E}-06$
I -133
8.64E-04
$\mathrm{XE}-133$
$6.39 \mathrm{E}+02$
$X E-133 M$
$2.83 \mathrm{E}+00$
CS -134
1.23E-06
$\mathrm{I}-135$
$3.57 \mathrm{E}-05$
$\mathrm{XE}-135$
$1.09 \mathrm{E}+01$
CS -137
$5.46 \mathrm{E}-06$
CS -138
$2.23 E-03$
$\mathrm{XE}-138$
$3.28 E-03$

TOTAL AIRBORNE TRITIUM RELEASE 1.83E+02

TOTAL LIQUID TRITIUM RELEASE $0.00 E+00$

$\begin{array}{llll}\text { VOLUME OF LIQUID WASTE RELEASED (PRIOR TO DILUTION) } & \text { LITERS } & 0.00 E+00 \\ \text { VOLUME OF DILUTION WATER USED DURING PERIOD } & \text { LITERS } & 0.00 \mathrm{E}+00\end{array}$ 
INSTALIATION:PALO VERDE $1,2 \& 3$

EFFLUENT AND WASTE DISPOSAL ANNUAL REPORT FOR YEAR 1989 SOLID EFFLUENTS

SOLID WASTE DISPOSITION

$\begin{array}{cl}\text { NUMBER OF SHIPMENTS MODE OF TRANSPORTATION } \\ 66 & \text { TRUCK }\end{array}$

DESTINATION

HANFORD WA

ESTIMATE OF MAJOR NUCLIDE COMPOSITION (BY TYPE OF WASTE)

JAN-JUNE JULY-DEC

A

AG-110M

$\mathrm{C}-14$

$\mathrm{CE}-144$

CO- 58

CO- 60

CR-51

CS -134

CS -137

FE- 55

FE- 59

$\mathrm{H}-3$

MN -54

NB -95

NI -63

PU $-239 / 240$

PU-241

RU -106

SB- 124

SB-125

SR -90

TC -99

ZR-95

B

AG-110M

C. 14

CE- 141

CE-144

CO- 58

CO- 60

CR - 51

CS -134

CS -137

FE -55

FE - 59

$\mathrm{H}-3$

I -131

MN- 54

NB-95

\begin{tabular}{|c|c|c|}
\hline & $7.98 \mathrm{E}-01$ & \\
\hline & $2.70 \mathrm{E}-01$ & 1. $42 E+00$ \\
\hline & $4.19 E-04$ & $5.12 \mathrm{E}-04$ \\
\hline$\frac{8}{8}$ & $6.21 E+00$ & $2.88 \mathrm{E}+01$ \\
\hline 8 & $1.08 \mathrm{E}+01$ & 1. $42 E+01$ \\
\hline 8 & $2.22 \mathrm{E}-01$ & $4.84 E-01$ \\
\hline 8 & $1.87 \mathrm{E}+01$ & $1.14 \mathrm{E}+01$ \\
\hline 8 & $2.60 E+01$ & $1.61 \mathrm{E}+01$ \\
\hline 8 & $1.50 \mathrm{E}+01$ & 1. $54 \mathrm{E}+01$ \\
\hline 8 & $1.44 \mathrm{E}-01$ & $3.28 \mathrm{E}-01$ \\
\hline 8 & $2.81 \mathrm{E}+00$ & $8.63 E-01$ \\
\hline 8 & $3.05 \mathrm{E}+00$ & $3.01 E+00$ \\
\hline 8 & $4.89 E-01$ & $1.09 E+00$ \\
\hline 8 & $2.31 E+00$ & $4.17 E+00$ \\
\hline 8 & $7.73 E-04$ & $4.25 E-05$ \\
\hline 8 & $2.70 E-02$ & $2.03 E-03$ \\
\hline 8 & $2.00 E-01$ & \\
\hline$\frac{8}{8}$ & 1. $21 E+01$ & $1.88 E+00$ \\
\hline 8 & $6.06 \mathrm{E}-0 \mathrm{I}$ & $3.55 E-02$ \\
\hline$\%$ & $9.75 \mathrm{E}-02$ & $2.74 \mathrm{E}-03$ \\
\hline$\%$ & $4.96 \mathrm{E}-05$ & $9.21 \mathrm{E}-05$ \\
\hline 8 & $3.02 \mathrm{E}-01$ & $7.05 \mathrm{E}-01$ \\
\hline 8 & $4.13 E-01$ & $8.87 \mathrm{E}-01$ \\
\hline \% & $3.53 \mathrm{E}-01$ & $7.29 \mathrm{E}-01$ \\
\hline$\frac{8}{8}$ & $9.52 \mathrm{E}-01$ & $2.70 \mathrm{E}-02$ \\
\hline 8 & $1.13 \mathrm{E}+00$ & $5.36 \mathrm{E}-01$ \\
\hline 욤 & $5.71 E+00$ & $7.95 E+00$ \\
\hline 8 & $8.66 \mathrm{E}+00$ & $4.50 E+00$ \\
\hline 8 & $9.38 E+00$ & $2.09 E+00$ \\
\hline 8 & $3.77 \mathrm{E}+00$ & $9.10 \mathrm{E}+00$ \\
\hline 8 & $1.06 \mathrm{E}+01$ & $2.32 E+0 I$ \\
\hline 8 & 3. $31 E+01$ & $1.47 \mathrm{E}+0$ \\
\hline 8 & $1.61 E+00$ & $5.31 \mathrm{E}-01$ \\
\hline 8 & $2.38 \mathrm{E}-01$ & $1.10 \mathrm{E}+00$ \\
\hline q & $2.70 \mathrm{E}-02$ & $1.04 \mathrm{E}+00$ \\
\hline 8 & $1.99 \mathrm{E}+00$ & $4.05 \mathrm{E}-01$ \\
\hline & $3.02 E+00$ & $3.36 \mathrm{E}+0$ \\
\hline
\end{tabular}


INSTALLATION:PALO VERDE $1,2 \& 3$

EFFLUENT AND WASTE DISPOSAL ANNUAL REPORT FOR YEAR 1989 SOLID EFFLUENTS

ESTIMATE OF MAJOR NUCLIDE COMPOSITION (BY TYPE OF WASTE)

JAN - JUNE JULY - DEC

B

NI -63

RU-103

SB-124

SR -90

TC -99

ZR-95

$\begin{array}{lll}8 & 5.78 \mathrm{E}-01 & 4.28 \mathrm{E}+00 \\ 8 & 1.72 \mathrm{E}+00 & 2.68 \mathrm{E}-01 \\ 8 & 1.57 \mathrm{E}+01 & 2.47 \mathrm{E}+01 \\ 8 & 3.00 \mathrm{E}-03 & 1.50 \mathrm{E}-02 \\ 8 & 1.00 \mathrm{E}-03 & 1.00 \mathrm{E}-03 \\ 8 & 1.20 \mathrm{E}+00 & 6.48 \mathrm{E}-01\end{array}$

TYPE OF WASTE

A. SPENT RESINS, FILTER SLUDGES, EVAPORATOR

UNIT YEAR TOTAL BOTTOMS, ETC.

M3 $5.01 \mathrm{E}+02$

CI $\quad 6.55 \mathrm{E}+02$

M3 $3.73 \mathrm{E}+02 \%$

B. DRY COMPRESSIBLE WASTE, CONTAMINATED

$3.73 \mathrm{E}+02$
$1.92 \mathrm{E}+01$

EQUIPMENT, ETC.

$\mathrm{CI}$

M3

C. IRRADIATED COMPONENTS, CONTROL

$\mathrm{CI}$

RODS, ETC.

D. OTHER

M3

CI

*AFTER VOLUME REDUCTION 
EFFLUENT AND WASTE DISPOSAL ANNUAL REPORT FOR YEAR 1989 AIRBORNE AND LIQUID EFFLUENTS

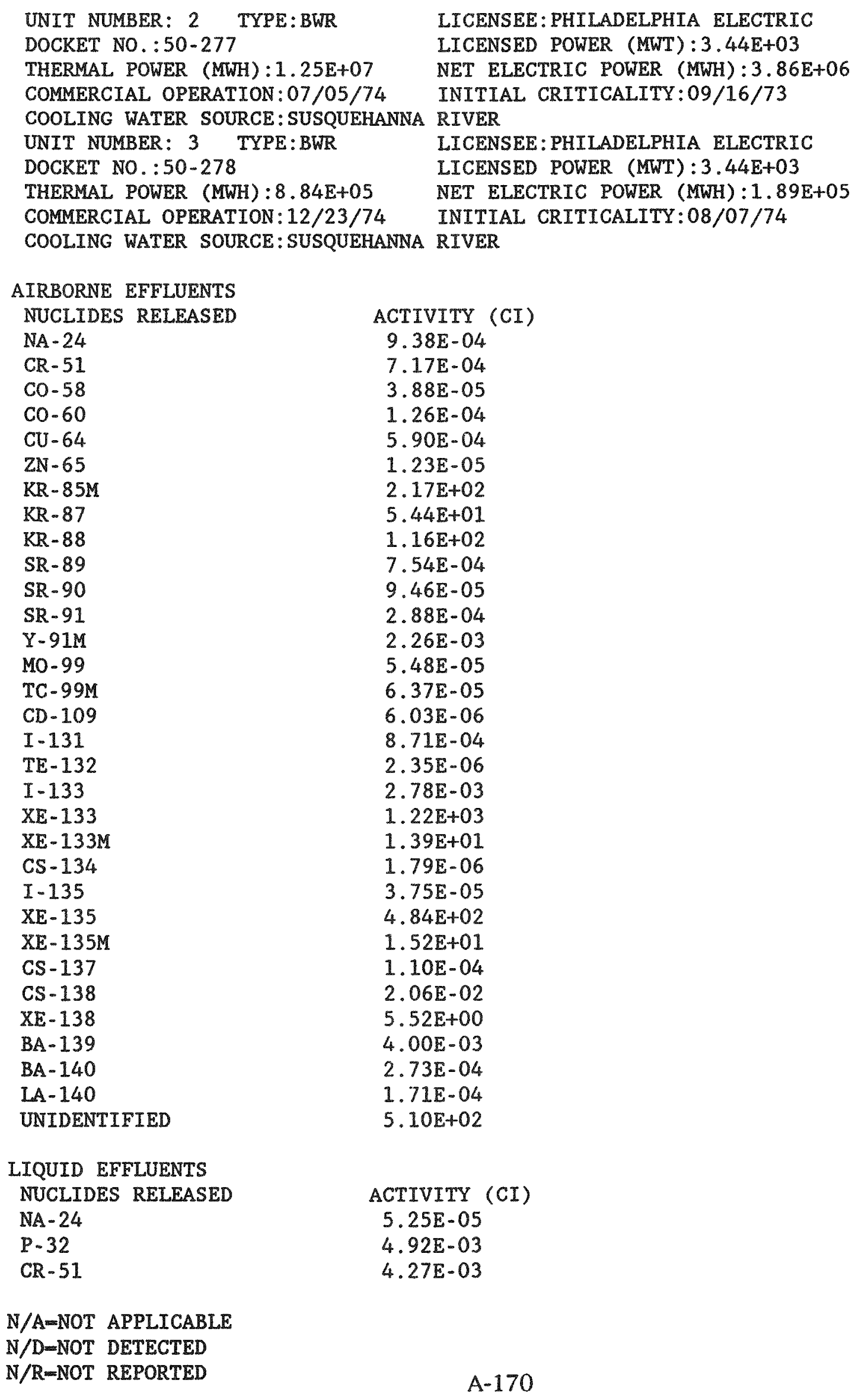


EFFLUENT AND WASTE DISPOSAL ANNUAL REPORT FOR YEAR 1989 AIRBORNE AND LIQUID EFFLUENTS

LIQUID EFFLUENTS
NUCLIDES RELEASED
MN-54
FE -55
CO-58
CO-60
ZN-65
SR-89
SR-90
SR-92
NB-95
MO-99
AG-110M
I-131
XE -133
CS -134
XE -135
XE -135 M
CS -137

LIQUID EFFLUENTS

NUCLIDES RELEASED

$\mathrm{MN}-54$

FE -55

$\mathrm{CO}-58$

SR- 89

SR -90

SR -92

NB -95

MO-99

AG-110M

$I-131$

$\mathrm{XE}-133$

CS-134

CS -137
ACTIVITY (CI)
4.12E-06
1. $23 E-02$
$9.05 \mathrm{E}-05$
1. $88 \mathrm{E}-02$
$3.29 E-03$
7.95E-04
$2.47 \mathrm{E}-04$
$5.53 \mathrm{E}-05$
1. $26 \mathrm{E}-04$
4.00E-06
1. $04 \mathrm{E}-03$
$6.68 \mathrm{E}-05$
$6.83 \mathrm{E}-03$
1. $83 \mathrm{E}-02$
8. $83 \mathrm{E}-03$
3. $32 \mathrm{E}-05$
4. $90 \mathrm{E}-02$

TOTAL AIRBORNE TRITIUM RELEASE $5.72 E+00$

TOTAL LIQUID TRITIUM RELEASE 2.00E+01

VOLUME OF LIQUID WASTE RELEASED (PRIOR TO DILUTION) LITERS 2.50E+07

VOLUME OF DILUTION WATER USED DURING PERIOD

LITERS $\quad 1.88 \mathrm{E}+11$ 
INSTALLATION : PEACH BOTTOM

EFFLUENT AND WASTE DISPOSAL ANNUAL REPORT FOR YEAR 1989 SOLID EFFLUENTS

SOLID WASTE DISPOSITION

NUMBER OF SHIPMENTS MODE OF TRANSPORTATION 238

$\mathrm{N} / \mathrm{R}$

DESTINATION

N/R

TYPE OF WASTE

A. SPENT RESINS, FILTER SLUDGES, EVAPORATOR

UNIT YEAR TOTAL BOTTOMS, ETC.

M3 $3.42 \mathrm{E}+02$

CI $\quad 8.04 \mathrm{E}+02$

B. DRY COMPRESSIBLE WASTE, CONTAMINATED

M3 4.95E+02 *

EQUIPMENT, ETC.

CI $\quad 2.34 \mathrm{E}+01$

C. IRRADIATED COMPONENTS, CONTROL

M3 1.63E +00 RODS, ETC.

CI $\quad 8.44 \mathrm{E}+02$

D. OTHER

M3 $5.37 \mathrm{E}+01$

MISC.

CI $\quad 6.35 \mathrm{E}+01$

*VOLUME AFTER REDUCTION 


\section{EFFLUENT AND WASTE DISPOSAL ANNUAL REPORT FOR YEAR 1989 AIRBORNE AND LIQUID EFFLUENTS}

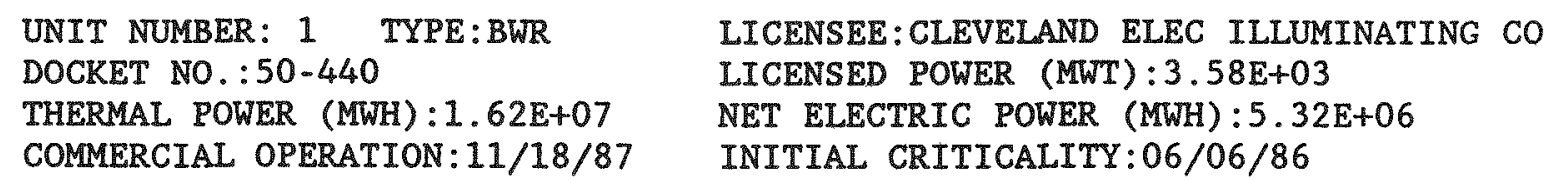

\section{LIQUID EFELUENTS}

NUCLIDES RELEASED

NA -24

SC -46

$\mathrm{CR}-51$

MN -54

FE -55

CO- 58

FE- 59

CO -60

$\mathrm{ZN}-65$

AS -76

SR -89

$\mathrm{SR}-92$

NB -95

ZR-95

TC-99M

AG-110M

SB -122

$S B-124$

SB-125

I -131

I -133

XE-133

CS -134

$\mathrm{XE}-135$

CS -137

$$
\begin{aligned}
& \text { ACTIVITY (CI) } \\
& 6.12 E-04 \\
& 4.31 E-05 \\
& 3.61 E-01 \\
& 1.92 E-01 \\
& 1.23 E-01 \\
& 3.73 E-02 \\
& 2.56 E-02 \\
& 3.75 E-01 \\
& 5.61 E-03 \\
& 6.41 E-04 \\
& 4.26 E-04 \\
& 6.31 E-04 \\
& 2.24 E-04 \\
& 3.96 E-05 \\
& 9.24 E-05 \\
& 1.87 E-02 \\
& 3.07 E-05 \\
& 3.74 E-04 \\
& 9.35 E-04 \\
& 1.10 E-03 \\
& 2.28 E-04 \\
& 9.20 E-03 \\
& 4.69 E-03 \\
& 2.26 E-03 \\
& 1.20 E-02
\end{aligned}
$$

$N / A=N O T$ APPLICABLE 
EFFLUENT AND WASTE DISPOSAL ANNUAL REPORT FOR YEAR 1989 AIRBORNE AND LIQUID EFFLUENTS

LIQUID EFFLUENTS

NUCLIDES RELEASED

LA -140

NP -239
ACTIVITY (CI)

$3.30 \mathrm{E}-03$

1. $30 \mathrm{E}-03$

TOTAL AIRBORNE TRITIUM RELEASE 0.00E +00

TOTAL LIQUID TRITIUM RELEASE $6.96 \mathrm{E}+00$

VOLUME OF LIQUID WASTE RELEASED (PRIOR TO DILUTION) LITERS $2.28 E+10$

VOLUME OF DILUTION WATER USED DURING PERIOD

LITERS $1.32 \mathrm{E}+11$ 
EFFLUENT AND WASTE DISPOSAL ANNUAL REPORT FOR YEAR 1989 SOLID EFFLUENTS

SOLID WASTE DISPOSITION

NUMBER OF SHIPMENTS 19

20
MODE OF TRANSPORTATION

TRUCK

TRUCK

ESTIMATE OF MAJOR NUCLIDE COMPOSITION (BY TYPE OF WASTE)

A

AG-110M

C. -14

$\mathrm{CO}-58$

$\mathrm{CO}-60$

CR -51

CS -137

FE -59

$\mathrm{H}-3$

MN- 54

NB -95

NI -63

SB-124

SR -90

TC- 99

$\mathrm{ZN}-65$

B

AG - $110 \mathrm{M}$

C -14

Co- 58

CO -60

CR- 51

CS -137

FE- 55

FE- 59

H- 3

MN -54

NB-95

NI -63

TYPE OF WASTE

A. SPENT RESINS, FILTER SLUDGES, EVAPORATOR BOTTOMS, ETC.

B. DRY COMPRESSIBLE WASTE, CONTAMINATED EQUIPMENT, ETC.

C. IRRADIATED COMPONENTS, CONTROL RODS, ETC.

D. OTHER

*VOLUME BEFORE COMPACTION

JAN-JUNE 2.54E+2 M3 SENT FOR COMPACTION; 8 TO 1 RED. FACTOR EXPECTED JULY-DEC 3.62E+2 M3 SENT FOR COMPACTION; 6 TO 1 RED. FACTOR EXPECTED

\section{DESTINATION \\ BARNWELL SC \\ RICHLAND WA}

$$
\text { JAN-JUNE JULY-DEC }
$$

$\begin{array}{lll}8 & 2.51 \mathrm{E}+00 & 2.72 \mathrm{E}+00 \\ 8 & 2.87 \mathrm{E}-01 & 1.49 \mathrm{E}-01 \\ 8 & 4.47 \mathrm{E}+00 & 3.26 \mathrm{E}+00 \\ 8 & 3.47 \mathrm{E}+01 & 4.24 \mathrm{E}+01 \\ 8 & 6.35 \mathrm{E}+00 & 1.56 \mathrm{E}+01 \\ 8 & 1.50 \mathrm{E}+00 & 6.27 \mathrm{E}-01 \\ 8 & 2.94 \mathrm{E}+00 & 7.59 \mathrm{E}+00 \\ 8 & 3.03 \mathrm{E}-01 & 3.53 \mathrm{E}-01 \\ 8 & 2.47 \mathrm{E}+01 & 1.64 \mathrm{E}+01 \\ 8 & 1.84 \mathrm{E}+01 & 7.70 \mathrm{E}+00 \\ 8 & 3.16 \mathrm{E}-01 & 7.52 \mathrm{E}-01 \\ 8 & 1.39 \mathrm{E}+00 & 1.08 \mathrm{E}+00 \\ 8 & 4.00 \mathrm{E}-03 & 4.00 \mathrm{E}-03 \\ 8 & 6.00 \mathrm{E}-03 & \\ 8 & 1.38 \mathrm{E}+00 & \\ & & \\ 8 & 1.03 \mathrm{E}+00 & 1.11 \mathrm{E}+00 \\ 8 & 1.00 \mathrm{E}-03 & 1.00 \mathrm{E}-03 \\ 8 & 1.54 \mathrm{E}+01 & 1.45 \mathrm{E}+01 \\ 8 & 1.26 \mathrm{E}+01 & 1.48 \mathrm{E}+01 \\ 8 & 3.39 \mathrm{E}+01 & 2.24 \mathrm{E}+01 \\ 8 & 7.90 \mathrm{E}-02 & 8.90 \mathrm{E}-02 \\ 8 & & 1.12 \mathrm{E}+01 \\ 8 & 4.48 \mathrm{E}+00 & 4.39 \mathrm{E}+00 \\ 8 & 2.00 \mathrm{E}-03 & 2.00 \mathrm{E}-03 \\ 8 & 2.93 \mathrm{E}+01 & 2.85 \mathrm{E}+01 \\ 8 & 2.24 \mathrm{E}+00 & 2.00 \mathrm{E}+00 \\ 8 & 1.37 \mathrm{E}-01 & 1.52 \mathrm{E}-01\end{array}$

UNIT YEAR TOTAL

$\begin{array}{ll}\text { M3 } & 1.39 \mathrm{E}+02 \\ \text { CI } & 8.95 \mathrm{E}+02 \\ \text { M3 } & 8.29 \mathrm{E}+02 \text { * } \\ \text { CI } & 2.25 \mathrm{E}+01 \\ \text { M3 } & \\ \text { CI } & \\ \text { M3 } & \\ \text { CI } & \end{array}$


EFFLUENT AND WASTE DISPOSAL ANNUAL REPORT FOR YEAR 1989 AIRBORNE AND LIQUID EFFLUENTS

\begin{tabular}{|c|c|}
\hline \multicolumn{2}{|l|}{ UNIT NUMBER: 1 TYPE:BWR } \\
\hline \multicolumn{2}{|r|}{ LICENSED POWER (MWT) $: 2.00 E+03$} \\
\hline \multicolumn{2}{|r|}{ NET ELECTRIC POWER (MWH): $1.71 \mathrm{E}+06$} \\
\hline \multicolumn{2}{|c|}{ COMMERCIAL OPERATION: $12 / 01 / 72$ INITIAL CRITICALITY:06/16/72 } \\
\hline \multicolumn{2}{|c|}{ COOLING WATER SOURCE: CAPE COD BAY } \\
\hline \multicolumn{2}{|l|}{ AIRBORNE EFFLUENTS } \\
\hline NUCLIDES RELEASED & ACTIVITY (CI) \\
\hline $\mathrm{CO}-60$ & $2.82 E-05$ \\
\hline $\mathrm{KR}-85 \mathrm{M}$ & $1.11 E+02$ \\
\hline $\mathrm{KR}-87$ & $7.54 E+01$ \\
\hline $\mathrm{KR}-88$ & $1.78 \mathrm{E}+02$ \\
\hline SR -89 & $2.47 E-04$ \\
\hline$S R-90$ & $2.05 E-06$ \\
\hline I-131 & $5.05 E-03$ \\
\hline$I-133$ & $3.16 E-02$ \\
\hline$X E-133$ & $7.96 E+01$ \\
\hline$I-135$ & $1.08 \mathrm{E}-02$ \\
\hline$X E-135$ & 1. $70 E+02$ \\
\hline $\mathrm{XE}-135 \mathrm{M}$ & $1.15 \mathrm{E}+01$ \\
\hline CS -137 & $9.32 E-06$ \\
\hline$X E-138$ & $5.17 E+01$ \\
\hline $\mathrm{BA} / \mathrm{LA}-140$ & $2.86 E-04$ \\
\hline \multicolumn{2}{|l|}{ LIQUID EFFLUENTS } \\
\hline NÜCLIDES RELEASED & ACTIVITY (CI) \\
\hline CR -51 & $5.68 E-04$ \\
\hline$M N-54$ & $3.74 E-04$ \\
\hline$F E-55$ & $9.47 E-03$ \\
\hline $\mathrm{CO}-58$ & $6.68 E-04$ \\
\hline$F E-59$ & $2.11 E-06$ \\
\hline$C 0-60$ & $8.65 E-03$ \\
\hline SR -89 & $4.57 E-05$ \\
\hline$S R-90$ & $4.26 E-05$ \\
\hline MO-99-TC-99M & $1.28 E-06$ \\
\hline $\mathrm{RH}-105$ & $2.78 E-05$ \\
\hline SB -122 & $4.58 E-07$ \\
\hline$I-131$ & $6.30 E-05$ \\
\hline$X E-133$ & $1.01 E-05$ \\
\hline $\operatorname{CS}-134$ & $2.26 E-05$ \\
\hline$X E-135$ & $1.24 E-04$ \\
\hline $\operatorname{CS}-137$ & $3.86 E-03$ \\
\hline BA/LA- 140 & $1.07 \mathrm{E}-03$ \\
\hline$C E-141$ & $9.60 E-07$ \\
\hline$C E-P R-144$ & $3.86 \mathrm{E}-06$ \\
\hline$H F-181$ & $3.93 E-06$ \\
\hline
\end{tabular}


EFFLUENT AND WASTE DISPOSAL ANNUAL REPORT FOR YEAR 1989 AIRBORNE AND LIQUID EFFLUENTS

TOTAL AIRBORNE TRITIUM RELEASE $4.85 \mathrm{E}+00$

TOTAL LIQUID TRITIUM RELEASE $2.37 \mathrm{E}+00$

VOLUME OF LIQUID WASTE RELEASED (PRIOR TO DILUTION) LITERS 2.73E+06

VOLUME OF DILUTION WATER USED DURING PERIOD

LITERS $\quad 6.33 \mathrm{E}+09$ 
EFFLUENT AND WASTE DISPOSAL ANNUAL REPORT FOR YEAR 1989 SOLID EFFLUENTS

SOLID WASTE DISPOSITION

$\begin{array}{lrl}\text { NUMBER OF SHIPMENTS } & \text { MODE OF TRANSPORTATION } \\ * & 2 & \text { TRACTOR-TRAILER } \\ & 21 & \text { TRACTOR - TRAILER } \\ * & 3 & \text { TRACTOR - TRAILER } \\ * & 16 & \text { TRACTOR - TRAILER }\end{array}$

*SHIPPED TO WASTE PROCESSOR FOR VOL. RED. BEFORE BURIAL

IRRADIATED FUEL SHIPMENTS (DISPOSITION)

NUMBER OF SHIPMENTS MODE OF TRANSPORTATION

0

$\mathrm{N} / \mathrm{A}$

ESTIMATE OF MAJOR NUCLIDE COMPOSITION (BY TYPE OF WASTE)

A

$\mathrm{BA}-140$

C -14

CM- 242

$\mathrm{CO}-58$

CO -60

CR- 51

CS -134

CS -137

FE -55

H-3

I -129

LA -140

$\mathrm{MN}-54$

NI -63

PU -241

SR -90

TC -99

TC- $99 \mathrm{M}$

TRU

B

C -14

CM- 242

CO -58

CO- 60

CS -134

CS -137

FE -55

$\mathrm{H}-3$

I -129

$\mathrm{MN}-54$
DESTINATION

ALARON-WAMPUM PA

BARNWELL SC

QUADREX-OAK RIDGE TN

SEG-OAK RIDGE TN

\section{DESTINATION}

N/A

JAN-JUNE JULY-DEC

$<1.00 E-02$

3. 10E- 02

$<1.00 \mathrm{E}-02$

$5.00 \mathrm{E}-03$

9. $70 \mathrm{E}-01$

$<1.00 \mathrm{E}-03$

3. $83 \mathrm{E}+01$

4. $17 \mathrm{E}+00$

4.07E+01

$4.85 \mathrm{E}+00$

9. 70E-01

$6.10 \mathrm{E}-01$

3. $29 \mathrm{E}+01$

2. $58 \mathrm{E}+01$

2. $12 \mathrm{E}+01$

1. $76 \mathrm{E}+01$

3. $00 \mathrm{E}-02$

1. $60 \mathrm{E}-02$

$<1.00 E-02$

1. $00 \mathrm{E}-03$

$2.89 \mathrm{E}+00$

3. 10E-02

$1.94 \mathrm{E}+00$

$2.97 \mathrm{E}+00$

2. $00 \mathrm{E}-02$

2. $70 \mathrm{E}+00$

$5.00 \mathrm{E}-02$

9. 20E-02

$<1.00 \mathrm{E}-02$

$2.99 E-01$

1. $00 \mathrm{E}-03$

$7.50 \mathrm{E}-02$

$<1.00 \mathrm{E}-02$

4. $00 \mathrm{E}-03$

$2.72 \mathrm{E}-01$

$9.00 \mathrm{E}-03$

$8.40 \mathrm{E}-02$

3. $38 \mathrm{E}+01$

1. $22 \mathrm{E}+00$

$3.05 E+01$

$2.73 \mathrm{E}+01$

2. $50 \mathrm{E}+00$

$9.00 \mathrm{E}-03$

1. $38 \mathrm{E}+00$ 
EFFLUENT AND WASTE DISPOSAL ANNUAL REPORT FOR YEAR 1989 SOLID EFFLUENTS

ESTIMATE OF MAJOR NUCLIDE COMPOSITION (BY TYPE OF WASTE)

$$
\text { JAN-JUNE JULY-DEC }
$$

B

NI -63

PU -241

SR -90

TC -99

TRU

$\begin{array}{ll}8 & 2.06 \mathrm{E}+00 \\ 8 & 4.33 \mathrm{E}-01 \\ 8 & 4.06 \mathrm{E}-01 \\ 8 & 3.80 \mathrm{E}-02 \\ 8 & 9.00 \mathrm{E}-03\end{array}$

TYPE OF WASTE

A. SPENT RESINS, FILTER SLUDGES, EVAPORATOR BOTTOMS, ETC.

B. DRY COMPRESSIBLE WASTE, CONTAMINATED EQUIPMENT, ETC.

C. IRRADIATED COMPONENTS, CONTROL RODS, ETC.

D. OTHER

UNIT YEAR TOTAL

M3 $1.25 E+02$

CI $\quad 2.75 \mathrm{E}+02$

M3 7.66E+01 \#

CI $\quad 6.11 \mathrm{E}-01$

M3

CI

M3

CI

\#VOLUME AFTER REDUCTION 
EFFLUENT AND WASTE DISPOSAL ANNUAL REPORT FOR YEAR 1989 AIRBORNE AND LIQUID EFFLUENTS

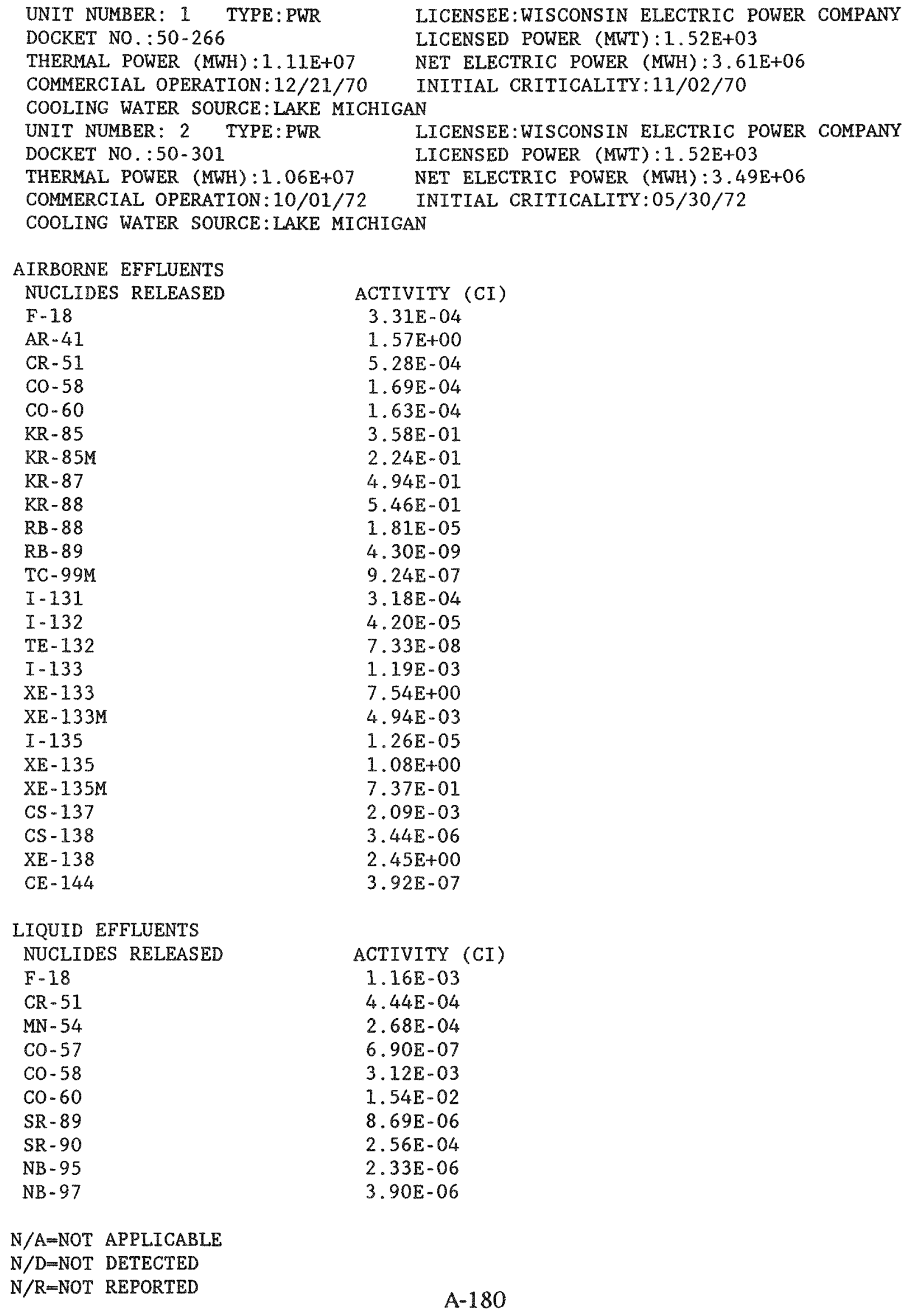

$N / A=N O T$ APPLICABLE 
EFFLUENT AND WASTE DISPOSAL ANNUAL REPORT FOR YEAR 1989 AIRBORNE AND LIQUID EFFLUENTS

LIQUID EFFLUENTS
NUCLIDES RELEASED
AG-110M
SB -125
$I-131$
$I-132$
$I-133$
CS $-134 M$
CS -137
CE -144
ACTIVITY (CI)
4. $70 \mathrm{E}-04$
2.12E-02
1. $77 \mathrm{E}-03$
$8.15 \mathrm{E}-04$
8. $11 E-03$
$5.97 \mathrm{E}-06$
2.80E-03
$1.59 \mathrm{E}-05$

TOTAL AIRBORNE TRITIUM RELEASE $1.42 \mathrm{E}+02$

TOTAL LIQUID TRITIUM RELEASE $5.59 \mathrm{E}+02$

VOLUME OF LIQUID WASTE RELEASED (PRIOR TO DILUTION) LITERS $1.82 E+08$

VOLUME OF DILUTION WATER USED DURING PERIOD

LITERS $3.60 \mathrm{E}+11$ 
INSTALLATION : POINT BEACH

EFFLUENT AND WASTE DISPOSAL ANNUAL REPORT FOR YEAR 1989 SOLID EFFLUENTS

SOLID WASTE DISPOSITION

NUMBER OF SHIPMENTS MODE OF TRANSPORTATION

27

$N / R$

DESTINATION

BARNWELL SC

TYPE OF WASTE
A. SPENT RESINS, FILTER SLUDGES, EVAPORATOR BOTTOMS, ETC.
B. DRY COMPRESSIBLE WASTE, CONTAMINATED EQUTPMENT, ETC.
C. IRRADIATED COMPONENTS, CONTROL RODS, ETC.
D. OTHER

UNIT YEAR TOTAL

M3 1.22E+01

CI $\quad 2.53 E+02$

M3 $9.35 E+01$

CI $\quad 1.54 \mathrm{E}+00$

M3

CI

M3

CI 
EFFLUENT AND WASTE DISPOSAL ANNUAL REPORT FOR YEAR 1989 AIRBORNE AND LIQUID EFFLUENTS
UNIT NUMBER: 1 TYPE:PWR
LICENSEE:NORTHERN STATES POWER
DOCKET NO : :50-282
LICENSED POWER (MWT) : $1.65 \mathrm{E}+03$
THERMAL POWER (MWH) $: 1.40 \mathrm{E}+07$
NET ELECTRIC POWER (MWH) $: 4.39 \mathrm{E}+06$
COMMERCIAL OPERATION: $12 / 16 / 73$
COOLING WATER SOURCE:MISSTS
UNIT NUMBER: 2 TYPE:PWR
DOCKET NO.:50-306
INITIAL CRITICALITY: $12 / 01 / 73$
RIVER
THERMAL POWER (MWH) : $1.25 E+07$
LICENSEE:NORTHERN STATES POWER
COMMERCIAL OPERATION : $12 / 21 / 74$
COOLING WATER SOURCE:MISSISSIPPI RIVER
LICENSED POWER (MWT) : $1.65 E+03$
NET ELECTRIC POWER (MWH) :3.89E+06
INITIAL CRITICALITY: $12 / 17 / 74$
AIRBORNE EFFLUENTS
NUCLIDES RELEASED
AR -41
$\mathrm{CO}-60$
ACTIVITY (CI)
$\mathrm{KR}-85$
$6.31 E-02$
$2.06 E-06$
$\mathrm{KR}-85 \mathrm{M}$
$2.10 \mathrm{E}-01$
SR -89
$2.37 E-03$
SR -90
$1.37 \mathrm{E}-07$
CD-109
$2.02 E-07$
I-131
9.17E-06
$\mathrm{XE}-131 \mathrm{M}$
4. $67 \mathrm{E}-06$
$\mathrm{XE}-133$
$\mathrm{XE}-133 \mathrm{M}$
$2.40 \mathrm{E}-02$
1. $69 \mathrm{E}+02$
$\mathrm{XE}-135$
$4.55 E-03$
CS -137
$3.64 \mathrm{E}+00$
LIQUID EFFLUENTS
NUCLIDES RELEASED
NA -24
AR -41
4. $71 \mathrm{E}-06$
SC -47
CR -51
ACTIVITY (CI)
MN -54
1.16E-06
$3.09 E-06$
1.18E-04
$5.55 E-04$
$1.03 \mathrm{E}-04$
FE- 55
1. $48 \mathrm{E}-01$
$\mathrm{CO}-58$
2. $72 \mathrm{E}-03$
$\mathrm{FE}-59$
2.13E-03
CO- 60
2. $39 \mathrm{E}-03$
$\mathrm{KR}-85 \mathrm{M}$
$5.58 \mathrm{E}-06$
SR -92
8. $90 \mathrm{E}-06$
NB- 95
3. 10E-05
NB -97
7. $48 \mathrm{E}-05$
ZR-97
3. $38 \mathrm{E}-04$
TC-99M
$9.37 \mathrm{E}-06$
$A G-110 M$
7. $39 \mathrm{E}-03$
$\mathrm{SN}-113$
$1.03 \mathrm{E}-03$
$\mathrm{SB}-122$
3. $58 \mathrm{E}-05$
SB -124
3. $02 \mathrm{E}-03$
SB -125
4. $20 \mathrm{E}-03$
I-131
2. $71 E-04$
$\mathrm{XE}-131 \mathrm{M}$
$6.28 \mathrm{E}-04$

$N / A=N O T$ APPLICABLE 
EFFLUENT AND WASTE DISPOSAL ANNUAL REPORT FOR YEAR 1989 AIRBORNE AND LIQUID EFFLUENTS

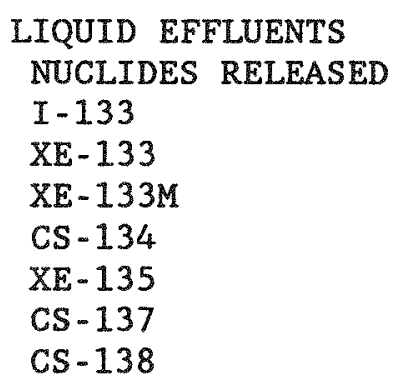
ACTIVITY (CI)
8.11E-05
4. 50E-02
$3.07 E-04$
$9.74 \mathrm{E}-08$
$6.28 \mathrm{E}-04$
$1.02 E-05$
2.28E-05

TOTAL AIRBORNE TRITIUM RELEASE $1.18 \mathrm{E}+02$

TOTAL LIQUID TRITIUM RELEASE $4.64 E+02$

VOLUME OF LIQUID WASTE RELEASED (PRIOR TO DILUTION) IITERS $1.48 E+08$

VOLUME OF DILUTION WATER USED DURING PERIOD 
INSTALLATION:PRAIRIE ISLAND

EFFLUENT AND WASTE DISPOSAL ANNUAL REPORT FOR YEAR 1989 SOLID EFFLUENTS

SOLID WASTE DISPOSITION NUMBER OF SHIPMENTS

$\begin{aligned} 1 & \text { TRUCK } \\ 2 & \text { TRUCK } \\ 2 & \text { TRUCK } \\ 14 & \text { TRUCK }\end{aligned}$

ESTIMATE OF MAJOR NUCLIDE COMPOSITION (BY TYPE OF WASTE)

A

$\mathrm{C}-14$

CO- 60

CS -134

CS -137

FE -55

$\mathrm{H}-3$

NI -63

B

AG-110M

C. 14

$\mathrm{CO}-58$

CO- 60

CS -134

CS -137

FE $=55$

$\mathrm{H}-3$

NB-95

NI -63

$S B-125$
MODE OF TRANSPORTATION

RUCK

TRUCK

RUCK

\author{
DESTINATION \\ BARNWELL SC \\ OAK RIDGE TN \\ QUADREX RECYCLE CENTER \\ RICHLAND WA
}

JAN - JUNE JULY-DEC

$\begin{array}{lll}8 & & 9.50 \mathrm{E}-01 \\ 8 & & 4.05 \mathrm{E}+01 \\ 8 & & 2.60 \mathrm{E}+00 \\ 8 & & 8.96 \mathrm{E}+00 \\ 8 & & 2.38 \mathrm{E}+01 \\ 8 & & 1.35 \mathrm{E}+00 \\ 8 & & 2.18 \mathrm{E}+01 \\ 8 & & \\ & & \\ 8 & 2.00 \mathrm{E}+00 & 2.35 \mathrm{E}-03 \\ 8 & 6.80 \mathrm{E}+01 & 1.08 \mathrm{E}+01 \\ 8 & 7.00 \mathrm{E}-01 & 8.23 \mathrm{E}-04 \\ 8 & 6.40 \mathrm{E}+00 & 2.63 \mathrm{E}+01 \\ 8 & & 1.30 \mathrm{E}+00 \\ 8 & 3.00 \mathrm{E}-01 & 3.80 \mathrm{E}+00 \\ 8 & 1.02 \mathrm{E}+01 & 4.16 \mathrm{E}+01 \\ 8 & 8.50 \mathrm{E}+00 & 2.91 \mathrm{E}+00 \\ 8 & & 1.10 \mathrm{E}+00 \\ 8 & 2.40 \mathrm{E}+00 & 9.89 \mathrm{E}+00 \\ 8 & 8.00 \mathrm{E}-01 & 9.40 \mathrm{E}-04\end{array}$

UNIT YEAR TOTAL

M3 3.71E+01

CI $\quad 6.52 \mathrm{E}+01$

M3 8.82E+01 *

CI $\quad 3.75 \mathrm{E}+01$

M3

CI

M3

CI

*VOLUME INCLUDES BOTH NON-COMPACTED(JAN-JUNE) AND COMPACTED(JULY-DEC) WASTE. 


\section{EFFLUENT AND WASTE DISPOSAL ANNUAL REPORT FOR YEAR 1989} AIRBORNE AND LIQUID EFFLUENTS

\author{
UNIT NUMBER: 1 TYPE:BWR \\ DOCKET NO. : 50-254 \\ THERMAL POWER (MWH) : $1.40 \mathrm{E}+07$ \\ COMMERCIAL OPERATION:02/18/73 \\ COOLING WATER SOURCE:MISSISSIPPI \\ UNIT NUMBER: 2 TYPE:BWR \\ DOCKET NO.: 50-265 \\ THERMAL POWER (MWH) $: 1.85 \mathrm{E}+07$
}

COMMERCIAL OPERATION:03/10/73

COOLING WATER SOURCE:MISSISSIPPI
LICENSEE: COMMONWEALTH EDISON CO. LICENSED POWER (MWT) $: 2.51 \mathrm{E}+03$ NET ELECTRIC POWER (MWH) :4.28E+06 INITIAL CRITICALITY: 10/18/71 RIVER

LICENSEE: COMMONWEALTH EDISON CO. LICENSED POWER (MWT) : $2.51 E+03$ NET ELECTRIC POWER (MWH) :5.74E+06 INITIAL CRITICALITY:04/26/72

\section{AIRBORNE EFFLUENTS \\ NUCLIDES RELEASED \\ CR -51 \\ MN -54 \\ $\mathrm{CO}-58$ \\ CO -60 \\ $\mathrm{ZN}-65$ \\ $\mathrm{KR}-85 \mathrm{M}$ \\ $\mathrm{KR}-87$ \\ $\mathrm{KR}-88$ \\ SR -89 \\ SR -90 \\ MO-99 \\ AG-110M \\ I -131 \\ I -133 \\ XE -133 \\ CS -134 \\ I- 135 \\ $\mathrm{XE}-135$ \\ $X E-135 M$ \\ CS -137 \\ $\mathrm{XE}-138$ \\ $\mathrm{BA}-140$ \\ IA -140}

\section{LIQUID EFFLUENTS}

NUCLIDES RELEASED

NA -24

CR -51

MN -54

FE -55

CO- 58

FE- 59

CO- 60

$\mathrm{ZN}-65$

SR -89

SR -90

MO-99

$A G-110 M$

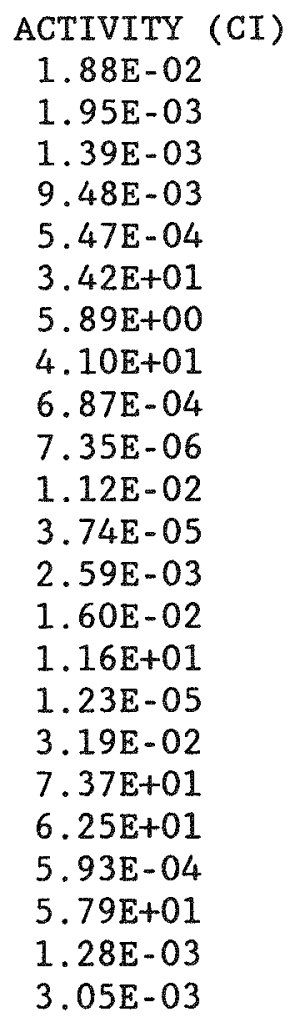

$5.79 E+01$

1. $28 \mathrm{E}-03$

$3.05 E-03$

ACTIVITY (CI)

5.82E-04

3. $05 \mathrm{E}-02$

4. $26 \mathrm{E}-02$

3. $81 E-02$

$8.52 \mathrm{E}-03$

$3.35 E-03$

3. $51 \mathrm{E}-01$

5. $51 E-04$

$8.40 \mathrm{E}-04$

$1.02 \mathrm{E}-04$

8. 39E-05

$8.74 E-04$

$N / A=N O T$ APPLICABLE

$N / D=N O T$ DETECTED

$N / R=N O T$ REPORTED 
EFFLUENT AND WASTE DISPOSAL ANNUAL REPORT FOR YEAR 1989 AIRBORNE AND LIQUID EFFLUENTS

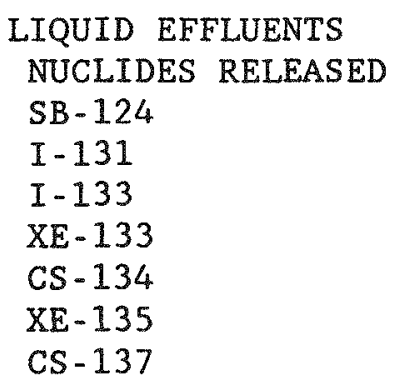

TOTAL AIRBORNE TRITIUM RELEASE 7.82E+01 TOTAL LIQUID TRITIUM RELEASE
ACTIVITY (CI)
$1.14 \mathrm{E}-05$
1. $10 \mathrm{E}-04$
$3.25 \mathrm{E}-05$
$9.28 \mathrm{E}-04$
1. $18 \mathrm{E}-04$
$1.36 \mathrm{E}-03$
$6.31 \mathrm{E}-03$

VOLUME OF LIQUID WASTE RELEASED (PRIOR TO DILUTION) VOLUME OF DILUTION WATER USED DURING PERIOD
LITERS

LITERS
$6.11 \mathrm{E}+06$

$1.58 \mathrm{E}+12$ 
EFFLUENT AND WASTE DISPOSAL ANNUAL REPORT FOR YEAR 1989 SOLID EFFLUENTS

SOLID WASTE DISPOSITION NUMBER OF SHIPMENTS

6
7
59
7
14

7

59

7

14
MODE OF TRANSPORTATION

A J METLER

A J METLER

CNSI

CNSI

HITTMAN
DESTINATION

SEG

U.S. ECOLOGY

BARNWELL SC

U.S. ECOLOGY

SEG

UNIT YEAR TOTAL

M3 $9.79 \mathrm{E}+02$

CI 1.33E+05

M3

CI

M3

$\mathrm{CI}$

M3

CI 
EFFLUENT AND WASTE DISPOSAL ANNUAL REPORT FOR YEAR 1989 AIRBORNE AND LIQUID EFFLUENTS

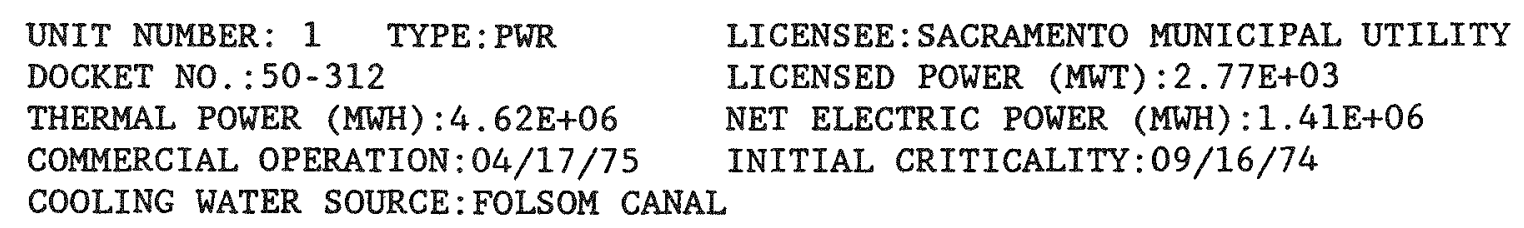

TOTAL AIRBORNE TRITIUM RELEASE 3.75E+01 TOTAL LIQUID TRITIUM RELEASE $7.29 \mathrm{E}+01$

$\begin{array}{llll}\text { VOLUME OF LIQUID WASTE RELEASED (PRIOR TO DILUTION) } & \text { LITERS } & 5.46 E+07 \\ \text { VOLUME OF DIIUTION WATER USED DURING PERIOD } & \text { LITERS } & 1.91 E+10\end{array}$ 
SOLID WASTE DISPOSITION

NUMBER OF SHIPMENTS MODE OF TRANSPORTATION

22

TRUCK(SOLE USE VEHICLE)

ESTIMATE OF MAJOR NUCLIDE COMPOSITION (BY TYPE OF WASTE)

A

$\mathrm{C}-14$

CO -58

$\mathrm{CO}-60$

CS -134

CS -137

FE -55

$\mathrm{H}-3$

$\mathrm{MN}-54$

NI -63

SB -125

SR -90

$B$

AG-110M

C -14

CE -141

CE -144

CO -58

Co- 60

CR -51

CS -134

CS -137

FE-55

$\mathrm{H}-3$

MN -54

NB-95

NI -63

SB- 125

$T E-125 \mathrm{M}$

ZR-95

\section{DESTINATION \\ RICHLAND WA}

JAN-JUNE JULY-DEC

$3.66 \mathrm{E}-01 \quad 1.18 \mathrm{E}-01$

$6.20 \mathrm{E}+00 \quad 1.70 \mathrm{E}+00$

$1.29 \mathrm{E}+01 \quad 3.32 \mathrm{E}+00$

$5.33 \mathrm{E}+01 \quad 1.34 \mathrm{E}+01$

$1.43 \mathrm{E}+01 \quad 1.59 \mathrm{E}+00$

$7.09 \mathrm{E}+01$

3. $34 \mathrm{E}-01$

1. $24 \mathrm{E}+01$

$3.20 E+00$

$2.79 \mathrm{E}-01$

1. $33 E-01$

$1.11 E+00$

1. $73 \mathrm{E}-01$

3. $42 \mathrm{E}-01$

1. $58 \mathrm{E}-01$

8.91E-01

$2.46 \mathrm{E}+01$

$6.38 \mathrm{E}+00$

$2.73 \mathrm{E}-01$

$6.13 \mathrm{E}+00$

2. $39 E+01$

1. $81 \mathrm{E}+01$

7. 29E-01

1. $26 \mathrm{E}-01$

1. $52 \mathrm{E}+00$

$1.43 \mathrm{E}+01$

$5.26 \mathrm{E}-01$

$1.16 \mathrm{E}-01$

7. $68 \mathrm{E}-01$

$7.08 \mathrm{E}+00$

$2.58 \mathrm{E}+00$

1. $13 \mathrm{E}+01$

7. $57 \mathrm{E}+00$

$5.90 \mathrm{E}+01$

1. $13 \mathrm{E}-01$

1. $12 \mathrm{E}+01$

3. $13 \mathrm{E}-01$

UNIT YEAR TOTAL

TYPE OF WASTE

A. SPENT RESINS, FILTER SLUDGES, EVAPORATOR BOTTOMS, ETC.

B. DRY COMPRESSIBLE WASTE, CONTAMINATED EQUIPMENT, ETC.

M3 $2.10 \mathrm{E}+02$

CI $\quad 2.83 \mathrm{E}+02$

M3 $\quad 3.48 \mathrm{E}+01$

CI $\quad 4.44 \mathrm{E}+01$

C. IRRADIATED COMPONENTS, CONTROL RODS, ETC.

D. OTHER
M3

CI

M3

CI 


\section{EFFLUENT AND WASTE DISPOSAL ANNUAL REPORT FOR YEAR 1989 AIRBORNE AND LIQUID EFFLUENTS}

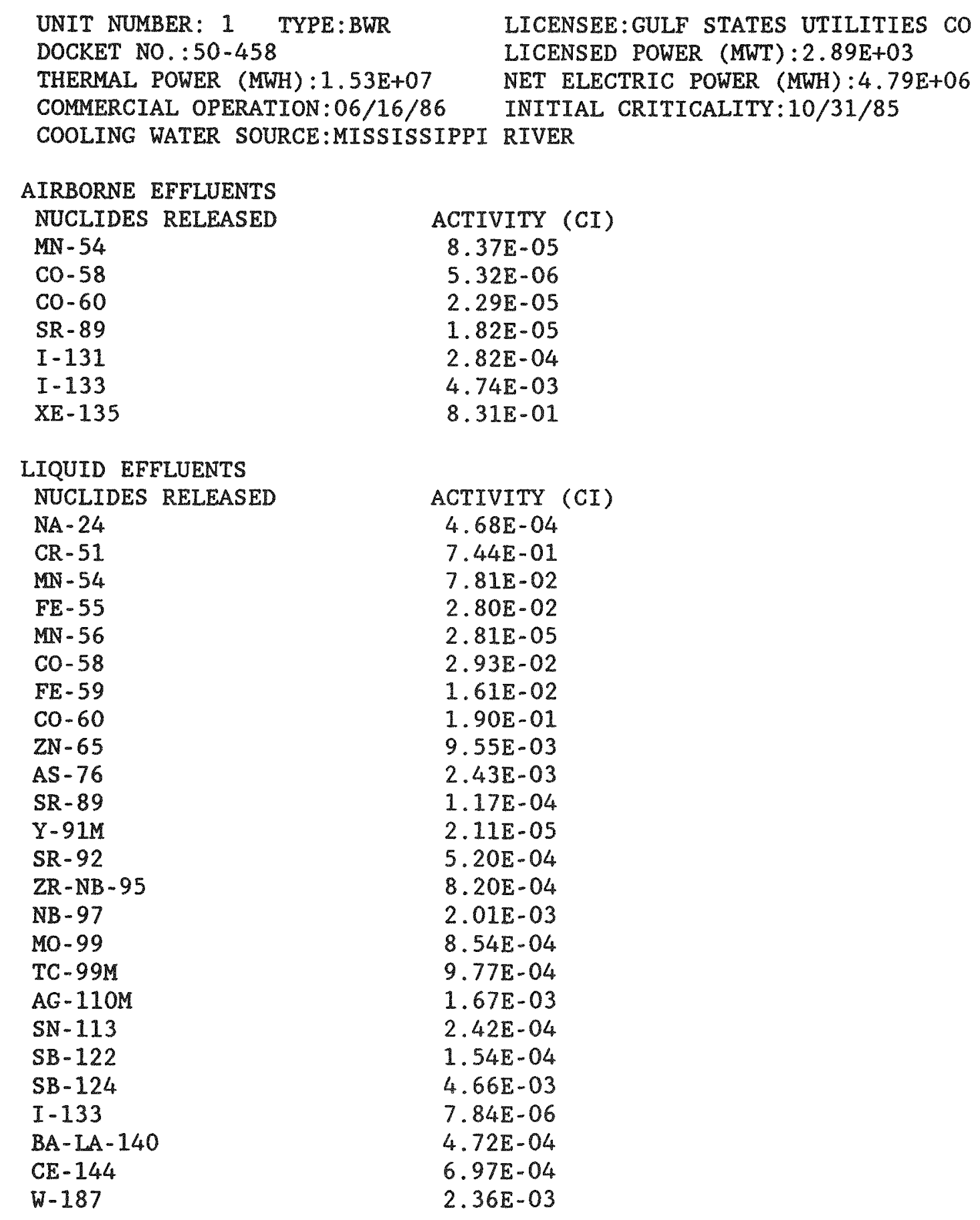

TOTAL AIRBORNE TRITIUM RELEASE 5.82E+00

TOTAL LIQUID TRITIUM RELEASE 1.60E+01

VOLUME OF LIQUID WASTE RELEASED (PRIOR TO DILUTION) LITERS 2.98E+07

VOLUME OF DILUTION WATER USED DURING PERIOD LITERS $4.88 \mathrm{E}+09$ 
INSTALLATION:RIVER BEND

EFFLUENT AND WASTE DISPOSAL ANNUAL REPORT FOR YEAR 1989

SOLID EFFLUENTS

SOLID WASTE DISPOSITION NUMBER OF SHTPMENTS 67

MODE OF TRANSPORTATION TRUCK EXCLUSIVE USE

IRRADIATED FUEL SHIPMENTS (DISPOSITION)

NUMBER OF SHIPMENTS MODE OF TRANSPORTATION

0

N/A

ESTIMATE OF MAJOR NUCLIDE COMPOSITION (BY TYPE OF WASTE)

A

$A G-110 \mathrm{M}$

AM -241

$\mathrm{C}-14$

CM-242

CM- 244

$\mathrm{CO}-57$

$\mathrm{CO}-58$

CO- 60

CR -51

FE -55

FE- 59

$\mathrm{H}-3$

I -131

LA - 140

MN -54

MO- 99

NA- 24

NB -95

NI -63

PU -238

PU - 239

PU -241

SB -122

SB -124

SC -46

$\mathrm{SN}-113$

SR -89

$\mathrm{ZN}-65$

ZR-95

B

AM- 241

C. 14

CM- 242
DESTINATION

CNSI-BARNWELL SC

DESTINATION

N/A

JAN-JUNE JULY-DEC

\begin{tabular}{|c|c|c|}
\hline 8 & $4.88 \mathrm{E}-02$ & $5.33 \mathrm{E}-02$ \\
\hline & $2.46 E-04$ & $3.11 \mathrm{E}-04$ \\
\hline & $1.59 \mathrm{E}-01$ & $7.17 \mathrm{E}-02$ \\
\hline & $3.34 E-04$ & $3.95 \mathrm{E}-0<$ \\
\hline & $2.86 \mathrm{E}-04$ & $3.76 \mathrm{E}-04$ \\
\hline & $1.85 \mathrm{E}-02$ & $7.65 \mathrm{E}-0$ \\
\hline & $5.48 \mathrm{E}+00$ & $1.83 \mathrm{E}+0$ \\
\hline 8 & $3.03 E+01$ & $3.06 \mathrm{E}+0$ \\
\hline 8 & 1. $38 \mathrm{E}+01$ & $1.16 \mathrm{E}+0$ \\
\hline 8 & $2.70 \mathrm{E}+01$ & $5.22 E+0$ \\
\hline$\frac{8}{8}$ & $3.49 E-01$ & $5.37 \mathrm{E}-0$ \\
\hline 8 & $1.71 \mathrm{E}-02$ & $1.07 \mathrm{E}-0$ \\
\hline 8 & $9.42 E-04$ & \\
\hline 8 & $3.68 \mathrm{E}-07$ & \\
\hline 8 & $1.89 \mathrm{E}+01$ & 1. $15 E+0$ \\
\hline 8 & $4.09 E-05$ & $9.35 E-0$ \\
\hline 8 & $4.48 E-27$ & 3. $34 E-1$ \\
\hline 8 & $4.00 \mathrm{E}-02$ & $5.02 \mathrm{E}-03$ \\
\hline 8 & $8.02 E-01$ & $5.07 E-01$ \\
\hline 8 & $5.42 E-04$ & $6.85 \mathrm{E}-0$ \\
\hline 8 & $3.97 E-04$ & $5.02 E-0$ \\
\hline 8 & $1.42 \mathrm{E}-02$ & 1. $78 \mathrm{E}-\mathrm{C}$ \\
\hline 8 & $7.04 E-07$ & \\
\hline 8 & $9.20 \mathrm{E}-02$ & $2.60 \mathrm{E}-0$ \\
\hline 8 & & $1.33 \mathrm{E}-0$ \\
\hline 8 & $7.16 \mathrm{E}-04$ & \\
\hline & $5.02 \mathrm{E}-01$ & \\
\hline 8 & $2.46 \mathrm{E}+00$ & $1.93 \mathrm{E}+0$ \\
\hline & $2.46 \mathrm{E}-02$ & $2.06 \mathrm{E}-0$ \\
\hline & $2.90 \mathrm{E}-03$ & \\
\hline & $1.26 E+00$ & $8.40 E-0$ \\
\hline & $4.43 E-03$ & \\
\hline
\end{tabular}


INSTALLATION:RIVER BEND

EFFLUENT AND WASTE DISPOSAL ANNUAL REPORT FOR YEAR 1989

SOLID EFFLUENTS

ESTIMATE OF MAJOR NUCLIDE COMPOSITION (BY TYPE OF WASTE)

JAN-JUNE JULY-DEC

B

CM -244

CO -58

CO- 60

CR -51

FE -55

FE -59

MN -54

NB -95

NI -63

PU -239

PU -241

$\mathrm{ZN}-65$

\section{TYPE OF WASTE}
A. SPENT RESINS, FILTER SLUDGES, EVAPORATOR BOTTOMS, ETC.
B. DRY COMPRESSIBLE WASTE, CONTAMINATED EQUIPMENT, ETC.
C. IRRADIATED COMPONENTS, CONTROL RODS, ETC.
D. OTHER

$\begin{array}{lll}8 & 3.51 \mathrm{E}-03 & \\ 8 & 2.97 \mathrm{E}+00 & 5.00 \mathrm{E}-01 \\ 8 & 4.59 \mathrm{E}+01 & 2.99 \mathrm{E}+01 \\ 8 & 2.68 \mathrm{E}+00 & 5.00 \mathrm{E}-02 \\ 8 & 1.61 \mathrm{E}+01 & 5.86 \mathrm{E}+01 \\ 8 & 1.23 \mathrm{E}+00 & \\ 8 & 2.13 \mathrm{E}+01 & 7.16 \mathrm{E}+00 \\ 8 & 4.48 \mathrm{E}-01 & \\ 8 & 9.54 \mathrm{E}-01 & 5.50 \mathrm{E}-01 \\ 8 & 4.68 \mathrm{E}-03 & \\ 8 & 1.70 \mathrm{E}-01 & 6.00 \mathrm{E}-02 \\ 8 & 6.97 \mathrm{E}+00 & 2.32 \mathrm{E}+00\end{array}$

UNIT YEAR TOTAL

M3 $3.22 \mathrm{E}+02$

CI $\quad 8.36 \mathrm{E}+02$

M3 $1.94 \mathrm{E}+02$

CI $\quad 4.57 \mathrm{E}+00$

M3

$\mathrm{CI}$

M3

CI 
EFFLUENT AND WASTE DISPOSAL ANNUAL REPORT FOR YEAR 1989 AIRBORNE AND LIQUID EFFLUENTS

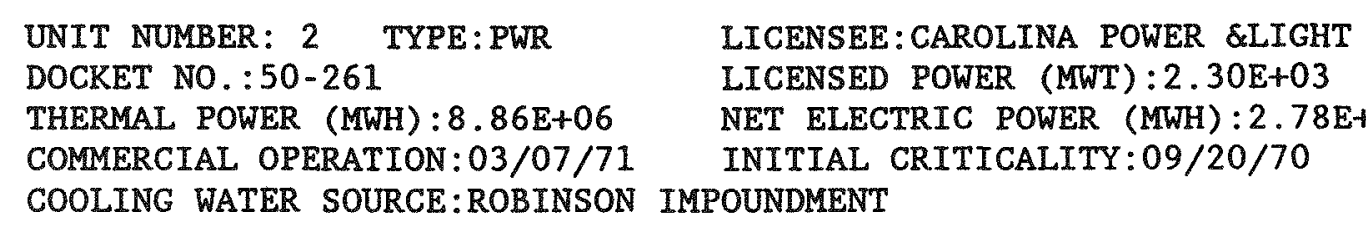


EFFLUENT AND WASTE DISPOSAL ANNUAL REPORT FOR YEAR 1989 AIRBORNE AND LIQUID EFFLUENTS

\section{LIQUID EFFLUENTS}

NUCLIDES RELEASED

ACTIVITY (CI)

SB- 125

$X E-127$

$9.54 \mathrm{E}-02$

$\mathrm{I}-131$

$3.33 E-05$

$\mathrm{TE}-132$

1. $57 \mathrm{E}-04$

$\mathrm{XE}-133$

$2.74 E-05$

$X E-133 M$

3.67E-02

CS -134

$2.04 \mathrm{E}-04$

$X E-135$

$2.20 \mathrm{E}-03$

CS -136

CS -137

$1.37 E-04$

$2.64 \mathrm{E}-05$

$4.28 \mathrm{E}-03$

TOTAL AIRBORNE TRITIUM RELEASE $1.68 \mathrm{E}+01$

TOTAL LIQUID TRITIUM RELEASE $1.64 \mathrm{E}+02$

$\begin{array}{lllll}\text { VOLUME OF LIQUID WASTE RELEASED (PRIOR TO DILUTION) } & \text { LITERS } & 3.65 E+06 \\ \text { VOLUME OF DILUTION WATER USED DURING PERTOD } & \text { LITERS } & 7.40 E+11\end{array}$ 
EFFLUENT AND WASTE DISPOSAL ANNUAL REPORT FOR YEAR 1989 SOLID EFFLUENTS

SOLID WASTE DISPOSITION NUMBER OF SHIPMENTS MODE OF TRANSPORTATION 47

IRRADIATED FUEL SHIPMENTS (DISPOSITION)

NUMBER OF SHIPMENTS MODE OF TRANSPORTATION 0 N/A

DESTINATION

N/A

ESTIMATE OF MAJOR NUCLIDE COMPOSITION (BY TYPE OF WASTE) JAN-JUNE JULY-DEC

A $\mathrm{CO}-58$ CO- -60 $\mathrm{CR}-51$ CS -134 CS -137 FE -55 $\mathrm{MN}-54$ NB -95 NI -63

$B$ OTHERS

$\begin{array}{lll}8 & 1.37 \mathrm{E}+00 & 3.86 \mathrm{E}+00 \\ 8 & 5.12 \mathrm{E}+00 & 1.80 \mathrm{E}+01 \\ 8 & 1.97 \mathrm{E}+00 & \\ 8 & 3.20 \mathrm{E}+01 & 1.50 \mathrm{E}+01 \\ 8 & 4.88 \mathrm{E}+01 & 2.82 \mathrm{E}+01 \\ 8 & 8.60 \mathrm{E}+00 & 7.19 \mathrm{E}+00 \\ 8 & 3.34 \mathrm{E}-01 & 4.24 \mathrm{E}+00 \\ 8 & 3.15 \mathrm{E}-01 & \\ 8 & 8.00 \mathrm{E}-01 & 2.32 \mathrm{E}+01 \\ 8 & 6.87 \mathrm{E}-01 & 1.76 \mathrm{E}-01 \\ & & \\ 8 & & 8.50 \mathrm{E}+00 \\ 8 & 4.88 \mathrm{E}+01 & 3.27 \mathrm{E}+01 \\ 8 & 3.33 \mathrm{E}+01 & 4.57 \mathrm{E}+01 \\ 8 & 1.19 \mathrm{E}+00 & 1.30 \mathrm{E}+00 \\ 8 & 1.55 \mathrm{E}+01 & 1.02 \mathrm{E}+01 \\ 8 & 1.17 \mathrm{E}+00 & 1.60 \mathrm{E}+00\end{array}$

\section{TYPE OF WASTE}

A. SPENT RESINS, FILTER SLUDGES, EVAPORATOR

UNIT YEAR TOTAL BOTTOMS, ETC.

$\begin{array}{ll}\text { M3 } & 1.59 E+01 \\ \text { CI } & 1.82 E+02 \\ \text { M3 } & 8.10 E+01 \\ \text { CI } & 4.29 E+00 \\ \text { M3 } & \\ \text { CI } & \\ \text { M3 } & \\ \text { CI } & \end{array}$

*AFTER VOLUME REDUCTION 
EFFLUENT AND WASTE DISPOSAL ANNUAL REPORT FOR YEAR 1989 AIRBORNE AND LIQUID EFFLUENTS

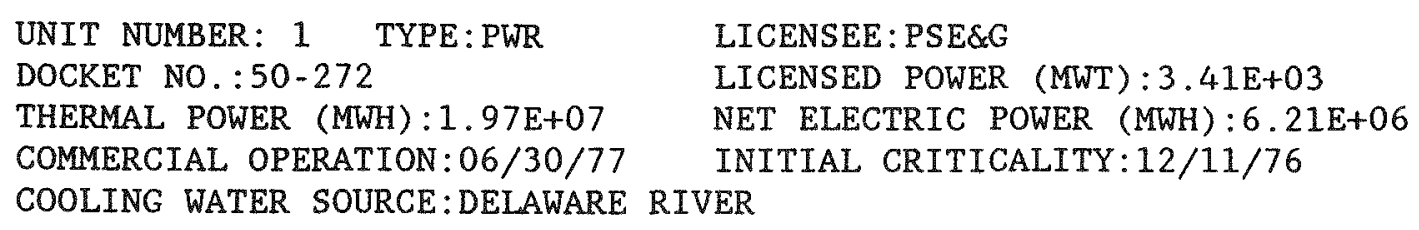

\section{LIQUID EFFLUENTS}

\section{NUCLIDES RELEASED}

F-18

NA -24

CR -51

$\mathrm{MN}-54$

FE- 55

CO- 57

CO- 58

FE -59

CO -60

$\mathrm{ZN}-65$

$\mathrm{BR}-82$

$\mathrm{KR}-85$

SR -89

SR -90

SR -92

NB-95

NB-95M

ZR-95

NB -97

TC- $99 \mathrm{M}$

RU -103

AG-110M

SN $-113 \mathrm{M}$

ACTIVITY (CI)

SB- 122

4. 35E-01

4. $70 \mathrm{E}-04$

5. 25E-03

1. $12 \mathrm{E}-01$

1. $75 \mathrm{E}-01$

$6.12 \mathrm{E}-03$

1. $82 \mathrm{E}+00$

1. $32 \mathrm{E}-03$

1. $78 \mathrm{E}-01$

3. $63 \mathrm{E}-04$

$1.80 \mathrm{E}-05$

1. $52 \mathrm{E}-04$

1. $54 \mathrm{E}-03$

6. $86 \mathrm{E}-04$

2. $30 \mathrm{E}-05$

3. $85 \mathrm{E}-03$

7. $15 E-05$

1. $53 \mathrm{E}-03$

7. $94 \mathrm{E}-05$

4. $63 \mathrm{E}-04$

3. $58 \mathrm{E}-05$

$2.69 \mathrm{E}-03$

$4.16 \mathrm{E}-05$

$3.46 \mathrm{E}-03$

1. $36 \mathrm{E}-02$

SB-124

6. 52E-02

$\mathrm{N} / \mathrm{A}=\mathrm{NOT}$ APPLICABLE 
EFFLUENT AND WASTE DISPOSAL ANNUAL REPORT FOR YEAR 1989 AIRBORNE AND LIQUID EFFLUENTS

\author{
LIQUTD EFFLUENTS \\ NUCLIDES RELEASED \\ I- 131 \\ $\mathrm{XE}-131$ \\ $I-133$ \\ TE-133M \\ $\mathrm{XE}-133$ \\ $\mathrm{XE}-133 \mathrm{M}$ \\ CS -134 \\ $I-135$ \\ $\mathrm{XE}-135$ \\ CS -136 \\ CS -137 \\ LA -140 \\ CE -144
}
ACTIVITY (CI)
2.99E-02
$3.09 \mathrm{E}-03$
$6.88 \mathrm{E}-03$
$6.36 \mathrm{E}-05$
$6.45 E-01$
$4.64 \mathrm{E}-03$
$1.16 \mathrm{E}-01$
1.94E-04
9.16E-03
$9.79 \mathrm{E}-04$
1. $28 \mathrm{E}-01$
$2.66 \mathrm{E}-04$
$1.19 \mathrm{E}-04$

TOTAL AIRBORNE TRITIUM RELEASE $1.99 \mathrm{E}+02$

TOTAL LIQUTD TRITIUM RELEASE $6.09 \mathrm{E}+02$

$\begin{array}{llll}\text { VOLUME OF LIQUID WASTE RELEASED (PRIOR TO DILUTION) } & \text { LITERS } & 1.16 E+07 \\ \text { VOLUME OF DILUTION WATER USED DURING PERIOD } & \text { LITERS } & 6.24 E+11\end{array}$ 
EFFLUENT AND WASTE DISPOSAL ANNUAL REPORT FOR YEAR 1989 AIRBORNE AND LIQUID EFFLUENTS

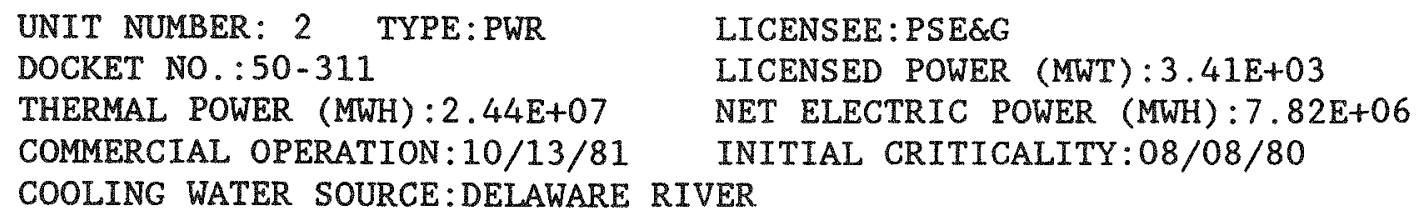

\title{
AIRBORNE EFFLUENTS
}

NUCLIDES RELEASED

AR - 41

CR -51

MN -54

CO- 58

$\mathrm{CO}-60$

$\mathrm{KR}-85$

KR $-85 \mathrm{M}$

KR -87

$\mathrm{KR}-88$

NB -95

ZR-95

I-131.

XE-131M

$X E-133$

$\mathrm{XE}-133 \mathrm{M}$

$X E-135$

$\mathrm{XE}-135 \mathrm{M}$

CS -137

\author{
ACTIVITY (CI) \\ 4. $32 E-03$ \\ 2.76E-05 \\ 1. $13 \mathrm{E}-06$ \\ 4. $76 \mathrm{E}-05$ \\ $6.69 \mathrm{E}-05$ \\ $1.46 \mathrm{E}-01$ \\ $5.74 \mathrm{E}-01$ \\ $1.07 \mathrm{E}-03$ \\ $1.08 \mathrm{E}+00$ \\ $5.95 \mathrm{E}-04$ \\ $6.41 \mathrm{E}-04$ \\ 8.07E-05 \\ 1. $34 \mathrm{E}-01$ \\ 4. $57 \mathrm{E}+01$ \\ 2. $13 \mathrm{E}-01$ \\ $2.08 \mathrm{E}+01$ \\ 4. $31 \mathrm{E}+00$ \\ 5. $30 \mathrm{E}-06$
}

\section{LIQUID EFFLUENTS}

NUCLIDES RELEASED

F-18

NA -24

CR -51

MN -54

FE -55

CO- 57

$\mathrm{CO}-58$

FE- 59

$\mathrm{CO}-60$

$\mathrm{ZN}-65$

$\mathrm{KR}-88$

SR -89

SR -90

SR -92

NB -95

ZR-95

NB -97

TC-99M

AG-110M

ACTIVITY (CI)

$5.34 \mathrm{E}-01$

$8.08 E-04$

1. $57 \mathrm{E}-02$

1. $18 \mathrm{E}-01$

$1.89 \mathrm{E}-01$

$6.70 \mathrm{E}-03$

$2.02 \mathrm{E}+00$

$3.00 \mathrm{E}-03$

$2.08 \mathrm{E}-01$

$1.41 E-04$

7. $35 \mathrm{E}-05$

$1.51 E-03$

$6.46 \mathrm{E}-04$

$3.11 E-05$

7. $41 E-03$

$3.40 \mathrm{E}-03$

2. $54 \mathrm{E}-04$

6. 64E-04

$6.42 \mathrm{E}-03$

SB-122

3. $58 \mathrm{E}-03$

SB -124

1. $89 \mathrm{E}-02$

SB-125

8.09E-02

$N / A=N O T$ APPLICABLE

$N / D=N O T$ DETECTED

$N / R=N O T$ REPORTED 
EFFLUENT AND WASTE DISPOSAL ANNUAL REPORT FOR YEAR 1989 AIRBORNE AND LIQUID EFFLUENTS

LIQUID EFFLUENTS
NUCLIDES RELEASED
I-131
XE $-131 M$
$I-133$
XE-133
XE $-133 M$
CS -134
I- 135
XE -135
CS -136
CS -137
LA -140
CE -143
CE-144
PR -147
HG -203
ACTIVITY (CI)
3. $80 \mathrm{E}-02$
$3.56 \mathrm{E}-03$
$8.64 \mathrm{E}-03$
$7.08 \mathrm{E}-01$
$6.02 \mathrm{E}-03$
$1.42 \mathrm{E}-01$
$5.17 \mathrm{E}-04$
1. $00 \mathrm{E}-02$
1. $39 \mathrm{E}-03$
$1.55 E-01$
$5.19 \mathrm{E}-04$
$4.92 \mathrm{E}-05$
$6.04 \mathrm{E}-04$
$2.56 \mathrm{E}-04$
$2.81 E-05$

TOTAL AIRBORNE TRITIUM RELEASE $2.18 \mathrm{E}+02$

TOTAL LIQUID TRITIUM RELEASE $5.11 E+02$

VOLUME OF LIQUID WASTE RELEASED (PRIOR TO DILUTION) LITERS $9.03 E+06$

VOLUME OF DILUTION WATER USED DURING PERIOD

LITERS $\quad 5.68 \mathrm{E}+11$ 
EFFLUENT AND WASTE DISPOSAL ANNUAL REPORT FOR YEAR 1989 SOLID EFFLUENTS

SOLID WASTE DISPOSITION NUMBER OF SHIPMENTS 43

MODE OF TRANSPORTATION TRUCK

ESTIMATE OF MAJOR NUCLIDE COMPOSITION (BY TYPE OF WASTE)

A

$\mathrm{CE}-144$

CO- 58

Co- 60

CS -134

CS -137

FE -55

FE -59

$\mathrm{H}-3$

MN -54

NI -63

SB- 125

B

CE -144

$\mathrm{CO}-58$

$\mathrm{CO}-60$

CS -134

CS -137

FE -55

H -3

MN -54

NI -63

PU -241
DESTINATION

BARNWELL SC

JAN-JUNE JULY-DEC

$\begin{array}{lll}8 & & 4.00 \mathrm{E}-01 \\ 8 & 4.00 \mathrm{E}-01 & 1.95 \mathrm{E}+01 \\ 8 & 2.84 \mathrm{E}+01 & 3.19 \mathrm{E}+01 \\ 8 & & 6.70 \mathrm{E}+00 \\ 8 & & 9.30 \mathrm{E}+00 \\ 8 & & 3.70 \mathrm{E}+00 \\ 8 & 6.41 \mathrm{E}+01 & 2.00 \mathrm{E}-01 \\ 8 & & \\ 8 & 1.20 \mathrm{E}+00 & \\ 8 & 3.50 \mathrm{E}+00 & 3.00 \mathrm{E}+00 \\ 8 & 2.30 \mathrm{E}+00 & 2.46 \mathrm{E}+01 \\ 8 & & 7.00 \mathrm{E}-01 \\ 8 & & \\ & & 2.00 \mathrm{E}-01 \\ 8 & & 5.66 \mathrm{E}+01 \\ 8 & & 9.40 \mathrm{E}+00 \\ 8 & & 2.10 \mathrm{E}+00 \\ 8 & & 4.20 \mathrm{E}+00 \\ 8 & & 1.57 \mathrm{E}+01 \\ 8 & & 1.00 \mathrm{E}-01 \\ 8 & & 1.20 \mathrm{E}+00 \\ 8 & & 9.10 \mathrm{E}+00 \\ 8 & & 1.30 \mathrm{E}+00\end{array}$

UNIT YEAR TOTAL

M3 $3.44 \mathrm{E}+01$

CI $1.14 \mathrm{E}+03$

M3 8.70E +01

CI $\quad 7.47 \mathrm{E}+00$

M3 $1.05 \mathrm{E}+00$

CI $5.54 E+04$

M3 $0.00 E+00$

CI $\quad 0.00 E+00$ 
EFFLUENT AND WASTE DISPOSAL ANNUAL REPORT FOR YEAR 1989 AIRBORNE AND LIQUID EFFLUENTS

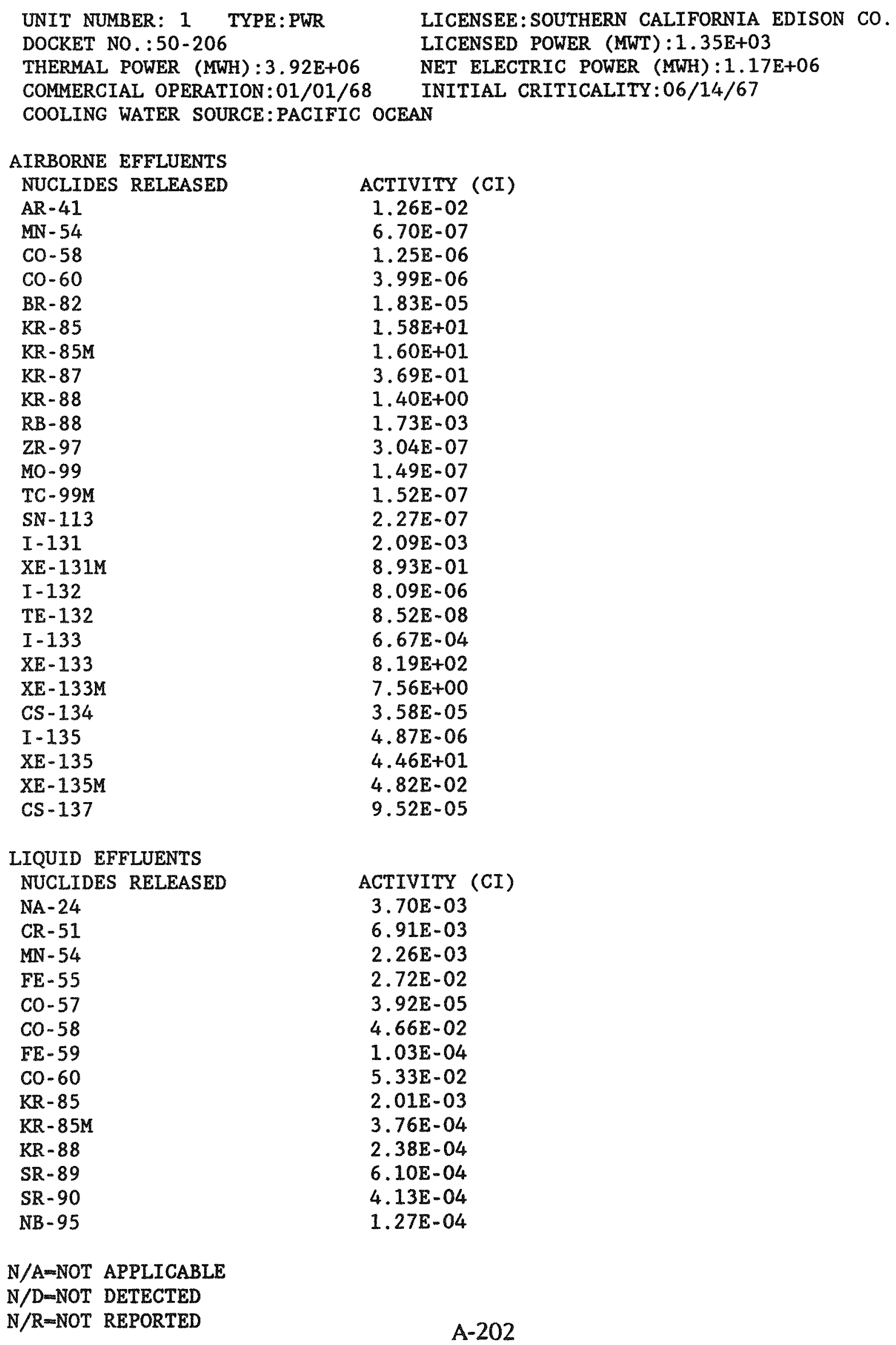


EFFLUENT AND WASTE DISPOSAL ANNUAL REPORT FOR YEAR 1989 AIRBORNE AND LIQUID EFFLUENTS

\section{LIQUID EFFLUENTS}

NUCLIDES RELEASED

MO-99

TC $-99 \mathrm{M}$

$\mathrm{RU}-103$

$\mathrm{RU}-106$

$A G-110 \mathrm{M}$

$S B-124$

SB -125

I- 131

$\mathrm{XE}-131 \mathrm{M}$

I -133

$\mathrm{XE}-133$

$\mathrm{XE}-133 \mathrm{M}$

CS -134

I- 135

$\mathrm{XE}-135$

CS -136

CS -137

BA- 140

LA -140

CE -141

CE- 143

CE -144
ACTIVITY (CI)

$3.75 \mathrm{E}-04$

3. $83 \mathrm{E}-04$

1. $89 \mathrm{E}-04$

$7.80 E-04$

$1.97 \mathrm{E}-04$

3. $33 \mathrm{E}-03$

2. $21 E-03$

1. $33 \mathrm{E}-02$

1. $24 \mathrm{E}-02$

1. $63 \mathrm{E}-02$

1. $42 \mathrm{E}-01$

4. $29 \mathrm{E}-03$

2.03E-01

3. $80 \mathrm{E}-04$

2. $63 \mathrm{E}-02$

2. $96 \mathrm{E}-03$

3. $00 \mathrm{E}-01$

2. $29 \mathrm{E}-06$

8. $66 \mathrm{E}-04$

$6.57 \mathrm{E}-04$

2. $64 \mathrm{E}-05$

1. $50 \mathrm{E}-03$

TOTAL AIRBORNE TRITIUM RELEASE 3.37E+01

TOTAL LIQUID TRITIUM RELEASE $9.62 E+02$

VOLUME OF LIQUID WASTE RELEASED (PRIOR TO DILUTION) LITERS 9.17E+06

VOLUME OF DILUTION WATER USED DURING PERIOD

LITERS $\quad 3.67 \mathrm{E}+11$ 
INSTALLATION: SAN ONOFRE 1

EFFLUENT AND WASTE DISPOSAL ANNUAL REPORT FOR YEAR 1989 SOLID EFFLUENTS

ESTIMATE OF MAJOR NUCLIDE COMPOSITION (BY TYPE OF WASTE)

JAN-JUNE JULY-DEC

A

AM -241

C. -14

CE-144

CM-242

$\mathrm{CO}-57$

Co- 58

Co- 60

CS -134

CS -137

$F E-55$

FE- 59

H -3

I -129

MN -54

NI -59

NI -63

PU -238

PU -241

SB-124

SR -89

SR -90

TC -99

$\mathrm{ZN}-65$

ZR-95

B

C. -14

Co- 58

$\mathrm{CO}-60$

CS -134

CS -137

FE- 55

$\mathrm{H}-3$

I -129

$\mathrm{MN}-54$

NI -63

PU-241

SR -90

TC -99

D

AG-110M

AM- 241

C- 14

CE-141

\begin{tabular}{|c|c|c|}
\hline 8 & $9.63 E-04$ & \\
\hline 8 & $8.16 \mathrm{E}-05$ & $1.46 \mathrm{E}-06$ \\
\hline 8 & $1.33 E-03$ & \\
\hline 8 & $1.48 \mathrm{E}-03$ & $2.51 E-03$ \\
\hline 8 & $7.65 E-02$ & $4.70 E-02$ \\
\hline 8 & $1.97 E+01$ & $4.73 E+00$ \\
\hline 8 & $1.57 E+01$ & $1.16 \mathrm{E}+01$ \\
\hline 8 & $2.30 E+01$ & 3. $71 E+01$ \\
\hline 8 & $2.97 E+01$ & $3.90 E+01$ \\
\hline 8 & $4.10 E+00$ & $3.02 E+00$ \\
\hline 8 & $3.70 E-02$ & \\
\hline 8 & $6.29 E-02$ & $8.15 \mathrm{E}-02$ \\
\hline 8 & $4.41 E-05$ & $1.25 E-04$ \\
\hline 8 & $2.21 \mathrm{E}+00$ & $7.53 \mathrm{E}-01$ \\
\hline 8 & $2.63 E-02$ & \\
\hline 8 & $5.18 \mathrm{E}+00$ & $3.50 \mathrm{E}+00$ \\
\hline 8 & $1.38 \mathrm{E}-03$ & \\
\hline 8 & $6.42 \mathrm{E}-02$ & $1.88 \mathrm{E}-02$ \\
\hline 8 & $1.42 \mathrm{E}-02$ & $7.96 \mathrm{E}-02$ \\
\hline 8 & $5.97 E-02$ & $5.56 \mathrm{E}-02$ \\
\hline 8 & $9.97 E-02$ & $1.04 E-01$ \\
\hline 8 & $5.09 E-05$ & $3.99 E-05$ \\
\hline 8 & & $1.68 E-03$ \\
\hline 8 & $2.11 \mathrm{E}-03$ & \\
\hline 8 & $5.75 \mathrm{E}-03$ & $3.49 E-01$ \\
\hline 8 & $3.35 E+00$ & $3.09 E+00$ \\
\hline 8 & $1.04 E+01$ & $1.53 E+01$ \\
\hline 8 & $1.04 E+01$ & $1.07 E+01$ \\
\hline 8 & $2.02 E+01$ & $2.46 E+01$ \\
\hline 8 & $3.11 E+01$ & $2.82 E+01$ \\
\hline 8 & $2.04 E+01$ & 1. $16 E+01$ \\
\hline 8 & $6.37 E-04$ & $7.81 \mathrm{E}-03$ \\
\hline 8 & $1.15 \mathrm{E}+00$ & $9.13 E-01$ \\
\hline 8 & $2.92 \mathrm{E}+00$ & $4.78 \mathrm{E}+00$ \\
\hline 8 & & $2.80 \mathrm{E}-01$ \\
\hline 8 & & $2.13 \mathrm{E}-02$ \\
\hline 8 & $1.79 E-04$ & $2.55 E-03$ \\
\hline 8 & & $2.23 E-02$ \\
\hline 8 & $9.31 E-03$ & $6.88 \mathrm{E}-03$ \\
\hline 8 & $6.38 \mathrm{E}-03$ & $1.95 E-03$ \\
\hline 8 & $2.91 \mathrm{E}-09$ & $2.35 E-01$ \\
\hline
\end{tabular}


INSTALLATION:SAN ONOFRE 1

EFFLUENT AND WASTE DISPOSAL ANNUAL REPORT FOR YEAR 1989 SOLID EFFLUENTS

ESTIMATE OF MAJOR NUCLIDE COMPOSITION (BY TYPE OF WASTE)

D

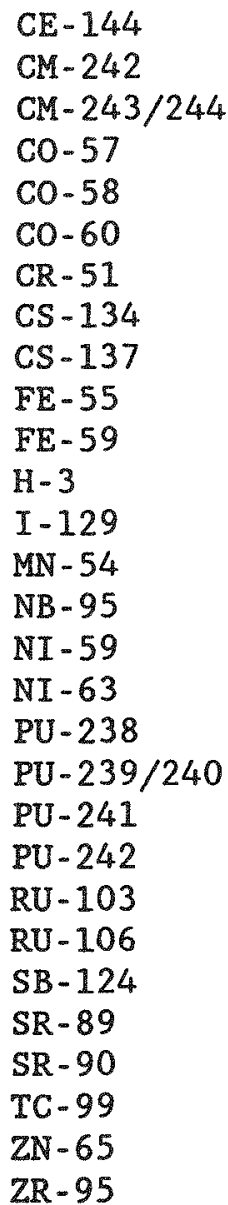

$\mathrm{CE}-144$

$\mathrm{CM}-243 / 244$

CO- 60

$\mathrm{CR}-51$

CS -134

CS -137

FE -55

FE -59

$\mathrm{H}-3$

I -129

$\mathrm{MN}-54$

NB -95

NI -59

NI -63

$\mathrm{PU}-239 / 240$

PU - 241

PU -242

$\mathrm{RU}-106$

SB- 124

SR -89

SR -90

TC -99

ZR-95
JAN-JUNE JULY-DEC

$\begin{array}{lll}8 & 6.40 E-02 & 6.18 E-01 \\ 8 & 9.77 E-02 & 9.62 E-02 \\ 8 & 1.06 \mathrm{E}-02 & 1.13 \mathrm{E}-02 \\ 8 & 1.00 \mathrm{E}-02 & 2.86 \mathrm{E}+00 \\ 8 & 3.08 \mathrm{E}-02 & 5.10 \mathrm{E}+00 \\ 8 & 3.14 \mathrm{E}+01 & 2.38 \mathrm{E}+01 \\ 8 & 4.12 \mathrm{E}-08 & 1.83 \mathrm{E}+01 \\ 8 & 6.19 \mathrm{E}-01 & 2.69 \mathrm{E}-01 \\ 8 & 2.31 \mathrm{E}+00 & 2.57 \mathrm{E}+00 \\ 8 & 2.83 \mathrm{E}+01 & 2.82 \mathrm{E}+01 \\ 8 & 3.83 & 3.83 \mathrm{E}-01 \\ 8 & 1.19 \mathrm{E}-07 & 3.32 \mathrm{E}+00 \\ 8 & 1.63 \mathrm{E}+01 & 5.11 \mathrm{E}-03 \\ 8 & 2.37 \mathrm{E}-03 & 1.03 \\ 8 & 6.30 \mathrm{E}-01 & 2.00 \mathrm{E}+00 \\ 8 & 1.40 \mathrm{E}-07 & 3.54 \mathrm{E}-01 \\ 8 & & 3.58 \mathrm{E}-02 \\ 8 & & 8.44 \mathrm{E}+00 \\ 8 & 9.69 \mathrm{E}+00 & 1.83 \mathrm{E}-02 \\ 8 & 1.09 \mathrm{E}-02 & 1.74 \mathrm{E}-03 \\ 8 & 4.43 \mathrm{E}-03 & 4.74 \\ 8 & 3.83 \mathrm{E}-01 & 5.60 \mathrm{E}-01 \\ 8 & & 1.46 \mathrm{E}-05 \\ 8 & & 2.39 \mathrm{E}-01 \\ 8 & & 2.69 \mathrm{E}-01 \\ 8 & & 3.49 \mathrm{E}-02 \\ 8 & & 4.11 \mathrm{E}-02 \\ 8 & 4.18 \mathrm{E}-05 & 5.31 \mathrm{E}-02 \\ 8 & 3.60 \mathrm{E}-08 & 3.57 \mathrm{E}-04 \\ 8 & 2.27 \mathrm{E}-02 & 3.57 \mathrm{E}-04 \\ 8 & 1.36 \mathrm{E}-03 & 2.07 \mathrm{E}-01 \\ 8 & & \end{array}$

UNIT YEAR TOTAL

M3 $3.94 \mathrm{E}+01$

CI $\quad 1.69 E+03$

M3 7.28E+01

CI $2.42 \mathrm{E}+00$

M3

$\mathrm{CI}$

M3 $6.48 E+00$

CI $\quad 2.38 \mathrm{E}+01$ 
EFFLUENT AND WASTE DISPOSAL ANNUAL REPORT FOR YEAR 1989 AIRBORNE AND LIQUID EFFLUENTS.

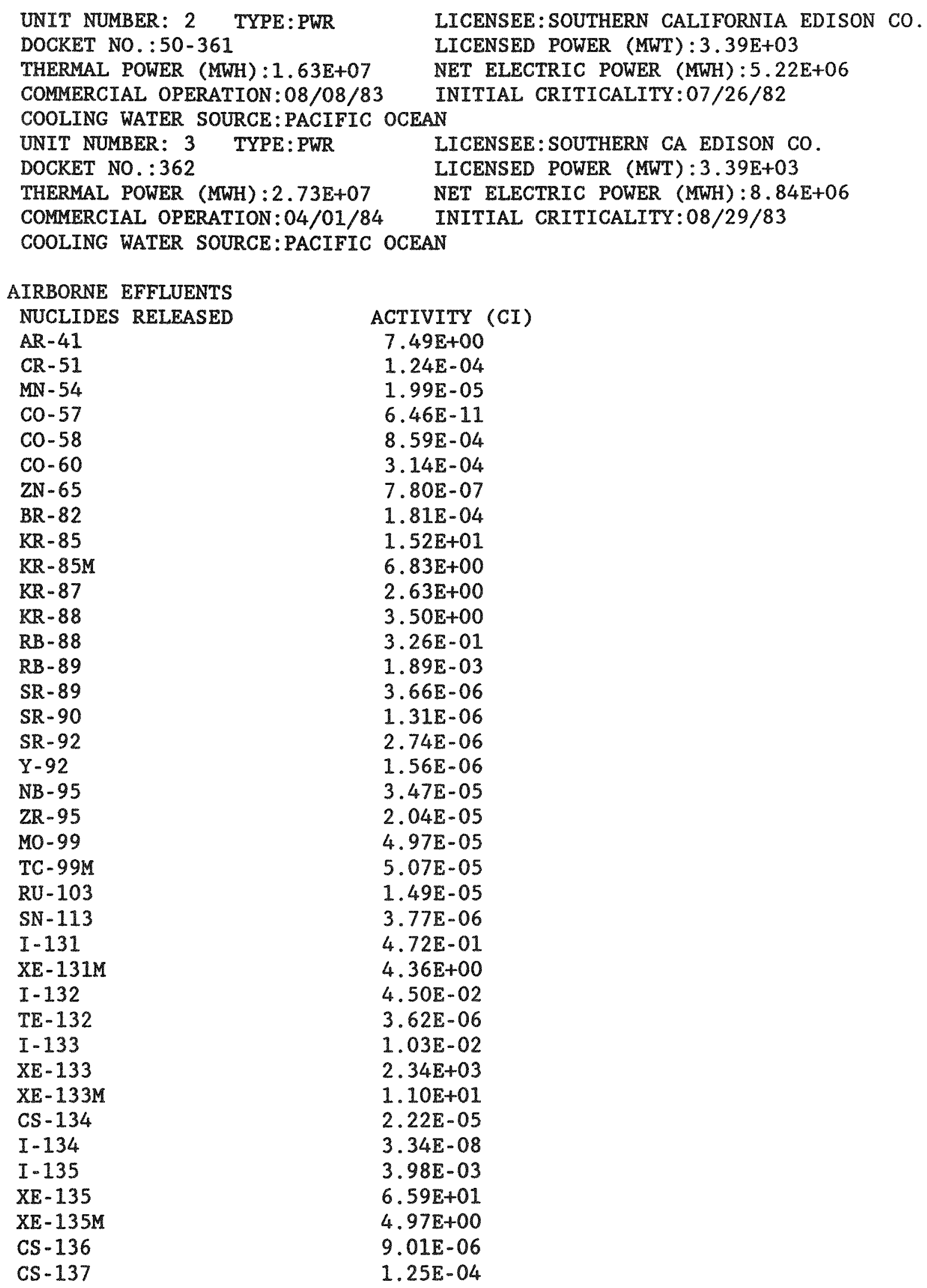


EFFLUENT AND WASTE DISPOSAL ANNUAL REPORT FOR YEAR 1989 AIRBORNE AND LIQUID EFFLUENTS

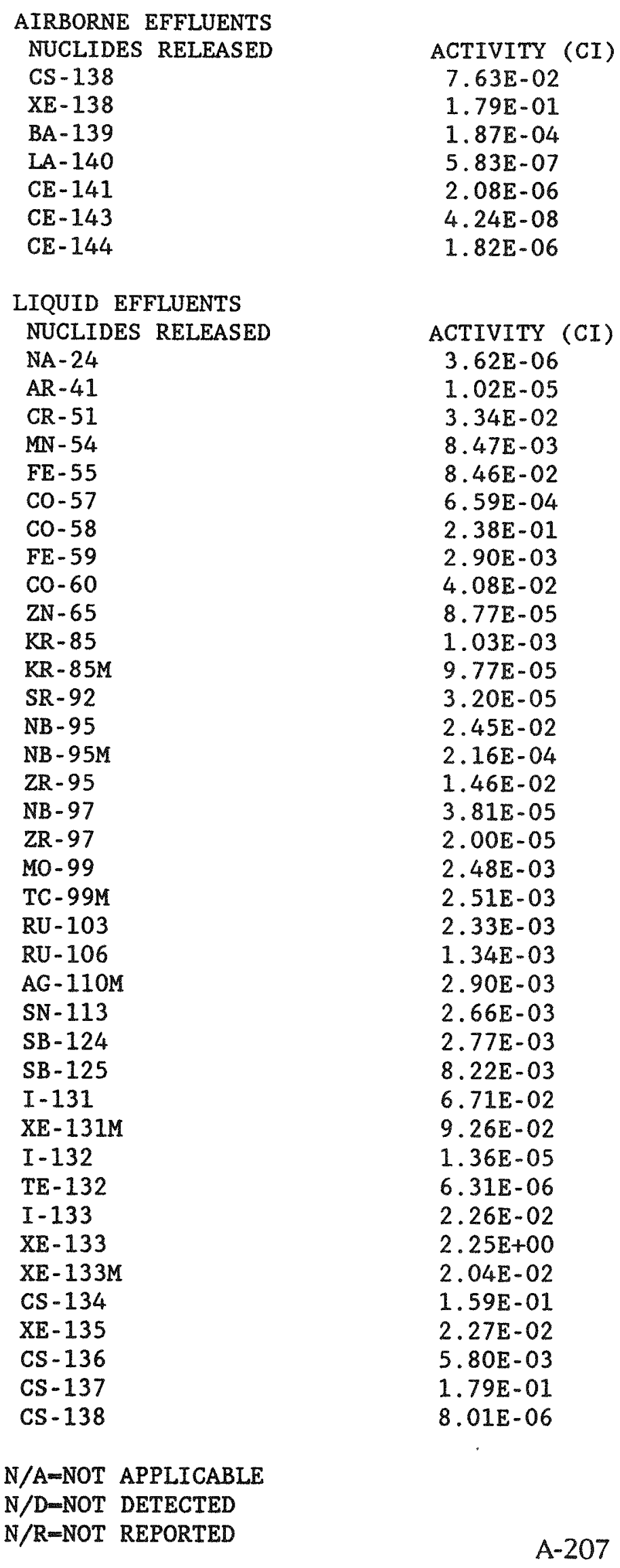


EFFLUENT AND WASTE DISPOSAL ANNUAL REPORT FOR YEAR 1989 AIRBORNE AND LIQUID EFFLUENTS

LIQUID EFFLUENTS
NUCLIDES RELEASED
BA-139
BA -140
LA -140
CE -141
CE -143
CE -144

LIQUID EFFLUENTS

NUCLIDES RELEASED

BA -139

BA -140

LA -140

$\mathrm{CE}-144$
ACTIVITY (CI)
$7.10 \mathrm{E}-05$
$1.43 E-03$
$5.81 \mathrm{E}-03$
$6.65 \mathrm{E}-04$
$9.63 \mathrm{E}-04$
$3.38 E-03$

$\begin{array}{ll}\text { TOTAL AIRBORNE TRITIUM RELEASE } & 4.05 E+01 \\ \text { TOTAL LIOUID TRITIUM RELEASE } & 1.30 E+03\end{array}$

VOLUME OF LIQUID WASTE RELEASED (PRIOR TO DILUTION) LITERS $4.79 E+07$

VOLUME OF DILUTION WATER USED DURING PERIOD

LITERS 2.32E+12 
INSTALLATION:SAN ONOFRE $2-3$

EFFLUENT AND WASTE DISPOSAL ANNUAL REPORT FOR YEAR 1989 SOLID EFFLUENTS

ESTIMATE OF MAJOR NUCLIDE COMPOSITION (BY TYPE OF WASTE)

JAN-JUNE JULY-DEC

A

C -14

$\mathrm{CE}-144$

CM -242

$\mathrm{CO}-57$

$\mathrm{CO}-58$

CO- 60

CS -134

CS -137

FE -55

H- 3

I -129

MN -54

NB- 95

NI -59

NI -63

PU-241

SB- 125

SR -89

SR -90

TC-99

ZR-95

B

AG-110M

BA- 140

C -14

CE- 144

CM- 242

$\mathrm{CO}-58$

CO- 60

CR -51

CS -134

CS -137

FE- 55

FE- 59

H- 3

I -129

I - 131

MN - 54

NB -95

NI -63

PU - 241

SB- 125

SR -89

\begin{tabular}{|c|c|c|}
\hline & $2.66 E-02$ & $4.79 \mathrm{E} .06$ \\
\hline & $9.34 E-04$ & $7.61 E-04$ \\
\hline & & $6.28 E-01$ \\
\hline & $2.38 \mathrm{E}-02$ & $5.87 \mathrm{E}-02$ \\
\hline & $2.31 E+00$ & $1.50 \mathrm{E}+00$ \\
\hline & $6.37 E+00$ & $2.34 E+00$ \\
\hline & $1.71 E+01$ & $2.24 E+01$ \\
\hline & $6.32 E+01$ & $5.74 \mathrm{E}+01$ \\
\hline & $3.39 E+00$ & $7.52 E+00$ \\
\hline & $2.86 E-02$ & $2.20 E-02$ \\
\hline & $2.26 E-05$ & $2.95 E-04$ \\
\hline & $7.64 E-01$ & $7.90 \mathrm{E}-01$ \\
\hline & 1. $13 \mathrm{E}-02$ & $9.74 E-02$ \\
\hline & $8.69 E-02$ & \\
\hline & $6.40 \mathrm{E}+00$ & $6.10 \mathrm{E}+00$ \\
\hline & $1.03 E-02$ & $6.28 \mathrm{E}-01$ \\
\hline & $9.22 E-02$ & $3.45 E-01$ \\
\hline & $6.60 E-03$ & $4.00 \mathrm{E}-02$ \\
\hline & $1.86 \mathrm{E}-01$ & $8.96 \mathrm{E}-02$ \\
\hline & $4.96 \mathrm{E}-05$ & $4.48 E-04$ \\
\hline & & $4.73 \mathrm{E}-02$ \\
\hline & & $3.62 \mathrm{E}-02$ \\
\hline & & $9.10 \mathrm{E}-02$ \\
\hline & $3.33 E-03$ & $2.20 \mathrm{E}-\mathrm{O} 3$ \\
\hline & & $9.37 \mathrm{E}-0]$ \\
\hline & & $3.46 \mathrm{E}-02$ \\
\hline & $3.24 E+00$ & $5.03 E+00$ \\
\hline & $1.29 \mathrm{E}+01$ & 1. $10 \mathrm{E}+01$ \\
\hline & & $9.80 \mathrm{E}-0$ \\
\hline & $7.28 \mathrm{E}+00$ & $8.89 E+00$ \\
\hline & $1.40 E+01$ & 1. $70 \mathrm{E}+01$ \\
\hline & $3.26 E+01$ & 3. $35 E+01$ \\
\hline & & $9.80 \mathrm{E}-02$ \\
\hline & $2.32 E+01$ & $9.44 E+00$ \\
\hline & $4.77 E-03$ & $4.18 \mathrm{E}-02$ \\
\hline & & $1.13 E-01$ \\
\hline & $1.82 \mathrm{E}+00$ & $2.12 \mathrm{E}+0 \mathrm{C}$ \\
\hline & $1.19 \mathrm{E}+00$ & $3.98 E+00$ \\
\hline & $3.64 E+00$ & $3.80 \mathrm{E}+0 \mathrm{C}$ \\
\hline & & $6.84 E-0$ \\
\hline & $1.52 \mathrm{E}-02$ & $3.08 \mathrm{E}-0$ \\
\hline & & $1.13 \mathrm{E}-0$ \\
\hline
\end{tabular}


INSTALLATION: SAN ONOFRE 2-3

EFFLUENT AND WASTE DISPOSAL ANNUAL REPORT FOR YEAR 1989 SOLID EFFLUENTS

ESTIMATE OF MAJOR NUCLIDE COMPOSITION (BY TYPE OF WASTE)

B

$S R=90$

TC -99

$\mathrm{ZR}-95$

D

AG- 110

AM- 241

$B A-140$

C. -14

CE-141

$\mathrm{CE}-144$

CM- 242

CM $-243 / 244$

$\mathrm{CO}-57$

CO. 58

$\mathrm{CO}-60$

CR -51

CS -134

CS -136

CS -137

$F E-55$

FE- 59

$\mathrm{H}-3$

I-129

$\mathrm{MN}-54$

NB- 95

NI -59

NI -63

PU -238

PU $-239 / 240$

PU-241

RU -103

RU. 106

SB- 124

SB- 125

SR- 89

$S R-90$

TC -99

$\mathrm{ZN}-65$

ZR-95
JAN-JUNE JULY-DEC

$6.15 \mathrm{E}-02$

3.43E-03 8.75E-02

$9.67 \mathrm{E}-02 \quad 1.67 \mathrm{E}+00$

6.76E-03 1.37E-01

5.79E-04 3.12E-06

1.16E-02 1.93E-05

1.83E-02 5.03E-04

4.89E-04 5.16E-03

$9.67 \mathrm{E}-02 \quad 1.96 \mathrm{E}-01$

$1.19 \mathrm{E}-02 \quad 8.07 \mathrm{E}-03$

$7.83 \mathrm{E}-04 \quad 8.74 \mathrm{E}-04$

$2.88 \mathrm{E}-02 \quad 6.89 \mathrm{E}-02$

$1.17 \mathrm{E}+00 \quad 1.36 \mathrm{E}+01$

$2.31 E+01 \quad 1.36 E+01$

$1.08 \mathrm{E}+00 \quad 3.02 \mathrm{E}+00$

1.54E-01 2.01E-01

7. $68 \mathrm{E}-03$

4. $62 \mathrm{E}-01$

$5.37 \mathrm{E}-01$

$6.36 E+01$

$5.34 \mathrm{E}+01$

4.46E-02 4.56E-01

$7.96 \mathrm{E}-01 \quad 1.09 \mathrm{E}+00$

$2.14 \mathrm{E}-03 \quad 3.46 \mathrm{E}-04$

1. $30 \mathrm{E}+00 \quad 1.77 \mathrm{E}+00$

$1.85 \mathrm{E}-01 \quad 3.83 \mathrm{E}+00$

8.80E-03 5.07E-05

$7.15 \mathrm{E}+00 \quad 5.11 \mathrm{E}+00$

$1.44 \mathrm{E}-03$

2.02E-03 3.93E-04

$1.58 \mathrm{E}-01 \quad 9.73 \mathrm{E}-02$

4. $35 \mathrm{E}-04 \quad 8.46 \mathrm{E}-02$

$1.05 \mathrm{E}-03 \quad 3.03 \mathrm{E}-02$

$1.87 \mathrm{E}-03 \quad 2.95 \mathrm{E}-02$

3.12E-01 2.12E-01

2.29E-03 2.63E-01

$8.59 E-02 \quad 3.87 E-02$

$4.87 \mathrm{E}-03 \quad 8.56 \mathrm{E}-04$

$1.02 \mathrm{E}-02 \quad 3.66 \mathrm{E}-02$

$1.20 \mathrm{E}-01 \quad 2.11 \mathrm{E}+00$ 
INSTALLATION:SAN ONOFRE $2-3$

EFFLUENT AND WASTE DISPOSAL ANNUAL REPORT FOR YEAR 1989

SOLID EFFLUENTS

TYPE OF WASTE

A. SPENT RESINS, FILTER SLUDGES, EVAPORATOR BOTTOMS, ETC.

B. DRY COMPRESSIBLE WASTE, CONTAMINATED EQUIPMENT, ETC.

C. IRRADIATED COMPONENTS, CONTROL RODS, ETG.

D. OTHER
UNIT YEAR TOTAL

M3 $7.37 \mathrm{E}+01$

CI $2.49 \mathrm{E}+03$

M3 $2.34 E+02$

CI $\quad 1.15 \mathrm{E}+01$

$\mathrm{M3}$

CI

M3 $1.97 \mathrm{E}+01$

CI $2.22 \mathrm{E}+02$ 
INSTALLATION:SAN ONOFRE $1,2 \& 3$

EFFLUENT AND WASTE DISPOSAL ANNUAL REPORT FOR YEAR 1989 SOLID EFFLUENTS

SOLID WASTE DISPOSITION NUMBER OF SHIPMENTS 5

$\begin{array}{rr} & 5 \\ & 35 \\ \# \quad 3 \\ & 15 \\ \# \quad 3\end{array}$

MODE OF TRANSPORTATION TRI - STATE TRUCK/CASK TRI-STATE TRUCK/CASK TRI-STATE TRUCK/TRAILER TRI-STATE TRUCK/TRAILER TRI-STATE TRUCK/TRAILER
DESTINATION BARNWELL SC RICHLAND WA BARNWELL SC BEATTY NV RICHLAND WA

\#THESE SHIPMENTS WERE MADE FROM VENDORS AFTER VOL. REDUCTION

IRRADIATED FUEL SHIPMENTS (DISPOSITION)

NUMBER OF SHIPMENTS MODE OF TRANSPORTATION

$$
0
$$

$\mathrm{N} / \mathrm{A}$

TYPE OF WASTE

A. SPENT RESINS, FILTER SLUDGES, EVAPORATOR BOTTOMS, ETC.

B. DRY COMPRESSIBLE WASTE, CONTAMINATED EQUIPMENT, ETC.

C. IRRADIATED COMPONENTS, CONTROL RODS, ETC.

D. OTHER
DESTINATION

$\mathrm{N} / \mathrm{A}$

UNIT YEAR TOTAL

M3 N/A

CI N/A

M3 N/A

CI N/A

M3

$\mathrm{CI}$

M3

CI 
EFFLUENT AND WASTE DISPOSAL ANNUAL REPORT FOR YEAR 1989 AIRBORNE AND LIQUID EFFLUENTS

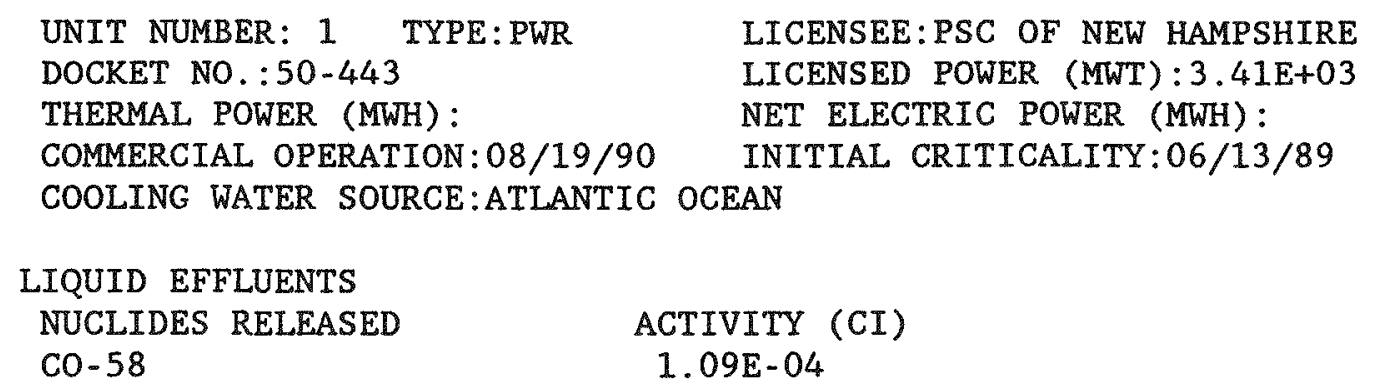

TOTAL AIRBORNE TRITIUM RELEASE $1.05 \mathrm{E}-05$

TOTAL LIQUID TRITIUM RELEASE 1.33E-03

VOLUME OF LIQUID WASTE RELEASED (PRIOR TO DILUTION) LITERS 3.02E+07

VOLUME OF DILUTION WATER USED DURING PERIOD $\quad$ LITERS $1.67 \mathrm{E}+11$ 
EFFLUENT AND WASTE DISPOSAL ANNUAL REPORT FOR YEAR 1989 AIRBORNE AND LIQUID EFFLUENTS

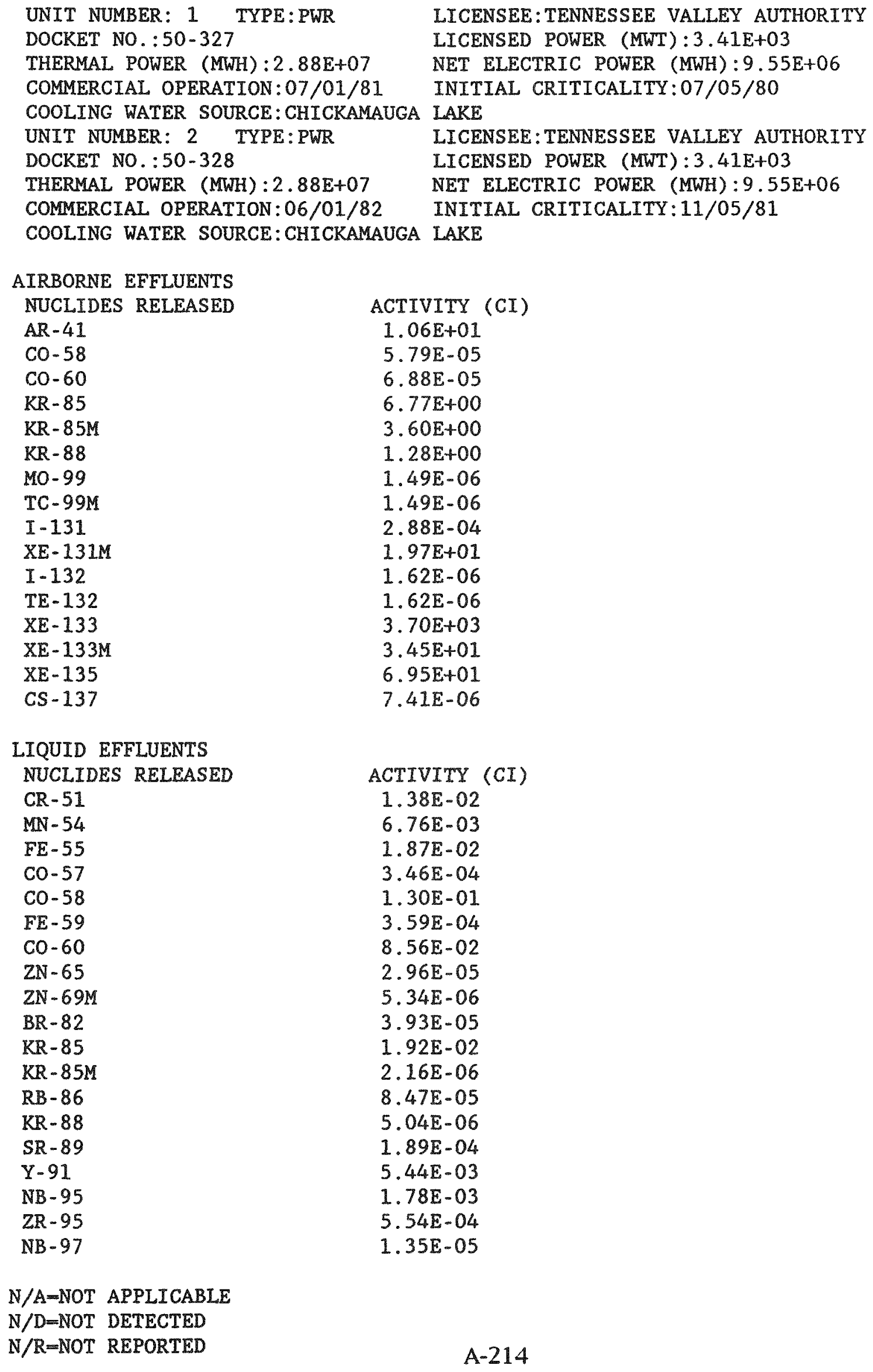


EFFLUENT AND WASTE DISPOSAL ANNUAL REPORT FOR YEAR 1989 AIRBORNE AND LIQUID EFFLUENTS

\begin{tabular}{|c|c|}
\hline & \\
\hline NUCLIDES RELEASED & ACTIVITY (CI) \\
\hline MO-99 & $1.55 E-02$ \\
\hline TC-99M & $1.69 E-02$ \\
\hline $\mathrm{RU}-103$ & $1.10 \mathrm{E}-03$ \\
\hline$S B-122$ & $2.77 E-06$ \\
\hline $\mathrm{SB}-124$ & $2.47 E-04$ \\
\hline$S B-125$ & $7.51 E-03$ \\
\hline $\mathrm{SN}-125$ & $2.86 E-05$ \\
\hline$I=131$ & $6.56 \mathrm{E}-03$ \\
\hline$X E-131 M$ & $2.53 E-02$ \\
\hline$I-132$ & $1.32 E-06$ \\
\hline$T E-132$ & $2.12 E-05$ \\
\hline$I-133$ & $2.10 E-04$ \\
\hline$X E-133$ & $1.94 E+00$ \\
\hline$X E-133 M$ & $1.70 E-02$ \\
\hline $\operatorname{CS}-134$ & $8.52 E-03$ \\
\hline $\mathrm{XE}-135$ & $8.66 E-03$ \\
\hline $\mathrm{XE}-135 \mathrm{M}$ & $7.62 E-11$ \\
\hline CS -136 & $6.02 E-05$ \\
\hline CS -137 & $2.74 E-02$ \\
\hline$B A-139$ & $2.72 E-05$ \\
\hline$B A-140$ & $6.53 E-04$ \\
\hline$L A-140$ & $3.08 E-03$ \\
\hline$C E-141$ & $1.56 \mathrm{E}-03$ \\
\hline$C E-143$ & $1.50 E-04$ \\
\hline$C E-144$ & $1.14 E-04$ \\
\hline$W-187$ & $4.05 E-05$ \\
\hline
\end{tabular}

TOTAL AIRBORNE TRITIUM RELEASE 5.62E+01

TOTAL LIQUID TRITIUM RELEASE $1.15 E+03$

VOLUME OF LIQUID WASTE RELEASED (PRIOR TO DILUTION) LITERS $4.40 E+07$ VOLUME OF DILUTION WATER USED DURING PERIOD

LITERS $\quad 5.08 \mathrm{E}+09$ 
EFFLUENT AND WASTE DISPOSAL ANNUAL REPORT FOR YEAR 1989 SOLID EFFLUENTS

SOLID WASTE DISPOSITION NUMBER OF SHIPMENTS 90

MODE OF TRANSPORTATION

DESTINATION MOTOR FREIGHT

1

MOTOR FREIGHT

BARNWELL SC

RICHLAND WA

IRRADIATED FUEL SHIPMENTS(DISPOSITION)

NUMBER OF SHIPMENTS MODE OF TRANSPORTATION

0

$\mathrm{N} / \mathrm{A}$

DESTINATION

N/A

ESTIMATE OF MAJOR NUCLIDE COMPOSITION (BY TYPE OF WASTE)

JAN-JUNE JULY-DEC

A

$\mathrm{C}-14$

Co- 58

CO- 60

CR -51

CS -134

CS -137

FE- 55

FE -59

$\mathrm{H}-3$

I -129

MN -54

NI -63

SR -90

TC -99

B

C -14

Co- 58

Co- 60

CS -137

FE- 55

H -3

I -129

I -131

NI -63

SR -90

TC- 99

$8 \quad 4.26 \mathrm{E}-01$

$8 \quad 3.30 \mathrm{E}+00$

\& $\quad 3.62 \mathrm{E}+01$

1. $70 \mathrm{E}+01$

$3.62 \mathrm{E}+01$
$2.58 \mathrm{E}-02$

$2.58 \mathrm{E}-02$
8

$3.08 \mathrm{E}+01$

2. $30 \mathrm{E}+00$

$6.29 \mathrm{E}+00$

$6.55 \mathrm{E}+00$

3. $91 \mathrm{E}+01$

3. $11 \mathrm{E}+01$

3. $56 \mathrm{E}-03$

2. 25E-02

8. $69 \mathrm{E}-06$

1. $26 \mathrm{E}-01$

1. $24 E+01$

1. $84 \mathrm{E}-03$

2.67E-05

1. $15 \mathrm{E}+01$

$8 \quad 2.58 \mathrm{E}-02$

8

$2.58 \mathrm{E}-02$

$2.49 E+00$

1. $97 \mathrm{E}+01$

1. $90 \mathrm{E}+01$

3. $38 \mathrm{E}-03$

7. $34 E+01$

$6.89 E+01$

8.18E-02

$7.11 \mathrm{E}-03$

1. $26 \mathrm{E}+00$

$5.50 \mathrm{E}+00$

9.91E-03

7. $95 \mathrm{E}-03$

$8.69 E+00$

D

C -14

CO- 58

Co- 60

CS -134

CS -137

5. 30E-02

2. $79 \mathrm{E}+01$

3. $94 \mathrm{E}+00$

1. $54 \mathrm{E}+01$

3. $37 \mathrm{E}+01$

$1.45 \mathrm{E}+00$

$2.04 \mathrm{E}+00$

$2.16 \mathrm{E}+00$ 
EFFLUENT AND WASTE DISPOSAL ANNUAL REPORT FOR YEAR 1989 SOLID EFFLUENTS

ESTIMATE OF MAJOR NUCLIDE COMPOSITION (BY TYPE OF WASTE) JAN-JUNE JULY-DEC

D

FE -55

$\mathrm{H}-3$

I -129

$\mathrm{MN}-54$

NI -63

TC -99

$\begin{array}{lll}8 & 4.57 \mathrm{E}+01 & 3.91 \mathrm{E}+01 \\ 8 & 5.16 \mathrm{E}-02 & \\ 8 & 4.31 \mathrm{E}-03 & \\ 8 & 2.88 \mathrm{E}+00 & \\ 8 & 4.50 \mathrm{E}+00 & 1.98 \mathrm{E}+01 \\ 8 & 4.83 \mathrm{E}-03 & \end{array}$

TYPE OF WASTE

A. SPENT RESINS, FILTER SLUDGES, EVAPORATOR

UNIT YEAR TOTAL BOTTOMS, ETC.

M3 $\quad 1.07 \mathrm{E}+02$

CI $\quad 2.47 \mathrm{E}+03$

B. DRY COMPRESSIBLE WASTE, CONTAMINATED

M3 $3.35 E+02$

EQUIPMENT, ETC.

CI $\quad 1.54 E+02$

C. IRRADIATED COMPONENTS, CONTROL

M3 RODS, ETC.

CI

M3 $2.27 \mathrm{E}+01$

D. OTHER

COMPOSITE LINERS \& OIL LINERS

CI $1.40 \mathrm{E}+01$ 
EFFLUENT AND WASTE DISPOSAL ANNUAL REPORT FOR YEAR 1989 AIRBORNE AND LIQUID EFFLUENTS

UNIT NUMBER: 1 TYPE:BWR DOCKET NO. : $50-322$

THERMAL POWER (MWH): COMMERCIAL OPERATION:

COOLING WATER SOURCE:LONG ISLAND SOUND

LIQUID EFFLUENTS

NUCLIDES RELEASED

$\mathrm{CO}-60$

ACTIVITY (CI)

1.78E-05
LICENSEE: LONG ISLAND LIGHTING CO LICENSED POWER (MWT) $: 1.22 E+02$ NET ELECTRIC POWER (MWH): INITIAL CRITICALITY:02/15/85
TOTAL AIRBORNE TRITIUM RELEASE N/D

TOTAL LIQUID TRITIUM RELEASE N/D

VOLUME OF LIQUID WASTE RELEASED (PRIOR TO DILUTION)

VOLUME OF DILUTION WATER USED DURING PERIOD
LITERS

LITERS

$8.51 \mathrm{E}+06$

1. $51 \mathrm{E}+10$ 
EFFLUENT AND WASTE DISPOSAL ANNUAL REPORT FOR YEAR 1989 SOLID EFFLUENTS

SOLID WASTE DISPOSITION $\begin{array}{cl}\text { NUMBER OF SHIPMENTS } & \text { MODE OF TRANSPORTATION } \\ 1 & \text { TRUCK }\end{array}$

ESTIMATE OF MAJOR NUCLIDE COMPOSITION (BY TYPE OF WASTE)

A

AG-110M

$\mathrm{CE}-141$

CE -144

$\mathrm{CO}-57$

$\mathrm{CO}-58$

CO- 60

$\mathrm{CR}-51$

CS -137

FE -55

FE -59

$\mathrm{H}-3$

MN -54

NB -95

NI -59

NI -63

PU -241

$\mathrm{SB}-124$

$S R-90$

$\mathrm{ZN}-65$

ZR-95
TYPE OF WASTE

A. SPENT RESINS, FILTER SLUDGES, EVAPORATOR BOTTOMS, ETC.

B. DRY COMPRESSIBLE WASTE, CONTAMINATED EQUIPMENT, ETC.

C. IRRADIATED COMPONENTS, CONTROL RODS, ETC.

D. OTHER
DESTINATION

BARNWELL SC

JAN-JUNE JULY-DEC

$3.69 E+00$

$5.00 \mathrm{E}-03$

$2.75 \mathrm{E}-01$

$5.50 \mathrm{E}-02$

1. $41 E+01$

$2.79 \mathrm{E}+01$

$1.86 \mathrm{E}+01$

8. $30 E-02$

2. $39 E+01$

1. $71 \mathrm{E}+00$

2. $81 \mathrm{E}-01$

$2.63 \mathrm{E}+00$

1. $16 \mathrm{E}-01$

$2.00 E-02$

$1.09 \mathrm{E}+00$

8. $17 E-01$

1. $38 \mathrm{E}-01$

2. $00 \mathrm{E}-03$

4. $59 \mathrm{E}+00$

2. 30E-02

UNIT YEAR TOTAL

M3 $1.79 \mathrm{E}+01$

CI $\quad 3.51 \mathrm{E}-02$

M3

CI

M3

CI

M3

CI 
EFFLUENT AND WASTE DISPOSAL ANNUAL REPORT FOR YEAR 1989 AIRBORNE AND LIQUID EFFLUENTS

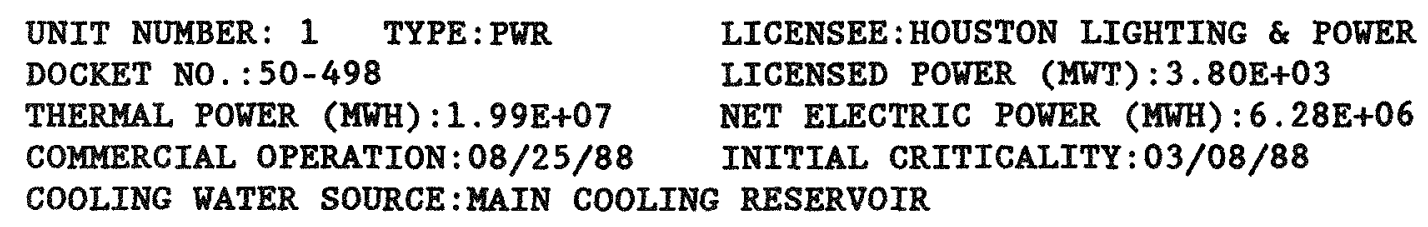

LIQUID EFFLUENTS NUCLIDES RELEASED

NA -24

CR -51

MN -54

FE -55

CO- 58

FE -59

CO- 60

ZN -65

SR-91

NB -95

ZR -95

MO- 99

TC-99M

AG-110M

$\mathrm{TE}-129 \mathrm{M}$

I-131

I-133

$\mathrm{XE}-133$

CS-134

$\mathrm{XE}-135$

CS -136

$$
\begin{aligned}
& \text { ACTIVITY (CI) } \\
& 4.18 \mathrm{E}-05 \\
& 6.88 \mathrm{E}-01 \\
& 8.00 \mathrm{E}-02 \\
& 5.13 \mathrm{E}-01 \\
& 1.21 \mathrm{E}+00 \\
& 5.63 \mathrm{E}-02 \\
& 2.02 \mathrm{E}-01 \\
& 6.64 \mathrm{E}-03 \\
& 3.09 \mathrm{E}-05 \\
& 7.88 \mathrm{E}-02 \\
& 4.29 \mathrm{E}-02 \\
& 4.94 \mathrm{E}-05 \\
& 7.29 \mathrm{E}-05 \\
& 2.90 \mathrm{E}-03 \\
& 2.35 \mathrm{E}-04 \\
& 9.93 \mathrm{E}-03 \\
& 1.34 \mathrm{E}-04 \\
& 9.16 \mathrm{E}-01 \\
& 1.13 \mathrm{E}-02 \\
& 5.05 \mathrm{E}-03 \\
& 5.86 \mathrm{E}-05
\end{aligned}
$$

$N / A=N O T$ APPLICABLE $N / D=N O T$ DETECTED 
EFFLUENT AND WASTE DISPOSAL ANNUAL REPORT FOR YEAR 1989 AIRBORNE AND LIQUID EFFLUENTS

\section{LIQUID EFFLUENTS}

NUCLIDES RELEASED

CS -137

$B A-140$

LA -140

CE-144

UNIDENTIFIED
ACTIVITY (CI)
$2.97 \mathrm{E}-02$
$1.67 \mathrm{E}-05$
$2.1 .9 E-04$
$2.70 E-04$
$8.57 \mathrm{E}-02$

TOTAL AIRBORNE TRITIUM RELEASE $6.33 E+00$

TOTAL LIQUID TRITIUM RELEASE $3.17 \mathrm{E}+02$

VOLUME OF LIQUID WASTE RELEASED (PRIOR TO DILUTION) LITERS 2.07E+07

VOLUME OF DILUTION WATER USED DURING PERIOD 
EFFLUENT AND WASTE DISPOSAL ANNUAL REPORT FOR YEAR 1989 AIRBORNE AND LIQUID EFFLUENTS

\begin{tabular}{|c|c|}
\hline UNIT NUMBER: 2 & LICENSEE \\
\hline DOCKET NO. : 50-499 & LICENSED \\
\hline $\begin{array}{l}\text { THERMAL POWER (MWH } \\
\text { COMMERCIAL OPERATI }\end{array}$ & $\begin{array}{l}\text { NET ELECT } \\
\text { INITIAL C }\end{array}$ \\
\hline COOLING WATER SOUR & COOLING RESERVC \\
\hline AIRBORNE EFFLUENTS & \\
\hline NUCLIDES RELEASED & ACTIVITY (CI) \\
\hline$A R-41$ & $8.88 \mathrm{E}+01$ \\
\hline $60-58$ & $1.60 \mathrm{E}-06$ \\
\hline$X E-133$ & $2.53 E+01$ \\
\hline$X E-135$ & $1.43 \mathrm{E}+00$ \\
\hline UNIDENTIFIED & $1.42 \mathrm{E}-03$ \\
\hline LIQUID EFFLUENTS & \\
\hline NUCLIDES RELEASED & ACTIVITY (CI) \\
\hline CR-51 & $7.02 \mathrm{E}-04$ \\
\hline$M N-54$ & $9.32 \mathrm{E}-05$ \\
\hline $\mathrm{CO}-58$ & $8.18 E-03$ \\
\hline FE -59 & $2.17 E-04$ \\
\hline $60-60$ & $2.07 E-04$ \\
\hline NB-95 & $8.16 E-06$ \\
\hline ZR -95 & $6.21 E-06$ \\
\hline$I-131$ & $2.69 E-06$ \\
\hline$I-133$ & $4.22 E-06$ \\
\hline$X E-133$ & $6.68 E-02$ \\
\hline$X E-135$ & $1.98 E-03$ \\
\hline LA-140 & $3.76 E-05$ \\
\hline UNIDENTIFIED & $2.26 E-03$ \\
\hline
\end{tabular}

TOTAL AIRBORNE TRITIUM RELEASE 1.20E+01

TOTAL LIQUID TRITIUM RELEASE $2.72 E+02$

VOLUME OF IIQUID WASTE RELEASED (PRIOR TO DILUTION) LITERS $1.26 E+07$

VOLUME OF DILUTION WATER USED DURING PERIOD 
EFFLUENT AND WASTE DISPOSAL ANNUAL REPORT FOR YEAR 1989 SOLID EFFLUENTS

SOLID WASTE DISPOSITION NUMBER OF SHIPMENTS MODE OF TRANSPORTATION 9 TRUCK

ESTIMATE OF MAJOR NUCLIDE COMPOSITION (BY TYPE OF WASTE)

A $\mathrm{CO}-58$

$\mathrm{CO}-60$

CS -137

$\mathrm{H}-3$

MN -54

NI -63

B

CO- 58

CR -51

FE -55

H -3

NB -95

ZR-95

\section{DESTINATION \\ SEG-OAK RIDGE TN}

JAN - JUNE JULY-DEC
8

8

8

8

8

8

$\frac{9}{8}$

8

8

8

8

8
$7.85 \mathrm{E}+01$

$5.00 \mathrm{E}+00$

1. $70 \mathrm{E}+00$

$7.00 \mathrm{E}+00$

$2.60 \mathrm{E}+00$

2. $10 \mathrm{E}+00$

1. $40 E+01$

2. $10 \mathrm{E}+01$

$3.60 \mathrm{E}+00$

$5.50 \mathrm{E}+01$

1. $90 \mathrm{E}+00$

1. $50 \mathrm{E}+00$
TYPE OF WASTE

A. SPENT RESINS, FILTER SLUDGES, EVAPORATOR BOTTOMS , ETC.

B. DRY COMPRESSIBLE WASTE, CONTAMINATED

EQUIPMENT, ETC.

UNIT YEAR TOTAL

M3 2.33E+01

CI $\quad 7.07 \mathrm{E}+00$

M3 2.70E+01

CI $\quad 1.69 \mathrm{E}-01$

C. IRRADIATED COMPONENTS, CONTROL

M3

RODS, ETC.

D. OTHER

$\mathrm{CI}$

M3

CI 
EFFLUENT AND WASTE DISPOSAL ANNUAL REPORT FOR YEAR 1989 AIRBORNE AND LIQUID EFFLUENTS

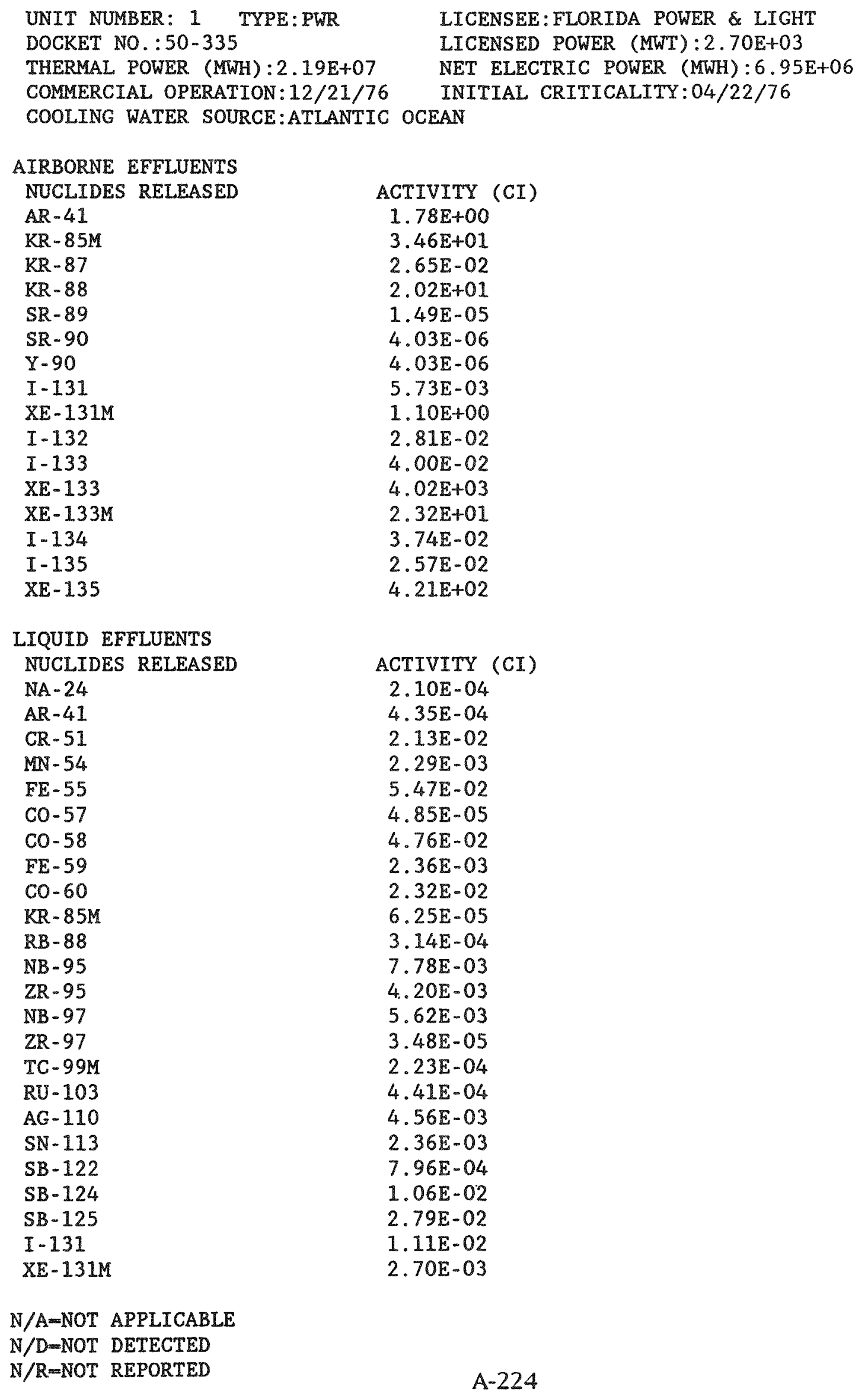


EFFLUENT AND WASTE DISPOSAL ANNUAL REPORT FOR YEAR 1989 AIRBORNE AND LIQUID EFFLUENTS

LIQUID EFFLUENTS
NUCLIDES RELEASED
$I-132$
$T E-132$
$I-133$
$X E-133$
$X E-133 M$
$C S-134$
$X E-135$
$C S-137$
$C S-138$
$B A-140$
LA -140
$C E-141$
$C E-144$
NP -239
ACTIVITY (CI)
4.70E- 04
4. 31E- 04
$1.42 \mathrm{E}-03$
4. $60 \mathrm{E}-01$
4. $10 \mathrm{E}-03$
$1.04 \mathrm{E}-02$
$3.33 E-03$
$1.41 \mathrm{E}-02$
$7.85 \mathrm{E}-05$
$4.09 \mathrm{E}-06$
$1.07 \mathrm{E}-03$
$2.69 \mathrm{E}-05$
$6.75 \mathrm{E}-04$
$3.64 \mathrm{E}-05$

TOTAL AIRBORNE TRITIUM RELEASE 3.10E+02

TOTAL LIQUID TRITIUM RELEASE $4.05 E+02$

VOLUME OF LIQUID WASTE RELEASED (PRIOR TO DILUTION) LITERS $3.66 E+07$

VOLUME OF DILUTION WATER USED DURING PERIOD

LITERS $1.78 \mathrm{E}+12$ 
EFFLUENT AND WASTE DISPOSAL ANNUAL REPORT FOR YEAR 1989 AIRBORNE AND LIQUID EFFLUENTS

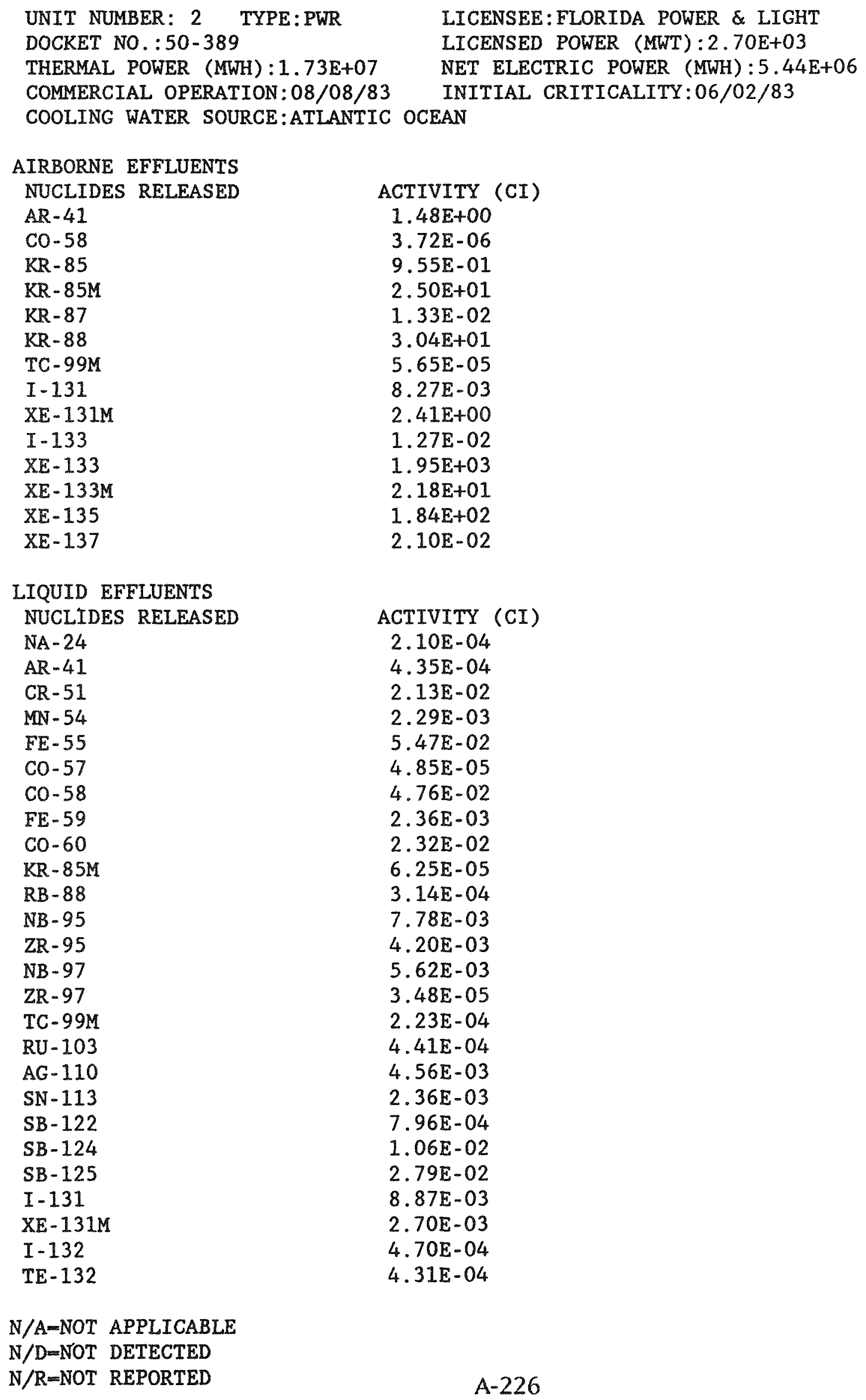


EFFLUENT AND WASTE DISPOSAL ANNUAL REPORT FOR YEAR 1989 AIRBORNE AND LIQUID EFFLUENTS

LIQUID EFFLUENTS
NUCLIDES RELEASED
I-133
XE-133
XE $-133 \mathrm{M}$
CS -134
XE -135
CS -137
CS -138
BA -140
LA -140
CE -141
CE -144
NP -239

ACTIVITY (CI)

$1.42 \mathrm{E}-03$

4. $60 \mathrm{E}-01$

4.10E-03

$9.95 \mathrm{E}-03$

3. $33 \mathrm{E}-03$

1. $32 \mathrm{E}-02$

$7.85 \mathrm{E}-05$

$4.09 \mathrm{E}-06$

1. $07 \mathrm{E}-03$

$2.69 \mathrm{E}-05$

$6.75 \mathrm{E}-04$

$3.64 \mathrm{E}-05$

TOTAL AIRBORNE TRITIUM RELEASE 7.78E+01

TOTAL LIQUID TRITIUM RELEASE $4.05 E+02$

VOLUME OF LIQUID WASTE RELEASED (PRIOR TO DILUTION) LITERS $5.98 \mathrm{E}+06$

VOLUME OF DILUTION WATER USED DURING PERIOD $\quad$ LITERS $1.78 E+12$ 
INSTALLATION:ST. LUCIE $1 \& 2$

EFFLUENT AND WASTE DISPOSAL ANNUAL REPORT FOR YEAR 1989 SOLID EFFLUENTS

SOLID WASTE DISPOSITION NUMBER OF SHIPMENTS 72

MODE OF TRANSPORTATION

DESTINATION

SOLE USE TRUCK

BARNWELL SC

IRRADIATED FUEL SHIPMENTS (DISPOSITION)

NUMBER OF SHIPMENTS MODE OF TRANSPORTATION

0

N/A

DESTINATION

N/A

JAN-JUNE JULY-DEC

ESTIMATE OF MAJOR NUCLIDE COMPOSITION (BY TYPE OF WASTE)

A

$\mathrm{CO}-58$

CO- 60

CR -51

CS -134

CS -137

$\mathrm{FE}-55$

FE -59

H- 3

MN -54

NB -95

NI -63

$\mathrm{SB}-122$

$\mathrm{SB}-125$

ZR-95

B

CE -144

$\mathrm{CO}-58$

CO- 60

CR- 51

CS -134

CS -137

FE- 55

$\mathrm{H}-3$

MN -54

NB -95

NI -63

SB -125

ZR-95

D

$\mathrm{CO}-58$

$\mathrm{CO}-60$

$\mathrm{CR}-51$

CS -134

$\begin{array}{lll}8 & 1.71 E+01 & 9.91 E+00 \\ 8 & 4.65 E+01 & 1.59 E+01 \\ 8 & 4.93 E+00 & \\ 8 & 8.02 E-01 & 1.57 E+01 \\ 8 & 2.53 E+00 & 3.57 E+01 \\ 8 & 1.20 E+01 & 5.05 E+00 \\ 8 & 7.51 E-01 & \\ 8 & & 1.08 E+00 \\ 8 & 2.21 E+00 & 1.10 E+00 \\ 8 & 2.24 E+00 & \\ 8 & 6.45 E+00 & 1.05 E+01 \\ 8 & & 1.18 E+00 \\ 8 & 1.52 E+00 & 2.85 E+00 \\ 8 & 1.32 E+00 & \\ & & \\ 8 & 3.02 E+00 & 2.09 E+00 \\ 8 & 7.27 E+00 & 4.77 E+00 \\ 8 & 2.26 E+01 & 2.11 E+01 \\ 8 & 1.06 E+00 & \\ 8 & 7.44 E+00 & 6.12 E+00 \\ 8 & 1.96 E+01 & 1.88 E+01 \\ 8 & 7.74 E+00 & 5.96 E+00 \\ 8 & 2.31 E+01 & 3.45 E+01 \\ 8 & 1.05 E+00 & 7.06 E-01 \\ 8 & 1.56 E+00 & \\ 8 & 2.54 E+00 & 2.46 E+00 \\ 8 & 1.07 E+00 & 1.01 E+00 \\ 8 & 1.57 E+00 & 8.09 E-01 \\ 8 & 4.85 E+00 & 1.54 E+00 \\ 8 & 2.80 E+01 & 4.11 E+01 \\ 8 & 1.08 E+00 & \\ 8 & 1.41 E+01 & 9.91 E+00 \\ 8 & 8\end{array}$


INSTALLATION:ST. LUCIE $1 \& 2$

EFFLUENT AND WASTE DISPOSAL ANNUAL REPORT FOR YEAR 1989 SOLID EFFLUENTS

ESTIMATE OF MAJOR NUCLIDE COMPOSITION (BY TYPE OF WASTE)

JAN-JUNE JULY-DEC

D
CS -137
FE -55
$\mathrm{MN}-54$
NB -95
NI -63
ZR -95

$\begin{array}{lll}8 & 3.68 \mathrm{E}+01 & 2.58 \mathrm{E}+01 \\ 8 & 5.93 \mathrm{E}+00 & 1.52 \mathrm{E}+01 \\ 8 & 1.74 \mathrm{E}+00 & 1.84 \mathrm{E}+00 \\ 8 & 2.85 \mathrm{E}+00 & \\ 8 & 2.87 \mathrm{E}+00 & 3.87 \mathrm{E}+00 \\ 8 & 1.64 \mathrm{E}+00 & \end{array}$

TYPE OF WASTE

A. SPENT RESINS, FILTER SLUDGES, EVAPORATOR

UNIT YEAR TOTAL BOTTOMS, ETC.

M3 $\quad 5.05 \mathrm{E}+01$

CI $\quad 1.66 \mathrm{E}+02$

B. DRY COMPRESSIBLE WASTE, CONTAMINATED

M3 $2.26 \mathrm{E}+02 *$

EQUIPMENT, ETC

CI $\quad 1.72 \mathrm{E}+00$

C. IRRADIATED COMPONENTS, CONTROL

M3 $0.00 E+00$

RODS, ETC.

CI $\quad 0.00 E+00$

D. OTHER

M3 4.14E+01 *

NON-COMPRESSIBLE METAL (DAW)

CI $\quad 6.43 \mathrm{E}-01$

*TYPE B \& D VOLUMES AFTER REDUCTION (3.31E2, 1.42E3 RESPECTIVELY, BEFORE RED.) 
EFFLUENT AND WASTE DISPOSAL ANNUAL REPORT FOR YEAR 1989 AIRBORNE AND LIQUID EFFLUENTS

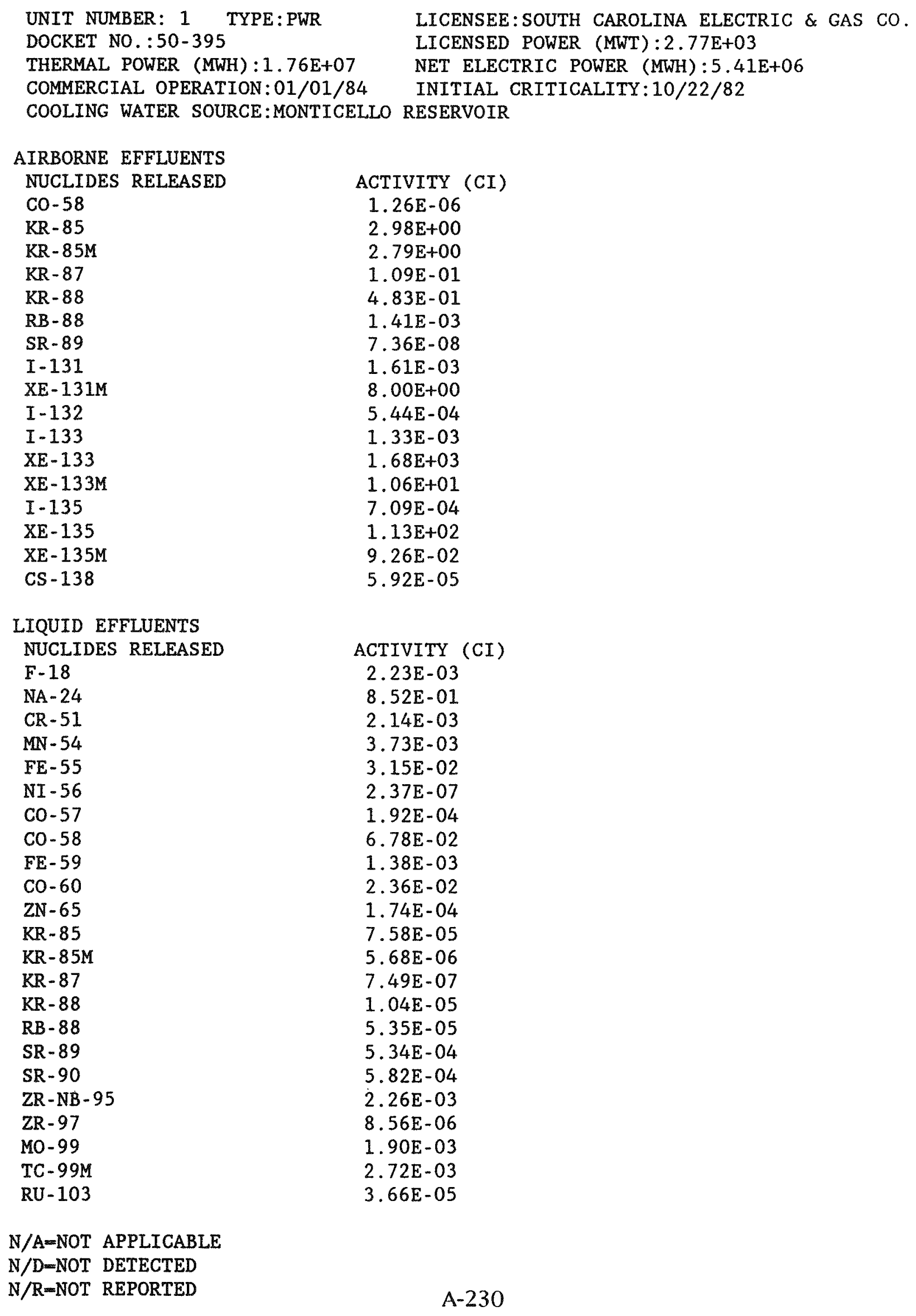


EFFLUENT AND WASTE DISPOSAL ANNUAL REPORT FOR YEAR 1989 AIRBORNE AND LIQUID EFFLUENTS

\begin{tabular}{|c|c|}
\hline NUCLIDES RELEASED & ACTIVITY (CI) \\
\hline $\mathrm{RU}-106$ & $6.35 E-04$ \\
\hline$A G-110 M$ & $1.40 E-04$ \\
\hline $\mathrm{SN}-113$ & $1.15 E-05$ \\
\hline$S B-124$ & $2.24 E-03$ \\
\hline$S B-125$ & $2.43 E-02$ \\
\hline$I-131$ & $1.22 \mathrm{E}-01$ \\
\hline$X E-131 M$ & $1.34 E-02$ \\
\hline$I-132$ & $2.38 E-02$ \\
\hline$I-133$ & $6.86 E-02$ \\
\hline$X E-133$ & $8.33 E-01$ \\
\hline$X E-133 M$ & $4.28 \mathrm{E}-03$ \\
\hline $\operatorname{CS}-134$ & $3.32 E-02$ \\
\hline$I-134$ & $1.27 \mathrm{E}-02$ \\
\hline$I-135$ & $3.73 E-02$ \\
\hline$X E-135$ & $3.39 E-03$ \\
\hline $\operatorname{CS}-136$ & $2.51 E-04$ \\
\hline $\operatorname{CS}-137$ & $4.19 E-02$ \\
\hline $\operatorname{CS}-138$ & $4.36 E-03$ \\
\hline$B A-L A-140$ & $6.11 E-04$ \\
\hline$C E-141$ & $4.97 E-06$ \\
\hline$C E-144$ & $5.79 E-04$ \\
\hline NP -239 & $3.89 E-06$ \\
\hline
\end{tabular}

TOTAL AIRBORNE TRITIUM RELEASE 1.05E-01

TOTAI LIQUID TRITIUM RELEASE $6.86 \mathrm{E}+02$

VOLUME OF LIQUID WASTE RELEASED (PRIOR TO DILUTION) LITERS $1.77 \mathrm{E}+08$

VOLUME OF DILUTION WATER USED DURING PERIOD

LITERS $\quad 1.57 \mathrm{E}+12$ 
EFFLUENT AND WASTE DISPOSAL ANNUAL REPORT FOR YEAR 1989 SOLID EFFLUENTS

SOLID WASTE DISPOSITION
NUMBER OF SHIPMENTS
* 60
MODE OF TRANSPORTATION
DESTINATION
TRUCK
BARNWELL SC

*INCLUDES 54 SHIPMENTS OF DAW FROM WASTE PROCESSOR TO BARNWELL

IRRADIATED FUEL SHIPMENTS (DISPOSITION)

NUMBER OF SHIPMENTS MODE OF TRANSPORTATION

0

N/A

DESTINATION
N/A

ESTIMATE OF MAJOR NUCLIDE COMPOSITION (BY TYPE OF WASTE)

JAN - JUNE JULY - DEC

A

$$
\begin{aligned}
& \text { CE }-144 \\
& \text { CO }-58 \\
& \text { CO }-60 \\
& \text { CS }-134 \\
& \text { CS }-137 \\
& \text { FE }-55 \\
& \text { H- } 3 \\
& \text { MN }-54 \\
& \text { NI }-63 \\
& \text { C- } 14 \\
& \text { CO- } 58 \\
& \text { CO }-60 \\
& \text { CR }-51 \\
& \text { CS }-134 \\
& \text { CS }-137 \\
& \text { FE }-55 \\
& \text { H- } 3 \\
& \text { MN }-54 \\
& \text { NB }-95
\end{aligned}
$$

B

$\begin{array}{lll}8 & & 1.13 \mathrm{E}+00 \\ 8 & 4.87 \mathrm{E}+01 & 6.57 \mathrm{E}+00 \\ 8 & 6.65 \mathrm{E}+00 & 2.11 \mathrm{E}+01 \\ 8 & 1.23 \mathrm{E}+01 & 2.24 \mathrm{E}+00 \\ 8 & 9.03 \mathrm{E}+00 & 3.81 \mathrm{E}+00 \\ 8 & 1.42 \mathrm{E}+01 & 4.96 \mathrm{E}+01 \\ 8 & 5.09 \mathrm{E}+00 & \\ 8 & & 2.36 \mathrm{E}+00 \\ 8 & 2.30 \mathrm{E}+00 & 1.08 \mathrm{E}+01 \\ & & \\ 8 & 1.42 \mathrm{E}+00 & 1.53 \mathrm{E}+00 \\ 8 & 2.14 \mathrm{E}+01 & 2.29 \mathrm{E}+01 \\ 8 & 7.55 \mathrm{E}+00 & 8.12 \mathrm{E}+00 \\ 8 & 3.69 \mathrm{E}+00 & 3.95 \mathrm{E}+00 \\ 8 & 5.99 \mathrm{E}+00 & 6.43 \mathrm{E}+00 \\ 8 & 5.10 \mathrm{E}+00 & 5.48 \mathrm{E}+00 \\ 8 & 4.56 \mathrm{E}+01 & 4.90 \mathrm{E}+01 \\ 8 & 3.41 \mathrm{E}+00 & \\ 8 & 1.14 \mathrm{E}+00 & 1.22 \mathrm{E}+00 \\ 8 & 1.23 \mathrm{E}+00 & 1.32 \mathrm{E}+00\end{array}$

TYPE OF WASTE

A. SPENT RESINS, FILTER SLUDGES, EVAPORATOR

UNIT YEAR TOTAL BOTTOMS, ETC.

M3 4.44E+01

CI $\quad 3.74 \mathrm{E}+02$

B. DRY COMPRESSIBLE WASTE, CONTAMINATED

M3 $9.60 \mathrm{E}+01$

EQUTPMENT, ETC.

C. IRRADIATED COMPONENTS, CONTROL

$1.85 E+00$

RODS, ETC.

M3

CI

D. OTHER

M3

CI 
EFFLUENT AND WASTE DISPOSAL ANNUAL REPORT FOR YEAR 1989 AIRBORNE AND LIQUID EFFLUENTS

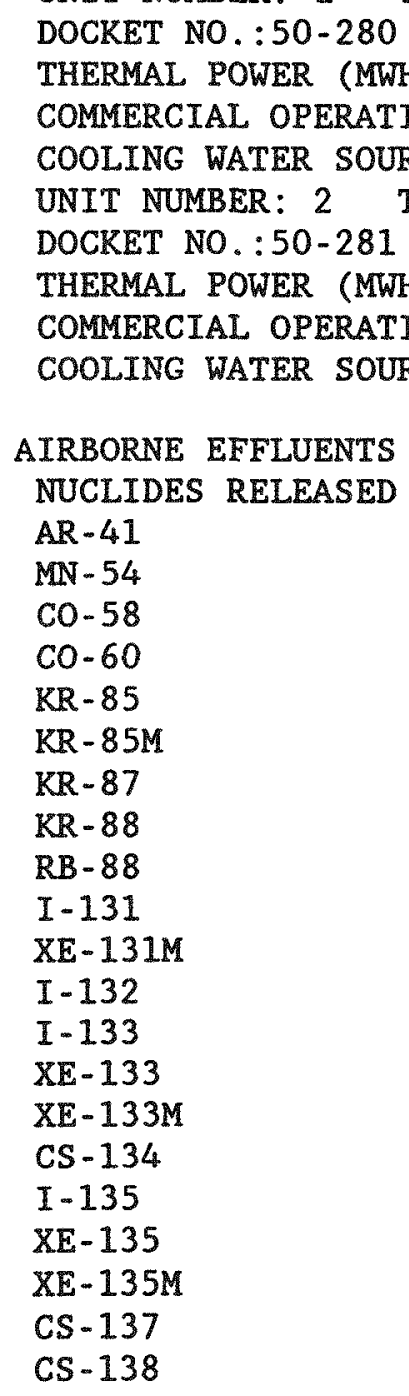

\section{AIRBORNE EFFLUENTS \\ NUCLIDES RELEASED \\ AR - 41 \\ MN -54 \\ CO- 58 \\ $\mathrm{CO}-60$ \\ $\mathrm{KR}-85$ \\ $\mathrm{KR}-85 \mathrm{M}$ \\ $\mathrm{KR}-87$ \\ $\mathrm{KR}-88$ \\ $\mathrm{RB}-88$ \\ I -131 \\ $\mathrm{XE}-131 \mathrm{M}$ \\ $I-132$ \\ I- 133 \\ $\mathrm{XE}-133$ \\ $\mathrm{XE}-133 \mathrm{M}$ \\ CS -134 \\ I-135 \\ $\mathrm{XE}-135$ \\ $\mathrm{XE}-135 \mathrm{M}$ \\ CS -137 \\ CS -138}

LICENSEE:VIRGINIA ELECTRIC \&POWER LICENSED POWER (MWT) :2.44E+03 NET ELECTRIC POWER (MWH) : $3.17 \mathrm{E}+06$ INITIAL CRITICALITY:07/01/72

LICENSEE:VIRGINIA ELECTRIC \&POWER LICENSED POWER (MWT) : $2.44 E+03$ NET ELECTRIC POWER (MWH) $: 8.94 E+05$ INITIAL CRITICALITY:03/07/73

\section{LIQUID EFFLUENTS}

NUCLIDES RELEASED

NA -22

NA -24

AR -41

CR -51

MN -54

FE -55

CO- 57

CO- 58

FE -59

CO- 60

ZN- 65

KR-85M

NB-95

ZR-95

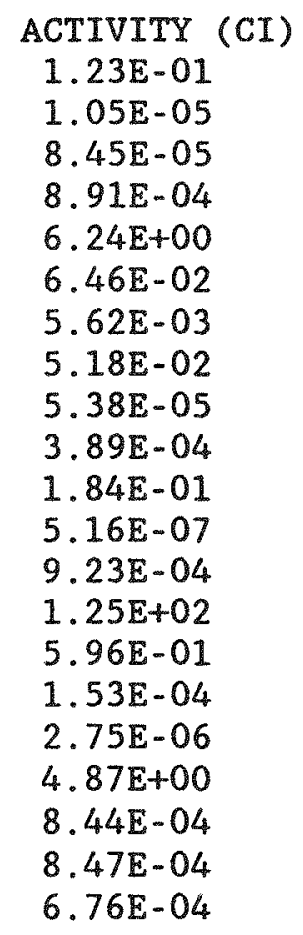

9.23E-04

1. $25 \mathrm{E}+02$

$5.96 \mathrm{E}-01$

1. $53 \mathrm{E}-04$

2. $75 \mathrm{E}-06$

$4.87 \mathrm{E}+00$

$8.44 \mathrm{E}-04$

8.47E-04

$6.76 \mathrm{E}-04$

ACTIVITY (CI)

5.95E-06

4. 32E-05

$6.42 E-05$

3. $54 \mathrm{E}-02$

1. 28E-02

4. $80 \mathrm{E}-01$

$5.54 \mathrm{E}-04$

$6.77 \mathrm{E}-02$

4. $19 E-04$

$5.45 \mathrm{E}-01$

1. $16 \mathrm{E}-04$

3. $76 \mathrm{E}-05$

$7.49 \mathrm{E}-03$

1. $67 \mathrm{E}-03$

$N / A=N O T$ APPLICABLE

$N / D=N O T$ DETECTED

$N / R=N O T$ REPORTED 
EFFLUENT AND WASTE DISPOSAL ANNUAL REPORT FOR YEAR 1989 AIRBORNE AND LIQUID EFFLUENTS

$\begin{array}{lc}\text { LIQUID EFFLUENTS } & \\ \text { NUCLIDES RELEASED } & \text { ACTIVITY (CI) } \\ \text { TC-99M } & 4.99 \mathrm{E}-05 \\ \text { RU-103 } & 6.18 \mathrm{E}-04 \\ \text { AG-110M } & 2.05 \mathrm{E}-02 \\ \text { SB }-124 & 1.19 \mathrm{E}-02 \\ \text { SB-125 } & 5.25 \mathrm{E}-01 \\ \mathrm{I}-131 & 8.74 \mathrm{E}-03 \\ \mathrm{TE}-132 & 1.62 \mathrm{E}-05 \\ \mathrm{I}-133 & 1.52 \mathrm{E}-03 \\ \mathrm{XE}-133 & 3.96 \mathrm{E}-01 \\ \mathrm{XE}-133 \mathrm{M} & 2.61 \mathrm{E}-03 \\ \mathrm{CS}-134 & 4.41 \mathrm{E}-01 \\ \mathrm{I}-134 & 4.63 \mathrm{E}-06 \\ \mathrm{I}-135 & 1.40 \mathrm{E}-04 \\ \mathrm{XE}-135 & 1.11 \mathrm{E}-01 \\ \text { XE }-135 \mathrm{M} & 7.80 \mathrm{E}-05 \\ \mathrm{CS}-137 & 1.71 \mathrm{E}+00 \\ \text { LA }-140 & 1.37 \mathrm{E}-04 \\ \mathrm{CE}-144 & 9.11 \mathrm{E}-05\end{array}$

TOTAL AIRBORNE TRITIUM RELEASE $2.75 \mathrm{E}+01$

TOTAL LIQUID TRITIUM RELEASE $4.29 \mathrm{E}+02$

VOLUME OF LIQUID WASTE RELEASED (PRIOR TO DILUTION) LITERS 2.95E+09

VOLUME OF DILUTION WATER USED DURING PERIOD

LITERS $1.13 \mathrm{E}+12$ 
EFFLUENT AND WASTE DISPOSAL ANNUAL REPORT FOR YEAR 1989 SOLID EFFLUENTS

SOLID WASTE DISPOSITION

$\begin{array}{cl}\text { NUMBER OF SHIPMENTS } & \text { MODE OF TRANSPORTATION } \\ 17 & \text { TRUCK } \\ 16 & \text { TRUCK } \\ 1 & \text { TRUCK }\end{array}$

ESTIMATE OF MAJOR NUCLIDE COMPOSITION (BY TYPE OF WASTE)

\author{
DESTINATION \\ BARNWELL SC \\ OAK RIDGE TN \\ RICHLAND WA
}

$$
\text { JAN-JUNE JULY-DEC }
$$

A

$\mathrm{CO}-58$

$\mathrm{CO}-60$

CS -134

CS -137

FE -55

MN -54

NI -63

B

CO- 58

CO- 60

CR -51 .

CS -134

CS -137

FE -55

$\mathrm{MN}-54$

NI -63

D

Co- 60

CS -137

FE- 55

NI -63

\section{TYPE OF WASTE}

A. SPENT RESINS, FILTER SLUDGES, EVAPORATOR BOTTOMS, ETC.

B. DRY COMPRESSIBLE WASTE, CONTAMINATED EQUTPMENT, ETC.

C. IRRADIATED COMPONENTS, CONTROL RODS, ETC.

D. OTHER

$\begin{array}{lll}8 & 6.38 \mathrm{E}+00 & 5.72 \mathrm{E}+00 \\ 8 & 3.19 \mathrm{E}+01 & 5.01 \mathrm{E}+01 \\ 8 & 2.22 \mathrm{E}+00 & 3.64 \mathrm{E}+00 \\ 8 & 5.05 \mathrm{E}+00 & 8.80 \mathrm{E}+00 \\ 8 & 1.47 \mathrm{E}+01 & 1.25 \mathrm{E}+01 \\ 8 & 2.63 \mathrm{E}+00 & \\ 8 & 3.60 \mathrm{E}+01 & 1.67 \mathrm{E}+01 \\ & & \\ 8 & 1.09 \mathrm{E}+01 & 1.12 \mathrm{E}+01 \\ 8 & 4.58 \mathrm{E}+01 & 5.25 \mathrm{E}+01 \\ 8 & 2.10 \mathrm{E}+00 & \\ 8 & 2.42 \mathrm{E}+00 & 3.34 \mathrm{E}+00 \\ 8 & 9.24 \mathrm{E}+00 & 1.36 \mathrm{E}+01 \\ 8 & 2.02 \mathrm{E}+01 & 9.31 \mathrm{E}+00 \\ 8 & 1.27 \mathrm{E}+00 & 1.55 \mathrm{E}+00 \\ 8 & 7.46 \mathrm{E}+00 & 7.72 \mathrm{E}+00 \\ & & \\ 8 & 3.59 \mathrm{E}+01 & \\ 8 & 1.57 \mathrm{E}+01 & \\ 8 & 9.03 \mathrm{E}+00 & \\ 8 & 3.70 \mathrm{E}+01 & \end{array}$

UNIT YEAR TOTAL

M3 $5.18 \mathrm{E}+01$

CI $\quad 1.28 \mathrm{E}+03$

M3 $4.82 E+02 *$

CI $\quad 2.90 \mathrm{E}+01$

M3 $0.00 E+00$

CI $\quad 0.00 \mathrm{E}+00$

M3 $3.70 \mathrm{E}+00$

CI $\quad 8.35 \mathrm{E}-01$

*NOT REPRESENTATIVE OF ACTUAL VOL. BURIED (4.68E+2 M3 SENT FOR VOL. RED.) 
EFFLUENT AND WASTE DISPOSAL ANNUAL REPORT FOR YEAR 1989 AIRBORNE AND LIQUID EFFLUENTS

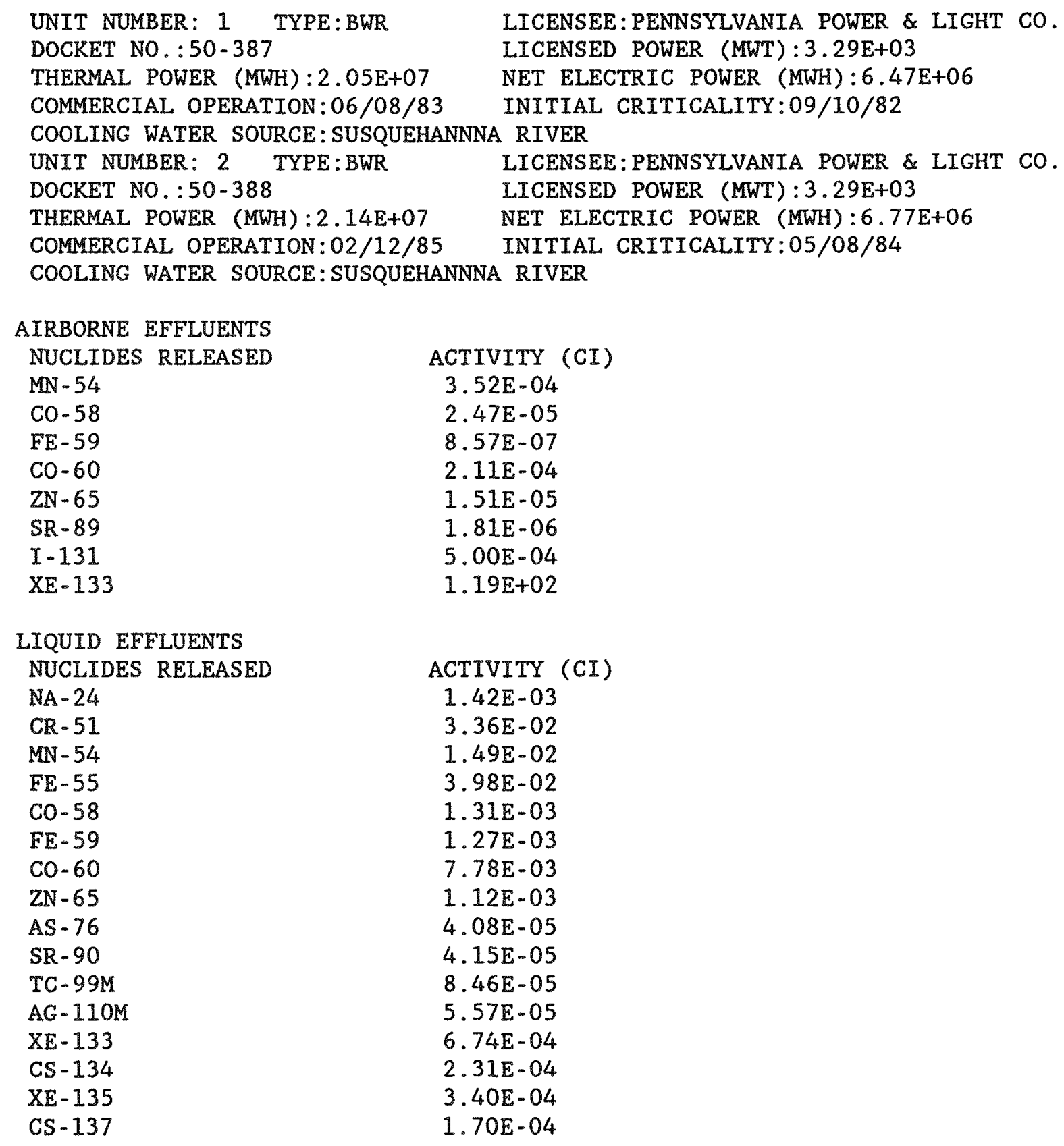

TOTAL AIRBORNE TRITIUM RELEASE $5.76 \mathrm{E}+01$

TOTAL LIQUID TRITIUM RELEASE $2.74 E+01$

VOLUME OF LIQUID WASTE RELEASED (PRIOR TO DILUTION) LITERS $1.03 E+07$ VOLUME OF DILUTION WATER USED DURING PERIOD IITERS $1.25 E+10$ 
EFFLUENT AND WASTE DISPOSAL ANNUAL REPORT FOR YEAR 1989 SOLID EFFLUENTS

SOLID WASTE DISPOSITION

NUMBER OF SHIPMENTS MODE OF TRANSPORTATION 45

TRUCK

ESTIMATE OF MAJOR NUCLIDE COMPOSITION (BY TYPE OF WASTE)

A

AG-110M

$\mathrm{C}-14$

$\mathrm{CO}-57$

CO- 58

$\mathrm{CO}-60$

CR -51

CS -134

CS -137

FE- 55

FE- 59

$\mathrm{H}-3$

I- 129

I- 131

KR -85

MN -54

NI -63

SB-124

SR -85

SR -90

TC- 99

$\mathrm{ZN}-65$

ZR-95

B

C. -14

CO- 58

CO- 60

CR -51

FE -55

FE- 59

H- 3

I -129

MN -54

NI -63

TC -99

ZN- 65

D

AG-110M

CO- 58
DESTINATION

BARNWELL SC

JAN-JUNE JULY-DEC

\begin{tabular}{|c|c|c|}
\hline & $1.72 \mathrm{E}-02$ & $2.41 E-02$ \\
\hline & $3.00 E-01$ & $5.64 E-02$ \\
\hline & $3.42 E-05$ & \\
\hline & $8.95 E-01$ & $6.68 \mathrm{E}-01$ \\
\hline & $9.64 E+00$ & $5.72 \mathrm{E}+00$ \\
\hline & $4.58 E+00$ & $2.83 E+00$ \\
\hline & $5.03 E-02$ & $4.29 \mathrm{E}-02$ \\
\hline & $3.11 E-02$ & $3.83 E-02$ \\
\hline & $6.57 E+01$ & 7. $31 E+01$ \\
\hline & $1.88 \mathrm{E}+00$ & $1.94 \mathrm{E}+00$ \\
\hline & $2.92 \mathrm{E}-02$ & $8.67 \mathrm{E}-03$ \\
\hline & $1.24 \mathrm{E}-02$ & $<6.57 \mathrm{E}-04$ \\
\hline 8 & $1.14 \mathrm{E}-03$ & $6.34 \mathrm{E}-04$ \\
\hline & $2.45 E+00$ & \\
\hline f & $1.23 E+01$ & $1.48 \mathrm{E}+01$ \\
\hline t & $3.26 E-01$ & $1.73 \mathrm{E}-01$ \\
\hline t & $3.00 E-03$ & \\
\hline 8 & $1.00 \mathrm{E}-02$ & \\
\hline 8 & $8.46 E-04$ & $1.07 \mathrm{E}-03$ \\
\hline 8 & $1.94 E-03$ & $<4.55 \mathrm{E}-04$ \\
\hline 8 & $1.69 E+00$ & $6.27 \mathrm{E}-01$ \\
\hline & $9.69 \mathrm{E}-03$ & \\
\hline 8 & $1.30 \mathrm{E}-02$ & $2.00 \mathrm{E}-02$ \\
\hline$\frac{8}{8}$ & $1.18 \mathrm{E}+00$ & $2.50 E-0$ \\
\hline 8 & $8.73 E+00$ & $7.84 E+0$ \\
\hline 8 & $2.55 E+00$ & $5.00 E-0$ \\
\hline 8 & $7.14 E+01$ & $7.95 \mathrm{E}+01$ \\
\hline 8 & $1.87 \mathrm{E}-01$ & $4.20 E-01$ \\
\hline 8 & $4.47 E-03$ & $<3.00 \mathrm{E}-03$ \\
\hline 8 & & $<3.00 \mathrm{E}-02$ \\
\hline 8 & $1.24 \mathrm{E}+01$ & $1.09 \mathrm{E}+01$ \\
\hline 8 & $3.15 \mathrm{E}-01$ & $1.00 \mathrm{E}-0$ \\
\hline 8 & $9.00 E-01$ & $<1.30 \mathrm{E}-0$ \\
\hline 8 & $2.37 E+00$ & $8.30 \mathrm{E}-0$ \\
\hline & $5.10 E-01$ & $2.10 \mathrm{E}-\mathrm{O}$ \\
\hline & $1.51 E+00$ & $1.48 \mathrm{E}+0$ \\
\hline
\end{tabular}


INSTALLATION: SUSQUEHANNA

EFFLUENT AND WASTE DISPOSAL ANNUAL REPORT FOR YEAR 1989 SOLID EFFLUENTS

ESTIMATE OF MAJOR NUCLIDE COMPOSITION (BY TYPE OF WASTE)

JAN-JUNE JULY-DEC

D

$\mathrm{CO}-60$

CR -51

CS -134

CS -137

FE -55

FE -59

MN -54

NI -63

SR -90

ZN -65

$\begin{array}{lll}8 & 1.52 E+01 & 1.29 E+01 \\ 8 & 1.57 E+01 & 1.01 E+00 \\ 8 & 3.20 E-01 & 6.90 E-01 \\ 8 & 2.90 E-01 & 4.60 E-01 \\ 8 & 3.80 E+01 & 5.81 E+01 \\ 8 & 1.86 E+00 & 8.70 E-01 \\ 8 & 2.29 E+01 & 1.76 E+01 \\ 8 & 4.70 E-01 & 4.30 E-01 \\ 8 & 2.00 E-02 & \\ 8 & 3.12 E+00 & 6.29 E+00\end{array}$

TYPE OF WASTE

A. SPENT RESINS, FILTER SLUDGES, EVAPORATOR

UNIT YEAR TOTAL BOTTOMS, ETC.

B. DRY COMPRESSIBLE WASTE, CONTAMINATED

M3 2.16E+02

CI $\quad 8.70 \mathrm{E}+02$

EQUIPMENT, ETC.

M3 $2.03 \mathrm{E}+02$

CI $2.70 E+01$

C. IRRADIATED COMPONENTS, CONTROL

M3 RODS, ETC.

D. OTHER

REACTOR WATER/FUEL CLEAN-UP

M3 $8.52 \mathrm{E}+00$

CI $\quad 4.41 \mathrm{E}+02$ 
INSTALLATION:THREE MILE ISLAND 1 LOCATION:10 MI SE HARRISBURG, PA

EFFLUENT AND WASTE DISPOSAL ANNUAL REPORT FOR YEAR 1989 AIRBORNE AND LIQUID EFFLUENTS

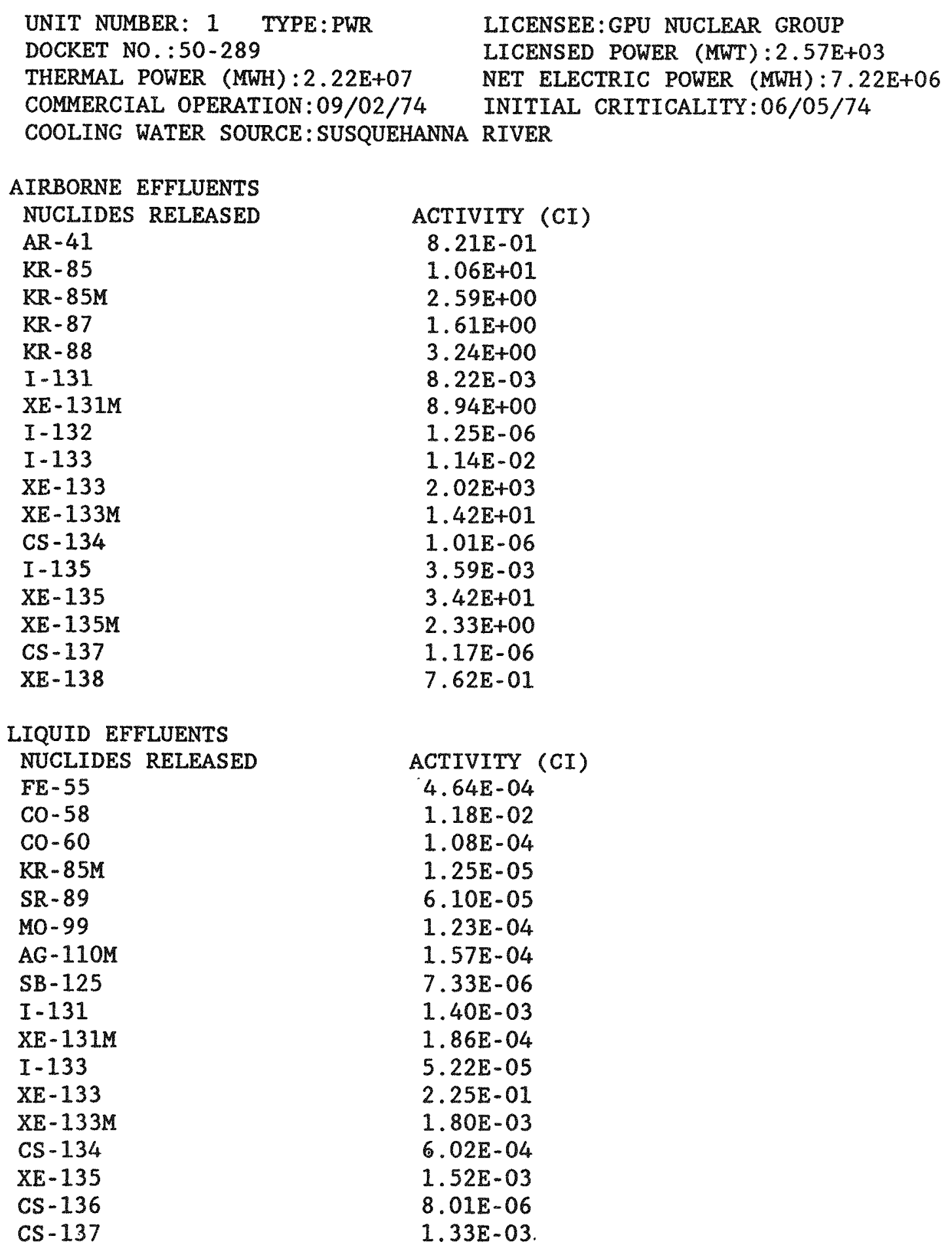

TOTAL AIRBORNE TRITIUM RELEASE 4.76E+00 TOTAL LIQUID TRITIUM RELEASE

$3.73 E+02$

VOLUME OF LIQUID WASTE RELEASED (PRIOR TO DILUTION) LITERS $3.94 \mathrm{E}+07$ VOLUME OF DILUTION WATER USED DURING PERIOD 
INSTALLATION:THREE MILE ISLAND 1

EFFLUENT AND WASTE DISPOSAL ANNUAL REPORT FOR YEAR 1989 SOLID EFFLUENTS

SOLID WASTE DISPOSITION NUMBER OF SHIPMENTS

$\begin{array}{rr} & 1 \\ & 1 \\ \# \quad 2 \\ \# \quad 1 \\ \# \quad 8\end{array}$

MODE OF TRANSPORTATION

TRACTOR-CASK

TRACTOR-CASK

TRACTOR-CLOSED VAN

TRACTOR-CLOSED VAN

TRACTOR - FLATBED

TRACTOR - FLATBED
DESTINATION

BARNWELL SC

HANFORD-RICHLAND WA

HANFORD-RICHLAND WA

SEG-OAK RIDGE TN

HANFORD-RICHLAND WA

SEG-OAK RIDGE TN

\#SENT TO WASTE PROCESSOR FOR VOL. RED.

ESTIMATE OF MAJOR NUCLIDE COMPOSITION (BY TYPE OF WASTE)

JAN-JUNE JULY-DEC

A

$\mathrm{CO}-58$

CR -51

CS -134

CS -137

$\mathrm{H}-3$

NI -63

B

CO- 58

CO- 60

CR -51

CS -134

CS -137

FE -55

NI -63

TYPE OF WASTE

A. SPENT RESINS, FILTER SLUDGES, EVAPORATOR BOTTOMS, ETC.

B. DRY COMPRESSIBLE WASTE, CONTAMINATED EQUTPMENT, ETC.

$$
\begin{aligned}
& 8 \\
& 8 \\
& 8 \\
& 8 \\
& 8
\end{aligned}
$$$$
8
$$$$
8
$$$$
8
$$$$
\text { 7. } 16 \mathrm{E}+01
$$$$
3.41 E+01
$$$$
6.30 \mathrm{E}+00
$$$$
8.86 \mathrm{E}+00
$$$$
5.33 E+00
$$$$
\text { 1. } 55 \mathrm{E}+01
$$$$
\text { 3. } 52 \mathrm{E}+00
$$$$
\text { 2. } 62 \mathrm{E}+01
$$

8

$\begin{array}{ll}3.96 \mathrm{E}+01 & 1.12 \mathrm{E}+01 \\ 6.47 \mathrm{E}+00 & 1.05 \mathrm{E}+01 \\ & 5.39 \mathrm{E}+00 \\ 1.65 \mathrm{E}+01 & 5.01 \mathrm{E}+01 \\ 5.99 \mathrm{E}+00 & \\ 1.07 \mathrm{E}+01 & 1.27 \mathrm{E}+01\end{array}$

UNIT YEAR TOTAL

M3 $9.48 \mathrm{E}+01$

CI $4.95 \mathrm{E}+01$

M3 2.37E+02 *

CI $\quad 9.87 \mathrm{E}-01$

M3

CI

M3

CI

$*$ VOLUME BEFORE REDUCTION 
EFFLUENT AND WASTE DISPOSAL ANNUAL REPORT FOR YEAR 1989

AIRBORNE AND LIQUID EFFLUENTS

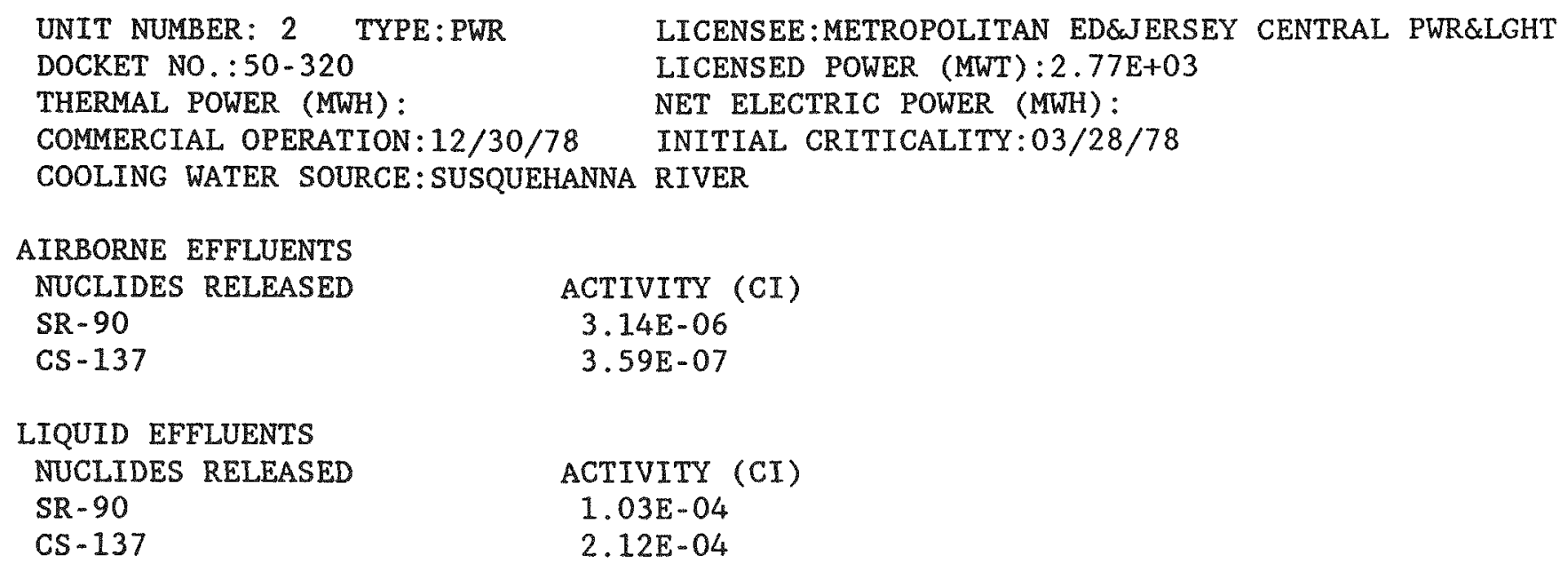

TOTAL AIRBORNE TRITIUM RELEASE $1.44 \mathrm{E}+01$

TOTAL LIQUID TRITIUM RELEASE 9.76E-04

VOLUME OF LIQUID WASTE RELEASED (PRIOR TO DILUTION) LITERS $4.55 E+05$

VOLUME OF DILUTION WATER USED DURING PERIOD LITERS $4.66 \mathrm{E}+10$ 
INSTALLATION:THREE MILE ISLAND $2 *$

EFFLUENT AND WASTE DISPOSAL ANNUAL REPORT FOR YEAR 1989 SOLID EFFLUENTS

SOLID WASTE DISPOSITION

NUMBER OF SHIPMENTS MODE OF TRANSPORTATION

$\begin{array}{rrl} & 11 & \text { TRACTOR - CASK } \\ & 10 & \text { TRACTOR-CASK } \\ & 2 & \text { TRACTOR-CLOSED VAN } \\ \# & 1 & \text { TRACTOR - CLOSED VAN } \\ & 2 & \text { TRACTOR-FLATBED } \\ & 16 & \text { TRACTOR - FLATBED }\end{array}$

\#SENT TO WASTE PROCESSOR FOR VOL. RED.

IRRADTATED FUEL SHIPMENTS (DISPOSITION)

NUMBER OF SHIPMENTS MODE OF TRANSPORTATION 12

RAILCAR-CASK

ESTIMATE OF MAJOR NUCLIDE COMPOSITION (BY TYPE OF WASTE)

A

CS -134

CS -137

NI -63

PM -147

SR -90

$B$

CS -137

PM-147

PU-241

SB -125

SR -90

\section{TYPE OF WASTE}
A. SPENT RESINS, FILTER SLUDGES, EVAPORATOR BOTTOMS, ETC.
B. DRY COMPRESSIBLE WASTE, CONTAMINATED EQUIPMENT, ETC.
C. IRRADIATED COMPONENTS, CONTROL RODS, ETC.
D. OTHER

\author{
DESTINATION \\ BARNWELL SC \\ HANFORD-RICHLAND WA \\ HANFORD - RICHLAND WA \\ SEG-OAK RIDGE TN \\ HANFORD-RICHLAND WA \\ SEG-OAK RIDGE TN
}

DESTINATION
INEL-SCOVILLE ID

JAN-JUNE JULY-DEC

$\begin{array}{lll}8 & 7.44 \mathrm{E}-01 & \\ 8 & 4.68 \mathrm{E}+01 & 1.34 \mathrm{E}+01 \\ 8 & & 1.15 \mathrm{E}+00 \\ 8 & 7.58 \mathrm{E}+00 & 1.06 \mathrm{E}+01 \\ 8 & 4.30 \mathrm{E}+01 & 7.33 \mathrm{E}+01 \\ & & \\ 8 & 2.61 \mathrm{E}+01 & 2.06 \mathrm{E}+01 \\ 8 & 8.63 \mathrm{E}+00 & 7.61 \mathrm{E}+00 \\ 8 & 3.98 \mathrm{E}+00 & 4.51 \mathrm{E}+00 \\ 8 & 1.97 \mathrm{E}+00 & 1.57 \mathrm{E}+00 \\ 8 & 5.33 \mathrm{E}+01 & 6.06 \mathrm{E}+01\end{array}$

UNIT YEAR TOTAL

M3 $5.58 \mathrm{E}+01$

CI $1.39 \mathrm{E}+04$

M3 1.12E+03

CI $1.53 \mathrm{E}+01$

M3

CI

M3

CI

*INCLUDING EPICOR II 
EFFLUENT AND WASTE DISPOSAL ANNUAL REPORT FOR YEAR 1989 AIRBORNE AND LIQUID EFFLUENTS

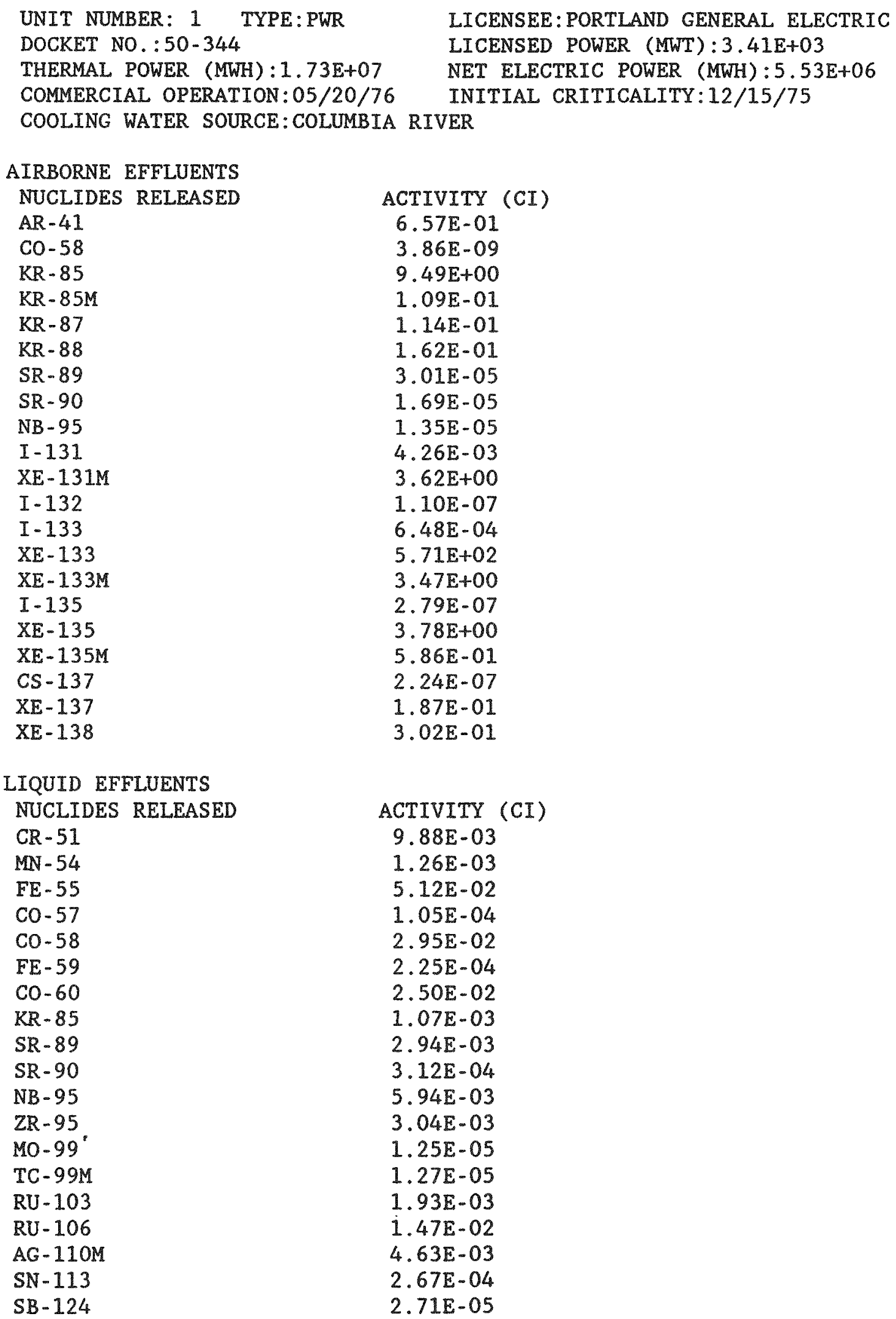

$N / A=N O T$ APPLICABLE $N / D=$ NOT DETECTED $\mathrm{N} / \mathrm{R}=\mathrm{NOT}$ REPORTED 
EFFLUENT AND WASTE DISPOSAL ANNUAL REPORT FOR YEAR 1989 AIRBORNE AND LIQUID EFFLUENTS

\author{
LIQUID EFFLUENTS \\ NUCLIDES RELEASED \\ SB -125 \\ $I-131$ \\ $\mathrm{XE}-131 \mathrm{M}$ \\ $I-133$ \\ $\mathrm{XE}-133$ \\ $\mathrm{XE}-133 \mathrm{M}$ \\ CS -134 \\ $\mathrm{XE}-135$ \\ CS -137 \\ LA -140 \\ CE -141 \\ CE -144 \\ W- 187 \\ UNIDENTIFIED
}
ACTIVITY (CI)
$2.01 E-03$
$7.14 \mathrm{E}-04$
$1.41 \mathrm{E}-03$
2. $24 \mathrm{E}-05$
2. $63 \mathrm{E}-01$
3. $72 \mathrm{E}-03$
$9.43 \mathrm{E}-04$
$4.95 E-03$
1. $50 \mathrm{E}-03$
2. $55 \mathrm{E}-04$
3. $24 \mathrm{E}-04$
$4.46 \mathrm{E}-03$
$1.69 \mathrm{E}-04$
$2.46 \mathrm{E}-05$

TOTAL AIRBORNE TRITIUM RELEASE 7.31E+01

TOTAL LIQUID TRITIUM RELEASE $3.18 E+02$

VOLUME OF LIQUID WASTE RELEASED (PRIOR TO DILUTION) LITERS 5.56E+07

VOLUME OF DILUTION WATER USED DURING PERIOD

LITERS $\quad 6.19 \mathrm{E}+10$ 
EFFLUENT AND WASTE DISPOSAL ANNUAL REPORT FOR YEAR 1989 SOLID EFFLUENTS

SOLID WASTE DISPOSITION NUMBER OF SHIPMENTS 2
14
2
MODE OF TRANSPORTATION
EXCLUSIVE TRUCK
EXCLUSIVE TRUCK
EXCLUSIVE TRUCK

ESTIMATE OF MAJOR NUCLIDE COMPOSITION (BY TYPE OF WASTE)

A
$A G-110 M$
C- 14
CE- 144
CM-242
$\mathrm{CO}-57$
CO- 58
CO- 60
CR -51
CS -134
CS -137
FE -55
FE- 59
H-3
I -131
MN -54
NB- 95
NI -63
PU- 238
PU- 239
PU -240
PU-241
RU-103
RU-106
SB-125
SR -89
SR -90
ZR-95
AG-110M
C. -14
CE -144
CO- 58
CO- 60
CR -51
CS -134
CS -137

B

\section{DESTINATION}

ALLIED NUCLEAR, RICHLAND WA RICHLAND WA

US ECOLOGY, RICHLAND WA

JAN-JUNE JULY-DEC

$$
\begin{array}{ll}
2.97 E-03 & 9.53 E-02 \\
8.45 E-02 & 1.49 E+00 \\
8.80 E-02 & 7.94 E-01 \\
& 3.18 E-02 \\
2.74 E-01 & \\
1.74 E+01 & 5.97 E+00 \\
2.83 E+01 & 6.00 E+00 \\
4.79 E-03 & 9.50 E-02 \\
4.10 E+00 & 2.48 E+00 \\
9.32 E+00 & 3.02 E+00 \\
1.10 E+01 & 1.39 E+01 \\
4.57 E-04 & \\
7.39 E-01 & 5.52 E+01 \\
2.28 E-04 & \\
9.44 E-01 & 6.35 E-01 \\
2.74 E-03 & 3.49 E-01 \\
2.55 E+01 & 5.27 E+00 \\
3.65 E-03 & 3.18 E-02 \\
4.11 E-03 & 3.18 E-02 \\
& 3.18 E-02 \\
2.75 E-01 & 2.32 E+00 \\
6.85 E-04 & 3.18 E-02 \\
2.56 E-01 & 1.30 E+00 \\
4.02 E-01 & 3.81 E-01 \\
2.28 E-04 & 3.18 E-02 \\
2.30 E+00 & 3.18 E-02 \\
2.97 E-03 & 5.08 E-01
\end{array}
$$

\% $\quad 1.25 \mathrm{E}-01$

8

8

1. $33 \mathrm{E}+01$

$5.45 E+00$

9. $65 \mathrm{E}-01$

$1.99 \mathrm{E}+00$

$4.17 \mathrm{E}+00$

$1.01 \mathrm{E}+01$

$5.70 \mathrm{E}+00$

1. $01 \mathrm{E}+01$

1. $34 \mathrm{E}+00$

3. $12 \mathrm{E}+00$

3. $74 \mathrm{E}-01$

$5.60 \mathrm{E}-01$

$4.13 E-01$ 
EFFLUENT AND WASTE DISPOSAL ANNUAL REPORT FOR YEAR 1989 SOLID EFFLUENTS

ESTIMATE OF MAJOR NUCLIDE COMPOSITION (BY TYPE OF WASTE)

JAN-JUNE JULY-DEC

B
FE -55
$\mathrm{H}-3$
$\mathrm{MN}-54$
NB -95
NI -63
$\mathrm{PU}-238$
PU -239
PU -240
PU -241
RU -103
$\mathrm{RU}-106$
$\mathrm{SB}-125$
SR -89
ZR-95

$\begin{array}{lll}8 & 1.46 \mathrm{E}+01 & 2.83 \mathrm{E}+01 \\ 8 & 4.05 \mathrm{E}+01 & 1.65 \mathrm{E}+01 \\ 8 & 2.74 \mathrm{E}+00 & 9.76 \mathrm{E}-01 \\ 8 & 1.15 \mathrm{E}+00 & 3.72 \mathrm{E}+00 \\ 8 & 2.55 \mathrm{E}+00 & 4.66 \mathrm{E}+00 \\ 8 & & 3.75 \mathrm{E}-02 \\ 8 & & 3.75 \mathrm{E}-02 \\ 8 & & 3.75 \mathrm{E}-02 \\ 8 & 1.37 \mathrm{E}+00 & 2.78 \mathrm{E}+00 \\ 8 & 4.36 \mathrm{E}-01 & 4.51 \mathrm{E}-01 \\ 8 & 1.81 \mathrm{E}+00 & 2.85 \mathrm{E}+00 \\ 8 & 6.20 \mathrm{E}-02 & 2.63 \mathrm{E}-01 \\ 8 & 7.38 \mathrm{E}+00 & 5.74 \mathrm{E}+00 \\ 8 & 9.02 \mathrm{E}-01 & 2.44 \mathrm{E}+00\end{array}$

TYPE OF WASTE

A. SPENT RESINS, FILTER SLUDGES, EVAPORATOR BOTTOMS, ETC.

B. DRY COMPRESSIBLE WASTE, CONTAMINATED EQUIPMENT, ETC.

UNIT YEAR TOTAL

M3 8.20E+01

CI $\quad 4.41 \mathrm{E}+02$

M3 $1.77 \mathrm{E}+02$

$\mathrm{CI}$

$5.86 \mathrm{E}+00$

C. IRRADIATED COMPONENTS, CONTROL

M3

RODS, ETC.

CI

M3

D. OTHER

CI 
EFFLUENT AND WASTE DISPOSAL ANNUAL REPORT FOR YEAR 1989 AIRBORNE AND LIQUID EFFLUENTS

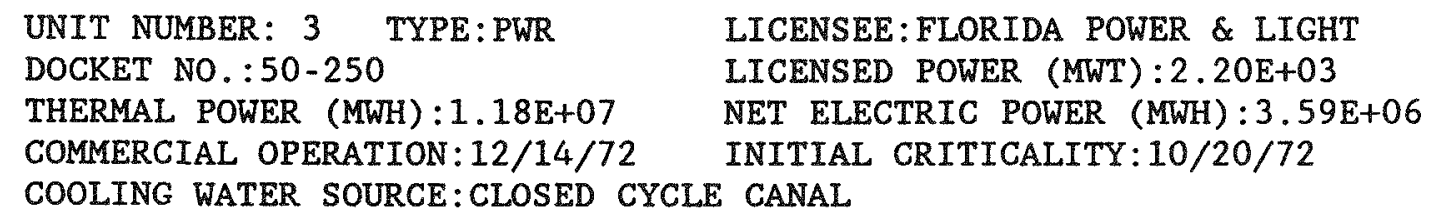

\section{LIQUID EFFLUENTS} NUCLIDES RELEASED

NA -24

CR -51

MN -54

FE- 55

CO -57

CO- 58

FE- 59

CO -60

$\mathrm{ZN}-65$

$\mathrm{KR}-85$

KR $-85 M$

SR -90

NB -95

ZR-97

AG-110

$\mathrm{SN}-117 \mathrm{M}$

SB- 124

$S B-125$

I- 131

$\mathrm{XE}-131 \mathrm{M}$

ACTIVITY (CI)

3. 36E-05

5. $24 \mathrm{E}-04$

$1.87 \mathrm{E}-02$

$3.49 \mathrm{E}-02$

$1.45 \mathrm{E}-05$

1. $26 \mathrm{E}-02$

1. $78 \mathrm{E}-05$

3. $13 \mathrm{E}-02$

3. $70 \mathrm{E}-05$

$7.55 \mathrm{E}-07$

3. $73 \mathrm{E}-05$

4. $85 \mathrm{E}-04$

8.12E-05

2. 58E-06

$2.12 \mathrm{E}-03$

3. $53 \mathrm{E}-06$

1. $02 \mathrm{E}-03$

$3.45 \mathrm{E}-02$

$5.56 E-04$

1. $32 E-02$

6. 71E-05

8. $82 \mathrm{E}-01$

4. $57 \mathrm{E}-03$

5. $32 E-03$

1. 15E-03

3. $78 \mathrm{E}-05$

$N / A=N O T$ APPLICABLE 
EFFLUENT AND WASTE DISPOSAL ANNUAL REPORT FOR YEAR 1989 AIRBORNE AND LIQUID EFFLUENTS

\section{LIQUID EFFLUENTS}

NUCLIDES RELEASED

CS. -137

$\mathrm{CE}-144$

W-187
ACTIVITY (CI)
$1.58 \mathrm{E}-02$
$4.56 \mathrm{E}-06$
$2.87 E-06$

TOTAL AIRBORNE TRITIUM RELEASE 4.23E +00

TOTAL LIQUID TRITIUM RELEASE $2.29 E+02$

VOLUME OF LIQUID WASTE RELEASED (PRIOR TO DILUTION) LITERS $7.02 E+06$

VOLUME OF DILUTION WATER USED DURING PERIOD

LITERS $\quad 2.62 \mathrm{E}+11$ 
EFFLUENT AND WASTE DISPOSAL ANNUAL REPORT FOR YEAR 1989 AIRBORNE AND LIQUID EFFLUENTS

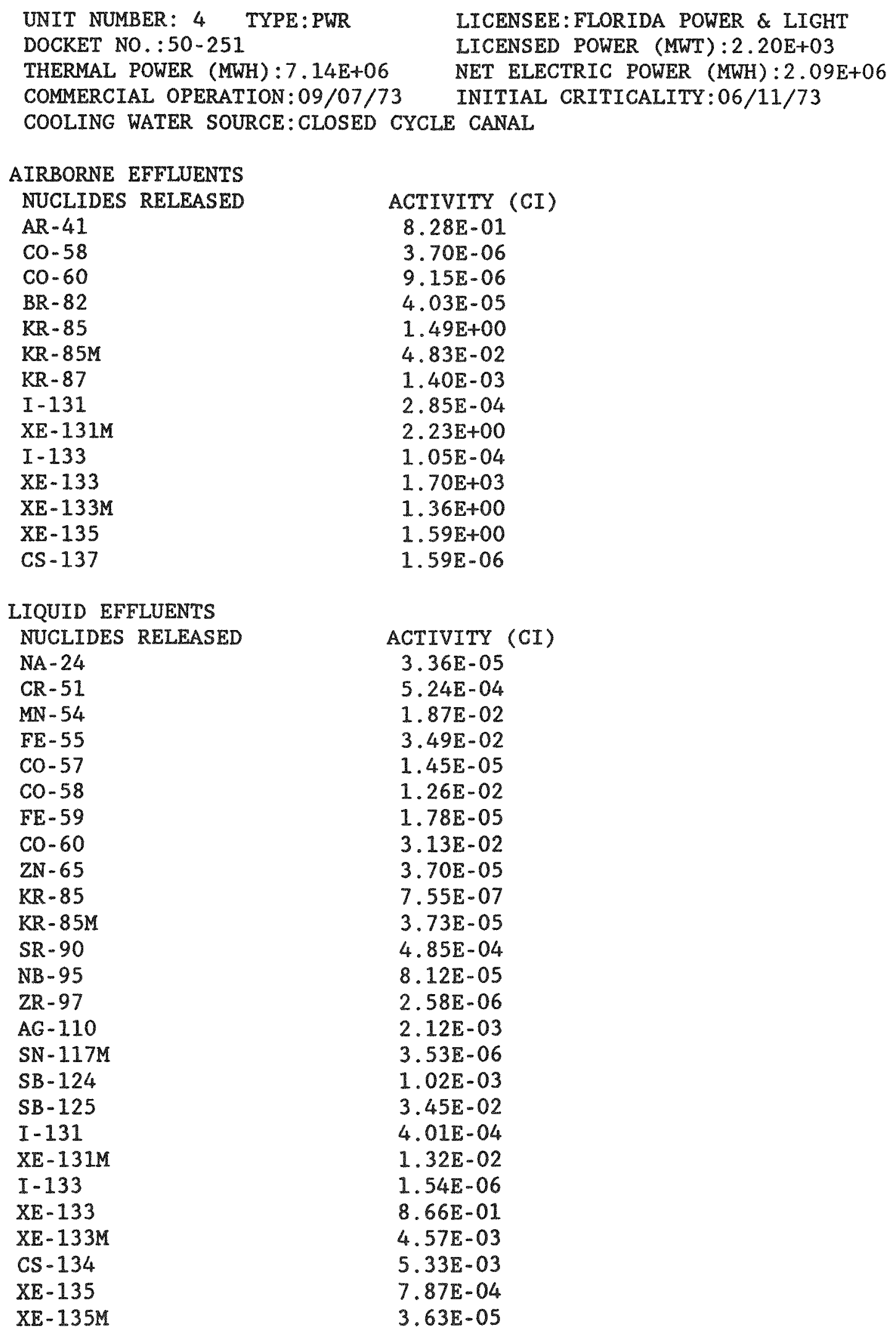

N/A $=$ NOT APPLICABLE

$N / D=N O T$ DETECTED

$N / R=N O T$ REPORTED 
EFFLUENT AND WASTE DISPOSAL ANNUAL REPORT FOR YEAR 1989 AIRBORNE AND LIQUID EFFLUENTS

LIQUID EFFLUENTS

NUCLIDES RELEASED

CS -137

CE -144

W-187
ACTIVITY (CI)

$1.58 \mathrm{E}-02$

4. $56 \mathrm{E}-06$

$2.87 \mathrm{E}-06$

TOTAL AIRBORNE TRITIUM RELEASE 4.36E+00

TOTAL LIQUID TRITIUM RELEASE

2. $29 \mathrm{E}+02$

VOLUME OF LIQUID WASTE RELEASED (PRIOR TO DILUTION)

LITERS

$7.02 \mathrm{E}+06$

VOLUME OF DILUTION WATER USED DURING PERIOD

LITERS

2. $62 \mathrm{E}+11$ 
EFFLUENT AND WASTE DISPOSAL ANNUAL REPORT FOR YEAR 1989 SOLID EFELUENTS

SOLID WASTE DISPOSITION NUMBER OF SHIPMENTS 35

MODE OF TRANSPORTATION SOLE USE TRUCK

ESTIMATE OF MAJOR NUCLIDE COMPOSITION (BY TYPE OF WASTE)

B

$\mathrm{C}-14$

$\mathrm{CO}-57$

$\mathrm{CO}-58$

$\mathrm{CO}-60$

CS -134

CS -137

FE -55

$\mathrm{H}-3$

MN -54

NB -95

NI -63

SB -125

D

AG-110M

C -14

$\mathrm{CO}-60$

CS -137

$F E-55$

NI -63
DESTINATION

OAK RIDGE TN

JAN-JUNE JULY-DEC

$\begin{array}{lll}8 & 1.00 \mathrm{E}+01 & \\ 8 & & 1.00 \mathrm{E}+00 \\ 8 & 1.40 \mathrm{E}+01 & 1.00 \mathrm{E}+00 \\ 8 & 4.00 \mathrm{E}+01 & 6.00 \mathrm{E}+01 \\ 8 & 2.00 \mathrm{E}+00 & 1.00 \mathrm{E}+00 \\ 8 & 5.00 \mathrm{E}+00 & 3.00 \mathrm{E}+00 \\ 8 & 1.70 \mathrm{E}+01 & 1.90 \mathrm{E}+01 \\ 8 & & 1.00 \mathrm{E}+00 \\ 8 & 1.00 \mathrm{E}+00 & 1.00 \mathrm{E}+00 \\ 8 & 1.00 \mathrm{E}+00 & \\ 8 & 7.00 \mathrm{E}+00 & 1.10 \mathrm{E}+01 \\ 8 & 2.00 \mathrm{E}+00 & 2.00 \mathrm{E}+00\end{array}$

8 $1.00 \mathrm{E}+00 \quad 1.00 \mathrm{E}+00$

8 $3.00 \mathrm{E}+00 \quad 2.00 \mathrm{E}+00$

\& $5.40 \mathrm{E}+01 \quad 4.60 \mathrm{E}+01$

\& $2.20 \mathrm{E}+01 \quad 3.60 \mathrm{E}+01$

$3.00 \mathrm{E}+00 \quad 3.00 \mathrm{E}+00$

$8 \quad 1.60 \mathrm{E}+01 \quad 1.20 \mathrm{E}+01$

UNIT YEAR TOTAL

M3 $0.00 E+00$

CI $\quad 0.00 \mathrm{E}+00$

M3 2.26E+02*

CI $\quad 8.90 \mathrm{E}-01$

M3 $0.00 \mathrm{E}+00$

CI $\quad 0.00 \mathrm{E}+00$

M3 1.21E+02*

CI $\quad 1.37 \mathrm{E}+00$

*TYPE B\&D WASTE ARE VOL. BURIED (259.9 M3, 596. M3 RESPECTIVELY; BEFORE REDUCTION) 
EFFLUENT AND WASTE DISPOSAL ANNUAL REPORT FOR YEAR 1989 AIRBORNE AND LIQUID EFFLUENTS

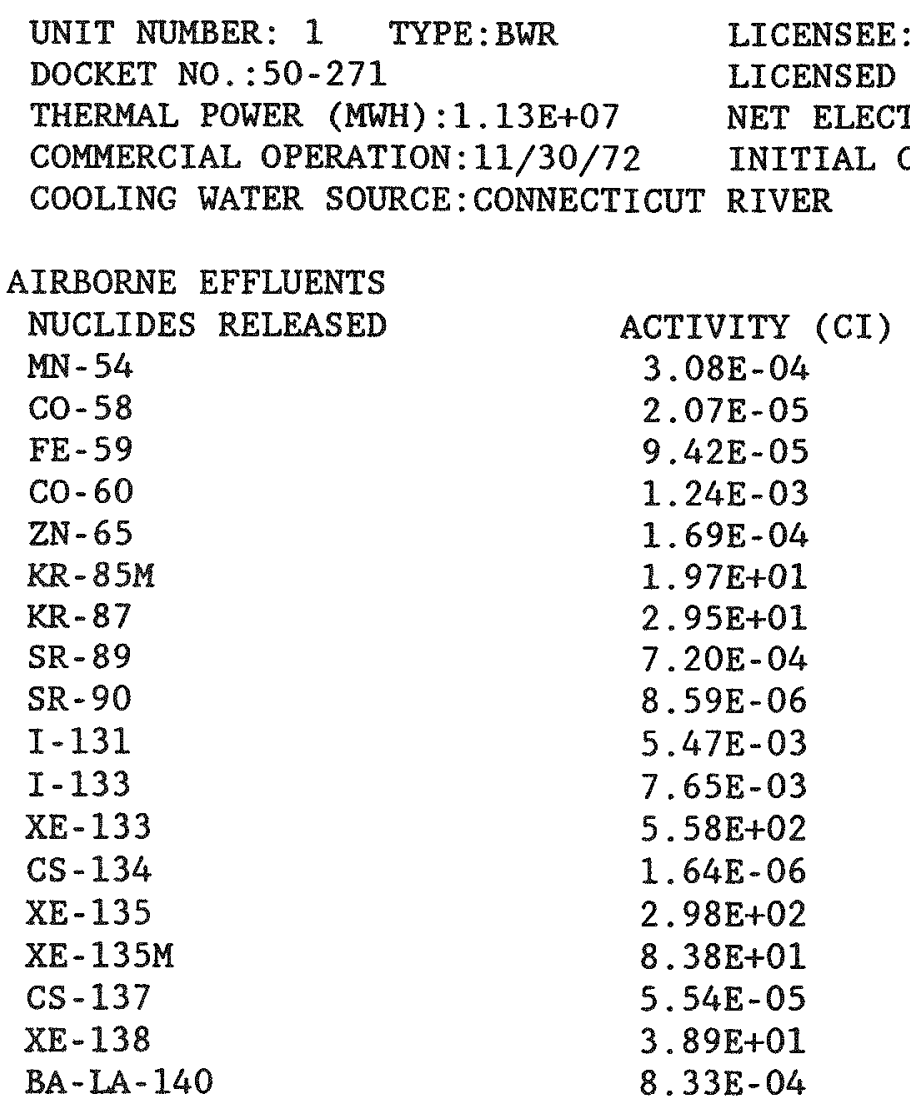

TOTAL AIRBORNE TRITIUM RELEASE 5.53E+01

TOTAL LIQUID TRITIUM RELEASE $0.00 E+00$
LITERS $\quad 0.00 \mathrm{E}+00$

LITERS $\quad 0.00 \mathrm{E}+00$ 
INSTALLATION:VERMONT YANKEE

EFFLUENT AND WASTE DISPOSAL ANNUAL REPORT FOR YEAR 1989 SOLID EFFLUENTS

SOLID WASTE DISPOSITION

NUMBER OF SHIPMENTS MODE OF TRANSPORTATION

TRUCK

DESTINATION

BARNWELL SC

ESTIMATE OF MAJOR NUCLIDE COMPOSITION (BY TYPE OF WASTE)

JAN-JUNE JULY-DEC

A

C -14

CM -242

$\mathrm{CO}-60$

CS -134

CS -137

FE -55

$\mathrm{H}-3$

I -129

I -131

$\mathrm{MN}-54$

NI -63

PU-241

TC- 99

TRANSURANICS

$\mathrm{ZN}-65$

$\begin{array}{ll}8 & 1.49 E-01 \\ 8 & 9.30 E-05 \\ 8 & 1.71 E+01 \\ 8 & 5.55 E+00 \\ 8 & 2.20 E+01 \\ 8 & 1.97 E+01 \\ 8 & 1.38 E+00 \\ 8 & 3.12 E-03 \\ 8 & 5.33 E-01 \\ 8 & 3.11 E+00 \\ 8 & 7.00 E+00 \\ 8 & 2.60 E-01 \\ 8 & 3.67 E-03 \\ 8 & 3.91 E-03 \\ 8 & 2.32 E+01\end{array}$

TYPE OF WASTE

A. SPENT RESINS, FILTER SLUDGES, EVAPORATOR BOTTOMS, ETC.

UNIT YEAR TOTAL

M3 4.84E+00

CI $\quad 2.15 \mathrm{E}+00$

B. DRY COMPRESSIBLE WASTE, CONTAMINATED

M3

EQUIPMENT, ETC.

CI

C. IRRADIATED COMPONENTS, CONTROL

M3

RODS, ETC.

CI

D. OTHER

M3

CI 
EFFLUENT AND WASTE DISPOSAL ANNUAL REPORT FOR YEAR 1989 AIRBORNE AND LIQUID EFFLUENTS

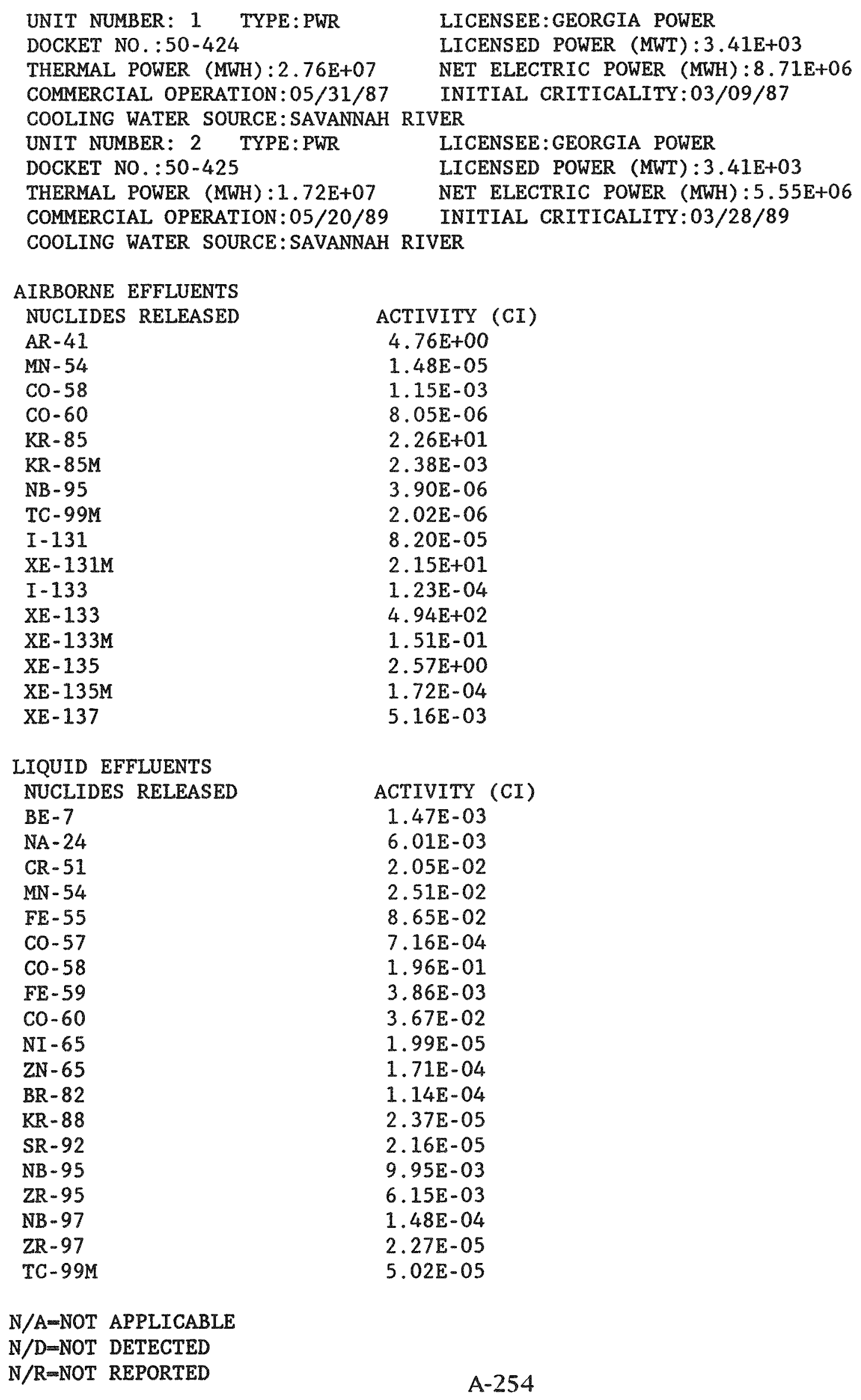

N/A $=$ NOT APPLICABLE 
EFFLUENT AND WASTE DISPOSAL ANNUAL REPORT FOR YEAR 1989 AIRBORNE AND LIQUID EFFLUENTS

LIQUID EFFLUENTS
NUCLIDES RELEASED
RU-103
AG-110M
SB-124
SB-125
TE-125M
I-131
XE-131M
TE-132
I-133
XE -133
XE $-133 M$
CS -134
XE-135
CS -137
BA-140
LA -140
CE -144
HF -181
W-187
AU -198

ACTIVITY (CI)
$7.50 E-06$
$3.70 E-05$
$1.10 E-03$
$4.03 E-03$
$7.21 E-04$
$1.09 E-03$
$8.77 E-05$
$2.78 E-08$
$6.37 E-04$
$1.49 E-01$
$3.36 E-04$
$8.00 E-04$
$8.62 E-03$
$1.52 E-03$
$5.47 E-08$
$1.13 E-04$
$3.47 E-05$
$1.55 E-04$
$4.36 E-05$
$1.30 E-05$

$7.50 \mathrm{E}-06$

$3.70 \mathrm{E}-05$

$1.10 \mathrm{E}-03$

$1.09 \mathrm{E}-03$

$8.77 \mathrm{E}-05$

$2.78 \mathrm{E}-08$

$6.37 E-04$

$1.49 \mathrm{E}-01$

$3.36 \mathrm{E}-04$

$8.00 E-04$

$8.62 E-03$

1. $52 \mathrm{E}-03$

$5.47 \mathrm{E}-08$

$1.13 \mathrm{E}-04$

$3.47 \mathrm{E}-05$

1.30E-05

TOTAL AIRBORNE TRITIUM RELEASE 9.10E+02

TOTAL LIQUID TRITIUM RELEASE $9.18 \mathrm{E}+02$

$\begin{array}{llll}\text { VOLUME OF LIQUID WASTE RELEASED (PRIOR TO DILUTION) } & \text { LITERS } & 9.94 \mathrm{E}+06 \\ \text { VOLUME OF DILUTION WATER USED DURING PERIOD } & \text { LITERS } & 5.19 \mathrm{E}+09\end{array}$ 
INSTALLATION:VOGTLE

EFFLUENT AND WASTE DISPOSAL ANNUAL REPORT FOR YEAR 1989 SOLID EFFLUENTS

SOLID WASTE DISPOSITION NUMBER OF SHIPMENTS 7

MODE OF TRANSPORTATION TRACTOR TRAILER

TRACTOR/SHIELDED CASK

DESTINATION

SEG, OAK RIDGE TN

BARNWELL SC

IRRADIATED FUEL SHIPMENTS (DISPOSITION)

NUMBER OF SHIPMENTS MODE OF TRANSPORTATION

0

$\mathrm{N} / \mathrm{A}$

DESTINATION

$\mathrm{N} / \mathrm{A}$

JAN-JUNE JULY-DEC

A

Co- 58

FE- 55

OTHERS

B

$\mathrm{CO}-58$

Co- 60

FE- 55

OTHERS

8 $\quad 3.02 \mathrm{E}+01$

3. $29 E+01$

8 $2.44 \mathrm{E}+01$

2. $38 \mathrm{E}+01$

\& $4.54 E+01$

4. $32 \mathrm{E}+01$

$8.15 \mathrm{E}+01$

\% $\quad 3.75 \mathrm{E}+01$

\& $2.65 \mathrm{E}+01$

\& $3.60 \mathrm{E}+01$

2. $59 \mathrm{E}+01$

3. $26 \mathrm{E}+01$

TYPE OF WASTE

A. SPENT RESINS, FILTER SLUDGES, EVAPORATOR

UNIT YEAR TOTAL BOTTOMS, ETC.

B. DRY COMPRESSIBLE WASTE, CONTAMINATED EQUTPMENT, ETC.

M3 $\quad 3.01 \mathrm{E}+01$

CI $\quad 1.49 \mathrm{E}+01$

M3 7.01E+01*

CI $\quad 2.07 \mathrm{E}-01$

C. IRRADIATED COMPONENTS, CONTROL

M3 RODS, ETC.

$\mathrm{CI}$

D. OTHER

M3

CI

*VOLUME AFTER COMPACTION 
EFFLUENT AND WASTE DISPOSAL ANNUAL REPORT FOR YEAR 1989 AIRBORNE AND LIQUID EFFLUENTS

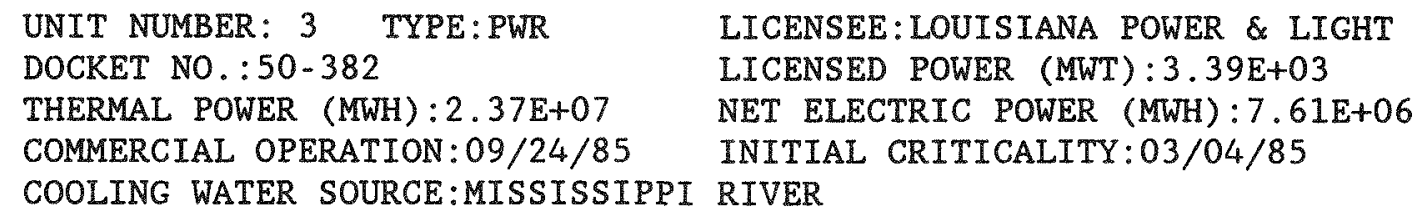

\section{LIQUID EFFLUENTS}

NUCLIDES RELEASED

ACTIVITY (CI)

NA - 24

AR -41

1. $34 \mathrm{E}-03$

4. $93 \mathrm{E}-05$

CR -51

4. $38 \mathrm{E}-02$

$M N-54$

8. $14 E-03$

FE- 55

1. $02 \mathrm{E}-01$

CO -57

$1.05 \mathrm{E}-03$

CO- 58

4. $59 \mathrm{E}-01$

FE -59

$2.28 \mathrm{E}-03$

CO- 60

4. $64 \mathrm{E}-02$

$\mathrm{KR}-85$

$3.49 \mathrm{E}-02$

KR $-85 M$

3. $24 E-04$

$\mathrm{KR}-88$

$6.24 \mathrm{E}-05$

$\mathrm{RB}-88$

$2.10 \mathrm{E}-04$

SR -92

$3.28 \mathrm{E}-04$

NB -95

3. $04 E-02$

ZR-95

1. $66 \mathrm{E}-02$

$\mathrm{NB}-97$

$1.96 \mathrm{E}-03$

ZR -97

$3.59 \mathrm{E}-04$

MO- 99

$2.59 \mathrm{E}-04$

TC-99M

1. $50 \mathrm{E}-04$

RU -103

1. $74 \mathrm{E}-03$

RU -106

$2.19 E-03$

$N / A=N O T$ APPLICABLE 
EFFLUENT AND WASTE DISPOSAL ANNUAL REPORT FOR YEAR 1989 AIRBORNE AND LIQUID EFFLUENTS

\begin{tabular}{|c|c|}
\hline \multicolumn{2}{|l|}{ LIQUID EFFLUENTS } \\
\hline NUCLIDES RELEASED & ACTIVITY (CI) \\
\hline$A G-110 M$ & $1.09 \mathrm{E}-02$ \\
\hline $\mathrm{SN}-113$ & $5.46 \mathrm{E}-03$ \\
\hline SB -122 & $8.27 \mathrm{E}-03$ \\
\hline SB- 124 & $4.16 \mathrm{E}-02$ \\
\hline SB -125 & $1.77 \mathrm{E}-01$ \\
\hline SB -126 & $2.54 \mathrm{E}-03$ \\
\hline$S B-127$ & $9.33 E-04$ \\
\hline I - 131 & $9.33 E-02$ \\
\hline $\mathrm{XE}-131 \mathrm{M}$ & $1.19 E-01$ \\
\hline$I-132$ & $2.40 \mathrm{E}-03$ \\
\hline $\mathrm{TE}-132$ & $1.48 \mathrm{E}-03$ \\
\hline$I-133$ & $7.51 E-04$ \\
\hline$X E-133$ & $8.27 E+00$ \\
\hline $\mathrm{XE}-133 \mathrm{M}$ & $5.10 \mathrm{E}-02$ \\
\hline CS - 134 & $8.14 E-02$ \\
\hline$X E-135$ & $2.89 E-02$ \\
\hline CS -136 & $1.08 E-04$ \\
\hline CS -137 & 1. $30 E-01$ \\
\hline BA -140 & $5.79 E-04$ \\
\hline LA -140 & $3.49 E-03$ \\
\hline$C E-141$ & $3.30 \mathrm{E}-05$ \\
\hline$C E-144$ & $8.12 E-04$ \\
\hline$W-187$ & $1.22 \mathrm{E}-03$ \\
\hline
\end{tabular}

TOTAL AIRBORNE TRITIUM RELEASE $1.26 \mathrm{E}+02$

TOTAL LIQUID TRITIUM RELEASE $3.58 \mathrm{E}+02$

VOLUME OF LIQUID WASTE RELEASED (PRIOR TO DILUTION) LITERS 7.69E+06 VOLUME OF DILUTION WATER USED DURING PERIOD 
EFFLUENT AND WASTE DISPOSAL ANNUAL REPORT FOR YEAR 1989 SOLID EFFLUENTS

SOLID WASTE DISPOSITION

$\begin{array}{cl}\text { NUMBER OF SHIPMENTS } & \text { MODE OF TRANSPORTATION } \\ 3 & \text { TRUCK } \\ 15 & \text { TRUCK } \\ 1 & \text { TRUCK }\end{array}$

ESTIMATE OF MAJOR NUCLIDE COMPOSITION (BY TYPE OF WASTE)

A

CO -58

$\mathrm{CO}-60$

CS -134

CS -137

FE -55

$\mathrm{H}-3$

MN -54

NI -63

B

CO- 58

CO- 60

CS -134

CS -137

FE- 55

MN -54

NI -63

SB- 124

SB- 125

D

CD -109

CO -58

CO -60

FE -55

MN -54

NB -95

NI -59

NI -63

SN -113

ZR-95

TYPE OF WASTE

A. SPENT RESINS, FILTER SLUDGES, EVAPORATOR BOTTOMS, ETC.

B. DRY COMPRESSIBLE WASTE, CONTAMINATED EQUIPMENT, ETC.

C. IRRADIATED COMPONENTS, CONTROL RODS, ETC.

D. OTHER

DEWATERED MECH. FTLTRS.

*VOLUME SHIPPED FOR REDUCTION(NOT FINAL BURIAL VOL.)

\section{DESTINATION \\ BARNWELL SC \\ OAK RIDGE TN \\ RICHLAND WA}

JAN-JUNE JULY-DEC

$\begin{array}{lll}8 & 6.94 \mathrm{E}+00 & 1.46 \mathrm{E}+01 \\ 8 & 3.72 \mathrm{E}+00 & 3.23 \mathrm{E}+00 \\ 8 & 2.72 \mathrm{E}+01 & 2.62 \mathrm{E}+01 \\ 8 & 4.74 \mathrm{E}+01 & 4.18 \mathrm{E}+01 \\ 8 & 3.66 \mathrm{E}+00 & 2.87 \mathrm{E}+00 \\ 8 & & 6.07 \mathrm{E}-03 \\ 8 & 1.64 \mathrm{E}+00 & 1.74 \mathrm{E}+00 \\ 8 & 1.00 \mathrm{E}+01 & 9.47 \mathrm{E}+00 \\ & & \\ 8 & 6.05 \mathrm{E}+01 & 1.52 \mathrm{E}+00 \\ 8 & 3.75 \mathrm{E}+00 & 2.70 \mathrm{E}-01 \\ 8 & 9.63 \mathrm{E}+00 & 4.02 \mathrm{E}-01 \\ 8 & 1.31 \mathrm{E}+01 & 6.23 \mathrm{E}-01 \\ 8 & 1.15 \mathrm{E}+01 & 5.20 \mathrm{E}-01 \\ 8 & 1.64 \mathrm{E}+00 & 1.83 \mathrm{E}-01 \\ 8 & 5.76 \mathrm{E}+00 & 3.66 \mathrm{E}-01 \\ 8 & 8.30 \mathrm{E}-02 & \\ 8 & 9.86 \mathrm{E}-01 & \\ & & \\ 8 & & 7.90 \mathrm{E}-01 \\ 8 & & 6.21 \mathrm{E}+00 \\ 8 & & 3.58 \mathrm{E}+00 \\ 8 & & 1.74 \mathrm{E}+01 \\ 8 & & 5.49 \mathrm{E}-01 \\ 8 & & 6.03 \mathrm{E}-01 \\ 8 & & 1.72 \mathrm{E}+00 \\ 8 & & 1.20 \mathrm{E}+00 \\ 8 & & 4.24 \mathrm{E}-01 \\ 8 & & 5.08 \mathrm{E}-01\end{array}$

UNIT YEAR TOTAL

M3 $3.14 \mathrm{E}+01$

CI $\quad 3.68 \mathrm{E}+02$

M3 7.26E+02*

CI $\quad 4.68 \mathrm{E}+00$

M3

CI

M3 $3.41 \mathrm{E}+00$

CI $3.39 E+01$ 

EFFLUENT AND WASTE DISPOSAL ANNUAL REPORT FOR YEAR 1989
AIRBORNE AND LIQUID EFFLUENTS

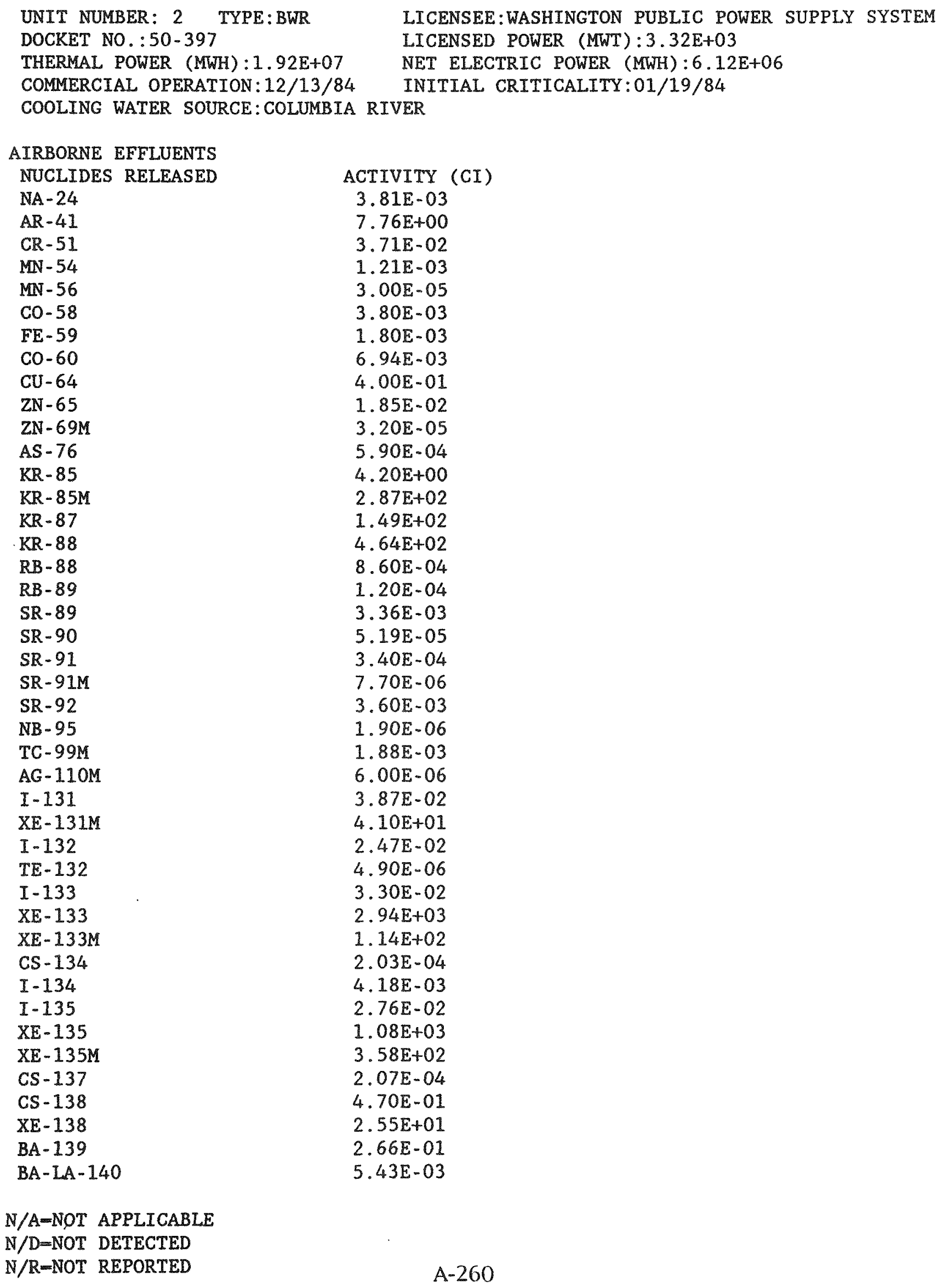


EFFLUENT AND WASTE DISPOSAL ANNUAL REPORT FOR YEAR 1989 AIRBORNE AND LIQUID EFFLUENTS

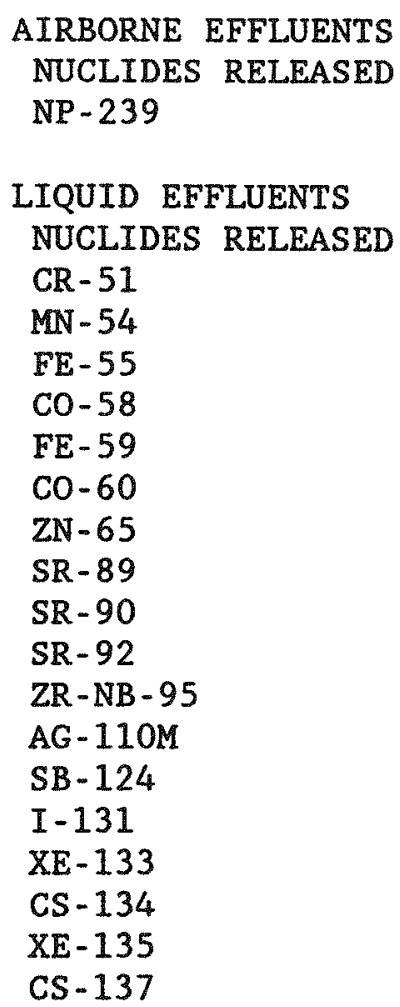

ACTIVITY (CI)

$2.90 E-03$

ACTIVITY (CI)

8.74E-03

2.12E-03

$1.92 E-03$

1.91E-03

1.20E-03

$1.92 \mathrm{E}-02$

$1.41 E-02$

$1.86 \mathrm{E}-05$

$1.44 \mathrm{E}-05$

2.10E-05

$5.00 \mathrm{E}-04$

1. 84E-04

4. 80E-05

9.90E-05

3. $90 \mathrm{E}-05$

1. $60 \mathrm{E}-04$

2. 55E-04

2.00E-04

TOTAL AIRBORNE TRITIUM RELEASE 1.68E+01

TOTAL LIQUID TRITIUM RELEASE $2.03 \mathrm{E}+00$

VOLUME OF LIQUID WASTE RELEASED (PRIOR TO DILUTION) LITERS 3.17 E+06 VOLUME OF DILUTION WATER USED DURING PERIOD

LITERS $1.18 \mathrm{E}+09$ 
INSTALIATION : WNP - 2

EFFLUENT AND WASTE DISPOSAL ANNUAL REPORT FOR YEAR 1989

SOLID EFFLUENTS

SOLID WASTE DISPOSITION

NUMBER OF SHIPMENTS MODE OF TRANSPORTATION

$\begin{array}{rl}2 & 10-142 \text { CASK } \\ 23 & 14-170 \text { CASK } \\ 11 & \text { FLAT BED TRAILER }\end{array}$

ESTIMATE OF MAJOR NUCLIDE COMPOSITION (BY TYPE OF WASTE)

DESTINATION
US ECOLOGY RICHLAND WA
US ECOLOGY RICHLAND WA
US ECOLOGY RICHLAND WA

JAN-JUNE JULY-DEC

A
AG-110M
$\mathrm{CO}-134$
CO- 58
CO- 60
CR -51
CS -137
FE -55
MN - 54
NB-95
ZN-65
ZR-95
CO -58
$\mathrm{CO}-60$
CR -51
FE -55
$\mathrm{H}-3$
MN -54
NB- 95
NI -63
SB- 125
$\mathrm{ZN}-65$
ZR-95

B

\section{TYPE OF WASTE}
A. SPENT RESINS, FILTER SLUDGES, EVAPORATOR BOTTOMS, ETC.
B. DRY COMPRESSIBLE WASTE, CONTAMINATED EQUIPMENT, ETC.
C. IRRADIATED COMPONENTS, CONTROL RODS, ETC.
D. OTHER

$\begin{array}{lll}8 & 5.50 \mathrm{E}-01 & \\ 8 & & 7.60 \mathrm{E}+00 \\ 8 & 5.22 \mathrm{E}+00 & 3.10 \mathrm{E}+00 \\ 8 & 1.90 \mathrm{E}+01 & 2.05 \mathrm{E}+01 \\ 8 & 1.80 \mathrm{E}+01 & 6.60 \mathrm{E}+00 \\ 8 & 5.00 \mathrm{E}-01 & 7.00 \mathrm{E}+00 \\ 8 & 5.38 \mathrm{E}+00 & 5.60 \mathrm{E}+00 \\ 8 & 2.99 \mathrm{E}+00 & 2.80 \mathrm{E}+00 \\ 8 & 3.78 \mathrm{E}+00 & 2.30 \mathrm{E}+00 \\ 8 & 4.10 \mathrm{E}+01 & 4.17 \mathrm{E}+01 \\ 8 & 2.49 \mathrm{E}+00 & 1.40 \mathrm{E}+00 \\ & & \\ 8 & 1.26 \mathrm{E}+00 & 4.62 \mathrm{E}-01 \\ 8 & 2.71 \mathrm{E}+01 & 4.18 \mathrm{E}+01 \\ 8 & 9.83 \mathrm{E}+00 & 3.38 \mathrm{E}-01 \\ 8 & 2.32 \mathrm{E}+01 & 3.33 \mathrm{E}+01 \\ 8 & 2.28 \mathrm{E}+00 & \\ 8 & 1.11 \mathrm{E}+00 & 1.60 \mathrm{E}+00 \\ 8 & 1.32 \mathrm{E}+00 & 8.13 \mathrm{E}-01 \\ 8 & 1.55 \mathrm{E}+00 & 2.70 \mathrm{E}+00 \\ 8 & & 2.00 \mathrm{E}-01 \\ 8 & 2.32 \mathrm{E}+01 & 1.80 \mathrm{E}+01 \\ 8 & 7.60 \mathrm{E}-01 & 4.00 \mathrm{E}-01\end{array}$

UNIT YEAR TOTAL

M3 $\quad 1.54 \mathrm{E}+02$

CI $\quad 7.08 \mathrm{E}+02$

M3 $2.10 \mathrm{E}+02$

CI $\quad 3.92 \mathrm{E}+02$

M3

CI

M3

CI 


\section{EFFLUENT AND WASTE DISPOSAL ANNUAL REPORT FOR YEAR 1989} AIRBORNE AND LIQUID EFFLUENTS

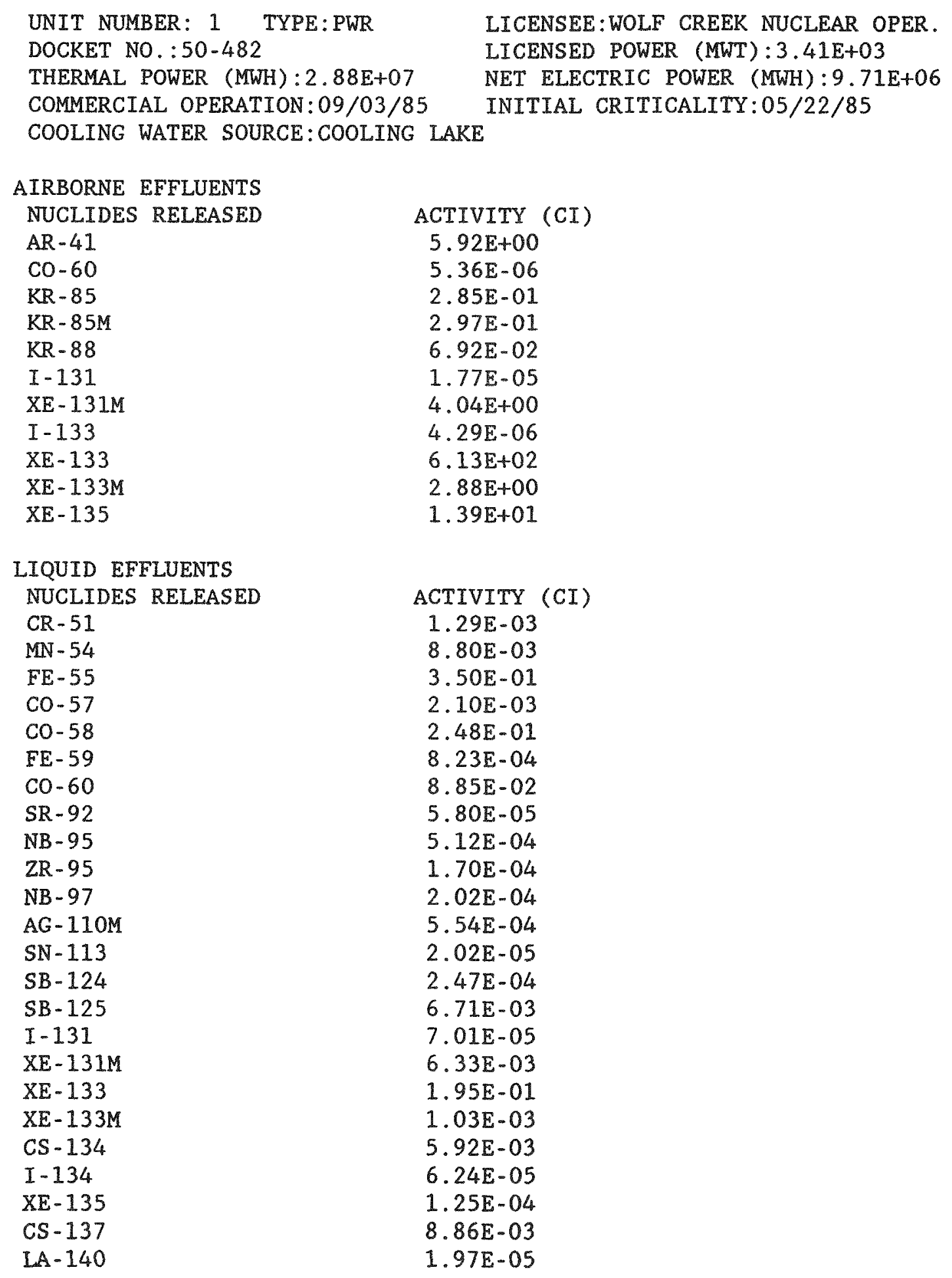


EFFLUENT AND WASTE DISPOSAL ANNUAL REPORT FOR YEAR 1989 AIRBORNE AND LIQUID EFFLUENTS

TOTAL AIRBORNE TRITIUM RELEASE 1.23E+02

TOTAL LIQUID TRITIUM RELEASE $5.88 \mathrm{E}+02$

VOLUME OF LIQUID WASTE RELEASED (PRIOR TO DILUTION) LITERS $\quad 8.85 \mathrm{E}+07$

VOLUME OF DILUTION WATER USED DURING PERIOD 
EFFLUENT AND WASTE DISPOSAL ANNUAL REPORT FOR YEAR 1989 SOLID EFFLUENTS

SOLID WASTE DISPOSITION

NUMBER OF SHIPMENTS MODE OF TRANSPORTATION 11 TRUCK

ESTIMATE OF MAJOR NUCLIDE COMPOSITION (BY TYPE OF WASTE)

A

$$
\text { C }-14
$$

$\mathrm{CO}-58$

$\mathrm{CO}-57$

CO- 58

CO- 60

CR -51

CS -134

CS -137

FE- 55

FE- 59

H -3

MN -54

NB -95

NI -63

RU - 106

SB-125

SN- 113

SR- 89

SR -90

ZR-95

$B$

AG-11OM

C. 14

CO- 57

CO- 58

CO- 60

CR -51

CS -134

CS -137

FE -55

FE -59

$\mathrm{MN}-54$

NI -63

SB- 125

SN-113

ZR-95
DESTINATION

BARNWELL SC

JAN-JUNE JULY-DEC

1. $62 \mathrm{E}-01$

$7.00 \mathrm{E}-03$

$8.00 \mathrm{E}-02$

$2.96 \mathrm{E}+00$

$2.14 \mathrm{E}+00 \quad 5.75 \mathrm{E}+00$

4. $61 \mathrm{E}+00$

$2.28 \mathrm{E}-01 \quad 1.27 \mathrm{E}+00$

$3.05 \mathrm{E}-01 \quad 1.80 \mathrm{E}+00$

$5.64 \mathrm{E}+01 \quad 7.37 \mathrm{E}+01$

2. $30 \mathrm{E}+00$

2. $95 \mathrm{E}+00$

$4.98 E-01 \quad 1.03 E+00$

$5.00 \mathrm{E}-03 \quad 6.20 \mathrm{E}-02$

$3.94 \mathrm{E}+01 \quad 3.05 \mathrm{E}+00$

9.22E-01 5.00E-02

1. $80 \mathrm{E}-02 \quad 1.65 \mathrm{E}-01$

$2.00 \mathrm{E}-03$

$8.65 \mathrm{E}-02$

$6.00 E-03$

$5.00 \mathrm{E}-03 \quad 3.60 \mathrm{E}-02$

$7.76 E-01 \quad 1.20 E-02$

5.50E-02 3.00E-02

$1.99 \mathrm{E}+00$

$2.02 \mathrm{E}-01$

2. $38 \mathrm{E}+01$

3. $62 \mathrm{E}+00$

$2.05 \mathrm{E}+01$

$1.67 \mathrm{E}+01$

1. $25 \mathrm{E}+00$

3. 20E-02

5. $27 \mathrm{E}-01$

1. $10 \mathrm{E}-02$

$2.00 \mathrm{E}-03$

3. $04 E+01$

5. $25 \mathrm{E}+01$

1. $35 E+00$

4. $39 \mathrm{E}+00$

$2.58 \mathrm{E}+00$

1. $23 \mathrm{E}+01$

2. $33 \mathrm{E}+01$

1. $79 \mathrm{E}+00$

1. $10 \mathrm{E}+00$

3. $84 \mathrm{E}-01$

$5.09 \mathrm{E}-01$ 
EFFLUENT AND WASTE DISPOSAL ANNUAL REPORT FOR YEAR 1989 SOLID EFFLUENTS

TYPE OF WASTE

A. SPENT RESINS, FILTER SLUDGES, EVAPORATOR BOTTOMS, ETC.

B. DRY COMPRESSIBLE WASTE, CONTAMINATED EQUIPMENT, ETC.

C. IRRADIATED COMPONENTS, CONTROL RODS, ETC.

D. OTHER
UNIT YEAR TOTAL

M3 $4.18 \mathrm{E}+01$

CI $1.26 \mathrm{E}+03$

M3 1.09E+02 *

CI $\quad 3.36 \mathrm{E}+00$

M3

CI

M3

CI

*VOLUME AFTER COMPACTION 
EFFLUENT AND WASTE DISPOSAL ANNUAL REPORT FOR YEAR 1989 AIRBORNE AND LIQUID EFFLUENTS

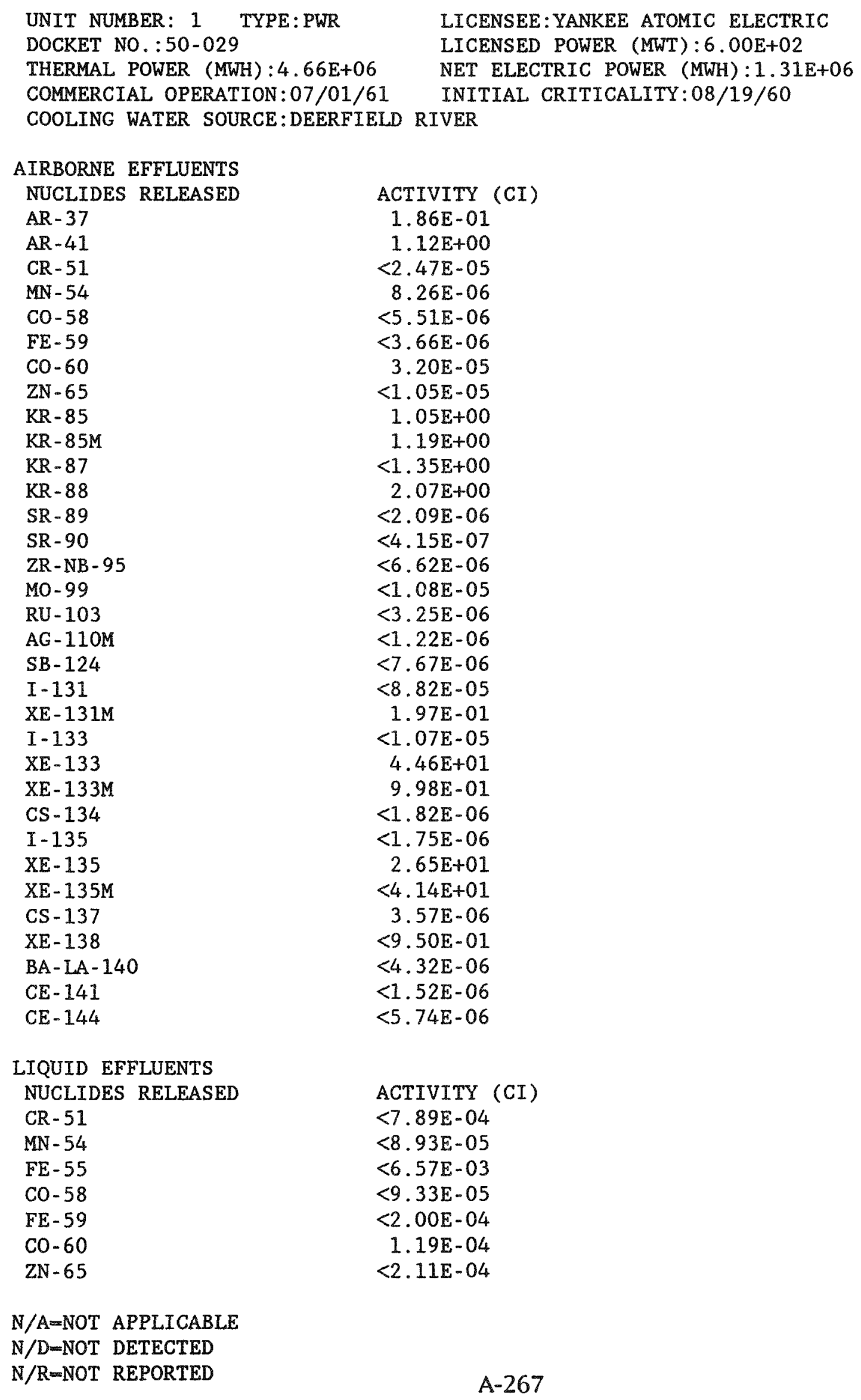

N/A=NOT APPLICABLE 
EFFLUENT AND WASTE DISPOSAL ANNUAL REPORT FOR YEAR 1989 AIRBORNE AND LIQUID EFFLUENTS

\author{
LIQUID EFFLUENTS \\ NUCLIDES RELEASED \\ $\mathrm{SE}-75$ \\ $\mathrm{KR}-85$ \\ SR -89 \\ SR -90 \\ ZR-NB-95 \\ MO -99 \\ TC $-99 \mathrm{M}$ \\ RU -103 \\ $A G-110 M$ \\ SB -124 \\ $S B-125$ \\ $\mathrm{I}-131$ \\ $\mathrm{XE}-131 \mathrm{M}$ \\ $I-133$ \\ $\mathrm{XE}-133$ \\ $\mathrm{XE}-133 \mathrm{M}$ \\ CS -134 \\ $\mathrm{XE}-135$ \\ CS -136 \\ CS -137 \\ BA-LA- 140 \\ CE- 141 \\ CE-144
}

ACTIVITY (CI)

$<1.12$ E-04

$<5.44 \mathrm{E}-02$

$<7.72 \mathrm{E}-04$

$<4.21 \mathrm{E}-04$

$<1.68 \mathrm{E}-04$

$<7.12 E-04$

$<3.01 \mathrm{E}-05$

$<9.82 \mathrm{E}-05$

$<9.92 \mathrm{E}-05$

$<8.60 \mathrm{E}-05$

$<2.82 \mathrm{E}-04$

$<1.11 \mathrm{E}-04$

$<3.58 \mathrm{E}-03$

$<8.17 \mathrm{E}-05$

$<3.24 \mathrm{E}-02$

$<6.08 \mathrm{E}-04$

$<3.92$ E-04

$<1.65 \mathrm{E}-04$

$<9.64 \mathrm{E}-05$

$7.36 \mathrm{E}-04$

$<3.45 \mathrm{E}-04$

$<1.39 E-04$

$<6.37 \mathrm{E}-04$

TOTAL AIRBORNE TRITIUM RELEASE $6.64 E+00$

TOTAL LIQUID TRITIUM RELEASE $1.68 E+02$

VOLUME OF LIQUID WASTE RELEASED (PRIOR TO DILUTION) LITERS $2.39 E+07$ VOLUME OF DILUTION WATER USED DURING PERIOD

LITERS $3.65 \mathrm{E}+11$ 
INSTALLATION:YANKEE ROWE

EFFLUENT AND WASTE DISPOSAL ANNUAL REPORT FOR YEAR 1989

SOLID EFFLUENTS

SOLID WASTE DISPOSITION

NUMBER OF SHIPMENTS MODE OF TRANSPORTATION

DESTINATION

19

TRUCK

BARNWELL SC

IRRADIATED FUEL SHIPMENTS (DISPOSITION)

NUMBER OF SHIPMENTS MODE OF TRANSPORTATION

0

N/A

DESTINATION

N/A

ESTIMATE OF MAJOR NUCLIDE COMPOSITION (BY TYPE OF WASTE)

JAN-JUNE JULY-DEC

A

C- 14

$\mathrm{CO}-60$

CS -134

CS -137

$\mathrm{FE}-55$

$\mathrm{H}-3$

NI -63

B

C -14

CO -60

CS -134

CS -137

FE -55

$\mathrm{MN}-54$

NB -95

NI -63

$\begin{array}{lll}8 & & 8.40 \mathrm{E}-02 \\ 8 & 2.92 \mathrm{E}+00 & 2.06 \mathrm{E}+00 \\ 8 & 1.71 \mathrm{E}+01 & 2.39 \mathrm{E}+01 \\ 8 & 2.41 \mathrm{E}+01 & 2.75 \mathrm{E}+01 \\ 8 & 1.71 \mathrm{E}+01 & 1.53 \mathrm{E}+01 \\ 8 & 3.69 \mathrm{E}+01 & 2.97 \mathrm{E}+01 \\ 8 & 1.70 \mathrm{E}+00 & 1.23 \mathrm{E}+00 \\ & & \\ 8 & & 1.13 \mathrm{E}-01 \\ 8 & 4.01 \mathrm{E}+00 & 2.84 \mathrm{E}+00 \\ 8 & 3.00 \mathrm{E}+01 & 3.42 \mathrm{E}+01 \\ 8 & 3.33 \mathrm{E}+01 & 3.74 \mathrm{E}+01 \\ 8 & 2.68 \mathrm{E}+01 & 2.17 \mathrm{E}+01 \\ 8 & 1.05 \mathrm{E}+00 & \\ 8 & 1.80 \mathrm{E}+00 & 2.01 \mathrm{E}+00 \\ 8 & 2.12 \mathrm{E}+00 & 1.66 \mathrm{E}+00\end{array}$

TYPE OF WASTE

A. SPENT RESINS, FILTER SLUDGES, EVAPORATOR

UNIT YEAR TOTAL BOTTOMS, ETC.

M3 $1.16 \mathrm{E}+02$

CI $\quad 1.19 \mathrm{E}+01$

M3 $1.82 \mathrm{E}+02$

CI $\quad 5.89 E+00$

EQUIPMENT, ETC.

M3

. IRRADIATED COMPONENTS, CONTROL

CI

RODS, ETC.

M3

D. OTHER

CI 
EFFLUENT AND WASTE DISPOSAL ANNUAL REPORT FOR YEAR 1989 AIRBORNE AND LIQUID EFFLUENTS

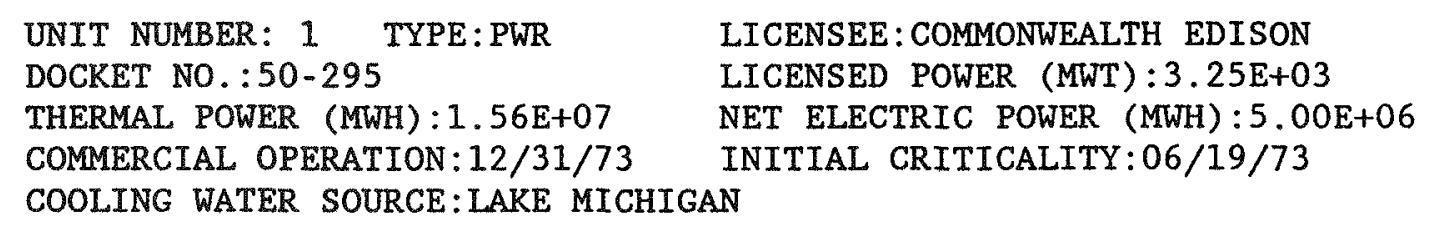

LIQUID EFFLUENTS

NA -24

CR -51

MN -54

$F E-55$

$\mathrm{CO}-57$

CO- 58

FE- 59

CO- 60

$\mathrm{KR}-85 \mathrm{M}$

$\mathrm{RB}-88$

SR -89

SR -90

NB- 95

ZR -95

MO-99

TC-99M

AG-110M

SN -113

SB- 124

$\mathrm{SB}-125$

SB- 126

I-13I

$\mathrm{XE}-131 \mathrm{M}$

$\mathrm{I}-132$

I-133

$\mathrm{XE}-133$

$\mathrm{XE}-133 \mathrm{M}$

CS -134

I -135

$\mathrm{XE}-135$

CS -137

IA -140

TOTAL LIQUID TRITIUM RELEASE
NUCLIDES RELEASED

$$
\begin{aligned}
& \text { ACTIVITY (CI) } \\
& 2.00 E-03 \\
& 3.50 E-02 \\
& 2.72 E-03 \\
& 8.42 E-02 \\
& 4.32 E-03 \\
& 2.18 E-01 \\
& 3.70 E-03 \\
& 1.41 E-01 \\
& 2.70 E-05 \\
& 2.10 E-04 \\
& 1.20 E-04 \\
& 8.80 E-06 \\
& 6.57 E-03 \\
& 5.74 E-03 \\
& 2.45 E-04 \\
& 2.50 E-05 \\
& 5.47 E-02 \\
& 6.20 E-05 \\
& 6.89 E-02 \\
& 1.78 E-01 \\
& 6.60 E-04 \\
& 2.43 E-02 \\
& 4.00 E-04 \\
& 3.60 E-03 \\
& 6.20 E-03 \\
& 3.91 E-01 \\
& 1.27 E-03 \\
& 3.15 E-02 \\
& 3.00 E-04 \\
& 3.47 E-03 \\
& 3.27 E-02 \\
& 1.19 E-03 \\
& 1.81 E+02 \\
&
\end{aligned}
$$

$\begin{array}{llll}\text { VOLUME OF LIQUID WASTE RELEASED (PRIOR TO DILUTION) } & \text { LITERS } & 4.96 \mathrm{E}+07 \\ \text { VOLUME OF DIIUTION WATER USED DURING PERIOD } & \text { LITERS } & 1.01 \mathrm{E}+12\end{array}$ 
EFFLUENT AND WASTE DISPOSAL ANNUAL REPORT FOR YEAR 1989 AIRBORNE AND LIQUID EFFLUENTS
UNIT NUMBER: 2 TYPE:PWR
DOCKET NO. : 50-304
THERMAI POWER (MWH) :2.40E+07
COMMERCIAL OPERATION : 09/17/74
COOLING WATER SOURCE:LAKE MICHIGAN
LICENSEE : COMMONWEALTH EDISON
LICENSED POWER (MWT) : $3.25 E+03$
NET ELECTRIC POWER (MWH): $7.69 E+06$
INITIAL CRITICAIITY: $12 / 24 / 73$

\section{LIQUTD EFFLUENTS}

NUCLIDES RELEASED

NA -24

CR -51

MN -54

FE- 55

CO- 57

Co- 58

FE -59

CO -60

ACTIVITY (CI)

$\mathrm{ZN}-65$

$\mathrm{SE}-75$

$\mathrm{KR}-85 \mathrm{M}$

3. $34 \mathrm{E}-03$

$1.35 \mathrm{E}-01$

1. $06 \mathrm{E}-02$

1. $60 \mathrm{E}-01$

2. $37 \mathrm{E}-03$

8. $80 \mathrm{E}-01$

$8.80 \mathrm{E}-03$

3. $54 \mathrm{E}-01$

$7.50 \mathrm{E}-05$

1. $60 \mathrm{E}-04$

KR -88

SR-90

NB- 95

ZR-95

MO- 99

TC-99M

$3.60 \mathrm{E}-05$

$2.60 \mathrm{E}-04$

$8.20 \mathrm{E}-06$

$4.52 \mathrm{E}-02$

$2.76 \mathrm{E}-02$

RU-103

$3.32 E-03$

$5.60 \mathrm{E}-05$

AG- $110 \mathrm{M}$

$4.40 E-04$

$\mathrm{SN}-113$

$2.29 E-01$

SN-117M

$6.29 E-03$

$\mathrm{SB}-124$

1. $83 \mathrm{E}-03$

$S B-125$

1. $69 \mathrm{E}-01$

$\mathrm{SB}-126$

2. $85 E-01$

$\mathrm{I}-131$

$6.35 E-03$

XE-131M

6.99E-02

I -132

$5.80 \mathrm{E}-03$

TE- 132

7. 20E-04

I -133

$3.90 E-04$

$\mathrm{XE}-133$

$2.85 \mathrm{E}-02$

$X E-133 M$

$1.64 \mathrm{E}+00$

CS -134

1. $77 \mathrm{E}-02$

$I-135$

$5.98 \mathrm{E}-02$

$\mathrm{XE}-135$

4. $95 \mathrm{E}-04$

CS -137

3. $15 \mathrm{E}-02$

IA -140

8.17E-02

$4.07 E-03$

TOTAL LIQUID TRITIUM RELEASE

8. $66 \mathrm{E}+02$

VOLUME OF LIQUID WASTE RELEASED (PRIOR TO DILUTION)

LITERS

$1.29 \mathrm{E}+08$

VOLUME OF DILUTION WATER USED DURING PERIOD

LITERS

1. $44 E+12$ 
EFFLUENT AND WASTE DISPOSAL ANNUAL REPORT FOR YEAR 1989 AIRBORNE AND LIQUID EFFLUENTS

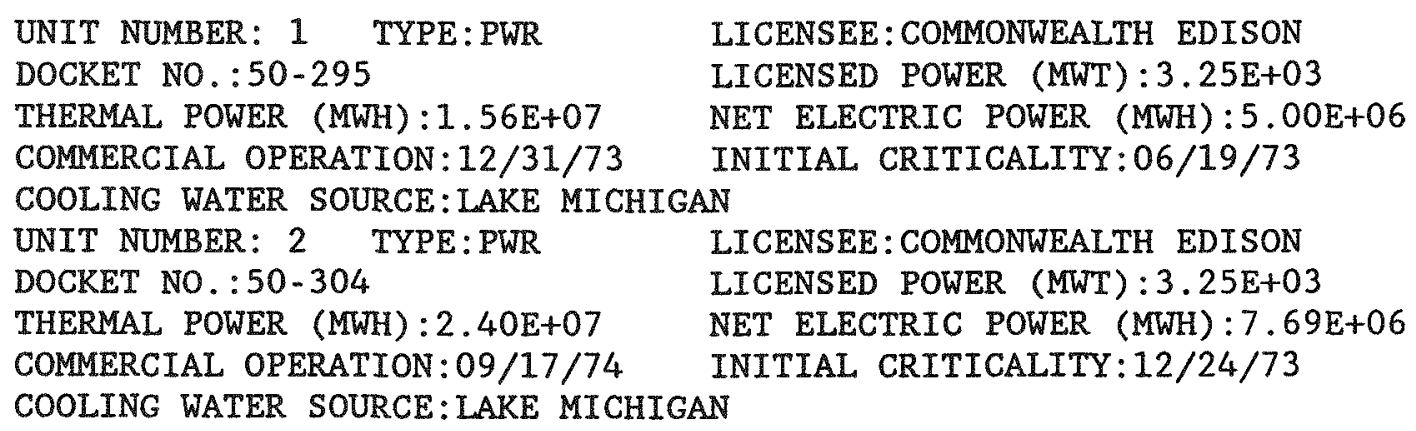

UNIT NUMBER: 1 TYPE:PWR DOCKET NO. : 50-295

THERMAL POWER (MWH) $: 1.56 \mathrm{E}+07$ COMMERCIAL OPERATION: $12 / 31 / 73$ COOLING WATER SOURCE:LAKE MICHIGAN UNIT NUMBER: 2 TYPE: PWR DOCKET NO. : $50-304$ THERMAL POWER (MWH) : $2.40 \mathrm{E}+07$ COMMERCIAL OPERATION:09/17/74 COOLING WATER SOURCE:LAKE MICHIGAN

LICENSEE: COMMONWEALTH EDISON

LICENSED POWER (MWT) $: 3.25 E+03$

NET ELECTRIC POWER (MWH):5.00E+06 INITIAL CRITICALITY:06/19/73

LICENSEE: COMMONWEALTH EDISON LICENSED POWER (MWT) : $3.25 E+03$ NET ELECTRIC POWER (MWH) : 7.69E+06 INITIAL CRITICALITY: $12 / 24 / 73$

\begin{tabular}{|c|c|}
\hline AIRBORNE EFFLUENTS & \\
\hline NUCLIDES RELEASED & ACTIVITY (CI) \\
\hline$N A-24$ & $5.74 E-05$ \\
\hline$A R-41$ & $7.62 E+00$ \\
\hline CR -51 & $3.40 E-06$ \\
\hline $\mathrm{MN}-54$ & $2.05 E-06$ \\
\hline$M N-56$ & $1.44 \mathrm{E}-07$ \\
\hline $\mathrm{CO}-58$ & $2.09 E-04$ \\
\hline$C 0-60$ & $8.78 E-05$ \\
\hline $\mathrm{BR}-82$ & $2.21 E-05$ \\
\hline $\mathrm{KR}-85$ & $2.10 E+00$ \\
\hline $\mathrm{KR}-85 \mathrm{M}$ & $3.45 E-01$ \\
\hline $\mathrm{KR}-87$ & $4.21 E-01$ \\
\hline$K R-88$ & $1.69 E+00$ \\
\hline$R B-88$ & $9.16 E-03$ \\
\hline SR -89 & $8.10 \mathrm{E}-08$ \\
\hline$S R-90$ & $1.87 E-08$ \\
\hline $\mathrm{SR}-91$ & 1. $30 \mathrm{E}-07$ \\
\hline $\mathrm{NB}-95$ & $5.61 E-06$ \\
\hline$Z R-95$ & $3.40 E-06$ \\
\hline $\mathrm{MO}-99$ & $2.30 \mathrm{E}-06$ \\
\hline $\mathrm{TC}-99 \mathrm{M}$ & $8.30 E-08$ \\
\hline $\mathrm{RU}-103$ & $4.60 E-08$ \\
\hline$A G-110 M$ & $8.21 E-08$ \\
\hline SN-113 & $4.40 E-10$ \\
\hline$I-131$ & $2.02 E-03$ \\
\hline$X E-131 M$ & $3.74 \mathrm{E}-01$ \\
\hline$I-132$ & $6.19 E-05$ \\
\hline$I-133$ & $7.62 \mathrm{E}-04$ \\
\hline $\mathrm{XE}-133$ & $1.05 E+03$ \\
\hline$X E-133 M$ & $96 F+00$ \\
\hline $\mathrm{CS}-134$ & $2.23 E-05$ \\
\hline$I-134$ & $9.83 E-05$ \\
\hline$I-135$ & $1.13 E-03$ \\
\hline $\mathrm{XE}-135$ & $5.32 E+01$ \\
\hline$X E-135 M$ & $2.05 \mathrm{E}+00$ \\
\hline CS -136 & $2.94 \mathrm{E}-06$ \\
\hline CS -137 & $8.92 E-04$ \\
\hline $\mathrm{CS}-138$ & $2.90 E-04$ \\
\hline$X E-138$ & $1.29 E+00$ \\
\hline
\end{tabular}
$5.74 E-05$
$7.62 \mathrm{E}+00$
$3.40 \mathrm{E}-06$
$2.05 E-06$
$1.44 \mathrm{E}-07$
$2.09 \mathrm{E}-04$
$8.78 E-05$
$2.21 E-05$
$2.10 E+00$
$3.45 \mathrm{E}-01$
4. $21 \mathrm{E}-01$
$1.69 \mathrm{E}+00$
$9.16 \mathrm{E}-03$
$8.10 \mathrm{E}-08$
$1.87 \mathrm{E}-08$
1. $30 \mathrm{E}-07$
$5.61 \mathrm{E}-06$
3. $40 E-06$
. $30 \mathrm{E}-06$
.30E-08
4. 40E -10
$.02 E-03$
$7.62 \mathrm{E}-04$
$.83 E-05$
$2.05 \mathrm{E}+00$
$8.92 \mathrm{E}-04$
1.29E+00

N/A=NOT APPLICABLE 
EFFLUENT AND WASTE DISPOSAL ANNUAL REPORT FOR YEAR 1989 AIRBORNE AND LIQUID EFFLUENTS
AIRBORNE EFFLUENTS
NUCLIDES RELEASED
BA -139
ACTIVITY (CI)
$\mathrm{BA}-140$
$1.44 \mathrm{E}-06$
$2.80 \mathrm{E}-07$
LA -140
$6.51 \mathrm{E}-08$
W- 187
$5.38 \mathrm{E}-07$

TOTAL AIRBORNE TRITIUM RELEASE 6.92E+01 
SOLID WASTE DISPOSITION NUMBER OF SHIPMENTS

MODE OF TRANSPORTATION CNSI

$\mathrm{N} / \mathrm{R}$

33

1
DESTINATION

CHANNAHON IL

BARNWELL SC

SEG, OAK RIDGE TN
TYPE OF WASTE

A. SPENT RESINS, FILTER SLUDGES, EVAPORATOR BOTTOMS, ETC.

B. DRY COMPRESSIBLE WASTE, CONTAMINATED EQUIPMENT, ETC.

C. IRRADIATED COMPONENTS, CONTROL RODS, ETC.

D. OTHER
UNIT YEAR TOTAL

M3 $1.45 \mathrm{E}+02$

CI $\quad 3.27 E+03$

M3 $6.89 \mathrm{E}+01$ *

CI $\quad 3.07 \mathrm{E}+02$

M3

CI

M3

CI

*VOLUME AFTER REDUCTION 
(See instructions on the neverse) NUREG/CR-2907

2. TITLE AND SUBTITLE

BIIL-NUREG-51531

Vo1. 10

Radioactive Materials Released from Nuclear Power Plants

Annual Report 1989

\section{AUTHOR(S)}

J. Tichler, K. Norden, J. Congeni

DATE REPORT PUBLISHED

\begin{tabular}{|l|l}
\hline MONTH & YEAR
\end{tabular}

September 1992 B2234

6. TYPE OF REPORT

Annual Technical

7. PERIOD COVERED (Inclusive Dates) 1989

8. PERFORMING ORGANIZATION - NAME AND ADDRESS /ff NRC, provide Division, Office or Aegion, U.S. Nuctear Regulatory Commission, and mailing address, if contractor, provide nome and mailing address?

Brookhaven National Laboratory

Upton, NY 11973

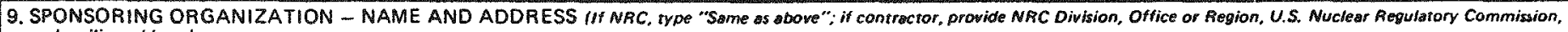
and mailing address.'

Office of Information Resources Management

U.S. Nuclear Regulatory Commission

Washington, DC 20555

\section{SUPPLEMENTARY NOTES}

\section{ABSTRACT 1200 words or less)}

Releases of radioactive materials in airborne and liquid effluents from commercial light water reactors during 1939 have been compiled and reported. Data on solid waste shipments as well as selected operating information have been included. This report supplements earlier annual reports issued by the former Atomic Energy Commission and the Nuclear Regulatory Commission. The 1989 release data are summarized in tabular form. Data covering specific radionuclides are summarized. 


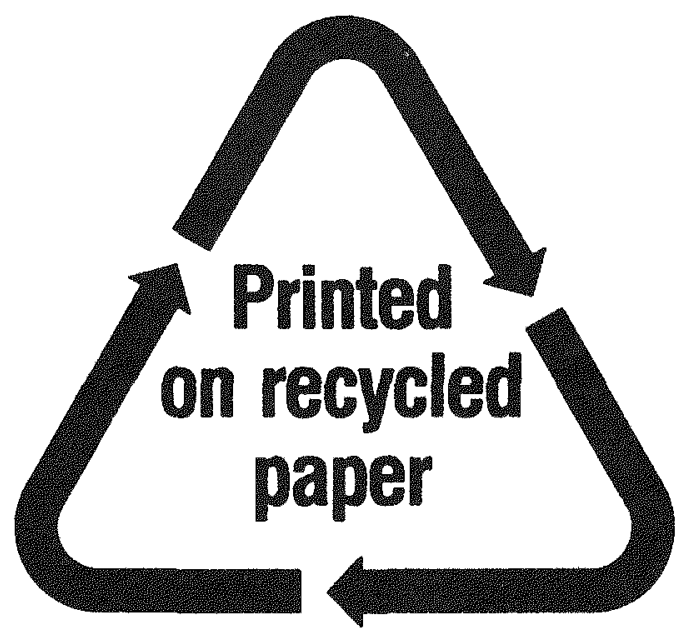

Federal Recycling Program 